\title{
Gespräche im Mentorat
}

Strukturlogiken und Interaktionsdynamiken eines Hochschulformats zur Unterstützung individueller Professionalisierung in der Lehrerinnen- und Lehrerbildung

\author{
Dissertation \\ zur Erlangung des Doktorgrades \\ der Sozialwissenschaftlichen Fakultät \\ der Georg-August-Universität Göttingen
}

vorgelegt von

Simone Herzog

geboren in Zürich

Göttingen, 2021 

Für meine Eltern 



\section{Danksagung}

Viele Menschen haben mich beim vorliegenden Forschungsvorhaben unterstützt und begleitet. Ein erstes Dankeschön geht an die Studierenden und die Mentorinnen und Mentoren, die ich an dieser Stelle zwar namentlich nicht nennen kann, es mir aber ermöglichten, ihre Gespräche im Rahmen dieser Forschungsarbeit auszuwerten.

Mein grösster Dank gilt meinen beiden Betreuungspersonen Katharina Kunze und Tobias Leonhard für ihre fortwährende Begleitung und grosse Unterstützung während der ganzen Promotionsphase. Die gemeinsamen Gespräche und Arbeitsphasen erlebte ich als sehr konstruktiv, wertvoll, wohlwollend und stets im Dienst der Sache. Ihre Begeisterung für die qualitative Sozialforschung ist ansteckend und fasziniert mich. Tobias Leonhard hat mich nicht nur dazu ermuntert, diese Arbeit in Angriff zu nehmen, und mir auch die Unterstützung seitens der Institution der Pädagogischen Hochschule der Fachhochschule Nordwestschweiz gesichert, sondern mich auch während der ganzen Promotionsphase institutionell und persönlich bestmöglich und mit grossem Engagement unterstützt.

Meine Daten konnte ich in verschiedenen Forschungswerkstätten in der Schweiz und in Deutschland vorlegen und gemeinsam mit den Teilnehmenden interpretieren. Besonderer Dank gilt hier den Kolleginnen und Kollegen der Forschungswerkstatt des Arbeitsbereichs "Erziehungswissenschaft» an der Georg-August-Universität in Göttingen. Das gemeinsame Rekonstruieren erlebte ich als sehr bereichernd. Auch möchte ich mich bei den Kolleginnen und Kollegen der Pädagogischen Hochschulen in Bern und Zürich wie auch der Fachhochschule Nordwestschweiz, der Peer-Mentoring-Gruppe «Qualizüri» der Universität Zürich sowie bei Forscherinnen und Forschern in Werkstätten an verschiedenen Tagungen im In- und Ausland für das gemeinsame Rekonstruieren bedanken.

Meiner Doktorandenkollegin Rosa Reinisch aus Göttingen verdanke ich fachlich und auch persönlich sehr viel Unterstützung. Unsere gemeinsamen Schreibwochen in den Schweizer Bergen waren für mich überaus wertvoll und ich werde diese schönen und verbindenden Momente vermissen.

Ferner bedanke ich mich bei allen Personen meines persönlichen und beruflichen Umfelds, meinen Freundinnen und Freunden und meinen Arbeitskolleginnen und Arbeits- 
kollegen für ihre Geduld, ihre Ermutigung, ihr Interesse und die Unterstützung während dieser Zeit.

Widmen möchte ich diese Arbeit meinen lieben Eltern. Schon sehr früh und über all die Jahre hinweg haben sie mir gezeigt und vorgelebt, dass man mit Ausdauer und dem nötigen Fokus auch grosse Vorhaben «schaffen` kann. Diese Ressource war für mich in den vergangenen Monaten ausserordentlich wichtig und wertvoll. Für ihr Vorbild bin ich ihnen zutiefst dankbar.

Simone Herzog, im März 2021 
Inhaltsverzeichnis

1 Einleitung

$\begin{array}{lll}1.1 & \text { Kontextualisierung der Untersuchung } & 2\end{array}$

1.2 Ziel der Untersuchung und Entwicklung der Fragestellung 6

$\begin{array}{lll}1.3 & \text { Aufbau der Arbeit } & 11\end{array}$

$\underline{2}$ Das Mentorat als Untersuchungsgegenstand 15

2.1 Annäherung an das Feld 15

2.1.1 Begriffliche Auseinandersetzung mit Mentoring 16

2.1.2 Formalstruktur und Konzeption des Mentorats am IKU der PH FHNW 19

2.1.3 Analyse von potenziellen Spannungsfeldern 22

2.1.3.1 Spannungsfeld zwischen Beratung, Beurteilung und Rechenschaftsablegung 23

2.1.3.2 Spannungsfeld zwischen berufspraktischen Bewältigungsanforderungen und reflexiver Distanz 24

2.1.3.3 Spannungsfeld zwischen Freiwilligkeitsansprüchen und Verpflichtung 25

2.1.3.4 Spannungsfeld zwischen Prozessoffenheit und institutionellen Vorgaben 26

2.2 Diskurslinien und Forschungsstand von Mentoring in der Lehrerinnen- und $\begin{array}{ll}\text { Lehrerbildung } & 27\end{array}$

2.3 Theoretische Rahmung 42

2.3.1 Perspektiven Professionalisierung 42

2.3.2 Bestimmungsansätze der Professionalisierungstheorie 43

2.3.2.1 Kompetenzorientierter Professionalisierungsansatz 44

2.3.2.2 Strukturtheoretischer Professionalisierungsansatz 45

2.3.2.3 Berufsbiografischer Professionalisierungsansatz 47

2.3.3 Strukturtheoretische Bezugskonzepte 49

2.3.3.1 Krise und Routine $\quad 50$

2.3.3.2 Antinomien professionellen Handelns 51

2.3.3.3 Arbeitsbündnis $\quad 55$ 
2.3.3.4 Pädagogische Permissivität

3.2 Theoretische und methodologische Rahmung

3.2.1 Gegenstandstheoretische Bestimmung 71

3.2.2 Methodologische Grundlagen der Objektiven Hermeneutik 72

3.3 Methodisches Vorgehen 76

3.3.1 Erhebung und Sampling 76

3.3.2 Methodische Prinzipien und Auswertung 79

4.1.1 Eröffnungssequenz: Ich zeige dir wo wir hingehen 87

4.1.1.1 Rekonstruktion der einzelnen Sequenzen

4.1.1.2 Zusammenfassung und riskante Fallstrukturhypothese der Eröffnungssequenz Ich zeige dir wo wir hingehen

4.1.2 Zweite Sequenz: Du sprichst von Struktur und Planung 104

4.1.2.1 Rekonstruktion der einzelnen Sequenzen 105

4.1.2.2 Zusammenfassung und riskante Fallstrukturhypothese der Sequenz Du sprichst von Struktur und Planung

4.1.3 Dritte Sequenz: Was ist aber auch dann eigentlich der noch wichtigere Schritt 122

4.1.3.1 Rekonstruktion der einzelnen Sequenzen

4.1.3.2 Zusammenfassung und riskante Fallstrukturhypothese der Sequenz Was ist aber auch dann eigentlich der noch wichtigere Schritt 
4.2.1.2 Zusammenfassung und riskante Fallstrukturhypothese der Eröffnungssequenz Ich begrüsse Sie recht herzlich

4.2.2 Zweite Sequenz: Aber eigentlich würde ich Ihrer These widersprechen

4.2.2.1 Rekonstruktion der einzelnen Sequenzen

4.2.2.2 Zusammenfassung und riskante Fallstrukturhypothese der Sequenz Aber eigentlich würde ich Ihrer These widersprechen

4.2.3 Dritte Sequenz: Sie verteidigen jetzt das Mentorat

4.2.3.1 Rekonstruktion der einzelnen Sequenzen

4.2.3.2 Zusammenfassung und riskante Fallstrukturhypothese der Sequenz Sie verteidigen jetzt das Mentorat

4.2.4 Fallzusammenfassung und finale Fallbestimmung: Frau Steffen \& Herr Bucher 194

4.3 Mentoratsgespräch Ingrid \& Lorenz

4.3.1 Eröffnungssequenz: Ich darf noch ein bisschen Kaffee trinken 198

4.3.1.1 Rekonstruktion der einzelnen Sequenzen

4.3.1.2 Zusammenfassung und riskante Fallstrukturhypothese der Eröffnungssequenz Ich darf noch ein bisschen Kaffee trinken

4.3.2 Zweite Sequenz: Aber das kostet nochmals deftig

4.3.2.1 Rekonstruktion der einzelnen Sequenzen

4.3.2.2 Zusammenfassung und riskante Fallstrukturhypothese der Sequenz Aber kostet nochmal deftig

4.3.3 Dritte Sequenz: Das wäre jetzt prinzipiell ein Angebot

4.3.3.1 Rekonstruktion der einzelnen Sequenzen

4.3.3.2 Zusammenfassung und riskante Fallstrukturhypothese der Sequenz Das wäre jetzt prinzipiell ein Angebot

4.3.4 Fallzusammenfassung und finale Fallbestimmung: Ingrid \& Lorenz

4.4.1 Eröffnungssequenz: Und wie läuft's im Partnerschulprojekt

4.4.1.1 Rekonstruktion der einzelnen Sequenzen

4.4.1.2 Zusammenfassung und riskante Fallstrukturhypothese der Eröffnungssequenz Und wie läuft's im Partnerschulprojekt? 
4.4.2 Zweite Sequenz: Also eigentlich sind jetzt alle Ziele abgearbeitet

4.4.2.1 Rekonstruktion der einzelnen Sequenzen

4.4.2.2 Zusammenfassung und riskante Fallstrukturhypothese der Sequenz Also eigentlich sind jetzt alle Ziele abgearbeitet

4.4.3 Dritte Sequenz: Das hab ich auch der Praxislehrperson zu verdanken

4.4.3.1 Rekonstruktion der einzelnen Sequenzen

4.4.3.2 Zusammenfassung und riskante Fallstrukturhypothese der Sequenz Das hab ich auch der Praxislehrperson zu verdanken

4.4.4 Vierte Sequenz: Was kannst du selber dafür tun

4.4.4.1 Rekonstruktion der einzelnen Sequenzen

4.4.4.2 Zusammenfassung und riskante Fallstrukturhypothese der Sequenz Was kannst du selber dafür tun

5.2.1 Aspekte und Modi der Bezugnahme auf das Setting

5.2.2 Aspekte und Modi der Bezugnahme aufeinander

5.2.2.1 Dimension «Informalisierung-Formalisierung»

5.2.2.2 Dimension «Diffusität-Spezifität»

5.2.2.3 Dimension «Symmetrie-Asymmetrie»

5.2.2.4 Tabellarische Darstellung der Aspekte und Modi der Bezugnahme 


\section{$1 \quad$ Einleitung}

«Aber das Mentorat könnte man ja auch abschaffen!» - Wenn man eine bestimmte Sache «auch abschaffen» könnte, scheint in dieser Sache für die Person, die diese Aussage tätigt, wenig Nutzen oder Gewinn erkennbar zu sein. Möglicherweise sind ihrer Einschätzung nach wertvolle Ressourcen wie Zeit oder Geld schlecht investiert oder sogar verschwendet. Auch denkbar ist, dass etwas einst Erschaffenes zum heutigen Zeitpunkt als überholt gilt, dadurch obsolet geworden ist und folglich abgeschafft werden könnte. Dennoch wurde die eingangs zitierte Aussage mit der Verwendung des Konjunktivs lediglich als Option im Modus von «man könnte, wenn man wollte» ausgesprochen. Das Zitat stammt aus einem Mentoratsgespräch am Institut Kindergarten- und Unterstufe (IKU) an der Pädagogischen Hochschule der Fachhochschule Nordwestschweiz (PH FHNW), an der die vorliegende Untersuchung durchgeführt wurde. Auf den ersten Blick scheint die Vermutung naheliegend, dass eine couragierte Studentin oder ein couragierter Student auszusprechen wagte, was andere Studierende vielleicht höchstens hinter vorgehaltener Hand gegenüber ihren Kommilitoninnen und Kommilitonen äussern ${ }^{1}$, nämlich dass das Mentorat eigentlich überflüssig sei.

Die Patenschaft im Mentorat, das heisst die Einführung einer neuen Person durch ein erfahrenes, hierarchisch höher gestelltes Organisationsmitglied, wie Lippmann (2006) Mentoring definiert, wäre mit der obigen Aussage delegitimiert. Allerdings trifft die Annahme, das Zitat stamme von einer oder einem Mentee, das heisst von einer Studentin oder eines Studenten der Pädagogischen Hochschule, nicht zu. Bei «Aber das [Mentorat; S.H.] könnte man ja auch abschaffen» handelt es sich um die im Rahmen eines Mentoratsgesprächs an eine Studentin gerichtete provokative Aussage eines Mentors, zu der sich die Studentin ihrerseits zu verhalten hatte. Die ausführliche Rekonstruktion des Sprechaktes wird in Kapitel 4.2 dieser Arbeit vorgenommen. Die darin aufscheinende Legitimationsfrage des Formats "Mentoratsgespräche» scheint die vorliegende Untersuchung zu prägen. Dennoch sei bereits an dieser Stelle explizit klargestellt, weder der Mentor noch die Studentin und erst recht nicht die Autorin dieser Forschungsarbeit

\footnotetext{
1 Die Forschungsarbeit unterliegt der Schweizer Rechtschreibung. In der Schweiz existiert das Eszett nicht mehr, sondern wurde durch das doppelte 's' ersetzt.
} 
gedenken, das Mentorat abzuschaffen, oder erachten dieses Format als überflüssig oder überholt. Ähnliches konstatiert auch Fraefel (2018, S. 43), der sich zusammenfassend und strukturierend auf Befunde und Überblickstudien zu Mentoring bezieht und aufzeigt, dass keine Studie die Bedeutung und die potenzielle Wirksamkeit des Mentorings infrage stelle. Welche Dynamik allerdings Gestalt gewinnt, wenn der Mentor die Studentin mit einer solchen Provokation konfrontiert, gilt es mit dem vorliegenden Forschungsvorhaben zu untersuchen.

Im Folgenden wird in einem ersten Schritt dargelegt, wo das Konzept des Mentorings seinen Ursprung hat und welche normativen Ziele damit verfolgt werden. Im Anschluss daran wird die Zielsetzung der Arbeit erläutert und die leitende Fragestellung der Untersuchung genauer expliziert, bevor in einem weiteren Schritt der Aufbau der Arbeit aufgezeigt wird.

\subsection{Kontextualisierung der Untersuchung}

Gängige Quellen in Bezug auf die Entstehung von Mentoring berufen sich auf ein Epos des Dichters Homer aus der griechischen Mythologie, auf die «Odyssee». Als Odysseus in den trojanischen Krieg ziehen muss, bittet er seinen Freund Mentor, sich seines Sohnes Telemachos anzunehmen und ihn auf die künftige Rolle als König von Ithaka vorzubereiten. Während der langen Abwesenheit Odysseus' infolge des Krieges kann Mentor Telemachos bei mehreren Gelegenheiten unterstützend unter die Arme greifen, beispielsweise indem er ihn berät, als er beim König vorsprechen muss und hinsichtlich der Wortwahl unsicher ist, oder indem er ihn auf seine Funktion vorbereitet respektive ihn in die Gepflogenheiten eines Königs einführt (z.B. Nienhaus et al., 2005).

Mit Blick auf den heute gebräuchlichen Mentoringbegriff lässt sich aus dem homerischen Epos der «Odyssee» Folgendes ableiten: Der Mentor ist der weise und wohlwollende Berater eines jungen, beruflich noch unerfahrenen Menschen, der diesem in kritischen Situationen den Rücken stärkt, ihn unterstützt und ermutigt, jedoch nicht an seiner Stelle handelt. In seinen Ursprüngen geht es bei Mentoring somit um die Begleitung und die Einführung des männlichen Nachwuchses in die Gesellschaft (Nienhaus et al., 2005, S. 18) 
Der erfahrene Mentor erhält dabei die Aufgabe, dem unerfahrenen Zögling als Berater, Begleiter und Förderer zur Seite zu stehen. Diese ursprüngliche Idee einer unterstützenden und persönlichen Beziehung zwischen zwei Menschen mit unterschiedlicher Lebensund Berufserfahrung bildet auch heute noch den Kern des Mentoringbegriffs (Haghanipour, 2013). Allerdings nimmt Mentor im Epos eine Form von Stellvertretung oder eines erweiterten Arms des abwesenden Vaters Odysseus ein, wenn auch ohne familiale Bande. Hier lässt sich eine Differenz beziehungsweise eine Bedeutungsverschiebung bezüglich des heutigen Verständnisses von Mentoring festmachen, das nicht auf Stellvertretung, sondern auf der Zuschreibung einer Begleitungsbedürftigkeit und der Herstellung von Verbindlichkeiten basiert.

In Homers Dichtung verkörpert Mentor je nach Erfordernis der Situation verschiedene Rollen und Funktionen, wodurch die Gestalt des Mentors zum «Prototypen des modernen Pädagogen» stilisiert wird (Nienhaus et al., 2005, S. 17). Sämtliche Aspekte der Idee, seinen Mentee in kritischen und anspruchsvollen Situationen zu unterstützen, ihm beratend und fürsorglich zur Seite zu stehen, seine Position und sein Selbstbewusstsein zu stärken, ihm ein Netzwerk und die eigene Erfahrung und das eigene Können zur Verfügung zu stellen, können unter das Konzept einer berufsbiografischen Unterstützung und Förderung subsumiert werden. Im griechischen Epos hat Mentor von Odysseus ein Mandat erhalten, das eine klare pädagogische ${ }^{2}$ Absicht verfolgt, nämlich Telemachos als künftigen Thronfolger zu coptimieren`.

Bei diesem Optimierungsprozess rückt die Interaktion zwischen der Mentoratsperson als Professionelle und der oder dem Mentee in der Rolle der Klientin oder des Klienten in den Vordergrund. Diese pädagogische Interaktion zielt auf eine Personenveränderung der Mentee oder des Mentees ab. Die professionelle Leistung der oder des Professionellen besteht dabei in der Unterstützung der Weiterentwicklung der zu professionalisierenden Person, wofür sich in der Professionsforschung der Begriff des Interaktionisten Hughes (1963), der hierbei von «people processing» spricht, eingebürgert hat. Beim «people processing» geht es darum, dass die rollenförmige Personalität in einem aktiven Prozess

\footnotetext{
2 Das Adjektiv «pädagogisch» wie auch das Substantiv «Pädagogik» sind etymologisch auf die griechischen Wörter "pais», was «Kind» oder "Jugendlicher» bedeutet, und "ago», was mit «ich führe», «ich leite» oder «ich ziehe» zu übersetzen ist, zurückzuführen. Unter «pädagogisch» wird in der vorliegenden Arbeit eine Bewegung verstanden, die als belehrend, bildend, erzieherisch, formend, prägend, zeigend und fürsorglich begleitend angesehen wird (vgl. auch Kernfall «Tina \& Michael» in Kapitel 4.1).
} 
sozial passfähiger gemacht werden soll. Nicht die Persönlichkeit wird verändert, sondern die soziale Form und die Passung der Person im Hinblick auf die Übernahme einer bestimmten Rolle. Diese Veränderung findet durch professionelle Hilfe und Unterstützung statt und lässt sich im Sinne von Personenförderung auffassen (Kurtz, 2011, S. 35). Diese Förderung basiert allerdings auf der Zuschreibung beziehungsweise der Unterstellung einer Entwicklungs- respektive Optimierungsbedürftigkeit. "People processing» beinhaltet demzufolge eine normative Vorstellung in Bezug darauf, wie eine Person sein müsste, und geht zugleich von der Annahme aus, dass sie dies noch nicht sei. Dieses SeinSollen und gleichzeitige Noch-nicht-Sein stellt die eigentliche Konstitutionsbedingung für «people processing» dar. Luhmann $(1983$, S. 172) merkt diesbezüglich an, dass es dabei meist um eine Selbständerung der Person durch Kommunikation gehe. Ergänzend zu Luhmanns Begriff der Selbständerung sei hier angemerkt, dass das Selbst im Begriff der Selbständerung konstitutiv ist, weil man jemand anderes nicht ändern, sondern bezogen auf die Selbständerung höchstens unterstützend, anregend, beratend oder begleitend agieren kann. Der Anspruch an diese Veränderung oder Entwicklung hat allerdings zumindest einer ersten Vermutung folgend - bei der unterstützenden Person selbst zu liegen.

Des Weiteren vollzieht sich «people processing» als Arbeit an der Person unter Rahmenbedingungen, die durch Zeit- und Entscheidungsdruck geprägt sind und als höchst ungewisse kontextuelle Umstände ein Misserfolgsrisiko in sich bergen (Stichweh, 2013, S. 296). Es ist jedoch genau diese Riskanz, die das Mentoring respektive die Unterstützung durch eine Mentorin oder einen Mentor erst legitimiert. Wie zuvor ausgeführt, weist diese professionelle Beziehung zwischen der Mentorin oder dem Mentor und der oder dem Mentee eine hohe pädagogische Implikation auf. In Anlehnung an Gieseckes Feststellung (2015, S. 43), dass keine Situation eine nur pädagogische sei, liesse sich an dieser Stelle folgende These aufzustellen: Auch die Beziehung zwischen der Mentorin oder dem Mentor und der oder dem Mentee ist nicht ausschliesslich pädagogischprofessionell, sondern enthält weitere Dimensionen und Facetten, möglicherweise auch solche, die sich im Widerspruch zu einer pädagogisch-professionellen Beziehungsstruktur verhalten. Ziel dieser Arbeit ist es, diese Beziehungsstruktur im Kontext der Hochschule empirisch zu untersuchen. Der Begriff «pädagogische Beziehung» wie auch einschlägige begriffliche Variationen davon bezogen sich traditionell auf ein Verhältnis von 
Erwachsenen und Kindern respektive Mündigen und Unmündigen (Giesecke, 2015, S. 108). Mittlerweile hat sich allerdings nicht nur ein professioneller pädagogischer Umgang mit Unmündigen etabliert, sondern auch ein ebensolcher Umgang mit Mündigen. Entsprechend gehen gemäss Giesecke «auch Professoren an der Hochschule [...] mit ihren Studenten eine pädagogische Beziehung ein» (Giesecke, 2015, S. 109). Die Beziehung zwischen der Mentorin oder dem Mentor und der Studentin oder dem Studenten kann folglich beinahe als prototypisch für eine professionell-pädagogische Beziehung im Modus von «people processing» betrachtet werden.

Grundsätzlich lässt sich feststellen, dass eine pädagogische Beziehung zwischen Lehrenden und Lernenden partikular ist (Giesecke, 2015, S. 109). Da die Beziehung zwischen der Mentorin oder dem Mentor und der oder dem Mentee programmatisch eine klare und dezidiert pädagogische Prägung enthält, verhält sie sich analog. Deshalb lässt sich auch diesbezüglich von einer partikularen Beziehung sprechen, die einem bestimmten Zweck, im Kontext des vorliegenden Vorhabens konkret der Veränderung der Studentin oder des Studenten im Sinne einer individuellen Förderung, dient. Diese individuelle Förderung lässt sich letztendlich unter den Grundgedanken der Professionalisierung als zentrales Programm und Ziel der Lehrerinnen- und Lehrerbildung subsumieren. Doch obgleich die Beziehung zwischen der Mentorin oder dem Mentor und der Studentin oder dem Studenten wegen ihrer Zweckbestimmung partikular ist, so treten sich die beteiligten Personen dennoch in ihrer ganzen Persönlichkeit gegenüber (Giesecke, 2015, S. 114). Diese Begegnung als ganze Personen dürfte durch die dyadische Gesprächssituation zusätzlich begünstigt werden, da sich die Mentorin oder der Mentor und die Studentin oder der Student in dieser Konstellation auch räumlich in einer Unausweichlichkeit gegenübertreten.

Wie und in welcher Form sich die beiden beteiligten Personen in dieser Gesprächssituation begegnen, wie sie die klare Zweckbestimmung und das Partikulare einerseits und die Begegnung als ganze Personen andererseits bearbeiten, oder anders formuliert, wie sie dieser Nähe zustimmen oder eine entsprechende Distanz aufbauen und stabilisieren, sind Fragen, die es in der vorliegenden Untersuchung empirisch zu klären gilt. Das konkrete Forschungsinteresse wie auch die Herleitung der Fragestellung des Vorhabens werden im nächsten Kapitel expliziert. 


\subsection{Ziel der Untersuchung und Entwicklung der Fragestellung}

In Analogie zum Titel des aus der hermeneutischen Schul- und Unterrichtsforschung stammenden Buches von Combe und Helsper (1994) «Was geschieht im Klassenzimmer?» bildet die alltagssprachlich ausgedrückte Frage «Was geschieht im Mentoratsgespräch?» den Ausgangspunkt der vorliegenden Untersuchung. Im Zentrum steht die Analyse konkreter Konstellationen der sozialen Praxis von Mentoratsgesprächen. Die Erfassung der Mikrostruktur dieser Gespräche wird mittels einer hermeneutischen, sequenzanalytischen Rekonstruktionsmethode durchgeführt, deren ausführliche Begründung und Beschreibung im Methodenkapitel (Kapitel 3) vorgenommen wird.

Das Forschungsvorhaben zielt darauf ab, die regelmässig stattfindenden Mentoratsgespräche zwischen den Mentorinnen oder den Mentoren als Mitarbeitenden der Pädagogischen Hochschule und den Studierenden zu beobachten, zu beschreiben und zu rekonstruieren. Mittels Rekonstruktionen von Transkripten solcher Mentoratsgespräche sollen empirisch begründete Aussagen über die Verfasstheit der sozialen Realität dieser Mentoratspraxis formuliert werden. Ziel der Untersuchung ist es, einen wissenschaftlich soliden, fundierten Einblick in dasjenige zu erlangen, was faktisch geschieht, wenn Mentorinnen oder Mentoren und Studierende in der Rolle der Mentees im Rahmen eines konzeptionellen Bestandteils der Lehrerinnen- und Lehrerbildung regelmässig und institutionalisiert dyadische Gespräche führen.

Die Untersuchung verortet sich ausdrücklich jenseits der Effektivitätslogik von Input und Output. Das bedeutet, sie verfolgt nicht das Ziel, Mentoring in der Lehrerinnen- und Lehrerbildung oder konkret Mentoratsgespräche in Bezug auf deren Nützlichkeit zu evaluieren. Auch geht es dezidiert nicht darum, die Arbeit der Mentorinnen und Mentoren oder die Reflexions- oder Kommunikationsfähigkeit der Studierenden zu messen und zu bewerten. Eine evaluative Feststellung hinsichtlich der Frage, ob die konzeptionellen Erwartungen an Mentoring in der Praxis erfüllt werden, würde eine

technische Vorstellung von gelingendem Mentoring voraussetzen, was mit dem Erkenntnisinteresse der Untersuchung nicht vereinbar wäre. Die Untersuchung fragt vielmehr nach der gemeinsamen Strukturlogik, die den empirisch dokumentierten Ausdrucksgestalten verschiedener Mentoratsgespräche zugrunde liegt. Mit dieser Absicht 
gilt es, die soziale Wirklichkeit dieser Mentoratsgespräche aufzuzeigen und die Sinnstruktur und den Sinngehalt dieser Gesprächsinteraktion von innen aufzuschliessen, oder alltagssprachlich formuliert, zu verstehen, was sich in solchen Gesprächen tatsächlich abspielt.

Aus den resultierenden Befunden erhoffe ich mir, in empirisch gesättigter Form einen Schritt in Richtung Theoriebildung der sozialen Praxis von Mentoratsgesprächen an Pädagogischen Hochschulen vornehmen und fallübergreifende Aussagen über die Charakteristika solcher Mentoratsgespräche formulieren zu können. Auf diese Weise möchte ich einen Beitrag zu einer empirisch-qualitativen Professionsforschung in der Lehrerinnen- und Lehrerbildung leisten und dabei die Spannungsfelder und Ambivalenzen von Mentoratsgesprächen in den Fokus nehmen. In der ausführlichen Analyse dieser Gespräche liegt neben der wissenschaftlichen Perspektive nicht zuletzt auch Praxisrelevanz, da es darum geht, das Format «Mentoratsgespräche» jenseits der programmatischen Vorstellungen oder «Fantasien` auf die tatsächlichen Prozesse hin zu untersuchen.

Mit dieser Ausrichtung schliesse ich an ein Forschungsdesiderat an, da die bislang vorliegenden empirischen Erkenntnisse zu Mentoratsgesprächen im Schweizer Ausbildungskontext die Dyade zwischen Studierenden der Pädagogischen Hochschule und Praxislehrpersonen ${ }^{3}$ betreffen und auf Selbstauskünften der befragten Personen - Studierenden wie auch Praxislehrpersonen - basieren. Untersuchungen, die sich auf die einphasige Lehrerinnen- und Lehrerbildung, wie sie im Schweizer Ausbildungskontext die Regel ist, beziehen und dyadische Konstellationen zwischen Studierenden und Mitarbeitenden von Pädagogischen Hochschulen analysieren, liegen meines Wissens hingegen noch nicht vor. In diesem Zusammenhang stellen insbesondere qualitativ-rekonstruktive Untersuchungen, die die Interaktionen in diesen Mentoratsgesprächen in situ untersuchen, nach wie vor ein Desiderat dar. Die vorliegende Untersuchung soll deshalb zur Bearbeitung dieser Forschungslücke beitragen.

Das Vorhaben wird als problemerschliessend und nicht als problemlösend gekennzeichnet, da es in erster Linie um eine Sensibilisierung für bestimmte Phänomene,

\footnotetext{
3 «Praxislehrpersonen» ist die im Schweizer Ausbildungskontext übliche Bezeichnung für Lehrpersonen, die Studierende in ihren Praktika betreuen und begleiten.
} 
Dynamiken und Spannungen geht. Meine offen formulierte, vorläufige These lautet, dass im Arrangement der Gesprächskonstellation zwischen der Mentorin oder dem Mentor und der Studentin oder dem Studenten transintentionale, das heisst nicht intendierte Effekte aufscheinen und dass strukturelle Spannungen für dieses Setting konstitutiv und als immanent anzusehen sind. Vor diesem Hintergrund gilt es die Fragen, welche transintentionalen Effekte dies sind, welche Spannungsmomente und Dynamiken sich konkret Geltung verschaffen und in welcher Form sie das tun, empirisch zu klären.

Der dem Mentoring zugrunde liegende Ursprungsgedanke aus der griechischen Mythologie, Berufsnovizinnen und Berufsnovizen durch die väterliche Figur eines Berufsexperten ${ }^{4}$ in die Berufswelt einzuführen und der Novizin oder dem Novizen unterstützend und beratend zur Seite zu stehen, ist ein hehrer Gedanke und dürfte wohl auf breite gesellschaftliche Zustimmung stossen. Dies gilt im Kontext des Bildungswesens umso mehr, als der Beruf der Lehrerin oder des Lehrers gemeinhin als anspruchsvoll betrachtet wird, die Anforderungen und Erwartungen der Gesellschaft an diese Tätigkeit zunehmen und gerade Berufseinsteigerinnen und Berufseinsteiger eine hohe Drop-out-Quote aufweisen (Wepfer, 2017, S. 11). Die Notwendigkeit einer intensiven und individuellen Betreuung und Begleitung als Vorbereitung auf die ersten Berufsjahre scheint das Format «Mentorat» folglich zu plausibilisieren. Gerade dort, wo programmatische Überlegungen eine breite Akzeptanz erfahren, mag es jedoch lohnenswert sein, eine möglicherweise normativ aufgeladene Vorstellung von Mentoring in der Ausbildungsphase empirisch zu hinterfragen und eine entsprechende Korrektur der Vorstellung oder Betrachtungsweise vorzunehmen. Dabei meine ich keineswegs, dass der Sinn eines Unterstützungsformats für Studierende im Kontext der Lehrerinnen- und Lehrerbildung grundsätzlich infrage zu stellen sei, sondern ich beziehe mich im Hinblick auf eine mögliche Korrektur primär auf die normativen Vorstellungen und Erwartungen an dieses Begleitformat. Denn, so plausibel ein Beratungs- und Begleitformat in Form von dyadischen Gesprächen hinsichtlich der Unterstützungsidee auch sein mag, diese Konstellation zwischen Mentorierenden und Studierenden erzeugt, wie die Rekonstruktionen der Gespräche zeigen, dennoch nicht unwesentliche Spannungen und Dynamiken, gegebenenfalls auch

\footnotetext{
${ }^{4}$ Aufgrund der väterlichen Figur aus der griechischen Mythologie wird hier konkret auf die Nennung der «mütterlichen Expertin» verzichtet.
} 
paradoxe Kommunikationsphänomene und transintentionale Effekte. Diese Spannungsfelder, die vorwiegend aus der Differenz zwischen dem programmatischen Anspruch der Mentoringkonzeption und deren Umsetzung hervorgehen (Herzog et al., 2017, S. 171), gilt es mit dem Vorhaben zu explizieren und zugänglich zu machen. Die Explikation und die Kenntnis der Spannungsfelder heben diese zwar weder auf, noch verhindern sie sie, aber im besten Fall beugt Transparenz einer Verschärfung vor (Bräu, 2008, S. 194). Des Weiteren ermöglichen diesbezügliche Erkenntnisse eine bewusste Gestaltung und Thematisierung der Spannungsfelder, beispielsweise auch in der Zusammenarbeit mit den Studierenden selbst (Herzog et al., 2017, S. 173). Nebst dem zu erzielenden Erkenntnisgewinn liegt im Vorhaben auch die Chance, Material zu gewinnen, mit dessen Hilfe eine Weiterentwicklung der Ausgestaltung des entsprechenden Formates empirisch fundiert möglich ist.

Die Untersuchung wird von den folgenden, heuristischen Fragestellungen geleitet:

- Welche Spannungen oder Paradoxien lassen sich als Folge der programmatischen Vorgaben in der sozialen Praxis von Mentoratsgesprächen im Rahmen eines Studiums zur Kindergarten- und Unterstufenlehrperson an der PH FHNW finden?

- Wie gehen die beteiligten Personen, das heisst die Studierenden und die Mentorinnen und Mentoren, mit diesen Spannungen und Paradoxien um?

- Welcher strukturellen Verfasstheit und fallspezifischen Interaktionsdynamik folgt das dabei hervorgebrachte Geschehen?

- Welche transintentionalen Effekte schreiben sich in die soziale Praxis der Mentoratsgespräche ein und wie werden diese von den beteiligten Akteurinnen und Akteuren bearbeitet?

Auf den Punkt gebracht lassen sich die heuristischen Fragen in der folgenden, untersuchungsleitenden Fragestellung zusammenführen:

\section{Welche Strukturlogiken und Interaktionsdynamiken prägen das Hochschulformat «Mentoratsgespräche»?}


Um Antworten auf diese Frage zu finden, stelle ich die Rekonstruktion von vier audiografierten Mentoratsgesprächen ins Zentrum dieser Arbeit. Die Rekonstruktionen der Ausdrucksgestalten aus diesen Gesprächen erscheinen mir vielversprechend, wenn es darum geht, Einblicke in die soziale Praxis von Mentoratsgesprächen zu erhalten. Insgesamt erhoffe ich mir, die von mir angenommenen Spannungsfelder in ihren eigensinnigen Umgangsweisen und ihrer Logik besser zu verstehen.

In der vorliegenden Untersuchung geht es primär um exemplarische Konstellationen der sozialen Praxis von Mentoratsgesprächen, nicht aber um eine Systematisierung von Formen solcher Gesprächspraktiken. Mit meinen Ergebnissen möchte ich einen konkreten Einblick in mögliche Dispositive vermitteln. Ich tue dies im Wissen darum, dass meinen Fällen lediglich exemplarischer Charakter zugeschrieben werden kann, während andere in der Praxis ebenfalls auftretende Dispositive nicht herausgearbeitet werden. Denn ich gehe davon aus, dass es so viele verschiedene Möglichkeiten oder Varianten gibt, diese Mentoratsgespräche zu gestalten und zu führen, wie sich unterschiedliche Konstellationen von Mentorinnen oder Mentoren und Studierenden finden lassen. Innerhalb dieses Möglichkeitsfeldes erachte ich jede einzelne Variante oder jeden Modus als analytische Einheit. Dies bereitet den Weg für eine Beschreibung und eine anschliessende Kontrastierung der sozialen Praxis von Mentoratsgesprächen entlang der Frage, welche unterschiedlichen Modi sich bezogen auf den Umgang mit Spannungsfeldern und Ambivalenzen dieser sozialen Praxis empirisch manifestieren und welche Dynamiken und Eigenlogiken sich dabei über den Einzelfall hinausgehend erkennen lassen. Die einzelnen Gesprächsmodi illustriere ich anhand von drei bis vier Fallrekonstruktionen aus einer konkreten Gesprächskonstellation zwischen einer Mentorin $Y$ oder einem Mentor $X$ und einer Studentin $\mathrm{Y}$.

Die Arbeit lässt sich in einem strukturtheoretischen rekonstruktiven Kontext verorten. Dabei gilt es, den Forschungsgegenstand der sozialen Praxis von Mentoratsgesprächen über die konkrete Fallrekonstruktion zu erfassen und strukturtheoretisch darzustellen (Combe \& Helsper, 1994). Den theoretischen Bezugsrahmen bilden die strukturtheoretischen Konzeptionen pädagogischer Professionalität und Professionalisierung nach Helsper (1996, 2002), Terhart (2000), Oevermann (1996b, 2002b) und Kolbe und Combe (2008). Diese Konzeptionen gehen von der Notwendigkeit der Ausbildung eines 
professionell-reflexiven Habitus als Grundlage für professionelle Handlungsfähigkeit im Lehrberuf aus (z.B. Helsper, 1996, 2000; Oevermann, 1996b, 2002b; Wernet, 2003). Die Ausbildung dieses professionell-reflexiven Habitus bedarf eines kommunikativen Austausches mit berufserfahrenen Personen über die berufsspezifischen Anforderungen und Handlungsprobleme (Reh, 2008). Das Mentorat stellt aus dieser Perspektive einen möglichen Ort dar, an dem sich Studierende in der Rolle von Berufsnovizinnen und Berufsnovizen handlungsentlastend berufsspezifischen Fragestellungen oder Herausforderungen zuwenden und im kommunikativen Austausch mit ihren Mentorinnen und Mentoren individuell ihren Professionalisierungsprozess vorantreiben können.

Die Anschlussfähigkeit der vorliegenden Untersuchung an den strukturtheoretischen Diskurs begründe ich mit der methodologischen Fundierung und gleichsam auch mit der Perspektivierung des Forschungsgegenstandes. Denn die Frage nach Strukturproblemen pädagogischen Handelns, die sich hier als Spannungsmomente in der sozialen Praxis von Mentoratsgesprächen manifestieren, impliziert einen strukturtheoretischen Ansatz. Mögliche Argumentationslinien bilden die Antinomien und Paradoxien professionellen Handelns nach Helsper (z.B. 2016) oder auch das therapeutische Arbeitsbündnis nach Oevermann (1996b, 2000, 2002b). Diese Linien gilt es für die Untersuchung auszuweisen und entlang deren Auffächerung die soziale Praxis der Mentoratsgespräche als Teil einer Ausbildungssituation zu analysieren. Mit einer strukturtheoretischen Perspektive lassen sich diese Gespräche als situativ sich ereignendes Geschehen und als Interaktion betrachten und diskutieren.

\subsection{Aufbau der Arbeit}

Die vorliegende Arbeit macht es sich zur Aufgabe, mittels empirischer Rekonstruktion und theoretischer Einordnung die strukturelle Verfasstheit und die entsprechenden fallspezifischen Interaktionsdynamiken von Mentoratsgesprächen aufzuzeigen. Eine Annäherung an das Mentorat und somit an den empirischen Untersuchungsgegenstand erfolgt in Kapitel 2 in einem ersten Schritt über eine begriffliche Auseinandersetzung mit Mentoring und eine Abgrenzung zu Coaching (Kapitel 2.1). Darauf folgt eine Diskussion der Formal- 
struktur und der Konzeption des Mentorats in seiner Funktion als individualisiertes Beratungs- und Begleitformat im Rahmen der Ausbildung zur Lehrperson. Des Weiteren gilt es, potenzielle Spannungsfelder im Mentorat, die sich aufgrund der programmatischen Bestimmungen ergeben können, zu analysieren. Daran anschliessend werden die Diskurslinien und der Forschungsstand zu Mentoring und Coaching in der Lehrerinnenund Lehrerbildung dargelegt (Kapitel 2.2). Ich nehme dabei eine Fokussierung vor, indem ich mich in erster Linie auf Mentoring und Coaching ${ }^{5}$ im Kontext der Schweizer Lehrerinnen- und Lehrerbildung beziehe. Studien aus Deutschland werden aufgrund der dort üblichen zweiphasigen Lehrerinnen- und Lehrerbildung mit Studium und Referendariat lediglich beigezogen, wenn sie für meine Arbeit eine gewisse inhaltliche Relevanz oder methodische Nähe aufweisen. Die theoretischen Bezugslinien werden im Anschluss daran im Kontext des Professionalisierungsdiskurses verortet (Kapitel 2.3). Zu diesem Zweck werden zuerst bedeutsame Bestimmungsansätze von Professionalisierung innerhalb des Ausbildungskontextes dargelegt und anschliessend strukturtheoretische Bezugskonzepte, wie beispielsweise das Verhältnis von "Krise und Routine», die "Antinomien professionellen Handelns» oder das «Arbeitsbündnis», erläutert. Die Annäherungen an das Feld schliesse ich mit Überlegungen zum Arbeitsbündnis in der Mentoratskonzeption ab, wo dieses in Relation zur Arbeitsbündniskonzeption der Therapie gesetzt wird (Kapitel 2.4).

Den Befunden der empirischen Analyse vorangestellt wird die Beschreibung des Forschungsdesigns und der methodischen Anlage der Untersuchung in Kapitel 3. Um die zentrale Frage der strukturellen Verfasstheit und der fallspezifischen Interaktionsdynamiken in Mentoratsgesprächen klären zu können (Kapitel 3.1), bedient sich die Arbeit eines qualitativ-rekonstruktiven und fallanalytischen Forschungsdesigns und folgt den methodologischen Grundannahmen und dem forschungsmethodischen Vorgehen der Objektiven Hermeneutik (z.B. Oevermann et al., 1979; Oevermann, 1983, 2000, 2013b; Wernet, 2009) (Kapitel 3.2). In diesem Zusammenhang werden die methodische Anlage des Forschungsvorhabens sowie die Begründung der Methode und der Methodologie diskutiert. Dabei nehme eine gegenstandstheoretische Bestimmung von Mentorats-

\footnotetext{
${ }^{5}$ Angesichts der unklaren Grenzen und der konzeptionellen Überschneidungen von Mentoring und Coaching werden Studien zitiert, die Mentoring oder Coaching in der Ausbildung von Lehrerinnen und Lehrern betreffen respektive den einen oder anderen Terminus in ihrem Titel tragen.
} 
gesprächen vor und erläutere in aller Kürze die methodologischen Grundlagen der Objektiven Hermeneutik. Im Anschluss daran stelle ich das methodische Vorgehen vor, beschreibe die Datenerhebung und das Sampling und zeige die methodischen Auswertungsprinzipien der Objektiven Hermeneutik auf (Kapitel 3.3).

Aufbauend auf den methodologischen und methodischen Bestimmungen stellt Kapitel 4 den empirischen Hauptteil meiner Studie dar: die objektiv-hermeneutisch angelegten Rekonstruktionen von vier Kernfällen, in denen die aufgeworfene Fragestellung der Untersuchung auf empirisch-rekonstruktive Weise nachgegangen wird (Kapitel 4.1 bis 4.4). Jede der vier Fallrekonstruktionen illustriert jeweils einen anderen Modus der spannungsvollen sozialen Praxis von Mentoratsgesprächen. Die Fallrekonstruktionen sollen es mir ermöglichen, ein klareres und präzises Bild der verschiedenen oder verschieden konturierten Modi oder Varietäten dieser Praxis zu erhalten. Diesbezüglich wird die These vertreten, dass sich durch die Fokussierung auf einzelne Fälle verallgemeinerbare Erkenntnisse zur strukturellen Verfasstheit von Mentoratsgesprächen gewinnen lassen. Auf der Grundlage transkribierter Audioprotokolle werden aus jeder In-situGesprächssituation drei bis vier Sequenzstellen in den Blick genommen, um die empirische Realität dieser Praxis zu rekonstruieren.

Kapitel 5 schliesst den empirischen Teil der Arbeit ab, indem die in Kapitel 4 dargestellten Befunde im Fallvergleich diskutiert und theoretisch eingeordnet werden. Ich bespreche die empirisch aufscheinende strukturelle Problemlage von Mentoratsgesprächen (Kapitel 5.1) und zeige auf der Basis von Fallkontrastierungen auf, welche differenten Modi der Bearbeitung sich im Umgang mit dieser strukturellen Problemlage beschreiben lassen (Kapitel 5.2). Die Fallkontrastierung wird mit einer Zusammenfassung und einer Verallgemeinerung der empirischen Ergebnisse abgeschlossen (Kapitel 5.3). Im dieses Forschungsvorhaben abschliessenden Kapitel 6 werden sodann die Grenzen der Arbeit reflektiert und es wird ein Ausblick auf mögliche Anschlussfragen, Folgestudien und Forschungsdesiderate vorgenommen. 



\section{Das Mentorat als Untersuchungsgegenstand}

\subsection{Annäherung an das Feld}

Die Entwicklung professioneller Handlungskompetenz angehender Lehrerinnen und Lehrer ist eine zentrale Aufgabe und Zielperspektive der Lehrerinnen- und Lehrerbildung (Reintjes et al., 2018). Als Mittel zur Förderung und Erreichung dieser Handlungskompetenzen, über welche die Studierenden bei ihrem Studienabschluss respektive zu Beginn des Berufseinstieges verfügen sollten, werden am IKU der PH FHNW, nebst Lehre sowie begleiteter und angeleiteter Unterrichtspraxis, auch regelmässig stattfindende dyadische Gespräche zwischen Studierenden und Personal der Hochschule, sogenannte Mentoratsgespräche, als individuelles Begleitformat eingesetzt.

Individuelle Förderbeziehungen haben im wissenschaftlichen Kontext eine lange Tradition (Petersen et al., 2017). Bereits bevor sich «Mentoring» als Bezeichnung für eine persönliche Beziehung zweier Menschen mit unterschiedlicher Berufserfahrung zum Zweck einer Unterstützung bezogen auf eine berufliche Entwicklung etabliert hatte, gab es auf informeller und nicht institutionalisierter Basis analoge Unterstützungsformate, die sich üblicherweise in einem One-to-one-Setting abspielen (Petersen et al., 2017).

Obwohl Mentor seinen Auftrag in der griechischen Antike von Odysseus und nicht von dessen Sohn Telemachos selbst erhalten hatte, geht man im heutigen Rollenverständnis einer klassischen Mentoratsbeziehung von einem proaktiven Verhalten und Engagement im Sinne einer Holschuld der Mentees wie auch von einer freiwilligen Basis bei der Errichtung dieses Mentoratsverhältnisses aus. In der spezifischen konzeptionellen Ausgestaltung der Mentoratskonstellation zwischen Studierenden und Mentorierenden der PH FHNW, auf die das Forschungsvorhaben ausgerichtet ist, ergibt sich bezogen auf die Holschuld und auf die Freiwilligkeit allerdings eine grundsätzliche Differenz zur klassischen Idee. Analog zur «ursprünglichen` Auffassung von Mentoring verhält es sich demgegenüber, dass das konstituierende Programm der Pädagogischen Hochschule ebenfalls darin besteht, Professionalisierungsprozesse angehender Lehrerinnen und Lehrer zu ermöglichen und diese in individuellen Formaten zu fördern (Herzog et al., 2017). Im Modus einer individuellen Unterstützung wird dabei dem Umstand Rechnung 
getragen, dass «Professionalisierung als berufs-biografischer Entwicklungsprozess» (Blömeke, 2002, S. 82) beziehungsweise als «biografisches Projekt» (Kunze, 2011) notwendigerweise individualisiert gedacht werden muss.

Im Folgenden werden zuerst begriffliche Klärungen zu Mentoring wie auch die entsprechenden Abgrenzungen zu Coaching vorgenommen. Daran anschliessend werden die Formalstruktur und die Konzeption des Mentorats am IKU der PH FHNW, das die Datenbasis für die vorliegende Untersuchung lieferte, aufgezeigt. Danach folgt eine Diskussion potenzieller Spannungsfelder, die sich aufgrund von strukturellen und organisatorischen Gegebenheiten bilden können.

\subsubsection{Begriffliche Auseinandersetzung mit Mentoring}

Unter «Mentoring» wird grundsätzlich eine zeitlich stabile Beziehung zwischen einer berufserfahrenen Mentorin oder einem berufserfahrenen Mentor und einer oder einem Mentee mit dem Ziel einer Förderung des Lernens und der Entwicklung und des Vorankommens der oder des Mentee verstanden (Ziegler et al., 2009, S. 11). Das dahinterliegende Prinzip geht hauptsächlich von einem informellen Lernen der berufsunerfahrenen Person von der berufserfahrenen Person aus und wird als professionelles Instrument der Personalentwicklung eingesetzt (Ziegler et al., 2009, S. 11). Ziele werden primär seitens der Mentee oder des Mentees definiert und können im Laufe der Zeit auch geändert oder angepasst werden. Auch das Einholen von Feedback geht von der Mentee oder vom Mentee aus. Mentorinnen und Mentoren verfügen mehrheitlich nicht über professionell ausgebildete Beratungskompetenzen und nehmen - im Gegensatz zu einer Coachin oder einem Coach - auch keine neutrale Rolle ein. Vielmehr führen Mentorinnen und Mentoren die Mentees in einer eher partnerschaftlichen Beziehung in bestehende Netzwerke ein und begleiten die Berufsnovizinnen und Berufsnovizen über einen längeren Zeitraum in ihrer Berufsbiografie (z.B. Peters et al., 2006). Im Zentrum des Mentoringmandats steht die Erfahrungsvermittlung.

Einige Elemente dieser Begriffsbestimmung, insbesondere die Zielperspektive der Förderung, gelten analog auch für eine Coachingbeziehung. Dies führt dazu, dass häufig eine Vermischung oder Verwirrung bei der Verwendung der Bezeichnungen «Mentoring» 
und «Coaching» feststellbar ist, was insbesondere auch darauf zurückzuführen sein dürfte, dass beide Begriffe in den 1980er-Jahren im deutschsprachigen Raum den Begriff «Praxisberatung» abgelöst haben (Ryter, 2018, S. 24). So konstatieren beispielsweise Ziegler et al. (2009, S. 19), dass der Mentoringbegriff in den letzten Jahren zunehmend aufgeweicht worden und die begriffliche Verwendungsvielfalt ${ }^{6}$ nur noch schwer überblickbar sei. Analog verhält es sich gemäss Rotering-Steinberg (2009) mit der Bezeichnung «Coaching».

Als mögliches Kriterium zur Abgrenzung von Mentoring und Coaching haben Megginson und Clutterbuck (2008, S. 4) vor diesem Hintergrund vorgeschlagen, eine Mentoratsbeziehung als längerfristig angelegtes Verhältnis zu betrachten, das sich in erster Linie auf die Identifikation der Stärken und des Potenzials der ganzen Person bezieht. Im Gegensatz dazu geht es beim Coaching stärker um Hilfe zur Selbsthilfe. Die Beziehung zwischen Coachin oder Coach und Coachee kann als unmittelbarer und direkter bezeichnet werden als diejenige zwischen einer Mentorin oder einem Mentor und der oder dem Mentee (Rauen, 2014). Auch ist das Coachingmandat zeitlich klar begrenzt und kurzfristiger angelegt; und während die Klientin oder der Klient letztlich den Coachingprozess bestimmt, verfügt die Coachin oder der Coach über Gestaltungskompetenz im Prozessverlauf (vgl. dazu ausführlich Megginson \& Clutterbuch, 2008, S. 5). Eine mögliche Definition für Coaching bietet Offermanns (2004) an. Sie schlägt vor, von einer freiwilligen, zeitlich begrenzten, methodengeleiteten und individuellen Beratung zu sprechen, bei der die zu beratende Person darin unterstützt wird, berufliche Ziele zu erreichen (Offermanns, 2004, S. 65). Die Behandlung von psychischen Störungen oder Pathologien schliesst Offermanns (2004, S. 65) in ihrer Definition explizit aus. Coaching bezieht sich somit primär auf die Verbesserung von Leistungen innerhalb eines bestimmten Kompetenzspektrums und fokussiert mit einer ressourcenorientierten Ausrichtung in jüngster Zeit auch zunehmend die Optimierung der Selbstreflexionsfähigkeit (Rotering-

\footnotetext{
6 Im erweiterten Spektrum der Beratung sind auch Dienstleitungsangebote wie psychologische Beratung, Familienberatung, Mediation, Supervision, Moderation, Training und andere mehr zu finden. Auch in diesen Fällen lässt sich feststellen, dass die einzelnen Angebote oftmals individuell beschrieben und nur schwach gegenüber benachbarten Formaten abgegrenzt werden. Eine Abgrenzung, vor allem bezogen auf Supervision und Coaching, nimmt beispielsweise Fietze (2011) vor. Grundsätzlich lässt sich diesbezüglich festhalten, dass sich Coaching an der Zielgruppe von Führungskräften orientiert, während sich Supervision an die Geführten richtet. Des Weiteren ist Coaching primär im Sinne von Personalentwicklung zu verstehen, wohingegen Supervision vielfach in Personenentwicklung besteht (Schreyögg, 2004).
} 
Steinberg, 2009, S. 32). In Anbetracht dieser begrifflichen Differenzierung ist die Bezeichnung «Mentoring» für institutionalisierte Begleitung von Studierenden durch Mitarbeitende der Pädagogischen Hochschule der Bezeichnung «Coaching» vorzuziehen, da die Beziehungskonstellation auf eine längerfristige Zusammenarbeit ausgerichtet ist. Eine weitere Herausforderung bei der begrifflichen Abgrenzung besteht darin, dass sich die Aufgabenbereiche von Mentorierenden und Coaches in weiten Bereichen überlappen. In beiden Konzepten lassen sich die Tätigkeitsbereiche mit den Verben «erklären», «zeigen», "vorschlagen» und "stimulieren» beschreiben (Rotering-Steinberg, 2009, S. 32). Mentorierende fungieren oftmals als Rollenmodelle oder Vorbilder sind organisationsintern angesiedelt und als Mitarbeitende der Organisation nicht neutral. In diesem Aspekt unterscheiden sie sich von den eher neutralen und externen Coaches (Lippmann, 2006).

Seit den letzten zwei Jahrzehnten erscheinen die Begriffe «Mentoring» und «Coaching» auch vermehrt im Kontext der Aus- und Weiterbildung von Lehrpersonen (Ryter, 2018, S. 26) und werden im Praxisfeld oft unspezifisch als Synonyme verwendet (Arnold et al., 2014). An der PH FHNW, und folglich auch im vorliegenden Forschungsvorhaben, bezieht sich die Bezeichnung «Mentorin» oder «Mentor» auf Hochschulmitarbeitende. Erfahrene Berufspersonen aus dem Schulfeld werden an der $\mathrm{PH}$ FHNW demgegenüber als «Praxislehrpersonen» bezeichnet, obwohl die Bezeichnung als Mentorin oder Mentor für Praxislehrpersonen - unter Berücksichtigung ihrer Funktion und Tätigkeit im Rahmen der Berufseinführung von Novizinnen und Novizen - ebenfalls passend wäre. Diese Begriffsunklarheit zeigt sich auch in Kapitel 2.2, da die Bezeichnung «Mentorin» oder "Mentor» in Studien und Forschungsarbeiten zu Mentoring hauptsächlich auf Praxislehrpersonen bezogen wird.

Die Mentorinnen und Mentoren in der der vorliegenden Untersuchung zugrunde liegenden Mentoratskonstellation sind Dozierende oder Lehrbeauftragte, die ein bestimmtes Mass an Erfahrung, Expertise und Wissen bezogen auf das künftige Berufsfeld der Studierenden aufweisen und darüber hinaus häufig, allerdings nicht zwingend, auch eigene Unterrichts- oder Berufsfelderfahrungen mitbringen. Aus diesem Grund beruht die Konstellation primär und vorwiegend auf einer Wissens- und Rollenasymmetrie, die der Mentorin oder dem Mentor für die Ausübung dieses Mandats institutionalisiert zuge- 
schrieben wird, und weniger auf einer persönlichen Berufserfahrungsasymmetrie im eigentlichen Sinne, die zwar bestehen kann, nicht aber notwendigerweise bestehen muss. Ryter (2018, S. 27) weist in ihrer Bestandsaufnahme zu Mentoring und Coaching als neuem Trend in der Lehrerinnen- und Lehrerbildung auf die dringliche Systematisierung der Begrifflichkeiten hin und schlägt vor, "Coaching» auf Formate zu beziehen, in denen die coachende Person die Prozessbegleitung übernimmt, Studierende die Ziele und Inhalte jedoch selbst bestimmen. Für alle übrigen individuellen Formate sei hingegen «Mentoring» zu verwenden. Folgt man Ryter, wären die Dyade zwischen Praxislehrperson und Studentin oder Student wie auch die Dyade zwischen Mitarbeitenden der Hochschule und Studentin oder Student somit als «Mentoring» zu bezeichnen (Ryter, 2018, S. 27). Gleichwohl differieren die Bezeichnungen «Coachin», «Coach» und «Coaching» respektive «Mentorin», «Mentor» und «Mentoring» je nach Institution und Kontext.

Die Diagnose der grundsätzlichen Unschärfe in der Gegenstandsabgrenzung von Mentoring und Coaching in der Lehrerinnen- und Lehrerbildung wie auch die doppelt belegte Bezeichnung «Mentorierende» für Praxislehrpersonen und Hochschulmitarbeitende erscheinen empirisch durchaus nachvollziehbar und lassen sich als Ausdruck einer Unklarheit verstehen, die sich hier auf einer ganz allgemeinen Ebene der Begriffsverwendung respektive Begriffsverwirrung zeigt. Welche potenziellen Spannungsfelder in dieser Unschärfe und Unklarheit aufscheinen, gilt es im nächsten Kapitel herauszuarbeiten.

\subsubsection{Formalstruktur und Konzeption des Mentorats am IKU der PH FHNW}

Die PH FHNW greift die Perspektive der individualisierten Professionsprozesse mit dem Format «Mentorat» konzeptionell auf und gewährleistet eine individuelle, jedoch verbindliche Begleitung von Studierenden. Die Konzeption ${ }^{7}$ basiert auf einer Reihe theoretischer Prämissen, die teilweise auf normativen Implikationen aufbauen. Den grundlegenden und normativen Ausgangspunkt bildet das humanistisch geprägte Menschenbild, «das von dem klassischen positiven Selbstbild des autonomen, handlungs-

\footnotetext{
7 Die Mentoratskonzeption ist auf der Homepage des IKU der PH FHNW unter https://www.fhnw.ch/de/diefhnw/hochschulen/ph/institute/institut-kindergarten-unterstufe/professuren-am-iku/professur-bpstprofessionalisierung/berufspraktische-studien/media/im-modus-individueller-unterstuetzung_mn.pdf_abrufbar.
} 
fähigen Menschen ausgeht. Der Mensch als bewusstes, intentionales, im kulturellen Kontext existierendes Wesen, das durch Wahlfreiheit, Entscheidungskraft und eine lebenslange Entwicklung gekennzeichnet ist» (Groeben \& Erb, 2005, S. 33), scheint die programmatische Ausgangsposition darzustellen, wenn Studierende zu eigenständigen, verantwortungsbewussten Lehrpersonen werden sollen und das Mentorat in diesem Prozess einen Beitrag leisten soll (Herzog et al., 2017, S. 166). Vor diesem Hintergrund scheint ein institutionelles, jedoch individuelles Unterstützungsangebot eine plausible Option für eine Ausbildungsinstitution zu bilden, deren Aufgabe darin besteht, die Studierenden zu einer elementaren Berufsfähigkeit im Sinne einer elementaren Professionalität hinzuführen (Herzog et al., 2017, S. 166).

Das Beratungsverständnis der Mentoratskonzeption des IKU lässt sich im MERID-Modell («Mentor teachers' roles in dialogues») (Crasborn \& Hennissen, 2010) präzise verorten. Crasborn und Hennissen unterscheiden auf der Basis zweier Dimensionen («nondirektives versus direktives Beratungshandeln» und «aktive versus re-aktive Setzung von Inputs») vier prototypische Rollen von Mentorinnen und Mentoren, nämlich den «Initiator», den «Encourager», den «Imperator» und den «Advisor». Die Mentoratskonzeption, die dieser Untersuchung zugrunde liegt, präferiert die Rolle des «Initiators» (Crasborn \& Hennissen, 2010, S. 66), der Themen der Studierenden identifiziert und aktiv anspricht, jedoch ohne die damit erfolgende Auseinandersetzung vorzuschreiben, und somit non-direktiv handelt (Herzog et al., 2017, S. 167). In der zweiten konzeptionell angedachten Rolle, derjenigen des «Encouragers» (Crasborn \& Hennissen, 2010, S. 66), agieren Mentorierende non-direktiv und reaktiv und schliessen unterstützend und ermutigend an das individuelle Lernen der Studierenden und ihre diesbezüglichen Erwartungen und Anliegen an. Die Beratungskonzeption im Mentorat des IKU der PH FHNW kann durch ein Primat eines non-direktiven, lösungsorientierten Zugangs gekennzeichnet werden und lehnt sich an systemische Beratungsansätze an (von Schlippe \& Schweitzer, 2011).

Aus programmatischer und konzeptioneller Perspektive bildet der Beratungsbedarf der Studierenden, der aus einem profunden Eigeninteresse an der Erreichung einer elementaren Berufsfähigkeit im Sinne elementarer Professionalität resultiert, den Anlass für Unterstützung im Rahmen des Mentorats (Herzog et al., 2017, S. 167 f.). 
Am IKU werden die Studierenden während ihrer regulären Studienzeit von sechs Semestern bis zum Bachelorabschluss in einer Gruppe von zehn bis zwölf Studierenden von einer Mentorin oder einem Mentor begleitet. Im Kontrast zu Mentoringkonzeptionen der Berufswelt, in denen die Mentorierenden in erster Linie einen Vorsprung an Erfahrung aufweisen, die sie bei Bedarf und im Sinne einer Holschuld an Berufseinsteigende weitergeben (von Schlippe \& Schweitzer, 2011), ist das Mentorat im Studiengang des IKU mit einem ECTS-Punkt pro Semester zwar ein kleines, jedoch obligatorisches Studienelement. Das Mentorat beinhaltet zusätzlich die Anfertigung eines Professionalisierungsportfolios. Dieses Professionalisierungsportfolio ist allerdings nicht Bestandteil der vorliegenden Untersuchung und wird deshalb nicht weiter ausgeführt. Dennoch sei hier angefügt, dass Mentorinnen und Mentoren teilweise Mentoratsgespräche führen, die sich explizit mit Themen oder Reflexionen aus diesen Portfolios befassen. In welcher Form nebst der Rechenschaftsablegung in der Portfolioarbeit auch innerhalb der Mentoratsgespräche eine Form von Rechenschaftsablegung stattfindet, gälte es empirisch zu überprüfen. Weder das Portfolio noch die Gespräche werden mit einer Benotung, sondern lediglich mit «pass» oder «fail» bewertet, wodurch der Selektionsauftrag abgefedert wird. Allerdings wird die Mentorin oder der Mentor beigezogen, falls die Leistungen der Studentin oder des Studenten in einem Praktikum in der berufspraktischen Ausbildung nur knapp ausreichen und die Gefahr besteht, dass sie nicht in genügendem Umfang erbracht werden. Im Leitfaden zur Vertiefungsphase, die im dritten und vierten Semester stattfindet, wird zu solchen Situationen Folgendes festgehalten: «Die Beurteilung der Praktikumsleistungen erfolgt durch die Praxislehrperson/en. Bestehen Zweifel daran, dass die Studienleistung erbracht werden kann, ist der Mentor oder die Mentorin frühzeitig zwingend zu beteiligen» (Leonhard et al., 2019, S. 14). Dies bedeutet, dass der Mentorin oder dem Mentor bei Bedenken betreffend Bestehen oder Nichtbestehen einer Praktikumsphase konstitutiv ein Mitspracherecht zugetragen wird.

Abschliessend lässt sich bezogen auf die Formalstruktur des Mentorats festhalten, dass es aus ausbildungsarchitektonischen Überlegungen Sinn ergibt, das Setting wie an der PH FHNW implementiert zu konzipieren. Denn die intendierten Professionalisierungsprozesse von angehenden Lehrpersonen müssen - trotz hoher Selbstverantwortung (Keller-Schneider, 2012) - institutionell angeleitet, begleitet und unterstützt werden 
(Reintjes et al., 2018, S. 10). Allerdings wird diese Konzeption auch durch Spannungen geprägt. Diese dürften jedoch nicht auflös-, sondern lediglich bearbeitbar zu sein. Wie diese Bearbeitung konkret erfolgt, gilt es im empirischen Teil dieser Arbeit zu klären.

\subsubsection{Analyse von potenziellen Spannungsfeldern}

Die Konzeption des Mentorats am IKU der PH FHNW ist theoretisch breit abgestützt und mehrfach erprobt. Dieses individuelle Unterstützungsformat wird zum jetzigen Zeitpunkt dezidiert nicht infrage gestellt (Herzog et al., 2017, S. 173). In einem idealtypischen Verlauf erhalten die Studierenden in Mentoratsgesprächen eine Plattform, die ihnen die Möglichkeit bietet, ihre individuellen Anliegen und Fragestellungen zu ihrem Professionalisierungsprozess mit einer Fach- und Vertrauensperson zu diskutieren und gemeinsam Handlungsoptionen $\mathrm{zu}$ erarbeiten. Allerdings können sich dabei programmatisch bedingt pragmatische Herausforderungen ergeben, nämlich dann, wenn sich die Bearbeitung eines bestimmten Themas oder die Nutzung der Gesprächszeit nicht bereits ausgehend von den Anliegen der Studierenden anbietet oder wenn den Studierenden der für die Platzierung ihrer Anliegen und Themen notwendige Raum nicht zur Verfügung gestellt wird. Nebst der Füllung des vorgesehenen Zeitfensters mit Gesprächsinhalten und -themen können auch weitere Aspekte der bestehenden Konzeption Spannungen und Ambivalenzen erzeugen. Diese sind auf einer institutionellen Ebene zu verorten und müssen beidseitig, das heisst von der Mentorin oder vom Mentor und von der Studentin oder vom Studenten, bearbeitet werden.

Von einer analytischen Betrachtung der im vorhergehenden Kapitel dargestellten konzeptionellen Überlegungen zum Format «Mentorat» ausgehend lassen sich erste Vermutungen und Rückschlüsse auf mögliche Spannungsfelder in diesem Setting vornehmen. Diese potenziellen Spannungen werden im Folgenden im Sinne von hypothetischen Überlegungen herausgearbeitet und erläutert. Ob und wie sie sich interaktiv in den Gesprächen manifestieren und wie die beteiligten Akteurinnen und Akteure sie bearbeiten, gilt es im empirischen Teil der Arbeit zu rekonstruieren. 


\subsubsection{Spannungsfeld zwischen Beratung, Beurteilung und Rechenschaftsablegung}

Die Koppelung von Beraten und Beurteilen lässt ein strategisches Verhalten oder Handeln seitens der Studierenden vermuten. Auf ein solches Kalkül weisen bereits Boettcher und Bemerich-Vos in ihrer Untersuchung aus dem Jahr 1985 hin, in der sie sich mit Unterrichtsnachbesprechungen in der zweiten Phase der Lehrerinnen- und Lehrerbildung befassen:

[...] diese Ambivalenz von beraten-beurteilen: Wie kann ich einen Referendar beraten, der offenbar gut daran tut, seine Probleme - soweit er sie für besonders bewertungsrelevant hält - gerade nicht zu exponieren, sondern sie in strategischer Absicht zu leugnen, zu bagatellisieren oder umzudeuten? Ist damit nicht - sozusagen schon vor Gesprächsbeginn - ein Problem konstituiert, dessen Lösung ähnlich verzweifeln lässt wie die Quadratur des Kreises? (Boettcher \& Bremerich-Vos, 1985, S. 247)

Wenngleich sich die Ausgangslage sowohl im Ausmass als auch in der Reichweite von derjenigen von Referendarinnen und Referendaren ${ }^{8}$ unterscheidet, dürfte in entsprechend abgeschwächter Form ein vergleichbar strategisches Verhalten auch bei den Studierenden der vorliegenden Untersuchung wahrscheinlich sein, obwohl der Beurteilungsaspekt im vorliegenden Setting mit «erfüllt» oder «nicht erfüllt» niederschwellig gehalten wird.

Des Weiteren unterläuft eine Dokumentationspflicht einer Rechenschaftslegung über die Arbeit im Mentorat strukturell die Beratungspflicht. Denn das Wissen darum, etwas im Modus der Selbstkundgabe dokumentieren zu müssen, das später möglicherweise explizit oder implizit Einfluss auf die Erfüllt-/Nicht-erfüllt-Bewertung nehmen wird, gefährdet oder behindert ein offenes Beratungsgespräch respektive die Thematisierung von selbst identifizierten Problemfeldern, die möglicherweise einen Beratungsbedarf nach sich ziehen. Selbstreflexion kann unter der Vorgabe der Selbstdarstellung zum «impression management» werden (Herzog et al., 2017, S. 172), da Studierende hierbei auch einer sozialen Kontrolle seitens der Mentorierenden nachkommen müssen. Dieses Dilemma

\footnotetext{
8 «Referendarin» respektive «Referendar» ist die Bezeichnung für Absolventinnen und Absolventen der zweiten Phase der Lehrerinnen- und Lehrerausbildung in Deutschland. Das Lehramt-Referendariat ist der sogenannte «Vorbereitungsdienst» für das Lehramt an allgemeinbildenden und beruflichen Schulen Deutschlands.
} 
spricht auch Kallmeyer an, wenn er dadurch das Vertrauensverhältnis der Mentees gegenüber ihren Mentorinnen und Mentoren gefährdet sieht:

Dabei spielt auch die Kombination von Beratung mit weiteren Handlungsschemata eine Rolle wie Prüfen und Bewerten [...] und soziale Kontrolle. Aufgrund der Asymmetrie und insbesondere im Zusammenhang mit der institutionenbedingten Dominanz von RG [Ratgebenden; S.H.] entsteht das Problem des Vertrauens von RS [Ratsuchenden; S.H.] in RG. (Kallmeyer, 2000, S. 230)

In Anbetracht der Koppelung oder Kombination verschiedener Handlungsschemata muss somit mit hoher Wahrscheinlichkeit davon ausgegangen werden, dass Studierende sich sehr genau überlegen, welche Themenfelder sie in Gesprächen aufgreifen und welche sie, trotz bestehender und individueller Relevanz, möglicherweise auch vermeiden.

\subsubsection{Spannungsfeld zwischen berufspraktischen Bewältigungsanforderungen und reflexiver Distanz}

Die berufspraktischen Bewältigungsanforderungen, die in der Berufspraxis an die Studierenden herangetragen werden, stehen potenziell in einem Spannungsfeld zur gleichzeitig geforderten reflexiven Distanz. Gerade im individuellen Begleitformat des Mentorats könnten unterschiedliche Erwartungen und Ansprüche als Spannungen aufscheinen, beispielsweise wenn Studierende Praxisexpertise suchen und diese einer theoretischen und wissenschaftlichen Expertise vorziehen. Die Mentorinnen und Mentoren verfügen allerdings primär über profunde akademische Kenntnisse und somit über theoretische und wissenschaftliche Expertise, die nur eine bedingte Passung für die Beratung und Begleitung praxisnaher Fragestellungen aufweist. Anders formuliert ergibt sich aus der Architektur dieses Formats eine Schwierigkeit, wenn die Mentorin oder der Mentor das Mandat aus einer wissenschaftstheoretischen Position heraus legitimiert, während die Studierenden als Mentees Erfahrungsexpertise präferieren dürften und dieser wohl auch eine höhere Legitimationsbasis zusprechen würden. Die auf beiden Seiten aufscheinenden unterschiedlichen Erwartungen können in der Folge zu Prekaritäten und Fragilitäten, vor allem in der Rolle der Mentorinnen und Mentoren, führen und entsprechend Potenzial für Spannungsmomente aufweisen. 


\subsubsection{Spannungsfeld zwischen Freiwilligkeitsansprüchen und Verpflichtung}

Wie bereits bei der Begriffsdefinition in Kapitel 2.1.1 aufgezeigt, weist eine Mentoratsbeziehung verschiedene Facetten wie beispielsweise Beraten, Begleiten, Belehren, Betreuen und andere mehr auf. Auf den Beratungsaspekt bezogen ist der Anspruch auf Freiwilligkeit für die ratsuchende Person eine Voraussetzung. Darauf wie auch auf einer Holschuld basierend und durch Eigenbemühungen der ratsuchenden Person motiviert, wird schliesslich ein gemeinsamer Problemlösungsprozess gestaltet.

In der Zielsetzung des Mentorats am IKU der PH FHNW ist konzeptionell ebenfalls angedacht, dass Studierende ihre Anliegen, das heisst ihren Beratungsbedarf, in ein Mentoratsgespräch einbringen und die Mentorierenden als «Initiators» und «Encouragers» non-direktiv und reaktiv darauf eingehen. Die konkreten Anliegen der Studierenden wären somit als Ausgangsbasis für eine gemeinsame Problemlösungserarbeitung zu betrachten. Allerdings finden die Mentoratsgespräche im Sinne einer Bringschuld auch ungeachtet eines konkreten Beratungs- oder Problemlösungsbedarfs seitens der Mentees institutionalisiert mindestens einmal pro Semester statt. Pointiert formuliert laufen diese Gespräche infolge der institutionellen Vorgaben Gefahr, zu 〈Pflichtübungen` ohne tatsächlich vorliegenden Beratungsbedarf zu werden. Die Studierenden werden von der Institution her als beratungsbedürftig konstituiert, was sich durch ihre Entscheidung, an dieser Institution ihre Ausbildung zur Lehrperson zu absolvieren, legitimieren lässt. Generelle Verpflichtungen, sich einer Beratung zu unterziehen, gibt es zudem auch in anderen gesellschaftlichen Bereichen. Dies kann beispielsweise der Fall sein, wenn ein substanzielles Risiko besteht, dass ein Individuum einer anspruchsvollen Situation nicht gewachsen sein könnte, sich jedoch auf freiwilliger Basis einer Beratung nicht unterziehen würde, oder wenn die auferlegte Beratung zur Bewältigung einer Herausforderung als unterstützend oder sinnvoll erachtet wird. Durch die Verordnung der Beratung wird eine Verbindlichkeit hergestellt. Auch ohne vertiefte Auseinandersetzung mit der Thematik der verbindlichen, verordneten Beratung lässt sich davon ausgehen, dass ein Zwang zur Beratung ein grundsätzliches Dilemma darstellt. Bezogen auf die Freiwilligkeit von Beratung halten unter anderem Boettcher und Bemerich-Vos (1987) fest, dass die Inanspruchnahme einer Beratung nicht verpflichtend 
sein dürfe. Zudem bestehe eine Abbruchfreiheit, da es keinen Zwang zur Fortführung einer begonnenen Beratung gebe. Dieses Freiwilligkeitsprinzip wird in der Mentoringkonstellation, die der vorliegenden Untersuchung zugrunde liegt, nicht eingelöst. Unter Berücksichtigung dieses Umstandes scheint die Hypothese zulässig, dass der «eigentliche» Beratungsaspekt zwar konzeptionell angedacht ist, sich empirisch aber möglicherweise different darstellt und gar nicht im Mittelpunkt der einzelnen Gespräche steht. Von der Strukturlogik her kann folglich von einem unfreiwilligen Beratungsverhältnis gesprochen werden, das von den Studierenden über die Ausbildungsentscheidung zumindest indirekt freiwillig gewählt wurde, aber in der Ausführung dennoch eine verpflichtende Komponente aufweist.

Dieser Aspekt der Beziehungslogik und der Mandatsbasis zwischen der Mentorin oder dem Mentor und der Studentin oder dem Studenten wird als ausschlaggebend und bedeutsam erachtet, wenn es darum geht, das Strukturproblem und die Interaktionsdynamiken dieser Mentoratsgespräche herauszuarbeiten. Deshalb wird er im Anschluss an die strukturtheoretische Fundierung in Kapitel 2.4 nochmals ausführlich diskutiert, und zwar in Abgrenzung zu einem therapeutischen Arbeitsbündnis, das hierbei als theoretische Vergleichsfolie dient.

\subsubsection{Spannungsfeld zwischen Prozessoffenheit und institutionellen Vorgaben}

Die Konzeption des Mentorats am IKU ist durch eine weitgehende Abwesenheit von institutionellen Vorgaben und Formblättern gekennzeichnet (Herzog et al., 2017, S. 172). Diese Offenheit ermöglicht individuelle Gestaltungsspielräume, stellt aber die Mentorierenden vor die Herausforderung, das notwendige Mass an Rahmung oder Orientierung im Sinne der Konzeption selbst zu entwickeln und festzulegen (Herzog et al., 2017, S. 172). Allfällige daraus hervorgehende Unklarheiten bezogen auf beidseitige Erwartungen und Verbindlichkeiten weisen Potenzial für Irritationen und Spannungen auf. Des Weiteren können die Mentorierenden infolge der Offenheit und der überwiegenden Abwesenheit von Vorgaben nicht auf einen klar umrissenen Auftrag für ihr Mandat zurückgreifen, was zu Unsicherheiten führen könnte, gerade auch wenn es um die Abbildung von zu erbringenden oder erbrachten Studienleistungen geht. 


\subsection{Diskurslinien und Forschungsstand von Mentoring in der Lehrerinnen- und Lehrerbildung}

Bevor ich auf die Argumentationslinien der vorliegenden Arbeit eingehe, skizziere ich im Folgenden aktuelle Diskurslinien und den Forschungsstand zu Mentoring und Coaching im Professionalisierungsprozess angehender Lehrpersonen. Unter «Diskurslinien» verstehe ich in diesem Zusammenhang theoretisch fundierte Arbeiten über Mentoringkonzeptionen.

Im Zusammenhang mit dem Aspekt «Forschungsstand» bespreche ich empirische Arbeiten zu Mentoring. Forschung zu einem spezifischen Ausbildungsformat ist in hohem Masse kontextabhängig. Deshalb begrenze ich mich bei der Rezeption und stelle lediglich Diskurslinien und Forschungsarbeiten dar, die eine gewisse Relevanz für das vorliegende Forschungsvorhaben aufweisen. Die Rezeption erhebt folglich keinen Anspruch auf Vollständigkeit und ist exemplarisch zu verstehen. Da ich mit meinem Vorhaben bis zu einem gewissen Grade (terra incognita) betrete, ist keine der aufgeführten Untersuchungen direkt mit der vorliegenden vergleichbar. Als relevante Studien führe ich deshalb Studien auf, die mindestens eines der folgenden Kriterien erfüllen:

- kontextbezogene Relevanz: einphasige Lehrerinnen- und Lehrerbildung, wie diese in der Schweiz vorliegt;

- gegenstandstheoretische Relevanz: Studien, in denen das Mentoratsverhältnis zum Gegenstand der Untersuchung wird;

- methodenbezogene Relevanz: Untersuchungen, die sich rekonstruktiver Methodologien, wie beispielsweise der Objektiven Hermeneutik bedienen.

In dieser Reihenfolge werden die Studien und Arbeiten nachstehend aufgeführt. Aus dem Stand der empirischen Forschung heraus leite ich schlussfolgernd die Forschungsanlage dieser Untersuchung ab.

Vorausschickend gilt festzuhalten, dass sich Mentoring oder Coaching im Kontext der Lehrerinnen- und Lehrerbildung meist auf erfahrene Lehrpersonen bezieht, die sich in der berufspraktischen Begleitung oder Betreuung von Lehrpersonennachwuchs engagieren. 
Aus diesem Grund überwiegen im deutschsprachigen Raum und somit auch im Schweizer Bildungskontext Untersuchungen, die sich auf die Dyade zwischen Mentorinnen oder Mentoren aus der Berufspraxis (Lehrpersonen) und Studierenden und nicht auf die Dyade zwischen Mentorinnen oder Mentoren der Pädagogischen Hochschulen und Studierenden beziehen (Kreis, 2012, S. 3). Im Gegensatz dazu zielt mein Promotionsvorhaben darauf ab, die Dyade von Hochschulmitarbeitenden als Mentorinnen oder Mentoren und Studierenden zu analysieren.

Forschungsvorhaben, die sich auf die Dyade zwischen Praxislehrpersonen, die in diesem Zusammenhang vielfach als «Mentorinnen» oder "Mentoren» bezeichnet werden, und Studierende beziehen, verfolgen oftmals einen kompetenzorientierten Ansatz (vgl. Kapitel 2.3.2.1). Sie zielen darauf ab, Kompetenzsteigerung unter Berücksichtigung von verschiedenen Ausgangsbedingungen, Kontextfaktoren, der Nutzung von Lerngelegenheiten oder Interventionen aufzuzeigen. Der Anspruch dieses Ansatzes liegt darin, Wirkungen und Gelingens- respektive Misslingensbedingungen zu erfassen. Die im Zentrum stehenden Kompetenzen werden aus forschungspragmatischen Gründen für gewöhnlich als Selbsteinschätzungen der Studierenden respektive Mentorinnen oder Mentoren oder in Form einer Wissensüberprüfung erhoben. Die Übereinstimmung dieser Selbstauskünfte oder Wissensfacetten mit den tatsächlichen Kompetenzen gilt es allerdings kritisch zu betrachten (Blömeke, 2014). Blömeke zufolge gelte es hier, die Genauigkeit von Selbsteinschätzungen detailliert zu beleuchten, um einerseits falsche Schlussfolgerungen zu vermeiden und andererseits verzerrende Einflüsse zu identifizieren (Blömeke, 2014, S. 126). Das vorliegende Forschungsvorhaben bewegt sich im Gegensatz dazu dezidiert jenseits der Dynamik von Input und Output und ist als In-situ-Untersuchung der sozialen Praxis von Mentoratsgesprächen aufgebaut.

Im Kontext der Schweizer Lehrerinnen- und Lehrerbildung ist die Mentoring- und Coachingidee weit verbreitet, weshalb es auch einige Forschungsarbeiten zu dieser Thematik gibt. Aufgrund der kontextbezogenen Relevanz (Einphasigkeit) werden diese nachfolgend auch dann berücksichtigt, wenn sie sich auf die Dyade zwischen Praxislehrperson und Studierenden beziehen. Zuerst führe ich Forschungsarbeiten auf, die sich mit dem Setting «Mentoratsgespräche» in situ befassen. Anschliessend gehe ich auf Studien ein, die Mentoratsgespräche und deren Wirksamkeit auf der Basis der Erhebung von Selbstauskünften untersuchen. Auf die Darstellung eines internationalen Überblicks 
über Wirksamkeitsstudien wird verzichtet. Ein entsprechender Überblick ist bei Futter (2016, S. 104 ff.) zu finden.

Forschungsbefunde, die sich sowohl auf den Kontext der Schweizer Lehrerinnen- und Lehrerbildung als auch auf die Dyade zwischen Mentorierenden als Hochschulmitarbeitenden und Studierenden beziehen, liegen bislang keine vor. Hingegen sind einige vorwiegend konzeptionell-programmatische Arbeiten verfügbar, die vielfältige Begründungen für die Etablierung von Mentoring und Coaching in der Lehrerinnen- und Lehrerbildung liefern und das Potenzial solcher Begleitformate aufzeigen. Über das Potential vom Mentorat als individuelles Begleitformat wird beispielsweise bei Ryter (2018) nachgedacht. Ihre Ausführungen stehen exemplarisch für einen Diskurs, der Coaching (und Mentoring ${ }^{9}$ ) als Import aus dem Managementbereich in die Lehrerbildung etabliert. Im Sinne einer Bestandsaufnahme referiert Ryter aktuelle Diskussionslinien und berichtet von eigenen Erfahrungen mit dem Beratungsformat von Coaching als neuem Trend in der Lehrerinnen- und Lehrerbildung der PH FHNW und bietet dabei einen fundierten Einblick in die Voraussetzungen für ein erfolgreiches Coaching. Sie spricht von einem Paradigmenwechsel in der Haltung von Lehrpersonenbilderinnen und Lehrpersonenbildnern als Voraussetzung für eine gelungene Umsetzung des Begleit- und Beratungsformates, da Studierende selbst als Expertinnen und Experten ihrer Situation angesehen werden müssten und die Aufgabe der Beraterinnen und Berater nicht darin bestehe, Lösungen oder Expertise zu vermitteln, sondern vielmehr darin, Entwicklungsprozesse anzuregen und den Studierenden viel zuzutrauen und zuzumuten. Wegen des hierarchischen Kontextes bestehe jedoch eine latente Gefahr, dass sich das Begleit- und Beratungsformat zu einem Kontrollinstrument entwickle, weshalb Studierende versuchen könnten, die von ihnen antizipierten Erwartungen strategisch zu befriedigen. Eine Prekarität sieht Ryter auch in den Doppel- oder Dreifachrollen, die Dozierende einnehmen, wenn sie Vorlesungen und Seminare halten, Studienleistungen beurteilen und Studierende gleichzeitig im Mentorat begleiten. Diesbezügliche Zuständigkeiten erfordern deshalb eine transparente und explizit kommunizierte Rollenklarheit.

\footnotetext{
${ }^{9}$ Ryter kommt in ihrer Bestandsaufnahme auf die begriffliche Überlappung von «Mentoring» und «Coaching» zu sprechen und stellt dabei fest, dass die Bezeichnung als «Coach» oder als «Mentorin» oder «Mentor» oftmals nach Institution und Kontext differiere (Ryter, 2018, S. 26 f.). Sie selbst spricht in diesem Zusammenhang von "Coaching», weshalb ich bei der Rezeption ihrer Bestandsaufnahme ebenfalls diesen Terminus verwende
} 
Ein weiteres Spannungsfeld macht Ryter in der Verbindlichkeit des Formats fest, die dann zum Problem werde, wenn aus der Sicht der Studierenden zuweilen kein dringender Beratungsbedarf vorliege und das Mentorat Gefahr laufe, einfach «erledigt» zu werden (Ryter, 2018, S. 34). Des Weiteren weist Ryter darauf hin, dass sie bei Studierenden Unsicherheiten in Bezug darauf festgestellt habe, was sie in den Gesprächen erwarte. Manchmal kämen Studierende auch als «Klagende» ins Mentorat, um sich über Personen, Situationen oder Studienelemente zu beschweren, oder sie erwarten eine konkrete Fachberatung (Ryter, 2018, S. 32). Ryters Erkenntnisse weisen einige Gemeinsamkeiten mit der im vorausgehenden Kapitel vorgenommenen Analyse von potenziellen Spannungsfeldern im Mentorat auf. Kritisch anzumerken ist jedoch, dass Ryters Erkenntnisse auf mehrjährigen eigenen Erfahrungen in der Coachingrolle oder auf bestehenden Konzepten beruhen, nicht aber als belastbare empirische Forschungsergebnisse eingestuft werden können. So stellt denn auch Ryter fest, dass eine systematische Evaluation der Wirkung von Mentoring wünschbar wäre, da sich diese Einzelgespräche nur rechtfertigen liessen, wenn sie tatsächlich eine neue Qualität brächten (Ryter, 2018, S. 32). Fachwissen könne auch in Seminaren oder Vorlesungen vermittelt werden und die Vermittlung allgemeingültiger Ratschläge bedürfe ebenso wenig eines individuellen Settings.

Auch Košinárs (2018) überwiegend programmatischer und gestaltungsbezogener Arbeit lässt sich eine zunehmende Etablierung von Coachingkonzepten in der Lehrerinnen- und Lehrerbildung entnehmen, die auch im schulischen Unterricht in Lerncoachkonzeptionen, beispielsweise bei Ribolits (2004) oder Struck und Würtl (2015), weite Verbreitung finden. Bei Fraefel (2018) wiederum findet sich ein Überblick zu empirischen Befunden, theoretischen Verortungen und Strategien der Transformation bezogen auf das kollaborative Mentoring in Schulpraktika.

Empirische Befunde zu Mentoratsgesprächen finden sich bei Kreis (2012). Sie hat in ihrer Studie Unterrichtsbesprechungen hinsichtlich ihres Lernertrags für die Studierenden untersucht und ist dabei der Frage nachgegangen, inwiefern sich Besprechungen mit hohem berichtetem Lernertrag in Bezug auf gesprächsanalytische Merkmale von Besprechungen mit geringem berichtetem Lernertrag unterscheiden. Ihre Untersuchung ist als Teilstudie einer quasi-experimentellen, multimethodischen Interventionsstudie angelegt, wobei die Intervention auf dem fachspezifischen und pädagogischen Coaching von Kreis und Staub (2011) basierte. Das Datenkorpus bestand aus videografierten 
Unterrichtsbesprechungen von 32 Dyaden aus einer Praxislehrperson und einer Studentin oder einem Studenten und deren Interviewaussagen über konkrete Lernereignisse dieser Besprechungen. Es wurde mit Fokus auf das Gesprächshandeln beider Interagierenden sowie deren Interaktion einer quantifizierenden Gesprächsanalyse unterzogen. Auf diese Weise konnte Kreis zeigen, dass Gespräche mit hohem berichtetem Lernertrag eine dialogische Folgestruktur mit dichterer Interaktionsfrequenz und häufiger eine dialogische Elaboration der Unterrichtsplanung oder Reflexion der Unterrichtsdurchführung aufweisen als Gespräche mit niedrigerem berichtetem Lernertrag. Auch liess sich nachweisen, dass gemeinsame Unterrichtsvorbesprechungen produktiver seien als Nachbesprechungen. Als Ergebnis ihrer Untersuchung formulierte Kreis Hypothesen bezüglich gesprächsanalytischer Merkmale von Unterrichtsbesprechungen, die mit einem hohen Lernertrag für die Studierenden einhergehen und folglich als "produktiv» bezeichnet werden können (Kreis, 2012, S. 289). Zu nennen sind diesbezüglich nebst Voranstelle von Nachbesprechungen beispielsweise auch das Führen eines längeren und kokonstruktiven Dialogs über Unterricht. Kreis' Untersuchung weist eine geografische Vergleichbarkeit und damit einhergehend eine kontextbezogene Relevanz im Hinblick auf mein Forschungsvorhaben auf. Des Weiteren basiert ihre Untersuchung in Teilen (videografierte Unterrichtsbesprechungen) auf In-situ-Daten und nicht ausschliesslich auf Selbstauskünften, die sich der Problematik der sozialen Erwünschtheit (z.B. Bortz \& Döring, 2015) stellen müssen. In Anbetracht ihrer Erkenntnisse weist Kreis bei den Forschungsdesideraten darauf hin, dass über eine Entkoppelung von Lern- und Bewertungssituationen nachgedacht werden solle. Eine solche Einschätzung habe ich bei der Analyse potenzieller Spannungsfelder im Mentorat in Kapitel 2.1.3 ebenfalls vorgenommen. In Differenz zu Kreis' Untersuchung, in der die Problemlösung im Zentrum steht, ist mein Vorhaben jedoch problemerschliessend konzipiert und basiert ausschliesslich auf In-situ-Daten. Vor allem aber beziehe ich mich in meiner Untersuchung auf die Dyade zwischen Hochschulpersonal und Studierenden und nicht auf diejenige zwischen Praxislehrperson und Studierenden.

Auch Futters (2016) Untersuchung bezieht sich auf die In-situ-Gesprächssituation zwischen Praxislehrpersonen und Lehramtsstudierenden. Diese Situationen werden bezüglich «potenzieller Lerngelegenheiten und deren Nutzung» analysiert. Anhand der Videoanalyse von 61 Besprechungen zwischen Studierenden und Praxislehrpersonen (30 
Unterrichts-Vorbesprechungen und 31 Unterrichts-Nachbesprechungen) konnte sie zeigen, dass nur knapp ein Drittel der insgesamt 781 potenziellen Lerngelegenheiten zum Lernen genutzt worden war (Futter, 2016, S. 131 ff.). Als weiteren Befund hält Futter fest, dass Lernen für die Studierenden eher möglich sein dürfte, wenn der Gesprächsstil der Praxislehrpersonen nicht zu stark direktiv und themenbestimmend sei. In einem solchen Modus seien Ko-Konstruktionen möglich, was zu potenziellen Lerngelegenheiten führe. Kritisch ist diesbezüglich allerdings anzumerken, dass solche eingeforderten KoKonstruktionen auch Gefahr laufen, der Logik eines mäeutisch-sokratischen Hervorlockens ${ }^{10}$ zu folgen (ausführlich zu mäeutisch-sokratisch vgl. Fallbesprechung «Tina \& Michael» in Kapitel 4.1). Hinweisstellen für Lerngelegenheiten innerhalb des Gespräches, von Futter «intention to change» genannt, werden in der Untersuchung im Sinne einer äusseren Zuschreibung vorgenommen. Die in diesem Zusammenhang einbezogenen Merkmale sind «Gesprächsstil», «Rolle», «Modaler Sprachgebrauch» und «Interaktionsmuster». Gerade das Merkmal «Modaler Sprachgebrauch», das heisst die Verwendung von Modalverben als Hinweis auf potenzielle Lerngelegenheiten, dürfte nicht ganz unproblematisch sein, weil es einen direkten, in allen Fällen geltenden Zusammenhang impliziert, der jedoch nicht notwendigerweise gegeben zu sein braucht. Diese «intentions to change» grenzt Futter von «descriptions», das heisst Situationsbeschreibungen von Studierenden bezogen auf zukünftiges Handeln ab, in denen sie keine Hinweisstellen auf Lerngelegenheiten identifizieren konnte. Die Forschungsergebnisse von Futter zeigen, dass in Nachbesprechungen mehr reflektiert wird als in Vorbesprechungen. Dieser Befund steht allerdings in einem gewissen Widerspruch zu den Befunden von Kreis (2012), in deren Studie sich gemeinsame Unterrichtsvorbesprechungen als produktiver herausgestellt hatten als Unterrichtsnachbesprechungen. Futter analysierte die Gesprächsstile in Unterrichtsbesprechungen anhand des MERIDModells (vgl. dazu Kapitel 2.1.2) und formulierte auf dieser Grundlage auch Implikationen für die Aus- und Weiterbildung von Praxislehrpersonen. Vor dem Hintergrund ihrer Befunde schlägt Futter vor, den Unterrichtsbesprechungen im Sinne eines partizipativen

\footnotetext{
${ }^{10}$ Bei der Mäeutik handelt sich um ein auf Sokrates zurückgeführtes methodisch-didaktisches Vorgehen. Mit dessen Hilfe werden durch produktive Verunsicherung (Fragen, Hinweise) Erkenntnisse gewonnen, die unbewusst bereits vorhanden waren. Das heisst, einer Person zu einer Erkenntnis verholfen, indem sie durch geeignete Fragen dazu veranlasst wird, den betreffenden Sachverhalt selbst herauszufinden und auf diese Weise Einsicht zu erlangen (Stangl, 2021).
} 
Ansatzes in der berufspraktischen Ausbildung ein neues Gewicht beizumessen und die Prinzipien einer ko-konstruktiven Gesprächsführung stärker in die Ausbildung angehender Lehrpersonen und in die Weiterbildung von Praxislehrpersonen zu integrieren. Dieses Desiderat liesse sich durchaus auch auf die Schulung von Mitarbeitenden der Hochschule in ihrer Rolle als Mentorinnen und Mentoren übertragen. Dieser Aspekt ist im Kontext meines Forschungsvorhabens jedoch nicht von direkter Relevanz.

Ein Modell im Kontext von Praktika in der einphasigen Lehrerinnen- und Lehrerausbildung, welches das gemeinsame Reflektieren von erfahrenen und angehenden Lehrpersonen ins Zentrum stellt, ist das Modell des reflexiven Praktikums (von Felten \& Herzog, 2001; von Felten, 2005). Da die Verbindung von Theorie und Praxis in herkömmlichen Praktika kaum umgesetzt wird, entwickelten von Felten und Herzog, basierend auf Schöns «reflection-on-action» (Schön, 1987), ein neues Konzept für die Zusammenarbeit von Studierenden und Praxislehrpersonen. Ziel dieses Modells ist es, dass sich angehende Lehrpersonen eine reflexive Grundhaltung aneignen und diese auch über die Grundausbildung hinaus beibehalten. Dies bedingt eine veränderte Rolle der Praxislehrpersonen: «Während die Hauptaufgabe der Praxislehrpersonen in einem herkömmlichen Praktikum darin besteht, den Studierenden Rückmeldungen zum Unterricht zu geben, müssen sie im reflexiven Praktikum die Reflexionsfähigkeit der Studierenden fördern und deren Lernprozess begleiten und anregen» (von Felten, 2005, S. 43). Praktisch umsetzen lässt sich diese Aufgabe, indem den Studierenden nicht lediglich erklärt wird, wie sie unterrichten sollen, sondern indem die Praxislehrperson dies in ihrem Unterricht auch selbst immer wieder handelnd vorzeigt, wodurch sich die Wirkungen dieses Handelns beobachten lassen.

Um die Wirkung des reflexiven Praktikums mit den Effekten eines herkömmlichen Praktikums zu vergleichen, führte von Felten eine quantitative Untersuchung als QuasiExperiment mit Studierenden und Praxislehrpersonen durch, wobei die Experimentalgruppe Weiterbildungsveranstaltungen zur Durchführung eines reflexiven Praktikums erhielt, während die Kontrollgruppe nicht geschult wurde. Auf der Grundlage dieses Vergleichs konnte sie zeigen, dass das reflexive Praktikum die Reflexion und die Entwicklung des Handelns stärker zu fördern vermag als das herkömmliche Praktikum. Neu an von Feltens Ansatz und inhaltlich nahe an den Aufgaben, die konzeptionell auch den Mentorinnen und Mentoren meines Forschungsvorhabens zugeschrieben werden, ist 
die Bedeutung, die der gemeinsamen Reflexion beigemessen wird. Weitere Anschlussmöglichkeiten für das vorliegende Forschungsvorhaben sind jedoch aus mehreren Gründen nicht erkennbar, da sich auch von Feltens Untersuchung auf Praxislehrpersonen bezieht und die Wirkung einer Intervention im Zentrum steht. Kritisch anzumerken ist bei von Feltens Forschungsdesign zudem, dass lediglich die sehr allgemein formulierte Variable "Weiterbildungsveranstaltung besucht» respektive «Keine Weiterbildungsveranstaltung besucht» einbezogen wird. Ob die Förderung der Reflexion tatsächlich den vermittelten Inhalten der Weiterbildungsveranstaltung zuzuschreiben ist oder ob allenfalls auch noch weitere Faktoren die Befunde beeinflussen, lässt sich nicht abschliessend klären.

Eine Brückenstudie, die eine qualitative und quantitative Untersuchung verbindet, ist diejenige von Schüpbach (2007). In seiner deskriptiv-empirischen Studie stellt Schüpbach die Frage, ob die Unterrichtsnachbesprechung der Verbindung von unterrichtlichen Handlungserfahrungen und beruflichem Wissen diene und insofern als «Nahtstelle von Theorie und Praxis» bezeichnet werden könne. Die Ergebnisse seiner Untersuchung zeigen, dass reflexive Theorie-Praxis-Bezüge in traditionellen Unterrichtsnachbesprechungen selten vorkommen und sich Nachbesprechungen oftmals auf kurze, evaluative Rückmeldungen mit praktischen Hinweisen und pädagogischen Tipps beschränken. Ausser einer kontextbezogenen Nähe lässt sich jedoch keine Verbindung zu meinem Vorhaben erkennen.

Niggli (2001, vgl. auch 2005) beschreibt in seinem «Drei-Ebenen-Modell» des Mentorings, wie Studierende durch Mentoring oder Coaching in ihrem professionellen Selbst gestärkt werden können. Niggli schlägt vor, Mentoring-Interaktionen auf drei inhaltlich unterschiedlichen Ebenen zu gestalten und auf jeder Ebene einen anderen Interaktionsmodus einzusetzen. Die erste Ebene ist diejenige des praktischen Tuns, des Vollzugs. Hier werden konkrete «Skills» bearbeitet und es wird auch die meiste Energie aufgewendet, damit das berufliche Tun erfolgreich gestaltet werden kann (Niggli, 2005, S. 17 ff.). Die zweite Ebene stellt die Ebene des Erkennens, des «Wissen, dass» beziehungsweise des «Wissen, warum» dar. Weil dieses Wissens noch nicht als Können im Sinne der Bewältigung konkreter Handlungssituationen verfügbar ist, gilt es, dieses getrennt von der ersten Ebene zu bearbeiten. Die dritte Ebene, diejenige der Orientierung, betrifft das Selbstsein im Kontext der eigenen Lebensgeschichte, das heisst die eigenen Überzeugungen, 
Werthaltungen und motivationale Befindlichkeiten, die das unterrichtliche Handeln beeinflussen (Niggli, 2005, S. 17 ff.).

Diese drei Bearbeitungsebenen sind miteinander verflochten, beeinflussen sich gegenseitig und sind lediglich in der in arbeitsanalytischer Absicht erfolgenden kommunikativen Begleitung voneinander zu trennen. Dies erfordert, dass sich die gesprächsführende Person jeweils Klarheit darüber verschafft, auf welcher Handlungsebene sie Entwicklungsanstösse tätigen möchte: durch das Anregen von Feedback (Feedbackgespräch), durch das Ausdifferenzieren der Reflexion (Praxisgespräch) oder durch begleitendes Coaching (Coachinggespräch) (Niggli, 2005, S. 73 ff.).

Niggli et al. (2008) überprüften anhand der Daten von 243 Studierenden und 198 Praxislehrpersonen aus drei Schweizer Pädagogischen Hochschulen, ob sich die den drei Ebenen zugeschriebenen Ansprüche diesen Gesprächstypen «Feedbackgespräch», «Praxisgespräch» und «Coachinggespräch» zuordnen lassen. Sie konnten nachweisen, dass diese drei theoretisch differenzierten Ebenen tatsächlich den kommunikativen Interessen der Studierenden und Praxislehrpersonen entsprechen, und das Modell somit verifizieren. ${ }^{11}$

Der heuristische Wert des Drei-Ebenen-Modells in Bezug auf die vorliegende Arbeit ist hingegen als gering einzuschätzen. Denn eine Analyse der rekonstruierten Gespräche anhand der vorgeschlagenen Systematik würde zwar eine elementare Differenzierung erlauben, ein vertiefter Einblick in die Strukturlogiken der Gespräche ergäbe sich daraus jedoch nicht.

Die Studie von Moroni et al. (2014) fragte nach den in Vor- und Nachbesprechungen verhandelten Themen und deren Passung zu den an der Hochschule vermittelten Inhalten. Diese Vor- und Nachbesprechungen weisen vom Gesprächssetting her (dyadisches Gespräch) eine gewisse Nähe zu dem von mir untersuchten Setting auf, allerdings lag das Forschungsinteresse von Moroni et al. (2014) auf der Ebene der verhandelten Inhalte, während mein Fokus auf der Interaktionsebene liegt.

\footnotetext{
${ }^{11}$ Gemäss den Angaben der für die Ausbildungsinhalte verantwortlichen Person ist das Drei-Ebenen-Modell von Niggli an der PH FHNW seit 2017 nicht mehr Bestandteil der Praxislehrpersonenaus- oder Weiterbildung. Ob das als Mentorierende fungierende Hochschulpersonal mit dem Modell vertraut ist und dieses in seiner Gesprächspraxis anwendet, lässt sich daher nicht abschliessend beurteilen.
} 
All diese in der Schweiz durchgeführten Untersuchungen operierten mit wenigen Ausnahmen (Futter, 2016; in Teilen Schüpbach, 2007; in Teilen Kreis; 2012) mit Befragungsdaten aus Selbst- und in Teilen auch Fremdbeschreibungen. Des Weiteren sind sie angesichts ihres Schwerpunkts auf der Ermittlung korrelativer Zusammenhänge weitgehend zur quantitativen Forschung zu zählen. Einzig die Studien von Schüpbach (2007) und Moroni et al. (2014) lassen sich teilweise auch der qualitativen Forschung zuordnen.

Untersuchungen, die das Augenmerk auf die Betreuung von Studierenden durch Hochschuldozierende legten, liegen auch für Deutschland nur in geringer Anzahl vor. Zu erwähnen ist in diesem Zusammenhang die Arbeit von Lazarides und Mohr. In ihrer 2015 durchgeführten quantitativen Online-Befragung von 56 Lehramtsstudierenden aus Deutschland beschäftigten sich Lazarides und Mohr mit der Frage, wie angehende Lehrpersonen die universitäre Betreuung und das Feedback wahrnehmen und welchen Nutzen sie in den Praxisbesuchen der Hochschuldozierenden sehen. Den Ergebnissen zufolge brachten die Befragten zwar eine hohe Zufriedenheit mit dem Feedback der Dozierenden zum Ausdruck, schätzten den Nutzen der Unterrichtsbesuche dennoch als gering ein. 44\% der Studierenden wünschten sich mehr Besuche und eine intensivere Betreuung durch die Dozierenden (Lazarides \& Mohr, 2015).

Auch diese Arbeit operierte mit Selbstzuschreibungen der befragten Studierenden. Was in diesem Betreuungsprozess tatsächlich stattfand, lässt sich auf dieser Datenbasis jedoch nicht klären, da nicht der tatsächliche Unterrichtsbesuch und das anschliessende Feedback untersucht wurden, sondern lediglich die Selbsteinschätzung dieser Gespräche ausgewertet wurde. Obwohl die Studie von Lazarides und Mohr (2015) die einzige Arbeit ist, die ebenfalls den Kontext «Hochschuldozierende und Studierende» in den Blick nahm, ergeben sich infolge des quantitativen Forschungszugangs wie auch hinsichtlich der untersuchten Population weitere Differenzen zu meinem Vorhaben, da sie sich auf die erste Phase der in Deutschland üblichen zweiphasigen Lehrerinnen- und Lehrerbildung bezog.

Schnebel (2009) zeigte in ihrer ebenfalls auf Deutschland bezogenen Studie mithilfe eines Mixed-Method-Verfahrens (qualitative Inhaltsanalyse und quantitative Codier- und Ratingverfahren) auf, dass in den dyadischen Mentoratsgesprächen zwischen Praxislehrpersonen und Studentin oder Student bestimmte Themenfelder wie beispielsweise 
Unterrichtsmethodik, Einsatz von Medien und Unterrichtsmaterial häufig diskutiert werden. Erst mit deutlichem Abstand folgen Themen wie das Handeln der Schülerinnen und Schüler oder die Lektionsziele. Organisatorische Aspekte oder Classroom-Management schliesslich sind gemäss Schnebel nur selten Thema in Mentoratsgesprächen. Des Weiteren konnte Schnebel nachweisen, dass Unterrichtsvorbesprechungen von Studierenden als hilfreicher eingestuft werden als entsprechende Nachbesprechungen. Gemäss Schnebel (2009) liegt das Verhältnis des Gesprächsanteils von Mentorinnen und Mentoren respektive Studierenden bei $80 \%$ zu 20\%, wobei die Studierenden kaum etwas zur Unterrichtsanalyse beitragen. Diese Befunde stehen im Einklang mit jenen von Schüpbach (2007), der ebenfalls zur Erkenntnis gelangte, dass in Unterrichtsnachbesprechungen konkrete Tipps und Handlungsanleitungen vermittelt würden, jedoch nur marginal eine gemeinsame Analyse oder Reflexion des Unterrichts stattfinde.

Liegmann et al. stellen in der Einleitung zum 2018 herausgegebenen Sammelband «Professionalisierung in Praxisphasen der Lehrerbildung» fest, dass kaum belastbare Forschungsbefunde zur ersten Ausbildungsphase an deutschen Universitäten vorlägen (Liegmann et al., 2018, S. 7). Die wenigen diesbezüglich verfügbaren Studien erheben Kompetenzen aus forschungspraktischen Gründen häufig in Form von Wissensfacetten oder sie erfassen die im Zentrum stehenden Kompetenzen als Selbsteinschätzungen der Studierenden. Ob diese Angaben mit den tatsächlich vorliegenden Kompetenzen übereinstimmen und diese adäquat abzubilden vermögen, dürfte infrage stellen zu sein (Blömeke, 2014). Mit Blick auf die Frage, wie Studierende in Praxisphasen lernen, was sie in diesen Praxisphasen genau tun und warum sie tun, was sie tun (Hascher, 2012), ist gar eine Forschungslücke festzustellen. Einen Beitrag zu deren Schliessung leistet der oben erwähnte Sammelband von Liegmann et al. (2018), der in methodisch fokussierter Perspektive Forschungslinien zu In-situ-Prozessen in der Referendariatsphase der Lehrerinnen- und Lehrerbildung aufzeigt. Die darin enthaltenen qualitativen Studien geben Aufschluss darüber, «wie Lehren, Lernen und Forschen in verlängerten Praxisphasen [im Referendariat; S.H.] jenseits intendierter Programmatiken realisiert und prozessiert werden, indem die soziale Praxis der an den Praxisphasen beteiligten Akteure [in ihrer Vollzugswirklichkeit; S.H.] in den Blick genommen wird» (Liegmann et al., 2018, S. 8). Mein Forschungsvorhaben schliesst präzise an die von Liegmann et al. aufgezeigte 
Forschungslücke an, da ich die Mentoratspraxis jenseits der intendierten Programmatik beschreiben möchte, indem ich sie in ihrer Vollzugswirklichkeit rekonstruiere.

Weitere, auch methodisch und methodologisch bedeutsame Referenzen sind die Forschungsarbeiten von Dzengel et al., die sich mit der übergeordneten Frage - «Was geschieht im Studienseminar?» - befassen und im Forschungsprojekt "AKuRat» eine umfassende Rekonstruktion von Ausbildungs- und Aneignungsstilen im Studienseminar vornehmen (Dzengel et al., 2011). Der Forschungsbericht zeichnet auf der Basis mikrologischer Fallrekonstruktionen ein empirisch fundiertes Bild der konkreten Problemlagen, mit denen sich die Ausbildungsinteraktion im Studienseminar konfrontiert sieht. In ihren Ergebnissen weisen Dzengel et al. insbesondere auf die spezifische Zwischenlage des Studienseminars als Ort hin, der sich weder eindeutig den theoretischen noch eindeutig den praktischen Ansprüchen, sondern beiden gleichzeitig verpflichtet fühlt.

Eine differenzierte Ausarbeitung eines zentralen Befundes aus der ersten Projektphase erfolgte im Rahmen der Dissertation von Dzengel (2016). In ihrer Untersuchung ist sie durch die Beobachtung von Interaktionen in Studienseminaren auf für sie unerwartete Problemdimensionen gestossen: «Die Ausbildungspraxis ist durch eine tiefe Verunsicherung gekennzeichnet, wie sie ihrem Auftrag - zugleich eine Reflexion über die pädagogische Praxis und eine Vermittlung von Handlungssicherheit für die pädagogische Praxis bereitzustellen - gerecht werden kann» (Dzengel, 2016, S. 15). Des Weiteren stellte sie fest, dass ein stabiler Orientierungsanker fehle, an dem sich die Studierenden während des kommunikativen Austauschs orientieren könnten, weshalb sie zwischen praktischem und theoretischem Diskurs hin und her gerissen seien.

Aus dieser Forschungsanlage heraus entstand auch die Arbeit von Kunze (2014), in der sie die Ausbildungsinteraktionen empirisch untersuchte und theoretische Überlegungen zu Perspektiven und Problemdimensionen des kasuistischen Arbeitens in der Lehrerinnenund Lehrerbildung diskutierte.

Eine weitere Untersuchung stellt diejenige von Dietrich (2014) dar. Sie nimmt ihren Ausgangspunkt bei den tradierten Klagen über die hohen Belastungen und den Druck, der die zweite Phase der Lehrerinnen- und Lehrerausbildung in Deutschland prägt. In einer objektiv-hermeneutischen Analyse von Interaktionsprotokollen aus Fallarbeitsseminaren arbeitet Dietrich die Strukturlogik der Krisenhaftigkeit innerhalb des Referendariats 
heraus. Dabei geht es ihm um die Frage, ob sich aus verschiedenen Umgangsweisen mit krisenhaften Situationen und Krisen induzierenden Momenten auch Möglichkeitsräume für Professionalisierungsprozesse ergeben. Mit seinen Befunden leistet er auf einer empirischen Ebene einen Beitrag zur Theoriebildung zum Thema «Professionalisierung qua Krisenbewältigung» und bietet Anschlussmöglichkeiten für weiterführende Untersuchungen individueller Professionalisierungsprozesse angehender Lehrpersonen.

Obwohl sich die Forschungsvorhaben von Dzengel et al. (2011), Dzengel (2016), Kunze (2014) und Dietrich (2014) alle auf das Referendariat als Berufseinstiegsphase beziehen, weisen sie aufgrund von ähnlichen oder vergleichbaren methodischen und methodologischen Untersuchungssettings eine Bedeutsamkeit und Orientierungshilfe für das methodische Vorgehen in meinem Vorhaben auf.

Pille (2013) wiederum geht mit seiner ethnografischen Studie zu den Praktiken der Subjektivierung im Referendariat der Frage nach, «wie angehende Lehrer in ihrer zweiten Ausbildungsphase [...] zu Agenten der Schule gemacht werden, bzw. wie sie sich selbständig in diesen Zustand bringen» (Pille, 2013, S. 12). Pille arbeitet Aspekte des Lehrpersonwerdens heraus und stellt dabei Selbst- und Fremdpositionierungen der Subjekte dar, ohne vorhandene Machtstrukturen auszublenden. In seiner Untersuchung zeigt er auf, welche grossen Herausforderungen Referendarinnen und Referendare ${ }^{12}$ im zweiphasigen System zu bewältigen haben, plädiert aber dennoch für das System der Zweiphasigkeit. Eine längsschnittlich angelegte Evaluationsstudie zur Professionalisierung im nordrheinwestfälischen Vorbereitungsdienst legten Reintjes und Bellenberg (2017) vor. Sie untersuchten auf der Basis von quantitativ erhobenen Daten an zwei unterschiedlichen Standorten, wie sich Lerngelegenheiten (Angebot) und deren Nutzung im verkürzten Vorbereitungsdienst von Nordrhein-Westfalen darstellen. Die Befunde ihrer Untersuchung deuten darauf hin, dass das Potenzial des Mentorings insgesamt nicht ausgeschöpft wird und die auf Reflexion abzielenden Lerngelegenheiten von den Referendarinnen und Referendaren als zu knapp und zu wenig differenziert bewertet werden (Reintjes \& Bellenberg, 2017, S. 130). Mentorinnen und Mentoren werden dann als positiv bewertet, wenn sie grosses persönliches Engagement zeigen, was jedoch gemäss dem

\footnotetext{
${ }^{12}$ Nicht nur aus Gründen der Gendergerechtigkeit, sondern auch mit Blick auf die inhaltliche Adäquatheit ist hier kritisch anzumerken, dass Pille konsequent lediglich von «Referendaren» spricht, obwohl $80 \%$ der in seiner Untersuchung begleiteten Novizinnen und Novizen Frauen waren und er explizit erwähnt, dass $85 \%$ der Lehrpersonen Frauen seien.
} 
Fazit von Reintjes und Bellenberg häufig nicht der Fall zu sein scheint. Des Weiteren haben der Autor und die Autorin eine internationale Bestandsaufnahme empirischer Befunde zu schulpraktischer Professionalisierung erstellt (Reintjes \& Bellenberg, 2017, S. 118 ff.), die sich jedoch vornehmlich auf die Phase des Berufseinstieges bezieht.

Aufgrund der grundsätzlich differenten Ausbildungsarchitektur - zweiphasig versus einphasig - in Deutschland und der Schweiz lassen sich die referierten Forschungsergebnisse aus Deutschland nur bedingt mit dem Schweizer Kontext vergleichen. Allenfalls wären zu diesem Zweck Forschungsergebnisse zum Berufseinstieg wie diejenigen von Keller-Schneider und Hericks (z.B. Keller-Schneider \& Hericks, 2011; KellerSchneider, 2012), die beide Länder einbezogen haben, heranzuziehen. Da sich ihre Studien nicht auf die Ausbildungsphase beziehen und sich dadurch markant von meinem Forschungsvorhaben unterscheiden, werden sie nachfolgend nicht explizit diskutiert.

Der Ende 2020 erschienene Sammelband von Dammerer et al. befasst sich mit der Professionalisierung wie auch mit der Qualifizierung von Lehrpersonen durch Mentoring in Niederösterreich (Dammerer et al., 2020, S. 11). Mit Ausnahme des Beitrags von Windl beziehen sich diese Arbeiten auf den Begleitprozess während der Berufseinstiegsphase und nicht auf die Ausbildungsphase wie in meinem Vorhaben, oder aber auf die Aus- und Weiterbildung von Lehrpersonen zu Mentorinnen und Mentoren. Windl ihrerseits arbeitet in ihrem Beitrag die Rolle und Funktion von Mentorinnen und Mentoren aus einer historischen Perspektive bis zur Gegenwart auf (Windl, 2020). Die legitimatorisch ausgerichtete Arbeit weist ebenfalls keine hohe Bedeutsamkeit für mein Vorhaben aus, weil auch Windl sich auf die Dyade Praxislehrperson und Studentin oder Student und nicht auf die Dyade Hochschulpersonal und Studentin oder Student bezieht und weil seit wenigen Jahren auch in Österreich die Studierenden eine Induktionsphase durchlaufen, die eher mit der zweiphasigen Ausbildung Deutschlands vergleichbar ist (Bundesministerium Bildung, Wissenschaft, Forschung, n.d.).

Resümierend lässt sich an dieser Stelle festhalten, dass eine fundierte und umfassende Auseinandersetzung damit, wie sich das Format der Praxisbegleitung von Studierenden international wie auch im deutschsprachigen Raum gestaltet, noch weitgehend aussteht. Zwar existieren Publikationen zu theoriebasierten Mentoring-Konzeptionen und Programmen und es bestehen Forschungsarbeiten zu Mentoring, das heisst zur individuellen Begleitung und Beratung von Studierenden. Im Kontext der Schweizer 
Lehrerinnen- und Lehrerbildung beziehen sich diese jedoch ausschliesslich auf die Dyade zwischen Mentorinnen und Mentoren aus dem Praxisfeld (Praxislehrpersonen) und Studierenden. Noch gänzlich unverfügbar sind empirische Befunde, die die Dyade von Hochschulmitarbeiten und Studierenden in den Blick nehmen. Auch eine Sichtung von Studien aus dem internationalen Raum würde sich diesbezüglich nicht als aufschlussreich erweisen, da sich auch Befunde wie beispielsweise diejenigen von Hobson et al. (2009) ebenfalls entweder auf die Dyade zwischen Praxislehrperson und Studentin oder Student beziehen oder den Fokus auf Lehrpersonen in der Rolle von Mentorierenden legen, die Berufseinsteigende in den ersten Berufsjahren begleiten und betreuen.

Zusammengefasst bedeutet dies, dass die an Pädagogischen Hochschulen in der Deutschschweiz zwar fest etablierte und konzeptionell detailliert ausgearbeitete Institution des Mentorats bisher kaum Gegenstand empirischer Forschungsbemühungen war. Insbesondere scheint vor allem die Frage, welche Interaktionsprozesse in den dyadischen Mentoratsgesprächen stattfinden, bislang zu wenig im Fokus gestanden zu haben. Daraus lässt sich das Desiderat ableiten, dass es Studien zu konzipieren gilt, die Mentoratsgespräche zwischen Lehramtsstudierenden und Vertreterinnen oder Vertretern von Pädagogischen Hochschulen in der einphasigen Lehrerinnen- und Lehrerbildung, wie sie im Schweizer Ausbildungskontext die Regel ist, untersuchen. Als aufschlussreich dürften sich diesbezüglich insbesondere qualitativ-rekonstruktive Untersuchungen erweisen, die die Interaktionsprozesse in diesen Mentoratsgesprächen in situ untersuchen, meines Wissens zurzeit jedoch noch nicht verfügbar sind. Vor diesem Hintergrund beabsichtige ich mit meiner Untersuchung, einen Beitrag zur Bearbeitung dieser Forschungslücke zu leisten. Statt Selbsteinschätzungen zu erheben und evaluative Kompetenzmessungen durchzuführen gilt es zu diesem Zweck, die Interaktionsdynamiken zu beobachten und zu beschreiben, die Strukturlogik dieser Interaktionen herauszuarbeiten und dabei zu klären, ob und allenfalls wie sich antizipierbare Spannungen im Datenmaterial empirisch abbilden. 


\subsection{Theoretische Rahmung}

Das Format «Mentorat» ist Bestandteil der Lehrerinnen- und Lehrerbildung und aus einer konzeptionellen Perspektive entsprechend der Förderung von Professionalisierungsprozessen verpflichtet. Aus diesem Grund werden in diesem Kapitel die Hauptlinien der Diskussion um Professionalisierungstheorien skizziert, wobei insbesondere der Ansatz der strukturtheoretischen Professionalisierungstheorie, in deren Horizont die vorliegende Studie verortet wird, in den Blick genommen wird.

\subsubsection{Perspektiven Professionalisierung}

Professionalisierung ${ }^{13}$ wird gemeinhin als Zielperspektive der Lehrerinnen- und Lehrerbildung verstanden (Baumert \& Kunter, 2006; Helsper, z.B. 2001; Hericks, 2006; Terhart, 2011).

Wie bereits in Kapitel 2.1.2 ausgeführt, finden am untersuchungsführenden Institut der PH FHNW zur Erreichung des bei Studienabschluss zu erwarteten Standes an Professionalität ${ }^{14}$ Mentoratsgespräche als individuelles Begleitformat dieses Professionalisierungsprozesses statt. Einer Klärung bedürfen hierbei der Begriff Profession und von ihm abgeleitete Begriffe ${ }^{15}$, die ihre Popularität nicht zuletzt ihrer Uneindeutigkeit verdanken (Dietrich, 2014, S. 33). Dies zeigt auch die pointierte Bezeichnung Radtkes (2004, S. 103), der im Zusammenhang mit dem Professionsbegriff von einer «Worthülse» spricht. In einem alltagsweltlichen Verständnis wird mit «Profession» in der Regel eine bedeutsame Könnerschaft assoziiert. In einem traditionellen Professionsmodell bezieht sich die Verwendung des Terminus hingegen auf eine spezifische Form der freien Beruflichkeit, die sich ursprünglich aus den klassischen akademischen Berufen der Fakultäten der Medizin, Theologie und Jurisprudenz heraus entwickelt hatte (Stichweh, 1996). Allerdings kommt dem Professionsbegriff im Kontext eines solchen traditionellen

\footnotetext{
13 Die Verwendung der Begriffe «Professionalisierung» und «Professionalität» wird nachfolgend aufgegriffen. 14 dito

${ }^{15}$ Für genauere Ausführungen zu den Termini «Profession», «Professionalität» und «Professionalisierung» wie auch zu «Deprofessionalisierung» oder «Semi-Professionalität» vgl. auch Helsper \& Tippelt (2011); Terhart (1992, 2011).
} 
Professionsmodells für den Lehrberuf keine analytische Relevanz zu, da es sich hierbei nicht um einen «freien Beruf» handelt (Terhart, 2011, S. 204).

Die Verwendung der Termini «Profession», «Professionalität» und «Professionalisierung» gestaltet sich innerhalb des wissenschaftlichen Diskurses different und divergierend, je nachdem zugrunde liegenden Professions- und Professionalisierungsverständnis und den spezifischen Konzeptionen von Sozialisations-, Bildungs- und Lernprozessen. Die Adaption des Professionsbegriffs für die Felder des pädagogischen Handelns wird im wissenschaftlichen Diskurs grundsätzlich kritisch eingeschätzt, wobei einige Forschende und Wissenschaftlerinnen und Wissenschaftler unterschiedlich deutlich für einen Verzicht auf die Verwendung des Professionsbegriffs für den Lehrberuf plädieren (Helsper \& Tippelt, 2011, S. 269). Ob allerdings und inwiefern für pädagogische Berufe die Bezeichnung «Profession» oder das Attribut «professionell» angemessen und gerechtfertigt ist, weist für die vorliegende Untersuchung keine hohe Relevanz auf, weshalb auf eine Aufarbeitung des diesbezüglichen Diskurses verzichtet und lediglich auf die ausführliche Diskussion bei Dietrich (2014) verwiesen wird.

Im vorliegenden Forschungsvorhaben wird an die Argumentationslinie von Helsper und Tippelt (2011, S. 275) angeschlossen und der Begriff der Professionalisierung anstelle von Professionalität verwendet. Denn Professionalisierung betont die Prozesshaftigkeit, was dem Setting der Lehrerinnen- und Lehrerausbildung entspricht, während dem Terminus der Professionalität eine Normativitätsvorstellung (d.h. eine höhere respektive geringer ausgeprägte Professionalität) anhaftet.

Im Folgenden werden die drei Hauptlinien der pädagogischen Professionalisierungstheorien skizziert.

\subsubsection{Bestimmungsansätze der Professionalisierungstheorie}

Folgt man Terhart (2001, S. 56), so lässt sich Professionalisierung als «berufsbiografisches Entwicklungsproblem» während des Ausbildungsprozesses zur Lehrperson verstehen. Im akademischen Diskurs darüber, welche Ansätze der Professionalisierungsforschung den Charakter der Ausbildung zum Lehrerberuf zu bestimmen vermögen, haben sich in den deutschsprachigen Erziehungswissenschaften vor allem drei Ansätze als wirkungsmächtig 
herauskristallisiert, die sich dem Gegenstand der Professionalisierung aus unterschiedlichen Perspektiven annähern. Es sind dies zum einen der kompetenzorientierte und zum anderen der strukturtheoretische Ansatz und als Mittelweg zwischen diesen beiden Positionen der berufsbiografische Ansatz (z.B. Terhart, 2000; vgl. auch Keller-Schneider \& Hericks, 2011, S. 299). Diese drei Bestimmungsversuche weisen unterschiedliche Akzentuierungen auf und beleuchten teils ungleiche, teils aber auch sich überschneidende Bereiche, tun dies jedoch in unterschiedlicher Terminologie und mit differenten methodischen Mitteln (Terhart, 2011, S. 209).

Den für die vorliegende Studie relevanten theoretischen Hintergrund bildet die strukturtheoretische Konzeption von Professionalisierung. Dies lässt sich in erste Linie mit einer heuristischen Negativbestimmung begründen, da die Untersuchung als Querschnittstudie keine Betrachtung der berufsbiografischen Verläufe (vgl. Kapitel 2.3.2.3) zulässt und sich als Interaktionsstudie auch nicht auf der Ebene von Wissen oder Einstellungen als Kernkompetenz (vgl. Kapitel 2.3.2.1) verorten lässt. Vielmehr bewegt sich das Mentorat - und das gilt es mit dem Forschungsvorhaben aufzuzeigen - in vergleichbaren antinomischen Strukturen, wie sie beispielsweise Helsper $(1996,2002)$ beschrieben hat, und es unterliegt widersprüchlichen Orientierungen, wie dies Oevermann (1996b, 2002b) diskutiert hat. Daher werden die beiden anderen theoretischen Ansätzen - kompetenzorientiert und berufsbiografisch - mit Blick auf die wenigen Untersuchungen aus der Schweiz, die eine thematische Annäherung an meine Fragestellung aufweisen, nachfolgend nur kurz und im Sinne einer Abgrenzung des vorliegenden Vorhabens aufgeführt.

\subsubsection{Kompetenzorientierter Professionalisierungsansatz}

Ausgangspunkt des kompetenzorientierten Ansatzes der pädagogischen Professionsforschung ist die Frage nach den notwendigen Kompetenzen und Einstellungen, die es für die erfolgreiche Bewältigung der Anforderungen des Lehrberufs braucht, sowie nach deren Entwicklung (z.B. Bauer, 1998; Baumert \& Kunter, 2006).

Der Ansatz basiert auf einer genauen Aufgabenbeschreibung der Tätigkeit der Lehrerinnen und Lehrer, die in Kompetenzbereiche und Wissensdimensionen gegliedert wird. Die Festlegung dieser Kompetenz- und Wissensbereiche erfolgt einerseits 
theoretisch-analytisch, das heisst deduktiv, und andererseits auf der Grundlage von empirischen Forschungsresultaten, das heisst induktiv (Terhart, 2011, S. 207).

Im kompetenzorientierten Ansatz gilt es für eine (angehende) Lehrperson, in den für als relevant erachteten Anforderungsbereichen möglichst hohe und weit entwickelte Kompetenzen aufzuweisen. Das Spektrum dieser Kompetenzen reicht von primär stofflich orientierten bis zu psychologisch und pädagogisch begründeten Kompetenzen und Standards. Die Überprüfung dieser Kompetenzen und Standards findet vornehmlich in quantitativen und hypothesenprüfenden Untersuchungen statt.

Im Schweizer Ausbildungskontext kommt der Studie zur Wirksamkeit der Lehrerinnenund Lehrerbildung von Oser und Oelkers (2001) eine wichtige Rolle zu, in deren Kontext diverse Entwürfe für Kompetenzmodelle und Ausbildungsstandards entstanden sind. Die in Kapitel 2.2 zum Stand der empirischen Forschung referierten Studien von Futter (2016), Kreis (2012) und Schüpbach (2007), die sich auf die einphasige Ausbildungssituation in der Schweiz beziehen, sind der kompetenzorientierten Perspektivierung zuzuordnen. Im Gegensatz dazu lassen sich mit dem vorliegenden Forschungsvorhaben, wie bereits erwähnt, keine Aussagen zu Kompetenzentwicklung oder -erwerb generieren, weshalb es sich jenseits eines kompetenzorientierten Bestimmungsansatzes positioniert.

\subsubsection{Strukturtheoretischer Professionalisierungsansatz}

Im strukturtheoretischen Ansatz (z.B. Helsper, 1996, 2000, 2004, 2011, 2014; Oevermann, 1992b, 2002b) wird davon ausgegangen, dass es Berufe gibt, die sich nicht durch Bürokratie oder den Markt regulieren lassen, sondern eine eigene, spezifische Strukturlogik besitzen (Parsons, 1964). Diese Eigenlogik respektive den Strukturkern professionellen Handelns gilt es für einen spezifischen Kontext zu bestimmen und deren Struktureigenschaften und -dynamiken als Idealtypus zu explizieren. Der im strukturtheoretischen Ansatz enthaltene Strukturbegriff verweist auf ein Modell der Zukunftsoffenheit von Lebenspraxis, wie es Mead (1968; vgl. auch Oevermann, 1991) entworfen hat. Strukturtheoretisch argumentierende Arbeiten beziehen sich in der Regel auf

Oevermanns Theorie des professionellen Handelns (Oevermann, 1996b) und sein Interaktionsmodell, das er ursprünglich für die psychoanalytische Therapie entwickelt hatte. Im Zentrum eines therapeutischen Interaktionsgeschehens stehen die beschädigte 
Integrität der Klientin oder des Klienten und der daraus resultierende Leidensdruck, den es im Rahmen der Therapie stellvertretend zu bewältigen gilt. Oevermanns These der strukturtheoretischen Bestimmung von Professionalisierung besagt dementsprechend, dass «alle professionalisierungsbedürftigen Berufspraxen im Kern mit der Aufgabe der stellvertretenden Krisenbewältigung für einen Klienten beschäftigt sind» (Oevermann, 2013, S. 119). Diese professionalisierte Praxis setzt genau dort ein, wo die primäre Lebenspraxis mit den zu bewältigenden Krisen nicht mehr selbst fertig wird und für deren Bewältigung eine Delegation an eine fremde Expertise vornehmen muss (Oevermann, 2013, S. 120). Diese Figur überträgt Oevermann auch auf das berufliche Handeln von Lehrpersonen.

Im Anschluss an Oevermann entwickelte Helsper (z.B. 1996, 2002, 2016) bezogen auf das Lehrhandeln eine Perspektive, in der er widersprüchliche Handlungen und Tätigkeiten herausarbeitete. Seinem strukturtheoretischen Bestimmungsansatz folgend weist professionelles Handeln in gewissen Kontexten in sich widersprüchliche, antinomische Strukturen und Spannungen auf. Die in diesen antinomischen Strukturen grundsätzlich enthaltenen und gleichwertigen Pole werden in verschiedenen Situationen unterschiedlich - teils dominant, teils rezessiv - bedient.

Nebst den antinomischen und widersprüchlichen Strukturen ist das Arbeitsbündnis zwischen der Therapeutin oder dem Therapeuten und der Klientin oder dem Klienten ein zentrales Konzept strukturtheoretischer Ansätze. Dieses Bündnis wird an anderer Stelle noch ausführlich diskutiert, in Kapitel 2.3.3.3 zuerst bezogen auf die soziale Situation der Psychotherapie, für die es, wie erwähnt, in seinen Ursprüngen von Oevermann entwickelt wurde, und im Anschluss daran in Kapitel 2.4 auch mit Blick auf die soziale Situation im Mentorat.

Wie bereits mehrfach festgehalten, lässt sich die vorliegende Untersuchung im strukturtheoretischen Diskurs verorten. Dies begründet sich mit der Anschlussfähigkeit des Forschungsgegenstands - den Strukturlogiken und Interaktionsdynamiken in Mentoratsgesprächen - an eine strukturtheoretische Betrachtung. Aus dieser Perspektive soll versucht werden, die Logiken und Dynamiken in Mentoratsgesprächen, die teilweise als Spannungsverhältnisse oder Paradoxien aufscheinen, zu rekonstruieren. Dabei richtet sich der Fokus der Analysen primär auf die institutionelle und nicht auf die individuelle Ebene dieses Professionalisierungsprozesses. In Kapitel 2.3.3 wird diese Ausrichtung 
bezogen auf die Aspekte Krise und Routine, auf die antinomischen Handlungsanforderungen und auf das hergestellte Arbeitsbündnis zwischen den beiden Akteurinnen respektive Akteuren noch ausführlicher erläutert.

Eine weitere Begründung für die Verortung im strukturtheoretischen Professionalisierungsansatz lässt sich in der methodologischen Fundierung der Untersuchung finden, da sich die Methodologie der Objektive Hermeneutik mit der Freilegung von latenten Sinnstrukturen befasst (ausführlich vgl. Kapitel 3).

\section{Anerkennungstheoretische Verschiebung im strukturtheoretischen Ansatz}

Die strukturtheoretische Bestimmung pädagogischer Professionalisierung, wie sie insbesondere in Arbeiten von Oevermann und Helsper diskutiert wird, erfährt bei Ricken (z.B. 2015), Reh und Ricken (2012) und weiteren Autorinnen und Autoren eine anerkennungstheoretische Verschiebung. Die Autorinnen und Autoren arbeiten mit der Heuristik der Adressierungsanalyse und knüpfen dabei an die strukturtheoretischen Grundüberlegungen an, weil jedes Interaktionsgeschehen auch als Anerkennungs- oder Adressierungsgeschehen strukturiert ist. Der zum Beispiel bei Helsper (2004) als antinomisch konzipierte Zusammenhang von Autonomie und Heteronomie wird bei Ricken (2015, S. 144) so verschoben, dass Heteronomie eine Konstitutionsbedingung für die Subjektwerdung und damit auch für die Autonomie des Subjekts darstellt. Die Anerkennungstheorie führt die Relationalität der oder des anderen respektive des heteronomischen Gegenübers als Voraussetzung für die Entwicklung der eigenen Autonomie als argumentative Rahmung pädagogischer Professionalisierung ein.

Entscheidend für die vorliegende Arbeit ist jedoch, dass dieser Gedanke meiner Kenntnis nach bisher nicht als alternative Perspektivierung zu Erklärungsansätzen der Professionalisierung von Lehrpersonen ausgearbeitet ist.

\subsubsection{Berufsbiografischer Professionalisierungsansatz}

Einen (Mittelweg) zwischen der kompetenzorientierten und der strukturtheoretischen Position stellt der von Keller-Schneider und Hericks (2011) genutzte Ansatz der Bildungsgangforschung dar. Dieser Ansatz richtet den Fokus auf individuelle Professionalisierungsprozesse von angehenden Lehrpersonen und versteht Professionalisierung als ein 
berufsbiografisches Entwicklungsprojekt, in dessen Zentrum die Auseinandersetzung mit sogenannten «Entwicklungsaufgaben» steht. Er geht davon aus, dass im Prozessverlauf des Lehrpersonwerdens ein permanenter Abgleich von beruflichen Anforderungen mit personenbezogenen und biografischen Ressourcen stattfindet (Keller-Schneider, 2010, S. 113), wobei auch Belastungserfahrungen und Belastungsbewältigung eine Rolle spielen. Im Mittelpunkt stehen die Prozesshaftigkeit des Kompetenzaufbaus und der Kompetenzentwicklung, die Übernahme des beruflichen Habitus und die Verknüpfung von privaten Lebensentwürfen mit einer beruflichen Karriere, worin eine stärker individualisierte, breiter kontextualisierte und zugleich lebensgeschichtlich-dynamische Sichtweise zum Ausdruck kommt (Terhart, 2011, S. 208).

Der berufsbiografische Ansatz weist in Bezug auf die längerfristig gedachte Kompetenzentwicklung eine gewisse Affinität zum kompetenzorientierten Ansatz auf, bei dem es ebenfalls zentral um die Entwicklung von Kompetenz und Expertise geht. Das berufsbiografischen Fragestellungen zugrunde liegende theoretische Problem besteht jedoch darin, Befunde zu empirisch rekonstruierbaren Berufsbiografien mit normativen Vorstellungen über gelungene Berufsbiografien zu verknüpfen (Terhart, 2011, S. 208).

Die Initiierung und die Unterstützung des Professionalisierungsprozesses angehender Lehrpersonen können als konstituierendes Programm einer Pädagogischen Hochschule gesehen werden (Herzog et al., 2017, S. 163). Weil davon auszugehen ist, dass «Professionalisierung als berufsbiografischer Entwicklungsprozess» (Blömeke, 2002, S. 253) beziehungsweise als «biografisches Projekt» (Kunze, 2011) notwendigerweise individualisiert gedacht werden muss, kommt Formaten individueller Unterstützung wie beispielsweise dem Mentoratsgespräch, die sich konzeptionell am berufsbiografischen Professionalisierungsansatz orientieren, zunehmende Bedeutung zu. Dennoch lassen sich aus meinen Erkenntnissen keine Aussagen zu konkreten berufsbiografischen Problemlagen ableiten, da die Orientierungs- und Deutungsmuster der beteiligten Akteurinnen und Akteure nicht Bestandteil dieser Forschungsarbeit sind. 


\subsubsection{Strukturtheoretische Bezugskonzepte}

Mit Blick auf eine Bestimmung von Professionalisierung aus strukturtheoretischer Perspektive in pädagogischen Handlungsfeldern können die Modelle und Konzeptionen von Oevermann (1996b, 2000, 2002b) und Helsper (1996, 2000, 2002, 2004, 2010, 2016) als die prominentesten und am breitesten rezipierten Ansätze betrachtet werden. Ausgangspunkt für die vorliegende Untersuchung bildet die in Kapitel 2.3.2.2 bereits angesprochene Grundfigur von Oevermanns «Arbeitsbündnis»-Konzeption zwischen Therapeutin oder Therapeut und Patientin oder Patient und deren Übertragung auf den Handlungskontext im Mentorat. Diese Konzeption und weitere im Rahmen des strukturtheoretischen Professionalisierungsansatzes entwickelte zentrale Konzepte wie «Krise und Routine» (z.B. Oevermann, 2000) und "Antinomien professionellen Handelns» (z.B. Helsper, 1996, 2002, 2004) bieten sich in meiner Untersuchung als Folien für die Auseinandersetzung mit den Mentoratsgesprächen an. Aus diesem Grund werden diese grundlegenden Konzepte der Strukturtheorie nachfolgend näher ausgeführt und im Anschluss daran in Kapitel 2.4 mit dem vorliegenden Forschungsvorhaben dazu in Verbindung gebracht.

In seiner Professionstheorie führt Oevermann zuerst eine allgemeine strukturtheoretische Bestimmung von professionellem Handeln aus. Von dieser konzeptuellen Grundlage ausgehend nimmt er sodann das pädagogische Handeln in den Blick. Dabei unterscheidet er zwischen einer faktischen Professionalisiertheit und einer Professionalisierungsbedürftigkeit (Oevermann, 1996b, S. 135 ff.). Zu den Berufen, die nicht professionalisiert, jedoch gemessen an den Handlungsproblemen, mit denen sie konfrontiert sind, als professionalisierungsbedürftig anzuerkennen sind, zählt er die pädagogischen Tätigkeiten, im Besonderen auch den Lehrberuf. Da das Mentorat ein Bestandteil der Ausbildung zum Lehrerberuf ist, lässt sich, Oevermann folgend, diese Professionalisierungsbedürftigkeit auch für dieses Format konstatieren.

Mit den folgenden Ausführungen lässt sich der strukturtheoretische Referenzrahmen von Professionalisierung bestimmen, vor dessen Hintergrund sich das Ziel des vorliegenden empirischen Forschungsvorhabens einordnen lässt. 


\subsubsection{Krise und Routine}

Sozialisationsprozesse werden in einem strukturtheoretischen Verständnis als Prozesse der Krisenbewältigung verstanden (Oevermann, 2000). Die Grundlage für diese soziologische Betrachtungsweise bildet Oevermann zufolge eine Umkehrung in der Betrachtungsweise des Begriffspaares «Krise und Routine». Krise oder krisenhaft versteht sich in diesem Zusammenhang nicht im Sinne von Stress, sondern im Sinne fehlender oder aufgebrochener Routine (Oevermann, 2002b). Diese (nicht traumatische) Krise als kontingente Praxis stellt den Normalfall dar und ist Ausgangspunkt für die Entwicklung oder Erzeugung von individuell, institutionell oder gesellschaftlich Neuem (Oevermann, 1996b, S. 82). Die alltägliche Handlungsroutine markiert demgegenüber den Grenzfall. Sie leitet sich aus einer vorgängigen Krisenkonstellation ab und geht aus der Bewältigung der Störung hervor. Es handelt sich somit um «Lösungen einer Krise, die sich bewährt haben und im Bewährungsprozess sich zu Routinen veralltäglichten» (Oevermann, 2002a, S. 10). Im Gegensatz dazu ist die Krise gemäss Oevermann ein «unvorhersehbares Öffnen eines Geschlossenen» (Oevermann, 1996a, S. 7).

Wenn Krisen naturwüchsig nicht mehr selbst gelöst werden können, weil die primäre Lebenspraxis in ihrer Autonomie eingeschränkt oder beschädigt ist, werden sie delegiert und der professionelle Handlungsprozess der stellvertretenden Krisenbewältigung setzt ein (Oevermann, 2002b). Dies bedeutet allerdings nicht, dass diejenige Person, an die die Krise delegiert wurde, diese stellvertretend und in Eigenregie löst oder bearbeitet. Vielmehr geht es darum, die sich in der Krise befindende Person mit fallsensiblen Deutungsangeboten bei der Erarbeitung von Lösungsoptionen zu unterstützen oder sie dazu zu befähigen. Auf diese Weise kann die sich in der Krise befindende Person Mitverantwortung für deren Bewältigung übernehmen (Oevermann, 2002b). Oevermann erachtet «die für die primäre konkrete Lebenspraxis stellvertretend expertenhaft vorzunehmende Krisenbewältigung" als Strukturkern des professionellen Handelns (Oevermann, 2002b, S. 24). Die hierfür erforderliche professionelle Tätigkeit könne nicht lediglich aus «Charisma» hervorgehen, sondern setze eine akademische, auf Wissenschaft basierende Ausbildung voraus (Oevermann, 1996b, S. 98), die einer systematischen und methodisch kontrollierten Bearbeitung von Geltungsfragen und dem Aufbau eines kritischen Habitus diene (Oevermann, 1996b). 


\subsubsection{Antinomien professionellen Handelns}

Freud bezeichnete das Erziehen und das Therapieren als «unmögliche» Berufe (Freud 1937, z.B. zitiert nach Britzmann, 2009). Vor dem Hintergrund dieser zugespitzten und metaphorischen Wendung betrachtet, zeigt sich Professionalisierung aus strukturtheoretischer Perspektive in der Fähigkeit, diese "Unmöglichkeit», die sich in Spannungen, widersprüchlichen Anforderungen und Antinomien manifestiert, sachgerecht, kompetent und reflektiert handhaben zu können (Terhart, 2011, S. 206). Hiermit wird ein weiteres professionstheoretisches Rahmenmodell angesprochen, das als zentrale Bezugstheorie des vorliegenden Forschungsvorhabens fungiert: das von Helsper (1996, 2002, 2004) über Jahre hinweg weiterentwickelte und empirisch gesättigte Modell der antinomischen Handlungsstrukturen in pädagogischen Kontexten. Das Modell differenziert die Spannungsproblematik aus und knüpft dabei direkt an Oevermanns strukturtheoretisches Konzept der «widersprüchlichen Einheit» an (Oevermann, 1996b, 2002b). Ebenfalls einbezogen werden die strukturtheoretischen Begründungsversuche aus den "pädagogischen Paradoxien», die Schütze zuerst für die Soziale Arbeit herausgearbeitet hatte (Schütze, 1996).

Helspers Modell bezieht sich auf das Lehrhandeln und auf das Unterrichten. Obwohl in der vorliegenden Studie ein Ausbildungssetting untersucht wird, das als Endziel auf die Befähigung zu kompetentem Lehrhandeln und Unterrichten ausgerichtet ist, unterscheidet sich die analysierte Konstellation zwischen Mentorin oder Mentor und Studentin oder Student in Form, Funktion und Programmatik vom Kontext, für den Helsper das Modell entwickelt hat. Im Sinne eines sensibilisierenden Konzepts wird es aber dennoch für die Besprechung herangezogen, dies unter anderem auch deshalb, weil es auf diese Weise möglich wird, Unterschiede und Übereinstimmungen der beiden Konstellationen aufzuzeigen.

Im Zentrum von Helspers Ansatz steht die These, dass pädagogische Arbeit konstitutiv widersprüchliche, antinomische beziehungsweise dilemmatische Handlungsanforderungen mit sich bringt. Die für diese antinomischen Figuren jeweils konstitutiven Anforderungen schliessen sich zwar gegenseitig aus oder widersprechen sich, 
beanspruchen aber dennoch gleichzeitig Gültigkeit (Helsper, 2002, S. 70 ff.). Helsper unterscheidet diesbezüglich elf nicht aufheb-, sondern lediglich reflexiv handhabbare Antinomien (Helsper, 2002, S. 75 ff.), die er zwei Ebenen zuteilt.

Auf einer ersten Ebene fasst er, in Bezugnahme auf Oevermanns Modell von «Krise und Routine», Antinomien zusammen, die auf die Strukturlogik des pädagogischen Handelns als stellvertretende Krisenlösung zurückzuführen sind und sich dem Hiatus von Entscheidungszwang und Begründungsverpflichtung zuschreiben lassen (Helsper, 2016, S. 54). Die konstitutiv widersprüchlichen Anforderungen, die sich in Krisensituationen stellen und auch für die (naturwüchsige) Lebenspraxis gelten, werden durch die Delegation an die Professionelle oder den Professionellen gesteigert und reproduzieren sich in den darauffolgenden Antinomien (Helsper, 2002, S. 75 ff.).

Die gesteigerten Anforderungen als Folge der Delegation an professionelle Pädagoginnen und Pädagogen manifestieren sich unmittelbar in der Begründungsantinomie: Professionelle unterliegen, im Gegensatz zur Lebenspraxis selbst, bei ihren Handlungen und Interventionen einem erhöhten Handlungsdruck und einer gesteigerten Begründungs- und Legitimationsverpflichtung, die sich gerade durch die stellvertretende Handlungspraxis ergibt. Eng mit der Begründungsantinomie verbunden ist die Praxisantinomie: Professionelles Handeln bedarf einer reflexiven und wissenschaftlich basierten Wissensgrundlage und damit einer Haltung, die einer möglichst weitreichenden praktischen Handlungsentlastung entspricht. Dieses theoretisch-reflexive Handeln steht jedoch in einem Kontrast und widersprüchlich zum praktischen Handeln, das aus einem das professionelle Handeln ebenfalls bestimmenden hohen Handlungs- und Entscheidungsdruck resultiert. Einen wichtigen Bestandteil des professionellen Wissens bildet deshalb ein praktisch-reflexives Wissen zum Umgang mit diesen Unvereinbarkeiten.

Im Zusammenhang mit der Subsumtionsantinomie beschreibt Helsper die Spannung zwischen der Rekonstruktion, das heisst dem «verstehenden Nachvollzug des singulären Falles», und der Subsumtion, der «abkürzungshaften Unterordnung des Einzelfalles unter einen Typus oder eine verallgemeinernde Kategorie» (Helsper, 2016, S. 55).

Diese notwendige «Einordnung des Falles unter wissenschaftliche, klassifikatorische Kategorien» (Helsper, 2002, S. 78) läuft Gefahr, der Spezifik des Einzelfalles nicht gerecht zu werden, und birgt ein konstitutives Risiko des Scheiterns, wodurch der Erfolg ungewiss 
wird. Deshalb führen die bisher skizzierten konstitutiven Antinomien zur Ungewissheitsantinomie (Helsper, 2000, S. 145 f.): Trotz struktureller Ungewissheit im Hinblick auf den Erfolg des Handelns besteht die Notwendigkeit eines Erfolgsversprechens. Diese Antinomie zwischen Erfolgsversprechen und Ungewissheit erfährt eine Steigerung in der professionellen Intervention, was Oevermann (1996, S. 32) als «Gift-Gegengift-Konstellation» bezeichnet, da eine Intervention immer auch einen Eingriff in die Person der Klientin oder des Klienten darstelle und eine weitere Destabilisierung nach sich ziehen könne (Helsper, 2016, S. 55). Diese Problematik weist gleichzeitig auch auf die überlegene Position der oder des Professionellen hin, die sich unter der Symmetrieantinomie beschreiben lässt: Zwischen Professionellen und ihren Klientinnen und Klienten besteht eine mehr oder weniger offensichtliche Asymmetrie und daraus folgend eine mehr oder weniger deutliche Verteilung von Macht. Gleichzeitig sind jedoch «alle wertbezogenen Ziele professionellen Handelns» (Helsper, 2016, S. 55) wie beispielsweise psychosoziale Integrität oder Bildung nicht über eine asymmetrische Machtstruktur erreichbar, sondern verlangen eine «zustimmende Haltung der Klienten selbst»(Helsper, 2016, S. 55). Professionelles Handeln hat folglich auf der Basis einer strukturellen Asymmetrie eine Symmetrie herzustellen. Für eine Gegenseitigkeit wiederum, die auf einer asymmetrischen Struktur basiert, ist ein Vertrauensverhältnis erforderlich, das Helsper mit der Vertrauensantinomie aufgreift: Voraussetzung für die Etablierung einer Arbeitsbeziehung zwischen Professionellen und Klientinnen oder Klienten ist ein Vertrauensverhältnis, das bei noch Unvertrauten zuerst aufgebaut werden muss. Je ausgeprägter die Abhängigkeit der Klientin oder des Klienten von der professionellen Person ist und je tiefgreifender die Interventionen die Integrität der Klientin oder des Klienten betreffen, desto mehr ist diese Vertrauensbasis gefährdet, «würde doch auf der Grundlage der skizzierten Asymmetrie und Abhängigkeit erst einmal Vieles für ein gesundes Misstrauen sprechen» (Helsper, 2016, S. 55).

Auf einer zweiten Ebene werden widersprüchliche Handlungsanforderungen ausdifferenziert, welche den Hiatus von universalistisch-spezifisch und partikular-diffusen Beziehungslogiken betreffen. Mit der Näheantinomie schliesst Helsper an das von Oevermann herausgearbeitete Arbeitsbündnismodell der «widersprüchlichen Einheit von diffusen und spezifischen Sozialbeziehungen» an (z.B. Oevermann, 1996b, S. 119), das in Kapitel 2.3.3.3 ausführlich dargelegt wird. Diffuse Sozialbeziehungen sind idealtypisch 
durch emotionale Nähe und intimes Vertrauen gekennzeichnet, während spezifische Sozialbeziehungen sich durch rollenförmig-distanzierte, universalistische und affektiv neutrale Elemente charakterisieren lassen. Eine handelnde Person bewegt sich in einem kontinuierlichen Spannungsfeld zwischen den Polen Diffusität (Adressierung des Gegenübers in der Gesamtheit der Person) und Spezifität (Adressierung des Gegenübers im Umfang begrenzt auf einen definierten Bereich) (Parsons, 1951, S. 65 f.; vgl. auch Parsons et al., 1954). Für Lehrende besteht hierdurch die widersprüchliche Anforderung, sowohl «emotional-diffuse, partikulare Haltung, als auch distanzierte, spezifische und universalistische Haltungen gegenüber den Schülern einzunehmen» (Helsper, 2002, S. 84). Die aus diesen sich widerstreitenden, nahen und zugleich distanzierten Handlungsformen resultierende Anforderung lässt sich darauf zurückführen, dass Schülerinnen und Schüler, (noch) nicht in der Lage sind, zwischen rollenförmigen und diffusen Sozialbeziehungen zu unterscheiden und ihren Lehrpersonen deshalb in der Diffusität ihrer ganzen Person begegnen.

Bei der Sachantinomie geht es um die Orientierung der Lehrenden sowohl an universalistisch gültigen Sachbezügen als auch an den lebensweltlich gültigen, biografischen Rahmungen des zu vermittelnden Gegenstandes (Helsper, 2002, S. 84). Ein weiteres Spannungsverhältnis, dasjenige zwischen Individualität und Kollektivität, lässt sich in der Differenzierungs- oder Pluralisierungsantinomie fassen. Unterschiedliche biografische Ausgangslagen und Ressourcen erfordern eine Ungleichbehandlung, während professionelles Handeln gleichzeitig einer universalistisch orientierten, homogenen Haltung gegenüber den Lernenden bedarf. Des Weiteren findet professionelles Handeln innerhalb einer Organisationsstruktur statt und steht unter grossem Einfluss von entsprechenden Regulierungen. Die Organisationsantinomie weist auf die daraus resultierenden Entlastungen durch Routinen und Regeln hin, zeigt aber auch die Gefahr von stark geregelten und festgelegten Abläufen in konstitutiv nicht standardisierbaren Interaktionen auf.

Als elfte und letzte der von Helsper beschriebenen Antinomien ist schliesslich die Autonomieantinomie aufzuführen, die eine enge Verbindungslinie zur Symmetrieantinomie aufweist und die wohl grundlegendste Spannung pädagogischen Handelns darstellt (Helsper, 2010, S. 19). Sie bezieht sich auf die Spannung zwischen der «Unterwerfung unter den gesetzlichen Zwang» und der Fähigkeit, sich «seiner Freiheit zu 
bedienen», wie Immanuel Kant (1803, S. 711) dies ausdrückte. Einerseits ist pädagogisches Handeln selbst nicht frei von Zwängen und findet im Rahmen sozialer Regelwerke und Normen statt. Andererseits beabsichtigt pädagogisches Handeln, die Heranwachsenden zum selbstverantwortlichen Gebrauch von Freiheit zu befähigen, was ihnen allerdings eine defizitäre und einer pädagogischen Führung bedürftige Position zuschreibt (Helsper, 2010, S. 19). Pädagoginnen und Pädagogen handeln diesbezüglich in einem Als-ob-Modus, indem sie den Heranwachsenden Autonomie ${ }^{16}$ und das Vermögen zu Verantwortungsübernahme unterstellen, obwohl sie pädagogischer Stützung bedürften.

Folgt man bezüglich dieser Autonomieantinomie und weiterer antinomischer Figurationen, die im pädagogischen Handeln eingeschrieben sind und zu dessen strukturellem Kern gehören, Helsper, so liegt die Vermutung nahe, dass diese auch in der Eigenlogik von Mentoratsgesprächen zum Ausdruck kommen dürften. Vor dem Hintergrund dieser Annahme scheint die Hypothese zulässig, dass Mentorierende wie auch Studierende mit einer Vielzahl von in sich widersprüchlichen normativen Anforderungen und Erwartungen konfrontiert sind oder sein könnten. Das Treffen konkreter Vorannahmen in Bezug darauf, welche der elf Antinomien sich in den Fallrekonstruktionen - dem Kernstück dieser Untersuchung - empirisch manifestieren, stünde hingegen im Widerspruch zu einer rekonstruktiven und ergebnisoffenen Vorgehensweise.

\subsubsection{Arbeitsbündnis}

Professionelle Beziehungen zwischen Professionellen und Klientinnen oder Klienten oder auch zwischen Expertinnen oder Experten und Laiinnen und Laien werden in der einschlägigen Literatur oftmals als «Arbeitsbündnis» bezeichnet. Die dahinterliegende Konzeption stammt aus der Psychotherapie und besagt, dass der Erfolg professionellen Handelns nicht lediglich von der richtigen Anwendung von Expertenwissen abhängt, sondern eine beidseitig gewollte Kooperation voraussetzt (Müller, 2011). Die Klientin oder der Klient trägt daher einen entscheidenden Anteil am Erfolg der Therapie.

\footnotetext{
16 Diesbezüglich sei auch auf die Ausführungen von Ricken (2015, S. 144 ff.) verwiesen. Ricken nimmt hier eine Verschiebung vor, indem er festhält, dass es sich bei der Autonomie nicht um einen Gegensatz zu Heteronomie handle, sondern sich Heteronomie und Autonomie im Prozess der Subjektivation vielmehr wechselseitig bedingen würden, da das Subjekt sich (nur) am Anderen erlerne.
} 
Namentlich Freud und seine Nachfolgerinnen und Nachfolger haben die Vorstellung geprägt, in diesem Arbeitsbündnis gehe es auch darum, die kranken, ausser Kontrolle geratenen Anteile mithilfe der gesunden Anteile wieder zu integrieren (Müller, 2011). Dies impliziert, dass dieses Arbeitsbündnis einen Leidensdruck voraussetzt und dieser grösser sein muss als der Krankheitsgewinn, da der Klientin oder dem Klienten sonst das Motiv für das Eingehen des Arbeitsbündnisses fehlt.

Wie in Kapitel 2.3.2.2 bereits festgehalten wurde, ist das Arbeitsbündnis, das von Oevermann (z.B. 1996b) beschrieben wurde, ein zentrales Konzept der strukturtheoretischen Perspektive. In einem ersten Zugriff wird dieses Konzept nachfolgend zunächst bezogen auf das therapeutische Setting dargelegt, wofür Oevermann es ursprünglich auch erarbeitet hatte. Im Anschluss an die Beschreibung der strukturlogischen Gestalt des therapeutischen Arbeitsbündnisses werden in Kapitel 2.4 sodann Gemeinsamkeiten zur Konstellation zwischen Mentorierenden und Studierenden aufgezeigt und ins Verhältnis zum therapeutischen Arbeitsbündnis gesetzt.

Durch die Entscheidung, ein professionelles Arbeitsbündnis einzugehen, bindet sich die Klientin oder der Klient an die betreffende Therapeutin oder den betreffenden Therapeuten. Diese Entscheidung geht daher mit einer Verletzbarkeit und Abhängigkeit einher. Die entscheidende Herausforderung in diesem Arbeitsbündnis besteht vor diesem Hintergrund darin, der Klientin oder dem Klienten die Autonomie der Lebenspraxis zurückzugeben, ohne ihre respektive seine Situation auszunutzen. Deshalb verpflichtet sich die Klientin oder der Klient, möglichst viele Eigenkräfte zu mobilisieren. Dies bedeutet, dass sie oder er aus der Hilfe der Therapeutin oder des Therapeuten eine Hilfe zur Selbsthilfe macht, wie dies in den Montessori-Kindergärten mit dem Leitspruch «Hilf mir, es selbst zu tun» angestrebt wird (Oevermann, 2013, S. 123).

Obwohl sich die Klientin oder der Klient durch das Eingehen eines Arbeitsbündnisses mit der Therapeutin oder dem Therapeuten in eine Abhängigkeit begibt, gelten für Oevermann bereits das Aufsuchen dieser Person und das Sich-Einlassen auf eine Therapie aufgrund des Leidensdrucks als ein Akt der Autonomie, da sich die Klientin oder der Klient einerseits freiwillig zur Therapeutin oder zum Therapeuten begeben hat und andererseits das Ziel des Wiedererlangens von Autonomie verfolgt (Oevermann, 1996b, S. 115). Würde im umgekehrten Falle die Therapeutin oder der Therapeut auf die Klientin oder den 
Klienten zugehen und ohne Aufforderung helfen, käme dies einer De-Autonomisierung gleich und würde Schaden anrichten (Oevermann, 1996b, S. 116).

Die am Therapieprozess beteiligten Personen bilden eine autonome Praxis und binden sich in dieser in der Logik diffuser Sozialbeziehungen aneinander, obwohl sie grundsätzlich in der spezifischen Sozialbeziehung von Vertragspartnerinnen und Vertragspartnern einer käuflichen Dienstleistung verbleiben. Aus diesem Grunde ist jedes Arbeitsbündnis einer professionalisierten Praxis durch die widersprüchliche Einheit von diffusen und spezifischen Beziehungskomponenten bestimmt (Oevermann, 2013, S. 123).

Aus dieser Figur des Arbeitsbündnisses hat Oevermann ein allgemeines Strukturmodell professionellen Handelns entwickelt. Dabei folgte er der grundlegenden These, wonach alle professionalisierungsbedürftigen Berufspraxen im Kern mit der Aufgabe der stellvertretenden Krisenbewältigung beschäftigt seien. Um stellvertretende Krisenbewältigung handle es sich deshalb, weil es nicht um die Bewältigung der eigenen, sondern um die Bewältigung der Krisen anderer gehe. Für die professionalisierte Praxis der stellvertretenden Krisenbewältigung «reicht eine ingenieuriale Wissensanwendung nicht mehr aus und muss durch eine interventionspraktische Anleitung ergänzt werden» (Oevermann, 2013, S. 121). Diese interventionspraktische Komponente macht den "Strukturkern aller professionalisierten Praxis» aus, so dass man auch sagen kann, dass die Einheit von Theorie und Praxis sich nicht in der Wissenschaft selbst, sondern erst in der professionalisierten Praxis der stellvertretenden Krisenbewältigung herstellt (Oevermann, 2013, S. 121).

Die Interventionspraxis im professionellen, therapeutischen Arbeitsbündnis ist auf der Seite der oder des Professionellen nicht ausschliesslich rollenförmig möglich, sondern setzt eine Mischung von Handeln in der Rolle wie auch Handeln als ganze Person dar (Oevermann, 2013, S. 134). Auf der Seite der Klientinnen und Klienten ergibt sich komplementär dazu Folgendes:

Für die Durchführung des Arbeitsbündnisses gilt auf der Patientenseite die sogenannte «Grundregel». Sie gibt dem Patienten auf, alles zu thematisieren, was ihm durch den Kopf geht und ihm einfällt, vor allem eben auch das, was er für ganz unwichtig hält und was ihm eher peinlich ist. Die Grundregel entspricht exakt der Struktur der diffusen Sozialbeziehung. Sie fordert den Patienten auf, sich gemäss der Strukturlogik dieser Sozialbeziehung in der therapeutischen Situation zu 
verhalten. Die Grundregel lässt sich also professionalisierungstheoretisch übersetzen als Repräsentanz der Komponente der «diffusen Sozialbeziehung». (Oevermann, 1996b, S. 116)

Gerade weil die Klientin oder der Klient sich mit der Aufforderung «sei diffus» zur Überwindung dieser Hemmschwelle verpflichtet, gilt für die Therapeutin oder den Therapeuten so etwas wie eine «Abstinenzregel, deren Einhaltung [...] das Belassen der therapeutischen Beziehung im Modell der spezifischen Rollenbeziehung sichert» (Oevermann, 1996b, S. 117).

Der Klientin oder dem Klienten muss ein geschützter Rahmen geboten werden, innerhalb dessen sie oder er die Position als ganzer Mensch in der uneingeschränkten Übertragung praktisch folgenlos und sanktionsfrei einnehmen kann. Demgegenüber nimmt die Therapeutin oder der Therapeut die diffuse Position gewissermassen nur passiv und weitgehend latent ein, öffnet sich zwar innerlich vollständig und mit allen Empfindungen und Reaktionen, vermeidet jedoch äusserlich und manifest ein entsprechendes Agieren, ohne ein eigenes Mitempfinden im Sinne eines empathischen Mitleidens zu unterdrücken (Oevermann, 2013, S. 127 f.). Oevermann spricht in diesem Zusammenhang von «Gegenübertragungsgefühlen»:

[Die Komponente der diffusen Sozialbeziehung] äussert sich dort in der Gestalt der Gegenübertragungsgefühle und -empfindungen. Der Patient überträgt nämlich, indem er die Grundregel einzuhalten versucht, in der therapeutischen Situation die pathogenen Konstellationen seiner ursprünglichen sozialisatorischen Interaktionspraxis auf die diffuse Beziehung mit dem Therapeuten. Dieser wird darin zum «Übertragungsobjekt». Indem der Therapeut in sich die entsprechenden Empfindungen und Gefühle «aufsteigen» lässt, was zum abgekürzten Sinnverständnis der Re-inszenierung und der Symptome des Patienten notwendig ist, partizipiert er im Unterschied zum Patienten - allein innerlich an der diffusen Sozialbeziehung. (Oevermann, 1996b, S. 116 f.)

Die Therapeutin oder der Therapeut muss somit einerseits in der Logik einer spezifischen Sozialbeziehung Distanz zur Klientin oder zum Klienten wahren, darf sich aber andererseits auch nicht gegenüber den Übertragungstendenzen verschliessen, da eine solche «selbstimmunisierende Abwehr» die «notwendige Empathie zerstören und damit eine Therapie verunmöglichen» würde (Wernet, 2014, S. 80). So gilt die widersprüchliche 
Einheit von diffusen und spezifischen Beziehungsanteilen für beide Beteiligten. Dieses Wechselspiel von Übertragung und Gegenübertragung lässt sich in allen professionalisierten Klientinnen- und Klientenbeziehungen, das heisst in allen auf stellvertretende Krisenbewältigung ausgerichtete Lebenspraxen, beobachten. Dies ist allein schon deshalb der Fall, weil die Klientin oder der Klient als ganze Person beteiligt und adressiert wird. In der psychoanalytischen Therapie vollzieht sich diese Wechselwirkung besonders elaboriert und ausführlich (Oevermann, 2013, S. 134).

Des Weiteren weist das therapeutische Arbeitsbündnis Oevermann zufolge eine symmetrisch-asymmetrische Struktur auf: symmetrisch, da beide beteiligten Akteurinnen und Akteure diffuse wie auch spezifische Beziehungsanteile einbringen, und asymmetrisch, da innen innerhalb der Symmetrie unterschiedliche Rollen und auch unterschiedliche Verpflichtungen zukommen. So folgt beispielsweise die Initiierung des Arbeitsbündnisses durch die Entscheidung der Klientin oder des Klienten, sich aufgrund eines Leidensdrucks in die Therapie zu begeben. Nach dem Inkrafttreten gestaltet sich das Arbeitsbündnis hingegen kontingent und wechselseitig, da sich ein «kunstgerechter Vollzug eines interventionspraktischen Arbeitsbündnisses» strukturlogisch grundsätzlich einer Standardisierung verweigert (Oevermann, 2013a, S. 124). Eine solche würde unweigerlich dazu führen, dass sich die Klientin oder der Klient, um die oder den es letztendlich geht, einem Schematismus unterordnen müsste, wodurch die Krise einer Routine angeglichen würde, noch bevor sie überhaupt verstanden werden könnte (Oevermann, 2013a, S. 124).

Auf organisatorischer Ebene gibt es für die Etablierung eines professionellen Arbeitsbündnisses demgegenüber gewisse Standardisierungen, die im Sinne von Rechten und Pflichten für beide Beteiligten zum Ausdruck kommen. Beispielsweise wird die Therapiestunde regelmässig vorgeplant und endet nach Ablauf der vereinbarten Gesprächsdauer. Auch muss die Therapeutin oder der Therapeut fristgerecht für die Dienstleistung bezahlt werden. Des Weiteren währt ein therapeutisches Verhältnis aus der Logik einer diffusen Sozialbeziehung heraus unendlich, weshalb aus pragmatischen Gründen im Sinne einer risikoabwägenden Entscheidung ein Ende gesucht werden muss. An diesem Endpunkt treten die spezifischen Rollenbeziehungen wieder in den Vordergrund (Oevermann, 1996b, S. 122). Allerdings lässt sich, im Gegensatz zu gewissen anderen vertraglichen Beziehungen, die Dauer einer Therapie nicht bereits zu Beginn befristen. Vielmehr kann sich die Beendigung eines therapeutischen Arbeitsbündnisses sachhaltig allein durch den 
«zukunftsoffenen Ablauf der Autonomie der therapeutischen Praxis ergeben» (Oevermann, 1996b, S. 122).

Zusammenfassend lässt sich festhalten, dass in einem therapeutischen Arbeitsbündnis die widersprüchliche Einheit von diffuser und spezifischer Sozialbeziehung, die widersprüchliche Einheit von Autonomie und Heteronomie und das Wechselspiel von Übertragung und Gegenübertragung im Zentrum stehen. In diesem Arbeitsbündnis kommt am deutlichsten zum Ausdruck, was für alle auf stellvertretende Krisenbewältigung ausgerichteten Lebenspraxen zwischen Klientinnen oder Klienten und Professionellen strukturlogisch generell zutrifft. Denn nach Oevermann geht es hierbei nicht um bewusst gewählte berufliche Strategien, sondern um eine Implikation der Pragmatik der Interventionspraxis in einem Arbeitsbündnis (Oevermann, 2013, S. 134).

Dieses Strukturmodell eines therapeutischen Grundmusters überträgt Oevermann (2002b, S. 35) auch auf die Pädagogik und hierbei auf die Schule und begründet dies mit einer strukturellen Ähnlichkeit von Therapie und Pädagogik. Diese Ähnlichkeit bezieht er beispielsweise auf die beiderseits angestrebte Autonomie und schliesst daraus, dass «das strukturanalytische Grundmodell von therapeutischer Praxis für die Bestimmung der pädagogischen Praxis einen geeigneten, sogar unabdingbaren Bezugsrahmen abgibt» ${ }^{17}$ (Oevermann, 1996b, S. 150). Für die Entlehnung und Übertragung des therapeutischen Arbeitsbündnismodells auf das Pädagogische wurde Oevermann vielfach und harsch kritisiert. Gruschka (2003, S. 77) beispielsweise bemängelt die zu wenig differenzierte Übernahme der Theorie der klassischen Professionen und die daraus folgende Gleichsetzung des Verhältnisses einer Lehrperson und ihren Schülerinnen und Schülern mit demjenigen einer Ärztin oder eines Arztes zu einer Patientin oder einem Patienten. Er kritisiert, dass Oevermann auf der Ebene der Modellbildung (Arzt-Patienten-Modell) bleibe und den Einbezug der empirischen Erfahrung im Verhältnis von Lehrperson und Lernenden nicht berücksichtige. Auch Baumert und Kunter (2006, S. 470) kritisieren, dass das Rollenbild des Psychoanalytikers in strukturtheoretisch geprägten Ansätzen auf das Rollenbild der Lehrperson übertragen werde, was sie als unangebracht taxieren. Wernet (2014) wiederum erachtet es als wesentliche Bruchstelle in Oevermanns Argumentation,

\footnotetext{
${ }^{17}$ Eine ausführliche Herleitung der von Oevermann angenommenen Analogien wird hier nicht vorgenommen. Vgl. dazu z.B. Oevermann (1996b).
} 
dass er den therapeutischen Komplex und den Wissenskomplex auf der gleichen Ebene ansiedle und den Leidensdruck der Klientinnen und Klienten mit der Neugier der Lernenden analogisiere und damit den Lehrberuf dem therapeutischen Komplex zuordne. Die Annahme, therapeutische und pädagogische Settings gleichzusetzen, sei zwar verlockend, gründe jedoch auf einer Utopie, nämlich einem Ideal einer Kooperation, in welcher Zwang wegdefiniert werde und sich Neugier und Selbsttätigkeit von allein entfalte (Wernet, 2014).

An dieser Stelle werden weder weitere Kritikpunkte eingebracht, die die bereits formulierte Kritik der Übertragung des therapeutischen Arbeitsbündnisses auf eine pädagogische Situation ergänzen oder stützen, noch soll Kritik an den exemplarisch aufgeführten Kritikpunkten geübt werden. Vielmehr beziehe ich die von Oevermann meines Erachtens überzeugend und stringent herausgearbeiteten Strukturmerkmale des therapeutischen Arbeitsbündnisses als Reflexionsschablone in meine Überlegungen zum Arbeitsbündnis im Mentorat ein, die ich in Kapitel 2.4 ausführe. ${ }^{18}$

\subsubsection{Pädagogische Permissivität}

Abschliessend und in grundsätzlicher Abgrenzung zu den strukturtheoretischen Bestimmungsansätzen pädagogischen Handelns von Oevermann und Helsper und vor allem als Einwand gegen eine Klassifikation des Lehrberufs als Profession sei nachstehend in aller Kürze und Knappheit auf Wernets Ansatz der pädagogischen Permissivität verwiesen (Wernet, 2003). Bei Dietrich (2014, S. 64 ff.) wird Wernets Konzept und seine Bezugnahme zu Oevermanns und Helspers Modell vertieft und gleichwohl im Sinne einer Zusammenfassung dargelegt.

\footnotetext{
${ }^{18}$ Auf Vergleiche oder Analogien des Arbeitsbündnisses im Mentorat mit dem pädagogischen Arbeitsbündnis wird bewusst verzichtet, weil letzteres theoriearchitektonisch nicht überzeugt und sich folglich nicht oder zu wenig als Erklärschablone eignet. Im kritischen Fokus steht insbesondere die von Oevermann vorgeschlagene Verortung pädagogischen Handelns im Feld der Therapie. Exemplarisch für Kritik an der Übertragung des therapeutischen auf ein pädagogisches Arbeitsbündnis sei hier Wagner zitiert: «Indem Oevermann in seinem Modell die Professionalität pädagogischen Handelns an die Wahrnehmung der therapeutischen Dimension bindet und von dieser abhängig macht, begeht er einen folgenreichen Kategorienfehler. Insofern nämlich pädagogisches Handeln es mit Lernen im Sinne des Machens neuer Erfahrungen zentral zu tun hat -und dies bestreitet Oevermann ja keineswegs -, kann der Bezugspunkt für dessen Professionalisierung nicht Therapie sein. Der Bezugspunkt zur Konstitution einer Professionstheorie pädagogischen Handelns muss demgegenüber die emergierende pädagogische Handlung unter der Perspektive des Lernens von Neuem sein. [...] Wissen, Normen und Therapie sind lediglich spezifische Ausprägungen des Lernens von Neuem» (Wagner, 1998, S. 96).
} 
Mit seiner Argumentationslinie verortet sich Wernet ausserhalb der Professionalisierungsdiskussion und sieht die Logik pädagogischen Handelns nicht primär in der Bearbeitung widersprüchlicher Anforderungen, sondern in erster Linie in deren Vermeidung. Für die strukturlogische rekonstruktive Entwicklung seines pädagogischpermissiven Ansatzes setzt Wernet bei Auffälligkeiten aus Alltagsbeispielen pädagogischer Interaktion an und stellt diesbezüglich «Entgrenzungen» seitens der Lehrpersonen fest (z.B. Wernet, 2003, S. 11 ff., 2018a). Solche üblichen Formen «taktlosen, indiskreten und demütigenden pädagogischen Handeln[s]» (Wernet, 2003, S. 17) reagieren Wernet zufolge nicht auf Widersprüchlichkeiten und Inkonsistenzen, sondern erzeugen diese erst. Auf diesen Erkenntnissen beruhend und in Bezugnahme auf Parsons Begriffssystem der «pattern variables» (Parsons, 1951) erachtet Wernet die Vereindeutigung der Situation durch ein rein rollenförmiges, spezifisches und an einer universalistisch-unpersönlichen Leistungsorientierung ausgerichtetes Verhalten als «eine den sozialisatorischen Prozess unterstützende Operation» (Wernet, 2003, S. 120).

Permissive Widerspruchs- und Entgrenzungsvermeidung sind Wernet zufolge Gelingensbedingungen für das Lehrhandeln. Knapp formuliert sieht Wernet die Logik pädagogischen Handelns nicht in der Vermittlung, sondern in der Vermeidung von widersprüchlichen Anforderungen. Das Heranziehen der Dynamik dieser «kunstvolle[n] Aufrechterhaltung von Distanz», wie Wernet dies nannte (2003, S. 168), als Vergleichsfolie für die Konstellation im Mentorat scheint mit Blick auf entsprechende Erkenntnisgewinne aussichtsreich zu sein und wird deshalb als sensibilisierendes Konzept weiterverfolgt. 


\section{4 Überlegungen zum Arbeitsbündnis in der Mentorats- konzeption}

Wie bei der Diskussion der Mentoratskonzeption in Kapitel 2.1.2 aufgezeigt wurde, zielt das Mentorat am IKU der PH FHNW programmatisch auf eine individuelle Förderbeziehung zwischen den Studierenden und den Mentorierenden zur Unterstützung des Professionalisierungsprozesses zur Lehrperson ab. Für die Bezeichnung dieser institutionalisierten und individuell ausgerichteten Beziehungskonstellation scheint sich der Terminus «Arbeitsbündnis» anzubieten, der sich, wie zuvor ausgeführt, auf einen vertraglich geregelten Zusammenschluss oder eine Zusammenarbeit von nicht zwingend formell gleichberechtigten Partnerinnen und Partnern zur Erreichung eines bestimmten, gemeinsamen Ziels bezieht.

Aus einer analytischen Perspektive betrachtet weist die arbeitsbündnishafte Beziehungsoder Interaktionsform im Setting «Mentorat» durchaus gewisse Ähnlichkeiten, gleichzeitig aber auch Differenzen zu einer therapeutischen Beziehungsform auf. Angesichts der Ähnlichkeiten, beispielsweise die dyadische Gesprächssituation zwischen Laiin oder Laie und Expertin oder Experte, scheint es aussichtsreich zu sein, das von Oevermann für den therapeutischen Kontext ausführlich beschriebene Arbeitsbündnismodell bei der Betrachtung der Beziehungskonstellation zwischen Mentorierenden und Studierenden im Sinne eines sensibilisierenden Konzepts zu verwenden. Der Terminus «sensitizing concepts» wird bei Blumer wie folgt erläutert: "Whereas definitive concepts provide prescriptions of what to see, sensitizing concepts merely suggest directions along which to look» (Blumer, 1954, S. 7, zitiert nach Kelle \& Kluge, 2010, S. 29). Unter einem sensibilisierenden Konzept wird expliziertes Wissen verstanden, das bestimmte Lebenserfahrungen, gezielt erhobenes Kontextwissen über das Feld und auch geeignete Theoriebezüge enthält (Glaser \& Strauss, 2010). Dieses Wissen wird im Sinne von heuristischen Vorannahmen für den Erschliessungsprozess beigezogen, ohne jedoch einen erkenntnisdeterminierenden Charakter anzunehmen. Vielmehr kommt ihm eine heuristische und in der Folge sensibilisierende Funktion zu (Strauss \& Corbin, 1996, S. 26). Vor diesem allgemeinen Hintergrund soll nun konkret geprüft werden, inwiefern sich die Arbeitsbündniskonzeption der Therapie auch als theoretischer Rahmen für das Mentorat 
fruchtbar machen lässt. Diesbezüglich stellt sich insbesondere die Frage, welche Aspekte des Arbeitsverhältnisses oder Arbeitsbündnisses zwischen der Mentorin oder dem Mentor und der Studentin oder dem Studenten sich aus einer pragmatischen Sichtweise analog und welche sich different zu einem therapeutischen Arbeitsbündnis gestalten. Um die entsprechenden Analogien und Differenzen zu markieren und darzustellen, werden die Strukturmerkmale des Therapiegesprächs denjenigen des Mentoratsgesprächs als Vergleichsfolie gegenübergestellt, wobei allerdings die Strukturprobleme des KrisenRoutinen-Modells wie auch die Strukturprobleme des Übertragung-GegenübertragungModells nicht explizit herangezogen werden. Begründen lässt sich diese Nichtberücksichtigung damit, dass beide Modelle eher die Individualebene betreffen, während sich das Forschungsvorhaben primär mit der institutionellen Ebene befasst und diesbezüglich das Strukturproblem des Arbeitsbündnisses in der Mentoratskonstellation im Mittelpunkt steht.

Aus einer etwas anders ausgerichteten Perspektive heraus kann jedoch in beiden Konstellationen, Therapie und Mentorat, die Rückgewinnung oder Stärkung der Autonomie als Ziel der Gespräche betrachtet werden. In der therapeutischen Konstellation ist die personale Autonomie der Klientin oder des Klienten beschädigt oder beeinträchtigt. Im Modus der stellvertretenden Krisenbewältigung gilt es, diese wiederherzustellen. Im Mentorat steht zwar nicht die personale Autonomie im Zentrum, aber es geht um die Entwicklung einer beruflichen oder professionellen Autonomie und Handlungsfähigkeit. Dieses Ziel zieht institutionalisiert die Unterstützung und Begleitung durch eine Mentorin oder einen Mentor nach sich.

Das therapeutische Arbeitsbündnis beruht wesentlich auf den Leidensdruck respektive auf der 〈Not〉 der Klientin oder des Klienten, weshalb der erste Schritt für das Arbeitsbündnis vonseiten der Klientin oder des Klienten vorgenommen wird. Im Mentorat hingegen erfolgt dieser erste Schritt institutionalisiert durch die hochschulseitige Zuteilung der Studierenden zu einer Mentorin oder einem Mentor. Der Studentin oder dem Studenten wird somit in gewisser Weise a priori eine Unterstützungsbedürftigkeit oder - in analoger und gewagter Formulierung - eine «Not` unterstellt. Das Setting basiert somit auf der Defizitannahme, dass aufseiten der Studierenden eine Hilfs- oder Unterstützungsbedürftigkeit vorliege, und zwar ungeachtet dessen, ob dies auch tatsächlich zutrifft oder nicht der Fall ist. Im Vergleich mit einer Therapie erfolgt die Initiative für das 
Arbeitsbündnis folglich gegenläufig: Während ein therapeutisches Setting bei entsprechendem Bedarf und auf Anfrage der Klientinnen und Klienten initiiert wird, ${ }^{19}$ geht im Mentorat die Hochschule konzeptionell von einem entsprechenden Bedarf aus und institutionalisiert das Mentorat als Unterstützungs- und Begleitformat ohne Berücksichtigung der individuellen Bedürfnislage der Studentin oder des Studenten. Anders formuliert ist im therapeutischen Setting der Anlass für das Arbeitsbündnis aufgrund der individuellen «Not` der Klientin oder des Klienten gegeben, wohingegen der Anlass für das Eingehen des Arbeitsbündnisses im Mentorat lediglich auf den formalen Vorgaben und der Verpflichtung, diese Gespräche zu führen, beruht. Diese markante Differenz zwischen Therapie und Mentorat lässt sich mit Häckers Unterscheidung zwischen Anlass und Veranlassung beschreiben (2017, S. 27 f.): Während der Anlass, das heisst das Eingeständnis einer Krise, die darauf zurückzuführen ist, dass etwas nicht mehr weitergehen kann wie bisher, (idealerweise) einer inneren Erkenntnis der Klientin oder des Klienten entspringt, kann eine Veranlassung auch von aussen erfolgen, wie dies beispielsweise für den vorliegenden Ausbildungskontext des Mentorats zutrifft. Diese Differenz hat auch bedeutsame Auswirkungen auf diffuse und spezifische Elemente in der Sozialbeziehung der beiden Vertrags- respektive Arbeitsbündnispartnerinnen oder Arbeitsbündnispartner. Diffuse und spezifische Sozialbeziehungen wurden bereits in Kapitel 2.3.3.2 ausgeführt und werden nachstehend kontextbezogen vertieft.

Im therapeutischen Arbeitsbündnis binden sich die Klientin oder der Klient und die Therapeutin oder der Therapeut in der Logik diffuser Spezialbeziehungen als ganze Person aneinander, obwohl sie grundsätzlich in der Logik einer spezifischen Sozialbeziehung von zwei Vertragsparteien einer Dienstleistung verbleiben (Oevermann, 2013, S. 123).

Infolgedessen ist das Arbeitsbündnis zwischen Therapeutin oder Therapeut und Klientin oder Klient geprägt durch die widersprüchliche Einheit von diffusen und spezifischen Beziehungskomponenten (Oevermann, 2013, S. 127). Eine diffuse Begegnung in diesem Arbeitsbündnis oder Setting wird möglich, da es qua Institutionalisierung einen sicheren und gefestigten Raum für die Klientin oder den Klienten wie auch für die Therapeutin oder den Therapeuten darstellt. Dadurch können die gleichzeitigen und widersprüchlichen

\footnotetext{
19 Das Setting einer verordneten Zwangstherapie wird hier ausgenommen, weil diese nicht dem Regelfall entspricht und der Zwang auch ein Problem dieses Settings darstellen dürfte.
} 
Ansprüche von Diffusität und Spezifität vom System her unter den Prämissen von Professionalisierung und Freiwilligkeit sichergestellt und gewährleistet werden. Für die Klientin oder den Klienten bedeutet dies, dass sie oder er ohne Folgen (Oevermann, 2013, S. 127) ihre oder seine Position als ganzer Mensch einnehmen kann und dies auch tun soll. In der Konstellation des Mentoratsgesprächs gestalten sich diese Sicherstellung und diese Gewährleistung bereits auf der konzeptionellen Ebene different. Denn während die Gesprächsanlage in der Therapie wie ausgeführt primär diffus angelegt ist und die Klientin oder den Klienten als ganze Person mit ihrer respektive seiner (Not) ins Zentrum stellt, scheint die Gesprächsanlage im Mentorat strukturell und ex negativo eher spezifisch disponiert zu sein, da die professionelle Legitimation zur diffusen Begegnung fehlt. Diese Differenz lässt sich beispielsweise an der Verpflichtung, in ein Arbeitsbündnis mit der Mentorin oder dem Mentor einzutreten, wie auch an der nicht vorhandenen Wahl, ob und mit wem die Studierenden diese Verpflichtung eingehen möchten, festmachen.

An dieser Stelle lohnt sich ein Kurzexkurs zu Oevermanns Argumentation zur Abschaffung der Schulpflicht (Oevermann, 2003, 2006). Darin führt Oevermann aus, dass die Gesellschaft davon ausgehe, Kinder und Jugendliche würden nicht freiwillig zur Schule gehen und lernen, sondern müssten dazu gezwungen werden. Oevermann zufolge sei allerdings gerade dieser Zwang kontraproduktiv und torpediere die grundsätzliche Neugier und einen entsprechenden Wissensdurst, die Kinder von Natur aus besässen. Vor diesem Hintergrund argumentiert er für den schulischen Kontext, dass die gesetzliche Schulpflicht nicht nur überflüssig geworden sei, sondern ihre Abschaffung aus soziologischer Sicht unter der Prämisse der «Professionalisierung von gewissen Dienstleistungen, denen gemeinsam ist, dass sie auf eine expertenhafte stellvertretende Krisenbewältigung für die primäre Lebenspraxis hinauslaufen» sogar als positiv zu bewerten wäre (Oevermann, 2003, S. 54). Als Gedankenkonstrukt ist diese Argumentationslinie auch für das Pflichtsetting «Mentorat» durchaus aufschlussreich und prüfenswert. Würde man die dahinterliegende Überlegung auf das Mentorat übertragen, hiesse dies Folgendes: Die Hochschule geht davon aus, dass die Studierenden die gebotene Unterstützung durch das Mentorat nicht beanspruchen würden, wenn sie nicht dazu angehalten und verpflichtet wären, dies zu tun. Alltagssprachlich und vereinfacht formuliert werden die Studierenden zu ihrem Glück gezwungen, weil grundsätzlich angenommen wird, dass sie das Potenzial und den Nutzen wie auch die Chancen des 
Begleitformats «Mentorat» von sich aus, das heisst ohne Verpflichtung, nicht erkennen würden. In Analogie zu Oevermanns Frage, welches die Vorzüge einer Abschaffung der gesetzlichen Schulpflicht wäre, ist die Frage, was die Vorzüge einer Abschaffung der institutionalisierten Mentoratspflicht wären, im Sinne einer sensibilisierenden Frage im Kontext des vorliegendes Forschungsvorhabens am Rande mitzudenken, wobei allerdings nicht Anspruch erhoben werden soll, diese Frage im Rahmen dieser Untersuchung zu beantworten.

Ein weiteres, markantes Unterscheidungsmerkmal ist die institutionalisierte Beurteilung, die die Mentorin oder der Mentor als Bedingung für die Kreditierung des Workloads vornehmen muss. Die Beurteilung wird im Modus von «pass» oder «fail» vorgenommen und ist damit inhaltlich bezüglich der Qualitätsaussage gering ausgeprägt, bezüglich des weiteren Studienverlaufs hingegen durchaus folgenreich. Des Weiteren gilt es den Aspekt zu berücksichtigen, dass Therapiegespräche wie auch Mentoratsgespräche einer formalen Rahmung unterliegen. Eckpfeiler dieser Formalstruktur sind in der Therapie nebst einer grundsätzlichen Freiwilligkeit auch die freie Wahl der Therapeutin oder des Therapeuten, die Bezahlung der Dienstleistung und die Unbestimmtheit der Therapiedauer. Überträgt man diese Eckpfeiler als Orientierungsrahmen auf die Mentoratsbeziehung, lassen sich wie teilweise bereits festgehalten und im Gegensatz zum therapeutischen Setting eine Gesprächsverpflichtung, eine fixe Zuteilung der Studierenden zu Mentorinnen und Mentoren, eine Bewertungsverpflichtung, mit der in der Folge auch Abhängigkeitsaspekte einhergehen, sowie eine klar absehbare Zeitdauer und Limitation des Arbeitsbündnisses, da das Ende bereits vor dessen Aufnahme festgelegt ist, feststellen. Als letzter Punkt sei angefügt, dass das Arbeitsbündnis, wenn es nicht auf Einwilligung oder Freiwilligkeit der Klientin oder des Klienten beruht, auch das Moment einer sozialen Kontrolle aufweist. Das Verhältnis von «Hilfe und Kontrolle» bleibt dabei jedoch ungeklärt (Oevermann, 2013a, S. 139), und zwar sowohl in Bezug auf die Frage, wie es auf der einen Seite intendiert, als auch hinsichtlich der Frage, wie es auf der anderen Seite aufgefasst respektive aufgenommen wird.

Im Sinne eines Fazits der konzeptionellen Rahmung des Arbeitsbündnisses im Mentorat gilt es resümierend Folgendes festzuhalten: Das Arbeitsbündnis im Mentorat weist aus einer konzeptionellen Disponiertheit heraus betrachtet Merkmale auf, die sich möglicherweise auch empirisch manifestieren könnten. Aus einer theoretischen 
Perspektive scheint die Legitimierung des Arbeitsbündnisses im Mentorat weniger gesichert und gefestigt zu sein als in der Therapie, wo die (Not) der Klientin oder des Klienten dieses Bündnis und auch die damit einhergehende Begegnung in der Diffusität der ganzen Person legitimiert. Im für das Mentorat charakteristischen Arbeitsbündnis scheint sich diese Konstellation, vor allem auch bezogen auf die Legitimierung des Settings, spannungsvoller und auch unsicherer zu gestalten, und dies, obwohl das Setting formal etabliert ist und in der Schweizer Lehrerinnen- und Lehrerbildung seit geraumer Zeit Anwendung findet. Im Sinne einer Sensibilisierung werden diese Überlegungen zu Differenzen und Gemeinsamkeiten des Arbeitsbündnisses im Mentorat und in der Therapie als Folie und theoretische Rahmung für die in Kapitel 4 erfolgenden Analysen von Mentoratsgesprächen verwendet und in Kapitel 5 zu den empirischen Erkenntnissen ins Verhältnis gesetzt. 


\section{$3 \quad$ Forschungsdesign und methodische Anlage der Untersuchung}

\subsection{Forschungsinteresse}

Das dieser Untersuchung zugrunde liegende Forschungsinteresse richtet sich auf die Frage, wie sich die soziale Interaktionspraxis in Mentoratsgesprächen zwischen den Studierenden und den Mentorierenden gestaltet. Mit einer offen formulierten Frage geht es in einem ersten Schritt darum, herauszuarbeiten und zu verstehen, wie solche Gespräche verlaufen und was sich in diesen Gesprächssituationen auf eine soziale Betrachtungsebene bezogen ereignet. Wie bereits in Kapitel 2.2 ausgeführt, verfolgten bisherige Untersuchungen zu Mentoring an Pädagogischen Hochschulen, sei dies zwischen Praxislehrpersonen und Studierenden oder zwischen Hochschulmitarbeitenden und Studierenden, vorwiegend eine befragungsbasierte Annäherung an das Format «Mentorat in der Lehrerinnen- und Lehrerbildung». ${ }^{20}$ Befragungen in schriftlicher wie auch mündlicher Form gelten als meistbeschrittener Weg in der Sozialforschung (Diekmann, 2017, S. 434) und ermöglichen die Erforschung von Einstellungen und Meinungen. Mit dem vorliegenden Forschungsvorhaben gilt es allerdings, die charakteristischen Strukturen von Mentoratsgesprächen zu rekonstruieren und die hinter diesem Phänomen operierenden Gesetzmässigkeiten herauszuarbeiten. Denn eine Untersuchung der sozialen Praxis solcher Gespräche steht noch aus und dieses Forschungsdesiderat bildet die Ausgangslage des vorliegenden Vorhabens. Konkretisieren lässt sich das Forschungsinteresse mit der Frage nach der sinnstrukturierten Verfasstheit der sozialen Praxis von Mentoratsgesprächen. Der in der Sinnstrukturiertheit enthaltene Sinnbegriff ist deskriptiv-analytisch zu verstehen. Es geht deshalb nicht um ein normatives Sinnverständnis, beispielweise um die Ermittlung der Sinnhaftigkeit solcher Gespräche, sondern darum, den Sinn zu klären, welcher der Ausdrucksgestalt dieser Gespräche

\footnotetext{
20 Eine genauere Bestimmung der Konstellation zwischen Mentorierenden und Studierenden, auf die sich die vorliegende Untersuchung richtet, und eine entsprechende Abgrenzung zu ähnlichen, jedoch differenten Konstellationen wurden in Kapitel 2.1.2 vorgenommen.
} 
zukommt (Oevermann, 2013a, S. 71). Ziel des Vorhabens ist es, auf der Grundlage einer Auseinandersetzung mit Protokollen von konkreten Gesprächssituationen Aussagen über die Eigenlogik und die fallspezifischen Interaktionsdynamiken von Mentoratsgesprächen zu treffen. Diese Gesprächsprotokolle können als ausdrucksmateriale Gegebenheiten der beziehungs- und interaktionsstrukturierten Praxis von Mentoratsgesprächen verstanden werden, da sich die entsprechende Eigenlogik und die Interaktionsdynamiken dieser sozialen Praxis in die Gesprächsprotokolle einschreiben und darin Ausdrucksgestalt gewinnen (Oevermann, 2013a, S. 73). Mentoratsgespräche werden folglich als interaktives Geschehen gefasst, in welchem sich die beteiligten Akteurinnen und Akteuren in Wechselwirkung aufeinander beziehen, das heisst aufeinander reagieren und einander beeinflussen respektive steuern. Wenn die soziale Praxis dieser Gespräche als Interaktionsgeschehen und nicht einfach nur als ein Verhalten von beteiligten Personen betrachtet wird, zieht dies eine Vorstellung von Regelgeleitetheit des sinnstrukturierten und reziproken sozialen Handelns nach sich. Dies bedeutet, dass sich soziales Handeln entlang von Regeln konturiert, weshalb die Interpretation eines Textprotokolls verbindlich unter Rückgriff auf dieses Regelwissen zu erfolgen hat (Wernet, 2009, S. 13).

Zusammengefasst lässt sich der Forschungsgegenstand des Vorhabens somit als Interaktionsgeschehen mit einer eigenlogischen Struktur und entsprechenden Dynamiken charakterisieren. Im Folgenden gilt es nun aufzuzeigen, wie und mit welchen methodologischen Prämissen sich diese Struktur und die dazugehörenden Dynamiken der Praxis von Mentoratsgesprächen untersuchen lassen. Die diesbezügliche gegenstandstheoretische Bestimmung wird in Kapitel 3.2.1 vorgenommen, bevor in Kapitel 3.2.2 die methodologischen Grundlagen der Objektiven Hermeneutik in aller Kürze ausgeführt werden. Das Folgekapitel 3.3 beinhaltet schliesslich eine Darstellung des methodischen Vorgehens, in der die Datenerhebung und das Sampling wie auch die sequenzanalytische Datenauswertung und die methodischen Prinzipien der Objektiven Hermeneutik beschrieben werden. 


\subsection{Theoretische und methodologische Rahmung}

\subsubsection{Gegenstandstheoretische Bestimmung}

Im vorausgehenden Kapitel wurde die Betrachtung der sozialen Praxis eines dyadischen Mentoratsgesprächs als Interaktionsgeschehen erläutert. Für die Erfassung der in diesen Interaktionen aufscheinenden Dynamiken bietet sich als theoretischer Bezugsrahmen ein strukturtheoretischer Ansatz mit der bereits in Kapitel 2.3 erläuterten strukturtheoretischen Konzeption der «Autonomie der Lebenspraxis» und den Grundbegriffen «Krise und Routine» an, da für eine autonome Lebenspraxis die selbstständige Bewältigung von Krisen konstitutiv ist. Denn wie ebenfalls bereits ausgeführt, besteht der programmatische Grundgedanke des Mentorats darin, die Studierenden in der Ausbildung bei der Bewältigung ihrer Krisen zu unterstützen und zu begleiten. Diesen strukturtheoretischen Krisenbegriff gilt es hierbei weit zu fassen und bezieht sich auf die Krisen des Bildungsprozesses, die sich daraus ergeben, dass die Studierenden noch nicht ausgebildet sind und sich noch in Ausbildung befinden (Oevermann, 1996b).

In dieser strukturtheoretischen Perspektive werden die Gesprächsprotokolle dieser Interaktionen in den Blick genommen, in denen sich zeigt, wie sich die soziale Wirklichkeit in diesen Gesprächen konstituiert. Auf dieser Grundlage lassen sich - mit Blick auf meine Fragestellung - die Gestalt gewinnenden Dynamiken aus den in sich widersprüchlichen Anforderungen identifizieren und herausarbeiten.

Für die intersubjektiv überprüfbare und methodisch kontrollierte Herausarbeitung der Eigenlogik der Interaktionsstruktur in Mentoratsgesprächen und der darin manifest werdenden Interaktionsdynamiken und Bezugnahmen der Akteurinnen und Akteuren auf das Setting und aufeinander bietet sich ein Forschungsansatz, wie inn die Objektive Hermeneutik bereitstellt, an. Die entsprechenden strukturtheoretischen Prämissen werden im nächsten Kapitel im Zusammenhang mit der Darlegung der Auswertung und der methodischen Prinzipien ausgeführt.

Wie in Kapitel 3.1 bereits festgehalten, wurde der Forschungsgegenstand, das heisst die Strukturlogik und die Interaktionsdynamiken der sozialen Praxis von Mentoratsgesprächen, in In-situ-Protokollen festgehalten, da davon auszugehen ist, dass er in den Gesprächen zum Ausdruck kommt. Mittels solcher Gesprächsprotokolle lässt sich die 
Wirklichkeit materialisieren und zugänglich machen (Wernet, 2009, S. 11). Dies bedeutet allerdings, dass in der vorliegenden Arbeit die Protokolle von Mentoratsgesprächen analysiert werden, die effektive soziale Praxis dieser Gespräche jedoch nicht untersucht werden kann. Denn «ein direkter Zugang zur protokollierten Wirklichkeit ist methodologisch prinzipiell nicht möglich, [sondern] vielmehr dem Hier und Jetzt der Lebenspraxis vorbehalten» (Oevermann, 1993, S. 132). Die Protokolle der Gespräche erlauben somit lediglich einen mittelbaren Einblick in die konkrete soziale Wirklichkeit oder Praxis. Solche In-situ-Daten haben jedoch den Vorteil, dass sie die Ebene der konkreten Handlungspraxis dokumentieren und weniger dem Phänomen der sozialen Erwünschtheit (z.B. Bortz \& Döring, 2015) unterliegen, als dies beispielsweise bei Selbstauskünften in Befragungen vermutbar wäre.

\subsubsection{Methodologische Grundlagen der Objektiven Hermeneutik}

Konkretisieren lässt sich die Analyse und Handhabbarmachung der Struktureigenschaften, das heisst der Sinn- und Bedeutungsstrukturen dieser sozialen Praxis, mit einem sequenzanalytischen Vorgehen, das die analytische Differenz zwischen dem Spielraum von Möglichkeiten und der faktisch getroffenen Auswahl in den Mittelpunkt rückt (Oevermann, 2013b, S. 75). Eine entsprechende sequenzanalytische Datenauswertungsmethode bietet die Methode der Objektiven Hermeneutik. Deren theoretische Konstitutionsprämissen der Regelgeleitetheit und Sequenzialität werden nachfolgend in Kürze dargelegt.

Die Erklärung der lebenspraktischen Wirklichkeit beruht auf zwei verschiedenen Parametern: Ein erster Parameter von bedeutungserzeugenden Regeln lässt an jeder Sequenzstelle Anschlussmöglichkeiten für die Zukunft eröffnen oder schliessen (Oevermann, 2002a, S. 7). Der tatsächliche Ablauf ist durch den zweiten Parameter bestimmt, der eine «Auswahl aus den durch Sequenzregeln eröffneten Möglichkeiten, also die Entscheidung trifft» (Oevermann, 2010, S. 31).

Sequenzanalyse bedeutet, an jeder Sequenzstelle beide Ebenen gleichzeitig abzudecken, «die der eröffneten Möglichkeiten und auf dieser Folie die Abbildung der tatsächlich vollzogenen [... ] Wirklichkeiten» (Oevermann, 2013b, S. 75). 
Das Konzept der Regelgeleitetheit aus dem ersten Parameter besagt nicht, was zu tun sei, sondern vielmehr was es bedeutet, wenn etwas so getan oder gesagt wird, wie es getan oder gesagt wird. Diese Regelgeleitetheit verleiht einer Handlung erst ihren Sinn. Auch ist der an einer Sequenzstelle eröffnete Möglichkeitsraum nicht beliebig, sondern durch diese Regelgeleitetheit vorstrukturiert. Diese Regeln operieren «wie Algorithmen unabhängig von den Intentionen und Absichten der konkret handelnden Subjekte» (Oevermann, 1996b, S. 76). Der zweite Parameter betrifft die Sequenzialität, da mit jeder Handlung an einer Sequenzstelle vorausgehend eröffnete und bis dahin noch offene Möglichkeiten geschlossen und neue Möglichkeiten eröffnet werden (Oevermann, 2002a, S. 7). Welche Auswahl konkret getroffen wird, ist kein bewusster Entscheidungsprozess. Vielmehr knüpft die Auswahl unbewusst und spontan an die Gesamtheit der Disposition einer Lebenspraxis, mit deren Eigenarten, Charakteren, Motivationen, Wertorientierungen, Normen, Bewusstseinsstrukturen und unbewussten Wünsche, an (Oevermann, 2000, S. 65). Diese Auswahl macht - sequenzanalytisch ausgedrückt - die Fallstruktur aus (Oevermann, 2002a, S. 8). Sequenzialität lässt sich somit nicht lediglich als ein temporales Nacheinander, sondern vielmehr als sinnlogische und bedeutungserzeugende Folge verstehen. Die Auswahl wird meist als Folge von Routinen, die sich bewährt haben und gewissermassen selbstverständlich sind, getroffen. Dies bedeutet, dass eine Entscheidung nur in den wenigsten Fällen bewusst vorgenommen wird (Oevermann, 2000). Dennoch bleibt die Entscheidung, objektiv betrachtet, eine solche.

Konstitutiv für die Lebenspraxis ist, dass ständig Auswahlen und Entscheidungen getroffen werden müssen, die «Lebenspraxis muss eine Entscheidung fällen, weil sie sich nicht nicht entscheiden kann» (Oevermann, 2000, S. 131). Eine Richtig-falsch-Beurteilung ist in einer Entscheidungssituation allerdings grundsätzlich nicht möglich, weil sie sonst keine Entscheidungssituation mehr darstellen würde. Dessen ungeachtet muss sich eine Entscheidung dem Anspruch einer rationalen Begründbarkeit stellen, denn andernfalls würde der Anspruch auf Vernunft bereits von vornherein aufgegeben, der «als Bedingung für den Entwurf eines Spielraums von Möglichkeiten eingegangen [worden] war» (Oevermann, 2000, S. 131). Unter diesen Bedingungen konstituiert sich die Autonomie der Lebenspraxis, als «notwendig widersprüchliche Einheit von Entscheidungszwang und Begründungsverpflichtung»(Oevermann, 2000, S. 131). Oevermann spricht hier von einer «widersprüchlichen Einheit», weil sich der Entscheidungszwang gerade daraus ergebe, 
dass Begründbarkeit zwar ausgeschlossen sei, die Verpflichtung zu einer Begründung aber dennoch nicht aufgehoben sei, sondern lediglich aufgeschoben werden könne (Oevermann, 2000, S. 131).

Das Verfahren der Sequenzanalyse lehnt sich in seiner Konstitution und Reproduktion als Praxis an diese widersprüchliche Einheit von Entscheidungszwang und Begründungsverpflichtung an. Darüber hinaus ist die Sequenzanalyse eine Methode, die an jeder einzelnen Sequenzstelle eine Entscheidung abruft und den «grundsätzlich krisenhaften Prozess der Reproduktion und Transformation von Lebenspraxis» sichtbar werden lässt (Oevermann, 2000, S. 132). Die unbewusst getroffene Handlungsentscheidung an einer Sequenzstelle stellt nach Wernet (2009, S. 18) die zentrale forschungslogische Ausrichtung dar. In diesem Zusammenhang spricht er das in der Objektiven Hermeneutik geläufige Begriffspaar von «latent» und «manifest» an (Wernet, 2009, S. 18). In der Forschungslogik der Objektiven Hermeneutik wird der Blick weder ausschliesslich auf die intentionalen und motivationalen Vorstellungen der Subjekte gerichtet, noch wird versucht, diese Ebene auszublenden. Ziel einer Rekonstruktion ist es vielmehr, genau dieses spannungsreiche Verhältnis der beiden Ebenen, die sich nicht komplementär zueinander verhalten, herauszuarbeiten und nachzuvollziehen: «Die Differenz zwischen der Ebene der objektiven latenten Sinnstrukturen und der Ebene der subjektivintentionalen Repräsentanz ist für die objektive Hermeneutik entscheidend» (Oevermann et al., 1979, S. 380). Stark vereinfacht lässt sich die Differenz an der Unterscheidung von Sagen und Meinen verdeutlichen: Meinen kann man nur subjektiv, weshalb «objektives Meinen» eine Contradictio in adiecto, das heisst einen Widerspruch in sich selbst, darstellt. Umgekehrt gilt, dass Sagen nur objektiv, nicht aber subjektiv möglich ist (Oevermann, 2013b, S. 72). Aus dieser kategorialen Differenz zwischen Sagen und Meinen, die sich beispielsweise darin manifestieren kann, dass wie etwas gesagt wird, nicht notwendigerweise damit übereinzustimmen braucht, wie es gemeint ist, ergibt sich eine Spannung. Deren methodische Untersuchung ist möglich, indem man sich an das objektiv Gesagte oder Ausgedrückte hält, worin zugleich auch das subjektiv Gemeinte zur Geltung gebracht wird. Alles andere bleibt methodisch verschlossen (Oevermann, 2013b, S. 72), denn erst über die Erfassung des objektiven Sinns lässt sich die darin repräsentierte Seite einer Handlung erschliessen. 
Genau diese latenten Sinnstrukturen, diese spezifischen Grammatiken oder eigenlogischen Verfasstheiten, die der sozialen Praxis von Interaktionen innewohnen, jedoch nicht unmittelbar sichtbar werden, gilt es mittels der Methode der Objektiven Hermeneutik empirisch in den Blick zu nehmen. Oevermann geht diesbezüglich davon aus, dass sich soziale Interaktionen durch das systematische Auseinandertreten manifester Sinnmotive und latenter Sinnstrukturen kennzeichnen. Wernet zufolge wird eine Interaktion geprägt durch das Spannungsverhältnis zwischen dem, was die sprechende Person mitzuteilen intendiert (Meinen), und dem, was sie in Protokollen zum Ausdruck bringt (Sagen), jedoch «nicht im intentionalen Horizont der Sprecher liegen» (Wernet, 2018b, S. 130). So könne das Gesagte «über die Intentionen hinausgehen, von ihnen abweichen, ihnen unter Umständen widersprechen» (Wernet, 2018b, S. 130).

Latente Sinnstrukturen, die in einem Spannungsverhältnis zu den manifesten Sinnbezügen stehen, führen zu aufschlussreichen, aber auch «unbequemen` Sichtweisen. Dieses «Kränkungspotential einer hinter die Bühne blickender Analyse» gilt es in Kauf zu nehmen (Wernet, 2018b, S. 130), da die Forschungs- oder Wissenschaftsperspektive eine andere ist als diejenige der Praxis. Für die geleistete Aufklärung darf die in der Wissenschaft tätige Person keinen Dank erwarten; vielmehr stösst sie damit bei den beteiligten Akteurinnen und Akteuren möglicherweise auf Empörung. Dies mag insofern verständlich sein, als die Rekonstruktion der latenten Sinnstrukturen das «Selbstbewusstsein» der Akteurinnen und Akteure irritiert (Wernet, 2018b, S. 132). Das ¿Unfaire`, das der wissenschaftlichen Analyse konstitutiv anhaftet, besteht in der Erkenntnis selbst und ist nur durch eine Verschleierung dieser Erkenntnis zu vermeiden (Wernet, 2018b, S. 133).

Die Fallstrukturgeneralisierung, «eine Theoriebildung in der Sprache des Falles» (Oevermann, 1981), bildet den Endpunkt eines Interpretationsprozesses und beansprucht ihre Gültigkeit über den Einzelfall hinaus. Basis eines solchen Anspruchs auf Generalisierbarkeit bildet die Prämisse, dass ein analysierter Fall immer schon das Allgemeine und das Besondere zugleich darstellt, da in jedem Protokoll sozialer Wirklichkeit das Allgemeine ebenso mitprotokolliert wird wie das Besondere (Wernet, 2009, S. 19). Deshalb lassen sich auch bei geringen Fallzahlen Verallgemeinerungen vornehmen: 
Jede konkrete Fallrekonstruktion führt also zu einer Strukturgeneralisierung bzw. der Bestimmung eines allgemeinen Typus. Dessen Allgemeinheit ist in gar keiner Weise von der Häufigkeit seines Vorkommens [...] abhängig. Selbst wenn er in einer «Grundgesamtheit» tatsächlich nur einmal vorkäme, hätte er diese Allgemeinheit eines Typus, die die Fallrekonstruktion in der logischen Form einer Strukturgeneralisierung bestimmt. (Oevermann, 2002a, S. 14)

Eine einzelne Fallrekonstruktion stellt folglich schon als solche eine Strukturgeneralisierung dar beziehungsweise leistet einen Beitrag zur Bestimmung eines allgemeinen Typus (Oevermann, 2002, S. 15), in der vorliegenden Arbeit im Konkreten zur Bestimmung der sozialen Praxis von Mentoratsgesprächen an Pädagogischen Hochschulen.

\subsection{Methodisches Vorgehen}

\subsubsection{Erhebung und Sampling}

Für die Beantwortung der Forschungsfrage braucht es Datenmaterial, das die soziale Praxis von Mentoratsgesprächen mit der darin enthaltenen Eigenlogik und den Interaktionsdynamiken abbildet. Dazu eignen sich In-situ-Protokolle aus natürlichen Gesprächssituationen, die als Ausdrucksgestalten und Träger der latenten Sinnstrukturen dieser Gesprächspraxis verstanden werden können. Denn aus strukturtheoretischer Perspektive ist erwartbar, dass sich die Strukturen dieser sozialen Praxis in die Gesprächstranskripte einschreiben und darin zum Ausdruck kommen.

Für das Forschungsvorhaben wurden an der PH FHNW zwischen Dezember 2016 und Mai 201725 Mentoratsgespräche zwischen Studentinnen ${ }^{21}$ des Bachelorstudiengangs «Kindergarten- und Unterstufe» und ihren jeweiligen Mentorinnen oder Mentoren in situ, das heisst während des im Semesterturnus durchgeführten obligatorischen Mentoratsgesprächs, von mir als Forscherin beobachtet und audiografiert. Meine Anwesenheit in der Rolle der teilnehmenden Beobachterin ist als minimale Intervention in das Gesprächs-

${ }^{21}$ An diesen 25 Gesprächen hatten auf der Seite der Mentees ausschliesslich Studentinnen teilgenommen. Dies repräsentiert allerdings durchaus die tatsächliche Situation am Institut Kindergarten- und Unterstufe, da der Männeranteil bei den Studierenden sehr klein ist. 
setting zu betrachten. Gleichwohl ist aus strukturtheoretischer Perspektive die Annahme zulässig, dass sich die Charakteristika der Strukturen dennoch in die Gesprächsinteraktionen eingeschrieben und in den Protokollen Ausdrucksgestalt gefunden haben.

Einige Studentinnen befanden sich zum Gesprächszeitpunkt am Anfang ihrer Ausbildung, andere standen kurz vor ihrem Studienabschluss. Von jeder Mentorin respektive jedem Mentor wurden mehrere Gespräche in unterschiedlichen Gesprächskonstellationen, das heisst mit verschiedenen Studentinnen, aufgezeichnet. Sämtliche Gespräche wurden in Standardsprache geführt. ${ }^{22}$ Im Datenkorpus finden sich nur Gesprächstandems, die der Datenerhebung zuvor beiderseitig freiwillig zugestimmt hatten. Da die Portfolioarbeit Bestandteil des Mentorats ist (vgl. ausführlich Kapitel 2.1.2), befassten sich Mentoratsgespräche teilweise auch mit Rückmeldungen zur Portfolioarbeit. Für die Zwecke der vorliegenden Untersuchung wurden jedoch einzig solche Gespräche als Datenbasis ausgewählt, die nicht oder nur am Rande auf das zu erstellende oder bereits erstellte Portfolio zu sprechen gekommen waren.

Damit eine gewisse Bandbreite an Daten zur Verfügung stand, wurden bewusst mehr Daten erhoben, als bearbeitet werden konnten. Diese Erhebung über die Bearbeitungskapazitätsgrenze hinaus ermöglichte es, der Logik des «theoretical sampling» von Glaser und Strauss (2010) folgend, eine empirische respektive materielle Sättigung zu gewährleisten. Eine solche Sättigung ist erreicht, wenn zusätzliches Datenmaterial keine neuen Aspekte für die Theoriebildung mehr liefert (Strübing et al., 2018, S. 85). Entsprechend dem Prinzip eines iterativen Prozesses war die Anzahl der Gespräche nicht bereits im Vorfeld bestimmt worden, sondern es wurden so viele Daten erhoben, das heisst so viele Mentoratsgespräche teilnehmend beobachtet und audiografiert, bis eine heterogene Auswahl an Gesprächen mit einer Varianz an Merkmalen zur Verfügung stand. Diese Vorgehensweise kam dem Prinzip der Offenheit in der qualitativen Sozialforschung

\footnotetext{
${ }^{22}$ Die Frage nach der Sprache, in der ein Gespräch stattfindet - Standardsprache oder Schweizer Mundart - ist für den Schweizer Diglossiekontext, wo die Daten erhoben wurden, von Bedeutung. Denn obwohl die deutsche Standardsprache an der PH FHNW die Ausbildungssprache ist, finden dyadische Gespräche oftmals im Schweizer Dialekt statt. Im Sample sprachen die Mentorin oder die Studentin in zwei Fällen Standardsprache, da sie in Deutschland aufgewachsen waren und die jeweils zweite Person die Standardsprache übernahm. Bei einem weiteren Gespräch hatte mich der Mentor zuvor wissen lassen, dass er Mentoratsgespräche in Standardsprache führe. Die beiden Beteiligten der vierten Dyade schliesslich hatten mich vor Gesprächsbeginn gefragt, ob sie wegen der Datenaufnahme Standardsprache sprechen sollten. Ich stellte innen die Sprachauswahl frei, worauf der Mentor anmerkte, dass Mundart für ein Forschungsvorhaben im deutschsprachigen Raum möglicherweise anspruchsvoll sei, weshalb sie sich auf Standardsprache einigten.
} 
nach.

Im Nachgang der Gespräche wurden Gesprächsnotizen erstellt und in einem weiteren Schritt Themenprotokolle angefertigt, die einen Überblick über die im Gespräch verhandelten Themen und Inhalte verschaffen sollten. Anschliessend wurden die Gespräche transkribiert. Die Transkription erfolgte in Originalsprache, weshalb keine sprachliche Glättung vorgenommen wurde. In einem nächsten Schritt galt es, aus den 25 Gesprächstranskripten ${ }^{23}$ ein Gespräch als Pilotfall auszuwählen, der den ersten Kernfall ${ }^{24}$ darstellt. Erneut der Logik der empirischen Sättigung folgend, wurden danach drei weitere Kernfälle bestimmt, die in relevanten Merkmalen maximale und in Teilen minimale Kontraste zum ersten Kernfall bildeten. Maximale und minimale Kontraste ermöglichen es, eine weitreichende materielle Sättigung bezogen auf die Ausdrucksgestalt zu erlangen. Die Transkriptionen der Gesprächsaudioaufnahmen der vier Kernfälle bildeten die Protokolle für die Rekonstruktionen.

Gemäss Oevermann (2000, S. 98) sind pro Fall maximal vier kurze Sequenzen einer Feinanalyse zu unterziehen. Basis für die Auswahl der Analysesequenzen bildete in erster Linie das Falsifikationsprinzip. Das heisst, es wurden grundsätzlich Sequenzstellen ausgewählt, die die zuvor bereits rekonstruierten bedeutsamen Strukturmomente zu widerlegen schienen. In einem weiteren Schritt galt das Interesse denjenigen Sequenzstellen, die Potenzial für eine Ausdifferenzierung und Erweiterung der bereits freigelegten Strukturmomente aufwiesen.

Die sequenzweisen Rekonstruktionen begannen in allen vier Kernfällen mit der Eröffnungssequenz, da der Anfang eines Protokolls als erste Analyseeinheit bedeutsam ist und im weiteren Verlauf nicht ignoriert werden kann:

Der Beginn des Protokolls sollte eigentlich immer analysiert werden, weil in den Eröffnungspassagen einer konkreten Praxis immer die entscheidenden Weichen gestellt werden [...], so dass ihre Analyse besonders aufschlussreich ist. (Oevermann, 2000, S. 98)

\footnotetext{
${ }^{23}$ Die vollständigen Gesprächstranskripte sind bei der Autorin erhältlich.

${ }^{24}$ Unter einem «Kernfall» wird im vorliegenden Forschungsvorhaben ein Mentoratsgespräch verstanden.
} 
Im Anschluss an die Analyse des Interaktionsbeginns wurden in der vorliegenden Untersuchung pro Kernfall drei bis vier Sequenzen für die Feinanalyse ausgewählt. Diese Auswahl erfolgte mit Blick auf das falsifizierende respektive schärfende Potenzial. Weiter wurde auf den Gehalt hinsichtlich des inhaltlichen Bezugs zur Forschungsfrage geachtet (Oevermann, 2000, S. 98). Die Bestimmung des genauen Endes einer Analyseeinheit ergab sich ebenfalls durch eine materielle Sättigung. Wenn sich Strukturmerkmale reproduzierten, liess sich an der betreffenden Stelle eine Fallstrukturhypothese formulieren. Oevermann zufolge reicht in der Regel die Rekonstruktion von insgesamt zehn bis zwölf Sequenzen auch für komplexe Fragestellungen aus, um eine materielle Sättigung zu erreichen:

Man ist dann in der Strukturerkenntnis und in der Strukturgeneralisierung so weit vorangekommen, dass die Erforschung von strukturtheoretischen Folgefragen ökonomischer ist, als die weitere Detaillierung der gesuchten Antworten. (Oevermann, 2002b, S. 17)

Das Sample umfasste schliesslich insgesamt 13 Rekonstruktionen (Sequenzstellen) aus vier Kernfällen.

\subsubsection{Methodische Prinzipien und Auswertung}

Im Folgenden werden die praktischen und methodischen Verfahrensschritte der Auswertung der Transkriptionsprotokolle dargestellt. Die objektiv-hermeneutische Textinterpretation folgte fünf Prinzipen, die «eine Brücke zwischen Methodologie und Methode» (Wernet, 2009, S. 21) schlagen und hier zusammenfassend beschrieben werden. Eine ausführliche Darstellung findet sich beispielsweise bei Wernet (2019) oder bei Garz (2015, S. 143 ff.)

Kontextfreiheit: Eine Interpretation hat in einem ersten Schritt unter Auslassung der Kontextuierung zu erfolgen. Das heisst, für die erste Begegnung mit dem Protokoll wird eine «künstliche Naivität» hergestellt (Wernet, 2009, S. 23). Dieses Prinzip der kontextfreien Interpretation bedeutet allerdings nicht, dass dem Kontext keine Bedeutung zukommt. Vielmehr besagt es, dass «die Einbeziehung des Kontextes erst dann 
eine gehaltvolle und strukturerschliessende, methodisch kontrollierte Bestimmung darstellt, wenn zuvor eine kontextunabhängige Bedeutungsexplikation vorgenommen wurde» (Wernet, 2009, S. 22).

Wörtlichkeit: Wird eine "Textanalyse als Wirklichkeitsanalyse» begriffen, ist eine wörtliche Interpretation zwingend (Wernet, 2009, S. 23). Die Bedeutungsrekonstruktion des tatsächlich artikulierten Textes darf dessen protokolliert vorliegende Gestalt nicht ignorieren, selbst wenn «innertextliche Widersprüche» auftreten (Wernet, 2009, S. 23). Denn das Prinzip der Wörtlichkeit eröffnet einen «interpretatorischen Zugang zur Explikation der Differenz zwischen manifesten Sinngehalten und latenten Sinnstrukturen eines Textes» (Wernet, 2009, S. 25).

Sequenzialität: Von zentraler Bedeutung für die Objektive Hermeneutik ist das Prinzip der Sequenzialität, das auf konstitutionstheoretische Vorüberlegungen verweist, die in Kapitel 3.2.2 ausgeführt wurden. Die Interpretation hat streng dem Ablauf zu folgen, den ein Text protokolliert. Diese Grundhaltung ist wie bereits beim Wörtlichkeitsprinzip darauf zurückzuführen, dass der Text als Text ernst zu nehmen ist (Wernet, 2009, S. 26 f.).

Extensivität: Ein markantes Charakteristikum der Objektiven Hermeneutik ist die Analyse einer geringen Textmenge, der man sich detailliert, geradezu akribisch widmet (Wernet, 2009, S. 32). Diesem Prinzip der Feinanalyse liegt die methodische Annahme zugrunde, dass sich in den «protokollierten Ausschnitten sozialer Realität ein Allgemeines rekonstruieren lasse», da «das Besondere erst auf der Folie des Allgemeinen sich bildet» (Wernet, 2009, S. 32). In der Terminologie der Objektiven Hermeneutik bedeutet dies, dass soziale Gebilde ihre Sinnstrukturiertheit nicht verlassen können. Deshalb lassen sich auch anhand von geringen Datenmengen die Strukturprinzipien eines Gebildes rekonstruieren (Wernet, 2009, S. 33).

Sparsamkeit: Dieses Prinzip lässt sich als begrenzendes Mittel für das Prinzip der Extensivität betrachten. Es schreibt vor, dass nur solche Lesarten gebildet werden dürfen, die ohne Zusatzannahmen auskommen und mit dem vorliegenden Text kompatibel sind (Wernet, 2009, S. 35). Dadurch soll eine ziellose Bedeutungssuche vermieden und eine Beschränkung auf sinnhafte Bedeutungsformen gewährleistet werden. 
Die eigentliche Interpretationspraxis stellt eine mehrschrittige Analyse dar, der die einzelnen Sequenzen unterzogen werden. In einem ersten Schritt werden Geschichten erzählt, in denen der Sprechakt, losgelöst vom eigentlichen Kontext, in seiner wörtlichen Gestalt auftreten könnte (Wernet, 2009, S. 39). Als nächsten Schritt gilt es, diese Geschichten auf strukturelle Gemeinsamkeiten und Differenzen hin zu sortieren und zu gruppieren, um so aus den Geschichten begründete Lesarten bilden zu können (Wernet, 2009, S. 39). Daran anschliessend findet die Konfrontation «mit dem tatsächlichen Äusserungskontext und der darin eingelassenen Aussageintention des Textes» statt (Wernet, 2009, S. 40).

Im Verlauf der Rekonstruktion werden diejenigen Lesarten ausgeschieden, die unter Hinzunahme weiterer Textstellen nicht mehr kompatibel sind, «bis letztendlich die eine objektive, bzw. intersubjektiv als treffend ausgewiesene Lesart verbleibt» (Garz, 2015, S. 144). Dabei lässt sich, wie in Kapitel 3.2.2 erläutert, die Differenz zwischen dem objektivlatent Gesagten und dem subjektiv-intentional Gemeinten feststellen. Auf diese Weise gelangt man zu einer Fallstrukturhypothese (Wernet, 2009, 2019). Eine solche bildet ab, wie sich die Grammatik der Selektion von Handlungsentscheidungen aus dem generierten Möglichkeitsraum darstellt. Die einzelnen Sequenzstellen werden anhand der beschriebenen Analyseschritte so lange interpretiert, bis sich die rekonstruierte Fallstrukturhypothese reproduziert (Wernet, 2009, S. 31). Die Fallstrukturhypothese, die am Ende jeder Sequenzanalyse steht, wird anschliessend an einer neuen respektive weiteren Sequenzstelle überprüft. Dies kann zu einer Modifikation, zu einer Verifikation oder auch zu einer Falsifikation der vorausgehenden Hypothese führen.

Ziel des Interpretationsprozesses bildet wie in Kapitel 3.2.2 bereits ausgeführt die Fallstrukturgeneralisierung, die Anspruch auf Gültigkeit über den Einzelfall hinaus erhebt. Wie auch Breidenstein et al. (2020, S. 139) festhalten, setzt die Fallanalyse zwar am Exemplarischen an, ist aber dennoch nicht bloss als Beispiel für oder von etwas aufzufassen. Vielmehr lässt sich im Partikularen immer auch Verallgemeinerbares erkennen, da sich in einer Fallanalyse auch die Dialektik von Allgemeinem und Besonderem widerspiegelt (vgl. dazu Kapitel 3.2.2).

Die Auswertungen des Datenmaterials erfolgten in einem ersten Zugriff, wann immer es die Situation erlaubte, in Interpretationsgruppen und Forschungswerkstätten an ver- 
schiedenen Standorten in der Schweiz und in Deutschland. Dies war für mich sehr gewinnbringend und hilfreich, um zum Ziel - zu einer intersubjektiven Übereinstimmung (Breidenstein et al., 2020) - zu gelangen. Die Entscheidung in Bezug auf eine abschliessende Argumentation und Begründung sowie der Versuch des Formulierens einer kohärenten Darstellung lagen in jedem Fall in meiner Verantwortung, wenngleich ich vielen Personen aus diesen Kollaborationen für ihre Deutungsangebote sehr dankbar bin.

Der Fokus der objektiv-hermeneutischen Analyse ist auf die strukturellen Gegebenheiten gerichtet. Zusätzlich ist meine Forschung inspiriert von der Re-Adressierungsanalyse nach Reh und Ricken (2012), die soziale Interaktionen als Re-Adressierungsgeschehen beschreiben. Dabei werden die in der Interaktion zur Aufführung gebrachten Positionierungen in den Blick genommen und es wird aufgezeigt, wie wer von wem und in welcher Form explizit oder implizit angesprochen und adressiert wird (Reh \& Ricken, 2012, $S$ 42). Eine Verbindung von Objektiver Hermeneutik und einer Betrachtung als Adressierungsgeschehen, wie sie auch von Kunze et al. (2019) vorgeschlagen wird, stellte eine zusätzliche Bezugsfolie für die Auswertung dar. Die ergänzende Analyse wurde allerdings nicht systematisch vorgenommen, sondern unterlegte lediglich den Diskussionsstrang mit einer anerkennungstheoretischen Perspektive. Denn die Idee der Adressierungsheuristik von Reh und Ricken ist subjektivationstheoretisch und praktikentheoretisch gerahmt. Diese Teile werden in meiner Auswertung nicht berücksichtigt und auch nicht in Aussicht gestellt, sondern die Analyse bedient sich lediglich des Grundgedankens und der Begrifflichkeiten der Adressierungsanalyse, die zudem auch etwas anders akzentuiert wird. Begründen lässt sich dies dadurch, dass sich die theoretischen Grundlagen und die theoretische Rahmung, die den Bezugspunkt meiner Arbeit darstellen, auf Akteurinnen und Akteure als Handelnde beziehen, was bei einer praktikentheoretischen Ausrichtung keinen Fokus darstellt. Kurz gefasst bedeutet dies, dass ich bei der Analyse auf die Grundidee von Reh und Ricken zurückgriff, indem ich die Interaktion zusätzlich als Geschehen verstand, in welchem Adressierungen, Re-Adressierungen und Positionierungen stattfinden. Aus diesem Grund werden in Kapitel 4 und Kapitel 5 die Begrifflichkeiten der Adressierungsheuristik flankierend verwendet, wodurch jedoch nicht der Anspruch einer Adressierungsanalyse erhoben werden soll. 


\section{$4 \quad$ Fallstudien}

In diesem Kapitel wird der empirische Hauptteil der Untersuchung dargestellt. Anhand der Rekonstruktion der Handlungspraxis von vier Fallstudien wird herausgearbeitet, welche Strukturlogiken und Interaktionsdynamiken im Hochschulformat «Mentoratsgespräche» Gestalt gewinnen. Die Analyse der Konzeption «Mentoratsgespräch» legt die Vermutung nahe, dass sich in der praktischen Umsetzung der Gespräche, das heisst in der Interaktion, einige Spannungsfelder manifestieren werden.

Die Darstellung der Rekonstruktion erfolgt bei jedem der vier Mentoratsgespräche nach demselben Muster: Nach einer knappen Beschreibung der Gesprächssituation werden drei bis vier Sequenz pro Gespräch feinanalytisch rekonstruiert. Zur besseren Übersicht werden zuerst die Sequenz als Ganzes wiedergegeben und daran anschliessend die ein einzelnen Gesprächssequenzen aufgeführt. Jeder der vier Kernfälle beginnt mit dem Anfang des Mentoratsgesprächs, da dort entscheidende Weichen für den weiteren Verlauf des Gesprächs und somit für die Interaktionsdynamik gestellt werden und noch kein «innerer Kontext» vorliegt (Oevermann, 2000, S. 98). Die Rekonstruktion einer Gesprächspassage wird so lange fortgeführt, bis diese wiedererkennbaren Mustern folgt. Diese Reproduktion stellt eine wichtige empirische Bewährung im Rekonstruktionsprozess dar und lässt die Formulierung einer riskanten Fallstrukturhypothese zu. Diese Reproduktion erfolgt nicht mechanisch, sondern als «kreativer Prozess der Erzeugung unvorhersehbarer, aber dennoch typischer Ausdrucksgestalten» (Wernet, 2019, S. 72, siehe zur Kreativität der Strukturreproduktion auch Wernet, 2012, S. 187 f.). Diese initiale Fallstrukturhypothese wird im Anschluss anhand der zweiten Sequenz auf Verifikation oder Falsifikation überprüft und allenfalls erweitert oder modifiziert, wobei sich dieses Vorgehen wiederholt, bis die Fallstrukturhypothesen zu einer abschliessenden Diskussion und einer Fallbestimmung verdichtet werden können.

Sämtliche aufgeführten Sequenzen der Mentoratsgespräche entsprechen den folgenden Transkriptionsrichtlinien: Die gesprochene Sprache bleibt in den Transkriptionen erhalten, das heisst, es werden keine logischen oder grammatikalischen Korrekturen vorgenommen. Schweizer Mundartausdrücke werden übernommen und in einer 
Fussnote als solche gekennzeichnet. Betonte Passagen werden durch Unterstreichung markiert und signifikante Veränderungen der Sprechweise wie beispielsweise «((lacht))» oder «((räuspert sich))» mit Doppelklammern vermerkt, wobei sich der Hinweis jeweils auf die Passage vor der Klammer bezieht. Auf Interpunktion wird in den Transkriptionen mit Ausnahme von Fragezeichen verzichtet. Zitate aus den Gesprächstranskriptionen werden im Fliesstext wie auch in Kombinationen mit Gedankenexperimenten, Beispielen und Analogien kursiv geschrieben, fiktive Zitate ${ }^{25}$ in Anführungs- und Schlusszeichen gesetzt.

Legende für weitere Vermerke:

- (.) Pause von einer Sekunde; (..) Pause von zwei Sekunden; (...) Pause von drei Sekunden

- Sprechüberschneidungen werden mit doppelten Vorwärtsschrägstrichen //...// angezeigt.

Dazu ein Beispiel: Person 1 spricht. Die Textstelle innerhalb der doppelten Schrägstriche wird gleichzeitig mit Textstelle der //zweiten Person gesprochen///Person 2://die Überschneidung wird in Klammer gesetzt//).

- Ein abgebrochenes Wort oder eine abgebrochene Textpassage wird mit einem Vorwärtsschrägstrich/ gekennzeichnet

- Besonders langgezogene Vokale werden mit Doppelpunkten hinter dem Vokal gekennzeichnet, wobei das «A» bei wa::s doppelt so lange ausgesprochen wird wie bei wa:s.

- Unverständliche Textpassagen werden mit «((unverständlich)» markiert.

Des Weiteren gilt es festzuhalten, dass vorgenommene Anonymisierungen insofern die Realität abbilden, als in Gesprächssituationen, in denen sich die Studentin und die Mentorin oder der Mentor mit Vornamen ansprechen, für die Vornamen Pseudonyme verwendet werden. Für die eine Gesprächskonstellation, in der sich die Studentin und der

\footnotetext{
25 Unter «fiktiven Zitaten» verstehe ich Zitate, die nicht tatsächlich in der Gesprächsinteraktion stattgefunden haben, jedoch vom Gesprächskontext, von der Strukturlogik und der Interaktionsdynamik möglich wären und als Vergleichsfolie zur realisierten Form herangezogen werden.
} 
Mentor mit dem Familiennamen ansprechen werden entsprechend Pseudonyme von Familiennamen aufgeführt.

In Diskussionen in Rekonstruktionsgruppen in der Schweiz und in Deutschland habe ich festgestellt, dass die Bezeichnungen «Kollegin» oder «Kollege» in den beiden Ländern different verwendet wird. Während in Deutschland mit «Kolleginnen und Kollegen» ein Personenkreis aus dem beruflichen Umfeld gemeint ist, wird dieser Ausdruck in der Schweiz alltagssprachlich auch für die Bezeichnung eines Beziehungsstatus verwendet, der loser als derjenige mit einer Freundin oder einem Freund, jedoch enger als derjenige mit einer Bekannten oder einem Bekannten ist. Genau diese Mittelposition zwischen Freundin und Freund und Bekannter oder Bekanntem ist in den Gesprächen gemeint, weshalb ich an der Schweizer Semantik des Begriffs «Kollegin» oder «Kollege» festhalte, ohne mich dabei auf den beruflichen Kontext zu beschränken. 


\subsection{Tina \& Michael}

Das Gespräch zwischen der Studentin Tina und dem Mentor Michael findet im Herbstsemester 2016 in einem Seminarraum der PH FHNW statt. Zu diesem Zeitpunkt befindet sich die Studentin im dritten von sechs Semestern im Bachelorstudium zur Kindergartenund Primar-Unterstufenlehrperson.

Der Mentor hat auf dem Tisch der Reihe nach von links nach rechts vier laminierte Karten mit blauen, unterstrichenen Titeln und roten Adjektiven hingelegt. Die Studentin und der Mentor sitzen ums Eck am Tisch.

Erste Karte:

Video-Analyse

präzis, prägnant, konkret

Zweite Karte:

Fallstudie

〈handlungs〉-orientiert

Dritte Karte:

Lern- und Entwicklungsziele

persönlich

Vierte Karte:

\section{Portfolio FS 17}

kreativ, innovativ 


\subsubsection{Eröffnungssequenz: Ich zeige dir wo wir hingehen}

Michael: Ich zeige dir wo wir hingehen so in den nächsten 20 Minuten 25

Tina: $\mathrm{mhm}$

Michael: erster Punkt Videoanalyse (.) Fallstudie wird ein Thema sein (.) so der

Rück- und Vorblick

Tina: $m h m$

Michael: die Lernentwicklungsziele und (.) je nach Zeit so (.)Portfolio (.) Frühlingssemester

Tina: $m h m$

Michael: ich starte nun an (.) Videoanalyse (.) ich habe da drei (.) Stichworte präzis prägnant konkret (.) versuch doch mal so das das Fazit ich habe dir den Auftrag gegeben //dein Fazit// (Tina: //genau//) (.) zu ziehen aus dieser (.) Analyse

Tina: ja also //ich habe das//( Michael: //und bevor//)

Michael: du das Fazit machst (.) äh (.) möchte ich von dir wissen wie war es für dich die Auseinandersetzung (.) oder dein dein Auftrag eine Videoanalyse durchzuführen war es total neu für dich? unerwartet? (.) speziell oder?

Tina: also (.) ich habe auch schon Videoaufnahmen von mir gemacht während dem Unterrichten (.) aber (.) zur Analyse war neu so dass es wirklich nur (.) oder (.) zu analytischen Zwecken (.) gefilmt wird das war neu (.) und das war für mich am Anfang auch ein bisschen so (.) hm will ich jetzt das? Also es war komisch und ich hatte auch ein bisschen so (.) Respekt also nicht Angst aber Respekt so (.) wie wirke ich wirklich oder (.) was wird das zeigen wird das Negatives zeigen oder sehe ich viel auch viel //Positives//( Michael: //mhm//)

Michael: $\mathrm{mhm}$ 


\subsubsection{Rekonstruktion der einzelnen Sequenzen}

Michael: Ich zeige dir wo wir hingehen

Die Eröffnungsfigur des Gesprächs lässt ausserhalb des realen Kontextes mehrere Passungsoptionen für die gewählte Ausdrucksform zu. Beispielsweise wäre diese Gesprächseröffnung bei einem Gespräch zwischen einem Bergführer und seinem Gast denkbar. Direktiv, sachlich, bestimmt und in der Funktion eines Briefings instruiert der Bergführer, auf eine Landkarte zeigend, welchen Plan er sich ausgedacht hat und verfolgen möchte. Die Führerschaft wird durch seine Wortwahl reklamiert und die Rollenverteilung von Experte und Laiin ${ }^{26}$ ist vollzogen. Sein Plan weist eine gewisse Relevanz auf, ist nicht im Sinne eines Vorschlages verhandelbar, sondern dient als Information. Gedankenexperimentell liesse sich die Geschichte mit dem Bergführer durch die Worte «mal» und «jetzt» ergänzen. Die Aussage würde dann «ich zeig dir mal, wo wir jetzt hingehen» lauten und im Kontrast zur tatsächlichen Aussage hinsichtlich der Gefolgschaft abgeschwächt und weniger absolut wirken. Eine Formulierung als Frage im Sinne von: «Soll ich dir mal zeigen, wo wir jetzt hingehen?» wäre als Vorschlag zu lesen und allenfalls seitens der angesprochenen Person kritisier- oder verhandelbar.

Systematisch erwartbar ist der obige Sprechakt auch in einem Versteckspiel von Kindern, wenn ein Kind ein anderes an der Hand nimmt und es in sein Versteck führt. Gerade zwischen Kindern finden oftmals keine Dynamiken von Abschwächungen statt, weshalb in dieser Lesart die Einnahme der Führerschaft und dementsprechend die zugeschriebene Gefolgschaft nicht mit einem asymmetrischen Verhältnis wie unter Erwachsenen oder zwischen Erwachsenen und Kindern gleichgesetzt werden muss. Während dem Bergführer die Rollenzuschreibung der Führerschaft qua Expertise zugeschrieben wird, erhält das Kind seine Führungsrolle qua Charisma. Eine weitere Situation, in welcher der Sprechakt sinnvoll anzutreffen wäre, ist beispielsweise eine Abklärung beim schulpsychologischen Dienst. Der Schulpsychologe könnte die Aussage an eine Schülerin richten und ihr erklären, was sie gemeinsam vorhaben.

\footnotetext{
${ }^{26}$ In den fiktiven Kontexten, in welchen der Sprechakt vorstellbar wäre, entsprechen die Gender denen der realen Situation. Deshalb wird hier Bergführer als Experte und Laiin gesprochen. Dieses Vorgehen gilt für alle fiktiven Kontexte der vier Kernfälle.
} 
Auf der Suche nach weiteren Gelingenskontexten für den Sprechakt stösst man auf ein experimentelles Setting jenseits einer sozialen Praxis, das Standardisierung als Notwendigkeit nach sich zieht, da nur so Vergleichbarkeit und Kontrollierbarkeit gewährleistet werden können. Denkbar wäre beispielsweise ein Labordesign für eine Studie zu Verhaltensforschung, bei dem die Probandin über die bevorstehende Untersuchung aufgeklärt und instruiert wird. In dieser Situation ist die Beziehungsvermeidung zwischen der Person, die das Experiment durchführt, und der Probandin eine Bedingung für nicht verzerrte Testdaten. Sie wird qua Formalisierung eingefordert, da eine solche für eine vorschriftsmässige, gewissenhafte und schematische Vorgehensweise notwendig ist. Beiden Situationen, der schulpsychologischen Abklärung wie auch der Studie für Verhaltensforschung, gemein sind universalistische und standardisierte Vorgehensweisen, da diese Gelingensbedingungen für die Reliabilität und die Validität der Untersuchung respektive Abklärung darstellen.

Die Bildsprache, die in der Raummetapher wo wir hingehen verwendet wird, sucht nach Materialität. Kontextungebunden wäre vorstellbar, dass sich wo wir hingehen auf die Landkarte bezieht, wie dies in der Geschichte mit dem Bergführer angedacht wurde. Auch denkbar wäre eine räumliche Auslegung wie beim Versteckspiel oder eine wörtliche wie im Laborexperiment oder bei der schulpsychologischen Untersuchung, wenn die Untersuchung oder Abklärung in einer anderen Räumlichkeit stattfindet. In allen Auslegungen weist dieser Ort des Hingehens eine unbekannte Komponente auf.

Michael: so in den nächsten 20 Minuten 25

Tina: $m h m$

Die Information wird durch eine Zeitangabe präzisiert (20 Minuten) und diese Präzisierung umgehend mit dem Nachtrag 25 wieder ausgedehnt. Durch das so liegt die gesamte Planung im Ermessensspielraum des Sprechers. Abgesehen vom Vagheitsmarkierer so kommt die Formulierung ohne Abschwächungspartikel oder Füllwörter aus und impliziert in dieser Sparsamkeit die Setzung des Settings im Sinne einer Gefolgschaft.

Unter Einbezug des realen Kontextes eines dyadischen Mentoratsgesprächs ist festzuhalten, dass das Setting formalbürokratisch elaboriert wird. Das so im Sinne von «ungefähr» im zweiten Satzteil ist der einzige Vagheitsmarkierer. Dadurch erhält die 
Länge des Gespräches minimalen Spielraum und das strukturierte Setting lässt eine geringfügige Variabilität und Aufweichung zu. Dies wird auch durch die nicht gänzlich festgelegte Zeitangabe (20 Minuten 25) verstärkt. Durch die Ansage wird die Gesprächsdauer limitiert und gleichzeitig ein Zeitpuffer eingebaut. Der Gesprächseinstieg von Michael ist universalistisch, direktiv und standardisiert. Dies wird durch die Nennung der bereits im Voraus bestimmten Zeitdauer zusätzlich unterstrichen.

Im vorgefundenen Kontext des Mentoratsgesprächs wird die Raummetapher - ich zeige dir wo wir hingehen - durch die Karten, die vor den beiden auf dem Tisch liegen, plausibilisiert und materialisiert. Die Karten unterstützen in ihrer Materialität die Strukturmerkmale des Standardisierungs- und Formalisierungseffektes und der Universalisierung, da sie mit grosser Wahrscheinlichkeit auch in anderen Mentoratsgesprächen von Michael Verwendung finden.

Mit diesem Eröffnungssprechakt erhält der Einstieg ins Mentoratsgespräch eine formalisierende, strukturierende und technokratisierende Komponente. Ebenfalls gewinnt im Gesprächsauftakt Gestalt, dass Michael die Gesprächsstrukturierung nicht aus den Händen gibt und diese folglich weder der Studentin Tina noch dem Zufall überlässt. Auf einer latenten Ebene wird dadurch eine Ungewissheit oder Unkontrollierbarkeit, die eine offene Gesprächseröffnung konstitutiv beinhalten würde, bereits potenziell bearbeitet respektive begrenzt.

Erwartbar wäre ein Anschluss der Studentin Tina im Sinne einer Bestätigung wie etwa «okay», «ja» oder "gut» oder auch nonverbal mit einem bestätigenden Kopfnicken. Etwas anderes als eine - auch stillschweigende - Einverständniserklärung zum erläuterten Vorgehen wird für die hier vorgefundene Gesprächskonstellation nicht vermutet und wäre irritierend. Erwartungsgemäss schliesst die Studentin daher mit $\mathrm{mhm}$, einer abgeschwächten Form von «okay» oder «ja», an und stimmt dem Vorgehen von Michael registrierend zu, bleibt jedoch in ihrer Äusserung defensiv und zurückhaltend.

Michael: erster Punkt Videoanalyse (.) Fallstudie wird ein Thema sein (.) so der Rück- und Vorblick

Tina: $\mathrm{mhm}$ 
Während des Sprechens zeigt Michael auf diese beiden Karten und stellt eine Aufzählungslogik respektive Planabarbeitungslogik her. Als zusätzliche Orientierung zu den Informationen, die auf den Karten aufgeführt sind, wird hier mitgeteilt, dass Videoanalyse als Rückblick und Fallstudie als Vorblick gedacht ist. Für einen dyadischen Kontext ist der Sprechakt ungewohnt, da kein personaler Bezug stattfindet. Erwartbar wäre der Sprechakt vor kleinem oder grösserem Publikum. Als Anschauungsbeispiel wäre ein Kontext aus der Lehre vorstellbar, wenn der Dozent im Sinne einer Vorschau das Programm der eben begonnenen Vorlesung aufzeigt. Das Publikum in der Rolle der Rezipierenden verfügt über keine Mitsprache bei der thematischen Festlegung der Inhalte, sondern wird lediglich in Kenntnis gesetzt, wodurch sich die Figur des Briefings aus dem vorausgehenden Sprechakt reproduziert. Zugespitzt wäre diese Einwegkommunikation in einem Setting vorstellbar, in dem der Radio- oder Fernsehmoderator zu Beginn einer Informationssendung über die Sendethemen informiert und dabei ein anonymes, möglicherweise lediglich potenziell vorhandenes Publikum anspricht. Überträgt man diese Überlegungen unter Einbezug des Kontextwissens auf das vorgefundene Mentoratsgespräch, so konturiert sich eine klare und spezifische Formalisierungsbewegung. Anteile einer diffusen, persönlichen Adressierung, wie sie für dyadische Gesprächen vermutet werden können, sind nicht erkennbar.

Analog zum Eröffnungssprechakt ist auch hier keine inhaltliche, sondern lediglich eine die Ausführungen akzeptierende oder allenfalls auch keine Äusserung von Tina erwartbar. Die Studentin schliesst tatsächlich erneut mit $\mathrm{mhm}$ im Sinne eines Hörerinnensignals, paraphrasiert mit «ich nehme es zur Kenntnis», an. Sie stimmt dem anmoderierten Vorgehen in minimalistischer Weise zu, lehnt dieses zumindest nicht ab oder behält sich eine Bewertung noch vor. 
Michael: die Lernentwicklungsziele und (.) je nach Zeit so (.) Portfolio ${ }^{27}$ (.)

Frühlingssemester

Tina: $m h m$

Die Aufzählung findet ihre Fortsetzung und die beiden auf dem Tisch verbleibenden Karten Lern- und Entwicklungsziele und Portfolio werden erwähnt. Um die Dynamik des Sprechaktes je nach Zeit so herauszuarbeiten, wird der vorgefundene Sprechakt mit der Variante «je nach Zeit noch» kontrastiert. In der zweiten Variante wird das noch folgende Thema als eines mit einer gewissen Relevanz markiert. Bei der vorgefundenen Variante je nach Zeit so scheint das noch Folgende hingegen weniger bedeutsam zu sein und eher als Zeitfüller zu dienen. In der Verwendung des so ist erneut ein gewisser Spielraum bezogen auf den Zeitfaktor erkennbar. Allerdings bezieht sich der Spielraum hier nicht auf eine Zeitspanne, sondern auf die Option, die darin besteht, dass ein Thema beispielsweise bei Zeitknappheit gar nicht behandelt wird. Das Adverb so kann demzufolge als Flexibilisierungsmarker gelesen werden. Es impliziert, dass es so «oder ähnlich oder aber auch ganz anders» sein könnte. Daher weist es eine unbestimmte Komponente auf.

Die universalistische Aufgleisung des Gesprächsablaufs wird von Neuem akzentuiert. Das Schema findet ungeachtet der Person der Protagonistin seine Anwendung. Eine Abweichung vom Schema ist lediglich mit Blick auf eine potenzielle Zeitknappheit und daraus folgende Streichung eines Gesprächsthemas angedacht und durch den Ausdruck so festgelegt. Starke, kleinschrittige Didaktisierung, Visualisierung und explizite Modellierung prägen den Auftakt des Mentoratsgesprächs und finden zusätzlich Ausdrucksgestalt in den sorgfältig gestalteten Karten, die aufliegen. Michael etabliert in seiner Gesprächseröffnung eine Konzeption eines Mentoratsgesprächs, die Ähnlichkeiten mit einem Briefing aufweisen. Auch in einem schulischen Setting wäre dieses Vorgehen

\footnotetext{
${ }^{27}$ Wie bereits im Kapitel 2.1.2 ausgeführt ist die Arbeit am Portfolio eine mögliche Arbeitsform im Mentorat. Die Portfoliokonzeption im IKU der PH FHNW bezieht sich auf eine klar bestimmte Bedeutung des inzwischen sehr breit verwendeten Portfoliobegriffs. In diesem Portfolio reflektieren und dokumentieren die Studierenden über sechs Semester hinweg ihren individuellen Professionalisierungsprozess anhand von aussagekräftigen Belegen aus allen Bereichen des Studiums. Alle Studierenden des Studiengangs erstellen verbindlich ein studienbegleitendes Professionalisierungsportfolio, dessen Zweck explizit das Lernen ist. Der damit verbundene Arbeitsaufwand ist in den Workloadberechnungen des Studiengangs berücksichtigt. Die regelmässige und kriterienbezogene Kommunikation zum Portfolio ist ein wesentliches Merkmal der Konzeption und Auftrag der Mentorinnen und Mentoren.
} 
vorstellbar, wenn die Lehrperson ein individuelles Lernstandsgespräch mit einer Schülerin führt.

Wie bereits ausgeführt ist die vorgefundene Steuerung eines klar strukturierten und formalisierten Settings risikoärmer und handhabbarer als diejenige eines diffusen oder offenen Settings, das möglicherweise auch unvorhersehbare und kontingente Elemente enthält. Wie in den ersten beiden Sprechpassagen schliesst die Studentin erneut mit einem $\mathrm{mhm}$ an, was sich in der Bedeutungsstruktur nicht von derjenigen, die in den vorhergehenden Ausführungen bereits dargelegt wurde, unterscheidet.

Michael: ich starte nun an (.) Videoanalyse ich habe da drei (.) Stichworte: präzis ${ }^{28}$, prägnant, konkret (.)

Erwartungsgemäss beginnt Michael mit dem Aufrufen und Vorlesen der ersten Karte. Die grammatikalisch inkorrekte und deshalb irritierende Formulierung ich starte nun an ist möglicherweise eine Kombination der beiden Verben «ich starte nun» und «ich fange nun an», die bedeutungsmässig nahe beieinanderliegen. "Anstarten» weist auch eine technische Komponente auf. Ein Motor oder ein Getriebe lässt sich «anstarten», in der Bedeutung von «den Prozess hochfahren». Mit der Formulierung ich starte nun an versprachlicht und kommentiert der Mentor auf einer manifesten Ebene seine Handlung; in der Latenz deutet sich in seiner Erklärung auch eine Bewegung in Richtung Legitimierung seiner Handlung des «Anstartens» an. Als gedankenexperimenteller Kontrast wäre auch eine Wir-Formulierung wie "wir starten nun» vorstellbar und würde das Gegenseitige in der Situation hervorheben. Dies wird im konkreten Sprechakt vermieden und lässt diesen ‘zackig` und bestimmt erscheinen.

Strukturell aufschlussreich, auch ohne inhaltliche Erschliessung des Sprechaktes, ist die Aufzählung von Adjektiven, da unter der Ankündigung ich habe da drei (.) Stichworte eher Substantive zu erwarten wären. Ein möglicher Kontext für die vorgefundene Formulierung wäre beispielsweise eine Besprechung, bei der der Art Director seiner Mitarbeiterin spezifische Kriterien in Form von Adjektiven unterbreitet. Das zu erstellende Produkt

\footnotetext{
${ }^{28}$ Im schweizerischen Kontext ist die Form «präzis» geläufig, während man in Deutschland «präzise» verwendet. Da die sprechende Person Hochdeutsch spricht, aber Schweizer ist, lässt sich die verwendete Form dadurch erklären.
} 
muss diesen Qualitätsanforderungen und Gelingensbedingungen standhalten und wird hier etwas (mantrahaft) vorgetragen. Dabei beansprucht die sprechende Person, diese Anforderung an etwas noch nicht Ausgesprochenes zu stellen und die adressierte Person damit zu konfrontieren. Die Briefing-Figur findet eine Fortsetzung. Durch die Unterbestimmung da wird nicht expliziert, woher die Stichworte stammen. Es scheint, als wäre ihre Genese aus der Sicht des Sprechers für die Angesprochene irrelevant. Im Kontext des Mentoratsgesprächs sind die Adjektive mit grosser Wahrscheinlichkeit als Indikatoren hinsichtlich der von Tina erwarteten Aussage zu verstehen. Einerseits dienen die drei Adjektive als Unterstützung und Hilfestellung für die von Tina zu formulierende Aussage, gleichzeitig zeigen sie auch Kriterien und Massstäbe auf, innerhalb derer sich die Aussage von Tina zu bewegen hat. Die Adjektive stecken den Rahmen ab und beinhalten dabei eine schliessende Komponente, das heisst, sie bieten zwar Sicherheit an, stellen aber auch eine Einschränkung dar.

Michael fährt mit seinen Ausführungen fort und erteilt Tina weitere Instruktionen.

Michael: versuch doch mal so das das Fazit

Die Aussage versuch doch mal so das das Fazit verweist mit der doppelten Verwendung des Artikels das auf eine Suchbewegung seitens des Sprechers und lässt sich im Sinne einer Ermutigung verstehen. Kontextungebunden wäre denkbar, dass die bislang angewendeten Strategien möglicherweise erfolglos geblieben sind oder aber eine Anforderungssteigerung angestrebt wird. Passend wäre die Aussage beispielsweise dann, wenn sie vom Vater an seine Tochter gerichtet würde, die das Radfahren ohne Stützräder übt. In dieser Situation ist eine Erfahrungsasymmetrie gegeben. Unter Auslassung von so und ohne die Suchbewegung durch den verdoppelten Artikel das wäre auch eine Geschichte denkbar, in der zwei befreundete Personen versuchen, ein Türschloss zu öffnen. "Versuch doch mal, den Schlüssel zuerst etwas anzuheben» wäre im Sinne eines Tipps egalitär und auf Augenhöhe angelegt. Das Erteilen des Ratschlages und die damit einhergehende Ermunterung wären diesem Fall symmetrisch unter der Überlegung «was ist für die Öffnung des Schlosses zielführend» zu verstehen.

In der vorgefundenen Version - versuch doch mal so das das Fazit - steht allerdings nicht lediglich das Zielführende im Fokus. Der Ratschlag und die dazugehörende Ermunterung 
erhalten zusätzlich eine anleitende Komponente. Diese wird jedoch sehr fürsorglich, oder alltagssprachlich formuliert (gut gemeint), vorgetragen. Durch dieses fürsorglichanleitende Moment wird die Angesprochene auch umgehend zur Hilfe- respektive Fürsorglichkeitsbedürftigen gemacht, die Situation ist nur noch in der Asymmetrie denkbar. Pointiert formuliert lässt sich bei dieser fürsorglichen Anleitung von einer pädagogischen ${ }^{29}$ Färbung sprechen. Dennoch ist die hier vorgefundene Figur nicht sonderlich prägnant im Aufforderungscharakter, sie bleibt ergebnisoffen und steht im Kontrast zur vorherigen Aussage, die eine klare Anforderung präsupponiert. Die sprechende Person anerkennt die Herausforderung und Schwierigkeit der gestellten Aufgabe und erwartet einen Versuch mit der Implikation eines potenziellen Scheiterns.

Diese Attribute lassen sich auch der kontextgebundenen Auslegung zuschreiben: Die Studentin Tina wird mit einer Ermutigung adressiert, einen Versuch zu wagen, die Aufgabenstellung zu lösen. Kurzfristig steht der Prozess des Versuches und nicht, wie zuvor angedeutet, die Anforderungen an das Produkt - präzis, prägnant, konkret - im Fokus. Zusammenfassend lässt sich festhalten, dass die zuvor etablierte, formalbürokratische und schliessende Adressierungsfigur durch die Aufforderung zum Versuch fürsorglichanleitende und belehrende, aber auch ergebnisoffene Anteile aufweist.

Michael: ich habe dir den Auftrag gegeben //dein Fazit//(Tina: //genau//) (.) zu ziehen aus dieser (.) Analyse

Umgehend wird die vorausgehende Öffnungsfigur versuch doch mal mit der Fortsetzung ich habe dir den Auftrag gegeben wieder zurückgenommen. Durch die klare Positionierung von Auftraggeber und Auftragnehmerin gewinnt eine Bewegung Gestalt, mit deren Hilfe die zuvor etablierte asymmetrische Konstellation performativ wiederhergestellt wird. Dabei wird nochmals vor Augen geführt, was - so die hier getroffene Annahme - beide beteiligten Personen bereits wissen. Nicht die Rekapitulation des

\footnotetext{
${ }^{29}$ Das Merkmal einer pädagogischen Färbung oder Implikation bezieht sich auf eine Bewegung des Zeigens in Analogie an Prange (2012, S. 65), die sich mit folgenden Attributen beschreiben lässt: fürsorglich-anleitend, wohlwollend, vermittelnd, asymmetrisch und gleichzeitig um Symmetrie bemüht, aber auch Autonomie absprechend. Dieser Modus findet sich vornehmlich in einem schulpädagogischen Setting, wenn die Lehrperson weiss, was für die Lernenden gut ist und was die Lernenden noch nicht wissen, jedoch wissen müssen.
} 
Vorgangs der Auftragserteilung, sondern vielmehr die Bewegung der Erzeugung eines Spannungsbogens scheint dabei im Zentrum zu stehen.

Um die Dynamik des Sprechaktes noch besser zu erfassen, wird im Sinne eines gedankenexperimentellen Kontrastes die Formulierung ich habe dir den Auftrag gegeben mit der Version «du hast den Auftrag erhalten» verglichen: Bei «du hast den Auftrag erhalten» wird die Verantwortung für das Ge- oder Misslingen des Auftrags der Auftragnehmerin zugesprochen, während der Auftraggeber diesbezüglich nur wenig oder zumindest in geringerem Masse involviert ist. Vorstellbar wäre der Sprechakt typischerweise in einem beruflichen Setting während eines Gesprächs zwischen einem Vorgesetzten und seiner Mitarbeiterin.

Im Gegensatz dazu ist die Involviertheit des Auftraggebers bei der Formulierung ich habe dir den Auftrag gegeben grösser und ein Misslingen könnte auf einer latenten Bedeutungsebene auch Elemente der persönlichen Kränkung für den Auftraggeber beinhalten. Denkbar wäre eine Eltern-Kind-Situation, in der ein Elternteil beispielsweise sagt: ich habe dir den Auftrag gegeben, «dein Zimmer aufzuräumen, aber du hast es nicht getan, was mich enttäuscht.» Die Aussage enthält ein zurechtweisendes, möglicherweise auch rügendes Element. Jedenfalls erscheint sie bezogen auf den Auftraggeber persönlich aufgeladener und beinhaltet mehr diffuse Anteile, als wenn eine Du-Formulierung ohne Ich-Bezug verwendet würde. Dies lässt sich auch daran festmachen, dass eine DuFormulierung mit Ich-Bezug wie beispielsweise in «du hast von mir den Auftrag erhalten» dieses zurechtweisende und rügende Element auch aufweist.

Unter Beizug des Kontextes scheinen jedoch nicht die primär die Zurechtweisung oder eine Rüge, sondern die Rekapitulation und die Fixierung der Positionen Auftraggeber und Auftragnehmerin bedeutsam zu sein. Der Sprechakt ist Ausdruck eines Versuches, die Situation formal und asymmetrisch zu halten. Dies lässt sich zugespitzt mit «ich gebe dir einen Auftrag, du führst ihn aus, das alles spielt sich in einem klaren Rahmen ab, wir beide können uns an dieser etablierten Konstellation festhalten und uns auf sie verlassen» paraphrasieren. Mit dieser klaren Rahmensetzung wird interaktiv an die Öffnungsfigur versuch doch mal [..] - angeschlossen.

Das sich akustisch überschneidende genau der Studentin lässt sich als Form einer Zustimmung deuten. Durch die Beipflichtung bestätigt Tina einerseits die Aussage von Michael und lässt dadurch auch verlauten, dass sie der Aufforderung in einer Form auch 
Folge leisten wird. Während in der vorausgehenden Aussage die Formulierung das Fazit verwendet wurde, spricht Michael hier von dein Fazit. Unter der Prämisse der kleinschrittigen Didaktisierung des Settings wird das zuerst allgemein Gehaltene - das Fazit durch die Verwendung des Possessivartikels dein konkret auf die Person bezogen und die Studentin wird zu einer Stellungnahme aufgefordert. Dabei steht das persönliche dein im Kontrast zur Formulierung aus dieser Analyse, die in der gewählten Formulierung kontextfrei und als mögliche Variante auch nicht Tinas eigene, sondern eine übernommene Analyse sein könnte. Tina hat das Fazit oder hier eben ihr Fazit aus dieser spezifischen Analyse zu ziehen. Die in sich brüchigen Formalisierungsbewegungen und Handhabbarmachungen durch klare Rahmensetzung scheinen sich zu reproduzieren.

Tina: ja also //ich habe das//(Michael: //und bevor//)

Michael: du das Fazit machst (.) möchte ich von dir wissen wie war es für dich die Auseinandersetzung (.) oder dein dein Auftrag eine Videoanalyse durchzuführen war es total neu für dich? unerwartet? (.) speziell oder?

Die Studentin beginnt ihre Äusserung mit ja also, was als Zäsur ein schliessendes wie auch ein eröffnendes Moment beinhaltet. Kontextfrei ist die Äusserung ja also in einer Situation denkbar, in der die Person, die soeben mit Sprechen begonnen hat, noch einen Moment zum Sortieren der Gedanken herausholt. Eine weitere Auslegung wäre, dass ein Resümee, eine Schlussfolgerung oder ein Fazit aus dem Vorangegangenen auf diese Weise eingeleitet wird. Auch eine ausholende Redewendung ist denkbar, in der möglicherweise eine einschneidende Aussage folgt, wodurch diese noch mehr Gewicht erhält. Vorstellbar wäre zudem, dass die sprechende Person mit dem Beginn ihrer Ausführungen aus einer Unsicherheit heraus noch zögert. In welche Richtung der Satzanfang ich habe das zielen sollte, bleibt unklar, weil Michael Tina unterbricht. Die Überschneidung Michaels liest sich, als ob er einen konkreten Ablauf - und bevor du das Fazit machst(.) mit einer spezifischen Reihenfolge im Kopf habe. Darin zeigt sich, dass er darauf bedacht ist, die Kontrolle nicht aus der Hand zu geben. Er hat genaue Vorstellungen bezüglich des Ablaufs und grenzt die Antwort von Tina auf diese Vorstellungen ausgerichtet ein.

Der Sprechakt möchte ich von dir wissen wird erneut aus der Ich-Perspektive formuliert, erinnert an einen Befehl oder ein Verhör und betont die asymmetrische Konstellation. Die 
daran anschliessende Frage wie war es für dich die Auseinandersetzung hingegen ist offen formuliert, spricht eine tiefgehende, auch persönliche und diffuse Ebene an. Mit der Frage öffnet sich ein relativ weiter Raum für potenzielle Antworten. Dies steht konträr zu den zuvor erteilten Anordnungen und zu präzis, prägnant und konkret, wie er zuvor ihr Fazit aus der Analyse angelegt hat. Im weiteren Verlauf der Formulierung wird diese Öffnung umgehend zurückgenommen, die Frage verengt sich und wird mit oder dein dein Auftrag eine Videoanalyse durchzuführen war es total neu für dich? Unerwartet? (.) speziell oder markant neutraler, spezifischer und distanzierter reformuliert. Tina erhält Anhaltspunkte darauf, welche Art von Entgegnungen von ihr erwartet werden, die ihr als Antwortangebote zur Verfügung gestellt werden. Die vorgegebenen Antworten ermöglichen es Michael, das Gespräch stärker zu lenken und die Kontrolle aufrechtzuerhalten.

Diese Lenkungsbewegung lässt sich als Ausdruck einer Eingrenzung und Handhabbarmachung der Diffusität, die potenziell im dyadischen Setting angelegt ist, lesen und sich mit 〈bloss nicht zu viel ganze Person`zuspitzen. Dadurch lässt sich diese potenzielle Diffusität und damit einhergehend ein Kontrollverlust für Michael vermeiden oder zumindest eingrenzen. Gleichzeitig gewinnt in den vorgefundenen Ich-du-Instruktionen Gestalt (z.B.: ich habe dir den Auftrag gegeben oder: möchte ich von dir wissen), dass er als ganze Person durchaus involviert ist und gerade dies durch die Formalisierungs- und Spezifizierungsbewegungen eingegrenzt und bearbeitet wird.

Um die strukturellen Besonderheiten bezogen auf Diffusität und Spezifität in der sozialen Situation von Mentoratsgesprächen herauszuarbeiten, wird erneut und in aller Kürze auf den Vergleich zu therapeutischen Konstellationen, wie sie von Oevermann (z.B. 2013) beschrieben und in Kapitel 2.3.3.3 im Zusammenhang mit dem Begriff des Arbeitsbündnisses diskutiert wurde, verwiesen: Ein therapeutisches Setting stellt qua Institutionalisierung einen «sicheren Raum` für die Begegnung zwischen einer Klientin oder einem Klienten und einer Therapeutin oder einem Therapeuten dar. Dieses Arbeitsbündnis beruht auf der stellvertretenden Krisenbewältigung und «bildet in sich eine autonome Praxis, deren Partizipant*innen sich als ganze Personen in der Logik diffuser Sozialbeziehungen aneinanderbinden, obwohl sie grundsätzlich in der spezifischen Sozialbeziehung von Vertragspartner*innen einer kaufbaren Dienstleistung verbleiben» (Oevermann, 2013, S. 123). Dies hat zur Folge, dass ein therapeutisches Arbeitsbündnis 
durch die widersprüchliche Einheit von diffusen und spezifischen Beziehungskomponenten bestimmt wird, die sich soziologisch für die Klientin oder den Klienten mit «sei diffus» und für die Therapeutin oder den Therapeuten mit «bleib spezifisch» übersetzen lassen. Die gleichzeitigen Ansprüche von Diffusität und Spezifität werden in einer therapeutischen Konstellation institutionell, das heisst von aussen her, und unter der Prämisse der Professionalisierung sichergestellt.

Anders gestaltet sich dies in der vorgefundenen Konstellation des Mentoratsgesprächs. Hier scheint diese Gewährleistung nicht bereits institutionell hervorgebracht zu sein, sondern muss interaktiv geschaffen, bearbeitet und vor allem legitimiert werden. In der konkreten Gesprächseröffnung zwischen Tina und Michael wird die Ambivalenz von gleichzeitiger Diffusität und Spezifität über Formalisierungsfiguren und Bewegungen, die den Pol der Spezifität bedienen, hergestellt (Briefing, Instruktionen, klare Auftragserteilung, Ablaufschemata). Diese Formalisierungsbewegungen sind allerdings in sich brüchig, wie sich beispielsweise in der Aussage versuch doch mal so zeigt.

Die laminierten Karten auf dem Tisch hingegen sind wieder Ausdruck eines klar vorgegebenen Rahmens, in dem sich das Gespräch abspielt. Dadurch wird transparent festgehalten, was im Mentoratsgespräch thematisiert wird, gleichzeitig aber auch aufgezeigt, was nicht aufgegriffen wird, weil es nicht auf einer Karte steht. Die Gesprächsanlage scheint strukturell spezifisch disponiert zu sein. Die dennoch aufscheinenden diffusen Ausdehnungen werden umgehend durch Formalisierungs- und Strukturierungsbewegungen eingegrenzt. Dies steht im Kontrast zu einer therapeutischen Gesprächsanlage. Diese ist diffus angelegt und stellt die Klientin oder den Klienten in ihrer oder seiner ganzen Person ins Zentrum. Das Arbeitsbündnis zwischen Therapeutin oder Therapeut und Klientin oder Klient und damit einhergehend die sozusagen <institutionalisierte> Diffusität ist bereits im Setting angelegt und wird auch durch das Setting gewährleistet. Mit anderen Worten können sich die Therapeutin oder der Therapeut und die Klientin oder der Klient innerhalb des etablierten und sicheren Raumes und aufgrund der institutionalisierten Absicherung oder Konstitution diffus und informell begegnen. Dieser «sichere Raum», der eine Begegnung in der Gleichzeitigkeit von Diffusität und Spezifität ermöglicht, scheint im vorgefundenen Setting nicht konstitutiv gegeben zu sein, sondern muss performativ hergestellt, legitimiert und permanent bearbeitet werden. Diese differente Konstellation bezogen auf das Arbeitsbündnis zwischen der Studentin 
und dem Mentor spiegelt sich in einer gewissen Fragilität wider, die aufscheint und bearbeitet werden muss. Abschliessend lässt sich in diesem Zusammenhang festhalten, dass die antinomische Figur von Diffusität und Spezifität in der fallspezifisch vorgefundenen Konstellation über Formalisierungsbewegungen seitens des Mentors bearbeitet wird. Auf diese Weise lassen sich die potenzielle Diffusität eines dyadischen Gespräches und die mit dieser Diffusität einhergehenden Risiken handhabbar machen. Der Anschluss von Tina wird nachfolgend kursorisch, nicht feinsequenzanalytisch, rekonstruiert.

Tina: also (.) ich habe auch schon Videoaufnahmen von mir gemacht während dem Unterrichten (.) aber (.) zur Analyse war neu also dass es wirklich nur (.) oder (.) zu analytischen Zwecken (.) gefilmt wird, das war neu (.) und das war für mich am Anfang auch ein bisschen so (.) hm will ich jetzt das also es war komisch und ich hatte auch ein bisschen so (.) Respekt also nicht Angst aber Respekt so (.) wie wirke ich wirklich oder (.) was wird das zeigen wird das Negatives zeigen oder sehe ich viel auch viel //Positives// (Michael: //mhm//)

Michael: $m h m$

Tina zeigt mit ihrem Anschluss auf einer manifesten Ebene auf, dass sie bereits gewisse Erfahrungen mit Videoaufnahmen hat. Auf einer latenten Bedeutungsebene findet auch eine richtigstellende oder verteidigende Dynamik Gestalt, die mit «also nicht dass du meinst, ich hätte noch nie Videoaufnahmen von mir gemacht, so ist es nämlich nicht» paraphrasiert werden könnte. Auffallend in ihren Ausführungen ist das zeitliche während dem Unterrichten, das den Fokus auf die Aktivität, nicht aber auf die Absicht richtet. Ihre Erfahrungen beziehen sich auf einer technischen Ebene auf die Paralleltätigkeit von Unterrichten und Filmen, nicht aber auf die Ebene der Analyse. Für Tina sind demzufolge Videoaufnahme und Videoaufnahme zur Analyse zwei differente Tätigkeiten. Mit der Wiederholung von neu entlastet sie sich potenziell vom Produkt oder Ergebnis der Analyse, indem sie sich Michael gegenüber als diesbezüglich, nicht aber grundsätzlich als bei Videoaufnahmen unerfahren positioniert. Gleichzeitig richtet sie damit auch einen leichten Appell an ihr Gegenüber, im Sinne von «berücksichtige bei der Beurteilung meiner Analyse auch den Umstand der Unerfahrenheit». Nach dem Abbruch eines 
Gedankens formuliert sie die Rekapitulation eines inneren Dialoges, der ausdrückt, dass sie die Ausführung des Auftrags mit $h m$ will ich jetzt das hinterfragt, als ob man ihr die Option eröffnet hätte, den Auftrag auch abzulehnen. Auf diese Weise deutet sie latent eine Kritik oder Widerstand an, ohne dies direkt auszusprechen. Mit am Anfang war es so relativiert sie ihre Aussage oder räumt zumindest die Möglichkeit ein, dass sich dies verändert habe. Im weiteren Verlauf spricht sie von Respekt und setzt diesen Begriff als Kontrast respektive in Relation zu Angst ein. Alltagssprachlich wird Respekt häufig als abgemilderte, positiv konnotierte Variante von Angst verwendet. In dieser Auslegung ist ihre Aussage als Paraphrase von «ich nehme den Auftrag nicht auf die leichte Schulter, lasse mich aber auch nicht einschüchtern» zu verstehen. Die von ihr vorgenommene Abgrenzung zur Angst lässt sich als aktive und bewusste Negation lesen, das heisst, sie zeigt und positioniert sich als nicht ängstliche Person. Ihre Darstellung liest sich, als ob die Videoanalyse - wie ein Hirnscan oder Lügendetektor - etwas zu Tage bringe, das sie nicht möchte oder ihr selbst nicht bewusst sei, sozusagen einen blinden Fleck. In ihrer Schilderung funktioniert die Videoanalyse wie ein Selbstläufer, sie lässt diese über sich ergehen und ist ihr ausgeliefert.

Abschliessend und im Sinne einer inhaltlichen Zusammenfassung lässt sich festhalten, dass Michael zuerst nach ihrem Befinden und anschliessend nach ihrem Umgang mit der Videoanalyse fragt. Auf einer latenten Eben zeigt sich, dass Tina eine Verbindung zwischen dieser Analyse und einer Bewertungsfunktion herstellt. Ihre Ausführungen deuten eher auf Angst, nicht aber auf Respekt hin, was sie jedoch explizit dementiert. Hierbei positioniert sie sich als Lernende, die sich den Erwartungen stellt und dabei auch Vorbehalte oder Bedenken ihren eigenen Leistungen gegenüber hat. Mit der Aussage hm will ich jetzt das als Metakommentar zu einer an sie gerichteten Frage übt sie zögerlich auch Kritik gegenüber dem ihr Zugetragenen aus. Auch zeigt sie auf, dass sie bezüglich der Videoanalyse keine Wahl hatte. 


\subsubsection{Zusammenfassung und riskante Fallstrukturhypothese der Eröffnungssequenz}

Ich zeige dir wo wir hingehen

Als primäres Strukturmerkmal des Auftaktes ist eine formalisierende und formalisierte Ausgestaltung der Gesprächssituation festzustellen. Diese wird technisch aufgezogen, folgt der Logik eines Briefings und beansprucht eine klare Situationsdefinition seitens des Mentors. Der Mentor zeigt der Studentin unmissverständlich auf, entlang welcher Themen das Gespräch ablaufen wird. Die Studentin trägt mit ihren minimalistischen Äusserungen zu Beginn die etablierte Gesprächssituation mit oder stellt diese mindestens nicht aktiv infrage oder leistet Opposition. Mit Michaels Sprechakt sieht sich die Studentin spannungsvoll adressiert: einerseits bestimmt und klar vorstrukturiert (ich zeige dir wo wir hingehen; ich starte nun an; und bevor du das Fazit machst), andererseits auch mit einer fürsorglich-anleitenden und Empathie bekundenden Formsprache - versuch doch mal so; wie war es für dich die Auseinandersetzung.

Die Studentin ihrerseits zeigt sich konform und willens bezüglich der Erfüllung der gestellten Anforderungen und den normativen Ansprüchen und positioniert sich implizit als Lernende. Mit der Aussage $h m$ will ich jetzt das übt sie auch zaghaft Kritik gegenüber dem ihr Zugetragenen aus. Während in einer rein formalbürokratischen Konstellation die Instruktionen vornehmlich Formalia betreffen, sind die Instruktionen des Eröffnungssprechakt nicht ausschliesslich formeller Natur, sondern betreffen auch Inhalte, beispielsweise wenn Kriterien wie präzis, prägnant und konkret aufgestellt werden oder auch ein Fazit gezogen werden soll. Folglich vermischen sich Strukturmerkmale einer Formalisierungsbewegung mit Strukturmerkmalen, die an eine pädagogische Konstellation erinnern, wie sie beispielsweise in einem schulischen Unterrichtssetting vorzufinden sind. Im Eröffnungssprechakt wird somit eine Gesprächskonstellation etabliert, die strukturelle Elemente eines formalistisch-technischen Ansatzes wie auch Elemente aus einem an Unterricht angenäherten Setting beinhalten. Die potenziell im Format «Mentoratsgespräche» angelegte Disposition für diffuse Bezugnahmen und Ausdehnungen, beispielsweise aufgrund des dyadischen Settings oder der normativen Erwartung an ein solches, wird in der Gesprächseröffnung durch Formalisierungsbewegungen handhabbar gemacht. Mittels einer Orientierung an einer vom Mentor hergestellten Verfahrens- 
förmigkeit wird das potenzielle Unbehagen respektive eine mögliche Prekarität dieser dyadischen Gesprächskonstellation bereits antizipierend bearbeitet.

Dies materialisiert sich beispielsweise in den auf dem Tisch ausgebreiteten Karten und verschafft sich auch in der klaren Situationsdefinition des Mentors - ich zeige dir wo wir hingehen so in den nächsten 20 Minuten 25 - Ausdruck. Die personenbezogene Nähe wie auch die diffuse Bearbeitung von Themen, die eine dyadische Gesprächskonstellation programmatisch eröffnen, werden gemässigt, kontrolliert oder bildhaft gesprochen «im Rahmen gehalten s.

Das zentrale Strukturmerkmal der Gesprächseröffnung ist demzufolge die Bearbeitung respektive Handhabbarmachung einer Gesprächsrahmung, welche die diffusen Anteile verfahrensförmig zurückfährt und in Beherrschbarkeit und Kontrollierbarkeit umsetzt. Mögliche Unsicherheiten oder Entgrenzungstendenzen lassen sich in «sicheres Fahrtwasser> überführen und stellen dadurch eine beidseitige Sicherheit her im Modus von «das Gespräch läuft nicht aus dem Ruder, sondern spielt sich in strukturierten Bahnen ab».

Diese Ausführungen führen zur folgenden, riskant formulierten Fallstrukturhypothese: Die Gesprächseröffnung zeigt einen Modus auf, mit dessen Hilfe Diffusitätspotenziale durch eine klar etablierte Gesprächsrahmung und Formalisierungsbewegungen handhabbar gemacht werden. Diese Formalisierungsdynamik bleibt allerdings fragil, was sich in den Daten in auch aufscheinenden diffusen Bezugnahmen zeigt. Im Gegensatz zu einem therapeutischen Setting, das einer institutionalisierten Gleichzeitigkeit von Diffusität und Spezifität folgt und diese sogar einfordert, lässt sich in der vorgefundenen Gesprächseröffnung ein tendenziell spezifisch institutionalisiertes Setting mit diffusen Ausdehnungstendenzen erkennen. Dabei gewinnt eine Figur Gestalt, in der diese widersprüchliche Disponiertheit während des Mentoratsgesprächs interaktiv und beidseitig bearbeitet wird. 


\subsubsection{Zweite Sequenz: Du sprichst von Struktur und Planung}

Im weiteren Gesprächsverlauf wird Tina dazu aufgefordert, als Konsequenz einer Videoanalyse ihres Unterrichts Positives wie auch Entwicklungspotenzial zu benennen. Als positive Aspekte erwähnt Tina, mit Körpersprache und Gesten gearbeitet zu haben. Entwicklungspotenzial erkennt sie bezüglich ihrer Wiederholungen von Antworten der Schülerinnen und Schüler. Sie bezeichnet diese als «Lehrer-Echo» und möchte sie künftig vermeiden. Daran anschliessend wird das zweite Thema auf einer laminierten Karte, die «Fallstudie», aufgegriffen. Tina wird gefragt, was sie aus der Fallbearbeitung für ihre künftige Tätigkeit als Lehrperson mitnehme. In Tinas Fallbearbeitung geht es um ein - in der Bezeichnung der Studentin - «trotzendes Kind» und die dadurch entstandenen Herausforderungen in der Klassenführung. An dieser Sequenzstelle setzt die feinsequenzielle Rekonstruktion für die Herausarbeitung der aufscheinenden Interaktionsdynamiken ein.

Michael: $m h m$ du sprichst eigentlich so zwei (.) zwei wichtige Punkte an (.) du sprichst von Struktur und Planung dass man das schon präventiv proaktiv/ ((räuspert sich)) das ist so der Bereich ähm Ziel (.) Zielorientierung Zielstrukturierung oder für den Unterricht (.) sich vornehmen das ist der Einstieg der läuft so ab und dann so (.) Formate/(.) oder so findet/ wann wann weiche ich jetzt ab von meiner Planung? (.) jetzt da war auch der Entscheid (.) das ist auch so eine eine (.) Zeitfrage (.) so dieses Spiel dauert jetzt plötzlich dann zwanzig Minuten so das ist auch dann (.) z.T. Spontaneität oder situativ ad hoc reagieren Tina: $m h m$ Michael: wie gehst/ also kannst du das auch ein bisschen auf/aufnehmen? Im Moment finden ja führe ich es trotzdem weiter eigentlich (.) oder gehe ich jetzt zurück zu meinem Plan? Ich habe meinen Plan ich habe meine Lernziele. (.) das ist die Struktur des Unterrichts Tina: ich denke es ist einfach (.) wichtig (.) seine Planung schon im Kopf zu haben aber (.) nicht starr darauf zu beharren (.) weil alles planen kann man nicht (.) und die Kinder kann man sowieso nicht planen (.) wie sie drauf sind was was sie heute machen (.) und da ist es wichtig (.) auch authentisch zu bleiben und zu sagen ich 
mache es jetzt oder ich möchte jetzt weiterfahren (.) wegen dem und dem und auch begründen ich denke Kinder haben ein Recht auf einen Grund sie sind ja dann eigentlich mir ausgeliefert auf eine Art und sie haben auch ein Recht auf eine Begründung wieso ich es jetzt so mache (.) und wieso es jetzt nicht für alle Kinder reicht (.) und da ist es auch wichtig die Kinder ernst zu nehmen und zu sagen ich (.) höre dich und ich weiss dass du das jetzt nicht toll findest oder ich weiss dass du jetzt enttäuscht bist (.) ich mache es so und so aus diesen und diesen Gründen

Michael: sehr gut

Tina: genau

\subsubsection{Rekonstruktion der einzelnen Sequenzen}

Michael: $\mathrm{mhm}$ du sprichst eigentlich so zwei (.)zwei wichtige Punkte an (.)

Ein $m h m$ zu Beginn eines Sprechaktes lässt sich grundsätzlich als zögernde Zustimmung oder als Indiz des Nachdenkens lesen. Je nach Sprechweise oder Intonation lassen sich etliche weitere mögliche Kontexte für $\mathrm{mhm}$ finden, weshalb hier der paraverbale Textmarker in Kombination mit dem anschliessenden Sprechakt rekonstruiert wird. Um die Sinnstrukturiertheit des gesamten Sprechaktes zu erfassen, wird im Sinne eines Gedankenexperimentes die Bedeutung ohne die Partikel eigentlich herausgearbeitet. Eine Beispielsgeschichte, in welcher der Sprechakt unter Auslassung von eigentlich als angemessene sprachliche Äusserung erscheinen würde, ist eine gemeinsame biblische Exegese, wenn die Lernende einen Text aus dem Alten Testament für die christliche Glaubenspraxis innerhalb der Glaubensgemeinschaft auslegt und der Lehrende die Auslegung validiert, systematisiert und würdigt, dass zwei wichtige Punkte angesprochen wurden. Auch in einem therapeutischen Setting wäre der Sprechakt erwartbar, wenn die Klientin einen Gedanken oder ein Thema darlegt und der Therapeut zwei Punkte nochmals aufgreifen und möglicherweise vertiefen oder diskutieren möchte. Ungewohnt, 
aber nicht grundsätzlich unvorstellbar ist, dass die Person mit $d u$ angesprochen wird, was im therapeutischen Kontext darauf hindeutet, dass die Klientin noch minderjährig ist. Beiden Kontexten gemein ist die Positionierung der sprechenden Person als diejenige, die über die Deutungshoheit bezogen auf Wichtiges und Unwichtiges verfügt und damit einhergehend eine Asymmetrie zwischen der sprechenden und der angesprochenen Person aufzeigt. Fragt man sich, ob der Sprechakt auch egalitär möglich wäre, beispielweise wenn Theologiestudierende gemeinsam eine biblische Stelle besprechen, wäre eine Ergänzung wie "für mich» oder «aus meiner Sicht» angemessen. Daraus lässt sich schliessen, dass in der vorliegenden sozialen Situation die Asymmetrie performativ hergestellt wird. Mit dem Zusatz eigentlich wird der Kern der Aussage abgeschwächt und Unsicherheitsmomente werden aufgezeigt. In der eigentlich-Aussage drückt sich auch eine Ambivalenz der sprechenden Person aus, da bei eigentlich implizit der Einwand, meist in Form eines "Abers», mitschwingt. Die eingenommene Position der Deutungshoheit weist eine Fragilität auf. Die Aussage wird im Präsens formuliert und erhält dadurch eine Gültigkeit, die anders als im Kontrastbeispiel «du hast im Vortrag eigentlich so zwei wichtige Punkte angesprochen» über eine bestimmte Zeitspanne hinausgeht und eine Universalisierung und Verallgemeinerung nach sich zieht. Die Betonung des Wortes an führt auf die Pause hin und gibt dem Folgenden mehr Gewicht. Würde, als Kontrast zum vorgefundenen Sprechakt, nur von zwei Punkten gesprochen und das wichtig wie auch das eigentlich weggelassen, käme die Aussage einem Gliederungskommentar gleich. Der Sprechakt weist demzufolge mit dem Adjektiv wichtig zusätzlich ein validierendes Element auf.

Aus diesen Überlegungen lassen sich drei Deutungsaspekte herausbilden, die zuerst isoliert voneinander betrachtet werden: Der erste Aspekt bezieht sich auf eine asymmetrische Struktur, die eine pädagogische Implikation aufweist. Dabei adressiert der Sprecher die Angesprochene im Modus des Zeigens. Verbunden wird diese Adressierung mit einer wertschätzenden und wohlwollenden Haltung, die sich mit «du erkennst das grundsätzlich schon gut. Ich zeige dir, wie man das, was du gemacht hast, nun noch besser darstellen könnte» paraphrasieren lässt. Die Kommentierung, die implizit im Sinne von «gut erkannt» vorgenommen wird, entspricht latent einer Evaluation. Mit dieser Deutung wird dem Sprecher implizit die Motivstruktur der Wissensvermittlung oder des Kompetenzaufbaus zugesprochen. 
Als Zweites lässt sich eine stärker evaluative und bewertende Deutung feststellen. In dieser wird eine Äusserung, eine Aktivität, ein Produkt oder auch ein Ergebnis eines Prozesses der Person vom Sprecher beurteilt oder hinsichtlich der Qualität eingeschätzt. Die Evaluation kann durchaus Ziele der Wissensvermittlung oder des Kompetenzaufbaus verfolgen, allerdings liegt der Fokus primär auf der Beurteilung. Deshalb ist hier die Deutung auf den evaluativen Aspekt ausgerichtet.

Als dritte Deutung lässt sich eine Struktur der Herstellung von Fürsorge, Gemeinsamkeit, Zusammengehörigkeit und Vergemeinschaftung ${ }^{30}$ erkennen, die über die Zustimmung der genannten Punkte erfolgt. Eine potenziell vorhandene Asymmetrie wird verschleiert oder mindestens nicht dominant gesetzt. Auch die Adressierung per Du in einer Konstellation, die dies nicht implizit voraussetzt, kann als Akt der Kollegialität und Vergemeinschaftung betrachtet werden.

Ein grundsätzlicher Gedanke bezogen auf die drei elaborierten Aspekte sei hier angefügt: Asymmetrie, wie sie in pädagogischen oder evaluativen Konstellationen konstitutiv vorhanden ist, schliesst Vergemeinschaftung nicht per se aus. Dennoch kann vermutet werden, dass gewissen Konstellationen, Kriterien oder Voraussetzungen eine gleichzeitige Installierung von Asymmetrie oder Vergemeinschaftung erleichtern respektive erschweren. Wie sich dies in der konkreten Gesprächssituation gestaltet, gilt es weiteren Verlauf zu überprüfen.

Für die Herausarbeitung der Gestalt des Sprechaktes werden an dieser Stelle die Anschlussoptionen hinsichtlich der drei Deutungsaspekte ausformuliert. Das relativierende und Unschärfe erzeugende Moment, das sich im eigentlich Ausdruck verschafft und auf eine Fortsetzung mit aber oder einem sinnähnlichen Wort hindeutet, wird in allen drei potenziellen Anschlüssen aufgegriffen.

In der Auslegung mit einer pädagogischen Implikation übernimmt der Anschluss die Vermittlungs- oder Zeigelogik und führt diese weiter aus, indem in der Fortsetzung verdeutlicht wird, welche Punkte oder Überlegungen für die adressierte Person noch anstehen. In dieser Auslegung könnte beispielsweise mit «Nun aber ist wichtig, dass du

\footnotetext{
${ }^{30}$ Vergemeinschaftung ist hier im Sinne von Max Weber zu verstehen und somit als soziale Beziehung, die auf subjektiv gefühlter Zusammengehörigkeit beruht und ein entsprechendes soziales Handeln hervorruft (vgl. Weber, 1925).
} 
dir konkret überlegst, ob allenfalls noch weitere wichtige Punkte dazukommen» angeschlossen werden.

Der Anschluss für eine evaluative Deutung wäre ein Urteil, bei dem das im eigentlich latent Angedeutete in den Fokus gelangt. Ein Anschluss wäre beispielsweise im Sinne von «beide Punkte erachte ich als sehr wichtig, allerdings käme auch noch der dritte und vielleicht wichtigste Punkt dazu, nämlich die Auswertung» denkbar.

In einer vergemeinschaftenden Deutungsversion könnte wie folgt angeschlossen und dadurch ein mögliches Hierarchiegefälle bearbeitet werden: «Spannend, ich hätte vermutlich die gleichen zwei Punkte ausgewählt, aber vielleicht gibt es noch weitere wichtige Punkte. Lass uns mal gemeinsam überlegen.» Die Relativierung durch eigentlich wird auch hier angetönt, steht aber nicht im Vordergrund respektive wird durch «vielleicht» und "gemeinsam» in Richtung Symmetrie bearbeitet.

\section{Michael: du sprichst von Struktur und Planung}

Der empirisch vorgefundene Anschluss von Michael löst die Vermutung einer AberStruktur nicht ein. Vielmehr werden diese zwei angekündigten wichtigen Punkte expliziert und als Gefäss mit Inhalt gefüllt. Allfällige Zweifel darüber, welche Punkte gemeint sind, werden auf diese Weise ausgeräumt. Der Sprechakt erhält durch die Wiederholung von du sprichst mehr Nachdruck, als wenn nur gesagt würde «du sprichst eigentlich zwei wichtige Punkte an, ¿Struktur» und 〈Planung»».

Losgelöst vom Kontext wäre der Sprechakt in einer Talkrunde vorstellbar. Der Sprecher schliesst auf einer metakommunikativen Ebene an eine Ausführung an: Du sprichst eigentlich zwei wichtige Punkte an, du sprichst von Struktur und Planung. "Ich allerdings bin überzeugt, dass das Projekt bereits auf einer politischen Ebene scheitert, wenn wir es nicht schaffen, die Gegnerinnen und Gegner auch an den Verhandlungstisch zu holen.» Wenngleich zugewandt und wertschätzend attestiert wird, dass grundsätzlich oder eben eigentlich zwei wichtige Punkte - Struktur und Planung - angesprochen werden, scheint die Funktion des Sprechaktes ein Überführen zu einem weiteren Thema zu sein. Die Aussage, und das ist im eigentlich angelegt, gleicht strukturell einem metakommunikativen Akt, in welchem das analytisch Verstandene abstrahiert, verdichtet und für eine Fortsetzung genutzt wird. Die zuvor getätigten Ausführungen der angesprochenen Person 
werden abstrahiert und den Begriffen Struktur und Planung zugeordnet. Diese Zuordnung wird im Ersetzungsargument deutlich. Hätte die angesprochene Person tatsächlich die Begriffe Struktur und Planung in ihren Ausführungen verwendet, würde der Sprechakt korrekterweise «du sprichst über Struktur und Planung» lauten. In der vorgefundenen Verwendung wird bei von Struktur und Planung hingegen eine Zuordnungsleistung vorgenommen. ${ }^{31}$

Diese manifeste Zuordnungsleistung kann auf einer latenten Ebene bedeuten, dass sich der Sprecher mit der Erwartung konfrontiert sieht, die Äusserungen des Gegenübers zu kommentieren, und er dieser Erwartung performativ im Modus einer Zuordnung nachkommt. Auch denkbar wäre, dass der Sprecher eine Bezugnahme zum bereits Gesagten vornimmt, um daran anschliessend oder (andockend) auf eine weitere Thematik zu sprechen zu kommen.

In der kontextbezogenen Situation wird ein Bemühen des Mentors sichtbar, auf Themen Bezug zu nehmen, die die Studentin bereits aufgegriffen hat. Die drei oben elaborierten Deutungsaspekte bleiben in beiden Auslegungen gedeckt und haltbar. Die bisherige Rekonstruktion des Sprechaktes lässt eine Lesart zu, die evaluative und vergemeinschaftende Strukturmomente und auch Strukturmomente mit pädagogischen Implikationen aufweist. Bezogen auf die Adressierung ist festzustellen, dass die Studentin in wertschätzender Art und Weise angesprochen wird. Dabei zeigt sich der Mentor als aufmerksamer Zuhörer, der das Gehörte einem gewissen Kontext zuordnet.

Michael: dass man das schon präventiv proaktiv/((räuspert sich))

Hier folgt der Anfang eines Nebensatzes, der mit dass eingeleitet wird und der Sinnlogik einer Setzung folgt. Erwartbar wäre die Fortsetzung mit einem Verb wie beispielsweise «überlegt», «einplant», «berücksichtigt» oder Ähnlichem. Die Aufforderung, etwas zu tun, wird abgeklemmt. Die Aussage endet mit einem Satzabbruch, gefolgt von einem Räuspern, was zweimal eine Suchbewegung andeutet. Die abgebrochene Aussage lässt sich als Hinweis verstehen, dass entweder das adäquate Verb fehlt oder die mit der vollständigen Formulierung einhergehende Aufforderung unpassend erscheint und

\footnotetext{
${ }^{31}$ Da das ganze Gesprächstranskript vorliegt, kann an dieser Stelle bestätigt werden, dass die Begriffe «Struktur» und «Planung» im bisherigen Gesprächsverlauf nicht verwendet wurden.
} 
deshalb vermieden wird. Gleichzeitig wechselt die Adressierung in diesem Sprechakt von du zu man, was einer Distanzierungsbewegung und/oder einem Verweis auf eine gültige Norm gleichkommt. Die beiden verwendeten Adjektive präventiv und proaktiv bedürfen einer isolierten Betrachtung: Das Präfix «prä-» kennzeichnet etwas als vorher erfolgend; «pro» steht ebenfalls für «vor» oder «für» und beide lateinischen Präfixe charakterisieren die Adjektive als Fremdwörter. Etwas präventiv zu tun dient der Verhinderung und Verhütung eines potenziellen «Unheils〉. Das Wort «proaktiv» ist derzeit (en vogue) und nimmt Machbarkeit und Beherrschung, im Sinne von selbstoptimierend salles im Griff haben` und aktiv auf den Gestaltungsprozess einwirken können, in den Fokus. Insgesamt erinnern beide Adjektive an einen Werbespruch oder an eine Plakatüberschrift.

Welche inhaltliche Bedeutung der Aneinanderreihung dieser Begriffe zukommt, ist nicht vollumfänglich zu erschliessen. Die Dynamik des Sprechaktes lässt sich mit «Assoziationen aufrufen» beschreiben: etwas soll bereits vor dem Eintreten bearbeitet werden. Die Funktion, durch die Verwendung von Fachvokabular Aufmerksamkeit und Zustimmung zu erzeugen und sich dadurch als kompetent und als Experte zu positionieren, haftet dem Sprechakt an und kann als erster Hinweis auf eine jargonhafte Sprechweise gelesen werden. Das Strukturmoment für die Verwendung von Jargon ${ }^{32}$ ist die Benennung von Dingen, das Aufrufen von Konzepten, ohne dabei eine Aussage zu treffen. Die jargonhafte Sprechweise lässt sich als Distinktionsfigur lesen und zieht eine Positionierung als jemand Bestimmtes nach sich.

Ob sich präventiv und proaktiv auf Struktur und Planung und demzufolge auf die zuvor geschilderte Gegebenheit oder auf noch Anstehendes beziehen, bleibt ungeklärt. Deshalb richtet sich der Fokus der Rekonstruktion auf die Unabgeschlossenheit und den Abbruch, die an dieser Stelle als symptomatisch erachtet werden. Auf einer latenten Ebene könnte der Abbruch auf Spannungsverhältnisse zwischen den unterschiedlichen Ansprüchen hinweisen, die der Mentor hier bearbeitet. Diese Spannungen beziehen sich auf Ansprüche, etwas zu zeigen oder zu vermitteln und auf Ansprüche zu evaluieren. Für die Einlösung dieser Ansprüche ist eine Asymmetrie in Form einer Expertise- und Erfahrungsdifferenz erforderlich, die performativ hergestellt wird. Auf der anderen Seite ist auch

\footnotetext{
32 Pädagogischer Jargon ist gemäss Dzengel et al. (2012) ein Austausch im Modus der Auseinandersetzung, der an der Klärung von pädagogischen Sachverhalten nicht interessiert ist, sondern in dessen Verlauf sich die Sprecherin oder der Sprecher als intellektuell versiert ausweist.
} 
eine soziale Anforderung dieses Formats erkennbar, die darin besteht, der Studentin wertschätzend und anerkennend zu begegnen. Diese Gleichzeitigkeit ruft strukturelle Unsicherheiten hervor, die sich beispielsweise im Abbruch Ausdruck verschaffen.

Michael: das ist so der Bereich ähm Ziel (.) Zielorientierung Zielstrukturierung oder für den Unterricht (.) sich vornehmen das ist der Einstieg der läuft so ab

Die Rekonstruktion des dem Sprechakt zugrunde liegenden Sinnes gestaltet sich als schwierig, da verschiedene Themen assoziativ aneinandergereiht, jedoch nicht vollendet werden. Die Vermutung scheint zulässig, dass Michael eine Hinführung zu den Bereichen Zielorientierung, Zielführung vorgenommen hat und diese performativ nicht geglückt ist. Die verwendeten Begriffe Zielorientierung und Zielstrukturierung sind dem Projektmanagement oder Controlling entlehnt und vermitteln dem Sprechakt in dieser «neopädagogischen` Verwendung einen elaborierten Anstrich. Mit Zielorientierung und Zielstrukturierung werden zu Beginn der Aussage weite Themenfelder elliptisch aufgegriffen, während der Sprechakt nach oder eine ganz andere Ebene bedient. Denn sich vornehmen steht noch vor dem konkreten Tun und schliesst ein mögliches Scheiterpotenzial latent mit ein. Diese Dynamik steht in markanter Differenz zur vorausgehenden Setzung der Bereiche Zielorientierung oder Zielstrukturierung.

Bemerkenswert scheint die Transformation, der die adressierte Person ausgesetzt ist. Sie wird mit Themen wie Struktur, Planung und präventiv und proaktiv auch mit Zielorientierung und Zielstrukturierung und am Schluss mit Vorsätzen bezogen auf Lektionseinstiege konfrontiert. Da jedoch die grammatikalische Struktur des Satzes nicht klar erkennbar ist und sich die Aussage inhaltlich nicht erschliessen lässt, richtet sich der Fokus der Rekonstruktion auf die hier anzutreffende Diffundierungslogik, die sich durch diese Vielfalt auch Optionen eröffnet. So ergibt sich für Tina bei einer potenziellen Wortmeldung die Möglichkeit, Themen herauszunehmen, die ihr entsprechen, und sie braucht sich in ihrer Bezugnahme lediglich zu diesen zu äussern.

Zusammenfassend lässt sich festhalten, dass im Sprechakt etwas dargelegt wird, was ein gewisses Mass an Verunklarung aufweist und sich inhaltlich bislang nicht erschliessen lässt. Deshalb wird eine Erschliessung auch nicht weiterverfolgt. Darin gewinnt ein vermuteter Geltungsanspruch Gestalt, der darin besteht, der angesprochenen Person 
etwas zu zeigen oder, alltagssprachlich formuliert, ihr für etwas «die Augen zu öffnen`. Der Sprecher positioniert sich als Zeigender und durch die Verwendung von Fachsprache auch als versiert und kompetent. Auf einer performativen Ebene betrachtet scheint es sich um eine jargonhafte Sprechweise zu handeln.

Die vorliegende Gesprächsinteraktion erinnert von ihrer Logik her an ein Lehr-LernSetting. Allerdings ist die Adressierung Tinas als Lernende brüchig, denn in der Verwendung von Jargon lässt sich auch eine Vergemeinschaftungsbewegung im Modus von «du verstehst schon, was ich meine, wenn ich dich auf diese Art anspreche» vermitteln. Die Ausführungen wechseln von einer abstrakteren (zwei Punkte ansprechen) auf eine konkretere Ebene (Ablauf des Einstiegs). Dies lässt sich als Versuch einer Vorwärtsbewegung lesen, in der möglicherweise auf die Verunklarung in den Äusserungen reagiert wird. Mit den verwendeten Begriffen wird ein assoziativer Raum aufgemacht; was Michael vermitteln möchte, lässt sich jedoch nicht genau erschliessen. Interaktionsdynamisch scheint dies Ausdruck von Unsicherheit zu sein, die in seiner Sprechweise Gestalt gewinnt.

Als Anschluss wäre eine Verständnisrückfrage Tinas angesichts der inhaltlichen Konfusion vorstellbar und sinnlogisch. Der wohlwollende Gesprächsverlauf und die vergemeinschaftende Art der Adressierung würden eine Rückfrage, trotz der Asymmetriedisposition, von der Beziehungsstruktur her zulassen. Konkret findet jedoch keine Rückfrage seitens der Studentin statt.

Inhaltlich wird an dieser Stelle das Thema «Unterrichtsvorbereitung» besprochen und Michael tritt als Experte auf. Sein Versuch einer Expertisierung wird performativ unterschiedlich sichtbar: zu Beginn relativ souverän und um Symmetrie und Vergemeinschaftung bemüht. Im weiteren Verlauf gewinnt eine Figur Gestalt, die sich mit einer zunehmenden Verunklarung seiner Aussagen beschreiben lässt. Bei sich vornehmen zeigt sich eine Dynamik hin zu einer Perspektivübernahme in die Situation hinein, die mit einer Selbstinvolvierung einhergeht, wie sie bereits in der Eröffnungssequenz sichtbar wurde. Die herausgearbeiteten strukturellen Merkmale führen zu einer ersten, riskanten Fallstrukturhypothese der zweiten Gesprächssequenz: Im Sprechakt werden Aspekte und Begrifflichkeiten der Thematik «Planung und Abweichung von dieser Planung» elliptisch aufgegriffen. Aus einer inhaltlichen Perspektive betrachtet bleiben die Aussagen vage. Auf der manifesten Ebene wird durch die Verwendung eines gewissen Vokabulars eine 
Expertise installiert, die allerdings in ihrer latenten Bedeutungsstruktur an eine jargonhafte Sprechweise erinnert. Die Äusserungen des Mentors Michael beinhalten an gewissen Stellen Elemente mit pädagogischen Implikationen, aber auch evaluative oder vergemeinschaftende Elemente scheinen auf, die sich allesamt wechselwirkend zueinander verhalten und durch diese Wechselseitigkeit ein Spannungsverhältnis erzeugen. Riskant formuliert gewinnt in der Art und Weise, in der Michael hier spricht, eine Notwendigkeit oder das Anliegen Gestalt, sich als wertschätzender, fürsorglicher, aber auch fachlich und in seinem Urteilsvermögen kompetenter Mentor in Geltung zu setzen. Die der Funktion des Mentors zugeschriebene Expertise- und Erfahrungsdifferenz ist fragil und muss performativ bearbeitet respektive bestätigt und legitimiert werden. In der Umsetzung dieser Ingeltungsetzung verkomplizieren sich seine Aussagen. Gerade in und durch diese Bewegungen, die Ausdruck der Bemühungen um die Stabilisierung der Expertise des Mentors sind, bestätigt und reproduziert sich die Fragilität des Status als Mentor.

Michael: und dann so (.) Formate/ (...) oder so findet/ wann wann weiche ich jetzt ab von meiner Planung? (.) jetzt da war auch der Entscheid (..) das ist auch so eine eine (.) Zeitfrage (.) so dieses Spiel dauert jetzt plötzlich dann zwanzig Minuten so das ist auch dann (.) z.T. Spontaneität oder situativ ad hoc reagieren

Michaels Sortierung und begriffliche Benennung lässt sich auch durch die hier vorgenommene Ergänzung nicht erschliessen. An dieser Stelle werden verschiedene Punkte erst einmal benannt. Die zuvor eröffnete Themenvielfalt - Zielorientierung, Zielstrukturierung, Unterricht, Einstieg - (aber doch einheitlich als unterrichtsvorbereitende Perspektive) allerdings wird durch die Fortsetzung des Sprechaktes deutlich begrenzt. Es scheint um Unvorhersehbarkeit im Verlauf von Unterrichtsinteraktionen und damit verbunden um zu treffende Entscheidungen während des Unterrichtens zu gehen. Hierbei findet ein Wechsel in der Zeitdimension statt; von einer prospektiven Perspektive wird in die Unterrichtsgegenwart gewechselt. Dabei bleibt offen, ob sich die Ausführungen auf Michaels eigenen Unterricht, auf den Unterricht von Tina oder ganz generell auf Unterricht beziehen. Dies zeigt sich beispielsweise auch in der Verwendung einer IchFormulierung anstelle des zuvor verwendeten man, die hier interaktionsdynamisch 
〈hineinrutscht〉. Dieses ich steht manifest für ein allgemeines Ich und ist auch als Hinweis für die vorgenommene Expertisierung und gleichzeitig auf einer latenten Ebene als Ausdruck des eigenen Bewährungsdrucks zu verstehen.

Michael zeigt sich als Experte oder Kenner der Thematik und expliziert mit seinen Selbstreflexionen, welche Fragen in einem solchen Entscheidungsprozess zu stellen seien. Performativ setzt er die Einnahme des Expertenstatus unterschiedlich um, was sich in den teilweise überschüssigen Formulierungen wie situativ ad hoc und auch in den Abbrüchen und den häufig vorgenommenen Sprechpausen zeigt. Gerade diese Pausen, die mit bis zu drei Sekunden auch lange anhalten, können als Ausdruck von Suchbewegungen gelesen werden.

Betrachtet man die Aussage als Ganzes, fallen zusätzlich die vielen so auf, die manifest eine gewisse Beliebigkeit und Vagheit darstellen, aber auch etwas Gönnerhaftes und Grosszügiges in der vorgenommenen Selbstexpertisierung unterstreichen. Auf einer latenten Ebene markieren sie aber auch eine Unsicherheit, gerade in Bezug auf die eigene Expertise. Besonders markant ist dies bei der Formulierung so (.) Formate/ (...) oder so. Hier wird angedeutet, dass es so oder ähnlich oder auch anders sein könnte und dies entweder wenig Relevanz oder aber eine diesbezügliche Unsicherheit aufweist. Insgesamt und riskant formuliert reproduziert sich in der Fortführung des Sprechaktes eine gewisse Virtuosität in seinen Aussagen, die inhaltlich ungefüllt bleiben, obwohl sie beispielsweise bei dieses Spiel dauert jetzt plötzlich dann zwanzig Minuten konkret formuliert werden. Relevant für die Deutung des Sprechaktes scheint die Frage, weshalb die transportierte Botschaft auf diese «umständliche` Art und Weise vermittelt wird. Als mögliche Erklärung lässt sich die Annahme verfolgen, dass der Fokus auf eine elaborierte, versierte und differenzierte Form oder (Verpackung) der Botschaft, mehr als auf inhaltliche Schlüssigkeit und Klarheit, gelegt wird. Diese Deutung kommt erneut dem Konzept des «pädagogischen Jargons» nahe, wie dies bei Dzengel et al. (2012) beschrieben wird. In einer noch pointierteren Formulierung stellt sich die Frage, ob es möglicherweise ein Strukturmerkmal der Sequenzstelle sein könnte, dass es gar keine eigentliche Botschaft gibt. Im Sprechakt des Mentors wird viel gesagt, denn es muss auch etwas gesagt werden. Gleichzeitig lässt sich inhaltlich nicht genau erschliessen, was gesagt wird, weil es möglicherweise gar nicht so viel zu sagen gibt. 
Im Sinne eines Gedankenexperimentes lässt sich nochmals ein professionell-therapeutisches Gesprächssetting als Vergleichsfolie herbeiziehen. In einem solchen wird die Klienten, falls kein Gesprächsbedarf mehr besteht, entlassen, wobei diese Entlassung in die zurückgewonnene Autonomie das eigentliche Therapieziel darstellt. Im vorliegenden Gesprächssetting des Mentorats scheint die Option, dass der Mentor die Studentin fragt, ob ihrerseits Gesprächsbedarf bestehe, und sie, falls sie verneint, entsprechend von der Verpflichtung des Mentoratsgesprächs befreit wird, programmatisch nicht vorgesehen. Dieser hier pointiert dargestellte Vergleich könnte allenfalls Hinweise darauf liefern, weshalb im Gesprächsverlauf nach Themen, Anliegen oder Problemen gesucht wird, die das Gespräch auch rechtfertigen und legitimieren. Auch die jargonhafte Sprechweise würde die Hypothese der Bearbeitung, Legitimation und Aufrechterhaltung der Form oder Hülle des gesprächsspezifischen Settings zwischen der Studentin und dem Mentor oder auch zwischen der Novizin und dem Experten stützen.

Auf einer manifesten Ebene lässt sich die Sprechweise somit mit dem Versuch einer souveränen und fachlich kompetenten Selbstpositionierung beschreiben, die aber latent eine gegenteilige Bewegung erfährt und durch die Verkomplizierungen, Pausen und Abbrüche Hinweise für Unsicherheiten und eine grundsätzliche Fragilität in der Disposition des Settings enthält.

Die Studentin ihrerseits muss sich in ihrem Anschluss zu dieser voraussetzungsreichen, teils auch unklaren Art und Weise des Angesprochen-Werdens verhalten.

Tina: $m h m$

Sprechaktlogisch bestünde hier die Möglichkeit, mit einer Nachfrage anzuschliessen; die Entgegnung mit einem zustimmenden $\mathrm{mhm}$ fällt allerdings analog zu zuvor minimal aus. Die Barrieren oder Hürden, die in einer Asymmetrie strukturell enthalten sind und eine Rückfrage allenfalls erschweren könnten, sind - wie bereits erwähnt - in der vorgefundenen Konstellation nicht erkennbar, trotz vorhandener struktureller Asymmetrie. Tinas Anschluss kommt an dieser Stelle de facto einem Nichteinsteigen, zumindest aber einem verzögerten Einsteigen in die Gesprächsinteraktion gleich. Die Frage stellt sich, ob die Studentin, trotz der wohlwollenden Adressierung des Mentors, sich nicht anzumerken getraut, den Ausführungen des Mentors nicht folgen zu können. Es fragt sich weiter, ob 
der Modus der Ansprache bei ihr kein Unbehagen oder Klärungsbedarf auslöst und ihr Anschluss im Modus einer Komplizenschaft im Sinne von «ich kann deinen Ausführungen zwar nicht folgen, frage aber dennoch nicht nach» zu verstehen ist. Auch denkbar ist, dass die Studentin den Ausführungen des Mentors nicht mehr folgt und mental bereits ausgestiegen ist, was allerdings in einer dyadischen Konstellation schwierig zu erkennen respektive zu verbergen ist. Würde sich die Interaktion - als kontrastiver Vergleichshorizont - in einer Schulklasse abspielen, könnte die Dynamik des Aussteigens mit grosser Wahrscheinlichkeit an einem steigenden Lärmpegel festgemacht werden. In einem dyadischen Setting erfährt die angesprochene Person keinen Schutz der Anonymität in der Masse und kann «nicht nicht reagieren`; die Reaktion $\mathrm{mhm}$ könnte in diesem Kontext daher im Sinne einer Nichtreaktion ausgelegt werden. ${ }^{33}$

Obwohl die Studentin bislang nicht explizit dazu aufgefordert wurde, bestünde für sie die Option, einen Gegenimpuls zu setzen, mit dem sie unterschiedlich moderat auf die Äusserungen des Mentors reagieren könnte. Diese Option wird nicht umgesetzt; ihr Anschluss fällt, wie bereits festgehalten, minimal aus. Das Mitspielen der Studentin lässt sich an dieser Stelle, zugespitzt formuliert, als (Komplizenschaft) deuten, die sich als Aushalten, ohne in Konfrontation zu gehen, Ausdruck verschafft, oder auch als Ausstiegsvariante lesen und kann als für die Interaktionsdynamik relevant angesehen werden.

Michael: wie gehst/ also kannst du das auch ein bisschen auf/ aufnehmen? im Moment finden ja führe ich es trotzdem weiter eigentlich (.) oder gehe ich jetzt zurück zu meinem Plan? ich habe meinen Plan ich habe meine Lernziele. (.) das ist die Struktur des Unterrichts

Die Angesprochene wird mit einer Frage konfrontiert, die allerdings abgebrochen wird. Nach der Satzplankorrektur wird ein neuer Gedanke ausgeführt respektive eine Frage formuliert, die eine Rückäusserung erfordert. Die Nachfrage, ob die Studentin die Ausführungen aufnehmen, also einordnen oder ihnen folgen könne, erinnert an eine für Ausbildungssituationen typische Formsprache. Gleichzeitig lässt sich in der Nachfrage

\footnotetext{
${ }^{33}$ Ein steigender Lärmpegel in der Schulklasse ist eigentlich auch als eine mögliche Reaktionsform zu verstehen. Dies würde heissen, dass auch in der Masse respektive in einer Schulklasse nicht nicht reagiert werden kann, was meine Hypothese allerdings stützt.
} 
auch die Legitimierungsbedürftigkeit von Michaels Mandat erkennen. Wäre er sich seiner Sache und der Funktion seiner Ansprache sicher, bräuchte er nicht nachzufragen. Hier jedoch bedarf die Situation einer entsprechenden Anerkennung seitens der Studentin.

Dabei bleibt der Mentor mit der Formulierung ein bisschen bescheiden. Auch ist als vergewisserndes «auch» zu verstehen. Der Umstand, dass dies überhaupt erfragt wird und sich nicht aus der Interaktion herauslesen lässt, ist bemerkenswert. Dies deutet zugleich auf eine gewisse Prekarität für den Mentor hin, da die Studentin nur minimal reagiert und partizipiert. Insgesamt zeigt sich in der Interaktion, dass der Status als Experte nicht ohne Weiteres eingenommen werden kann. Vielmehr sind Bemühungsbewegungen und ein beachtlich betriebener Aufwand zu erkennen, die darauf ausgerichtet sind, dass Michael von Tina als Mentor und als Experte für Unterrichtsfragen anerkannt wird. Die hier gezeigte Expertise lässt sich verstehen als: «Ich vermittle dir hier Wissen zu Unterricht, die deine Ausführungen, welche ich Struktur und Planung zuschreibe, betreffen. Kannst du dieses Wissen auch ein bisschen aufnehmen und zu meinen Ausführungen Stellung nehmen?» Falls Tina seine Ausführungen ein bisschen aufnehmen kann, ist es ihm gelungen, ihr mithilfe seiner Expertise Wissen zu vermitteln. Falls sie mit Nein antwortet, weil sie seinen Ausführungen nicht Folge leisten kann, ergibt sich für ihn die Möglichkeit, sie als Mentor fürsorglich zu unterstützen.

Als Zwischenfazit kann festgehalten werden, dass sich der Modus der brüchigen und hülsenhaften Sprechweise des Mentors reproduziert. Die hier etablierte Gesprächsanlage scheint der Logik zu folgen, etwas zu lehren und zu zeigen. Der entsprechende Modus beruht auf der Asymmetriedisposition zwischen den beiden am Gespräch partizipierenden Personen und bearbeitet die Erfahrungsdifferenz zwischen der Novizin und dem Experten. Der Modus erinnert an schulischen Unterricht, der hier in einer dyadischen Form umgesetzt wird.

Im real vorgefundenen Kontext des Mentoratsgesprächs zeigt sich eine soziale Notwendigkeit, die darin besteht, diese Asymmetrie interaktiv herzustellen und aufrechtzuerhalten. Hierbei scheint es wichtig zu sein, in dieser Asymmetrie von der Studentin anerkannt zu werden. Dies lässt Rückschlüsse auf eine strukturelle Unsicherheit zu. Denn wäre die Disposition klar und gefestigt, müsste sie nicht in Geltung gesetzt werden respektive bedürfte sie keiner Bearbeitung. Gleichzeitig steht die hergestellte Asymmetrie in einem Spannungsverhältnis zu den Fürsorglichkeitsansprüchen, die ebenfalls als 
Bestandteile der Gesprächsdynamik erkennbar sind. Diese Spannungen führen dazu, dass ein grosser Aufwand für die Legitimierung, die Aufrechterhaltung und die Ingeltungsetzung des Formats wie auch für die Festigung der Position des Mentors als Hüter des Formats betrieben werden muss. Dadurch werden Bezugnahmen auf die Thematik und die Klarheit in den Ausführungen zur Nebensache, obwohl das Format als ein an Unterricht angenähertes Wissensvermittlungs- oder Zeigeformat etabliert wird. Bestünde ein hoher und expliziter Anspruch, stofflich etwas zu vermitteln und gleichzeitig den Wissensstand der Studentin zu überprüfen, lägen möglicherweise different formulierte Sprechakte näher als die hier verwendeten.

Tina: ich denke es ist einfach (.) wichtig (.) seine Planung schon im Kopf zu haben aber (.) nicht starr darauf zu beharren (.) weil alles planen kann man nicht (.) und die Kinder kann man sowieso nicht planen (.) wie sie drauf sind was was sie heute machen (.) und da ist es wichtig (.) auch authentisch zu bleiben und zu sagen ich mache es jetzt oder ich möchte jetzt weiterfahren (.) wegen dem und dem und auch begründen ich denke Kinder haben ein Recht auf einen Grund sie sind ja dann eigentlich mir ausgeliefert auf eine Art und sie haben auch ein Recht auf eine Begründung wieso ich es jetzt so mache (.) und wieso es jetzt nicht für alle Kinder reicht (.) und da ist es auch wichtig die Kinder ernst zu nehmen und zu sagen ich (.) höre dich und ich weiss dass du das jetzt nicht toll findest oder ich weiss dass du jetzt enttäuscht bist (.) ich mache es so und so aus diesen und diesen Gründen

Der zuvor gegenüber der Studentin geöffnete assoziative Raum bietet ihr etliche Möglichkeiten, sich zu positionieren und sichtbar zu werden. Tina reagiert primär kooperativ und beantwortet manifest die konkrete Frage, ob sie etwas weiter ausführe oder zu ihrem Plan zurückkehre. Dabei zeigt sie performativ auf, dass es ihr gelingt, dies auch ein bisschen aufnehmen zu können, indem sie auf eine nicht klar formulierte Aufforderung elaboriert und differenziert anschliesst. Im Vergleich zu Michaels Ausführungen lässt sich ein Gefälle betreffend Klarheit und Verständlichkeit feststellen. Die Studentin Tina scheint aus einer gesicherten oder gesicherteren Position heraus agieren zu können. Diese Position lässt sich als diejenige der Praktikerin mit normativen Sollensvorstellungen 
beschreiben. Dabei verwendet sie den Praktikerinnenjargon souverän. In ihrem Beitrag finden sich Allgemeinplätze, Lebensweisheiten und Common-Sense-Aussagen. Dies lässt sich beispielsweise an der Aussage Kinder haben ein Recht auf einen Grund aufzeigen, die im Modus eines Glaubenssatzes formuliert wird.

Tina greift aus der Vielfalt aus Fachvokabular und Themenbereichen, die ihr entgegengebracht werden, zuerst das Thema «Planung und Kontingenz» auf und äussert diesbezüglich ihre pädagogische Überzeugung. Anschliessend bringt sie weitere Themenfelder, ebenfalls elliptisch, ein, beispielsweise «Authentizität», "Legitimation», «Macht» und «Machtmissbrauch». Ein weiteres aufschlussreiches Datum zeigt sich in der Verwendung von authentisch, das auf eine vollzogene Einsozialisation hinweist. Mit ihren Ausführungen schliesst sie aus der Perspektive einer bereits einsozialisierten Praktikerin an und präsentiert diese in einer selbstbehauptenden Form. Ihre Ausführungen lassen sich eher nicht auf die Vermittlungserfolge aus dem Mentoratsgespräch zurückzuführen, sondern scheinen ihr auch ohne Zutun des Mentors möglich zu sein. Jedenfalls lässt sich empirisch kein Indiz dafür finden, dass sich ihre Äusserungen aus seinen Vermittlungen speisen. Auf einer latenten Ebene geht dies mit einer Dynamik einher, die dem Mentor Anerkennung eher entzieht als zuschreibt.

Michael: sehr gut

Tina: genau

Mit sehr gut werden die Ausführungen der Studentin evaluiert und als überdurchschnittlich quittiert. Diese Bewertung fungiert als Bestätigung der asymmetrischen Position des Mentors. Obwohl diese wie bereits dargelegt zu Beginn der Gesprächsinteraktion von Michael hergestellt wurde und durch die kooperative Art von Tina auch nicht unterlaufen wird, gewinnt durch die vorgenommene Evaluation sehr gut erneut eine Bekräftigung der Asymmetrie Gestalt, die auf Tinas souveräne, selbstsichere und klare(re) Selbstkundgabe reagiert. Michael wendet ihre Selbstbehauptung somit dahingehend, dass er diese als sein Verdienst betrachtet und aus der Asymmetrie des Die-Novizinlobenden-Experten befestigt. Erneut gewinnt Gestalt, dass die Position des Mentors nicht per se als installiert oder etabliert betrachtet werden kann und eine gewisse Prekarität 
aufweist, die innerhalb des Gespräches performativ und fortwährend bearbeitet werden muss.

Genau als Anschluss von Tina ist irritierend. Aus der Interaktionsabfolge betrachtet wird mit genau das sehr gut von Michael kommentiert. Möglicherweise bezieht sich genau jedoch nicht primär auf sehr gut, sondern ist im Sinne von "so sehe ich das», "so denke ich über Planung und Abweichung» oder «so habe ich das aufgenommen» zu verstehen. Als weitere Lesart wäre denkbar, dass Tina mit ihren Ausführungen im von Michael gewünschten Modus geantwortet hat, das heisst, dass sie das Wesentliche oder Entscheidende ausgeführt oder eben genau getroffen hat und dies in ihrem Anschluss latent durchscheint. In dieser Lesart würde Tina nicht mehr selbstbehauptend agieren, sondern die Position der Schülerin einnehmen. Des Weiteren teilt sie mit genau mit, dass ihre Ausführungen beendet seien, keine weiteren Ergänzungen folgen würden und das Thema ad acta gelegt werden könne oder solle. Da diese Gesprächssequenz zu einem inhaltlichen Abschluss kommt und anschliessend ein neues Thema verhandelt wird, kann nachfolgend eine riskante Fallstrukturhypothese formuliert werden.

\subsubsection{Zusammenfassung und riskante Fallstrukturhypothese der Sequenz Du sprichst von Struktur und Planung}

Die Interaktion zeigt in dieser Sequenz einen Mentoratsgesprächsausschnitt, in dem eine Asymmetriedisposition und eine Fürsorge- und Wertschätzungsdisposition gleichzeitig bearbeitet werden, was zu Spannungsverhältnissen und Unsicherheiten führt. Die Installation und die Aufrechterhaltung der Asymmetriedisposition auf der einen Seite erfordern sowohl eine Erfahrungs- als auch eine Expertisedifferenz. Diese Differenzen legitimieren den pädagogischen Anspruch, etwas zu zeigen und auch zu evaluieren. In der vorgefundenen Interaktion kann diese Disposition nicht bereits als vom Setting her installiert und gesichert erachtet werden. Sie wird deshalb vonseiten des Mentors performativ hergestellt und bearbeitet. Die entsprechende Dynamik bedient dabei die strukturelle Notwendigkeit der Anerkennung, Ingeltungsetzung und Bekräftigung der Expertise des Mentors. Auf der anderen Seite ist auch die mit dem Format verbundene Anforderung erkennbar, der Studentin vergemeinschaftend und fürsorglich zu begegnen. 
Die pädagogischen, evaluativen und vergemeinschaftenden Aspekte, die sich interaktionsdynamisch in Michaels Adressierung finden, werden von Tina in ihrer komplementären Rolle mitreproduziert. Dies geschieht, indem sie ihrer Adressierung zustimmt oder diese zumindest nicht infrage stellt, sondern entsprechend daran anschliesst. Die Studentin Tina zeigt sich kooperativ, souverän und als bereits einsozialisierte Berufsperson mit klaren pädagogischen Prämissen zu berufsethischen Themen, wie die Kinder kann man sowieso nicht planen, Kinder haben ein Recht auf einen Grund oder es ist wichtig die Kinder ernst zu nehmen. Diese an normativem Gedankengut orientierten Alltagskonzepte bringt sie selbstbewusst, selbstbehauptend, souverän und von sich aus ins Gespräch ein. Sie werden von Michael mit sehr gut ratifiziert, was den Eindruck erweckt, er habe sie Tina vermittelt.

Die grundsätzliche, dem Setting immanent anhaftende Asymmetriedisposition erfährt durch den selbstbehauptenden Auftritt der Studentin im Modus einer bereits einsozialisierten Lehrperson möglicherweise eine Bewegung in Richtung Kollegialitätsdisposition. Eine dadurch potenziell aufgeweichte oder aufgehobene Asymmetrie wird in der Interaktionsdynamik durch Michaels evaluativen Sprechakt jedoch umgehend wieder hergestellt. Des Weiteren findet in der Gesprächsinteraktion - in Analogie zur Metapher des Name-Droppings - ein beidseitiger Austausch von pädagogischen Schlagwörtern, Common-Sense Aussagen und normativen Überzeugungen statt, die den Anschein einer Versiertheit vermitteln. Trotz beidseitiger Dynamiken, die darauf ausgerichtet sind, die Fragilität des Settings zu bearbeiten, gestaltet sich die Anerkennbarkeit des Status des Mentors im Format prekärer als diejenige der Studentin. Dies führt zu Dynamiken, die als Legitimations- und Aufrechterhaltungsbemühungen gedeutet werden können und in einem relativ aufwendigen Spiel vorgenommen werden.

Dies lässt die folgende riskante Hypothese der zweiten Sequenz zu: Das Mentoratsgespräch gestaltet sich in der vorliegenden Gesprächsinteraktion als legitimierungsbedürftiges Setting. Die Legitimierungsbedürftigkeit mündet nicht in eine produktive Offenheit, sondern führt zu Unsicherheiten und Prekaritäten. Im Gegensatz zur Eröffnungssequenz spielt diese Dynamik nicht primär auf der Ebene von Diffusität und Spezifität, sondern auf der Ebene von Bemühungen um die Legitimation des Mentorenstatus als Experte. Die asymmetrische Disposition und damit einhergehend die Expertise- und Erfahrungsdifferenz können nicht bereits als stabil und aus der Struktur 
des Settings im Sinne einer Voraussetzung angenommen werden, sondern bedürfen einer interaktiven Herstellung und Bekräftigung. Infolgedessen reproduzieren sich die settingsspezifische strukturelle Prekarität und Fragilität der Mentorendisposition. Beide beteiligten Personen, im Speziellen jedoch der Mentor, betreiben einen relativ hohen Aufwand, um das Setting zu gewährleisten. Daraus lässt sich schliessen, dass es sich hierbei um ein soziales Format handelt, dessen Rahmung nicht strukturell vorausgesetzt werden kann, sondern sich performativ selbst gewährleisten muss.

In der Annäherung des Settings an schulischen Unterricht mit Bestandteilen wie Zeigen, Vermitteln und Evaluieren verschafft sich die strukturelle Unsicherheit Ausdruck, die sich darauf zurückführen lässt, dass es einem (vielleicht auch herangetragenen) Anspruch nachzukommen und die Asymmetrie zwischen der Novizin und dem Experten aufrechtzuerhalten gilt. Diese Positionierung, sich als Experte Geltung zu verschaffen und gleichzeitig vergemeinschaftende und fürsorgliche Ansprüche zu bedienen, zeigt sich in der performativen Ausführung unterschiedlich. Die Fragilität der Erfahrungs- und Expertisedifferenz kann als Strukturmerkmal des Formats betrachtet werden. Der fallspezifische Umgang mit dieser Fragilität und Prekarität, der in Teilen an die Logik einer schulischen Unterrichtssituation erinnert, lässt sich als ein möglicher Interaktionsmodus im Mentoratsgesprächssetting beschreiben.

\subsubsection{Dritte Sequenz: Was ist aber auch dann eigentlich der noch wichtigere} Schritt

In der nächsten Gesprächspassage ruft der Mentor Michael diverse Themen wie "Qualitätsmerkmale von Unterricht», «Lernatmosphäre», "offene Kommunikation», «Kinder mit ihren Anliegen ernst nehmen» und «Partizipation» auf. Daran anschliessend schildert die Studentin Tina eine Idee für die Kindergartenstufe, die sie bei ihrer Praxislehrperson beobachtet hat: eine sogenannte "Sprechregel», die zum Einsatz gelangt, wenn alle Kinder durcheinandersprechen. An dieser Stelle setzt die feinsequenzanalytische Rekonstruktion der dritten und letzten Sequenz im Gespräch zwischen Tina und Michael ein. 
Michael: und was ist dann aber wichtig für dich als Lehrperson

Tina: dass ich (.) das nochmals mit den Kindern bespreche und auch sage ok diese Regeln wählen wir aus oder diese Regel ist wirklich wichtig und diese ist vielleicht (.) nur für dich wichtig und und (.) geht aber nicht weil die anderen Kinder das nicht so machen können oder so also //einfach//(Michael: //genau//) (.) das nochmals wirklich mit den Kindern durchbesprechen (.) und dann vielleicht auch ein Plakat gestalten wo dann alle Kinder unterschreiben können (.) oder einfach dass es noch visuell (.) irgendwo im Klassenzimmer ist Michael: $\mathrm{mhm}$ und was ist aber auch dann eigentlich (.) der noch wichtigere Schritt als nur (.) das (.)Veranschaulichende und Unterschreibende? (.) was ist nachher so (.) die Essenz nachher für drei Klassen (.) Klassenregeln? Tina: $\mathrm{mhm}$ die Essenz ((lacht)) dass dass

Michael: die werden ausgehandelt stehen da (.) alle haben unterschrieben Tina: dass sie auch //durchgesetzt//(Michael: //genau//) werden also dass sie konsequent (.) sind oder

Michael: genau

Tina: genau also dass dass allen (.) die Regeln klar sind (.) dass dass sie alle verstanden haben was jetzt genau die Regeln sind (.) und dass sie dann auch durchgesetzt werden oder die Kinder daran erinnert werden Michael: genau das ist dann deine Aufgabe natürlich und auch die der Kinder die auch mit/ mithelfen immer Regeln einzuhalten konsequent oder auch dann (.) Sanktionen also was passiert bei bei Regelverstössen dass es den Kindern von Anfang an klar ist und auch für dich was sind so Ablauf/ Ablauf//schemen// Tina://genau// 


\subsubsection{Rekonstruktion der einzelnen Sequenzen}

\section{Michael: und was ist dann aber wichtig}

Mit diesem Satz schliesst Michael an Tinas Ausführungen an und fragt nach der Benennung von Wichtigem. Aus dem und zu Beginn seines Sprechaktes lässt sich schliessen, dass Michael Bezug auf die vorausgehende Aussage von Tina nimmt und mit dann aber eine neue Perspektive oder eine Präzisierung erfragt, da die vorausgehende Aussage eine solche erfordert oder zulässt. Kontextfrei wäre der Sprechakt beispielsweise im Rahmen einer Umfrage zum Reiseverhalten von Personen mittleren Alters vorstellbar, wenn die befragte Person zuerst mehrere Punkte aufzählt, die für sie bei der Suche nach einer geeigneten Unterkunft kein Auswahlkriterium darstellen, etwa: «Ratingpunkte im Internet interessieren mich nicht, es muss kein Fünfsternhotel sein, ich brauche kein Animationsprogramm am Abend und auch keine Minibar im Zimmer.» Hier könnte der Fragesteller einhaken und fragen: und was ist dann aber wichtig? Im Sprechakt, der einer Nachfrage gleichkommt, würde die Angesprochene dazu angehalten werden, ihre auf Ausschusskriterien bezogenen Ausführungen nicht fortzusetzen, sondern den Fokus neu oder anders zu legen. Strukturell nimmt der Sprecher eine Korrekturbewegung oder Hinführung vor. Die Frage enthält eine Aufforderung zu einer Ergänzung im Modus einer Priorisierung.

Kontextgebunden lässt das aber in der Frage des Mentors darauf schliessen, dass sich die Studentin bereits geäussert hat. In der hier vorgefundenen Satzstruktur hat das aber eine lenkende und korrigierende Wirkung, die vom bereits Erwähnten - vom Fragesteller möglicherweise als weniger wichtig erachtet - zum Wichtigen, jedoch noch nicht Erwähnten überleitet. Dabei präsentiert sich der Mentor als jemand, der eine solche Lenkung vornehmen kann, dem dies qua Rolle zusteht und gleichzeitig auch Interesse an der von Tina vorgenommenen Priorisierung bekundet.

Ebenfalls passend wäre der Sprechakt im Rahmen einer Prüfungssituation. Der Prüfungsexperte kennt die (gesuchte` und (richtige` Antwort und überprüft, ob die Prüfungskandidatin diese auch weiss. Möglich wäre, dass die Prüfungskandidatin bereits einige Punkte aufgezählt hat. Das und zu Beginn des Sprechaktes wie auch das aber implizieren 
eine gewisse Kritik oder Korrektur an der vorhergehenden Aussage, wodurch das Asymmetrische in der hier vorgefundenen sozialen Situation unterstrichen wird. Für die ausführliche Rekonstruktion wird der anschliessende Sprechakt hinzugenommen.

\section{Michael: für dich als Lehrperson}

Für die Herausarbeitung der dem Sprechakt zugrunde liegende Struktur wird dieser kontextungebunden betrachtet, wozu Lehrperson durch einen Platzhalter ersetzt wird. Mit dem Sprechakt für dich als «X» wird der Fokus der antizipierten Positionierung präzisiert und eine subjektive Perspektivübernahme in einer spezifischen Rolle erfragt. Dies geht mit einer Abgrenzung zu anderen Rollen einher. Wenn allerdings die Rollenspezifik im Fokus stünde, wäre eher eine Formulierung wie «was ist dann aber für eine $X$ (beispielsweise eine Architektin, eine Mutter, eine Lehrperson) wichtig» erwartbar. In die Formulierung «für dich als X» gewinnt die explizite Aufforderung Gestalt, als ganze Person in der Rolle sichtbar zu werden. Mit für dich wird somit die Involvierung der ganzen Person in dieser Rolle angesprochen. Dabei scheint die Identifikation mit der Rolle eine gewisse Brisanz aufzuweisen. Beispielsweise könnte sich der Sprechakt im folgenden Ausbildungssetting abspielen: Der Fluglehrer im Flugsimulator richtet die folgende Frage an die Flugschülerin: «Und was ist dann aber wichtig für dich als Co-Pilotin?» Die Rolle wird hier präzisiert, die Flugschülerin soll in dieser Rolle antworten. Gleichzeitig scheint klar, dass sie die Rolle noch nicht innehat und deshalb die Identifikation mit der Rolle gerade auch hervorgehoben wird. Die Frage und die erwartete Antwort zielen auf das Elizitieren oder die Überprüfung von Wissen ab. Durch die Positionierung des Fragestellers als Experte und durch die explizite Rollenzuschreibung für dich als $X$ in Kombination mit dann aber ist ein asymmetrisches Gefälle in der Adressierung grundsätzlich gegeben.

Kontrastiert man die realisierte Variante mit einer Version, die ohne dann aber auskommt, wäre der Sprechakt in einer symmetrischen Konstellation vorstellbar. Beispielsweise, wenn sich ein Architekt und eine Ingenieurin gemeinsam über ein Projekt beugen und der Architekt aus Interesse und vor dem Hintergrund einer gemeinsamen verfolgten Idee fragt: "Und was ist wichtig für dich als Ingenieurin?» Mit der expliziten Benennung der Rolle rückt der Architekt das Spezialgebiet der Ingenieurin in den Fokus und spricht sie gleichzeitig in der Diffusität ihrer ganzen Person an. Unter Zuzug des in den 
Daten vorgefundenen Zusatzes dann aber trägt dieses Verfolgen einer gemeinsamen Idee nicht mehr, da der Sprechakt eine starke Intentionalität und eine mäeutisch-sokratische ${ }^{34}$ Form enthält. Hierdurch wird eine Asymmetriedifferenz aufzeigt, da sich die sprechende und die angesprochene Person strukturell nicht auf Augenhöhe befinden. Durch dann aber wird die Frage zu einer Prüfungs- respektive Überprüfungsfrage, bei der die Antwort mit der bereits definierten Antwort des Fragestellers verglichen wird.

Mit dem realisierten Sprechakt und was ist dann aber wichtig für dich als Lehrperson wird Tina dazu aufgefordert, als ganze Person ihre künftige Rolle zu imaginieren und aus dieser heraus zu antworten. Denn unter Zuzug des Kontextes ist bekannt, dass sich Tina noch in der Ausbildung zur Lehrperson befindet und eine zum gegebenen Zeitpunkt noch hypothetische Perspektive im Interesse des Fragestellers liegt. Dieses hypothetische Element verschafft sich auch im dann aber Ausdruck. Zugleich wird jedoch nicht die künftige Lehrerin, sondern die künftige Lehrperson im Neutrum angesprochen, was einer leichten Distanzierungsbewegung gleichkommt und einer Verallgemeinerungsimplikation enthält. Mit dieser Aufforderung adressiert er Tina als Lehrpersonennovizin und positioniert sich selbst erneut als Experten dieser Rolle. Diese Adressierung und die Positionierung verfestigen nochmals die Asymmetriedisposition. Zudem weist der Sprechakt ein korrigierendes Moment auf, was sich strukturell vor allem im und wie auch im dann aber zeigt.

Tina: dass ich (.) das nochmals mit den Kindern ${ }^{35}$ bespreche

Nach der Signalisierung, eine Antwort auf die Frage zu haben (dass ich), zögert Tina einen Augenblick, bis sie diese ausführt. Anschliessend vervollständigt sie mit einem Nebensatz die von Michael anmoderierte Aussage. Mit ihren Ausführungen nimmt sie die ihr zugetragene Rolle der Lehrpersonennovizin respektive der Lernenden an und gesteht ihm entsprechend seinen Expertenstatus zu. Da die feinanalytische Interpretation an dieser

\footnotetext{
34 Die hier erkennbare mäeutisch- sokratische Form wird bei der Analyse des nächsten Sprechakts des Mentors ausführlich beschrieben. Hier sei vorerst lediglich auf die noch folgenden Ausführungen oder auf Oevermann (1996b, S. $156 \mathrm{ff}$.) verwiesen.

${ }^{35}$ Bemerkenswert in Tinas Formulierung ist, dass sie von Kindern, nicht von «Schülerinnen und Schülern», spricht und somit weg von der Rolle («Schülerinnen und Schüler») zum Status (Kinder) wechselt, und dies, obwohl sie von Michael in der Rolle der Lehrperson angesprochen wird. Die Verwendung des Terminus «Kinder» anstelle von "Schülerinnen und Schüler» lässt sich als Hinweis lesen, dass sie ihre Rolle noch nicht vollständig verinnerlicht hat und diese noch bruchhaft ist. Obwohl ich diese Differenz als aussagekräftig erachte, wird sie hier nicht weiterverfolgt, da sie für mich nicht erkenntnisleitend ist.
} 
Sequenzstelle keine neuen Erkenntnisse zur Eigenlogik des Gesprächs zwischen Tina und Michael liefert, wird lediglich summarisch darauf hingewiesen, dass Tina erneut mit alltäglichen Maximen wie etwas nochmals zu besprechen argumentiert, falls die erste oder die vorausgehende Besprechung noch nicht die gewünschte Wirkung gezeigt habe und deswegen eine Wiederholung angesagt sei.

Demgegenüber ist die Spezifik des Verhältnisses Novizin-Experte für die Interaktionsdynamik als relevant zu erachten. Zu deren Verdeutlichung wird im Sinne eines gedankenexperimentellen Kontrastes die realisierte Antwort mit einer Antwort wie «meine Erfahrungen haben gezeigt, dass ich, wenn ich jeweils mit den Schülerinnen und Schülern ...» verglichen. Diese Alternative beinhaltet eine Selbstbehauptungsgeste, das heisst, die der Frage innewohnende Asymmetrieimplikation wird selbstbehauptend und selbstanerkennend bearbeitet. In der realisierten Antwort findet keine entsprechende Bearbeitung statt, sondern die Studentin antwortet aus der Asymmetrie heraus, aus der sie auch angesprochen wird, und fügt sich somit der Positionierung als Lehrpersonennovizin.

Tina: und auch sage ok diese Regeln wählen wir aus oder diese Regel ist wirklich wichtig und diese ist vielleicht (.) nur für dich wichtig und und (.) geht aber nicht weil die anderen Kinder das nicht so machen können oder so also //einfach// (Michael: //genau//) (.) das nochmals wirklich mit den Kindern durchbesprechen (.) und dann vielleicht auch ein Plakat gestalten wo dann alle Kinder unterschreiben können (.) oder einfach dass es noch visuell (.) irgendwo im Klassenzimmer ist

Für die Rekonstruktion dieser und der noch folgenden Gesprächspassage wird zwischen Interaktionsebene und Projektionsebene unterschieden. Die Interaktionsebene betrifft die Gesprächsinteraktion zwischen der Studentin und dem Mentor, die Projektionsebene bezieht sich auf die antizipierte oder eben projizierte Ebene der künftigen Lehrerperson, auf der die Studentin, als ob sie bereits Lehrerin wäre, adressiert wird. Im gesamten Sprechakt präsentiert Tina auf der Projektionsebene den Handlungsplan einer Lehrperson, der in der vorgefundenen Form einen gewissen Generalisierungsanspruch zu haben scheint. Die Schilderungen des Regelfindungsprozesses werden nachfolgend nicht feinsequenzanalytisch, sondern kursorisch rekonstruiert, da sie in erster Linie eine 
Unterrichtshandlung auf der Projektionsebene, nicht aber die Interaktionsebene im Gespräch mit Michael betreffen. Dennoch sei auf die nicht vorhandene Stringenz in ihren Ausführungen hingewiesen, denn der Regelfindungsprozess wird nur vermeintlich partizipativ und demokratisch gestaltet und lässt den Führungsanspruch der pädagogischen Situation durchscheinen.

Bei der Erstellung von Klassenregeln als Disziplinierungsmassnahme positioniert sich Tina als handlungsfähige Lehrperson, stolpert aber sprachlich in ihren Ausführungen an mehreren Stellen, was sich unter anderem in den Sprechpausen zeigt. Interaktionslogisch aufschlussreich ist das von Michael mehrfach verwendete genau. Genau scheint ein ¿Gewohnheitswort) von Michael zu sein. Um die dynamische Implikation dieses ¿Gewohnheitsworts〉 herauszuarbeiten, lässt sich dieses im Sinne eines Gedankenexperimentes mit der ebenso denkbaren Variante von «mhm» kontrastieren. Während «mhm» die Positionierung der anderen Person anerkennt und diese Anerkennung zum Ausdruck bringt, nimmt genau im Austauschprozess eine orientierende und validierende Funktion in einer wertschätzenden Gesprächsführung ein. Dies impliziert auch eine vorhandene Norm, an der Tinas Äusserungen gemessen werden. Tina scheint mit ihren Ausführungen die Erwartungen zu erfüllen und erhält diesbezüglich eine Validierung. Mit der Validierung lassen sich zugleich die Rolle des Validierers und die darin enthaltene asymmetrische Position bekräftigen.

In den weiteren Ausführungen greift Tina die Möglichkeit der visuellen Gestaltung eines Regelplakates auf. ${ }^{36}$ Darin wird die Übernahme einer normativen Ordnung der didaktischen Programmatik auf der Ebene einer Handlungsanleitung sichtbar, die der Studentin möglicherweise im Praxisfeld begegnet ist und die sie hier aufzeigt. Mit Blick auf Strukturmerkmale gleicht die Sequenz einer Überprüfung, die darin besteht, herauszufinden, welcher pädagogischen Überzeugung Tina folgt und welche diesbezüglichen

\footnotetext{
${ }^{36}$ Ohne inhaltlich vertiefte Thematisierung dieser Visualisierung sei nur am Rande erwähnt, dass dieser erzieherische Akt eine prekäre Komponente enthält. Schulregeln wie auch Regeln allgemein gelten auch ohne Unterschrift; eine solche bestätigt lediglich die Kenntnisnahme der Regeln. Im konkreten Fall werden Regeln zum Bestandteil eines Vertrags, der gemeinsam und formschön gestaltet und anschliessend von allen unterschrieben wird. Insofern wird hier ein Vertragsabschluss zwischen Lehrperson und Lernenden simuliert. Kontextungebunden beruht ein Vertrag auf der Freiwilligkeit aller beteiligten Parteien. In der vorliegenden Situation ist die Unterschrift jedoch nicht verhandelbar. Mit der Signatur wird ein Beteiligungsverfahren angedacht, das durch den Pflichtcharakter nicht eingelöst und so zu einer Scheinbeteiligung wird. Gerade die visuelle Ausarbeitung und der förmliche Akt der Unterschrift fungieren hier, zugespitzt formuliert, als (Köder), da sie die Wichtigkeit jeder und jedes Einzelnen betonen, was aber nur durch die Freiwilligkeit der Unterschrift eingelöst werden könnte.
} 
Normen sie aufrufen kann. Die darin enthaltene unausgesprochene Frage lautet «Was ist eine gute oder die richtige Handlung einer Lehrperson in der Situation X?» Tina stellt sich der Herausforderung kooperativ und führt in ihrer Antwort ihre Handlungsmaxime aus. Das parallel gesprochenen genau von Michael fungiert dabei als Bekräftigung der Asymmetrie und gleichzeitig auch als Bestärkung, da ihre Ausführungen mit seinen eigenen pädagogischen Überzeugungen kompatibel zu sein scheinen. Dies kommt einem Phänomen gleich, mit dem Studierende im Praxisfeld häufig konfrontiert werden, wenn sie in Unterrichtsnachbesprechungen Lob und Anerkennung für Handlungen oder Überzeugungen erhalten, die mit denjenigen der Praxislehrperson übereinstimmen. In diesem anerkennenden Modus in Bezug auf geteilte Gesinnung und pädagogische Ansichten erfährt die Studentin hier vom Mentor mit genau eine Bestätigung.

In einer manifesten Bedeutungsschicht wird Tina an dieser Gesprächsstelle auf der Projektionsebene einer bereits ausgebildeten und sich im Beruf befindenden Lehrperson angesprochen. Auf dieser Ebene weist die Gesprächsdynamik etwas Kollegiales und Heterarchisches auf. Des Weiteren enthält die Gesprächsinteraktion an dieser Stelle eine supervisorische Implikation. Jenseits der manifest vorgenommenen Adressierung konturiert sich auf der latenten Ebene jedoch eine andere Beziehungskonstellation: Der Mentor agiert im Modus eines Experten oder Lehrers, der Fragen stellt, deren Antworten er kennt und die hier überprüft werden. In dieser asymmetrischen Konstellation sind auch Elemente der Fürsorge und wohlwollender Zuneigung zu erkennen. Erneut erinnert diese soziale Situation an ein Lehrpersonen-Lernenden- oder Weiterbildungssetting. Die entsprechende Zuschreibung des Schülerinnenstatus findet auch auf Tinas Seite Resonanz: Mit ihrem Redebeitrag antwortet sie auf der manifesten Ebene zwar als bereits etablierte und einsozialisierte Lehrperson und gibt Einblick in ihre Handlungsmaxime, in der latenten Bedeutungsschicht nimmt sie die ihr zugewiesene Position der Schülerin jedoch an.

Michael: mhm und was ist aber auch dann eigentlich (.) der noch wichtigere Schritt als nur (.) das (.)Veranschaulichende und Unterschreibende? (.) was ist nachher so (.) die Essenz nachher für drei Klassen (.) Klassenregeln? 
Michael fragt nach einer Ergänzung, nach dem noch wichtigeren Schritt. Tina scheint, trotz des validierenden genau, noch nicht die vollständige gesuchte Antwort geliefert zu haben und wird deshalb lenkend auf die von ihm gewünschte Antwort hingeführt. Die Hinführung erinnert auf den ersten Blick an die mäeutisch-sokratische Gesprächsführung. Dieses didaktisch-methodische Vorgehen ist auf Sokrates zurückzuführen. Durch produktive Verunsicherung wird in Form von Fragen zu Erkenntnissen verholfen, die unbewusst bereits vorhanden sind. Die mäeutisch-sokratische Fragetechnik als «didaktischer Königsweg» folgt in ihren Grundsätzen und Ursprüngen der Idee einer diskursiven, gemeinsamen Erörterung von Fragen. Wie Wenzl ausführlich herausarbeitet, zeigt eine genaue Betrachtung jedoch, dass dem mäeutisch-sokratischen Fragen ein diskursives Element völlig fremd ist und diese Fragetechnik «sogar in einem besonderen Masse kommunikativ auf eine Unterwerfung angewiesen ist» (Wenzl, 2014, S. 138), da sich die mäeutisch-sokratisch Befragten im Vertrauen auf die Überlegenheit der fragestellenden Person darauf beschränken, auf deren Fragen zu antworten, um dadurch zu einer Erkenntnis hingeführt zu werden.

Auch für die vorgefundene Gesprächsinteraktion lässt sich - pointiert formuliert festhalten, dass eine Figur Gestalt gewinnt, die zwar einen mäeutisch-sokratischen Charakter aufzeigt, jedoch vor allem doktrinale Elemente enthält. ${ }^{37}$ Somit scheint eine korrekte Antwort zu existieren, die auf diese Weise gesucht wird. Wenn im weiteren Verlauf dieser Untersuchung von «mäeutisch-sokratischer Fragetechnik» oder «mäeutisch-sokratisch» gesprochen wird, ist damit genau diese Dynamik gemeint, in der manifest diskursiv und im Dialog zwischen der fragestellenden und befragten Person eine Thematik erörtert wird, die Geltungshoheit allerdings latent bei der fragestellenden Person liegt und die beiden Gesprächsparteien keine ebenbürtige Position einnehmen. Eine mäeutisch-sokratische Frage unterscheidet sich insofern und lediglich dadurch von einer Prüfungsfrage, als Erstere den Anspruch erhebt, zu einer Erkenntnis hinzuführen, während Letztere eine Erkenntnis voraussetzt und diese mit der Frage überprüft wird (Wenzl, 2014, S. 137). Diese Vorgehensweise ist gerade im Unterrichtskontext häufig anzutreffen und wird in ironischer Weise als «Osterhasenpädagogik» bezeichnet, mittels

\footnotetext{
${ }^{37}$ Wie genau sich Doktrinalität zur mäeutisch-sokratischen Fragetechnik verhält, lässt sich in Pranges Auseinandersetzung mit dem Menon-Dialog nachlesen (Prange, 1986, S. 30 f.).
} 
der die Lernenden dazu angehalten werden, das (verstecktes Wissen der Lehrperson in Analogie zum iversteckten Osterei) zu suchen (Wahl, 2013).

In der Gesprächspassage, in der das Gespräch zwischen der Studentin Tina und dem Mentor Michael über die Thematik der Installation und der Visualisierung von Klassenregeln in seiner latenten Bedeutungsschicht einen ‘osterhasenpädagogischen` Anstrich erhält, wird der noch wichtigere Schritt durch die Verwendung von eigentlich wieder relativiert. Die bereits getätigte Aussage Tinas scheint inhaltlich nicht falsch, jedoch noch nicht ausreichend zu sein. Im Prüfungsmodus würde Tina lediglich Teilpunkte erhalten, weshalb zwei weitere Anschlussfragen eingebracht werden - die Frage nach dem noch wichtigeren und die Frage nach der Essenz. Dabei werden die von der Studentin aufgeführten Punkte nochmals aufgegriffen und als das Veranschaulichende und Unterschreibende bezeichnet. Durch die Substantivierung der Verben wird der Prozess der Veranschaulichung und des Unterschreibens und nicht das Produkt betont. Kontextungebunden betrachtet verschiebt sich die Wortbedeutung durch die Substantivierung von einer konkreten auf eine abstraktere oder auch von einer individuellen auf eine allgemeinere Ebene. Dieses Stilmittel findet oftmals Verwendung um einer Formulierung mehr Objektivität und eine allgemeine Gültigkeit zu verleihen. Die Annahme, dass sich auch diese Implikation in die Daten hineinschreibt, um die Positionierung im Gestus des Experten und die Asymmetrie der sozialen Situation aufrechtzuerhalten und zu sichern, ist hierbei plausibel. Zu diesem Zweck beharrt der Mentor hier auf Präzision und Vollständigkeit. Das Veranschaulichende und Unterschreibende scheint aus der Sicht des Sprechers nicht das 'wirklich Wichtiges zu sein; dieses wurde noch nicht genannt.

Inhaltlich ist die Sequenzstelle nicht vollständig auflösbar. Es bleibt beispielsweise unklar, weshalb Michael von drei Klassenregeln spricht, oder auch, ob er bereits bei Klassen (.) nach dem Wort Klassenregeln sucht und dieses erst im zweiten Anlauf findet. Strukturell und interaktionslogisch lässt sich hingegen festhalten, dass ein mäeutisch-sokratisches oder wie zuvor ausgeführt ‘osterhasenpädagogisches` Hervorlocken von (gesichertem〉 Wissen stattfindet, das in dieser Form ebenfalls an schulischen Unterricht erinnert. Dadurch steigt für Tina die Bewährungshürde, die darin besteht, die von Michael erwartete, gesuchte Antwort zu geben und die Essenz zu erkennen.

Zusammenfassend lässt sich festhalten, dass die eingangs formulierte Frage und was ist dann aber wichtig für dich als Lehrperson, die manifest auf eine subjektive Relevanz- 
setzung ausgerichtet ist, an dieser Stelle eine Modifikation zu einer Wissensabfrage respektive Prüfungsfrage erfährt.

Tina: $m h m$ die Essenz ((lacht)) dass dass/

Der von Tina gewählte Anschluss enthält mehrere Hinweise auf ihre Unsicherheit bezogen auf die von Michael formulierte Frage. Sie beginnt mit $m h m$, möglicherweise um Zeit zu gewinnen, wiederholt anschliessend das Schlüsselwort, die Essenz, das in der Metapher der Osterhasenpädagogik für das gesuchte Ei steht. Das Lachen lässt sich in der weniger selbstsicheren Version als Verlegenheitslachen, in der selbstsicheren Version als überspielendes Lachen deuten. Beiden Auslegungen gemein ist, dass über das Lachen die Situationsunsicherheit und die Aufforderung zur Stellungnahme bearbeitet werden. Michaels Prüfungsfrage kann als für Tina voraussetzungsvoll betrachtet werden, was sich auch in der Wiederholung von dass und im darauffolgenden Satzabbruch zeigt.

Michael: die werden ausgehandelt stehen da (.) alle haben unterschrieben

Michael bietet Tina Unterstützung an. Er bearbeitet die Situationsunsicherheit, indem er ihren Anschluss als Aufforderung zu einer Hilfestellung rahmt und eine Rekapitulation vornimmt. Im Sinne eines Scaffoldings (Stender, 2016) benennt er den Prozess, der Tina zur gesuchten Essenz führen soll: das Aushandeln und Unterschreiben der Regeln. Der von Michael modellierte Sprechakt kann zugespitzt mit «vervollständige den Satz: Die werden ausgehandelt stehen da alle haben unterschrieben ...» wiedergegeben werden. Tina erhält die Aufgabe, seinen Gedanken zu vollenden. Dieser Auftrag wird jedoch nicht ausgesprochen, sondern muss von Tina implizit als solcher erkannt werden. Michael positioniert sich als Unterstützer, der erkennt, welche Hilfestellung Tina benötigt, um die ihr gestellte Aufgabe zu bewältigen. Diese zeigende, pädagogische Geste kann als Hinweis für eine Bemühung um eine Legitimierung seines unsicheren Status gelesen werden.

Tina: dass sie auch //durchgesetzt//(Michael: //genau//) werden also dass sie konsequent (.) sind oder

Michael: genau 
Wie eine chronologische Abfolge werden die einzelnen Handlungsschritte zur Etablierung dieser Klassenregeln im Gesprächsabschnitt auf der Projektionsebene thematisiert: zuerst gilt es, die Regeln zu besprechen. Danach muss man sie niederschreiben, anschliessend unterschreiben und, als letztes Traktandum in der Chronologie, durchsetzen.

Präzise gesprochen können Regeln selbst nicht konsequent sein, sondern nur konsequent umgesetzt werden. In Tinas Formulierung erhalten die Regeln allerdings eine eigene Autorität und agieren als (Selbstläufer) wie eine zweite Lehrperson. Gleich zweimal umgeht Tina es, sich selbst als Akteurin dieser Umsetzung zu nennen: In der ersten Teilaussage verwendet sie eine Passivkonstruktion und im zweiten Teil macht sie die Regeln selbst zu Akteuren, die konsequent zu sein haben. Auf einer manifesten Ebene lässt sich Tinas Sprechakt als performatives Aufzeigen lesen, mit dessen Hilfe sie zum Ausdruck bringt, dass sie die Normen und Erwartungen der Lehrpersonenrolle kennt. Zugleich konturiert sich auf einer latenten Ebene eine Distanzierungsbewegung. Diese Distanzierung indiziert auch Schwierigkeiten und Widerstände hinsichtlich der Rollenübernahme oder der Erfüllung der Erwartungsanforderungen. Dies lässt sich als weiterer Hinweis für eine Lehrperson-Lernenden-Konstellation lesen, da solche Dynamiken in dieser sozialen Situation oftmals vorzufinden sind. Auf den Kontext des Mentoratsgesprächs bezogen scheint hier, wie auch bei der Ausgestaltung des Plakates oder bei anderen Gesprächspassagen, durch, dass die Adaption von pädagogisch-normativen Orientierungen und Betrachtungsweisen strategisch zielführend ist, auf einer latenten Ebene aber dennoch nicht friktionsfrei übernommen werden kann.

Wiederholt und gleich doppelt wird Tinas Aussage von Michael mit dem «Gewohnheitswort` genau validiert, die gestellte Aufgabe gilt somit als erwartungsgemäss und korrekt gelöst.

Tina: genau also dass dass allen (.) die Regeln klar sind (.) dass dass sie alle verstanden haben was jetzt genau die Regeln sind (.) und dass sie dann auch durchgesetzt werden oder die Kinder daran erinnert werden

Tina steigt mit Michaels gewohnter Form der Validierung, mit genau, in die Fortsetzung ihrer Ausführung ein. Sie scheint nicht nur eine inhaltliche Annäherung an seine Haltung 
vorzunehmen, sondern auch performativ seine Sprachgewohnheiten zu übernehmen. Ihre Wiederholung des bereits Elaborierten lässt sich als Indiz ihrer Erleichterung lesen, die gewünschte Antwort respektive die Essenz gefunden zu haben. Die sprachliche Formulierung weist erneut auf eine Distanzierungsbewegung zur Rolle der ausführenden Person dieses Regeldurchsetzungsvorgangs hin. Dies verdeutlicht sich durch die konsequente und in dieser Konsequenz auffällige Anwendung des Passivs. Fragt man sich, was die Differenz ausmacht, ob Regeln durchgesetzt werden oder die Kinder daran erinnert werden, scheint eine Durchsetzung auch gegen den Willen der Kinder zu erfolgen, während die zweite Option sanfter und weniger autoritär ist. Bei Letzterer genügt die Erinnerung daran, damit die Kinder die Regeln wieder freiwillig befolgen. Tina lässt beide Strategien nebeneinander bestehen. Dies lässt sich deuten, als wolle sie aufzeigen, dass sie die womöglich von ihr erwartete Durchsetzungsfähigkeit anerkennt, ohne sich vollständig damit zu identifizieren. Erneut reproduziert sich das in der bisherigen Interpretation gewonnene Strukturmerkmal der Prekarität zwischen der (antizipierten) Erwartung, Normen zu entsprechen, und der Etablierung der eigenen Überzeugungen.

Aus einer professionalisierungstheoretischen ${ }^{38}$ Perspektive betrachtet und unter Beizug des realen Kontextes einer Ausbildungssituation gilt es diesbezüglich festzuhalten, dass die Studentin aufgrund ihres Ausbildungsstatus überwiegend über Theoriewissen verfügt. Ihr Fallwissen beruht auf Erfahrungen aus Praktika und auf diesen aufbauend auf Imagination. Die Generierung von eigenem und eigentlichem Handlungswissen ist deshalb nur beschränkt möglich. Das an dieser Stelle mit viel Eifer demonstrierte Handlungswissen scheint eher das Handlungswissen des Mentors zu sein, das er ihr mäeutisch-sokratisch entlockt und das die Studentin im Modus der Anforderungserfüllung präsentiert. Diese Dynamik - die Übernahme und die Präsentation von

\footnotetext{
${ }^{38}$ An dieser Stelle scheint das Einbringen von Oevermanns Betrachtungsweise einer professionalisierungstheoretischen Perspektive von Wissensformen, im Modus eines sehr verkürzten und knappen Exkurses, lohnenswert: In seiner revidierten Professionalisierungstheorie nimmt Oevermann (z.B. 2009, S. 114 ff.) Bezug auf die klassischen Theorien der Berufssoziologie und entwickelt einen Ansatz einer Theorie professionalisierten Handelns, die durch die Dialektik der beiden Komponenten "Theorie» und "Praxis» bestimmt ist. Die erste Komponente (Theorie) zeichnet sich durch wissenschaftlich-methodische Kompetenz (Theorieverstehen/Fachwissen) und rekonstruktiv-diagnostische Kompetenz (Fallverstehen/Fallwissen) aus. Die professionelle Expertin oder der professionelle Experte muss diese erste Komponente durch eine zweite, die interventionspraktische Kompetenz erweitern können, um professionell handlungsfähig zu sein und allgemeingültige Wissensbestände in krisenbewältigendes Handlungswissen zu transformieren. Professionelle Handlungskompetenz bezieht sich folglich auf jenes Vermögen von Professionellen, der Dialektik von Theorie und Praxis zu genügen oder - wie Oevermann sagt - ingenieuriales Wissen fallangemessen in interventionspraktisches Wissen übersetzen zu können (Garz \& Raven, 2015, S. 117 f.).
} 
Handlungswissen, als ob es ihr eigenes sei - vollzieht sich nicht friktionsfrei. Dies scheint beispielsweise in der Distanzierungsfigur - und dass sie dann auch durchgesetzt werden latent durch.

Michael: genau das ist dann deine Aufgabe natürlich und auch die der Kinder die auch mit/ mithelfen immer Regeln einzuhalten konsequent oder auch dann (.) Sanktionen also was passiert bei bei Regelverstössen dass es den Kindern von Anfang an klar ist und auch für dich was sind so Ablauf/ Ablauf//schemen// Tina: //genau//

Michael reagiert umgehend auf Tinas Distanzierungsbewegung und zeigt auf, dass die Aufgaben des Durchsetzens und Erinnerns ihr als Rollenträgerin zugeschrieben werden. Mit der Verwendung von natürlich in der Bedeutung von «selbstverständlich» oder «das weiss man ja» verleiht Michael der Übernahme der Aufgabe Nachdruck. Interaktionsdynamisch befestigt er erneut seinen Status als Experte und weist ihr den Novizinnenstatus zu. Manifest lässt sich ihr anschliessendes genau als Bestätigungssprechakt lesen, in der Latenz allerdings gewinnt im genau eine Selbstbehauptungsgeste statt, in der sie die Asymmetrie negiert und aus der Symmetrie heraus eine Bestätigung oder Evaluation vornimmt, wie er dies jeweils ihr gegenüber getan hat. Bereits zuvor waren seitens der Studentin Selbstbehauptungsdynamiken erkennbar, aber auch gegenteilige Bewegungen, in denen sie sich gerade nicht selbst behauptet, sondern die ihr zugetragene Position der Schülerin annimmt und aus dieser heraus reagiert. Vor diesem Hintergrund lässt sich nachfolgend eine Zusammenfassung vornehmen und eine Fallstrukturhypothese für die dritte Sequenz formulieren.

\subsubsection{Zusammenfassung und riskante Fallstrukturhypothese der Sequenz Was ist} aber auch dann eigentlich der noch wichtigere Schritt

Anhand der Thematik «Herstellung von Disziplin mittels Installation von Regeln» und im Modus des Abfragens ermittelt der Mentor Normen, Prinzipien, Regulative und Doktrinen, die für ihn selbst als relevant und handlungsleitend vermutet werden können. Von der Strukturlogik her operiert Michael mit einer mäeutisch-sokratisch Fragetechnik 
(Oevermann, 1996b, S. 156 ff.), die sich durch den doktrinalen Anstrich als «Osterhasenpädagogik» beschreiben lässt und oftmals in schulischen Kontexten Anwendung findet. Grundsätzlich betrachtet kann diese mäeutisch-sokratische Fragetechnik als sozial unproblematischer, kalkulier- und planbarer Modus betrachtet werden, da über die Richtig-falsch-Logik der Bewertung des in den Antworten zum Ausdruck kommenden Wissens potenzielle Riskanz und Kontingenz handhabbar und eingrenzbar gemacht werden. In der hier vorgefundenen doktrinalen Anwendung wird jedoch nicht gesichertes, deklaratives Fachwissen, sondern Fallwissen (die Herstellung von Regeln entspricht hier dem Fall) im Modus einer Schliessfigur verhandelt. Durch das Prozedieren dieses FrageAntwort-Spiels wird die Asymmetriedisposition und -inanspruchnahme beidseitig, das heisst sowohl von der Studentin als auch vom Mentor bekräftigt und gesichert, da dieses Vorgehen von der Studentin mitgetragen wird. Dies obwohl Tina in der Rolle der Lehrperson und dem Imperativ, sich als solche zu zeigen, aufgefordert wird. Denn das mäeutisch-sokratische Hervorlocken kommt auf einer latenten Ebene einer typischen Lehrer-Schülerin-Konstellation nahe. Die asymmetrische Interaktionsstruktur wird in dieser Sequenzstelle somit nicht verlassen und kann als ein Strukturmerkmal der Sequenz betrachtet werden. Die in der Eröffnungssequenz noch festgestellten Diffusitätspotenziale werden im Verlauf des Gespräches somit zunehmend eingegrenzt, obwohl sie an gewissen Stellen, beispielsweise in der sowohl diffusen als auch spezifischen Adressierung für dich als Lehrperson, durchscheinen.

Insgesamt zeigt sich in dieser dritten Sequenz für Tina ein Bewährungsdruck, der darin besteht, dass sie die Überzeugungen und Normen des Mentors zu erkennen und diesen zu entsprechen hat. Dies steht im Gegensatz zur eingangs der Sequenz formulierten Frage, was für sie (persönlich) als Lehrperson wichtig sei. Dieser Modus der Ansprache wird nicht aufrechterhalten, was sich bereits mit dem ersten evaluativen genau zeigt, da genau keinen adäquater Anschluss an die Beantwortung der Frage nach persönlichen Präferenzen und Prioritäten darstellt.

Die Art und Weise, mit der die Studentin zu den gesuchten Antworten hingeführt wird, enthält klar lenkende Elemente, die allerdings fürsorglich, wohlwollend und anerkennend vorgetragen werden. Die für die Struktur der Dyade zwischen Mentor und Studentin aus programmatischer Sicht vermutbare Erfahrungs- und Expertiseasymmetrie wird wiederholt interaktiv befestigt. Es scheint plausibel, diese interaktive Befestigung als Not- 
wendigkeit zu interpretieren, da das Setting als solches dies nicht bereits gewährleistet. Im Vergleich dazu wäre die Asymmetrie in einem pädagogischen Verhältnis, wie dies beispielsweise in der prototypischen Dyade Lehrperson-Lernende der Fall ist, offensichtlich und deutlich erkennbar. Die im Mentoratsgespräch etablierte, settingsspezifische Rahmung gestaltet sich diesbezüglich prekär und unsicher. Diese strukturelle Unsicherheit führt in der fallspezifischen Bearbeitung zu Legitimationsdynamiken, die sich in ihrer Eigenlogik an die Anderslogik einer Interaktion zwischen Lehrenden und Lernenden annähern. Der Lehrer ist einerseits Experte für gesichertes Wissen, das er seiner Schülerin im Modus des Zeigens vermittelt. Andererseits ist der Lehrer nebst der Wissensvermittlung auch für den Lernprozess der Schülerin, wie auch für ihr Wohl verantwortlich. Eine entsprechende Figur - Wissensvermittlung, Verantwortung für den Lernprozess und Fürsorge - ist fallspezifisch auch in der vorgefundenen Interaktion erkennbar.

Aus diesen Überlegungen heraus lässt sich folgende Fortschreibung der Fallstrukturhypothese für die dritte rekonstruierte Sequenz bilden: Im Mentoratsgespräch zwischen Tina und Michael zeigt sich eine Gleichzeitigkeit aus einer Asymmetriedisposition und Fürsorgeansprüchen. Dies führt beidseitig zu Unsicherheiten und Spannungen. Spannungsvoll gestaltet sich hierbei vor allem die Differenz zwischen der manifesten und der latenten Adressierung der Studentin. Manifest wird die Studentin in einem Als-obModus adressiert, das heisst so, als ob sie bereits in den Lehrerberuf einsozialisiert worden sei und hier ihr Erfahrungs- und Handlungswissen und ihren Lehrerinnenhabitus präsentiere. Latent allerdings zeigt sich gerade in dieser Adressierung eine Lehr-LernStruktur, in welcher der Mentor die Studentin zur Lernenden, zur Schülerin oder zur Novizin macht und sich selbst entsprechend als Lehrer oder Experte positioniert und ihr aus dieser Positionierung heraus und im Modus des mäeutisch-sokratischen Fragens sein Handlungswissen «überstülpt». 


\subsubsection{Fallzusammenfassung und finale Fallbestimmung: Tina \& Michael}

Das Mentoratsgespräch zwischen Tina und Michael folgt einer formalisierenden und formalisierten Etablierung der Gesprächssituation. Das Gespräch wird technisch aufgezogen und erinnert zu Gesprächsbeginn an die Logik eines Briefings oder einer Instruktion. Dabei beansprucht der Mentor eine klare Situationsdefinition. Dennoch gestaltet sich die Adressierung der Studentin spannungsvoll, einerseits bestimmt und klar vorstrukturiert, andererseits mit einer fürsorglich-anleitenden Ansprache. Folglich vermischen sich Strukturmerkmale, die sich einer pädagogisch-zeigenden Konstellation annähern, mit Strukturmerkmalen, die Formalisierungsbewegungen beinhalten. Durch solche formalisierenden und formalisierten Bewegungen lassen sich im Format «Mentoratsgespräche» potenziell angelegte diffuse Dispositionen - beispielsweise aufgrund der normativen Erwartungen einer dyadischen Situation - kontrollieren und eingrenzen.

Auch im weiteren Gesprächsverlauf ist eine Gleichzeitigkeit von Asymmetrie- und Fürsorgedispositionen zu erkennen. Diese führt zu Spannungsverhältnissen und Unsicherheiten. Diese Spannungen resultieren daraus, dass sich einerseits Zeige- und Vermittlungsansprüche aus der Asymmetrie- und Expertisedifferenz legitimieren. Andererseits sind auch Ansprüchlichkeiten und Anforderungen erkennbar, die Studentin weiterhin vergemeinschaftend und fürsorglich zu adressieren.

Die Studentin ihrerseits positioniert sich als bereits einsozialisierte Lehrperson, die klare pädagogische Vorstellungen aufweist und Alltagskonzepte zu Unterricht kennt. Der souveräne und selbstbehauptende Auftritt der Studentin kann als Gefahr für die vom Mentor etablierte Asymmetrie- und Expertisedisposition betrachtet werden. Der Mentor bearbeitet ihren Auftritt mit evaluativen Sprechakten und stellt die Asymmetrie umgehend wieder her. Dennoch gestaltet sich insgesamt der Status des Mentors fragiler als derjenige der Studentin. Dies gewinnt in seinen Legitimations- und Aufrechterhaltungsbemühungen Gestalt.

Die Gesprächsinteraktion folgt mehrheitlich der Strukturlogik der mäeutisch-sokratisch Fragetechnik. In dieser Logik ermittelt und erfragt der Mentor handlungsleitende Normen und pädagogische Prinzipien. Die asymmetrische Interaktionsstruktur lässt sich in diesem Modus mühelos aufrechterhalten, ermöglicht eine klare Lenkung und Handhabbar- 
machung des Gesprächs und festigt die installierte und performativ hergestellte Asymmetrie- und Expertisedifferenz.

Die Fallstrukturhypothesen der drei rekonstruierten Sequenzen lassen sich in der folgenden Gesamtstruktur der Interaktionsdynamik im Gespräch zwischen der Studentin und dem Mentor verdichten:

Mit Blick auf die fallspezifische Bearbeitung des Formats «Mentoratsgespräche» zeigt sich eine Variante, die auf eine strukturelle Schwierigkeit in der Erfahrungs- und ExpertiseAsymmetrie hinweist. Diese asymmetrische Konstellation wird während des Gespräches wiederholt interaktiv hergestellt und befestigt. Die strukturelle Unsicherheit oder Schwierigkeit führt in der fallspezifischen Bearbeitung zu Legitimationsdynamiken, die der Formsprache nach an die Anderslogik schulischen Unterrichts respektive an die soziale Situation zwischen Lehrenden und Lernenden erinnern. Allerdings zeigt sich eine Schwierigkeit, da die im schulischen Kontext gegebene Legitimationsbasis nicht beansprucht werden kann.

Die bisherigen Befunde machen darauf aufmerksam, dass in Mentoratsinteraktionen pädagogische respektive zeigende Dynamiken Gestalt gewinnen und in Suchbewegungen zur Herstellung eines an Schule oder Unterricht angenäherten Arbeitsbündnisses zwischen der Studentin und dem Mentor münden. Diese Zeigefigur wird bereits in der Eingangssequenz mit ich zeige dir aufgegleist und enthält eine doppelte Zeigebewegung: der angesprochenen Person etwas zeigen und zugleich die Absicht oder gar die Notwendigkeit, sich selbst zu zeigen. 


\subsection{Frau Steffen \& Herr Bucher}

Der zweite Kernfall ist das Gespräch zwischen der Studentin Frau Steffen und dem Mentor Herr Bucher. Es findet an einem Vormittag im Frühlingssemester 2017 in einem Seminarraum an der PH FHNW statt und dauert insgesamt eine halbe Stunde. Die Studentin Frau Steffen befindet sich zu diesem Zeitpunkt im zweiten von sechs Semestern. Im Gegensatz zu den anderen drei Gesprächen siezen sich die Studentin und der Mentor.

\subsubsection{Eröffnungssequenz: Ich begrüsse Sie recht herzlich}

Herr Bucher: ich begrüsse Sie recht herzlich zum Mentoratsgespräch es dauert ungefähr (.) eine halbe Stunde wir würden eben durch drei Bereiche gehen das Erste wäre so eine allgemeine Einschätzung Stand der Dinge ((Bucher räuspert sich)) zu verschiedenen Fragen rund ums Mentorat das Zweite wäre die Planungs/ der Planungshorizont für den zweiten Studienabschnitt und das Dritte die Einschätzung des ausgebild/ Ausbildungsangebots an der PH hier in [Studienort] (.) zum Ersten wenn es um die Orientierung an Professionalisierungsstandards geht (.) welche äh sind für Sie besprechenswert zu diskutieren (.) wie schätzen Sie äh wie ist Ihre Einschätzung der Kompetenzbereiche jetzt in Bezug auf Sie (.) selber Sie können die auswählen die für Sie wichtig sind Frau Steffen: also ich habe im (.) in diesem Dokument dass wir zwei auswählen mussten hab ich die Planung und Durchführung für mich ausgewählt auf das ich mich konzentrieren möchte weil ich finde die Planung ist noch für uns jetzt so am Anfang sehr schwierig 


\subsubsection{Rekonstruktion der einzelnen Sequenzen}

Herr Bucher: ich begrüsse Sie recht herzlich zum Mentoratsgespräch

Der Gesprächseinstieg weist einen hohen Grad an Formalisierung auf. Manifest liegt ein freundlicher, verbindlicher Begrüssungssprechakt vor, der die Wechselseitigkeit der Situation zum Ausdruck bringt. Vorstellbar wäre eine solche Begrüssung beispielsweise bei einem Vortrag. Neben der vortragenden Person ist auch eine Zuhörerschaft anwesend und diese wird mit einer förmlichen Anrede begrüsst. Falls die Aufmerksamkeit für den bevorstehenden Anlass noch nicht hergestellt ist, wird diese mit der Begrüssung eingefordert. Dies geschieht, indem dem Publikum freundlich aber dennoch bestimmt und unwiderruflich signalisiert wird, dass der offizielle Teil des Anlasses eben begonnen hat. Obwohl der Sprechakt den Anfangspunkt markiert, beinhaltet er neben dem eröffnenden Element auch ein schliessendes: Die laufenden Gespräche und Tätigkeiten sind zu einem Abschluss zu bringen. Insofern stellt er eine Zäsur vom informellen zum formellen Teil des Anlasses dar. Fände der Sprechakt vor einem grossen Publikum - beispielsweise im Zirkus oder an einer Galaveranstaltung - statt, würde eine noch förmlichere Begrüssung wie «sehr verehrtes Publikum» erwartet. Ebenfalls ausschliessen lässt sich angesichts des Wohlgeformtheitskontextes ein imaginäres Publikum wie in einer Fernseh- oder Radiosendung. Auch da würde die sprechende Person die Rezipienten explizit mit «liebe Zuschauerinnen, liebe Zuschauer» oder ähnlichem ansprechen. Der Eröffnungssprechakt strukturiert den Übergang hinzu einer formellen Rahmung, schafft in dieser Auslegung eine Bühne mit dem entsprechenden Publikum und stellt eine Öffentlichkeit her.

Ebenso wäre die Gesprächseröffnung in einem formalen Gespräch, beispielsweise einem Bewerbungsgespräch, mit verschiedenen Akteuren (Bewerberin, Vertretung aus der Geschäftsleitung, Personalfachperson etc.) formgerecht und vorstellbar. Hierbei wird bewusst eine formelle Gesprächssituation herbeigeführt. Analog zur obigen Vortragssituation leitet der Sprechakt freundlich und bestimmt den offiziellen Startpunkt des Bewerbungsgespräches ein. Ein weiterer Kontext, in welchem der Sprechakt wohlgeformt und erwartbar wäre, ist die Begrüssung zu einem Elternabend. Strukturell unterscheidet sich der Kontext nicht massgeblich vom Vortrag oder vom Bewerbungsgespräch. Auch 
hier ist das Setting formal, nicht aber hochformalisiert. In allen Situationen wird die schnörkellose und förmliche Gesprächseröffnung durch recht herzlich geringfügig aufgeweicht. Der Sprechakt ist freundlich, bleibt aber zugleich universalistisch und formalisiert.

Zusammenfassend lässt sich sagen, dass es sich um einen formalisierenden und strukturierenden Sprechakt handelt, der den Übergang zu einem offiziellen Teil des Geschehens markiert.

Herr Bucher: es dauert ungefähr (.) eine halbe Stunde

Anschliessend an die Begrüssung findet eine Absteckung des zeitlichen Rahmens statt. Durch den Vagheitsmarkierer ungefähr wird minimale Flexibilität eingeräumt, aber auch signalisiert, dass die Steuerung des Gespräches über die zeitliche Dimension veranschlagt wird. Es wird klar, dass kein Verhandlungsspielraum über die Form vorgesehen ist und das Gespräch, unabhängig von der inhaltlichen Problemlage, nach ungefähr einer halben Stunde endet. Der Strukturierungsanspruch liegt ausschliesslich beim Sprecher. Im Sinne eines kontrastierenden Gedankenexperimentes wäre in einer dyadischen Gesprächssituation auch vorstellbar, dass für die Festlegung des zeitlichen Rahmens die Frage «Was denken Sie, wie lange sollen wir uns Zeit nehmen?» gestellt würde. Dieses dyadische Element wird mit der klaren, zeitlichen Angabe insofern unterlaufen, als dass die Gestalt des Gesprächs bestimmt wird, die kommunikative, inhaltliche Auseinandersetzung jedoch unthematisiert bleibt.

Für den bisherigen Gesprächseinstieg ist festzuhalten, dass es sich um einen moderierenden Sprechakt handelt, der kontextfrei für die Begrüssung eines potenziell heterogenen und mittelgrossen Publikums erwartbar wäre. Wie zuvor erwähnt, schliesst die fehlende direkte Adressierung ein hochoffizielles Setting aus. Gleichzeitig erscheint die Begrüssung für eine dyadische Situation ungewohnt offiziell und auffallend distanziert. Der Sprecher nimmt qua Begrüssung eine Strukturierung des Settings vor, indem er vom informellen in den formellen Teil überleitet. Mit Blick auf seine Position bedeutet dies, dass er eine Strukturierungsbefugnis reklamiert und ein als Präsentieren-Rezipieren gerahmtes Setting konstruiert. 
Im real vorgefundenen Kontext der Gesprächseröffnung eines Mentoratsgesprächs lässt Herr Bucher durch die formelle, offizielle und universalistische Art ins Gespräch einzusteigen die Rahmung erkennen, die er für das Setting vornimmt. Spätestens mit der Nennung des vorgesehenen Zeitrahmens geht die Strukturierungsbefugnis in eine Strukturierungsmacht über, da Herr Bucher Frau Steffen diesbezüglich lediglich in Kenntnis setzt. Der zeitliche Rahmen wird nicht zur Diskussion gestellt. Hier deutet sich die Vermeidung einer dyadischen Situation sowie eine markante Formalisierung an.

Erwartbar ist an dieser Stelle, dass der Sprecher Informationen zum weiteren Verlauf anfügt und die Strukturierung des Anlasses fortführt.

Herr Bucher: wir würden eben durch drei Bereiche gehen das Erste wäre so eine allgemeine Einschätzung Stand der Dinge ((Bucher räuspert sich)) zu verschiedenen Fragen rund ums Mentorat das Zweite wäre die Planungs/der Planungshorizont für den zweiten Studienabschnitt und das Dritte die Einschätzung des ausgebild/ Ausbildungsangebots an der PH hier in [Studienort] (.)

Mit diesem Sprechakt wird die Agenda für die nächste halbe Stunde begründet und eine thematische Setzung anhand der drei zu verhandelnden Bereiche vorgenommen. Die Verwendung des Konjunktives wir würden kann als Andeutung auf eine implizit erforderliche Zustimmung der anderen Partei - im Sinne von "falls keinen Einwand erfolgt» gelesen werden. Auch die Verwendung des Konjunktivs als eine helvetische Höflichkeitsform $^{39}$, die zwischen formell und informell liegt, wäre denkbar.

In beiden Lesarten wird mit der Benutzung des Konjunktivs die rigide Festsetzung der ersten beiden Agendapunkte minimal aufgeweicht und zur Diskussion gestellt. Der dritte Punkt, die Einschätzung des ausgebild / Ausbildungsangebots an der PH hier in [Studienort], wird ohne wäre angeführt. Auf einer latenten Bedeutungsebene scheint dieser Punkt im Programm fester verankert als die beiden vorangegangenen. Auffällig bleibt der Einstieg mit dem Personalpronomen wir, wodurch das Ganze auf einer sprachlichen Ebene implizit zu einem gemeinsamen Vorhaben gemacht wird.

\footnotetext{
${ }^{39}$ In der Schweizer Mundart dienen Konjunktive oftmals als Höflichkeitsfloskel.
} 
Die eingangs elaborierte Lesart, die mit Vortrag gekennzeichnet wurde, hält weiterhin Bestand. Nach dieser Lesart ist beispielsweise vorstellbar, dass die vortragende Person auf einer Power-Point-Slide mit dem Cursor auf die drei Programmpunkte zeigt. Auch ein Bewerbungsgespräch oder ein Elternabend bleiben als potenzielle Settings gestützt, wenn die Agendapunkte als Platzhalter für spezifische, kontextabhängige Themen fungieren. Bewerbungsgespräch und Elternabend unterscheiden sich strukturell nicht grundsätzlich vom Vortrag. Die Öffentlichkeit ist zwar eine andere und die Rollenzuschreibung ist vor allem im Bewerbungsgespräch nicht von der Dichotomie produzierend/rezipierend geprägt, dennoch finden sich strukturell keine grundlegenden Differenzen.

Während bis anhin die Äusserungen sprachlich einwandfrei formuliert wurden, wird bei die Planungs abgebrochen und neu angesetzt. Es stellt sich die Frage, welcher Begriff ursprünglich angedacht war und durch Planungshorizont ersetzt wird. Passend wäre der Terminus die Planungsperspektive, der stärker personalisiert ist als der Terminus Planungshorizont, der stärker das Allgemeinmögliche repräsentiert. Träfe diese Einschätzung zu, kommt dies einer Vermeidung personalisierter Elemente gleich. Dies ist anschlussfähig an das bereits elaborierte Strukturmerkmal der Vermeidung des Dyadischen.

Gleichzeitig lassen sich Worte wie Planungshorizont, als der Zeitraum für den geplant werden soll, und zweiter Studienabschnitt, anstelle der Bezeichnung Semester oder Jahr, als Indizien für Bemühungsdynamiken zur Etablierung einer Fachsprache lesen. Der Sache nach reproduziert sich die Formalisierungslogik. Pointierter formuliert: Die sichtbar werdenden Formalisierungsbestrebungen sind auffallend und konturieren ein Vermeidungsmotiv möglicher diffuser Anteile und der Involviertheit der ganzen Person, die häufig in Gesprächen zu zweit vorzufinden sind.

An einer weiteren Stelle wird die Aussage abgebrochen und reformuliert. Aus die Einschätzung des Ausgebild wird Ausbildungsangebots. Ausgebild ist das Partizip II des Verbs ausbilden ohne die Endsilbe. Eine Person wird beispielsweise durch eine Institution ausgebildet. Im Kontext des Gesprächs wäre dies die Studentin Frau Steffen durch die PH. Die Frage nach dieser personifizierten Einschätzung bricht Herr Bucher ab und ersetzt sie durch Ausbildungsangebot, was erneut einer Entpersonalisierungsbewegung gleichkommt. 
Die Offenlegung der Agendasetzung kommt mit der Einschätzung des Ausbildungsangebotes zu einem Ende.

Herr Bucher: zum Ersten wenn es um die Orientierung an Professionalisierungsstandards geht (.) welche äh sind für Sie besprechenswert zu diskutieren (.)

Sowohl im Kontext eines Vortrags, der halböffentlich zugänglich ist, als auch eines Elternabends, zu dem nur ein bestimmter Personenkreis eingeladen wird, wäre der Sprechakt wohlgeformt. Für das Setting eines Bewerbungsgespräches passt er nicht mehr, da in diesem Kontext die Bewerberin kaum nach für sie besprechenswerten respektive zu diskutierenden Traktanden gefragt wird.

Im realen Kontext steigt Herr Bucher in den ersten Themenblock ein. Allerdings geht es nicht, wie zuvor angekündigt, um eine allgemeine Einschätzung [zum] Stand der Dinge zu verschiedenen Fragen rund ums Mentorat, sondern um besprechenswerte Professionalisierungsstandards (oder um besprechenswerte Orientierungen an solchen). Dabei wird besprechenswert mit zu diskutieren ergänzt. Als eine mögliche Lesart handelt es sich dabei um eine Aufzählung: zuerst besprechenswert; dann zu diskutieren. Aus einer anderen Perspektive ist zu diskutieren eine Korrektur von besprechenswert. Die beiden Tätigkeiten besprechen und diskutieren stellen zwei unterschiedliche Formen der kommunikativen Bezugnahme dar, die der Struktur nach nicht ohne weiteres miteinander vereinbar sind. In einer Besprechung werden Informationen ausgetauscht, Traktanden abgearbeitet, Absprachen getroffen. Endpunkt der Besprechung stellen im Idealfall Abmachungen für die beteiligten Parteien dar. Eine Diskussion hingegen ist ergebnisoffen und folgt der Logik des Austauschs von Argumenten und Gegenargumenten. In einer solchen sind unterschiedliche Standpunkte möglich. Diese Unterscheidung gilt es in der obigen Sequenzstelle zu berücksichtigen.

Der Sprechakt bewegt sich hin zu der Aufforderung, die Professionalisierungsstandards einzuschalten beziehungsweise zu diskutieren. Seitens des Sprechers scheint ein Unbehagen gegenüber den Professionalisierungsstandards durch, was sich in der latenten Aufforderung zur Kritik oder mindestens zur kritischen Stellungnahme Ausdruck verschafft. 
Für den hier beschriebenen Vorgang führe ich den Terminus «Diskurs-Move» ein. Damit wird eine Bewegung beschrieben, die eine Positionierung oder Stellungnahme der Gegenpartei provoziert, um eine (Schein-)Diskussion zu initiieren. Unter diesem Gesichtspunkt ist die Aufforderung Herr Buchers als potenzieller Diskurs-Move zu betrachten. Von der Interaktionslogik und des pausierenden Sprachflusses her wäre erwartbar, dass die Studentin auf den Diskurs-Move reagiert. Dazu kommt es allerdings nicht, sondern der Mentor fährt mit einer nächsten Frage fort.

Herr Bucher: wie schätzen Sie äh wie ist Ihre Einschätzung der Kompetenzbereiche jetzt in Bezug auf Sie (.) selber Sie können die auswählen die für Sie wichtig sind

Der Sprechakt beginnt mit einer Frage, die mithilfe eines Verbes formuliert und bei äh abgebrochen wird. In der Reformulierung wird die Aussage nominalisiert. Die Verwendung von Nominalisierungen ist als Positionierung einer bestimmten Fachlichkeit zu lesen und zieht eine erneute Entpersonalisierungsbewegung nach sich.

Der erste Teil - die Frage nach der Einschätzung - wäre beispielsweise als journalistische Frage vorstellbar, mit Hilfe derer eine als kompetent erachtete Person um ihre Einschätzung gebeten wird. Analog wäre die Frage in einem Gespräch zwischen einem Patienten und einer Ärztin erwartbar. Nach beiden Auslegungen fragt eine weniger wissende Person eine wissendere um eine Lagebeurteilung. Mit dem Zusatz jetzt in Bezug auf Sie (.) selber bricht diese Ordnung allerdings auf und das Wissens- respektive Kompetenzverhältnis dreht sich um. Der Fragesteller wird zur hierarchisch höher gestellten Person und die Frage erhält eine Überprüfungsimplikation. Diese wird mit dem Ausdruck Sie können die auswählen die für Sie wichtig sind zusätzlich verschärft. Die Prüfungskandidatin erhält hierbei die Option mit einem Thema ihrer Wahl oder nach persönlicher Wichtigkeit einzusteigen, wodurch dem Sprechakt auch eine pädagogische Note anhaftet. 
Frau Steffen: also ich habe im (.) in diesem Dokument dass wir zwei auswählen mussten hab ich die Planung und Durchführung für mich ausgewählt auf das ich mich konzentrieren möchte weil ich finde die Planung ist noch für uns jetzt so am Anfang sehr schwierig

Die gefragte Person steigt mit dem Adverb also ein, was als Anlauf nehmen für eine längere Ausführung ausgelegt werden kann. Mit dem Sprechakt wird der Aufforderung zu einer Wahl gefolgt. Dass wir zwei auswählen mussten markiert sprachlich die Tatsache, dass sie auswählen mussten anstelle von «auswählen zu dürfen». Die Existenz des Einschubes ist markant. Er betont den Pflichtcharakter der zu treffenden Auswahl und stellt klar, dass der Entscheidungsspielraum begrenzt war.

Jenseits dieser Klarstellung zeigt der Sprechakt auf, dass die Studentin die ihr zugetragene Aufgabe kooperativ annimmt. Mit der Aussage Planung ist noch für uns jetzt so am Anfang sehr schwierig thematisiert sie nicht ihr eigenes Empfinden, sondern nimmt die Position einer Sprecherin für das Studierendenkollektiv ein. Dadurch wird eine Ich-duKonstellation vermieden.

Mit der Verwendung von möchte wird ein Vorhaben oder eine Intention aufgezeigt. Mit anderen Worten wird weder auf die Frage wie ist Ihre Einschätzung der Kompetenzbereiche jetzt in Bezug auf Sie (.) selber, die auf eine Selbsteinschätzung zielt, noch auf den Diskurs-Move eingegangen. Dementgegen folgt die Studentin mit ihrer Antwort dem Aspekt der Überprüfung. Sie greift folglich den latenten Inhalt der Frage auf, der auf dieser Ebene für sie die Aufforderung, ihre Schwächen zu benennen und daraus ein Vorhaben zu formulieren, beinhaltet. Zugespitzt formuliert kauft sie Herrn Bucher an dieser Stelle die geforderte Einschätzung der Kompetenzbereiche nicht ab, sondern sieht sich aufgefordert eigene Defizite zu thematisieren. Dies bezieht sie allerdings nicht auf sich persönlich, sondern auf ihren Status als Studentin.

Der eigentliche Begrüssungsakt ist an dieser Stelle beendet. Die Studentin führt in der Fortsetzung inhaltlich ihre Wahl weiter aus. Deshalb wird an dieser Stelle eine Fallstrukturhypothese für die Eröffnungssequenz formuliert. 


\subsubsection{Zusammenfassung und riskante Fallstrukturhypothese der Eröffnungssequenz Ich begrüsse Sie recht herzlich}

Die höfliche Ansprache mit einer Begrüssungsformel aus einer halböffentlichen Sphäre ist kontextfrei in einer Vortagssituation vor Publikum zu erwarten. Die Gesprächseröffnung lässt erkennen, dass sowohl die formalen wie auch die inhaltlichen Eckpfeiler gesetzt werden. Führung und Ablauf des Gespräches werden weder dem Zufall noch der Gesprächsdynamik überlassen. Mit der getätigten Begrüssung positioniert sich der Sprecher als Person mit Strukturierungsbefugnis und nimmt die Gesprächsführung in Anspruch. Gleichzeitig wird das Gespräch als institutioneller, zweckgebundener Anlass gekennzeichnet.

Beim realen Sprechakt handelt es sich allerdings um den Gesprächseinstieg eines VierAugen-Gespräches. Das strukturlogisch markante Merkmal der Eröffnungssequenz ist die in ihr aufscheinende Formalisierungsbewegung. Mit dem an eine Öffentlichkeit oder Halböffentlichkeit gerichteten Sprechakt gewinnt etwas Überschüssiges, Nähe-Vermeidendes oder ein Motiv der Distanzwahrnehmung Gestalt. Dieses Motiv entlastet die Gesprächssituation von potenzieller Diffusität, dyadischen Anteilen und dem Anspruch auf Involviertheit als ganze Person. Ferner gewinnen in der Sprechweise des Mentors Bemühungen Gestalt, ein Fachvokabular anzuwenden, was sich beispielsweise in der Verwendung von Termini wie eine allgemeine Einschätzung [zum] Stand der Dinge, Planungshorizont und Orientierung an zeigt. Des Weiteren werden personalisierte Elemente vermieden oder sprachlich in weniger personalisierende umgeformt. Mit Blick auf die Interaktionsdynamik wird das Gespräch auf eine Ebene gehoben, die sich von einer alltagssprachlichen unterscheidet.

Der formalisierte, mit einem klaren Führungsanspruch unterlegte Gesprächseinstieg des Mentors steht insgesamt in einem Spannungsverhältnis zur manifesten Aufforderung an die Studentin Stellung zum Ausbildungsangebot der PH zu nehmen sowie zur latenten Aufforderung Kritik daran zu üben. Letztere tritt als Frage nach besprechenswert zu diskutierenden Standards in Erscheinung. Die Studentin nimmt die ihr zugetragene Aufgabe formal und von der Interaktionsstruktur des Gesprächs her betrachtet an. In ihren Ausführungen geht sie allerdings nicht auf die geforderte Einschätzung ein, das heisst sie folgt dem Diskurs-Move nicht. Vielmehr kommt sie der für sie erkennbaren 
(Über-)Prüfungsimplikation nach und benennt Schwierigkeiten, die sie aus ihrer Rolle als Novizin ableitet. Mit der Verwendung der Pluralform uns bei die Planung ist noch für uns jetzt so am Anfang sehr schwierig spricht sie für sich und für ihre Kommilitoninnen und Kommilitonen. Dabei gewinnt auch eine Dynamik der Schutzsuche im Kollektiv an Gestalt und sie entzieht sich der direkten Ich-du-Konstellation.

Das zentrale Merkmal der Interaktionsdynamik der Eröffnungssequenz ist die Vermeidung einer Beziehungsstruktur. Dies führt zur folgenden Fallstrukturhypothese der Eröffnungssequenz im Mentoratsgespräch zwischen Frau Steffen und Herrn Bucher:

Die spezifische Figur des vorliegenden Eröffnungsaktes ist die Vermeidung dyadischer und diffuser Beziehungsanteile, die potenziell in einem Vier-Augen-Gespräch - wie dem vorliegenden Mentoratsgespräch - angelegt sind.

\subsubsection{Zweite Sequenz: Aber eigentlich würde ich Ihrer These widersprechen}

Im weiteren Gesprächsverlauf spricht die Studentin von der Schwierigkeit, die von den Schülerinnen und Schülern benötigte Zeit für die Erfüllung einer Aufgabe korrekt einzuschätzen, und von der Herausforderung, einen Auftrag verständlich zu erteilen. Sie wird vom Mentor aufgefordert diese Aspekte zu präzisieren. Ihre diesbezüglichen Schilderungen beziehen sich hauptsächlich auf die Problematik der Zeiteinschätzung und wie sie dieser durch die Erstellung mehrerer Arbeitsblätter mit unterschiedlichem Umfang und gestuften Anspruchsniveaus begegnet. Der Mentor geht nicht auf ihre ausführlichen Schilderungen ein, sondern formuliert erneut eine Frage nach einer Einschätzung, die inhaltlich nicht im direkten Zusammenhang mit ihren Ausführungen steht. Unter Zuzug einer sequenzübergreifenden, fallimmanenten Betrachtung ist bemerkenswert, dass Herr Bucher im gesamten Gesprächsverlauf den Terminus Einschätzung fünfmal in eine Frage einbindet. Die ausformulierte Variante wie schätzen Sie ein tritt sogar in elf Fällen auf, weshalb sich eine genaue Rekonstruktion einer solchen Fragekonstruktion lohnt. An einer entsprechenden Stelle setzt die ausführliche, sequenzanalytische Rekonstruktion wieder ein. 
Herr Bucher: wie schätzen Sie den zweiten Kompetenzbereich ein wenn's um die Sprache geht wenn's darum geht Kinder in ihrer Lebens/in ihrem Lebensalter in ihrer Sprache zu treffen dass es verständlich ist dass man eine pädagogische Sprache führt die auch der Situation nützt und für die Kinder gerecht ist dass man sie eben nicht überfordert oder nicht unterfordert wo liegen dort die Probleme? wie zeigen sich Probleme in diesem Kompetenzbereich? Frau Steffen: ja also oftmals da hab ich gedacht ich hab jetzt etwas gut erklärt (.) und dann sieht man bei den schwächeren Kindern dass sie es noch gar nicht begriffen haben aso man muss sich immer (.) vorher ganz genau überlegen wie man etwas ausdrücken möchte (.) aso mit der Zeit muss man das dann nicht mehr aber am Anfang (.) und es ist halt schwierig //weil// Herr Bucher: //wo setzen Sie da an beim Training?// Frau Steffen: aso wie meinen Sie das?

Herr Bucher: dass man Kinder eben dort trifft und dort abholt wo sie wo sie es verstehen können dass man eine adäquaten Sprache hat trainiert man dann die Sprache oder trainiert man die Planung und die Vorbereitung?

Frau Steffen: ich denke die Planung und die Vorbereitung dass man eben weiss wie man sich ausdrücken möchte und welche Wörter man verwenden möchte dass es die Kinder verstehen aber auch muss man die Sprache in der Schule so anpassen dass man eigentlich immer dann so spricht dass es für die Kinder verständlich und dass so wie auf ihrem Sprachniveau ist Herr Bucher: wie realisiert man ein Sprachniveau Frau Steffen: ja man hört ja selber welche Wörter dass sie verwenden und was sie alles wissen können aso auch von zu Hause mitbringen und da gibt es einige die sicherlich mehr Wissen haben und mehr Fremdwörter kennen und andere die halt wenn sie eine andere Muttersprache haben oder so da vielleicht Defizite haben und da könnte man auch wenn man solche Kinder hat die nicht super Deutsch können zuerst eine normale also normale ((Studentin formt mit Fingern Anführungszeichen)) Einführung geben und dann noch zu diesen Kindern gehen und es ihnen noch einmal ganz langsam und ruhig nochmals erklären und ich glaube es ist auch immer wichtig dass man nicht zu viel auf einmal sagt sondern nur die nächste Anweisung und dann wieder die nächste weil wenn man sagt das 
und das und das und das und das dann wissen sie schon nicht mehr was sie als erstes machen müssen und wenn man ihnen einfach den nächsten Auftrag gibt und sie sich nur auf das konzentrieren müssen dann (.) glaub ich ist das Gelingen auch besser dass dann alle das Richtige machen

Herr Bucher: eine theoretische Überlegung ich geb's zu dass die nicht so praktisch ist aber wie wie lösen Sie das Problem wenn ich definiere Lern/Lernen bedeutet Schwierigkeiten überwinden was müssten Sie eigentlich in der Sprache immer ein bisschen voraus sein oder vom Niveau her angehoben sein natürlich auch unterschiedlich wenn die Kinder unterschiedliche Niveaus haben aber eigentlich würde ich Ihrer These widersprechen dass man die Kinder treffen muss Frau Steffen: aso //man kann sicher//

Herr Bucher: //Sie müssen// ja im Pädagogischen immer einen kleinen Schritt Frau Steffen: ja einen kleinen sicherlich Herr Bucher: voraus sein Frau Steffen: das finde ich auch Herr Bucher: was würde das wenn das theoretisch wenn meine These stimmt (.) was würde das für Ihre Aussagen vorhin für Konsequenzen haben Frau Steffen: aso für die Aussagen ist es eigentlich noch dasselbe

\subsubsection{Rekonstruktion der einzelnen Sequenzen}

Herr Bucher: wie schätzen Sie den zweiten Kompetenzbereich ein

Fragt man sich kontextfrei für welche soziale Situation die hier formulierte Frage angemessen wäre, lässt sich ein Gespräch zwischen zwei (Fach-)Personen mit unterschiedlicher oder geteilter Expertise vorstellen. Hier würde eine fragende Person die Einschätzung einer befragten Person einholen. Möglicherweise lässt der Fragesteller diese Einschätzung in die eigene Entscheidungsfindung mit einfliessen. Letztendlich muss er aber sein eigenes Urteil fällen und auch die Verantwortung für dieses übernehmen. Systematisch könnte der Sprechakt beispielsweise in einem Beratungskontext fallen, 
wenn der Bürgermeister (D) respektive der Gemeindepräsident $(\mathrm{CH})$ die Meteorologin fragt: «Wie schätzen Sie die Waldbrandgefahr ein? Ist es verantwortbar, die Erlaubnis für Grillfeuer zu erteilen?» In dem Sprechakt wird die angesprochene Person als Expertin adressiert und zu einem Gegenstand befragt. Der Sprecher holt mit seiner Positionierung pflichtbefreit eine Expertise für die eigene Lageanalyse ein.

Ebenfalls passend wäre der Sprechakt in einer mündlichen Prüfungssituation, wenn Informationen interpretiert werden sollen. Obwohl der Fragesteller die Befragte der Form nach als Expertin anspricht, ist - im Gegensatz zum Beratungskontext - der Fragesteller hier der Experte und adressiert die Prüfungskandidatin als Teil seiner didaktisierten Fragestrategie als ob sie Expertin wäre. Die Prüfungskandidatin hat ihre Expertise zum Zwecke einer Selektionsentscheidung unter Beweis zu stellen. Dabei positioniert sich der Sprecher als Experte für die Materie und demzufolge als Selektionsentscheidungsträger. Obwohl in Prüfungs- oder Selektionskontexten üblicherweise mindestens zwei Expertinnen oder Experten anwesend sind, erscheint der vorgefundene Sprechakt in seiner strukturellen Form nicht weniger plausibel. In der empirischen Sequenz stellt die Frage allerdings keine offensichtliche oder klassische Prüfungsfrage dar, da konkret nach einer Einschätzung und nicht nach Wissen gefragt wird.

Eine weitere, kontextfreie Auslegung des Sprechaktes liesse sich in einem Polittalk vor fiktivem oder realem Publikum finden, etwa wenn der Moderator eine Politikerin in der Fernsehdebatte fragt: «Wie schätzen Sie die Chancen für einen Wahlsieg ein?» Im Unterschied zur an die Meteorologin gerichtete Frage wird hier nicht aus Eigeninteresse gefragt, sondern die angefragte Person wird für ein Publikum oder eine Öffentlichkeit sichtbar gemacht. Die Frage dient folglich der Meinungsbildung oder der Unterhaltung.

Das gemeinsame Bedeutungsmoment der drei elaborierten Situationen ist ein Rekurs auf die Expertise der angefragten Person. Die strukturellen Unterschiede der drei Kontexte, in welchen der Sprechakt systematisch erwartbar wäre, werden nachfolgend nochmals aufgezeigt und in ihren Unterscheidungsmerkmalen in Beziehung gesetzt.

Der Sprechakt lässt sich in einer ersten Lesart als Anfrage oder Einholung einer Expertise verstehen, in der der Fragesteller die Antwort nicht kennt und folglich eine echte Frage stellt. Für die befragte Person steht möglicherweise ihre Reputation auf dem Spiel. Die finale Verantwortung für den Umgang mit den gelieferten Informationen liegt dennoch 
beim Fragesteller. Die Antwort wird als Expertise registriert, muss jedoch nicht evaluiert werden.

Als zweite Lesart lässt sich der Sprechakt als Prüfungsfrage respektive Prüfungsaufgabe deuten. In der Prüfungssituation wird die Antwort der Kandidatin qua Expertise des Fragenden mit der erwarteten Antwort verglichen. Die Expertise der Kandidatin wird zum Zwecke einer Selektionsentscheidung unter Beweis gestellt. Eine Evaluation der Antwort seitens des Fragestellers erfolgt unmittelbar oder zeitverschoben. Der Bewährungsdruck respektive das Potenzial zum Scheitern kann für die befragte Person, je nach Art der Prüfung und Grad der Selektion, hoch ausfallen. Im Gegensatz zum Kontext der ersten Lesart handelt es sich bei einer Prüfung um eine arrangierte Situation. Die angesprochene Person wird mit Erwartungen konfrontiert, gegenüber denen sie sich bewähren kann und muss.

Eine dritte Lesart, die sich aus der Polittalk-Szene herausbildet, folgt der Logik einer Sichtbarmachung der angefragten Person. Ihre Performanz lässt sich einerseits über die Inhalte messen, andererseits über ihren Auftritt, das heisst: wie professionell, souverän und charismatisch sie sich präsentiert, Fragen beantwortet oder Provokationen paroli bietet. Die angesprochene Person erhält eine Plattform zur Selbstdarstellung. Dabei steht nicht der argumentative Austausch, sondern die Akklamationsfähigkeit im Fokus. Mit anderen Worten soll Zustimmung vom Publikum für die eigene Position oder Person gewonnen werden. Der Fragesteller ist nicht primär an inhaltlichen Aspekten interessiert und nimmt auch keine Evaluation vor. Eine allfällige Evaluation oder Bezugnahme zum Gesagten dient einem fürs Publikum aufschlussreichen und informativen Gesprächsverlauf, auch unter Einsatz von rhetorischen Mitteln. Das Gespräch folgt dem Modus einer Inszenierung. Für die befragte Person stehen bisweilen ihre Reputation und daran anknüpfend auch ihre Politkarriere auf dem Spiel.

Abschliessend scheint es lohnenswert die Strukturlogik einer Einschätzung aus einer grundsätzlichen Perspektive zu betrachten. Einer Einschätzung liegen Abwägungen und Mehrperspektivität zu Grunde. Final wird sie dennoch holistisch getroffen. Für eine Einschätzung sind Unschärfen und Spielräume konstitutiv, was der Wortbaustein schätzen bereits nahelegt. Wäre eine eindeutige, präzise Antwort erwartbar oder überhaupt möglich, würde nicht nach einer Einschätzung gefragt. Die vorgefundene Fragestellung nach der Einschätzung konstituiert den ihr zugrunde liegenden Kontext folglich als einen, 
der sich allein durch Interpretationsleistung erschliesst. Die Einschätzung im Modus einer Überprüfung vorzunehmen weist ein in sich widersprüchliches Moment auf. In diesem Modus geht es dem Fragesteller nicht primär um die Inhalte der Antwort, sondern vielmehr um die Argumentation der befragten Person.

Mit der vorgefundenen Frage wird das Strukturmerkmal der distanzierten und formellen Sprechweise, welches bereits in der Eröffnungssequenz zu Tage tritt, reproduziert.

Herr Bucher: wenn's um die Sprache geht ${ }^{40}$

Mit den weiteren Ausführungen wird eine Präzisierung vorgenommen. Die Einschätzung hat sich auf einen spezifischen Aspekt zu beziehen. Entweder geht es dem Sprecher bei der erfragten Einschätzung lediglich um einen Teilaspekt nämlich um die Sprache oder der Aspekt die Sprache wird konkretisiert und allfällige Zweifel, welcher Aspekt gemeint sei, werden ausgeräumt.

Alle bisher elaborierten Lesarten halten auch mit diesem Zusatz weiterhin stand. Allerdings können die hier angefügten Informationen oder Präzisierungen als erste Hinweise für ein Wanken der Lesart Expertiseanfrage gedeutet werden, da entsprechende Zusatzinformationen in einer solchen eher nicht erwartbar wären.

In der real vorgefundenen Interaktion des Mentoratsgesprächs bedeutet der Sprechakt, dass der zweite Kompetenzbereich die Sprache betrifft und Herr Bucher dies als Gedächtnisstütze nochmals in Erinnerung ruft oder aber, dass Frau Steffen sich nur zum Teilaspekt der Sprache innerhalb des zweiten Kompetenzbereiches äussern soll.

Herr Bucher: wenn's darum geht Kinder in ihrer Lebens/in ihrem Lebensalter in ihrer Sprache zu treffen

In der Fortsetzung der Ausführungen wird zu einer Begriffsbestimmung angesetzt, die mit in ihrer Lebens/ beginnt und danach zum Abbruch kommt. Hier gilt es zu überlegen, welcher Begriff abgebrochen und anschliessend korrigiert wird. Denkbar wären beispiels-

\footnotetext{
${ }^{40}$ Unter Zuzug des Kontexts weiss man, dass sich der zweite Kompetenzbereich auf Planung und Durchführung bezieht und nicht - wie sich der konkrete Sprechakt auch deuten liesse - den Bereich Sprache betrifft. Diese zweite Auslegung kann ausgeschlossen werden.
} 
weise «Lebenssituation», "Lebenswelt» oder "Lebensphase». Bucher setzt neu an und verwendet den Terminus Lebensalter, ein soziologischer oder statistischer Fachbegriff, der für die gelebten Jahre steht. Verwendung findet er etwa im Zusammenhang mit älteren Menschen, wenn man «vom dritten oder vierten Lebensalter» spricht.

In Anbetracht des Themas einer angemessenen Sprache, das weniger ein soziologisches als vielmehr ein dezidiert pädagogisches und normatives Konzept darstellt, wäre «altersgemäss» der adäquate Begriff. Die abgebrochenen Begriffe «Lebenssituation», «Lebenswelt» oder "Lebensphase» wären allesamt passender als die vorgenommene Korrektur Kinder in ihrem Lebensalter in ihrer Sprache zu treffen. Fragt man sich, welche Dynamik in dieser Sprechaktfigur enthalten ist, respektive was durch die Korrektur Gestalt gewinnt, ist die Figur als Präzisierungsversuch zu lesen, der performativ den Eindruck von Fachlichkeit erzeugt.

Wäre dieser Versuch erfolgreich, ginge das mit einer dezidierten Distanzierung von einer praktisch-normativen Orientierung einher. Diese hier brüchige Ambition, den Anspruch auf Fachlichkeit im Gespräch anzuheben, lässt sich an zwei Daten festmachen. Einerseits an der Korrektur von Lebens/mit dem wissenschaftlicheren Begriff des Lebensalters, der im gegebenen Kontext wenig erwartbar ist, andererseits in der Vermeidung von «altersgemäss». Beide Daten weisen auf das Umgehen einer für die pädagogische Formsprache typischen Sprechweise und Argumentation hin. Im Gegensatz dazu evoziert das Verb treffen eine Vorstellung von «erreichen», «abholen», «betroffen machen» oder auch «erschüttern». Die Idee des Treffens weist im Gegensatz erneut eine pädagogische Implikation auf respektive markiert einen normativen Anspruch. Dies steht im Widerspruch zu Lebensalter, als Terminus aus einem fachsprachlichen Diskurs. Die vorgenommene Selbstpositionierung changiert zwischen einem Fachsprachediskurs und normativen Vorstellungen. Als Hypothese lässt sich eine Situationsunsicherheit festhalten, die sich in der Vermeidung einer normativ-pädagogischen Haltung zeigt.

Der angefügte Zusatz impliziert ein Erklärungsmoment und lässt es unwahrscheinlich erscheinen, dass jemand als Expertin adressiert wird. Die Lesart der Expertiseanfrage kann damit nicht mehr aufrechterhalten werden. Viel eher ist der Sprechakt in einem Prüfungskontext vorstellbar oder aber bei einer Befragung zwecks Sichtbarwerdung der Befragten. Herr Bucher führt seine Ausführungen fort: 
Herr Bucher: dass es verständlich ist dass man eine pädagogische Sprache führt die auch der Situation nützt und für die Kinder gerecht ist dass man sie eben nicht überfordert oder nicht unterfordert wo liegen dort die Probleme? wie zeigen sich Probleme in diesem Kompetenzbereich?

Nach einer weiteren Ausdifferenzierung des Problems, zu dem Frau Steffen ihre Einschätzung abgeben soll, folgen die eigentlichen Fragen. Die Studentin wird aufgefordert anhand ihrer Einschätzung und ihrem Wissen sichtbar zu werden. Herr Bucher positioniert sich als Experte auf diesem Gebiet, was unter anderem durch die kleinschrittig erläuterten Zusatzinformationen Gestalt gewinnt. Bezeichnend ist wie der Gegenstand, zu dem sich Frau Steffen verhalten soll, in bedeutsamer Dauer und einem kommunikativen Prozess konkretisiert wird. Die Ausführungen des Mentors markieren zunehmend einen normativen Anspruch. Damit wird der zunächst brüchig und zaghaft angesteuerte Modus eines Fachgespräches in Richtung eines Austauschs über praktische Problemstellungen gelenkt. Anders gesagt führen die Formulierungen von einer wissenschaftlichen Perspektive hin zu einer Sichtweise mit pädagogischen Implikationen und einer Positionierung von Normen (altersgemäss, weder über-, noch unterfordern), die eben gerade keiner Fachgesprächslogik, sondern vielmehr einer Zeigelogik, folgen (für Zeigelogik vgl. Prange, 2012, S. 65). Es gilt nun zu klären, welche spezifische Struktur in Bezug auf die elaborierten Lesarten Gestalt gewinnt. Im Falle einer Prüfungsfrage ist der Expertisevorsprung des Fragestellers konstitutiv und die Sichtbarkeit der eigenen, normativen Anteile zwar nicht zwingend, aber dennoch nicht ungewohnt. Die Lesart kann folglich aufrechterhalten werden. Dennoch sprechen die extensiven Ausführungen als Hinführung zur eigentlichen Prüfungsfrage eher für eine Prüfung ohne weitreichende Konsequenzen. Stände für die Prüfungskandidatin sehr viel auf dem Spiel, wäre eine kürzere Formulierung, mit eher verdeckten Normativitätsvorstellungen, erwartbar.

Für die Lesart der Befragung zwecks Sichtbarwerdung lassen sich die vorgefundenen Ausführungen als Selbstpositionierung des Fragestellers interpretieren. Diese beinhaltet neben der Einnahme der Moderatorenrolle auch sich als mit der Thematik vertraut zu zeigen. Die hier durchscheinenden Normativitätsvorstellungen lassen sich different deuten: In einer ersten möglichen Auslegung entsprechen sie den Normativitätsvorstellungen des Sprechers und werden (un-)beabsichtigt im Sprechakt sichtbar. Oder 
aber - so ein zweites Deutungsangebot - der Sprecher zeigt, dass er mit den Normativitätsvorstellungen, welche seiner Einschätzung nach den Common-Sense abbilden, vertraut ist. Als Teil seiner Gesprächsstrategie konfrontiert er die Befragte mit diesen.

In einem weiteren Deutungsangebot lassen sich die Ausführungen des Fragestellers als Hilfestellung lesen, mit denen die Erfolgschancen der Prüfungskandidatin erhöht werden, insbesondere wenn die Prüfungsfrage voraussetzungsreich ist und dennoch nicht von dieser abgesehen wird.

Gewendet auf den Gesprächskontext wird mit einer doppelten Frage - wo liegen dort die Probleme; wie zeigen sich die Probleme in diesem Kompetenzbereich - nach den Schwierigkeiten einer kindgerechten Sprache gefragt. Zu dieser Problemstellung soll sich Frau Steffen allerdings nicht mit Handlungsanleitungen - «Wie mache ich es?»-, sondern in Form einer Vergegenwärtigung, positionieren. Dazu breitet der Mentor vor der Studentin nochmals einige Aspekte als Grundlage aus, auf die sich die Studentin in ihrer Antwort beziehen kann. Das Gerüst dient der Unterstützung. Gleichzeitig wird dadurch das Feld der antizipierten Antwort abgesteckt. Mit dieser Bewegung wird allerdings eine gewisse Widersprüchlichkeit erzeugt. Einerseits wird Frau Steffen vordergründig aufgefordert ihr Fachwissen aufzuzeigen und sich als kompetente Gesprächspartnerin zu präsentieren. Andererseits wird die gestellte Aufgabe aus Sicht des Fragestellers als voraussetzungsreich gekennzeichnet und der Studentin Erläuterungen und Zusatzinformationen für die Bearbeitung zur Verfügung gestellt.

Das Zusammenspiel des Imperativs «Zeige dich als fachlich kompetent!» mit den Hilfestellungen stellt dabei ein Spannungsfeld dar. Dies lässt sich als Hinweis lesen, dass ihr eine fachliche Kompetenz nicht per se zugesprochen wird. Ihr Sich-Zeigen bedarf einer Unterstützung.

Zusammenfassend ist festzuhalten, dass die Figur der Anforderungs- und Bewährungssituation, die die Grundlage der beiden Lesarten bildet, als voraussetzungsreich antizipiert wird. Die Studentin wird mit einer Problemeinschätzung konfrontiert und herausgefordert sich auf einer fachlichen Ebene Geltung zu verschaffen.

Als Anschlussoption wäre eine Einlassung auf die vom Mentor in der Frageformulierung bereits vorgespurte Perspektive denk- und erwartbar. Diese könnte beispielsweise lauten: «Also aus meiner Erfahrung heraus besteht das grösste Problem in der Bandbreite der 
jeweils adäquaten Sprache.» Die Studentin würde der Aufforderung sich zu zeigen nachkommen und dabei die Hilfestellung des Mentors annehmen.

Frau Steffen: ja also oftmals da hab ich gedacht ich hab jetzt etwas gut erklärt (.) und dann sieht man bei den schwächeren Kindern dass sie es noch gar nicht begriffen haben aso ${ }^{41}$ man muss sich immer (.) vorher ganz genau überlegen wie man etwas ausdrücken möchte (.) aso mit der Zeit muss man das dann nicht mehr aber am Anfang (.) und es ist halt schwierig //weil// (Herr Bucher: //wo setzen Sie da an beim Training?//) ${ }^{42}$

Der anschliessende Sprechakt ist keine Antwort auf die gestellten Fragen, sondern lässt sich in erster Linie als Selbstbezug verstehen. Dabei zeigt die Studentin ihre gemachten Erfahrungen auf. In der Antwort ist erkennbar, dass das Einlösen der geforderten Bewährungsansprüche einer Problemanalyse tatsächlich anspruchsvoll ist und nicht ohne weiteres realisiert werden kann. Ihre Aussage beginnt Frau Steffen mit ja also. Dies ist als Indiz für eine ausführliche Erläuterung zu lesen. Ihre daran anschliessende Einschätzung folgt der Logik einer alltagsweltlichen Selbstreflexion im Erzählmodus. Mit oftmals da spricht sie eine Routine oder sich wiederholende Erfahrungen an und schildert in der Dynamik eines Bekenntnisses den Prozess ihrer Fehleinschätzung in Bezug auf etwas gut erklärt zu haben.

Den Anspruch auf Fachlichkeit bearbeitet sie anschliessend, indem sie mit einer proklamierten Handlungsmaxime für Novizinnen und Novizen - man muss sich vorher immer genau überlegen wie man [...] - auf eine allgemeine Ebene wechselt. Im Sprechakt führt sie die Erfahrung des Scheiterns aus und zeigt sich gegenüber dem Mentor als Person, der Dinge mehrfach missglückt sind. Dieses Bekenntnis und die Form der Offenheit überraschen in einer Gesprächsdynamik, die auf einer Bewährung- und Anforderungsstruktur basiert. Dies deutet möglicherweise auf ein strategisches Moment hin, mit welchem die Prekarität der Bewährung bearbeitet wird. Es stellt sich die Frage, weshalb sie wie vor-

\footnotetext{
${ }^{41}$ Aso ist eine Mundartversion von also. Es ist keine Systematik zu erkennen in welchen Fällen Frau Steffen aso anstelle von also verwendet. Die Vermutung, dass aso mit einer Informalisierung einhergeht, muss als Vermutung stehen bleiben.

42 Die Unterbrechung Herrn Buchers mit V/wo setzen Sie da an beim Training//) wird hier lediglich aufgeführt und erst im Anschluss als eine Sequenz rekonstruiert.
} 
gefunden vorgeht und warum sie eine kategorial andere Antwort als die angefragte erteilt. Eine naheliegende Erklärung wäre, dass sie sich über das Nichtbeantworten der Ursprungsfrage nicht bewusst ist. Mit der Aussage aso mit der Zeit muss man das dann nicht mehr zeigt Frau Steffen, dass sie als Studentin diesen Lern- und Entwicklungsprozess erfolgreich durchlaufen hat. Im Sprechakt gewinnt eine Figur Kontur, in der zunächst über eine (negative) Erfahrung berichtet wird. Anschliessend positioniert sich Frau Steffen als Daraus-gelernt-Habende und gleichzeitig auch als Noch-in-Entwicklung-Begriffene. In ihrem Redebeitrag zeigt sie die eigenen Unzulänglichkeiten auf und lässt dabei ebenso ihre Bescheidenheit, ihre Lernbereitschaft und die Prozesshaftigkeit ihrer Ausbildungssituation sichtbar werden. Ihre Ausführungen betonen den Prozess mehr als die bereits erreichte Expertise. Dies deutet darauf hin, dass sie die Situation nicht explizit als Prüfungssituation rahmt.

Aus einer gesprächsdynamischen Perspektive betrachtet ist der Zeitpunkt bemerkenswert, an dem Herr Bucher Frau Steffen ins Wort fällt. Interaktionslogisch leitet sie an dieser Stelle mit weil eine Begründung ein, die von ihm jedoch abgeklemmt wird. Mit der Unterbrechung - so die naheliegende Vermutung - reagiert er auf und es ist halt schwierig. Die Antwort der Studentin auf die Frage wie zeigen sich Probleme in diesem Kompetenzbereich führt beim Mentor, bewusst oder unbewusst, zu einer Sprechüberschneidung, so dass ihre angefangene Begründung nicht weiter ausgeführt wird.

Insgesamt weist die Gestalt gewinnende Figur eine ambivalente, uneindeutige Selbstpositionierung auf. Das Prozesshafte im Modus von «am Anfang war es schwer aber mittlerweile beherrsche ich es» dominiert. Gleichzeitig zeigt sie mit der Formulierung ich hab jetzt etwas gut erklärt auf, dass sie sich noch im Prozess befindet. Der zeitliche Begriff jetzt unterstreicht die Aktualität ihrer Erläuterung.

Herr Bucher: //wo setzen Sie da an beim Training?//

Losgelöst vom Kontext ist die Unterbrechung eines Redebeitrages mit der Frage wo setzen Sie da an beim Training vorstellbar, wenn etwa der Fragesteller mit dieser Frage die angesprochene Person zu einer eigenen Erkenntnis hinführen möchte. Würde die Frage der Logik einer Fachwissensüberprüfung folgen, wäre im Kontrast zur hier realisierten Variante eine Formulierung unter Auslassung des fallspezifischen da wie etwa in "Wo 
setzen Sie an beim Training?» oder «Wo würden Sie beim Training ansetzen?» erwartbar. Die vorgefundene Variante zielt allerdings nicht primär auf eine Wissensantwort ab. Da fungiert als Hinweis auf eine konkrete Situation. Die entsprechende Auseinandersetzung erfordert folglich eher Handlungskompetenz und Praxiswissen anstelle von Fachwissen. Die Frage wo setzen Sie da an beim Training scheint auf den ersten Blick indikativ für ein mäeutisch-sokratisches Hervorlocken einer Handlungsanleitung (Oevermann, 1996b, S. $156 \mathrm{ff}$.$) . Eine latente Prüfungsimplikation haftet der Frage weiter an und gewinnt in$ diesem Sprechakt erneut Gestalt, insbesondere weil sich die Frage kompetent oder auch weniger kompetent beantworten lässt. Dennoch gilt es zu konstatieren, dass der Prüfungsduktus nicht auf eine Wissensabfrage abzielt, sondern als Quasi-Fallbezug zu lesen ist.

Die Frage nach einem Entwicklungsimpuls übt implizit Druck auf die gefragte Person aus, da sie im Modus einer Aufforderung erfolgt. Der entsprechende Imperativ ist «Zeig dich als Person, die aus ihren Erfahrungen lernt, entsprechende Konsequenzen zieht und sich nicht in der Aussage es ist halt schwierig verliert!»

Inhaltlich wird durch die der Trainingslogik zugrunde liegenden Struktur eine Behebbarkeit oder Bearbeitbarkeit der Schwierigkeit suggeriert. Die angesprochene Person sieht sich mit einer Anstrengungszumutung konfrontiert. Die emergierende Gesprächsdynamik lässt an dieser Stelle eine Zunahme im Bewährungsimperativ erkennen. Die direkte Ansprache wo setzen Sie da an beim Training erhält durch das Sie auch eine subtile Provokation, die sich über die neutrale Kontrastformulierung «wo kann man da beim Training ansetzen» aufzeigen lässt.

Unter Berücksichtigung dieser Überlegungen bedarf die Prüfungslesart einer Modifikation. Nicht die Abfrage des Richtigen, wie bei der klassischen Prüfungsidee, sondern die Hinführung zum Richtigen erscheint als zentrale Interaktionsfigur. Diese Figur erinnert an die «Hebammenkunst», wie die mäeutisch-sokratische Fragetechnik von Sokrates selbst beschrieben wurde (Bühler, 2012). Mit dieser Fragetechnik wird die befragte Person zu einer Einsicht hingeführt (Wenzl, 2014, S. 131 ff.). Auch in diese Gesprächsdynamik - in Analogie zum Mentoratsgespräch zwischen der Studentin Tina und dem Mentor Michael - schreibt sich eine Richtig-falsch-Logik als zentrales Merkmal der Erkenntnisgewinnung 
und Richtungsweisung in die Struktur ein. Der mäeutisch-sokratischen Fragetechnik haftet damit eine Prüfungsimplikation $\mathrm{an}^{43}$.

Die Dynamik, die hier Gestalt gewinnt, lässt sich als «ich bringe dich dahin, wo du das Richtige selber erkennst» umreissen. Dies erinnert an die Konzeption von "people processing», die bereits in der Einleitung erläutert wurde. Es gilt im weiteren Gesprächsverlauf zu klären, inwiefern die vorgefundene Dynamik Parallelen zur «people processing»-Figur aufweist, die Hughes (1963) in Hinblick auf professionelle Praktikerinnen und Praktiker und ihre Klientinnen und Klienten beschreibt. Weiter gilt es zu ergründen, ob der Zugriff, wie im «people processing» angedacht, auf die Rollenspezifik begrenzt ist oder ob sich diffus auf die ganze Person ausdehnt.

Es gilt nun aufzuzeigen, welche Implikationen die Frage nach dem Ansatzpunkt des Trainings für die zweite noch vorhandene Lesart, die der Sichtbarwerdung, hat. Im Unterschied zu einem wissenschaftlichen Diskurs, in dem sich Proposition und Opposition aufeinander beziehen, geht es beispielsweise bei einem Polittalk nicht primär um den Austausch von Argumenten, sondern vorrangig um ein charismatisches Sich-Zeigen, das im Idealfall Zustimmung auf Seiten des Publikums hervorruft. Der Moderator agiert dabei in seiner Rolle, ein Sichtbarwerden der eigenen Person kann, muss aber nicht stattfinden. Im vorliegenden Mentoratsgespräch erlangt das fiktive Publikum, das bereits zu Gesprächsbeginn mit ich begrüsse Sie recht herzlich zum Mentoratsgespräch adressiert wurde, erneut Raum. Dies würde die Hypothese der Inszenierung stützen. Während die mäeutisch-sokratische Fragetechnik auf eine eigene Erkenntnis der befragten Person als Endpunkt abzielt, steht bei der zweiten Lesart nicht der Endpunkt, sondern der Prozess an sich und die Sichtbarwerdung in dessen Rahmen im Vordergrund. Die Frage wo setzen Sie da an beim Training weist im realen Mentoratsgesprächskontext einen Interventionscharakter auf und stellt einen Impuls mit dem Imperativ «Machen Sie mal bei diesem Thema weiter!» dar. Sie ist Ausdruck des (un-)bewussten Anliegens auf die Studentin einzuwirken.

Grundsätzlich bestünde in der Situation die Möglichkeit einen Interventionsimpuls im Modus des Zeigens zu vermitteln und der angesprochenen Person die eigene Expertise

\footnotetext{
${ }^{43}$ Für die mäeutisch-sokratische oder eben nur vermeintlich mäeutisch-sokratische Fragetechnik siehe ausführlich die vorausgehende Fallrekonstruktion des Mentoratsgesprächs zwischen der Studentin Tina und dem Mentor Michael.
} 
zur Verfügung zu stellen. Hierbei könnte auf die Bemerkung es sei halt schwierig reagiert und aufgezeigt werden, welche Optionen für die Problembearbeitung zur Verfügung stünden. Stattdessen wird faktisch eine Frage formuliert, die im Modus des mäeutischsokratischen Hervorlockens die Studentin anhält, die Intervention selbst zu artikulieren und zu gestalten.

Die Dynamik der Gesprächsinteraktion lässt sich unter dem Anspruch oder der Ausbildungsidee des Mentors wie folgt fassen: Sein Sprechakt stellt eine Aufforderung zur Sichtbarwerdung dar. Der Mentor - in der spezifischen Rolle des Aufforderers - greift einen Punkt aus den vorausgehenden Ausführungen der Studentin auf. Er hakt nach und formuliert eine Anschlussfrage. Auf einer latenten Bedeutungsebene impliziert diese Anschlussfrage eine Dekonstruktion, anhand derer Vermittlungs- respektive Zeigeansprüche eingelöst werden.

Im Anschluss wäre zu erwarten, dass auf die Frage Bezug genommen und konkret und praxisorientiert erläutert wird, welche diesbezüglichen Strategien verfolgt werden. Dieser Anschluss käme der anmoderierten Interventionslogik nach. Dabei würde sich die Angesprochene der Bewährungsdynamik aussetzen und die ihr zugeteilte Position bestätigen.

Ebenfalls denkbar wäre ein Aussteigen aus der anmoderierten Interventionslogik respektive der Versuch eines kooperativen Entziehens. Dies wäre beispielsweise durch eine Zustimmung ohne eine potenzielle Dekonstruktionsfigur möglich und könnte mit einem Anschluss wie «das hätte ich gerne mit Ihnen besprochen, was es da für Optionen gäbe» erfolgen. In dieser Anschlussoption würde Herr Bucher als Coach angesprochen.

Frau Steffen: aso wie meinen Sie das?

Herr Bucher: dass man Kinder eben dort trifft und dort abholt wo sie wo sie es verstehen können dass man eine adäquate Sprache hat trainiert man dann die Sprache oder trainiert man die Planung und die Vorbereitung?

Die Rückfrage, wie das gemeint sei, signalisiert eine Irritation. Frau Steffen zeigt, dass die Aussage nicht eindeutig verständlich ist. Sie fragt selbstbewusst zurück und bringt Herrn Bucher dadurch in Zugzwang. Die Rückfrage wird seitens des Mentors bearbeitet, indem eigene Ideen im Modus von Handlungsanleitungen aufgezeigt werden. Auf diese Weise 
wird die Studentin zu einer potenziellen Antwort hingeführt. Die Erläuterungen stehen programmatisch für den vermittelten Inhalt. Sie lassen sich als Versuch - konkret: als Training - lesen Frau Steffen mit einer adäquaten Sprache an ihrem derzeitigen Standort abzuholen. Im zweiten Teil seiner Aussage wird erneut eine Frage formuliert und inhaltlich nach dem passenden Trainingskonzept gefragt, welches zu einer adäquaten Sprache verhilft. Dabei wird ein Scaffolding mit zwei Auswahlantworten angeboten.

Generell lässt sich feststellen, dass die Bewährungsdynamik anhält. In dieser Dynamik zeigen sich sowohl Strukturelemente der Logik der Befragung zwecks Sichtbarwerdung wie auch Strukturelemente der Prüfungslogik.

Die Überlegungen lassen es zu die zwei noch verbleibenden, bereits spezifizierten Lesarten - Befragung zwecks Positionierung und Prüfung respektive Hinführung zum Richtigen - unter einer Figur zu subsummieren. Diese Figur einer Prüfung und Bewährung in der Anfechtung bedient sowohl das Strukturmerkmal der Prüfungs- und Hinführungsimplikation, als auch das des Sich-Zeigens, das mit der Anfechtung weiter beschrieben wird.

Die Interaktionsdynamik lässt sich in folgender Weise beschreiben: Die Studentin wird geprüft. Es wird aber nicht primär Wissen, sondern die Standhaftigkeit der Studentin, geprüft. Alltagssprachlich formuliert wird im Fragemodus getestet wie sich Frau Steffen in der Konfrontation respektive in der Anfechtung (schlägt). Diese Dynamik beinhaltet auch eine Positionierungs- und Sichtbarwerdungsimplikation.

Zurück zum konkreten Gesprächsverlauf und zu Frau Steffens Antwort auf die Frage, ob man dann die Sprache oder die Planung und die Vorbereitung trainiere.

Frau Steffen: ich denke die Planung und die Vorbereitung dass man eben weiss wie man sich ausdrücken möchte und welche Wörter man verwenden möchte dass es die Kinder verstehen aber auch muss man die Sprache in der Schule so anpassen dass man eigentlich immer dann so spricht dass es für die Kinder verständlich und dass sowie auf ihrem Sprachniveau ist

Statt der Auswahl einer der beiden Optionen werden beide Aspekte in die Antwort aufgenommen. Diese wird mit der Einleitung ich denke vorsichtig und subjektiviert artikuliert. Dadurch wird die Bereitschaft hergestellt sich eines Besseren belehren zu 
lassen, falls die Antwort nicht der gesuchten entspricht. Die Aussagen werden unter der Verwendung von man generalisiert und als universell gerahmt. Die eigene Person wird aus der Schusslinie genommen und es zeigt sich eine Distanzierungsbewegung zum Gesagten. Der Sprechakt weist sprachliche Holprigkeiten und redundante Teile auf. Inhaltlich entspricht er einer Schilderung von konkreten Handlungsanleitungen, wie die Entwicklungsaufgabe - in der Planung und Vorbereitung eine adäquate Sprache zu trainieren (Zitat Bucher im vorausgehenden Sprechakt) - zu bewerkstelligen sei. Im Sprechakt zeigt sich Frau Steffen kooperativ, liefert kaum Angriffsfläche und führt die ihr zugetragene Aufgabe einvernehmlich aus.

Herr Bucher: wie realisiert man ein Sprachniveau

Die Frage folgt einer ähnlichen Struktur wie die vorangehende - wo setzen Sie da an beim Training -, mit der Frau Steffen direkt und fallspezifisch angesprochen wurde. In dieser Formulierung findet eine Wegbewegung von der Fallspezifik statt und mit man wird nach einer generalisierten Art und Weise ein Sprachniveau zu realisieren gefragt. Die Frage zielt auf praktischen Konsequenzen und enthält ein imperatives Momentum, das sich mit «Werden Sie konkret!» umschreiben lässt. Inhaltlich wird an dieser Stelle nach einer Intervention gefragt. Der Studentin wird erneut durch mäeutisches Hervorlocken zu einer Selbsterkenntnis verholfen. Um die Struktur der Frage herauszuarbeiten, wird abermals auf das Mittel des gedankenexperimentellen Kontrastes zurückgegriffen. Die Frage wird mit einer Formulierung verglichen, die ein Füllwort im Sinne eines \&Geschmeidigmachers` enthält. Die Frage hiesse dann beispielsweise «Wie realisiert man eigentlich ein Sprachniveau?» Sie wäre in dieser Form abgemildert und eher auf Diskurs oder Interesse des Fragestellers ausgerichtet. Die vorgefundene Kurzversion wie realisiert man ein Sprachniveau hat die Funktion der Person auf den Zahn zu fühlen, da der Frage immanent eine (subtile) Provokation anhaftet. Zugespitzt formuliert zeigt sich hier die Figur einer anhaltenden Irritation oder auch Provokation, der die Studentin interaktionsdynamisch ausgesetzt wird.

Angesichts des Faktums, dass sich die bereits freigelegten Strukturmerkmale reproduzieren, wird die nächste Sequenz, die Antwort Frau Steffens auf die ihr gestellte Frage, grossflächig rekonstruiert. 
Frau Steffen: ja man hört ja selber welche Wörter dass sie verwenden und was sie alles wissen können aso auch von zu Hause mitbringen und da gibt es einige die sicherlich mehr Wissen haben und mehr Fremdwörter kennen und andere die halt wenn sie eine andere Muttersprache haben oder so da vielleicht Defizite haben und da könnte man auch wenn man solche Kinder hat die nicht super Deutsch können zuerst eine normale also normale ((Studentin formt mit Fingern Anführungszeichen)) Einführung geben und dann noch zu diesen Kindern gehen und es ihnen noch einmal ganz langsam und ruhig nochmals erklären und ich glaube es ist auch immer wichtig dass man nicht zu viel auf einmal sagt sondern nur die nächste Anweisung und dann wieder die nächste weil wenn man sagt das und das und das und das und das dann wissen sie schon nicht mehr was sie als erstes machen müssen und wenn man ihnen einfach den nächsten Auftrag gibt und sie sich nur auf das konzentrieren müssen dann (.) glaub ich ist das Gelingen auch besser dass dann alle das Richtige machen.

Mit der Formulierung ja man hört ja selber - in der Bedeutung von «das merkt man ja selber» - reagiert Frau Steffen mit einer Selbstbehauptungsgeste auf die subtile Provokation, wobei ihrer Sprechweise ein Unterton anhaftet. Dieser verschafft sich in den beiden Jas und dem Selbstbezug durch selber Ausdruck. Es wird eine Offensichtlichkeit angesprochen, die man aus ihrer Perspektive nicht übersehen kann. Die Studentin (fängt) sich umgehend und zeigt in der Fortsetzung auf, dass sie bei der Realisierung eines Sprachniveaus über Faustregeln verfügt. Diese führt sie im Sprechakt konkret aus. Ihre Darstellungen weisen erneut den Charakter von Handlungsanweisungen und praktischen Tipps auf, die ihr die Einsozialisation und den Einstieg in ihren künftigen Beruf erleichtern. An dieser Stelle scheint es lohnenswert, sich die Gestaltung der Sichtbarkeit des Mentors - in seiner Rolle und als Person - anzuschauen. In einer alltäglichen Formulierung lauten die Imperative seiner Frage nach dem Trainingsansatz wie auch nach der Realisierung des Sprachniveaus «Zeig dich, wie du dich in der antizipierten Situation bewährst!» Interaktionslogisch wäre als Reaktion seinerseits eine (minimale) Form der Evaluation oder Kommentierung der studentischen Ausführungen erwartbar. Eine solche bleibt allerdings bei den bisher gestellten Fragen aus. Dieses Ausbleiben scheint als Befund bedeutsam. 
Um die Dynamik der konsequenten Nicht-Reaktion ${ }^{44}$ des Mentors zu erfassen, wird erneut die mäeutisch-sokratische Fragetechnik als Vergleichsfolie herangezogen. Bisher wurde diese vor allem in Hinblick auf die Selbsterkenntnis der befragten Person als Referenzpunkt genutzt. An dieser Stelle geht es allerdings nicht primär und die Hinführung zur Selbsterkenntnis, sondern mehr um das interaktive Erschliessen des Themas zwischen der fragestellenden und der befragten Person in der Dialogpraxis an sich (Schäfer, 2013). Herr Bucher nimmt die Rolle eines entsprechenden Dialogpartners nicht ein. Seine Interventionen - mittels Fragen oder Provokationen - können als Verweigerung einer diskursiven Gesprächspraxis bezeichnet werden. Seinerseits ist keine Bereitschaft erkennbar, sich selbst als Mentor zu positionieren und einen Standpunkt, zu dem sich die Studentin ihrerseits wieder verhalten könnte, einzunehmen. Die Aufforderungen an die Studentin, sich als Person zeigen, gehen folglich mit einer Verweigerung des Mentors einher, als eigene Person sichtbar zu werden und selbst in Beziehung zu treten.

Anhand der erkennbaren Dynamik kann ein weiteres Strukturmerkmal der Interaktion formuliert werden. Die Studentin wird einseitig adressiert und aufgefordert sichtbar zu werden und sich zu positionieren. Währenddessen bleibt der Mentor strenggenommen positionslos und entzieht sich dadurch der Rolle eines Gegenübers. Wie bereits ausgeführt weisen die Fragen und Aussagen des Mentors implizit auch eine mehr oder weniger starke Provokation auf. Eine Provokation zielt konstitutiv auf die ganze Person ab. Die Studentin wird aus einer interaktionslogischen Betrachtungsweise in ihrer ganzen Person adressiert und irritiert. Um die Dynamik des Auf-die-ganze-Person-Spielens fassbar zu machen, lässt sie sich in einem Gedankenexperiment mit der Dynamik eins therapeutischen Settings kontrastieren. Ein Therapeut geht mit seiner Klientin eine diffus-spezifische Sozialbeziehung ein. Er fordert die Klientin auf sich gemäss der Strukturlogik dieser Sozialbeziehung zu verhalten und alles zu thematisieren, was ihr durch den Kopf gehe, selbst wenn ihr dies peinlich sei. Diese Grundregel gilt allerdings nicht nur für die Klientin, sondern ebenso für den Therapeuten. Das äussert sich in Gestalt von Gegenübertragungsgefühlen. Auf dieser Gegenseitigkeit beruht das Arbeitsbündnis zwischen dem Therapeuten und der Klientin (Oevermann, 1996b, S. 117, vgl. auch Oevermann, 2002b,

\footnotetext{
44 In Analogie zu Watzlawiks geflügeltem Wort «Man kann nicht nicht kommunizieren» (Watzlawik zitiert nach Jackson et al., 1996, S. 53) ist hier die Nicht-Reaktion als Reaktion zu verstehen.
} 
S. 42 ff.). Eine analoge Gegenseitigkeit ist im Gespräch zwischen Frau Steffen und Herrn Bucher nicht erkennbar. Es konturiert sich an dieser Sequenzstelle ein bedeutsames Strukturmoment: eine Vermeidungsfigur von Gegenseitigkeit. Dies lässt die Konstellation notgedrungen einseitig und auch machtvoll erscheinen. Was sich in der Therapie als gegenseitig verpflichtendes Arbeitsbündnis beschreiben lässt, ist hier im Gegensatz eine einseitige Intervention, die keiner Zustimmung der Betroffenen bedarf.

Herr Bucher: eine theoretische Überlegung ich geb's zu dass die nicht so praktisch ist aber wie wie lösen Sie das Problem wenn ich definiere Lern/Lernen bedeutet Schwierigkeiten überwinden was müssten Sie eigentlich in der Sprache immer ein bisschen voraus sein oder vom Niveau her angehoben sein natürlich auch unterschiedlich wenn die Kinder unterschiedliche Niveaus haben aber eigentlich würde ich Ihrer These widersprechen dass man die Kinder treffen muss

Die Bewegung weg von den ausführlichen Erläuterungen Frau Steffens - ohne jegliche Form der Bezugnahme - stellt einen Bruch dar. Herr Bucher leitet die Bewegung mit dem Metakommentar eine theoretische Überlegung ein. Sein Sprechakt bleibt im Duktus der Aufforderung sich zu zeigen verhaftet. Die Bemerkung - eine theoretische Überlegung die zugegebenermassen nicht so praktisch ist - lässt auf einer latenten Ebene Kritik an der handlungspraktisch orientierten Haltung von Frau Steffen zum Ausdruck kommen. Sie kann als implizite Andeutung verstanden werden, sich auch für theoretische Überlegungen zu öffnen. Die Figur der angestrebten Fachlichkeit manifestiert sich sprachlich in der Kennzeichnung ihrer vorherigen Aussage als These. Damit erkennt er Frau Steffen als Gesprächspartnerin an und versteht das Gespräch als Diskussion, in der er ihrer These widerspricht respektive widersprechen würde.

Die einleitende Aussage ich definiere kündigt eine relevante, wissenschaftliche Festlegung an. In der Fortsetzung - Lernen bedeutet Schwierigkeiten überwinden - weist der Sprechakt die Gestalt einer Parole auf, da kein fachlicher Hintergrund für die Definition berücksichtigt wird. Er enthält erneut provokative Anteile und stellt Frau Steffen die Aufgabe, sich zu dessen Inhalt zu verhalten. In den Formulierungen ich definiere wie auch theoretische Überlegung gewinnt wiederholt das Strukturmerkmal der angestrebten Fachlichkeit Gestalt. Diese wechselt an mehreren Gesprächsstellen auf die Ebene einer 
Praxisbetrachtung. Im Hin- und Herwechseln zwischen Ebenen und Themen scheint eine Figur auf, in der das Versetzen in einen Argumentationsmodus ein Primat über die sorgfältige Bearbeitung von Themen erlangt. Gewagt formuliert kann die Dynamik des Vorgehens des Mentors als Intervention gedeutet werden, um Frau Steffen in Anlehnung an die Propädeutik, also an die Einführung in die Vorkenntnisse eines Studiums - in die Methodik und Sprache des Argumentierens einzuführen. Dabei scheint die Form wichtiger als der Inhalt. Der zentrale Imperativ der Intervention «Denk nach, bezieh Stellung, rechtfertige dich und werde sichtbar!» erinnert an den Kant'schen Leitspruch der Aufklärung «habe Mut, dich deines eigenen Verstandes zu bedienen» (Büchmann et al., 2007, S. 330). Sich «im stillen Kämmerlein` seines Verstandes zu bedienen reicht nicht aus. Es geht um Sichtbarkeit, Positionierung und Bewährung. Das bedeutsame Moment, das die zentrale Interaktionslogik der realisierten Figur beschreibt, ist die bemerkenswert persistente Aufforderung sich zu zeigen. Durch Fragen, theoretische Überlegungen, subtile Provokationen oder Widersprechen einer unterstellten These wird dem Gegenüber eine Stellungnahme entlockt, anhand derer sich etwas (auf-)zeigen lässt. Diese Dekonstruktion der studentischen Aussagen erinnert an einen Modus von «people processing», in dem die Selbstreflexionen und subjektiven Dispositionen der Studentin offengelegt werden. Die dahinterliegende Annahme ist, dass über diese Irritationen und Provokationen Erkenntnisprozesse angeregt, beschleunigt und Einsichten gewonnen werden. Das eigene (studentische) Tun und Denken gilt es grundsätzlich zu hinterfragen. In der resultierenden, skeptischen Haltung wird Selbstreflexivität zu einer Zentralkompetenz. Vergleichbare Ziele verfolgt auch die Entwicklung eines forschenden Habitus (z.B. Neuweg, 2013) oder die Kasuistik (z.B. Hummrich, 2016). Im Gegensatz zur vorgefundenen Interaktionsfigur liegt in diesen beiden Formaten der Fokus auf der Bearbeitung eines externen Falls. Hier allerdings muss sich die Studentin selbst zum Fall machen. Insgesamt verschafft sich ein hoher Anspruch von Herrn Bucher Ausdruck, Frau Steffen in die Welt der (Academia), der Gemeinschaft der Studierten und Gelehrten im Bereich der höheren Bildung einzuführen. Die entsprechenden Bemühungen und das hohe Engagement seitens des Mentors lassen sich als klares Indiz lesen, dass die angestrebte Performanz nicht lediglich der aktuellen Sichtbarwerdung dient, sondern eine weiterreichende Wirkkraft für zukünftige Herausforderungen entfalten soll. Dies deckt sich mit der «people processing»-Konzeption. 
Zurück zur konkreten Gesprächspassage. Auf einer interaktionslogischen Ebene ist der Sprechakt dahingehend bemerkenswert, dass Herrn Buchers Gedanken in einem ersten Schritt zu Frau Steffens These gemacht werden und in einem zweiten Schritt dieser These von ihm selbst widersprochen, respektive diese dekonstruiert wird. Dieses Vorgehen lässt sich als Versuch deuten, eine Positionierung aus Frau Steffen ‘herauszukitzeln ) und dadurch ihre Professionalität und Sattelfestigkeit zu überprüfen.

Diese Inszenierungslogik, wie auch die Vermeidung, eine eigene Position zu beziehen, scheinen auch in der Formulierung eigentlich würde ich Ihrer These widersprechen durch. Der Mentor widerspricht nicht wirklich, denn mit einem Widerspruch würde er seinerseits eine diskursive Position einnehmen und die Studentin könnte sich ihrerseits darauf beziehen. Vielmehr formuliert er den Widerspruch als Option, derer er sich entzieht und dabei seine Inszenierung entlarvt.

In Summe ist Herr Buchers Aussage, die er mit einer vermeintlich theoretischen Überlegung beginnt und einem optionalen Widerspruch beendet, inhaltlich nicht vollständig zu erschliessen. Überlegungen, Definitionen und Thesen beziehen sich nur teilweise aufeinander.

Frau Steffen: aso //man kann sicher//

Herr Bucher: //Sie müssen// ja im Pädagogischen immer einen kleinen Schritt

Frau Steffen: ja einen kleinen sicherlich

Herr Bucher: voraus sein

Frau Steffen: das finde ich auch

Frau Steffen steigt abermals mit aso ein, nimmt das Vorausgegangene auf und führt es, angelehnt an "also es ist so dass...», weiter aus. Ihre Aussage beginnt sie mit einer neutralen und universellen man-Formulierung. Sie kann diese nicht vollenden, da sie von Herrn Bucher mit einer ähnlichen Metapher wie zuvor unterbrochen wird. Vorher sprach er davon eigentlich in der Sprache immer ein bisschen voraus sein zu müssen. An dieser Stelle sagt er, dass man im Pädagogischen ja immer einen kleinen Schritt voraus sein muss. In beiden Aussagen wird mit eigentlich oder ja auf einer latenten Ebene der argumentative Punkt, an dem Frau Steffen anzusetzen hätte, aufgezeigt, damit er seinerseits dann wieder daran anknüpfen kann. 
Die Antwort Frau Steffens - ja einen kleinen sicherlich - sorgt für die Anschlussfähigkeit an ihr eigenes Statement - die Kinder in einer möglichst für sie verständlichen Sprache anzusprechen. Hier ergibt sich für sie die Möglichkeit zuzustimmen, ohne dabei die eigene, vorangegangene Aussage zu dementieren, da der diskutierte pädagogische Vorsprung nur klein ist und dies insgesamt nicht im Widerspruch zu ihrer Ausführung steht. Auf einer manifesten Betrachtungsebene agiert sie kooperativ, indem sie die Güte seines Argumentes anerkennt. Da sie jedoch nicht in eine Diskussion einsteigt, verweigert sie sich auf einer latenten Ebene jedoch seiner Aufforderung.

Auffallend ist in der Fortsetzung, dass Herr Bucher, obwohl Frau Steffen ihm bereits zugestimmt hat, seinen Gedanken weiter ausführt. Dies lässt sich als Reaktion auf das Nichteinsteigen von Frau Steffen lesen. Seine Bemühungen und Anstrengungen Frau Steffen in einen reflexiven, vor allem aber ihm widersprechenden Modus zu versetzen, setzt er fort. Dieser Aufforderung kommt sie erneut nicht nach, sondern führt eine Schliessbewegung aus, indem sie mit der minimalen, nicht reflexiven Einschätzung das finde ich auch zustimmt.

Frau Steffens verhaltene Reaktion deutet an, dass sie Herrn Buchers Vorgehen oder gewagter formuliert sein Spiel mit zunehmender Gesprächsdauer immer mehr durchschaut. Sie will ihm nicht ins Messer laufen und entzieht sich taktisch. Ihre Zustimmung lässt sich als Absage an das vermeintliche Streitgespräch deuten, wodurch sie sich vor einer möglichen Dekonstruktion ihrer Aussage schützt. Allegorisch lässt sich dies als individueller Entzug aus einer Spielpraxis beschreiben, in der die Regeln entweder nicht bekannt sind oder diesen nicht zugestimmt wird.

In einem supervisorischen oder therapeutischen Setting - als Kontrastfolie - wäre die Offenlegung der Spielregeln sowie die Zustimmung aller Beteiligter unumgänglich. Dies ist in der vorliegenden Interaktion nicht gegeben und stellt eine Erklärung für Frau Steffens Schliessbewegung qua Zustimmung dar.

Herr Bucher: was würde das wenn das theoretisch wenn meine These stimmt (.) was würde das für Ihre Aussagen vorhin für Konsequenzen haben Frau Steffen: aso für die Aussagen ist es eigentlich noch dasselbe 
Bleibt man bei der Allegorie des Spiels, zeigt sich mit Herrn Buchers nächstem Sprechakt, dass er nicht aufgibt, Frau Steffen in sein Spiel zu verwickeln. Er stellt die hypothetische Frage - wenn meine These stimmt -, räumt also die Option ein, dass diese nicht stimmen könnte. Dabei verrät er erneut seine didaktische Inszenierung. Wenn er diese These wirklich verträte, würde er die Negation nicht selber andenken.

Frau Steffen weicht erneut dem Streitgespräch aus. Herr Bucher spricht von Ihre Aussage. Frau Steffen modifiziert Ihre Aussage zu die Aussage und distanziert sich von der Zuschreibung, dass diese die ihre sei. Sie bietet keinen Widerstand und keine weitere Dekonstruktionsfläche. Ihre Antwort lässt sich zusammenfassend als kooperative Zurückweisung seiner Aufforderung, sich selbst erneut infrage zu stellen, lesen.

Da sich in der Interaktionsdynamik gewisse Strukturmerkmale reproduzieren, scheint es angebracht ein Zwischenfazit zu ziehen und anschliessend eine riskante Fallstrukturhypothese der zweiten Sequenz zu formulieren.

\subsubsection{Zusammenfassung und riskante Fallstrukturhypothese der Sequenz Aber eigentlich würde ich Ihrer These widersprechen}

Die Eigenlogik der Strukturdynamik der zweiten, rekonstruierten Gesprächspassage lässt sich wie folgt beschreiben: Frau Steffen und Herr Bucher sind bezogen auf den zweiten Kompetenzbereich eines Ausbildungskonzepts miteinander im Gespräch. Frau Steffen wird in einer Art und Weise adressiert, die sie argumentativ herausfordert, als gelte es die Studentin in einen selbstreflexiven Zustand zu versetzen. Ihre diesbezüglichen Fähigkeiten und Kompetenzen sollen hervorgelockt und sichtbar gemacht werden. Diese Vorgehensweise erinnert an die Figur von «people processing», in der die rollenförmige Personalität sozial passfähiger gemacht wird. «People processing» findet hierbei durch die Konfrontation mit Provokationen, unterstellten Thesen, eingeforderten Stellungnahmen zu pädagogischen Konzepten und mäeutisch-sokratischem Hervorlocken statt. Dadurch wird Frau Steffen in Begründungspflicht gebracht. Sie muss sich in der Gesprächsinteraktion gegenüber Herrn Bucher bewähren. Der Mentor begegnet der Studentin im Gespräch mit einem Interaktionsmuster, das durch Intervention die Aktivierung von personalen Ressourcen anregt, um im Sinne eines Ausbildungsanspruchs ein Sich-Zeigen, Sich-Verteidigen und Sich-Positionieren hervorzurufen. 
Im Gegensatz zum konzeptionell angedachten Modus des «people processing», in dem ein spezifisch rollenförmiger Zugriff auf die Person erfolgt, zeigt sich empirisch, dass auf die Person der Studentin in ihrer ganzen Diffusität zugegriffen wird. Diesem Zugriff kann sie sich nur mit Aufwand entziehen. Er setzt die Einsicht und Zustimmung der betroffenen Person nicht voraus, sondern kann als notwendiges Übel einseitig vorgenommen werden. In der vorliegenden Gesprächsinteraktion wird die Studentin mit Fragen und Thesen konfrontiert, zu denen sie sich verhalten muss. Sie ist aufgefordert Verteidigungs- wie auch Argumentationsfähigkeit unter Beweis zu stellen. Eine zugrunde liegende Orientierung könnte sein, dass sich durch Dauerirritation ein Erkenntnisprozess auslösen oder beschleunigen lässt. Da diese Strategie nicht artikuliert oder offengelegt wird, erhält sie in der Umsetzung auch einen Aspekt des Vorgeführt-Werdens, dem zugespitzt formuliert auch etwas Unfaires anhaftet.

Die Aussagen und Antworten der Studentin werden in der vorliegenden Gesprächsinteraktion seitens des Mentors weder kommentiert noch evaluiert. Herr Bucher ist bemüht, die Studentin in ihrem Handeln und ihrer Selbsthaltung prinzipiell infrage zu stellen. Diese Strategie lässt sich riskant als Dauerirritation und Zwang zur Dauerselbstinfragestellung beschreiben.

Im Gesprächsverlauf wechselt der Mentor von der Ebene des Fachgespräches zunehmend zu einer Ebene der Konfrontation mit praktischen Problemstellungen. Der Sprechmodus des Mentors erweist sich als brüchig und changiert zwischen formal-distanziert und alltagssprachlich-praxisnah. Auf einer manifesten Ebene kann die Adressierung der Studentin als weitgehend spezifisch und rollenförmig angesehen werden. Dennoch weist der Adressierungsmodus in der Latenz auch diffuse Implikationen auf und zwar dann wenn mit Provokationen, Irritationen und Dekonstruktionen auf die ganze Person der Studentin gespielt wird. Der Mentor agiert seinerseits rollenspezifisch und wird als Gesprächspartner und Person nicht fassbar.

Die Logik der Gesprächsinteraktion wechselt von Abfrage zu Beginn der Sequenz - wo liegen dort die Probleme; wie zeigen sich Probleme in diesem Kompetenzbereich - hin zu Intervention - wo setzen Sie da an beim Training oder wie realisiert man ein Sprachniveau - und Provokation - aber eigentlich würde ich Ihrer These widersprechen dass man die Kinder treffen muss. Die Möglichkeit für die Studentin, dem Mentor ebenfalls durch Provokationen zu begegnen, gestaltet sich einerseits aufgrund des hierarchischen Gefälles 
resultierend aus der Ausbildungssituation, andererseits auch durch die Positionslosigkeit des Provokateurs als kaum realisierbar. Äusserlich betrachtet scheint die etablierte Gesprächsanlage über weite Strecken zu funktionieren. Frau Steffen bezieht Position und teilt ihr Wissen und ihre Problemlösestrategien mit. Dabei werden die anspruchsvollen Fragen Herrn Buchers teilweise mit seiner Unterstützung beantwortet. An gewissen Stellen im Gespräch wechselt Herr Bucher in einen Monologmodus, führt seine Fragen im Sinne einer Hilfestellung erklärend aus und beantwortet diese streckenweise selbst. In der Latenz werden an diesen Gesprächspassagen seine eigenen Konzepte und Normativitätsvorstellungen erkennbar. Die Dynamik, in der Interaktion keine eigene, streitbare Position zu beziehen, wird nicht durchgängig aufrechterhalten. An gewissen Stellen wird sie brüchig, beispielsweise wenn das Hervorlocken einer Erkenntnis anspruchsvoll ist und die mäeutisch-sokratisch formulierte Frage respektive Provokation die eigenen Normativitätsvorstellungen durchscheinen lässt.

An genau diesen Stellen wäre ein echter mäeutisch-sokratischer Dialog möglich. Dafür müsste der Mentor allerdings selbst eine Position beziehen und die Studentin bräuchte Raum, um sich ihrerseits dazu verhalten zu können. Voraussetzung dafür wäre, dass die Studentin innerhalb der sozialen Situation eine eigene Position vertreten und dem Mentor auch widersprechen könnte. Allerdings wäre es in diesem echten sokratischen Dialog auch denkbar, dass die gemeinsam gefundenen Erkenntnisse nicht identisch mit den Erkenntnissen sind, die der Mentor zuvor bestimmt hat. Von der Gesprächsdynamik her betrachtet scheint jedoch die Hypothese zulässig, dass dieser echte sokratische Dialog seitens des Mentors nicht angedacht ist und deshalb auch nicht ausgeführt wird.

Als weiteres Strukturmerkmal gilt festzuhalten, dass sich im Gesprächsverlauf die Selbstpositionierung der Studentin verschiebt. Während Frau Steffen zu Beginn mitspielt, antwortet sie später zunehmend bejahend. Die lässt sich als Ausstieg aus dem Sich-Zeigen und als Verweigerung der Selbstpositionierung deuten. Auf einer manifesten Ebene heisst sie Herrn Buchers Aussagen am Schluss der Gesprächspassage durchweg gut und bietet somit keine Dekonstruktionsfläche an. Auf der latenten Ebene entzieht sie sich der Praxis der Selbstbehauptung in der «Spielanlage», deren Regeln sie weder kennt noch zugestimmt hat.

Um die Dynamik des Gesprächssettings noch besser zu erfassen, lässt sich das vorgefundene Setting mit einem diskursiven vergleichen. Ein diskursives Setting stellt eine 
durch Argumentationen charakterisierte Form der Kommunikation dar, das die Geltungsansprüche der am Diskurs Beteiligten zum Thema macht (Habermas, 1998, S. 130).

Dieses diskursive Fachgespräch - der kollegiale Austausch von kognitivem Wissen - ist folglich durch Position und Gegenposition gekennzeichnet und als prototypisches, akademisches Setting zu betrachten. Im Gegensatz zum diskursiven Fachgespräch erinnern die Interaktionsdynamiken im Mentoratsgespräch zwischen Frau Steffen und Herrn Bucher an das Einüben der von Helsper (z.B. 2004, S. 72) entworfenen Figur der Subsumtionsantinomie oder auch der Ungewissheitsantinomie pädagogischen Handelns. Die Subsumtionsantinomie besagt, dass, obwohl professionelles Handeln gesicherter Routinen bedarf, zugleich auch eine bewusste Haltung der Skepsis gegenüber jeder Form von Routinen und damit einer habitualisierten Unterstellung der Krise als Normalfall bedarf (Helsper, 2004, S. 72). In der Ungewissheitsantinomie irritiert die Lehrperson die Lernenden bewusst, bringt sie in Krise, initiiert eine Destabilisierung und greift in ihre soziale Integrität ein, um emergente Lernprozesse zu ermöglichen (Helsper, 2004, S. 73 f.). Das Eingreifen und die damit einhergehende Destabilisierung eines psychosozialen Zustandes beschreibt Oevermann in seinem «pädagogischen Gift-Gegengift-Modell» (Oevermann, 1996b, S. 154) als Weiterführung des Prinzips «Heilung durch Verwundung». Riskant formuliert wird der Studentin mittels Irritation von Sicherheiten und Routinen konkret durch Provokation und Infragestellung - ein pädagogisch wertvoller Schmerz zugefügt. Heilung wird anschliessend durch die Herausbildung von Selbstreflexivität als Zentralkompetenz erreicht.

Ohne dem Mentor zu unterstellen, dass er dieses «pädagogische Gift-Gegengift-Modell» im Kopf hat, scheint es Parallelen zwischen der Eigenlogik der Gesprächsinteraktion und dieser Figur zu geben. Die Provokationen, die permanente Infragestellung der Person sowie die Erzeugung von Unsicherheiten sind als Versuche zu lesen eine grundlegende Skepsis der Studentin gegenüber ihrem eigenen Handeln anzuregen. Die Erzeugung von Unsicherheiten wäre mit dem «Gift» und die grundlegende Skepsis mit dem «Gegengift» gleichzusetzen.

Prinzipiell wäre das Erzeugen einer grundlegenden Skepsis und das Einüben einer entsprechenden Art zu Denken auch über die Etablierung eines forschenden Habitus möglich. Die hier Gestalt gewinnende Form von «people processing» unterscheidet sich jedoch maximal von einem forschenden Habitus. Sie scheint ein Machtgefüge zwischen der 
Studentin und dem Mentor zu bedienen und greift auf eine Dekonstruktion der bei der Studentin antizipierten Haltung zurück.

Der Studentin wird nicht ausschliesslich zu einer skeptischen Haltung verholfen, sondern ihr wird ebenso aufgezeigt, dass ihre bisherige Haltung einer Korrektur bedarf. Diese Korrektur lässt sich mit «Einsicht und Belehrung zu etwas Besserem hin» paraphrasieren und zeigt sich vor allem dann, wenn Normativitätsvorstellungen des Mentors durchscheinen. Im Fokus steht nicht die Hinführung zu einer, sondern die Hinführung zur 〈richtigen〉 Erkenntnis.

Diese Überlegungen führen zur Formulierung folgender Fallstrukturhypothese: In der Gesprächsdynamik gewinnt eine Figur Gestalt, in der sich die Studentin Frau Steffen mit Provokationen, Irritationen, permanenter Infragestellung ihrer Person und Dekonstruktionen ihrer Antworten konfrontiert sieht. Zu diesen muss sie sich in der Diffusität ihrer ganzen Person verhalten respektive bewähren.

Im Verlauf des Gesprächs zeigt sich, dass die Studentin, die zu Beginn das hier inszenierte Selbstbehauptungsspiel weitgehend mitgespielt hat, mehr und mehr aussteigt, keine eigene Position mehr bezieht und weniger Angriffsfläche bietet. Der Mentor agiert seinerseits spezifisch in seiner Rolle und wird als Person oder Gesprächspartner nicht fassbar.

\subsubsection{Dritte Sequenz: Sie verteidigen jetzt das Mentorat}

Während des Gesprächsverlaufs wird Frau Steffen immer wieder mit Fragen nach ihrer Einschätzung, oftmals zu curricularen Themen konfrontiert. An dieser Sequenzstelle leitet der Mentor das nächste Gesprächstraktandum mit dem Metakommentar ich würde überleiten zu Portfolioarbeit ${ }^{45}$ ein. Er fragt anschliessend Frau Steffen nach den wichtigsten Ergebnissen und Erkenntnissen aus ihrer Portfolioarbeit. Bei ihrer Antwort auf diese Frage wird die Feinanalyse fortgesetzt.

\footnotetext{
${ }^{45}$ Die Portfolioarbeit als zentrale Arbeitsform im Mentorat wurde bereits bei der ersten Fallrekonstruktion Tina \& Michael erläutert, weshalb hier lediglich auf die Ausführungen in der Mentoratskonzeption des IKU der PH FHNW verwiesen wird.
} 
Frau Steffen: also ich finde vor allem wichtig dass es so ein breites Spektrum ist was wir darüber schreiben mussten und vor allem worüber wir lesen mussten weil ich hätte von mir aus jetzt nie solche Texte gelesen und die bringen einem sicherlich etwas weil auch den Hori/ Horizont erweitert dass man sich überhaupt Gedanken über solche Themen macht wie Autonomie oder// Herr Bucher: //aber Sie// werden ja nicht besser im Unterrichten (.) eh Sie haben es ja vorhin eh eigentlich gesagt ich müsste eigentlich noch viel genauer wissen was die Kinder wann in welchem Tempo das müssen Sie ja eigentlich technisch können

Frau Steffen: (atmet hörbar aus) ja das lernen wir//ja//

Herr Bucher: //da// werden Sie mit Fragen zugeschüttet die auch nicht direkt in den Unterricht einfliessen

Frau Steffen: ich denke schon dass es in den Unterricht einfliesst weil das ist so wie (..) aso ich ich glaube mit diesen Texten haben wir uns so wie unsere eigene Meinung bilden können wie wir als Lehrperson auftreten möchten und wie wir (.) zum Beispiel mit den Eltern umgehen möchten oder wie wir mit den Kindern umgehen möchten (.) und ich denke nicht dass das jetzt auf das Fachdidaktische oder so einen Einfluss hat aber es hat auf die Haltung der Lehrperson also auf meine Haltung hat es einen Einfluss (.) je mehr ich über solche Themen weiss und je breiter dass ich ich in all diese Themen etwas darüber gelesen hab Herr Bucher: Sie verteidigen jetzt das Mentorat aber das könnte man ja auch abschaffen

Frau Steffen: das könnte man schon ((lacht)) (.) aber also ich finde man hat jetzt auch vor allem durch die Gespräche dass wir unsere Meinung eben verteidigen müssen und (.) auch wenn jemand uns das Wort im Mund herumdreht dass man immer noch (.) zu seiner Meinung steht und sagen kann nein ich habe es so und so gemeint und das wird mir sicher späterlich später auch (.) auch brauchen wenn man Eltern hat die (.) irgendwie finden dass man etwas nicht gut macht muss man auch zu seiner Meinung stehen und diese vertreten können 


\subsubsection{Rekonstruktion der einzelnen Sequenzen}

Frau Steffen: also ich finde vor allem wichtig

Mit also zu Beginn des Sprechaktes wird eine Zäsur signalisiert. Erwartbar wäre der gesamte Sprechakt in einer politischen Diskussion, wenn die Sprecherin auf das Argument des Vorredners nicht erneut Bezug nimmt, sondern ein ergänzendes Argument im Modus der Selbstbezogenheit einbringt. Der Sprechakt leitet keinen Konter ein, sondern eröffnet eine neue Perspektive. Er beansprucht keine Ausschliesslichkeit, was sich im vor allem zeigt. Die hier begonnene Aussage könnte beispielsweise mit «dass wir uns auch Gedanken darüber machen, wie wir mit der Presse kommunizieren» fortgesetzt werden.

Frau Steffen: dass es so ein breites Spektrum ist was wir darüber schreiben mussten

In der Fortsetzung wird ausgeführt, was für die Sprecherin vor allem wichtig ist: Dass sie entweder über ein breites Spektrum oder aber breit über etwas schreiben mussten. Der Terminus Spektrum tritt kontextfrei und alltagssprachlich meist mit dem Adjektiv breit in Erscheinung. Die Bezeichnung ein breites Spektrum ist wenig differenziert und lässt einen gewissen Deutungsspielraum zu. Die Aussage weist mit Spektrum, was und darüber drei vage Bestimmungen auf, die nicht weiter beschrieben werden. Das Produkt bleibt in den Äusserungen von Frau Steffen unterbestimmt, wohingegen dem Prozess mit mussten ein Zwang oder eine Pflicht zugeschrieben wird.

Würde Frau Steffen nicht von schreiben mussten sprechen, wäre der Sprechakt - abgesehen von der Verwendung von was anstelle von worüber - eloquent formuliert und würde erneut zu einem Polittalk passen. Die Verwendung von mussten weist allerdings auf einen Zwang hin, der aus einer hierarchischen Situation oder einem Machtgefüge resultiert. An dieser Stelle unterläuft Frau Steffen etwas. Ihre Aussage transformiert sich zu einem typischen Lernendensprechakt in einer Erziehungs- oder Ausbildungskonstellation, da man gewöhnlich in solchen pädagogischen Settings über etwas schreiben muss. 
Frau Steffen: und vor allem worüber wir lesen mussten

Darüber schreiben (zu) müssen wird mit der eigentlichen Aufgabe - vor allem worüber wir lesen mussten - ergänzt. Im Sprechakt wird die zuvor vermutete Lernendenrolle betont und erneut auf den dabei erlebten Zwang hingewiesen. Der Sprechakt zeigt auf, dass dem Auftrag Folge geleistet wurde. Eine erkennbare Identifikation mit den Inhalten des Geschriebenen oder Gelesenen erschliesst sich nicht. Vorstellbar wäre die ganze bisherige Aussage in einer Situation, in der die sprechende Person zwar höflich und pflichtgemäss einem Auftrag nachkommt, dabei jedoch nichts über sich selbst oder die gelesenen respektive geschriebenen Inhalte preisgibt. Diese - riskant formuliert - Kaschierung lässt sich als Hinweis deuten, dass für die sprechende Person die Frage keine echte Neugier aufweist. Folglich gibt sie auch nicht mehr als das hier Gesagte preis.

Unter einer zweiten Lesart könnte im Modus der politischen Korrektheit oder der sozialen Erwünschtheit agiert werden. Diese Dynamik könnte bewusster oder weniger bewusst stattfinden beziehungsweise unterschiedlich strategisch motiviert sein.

Im Sprechakt bildet die Positionslosigkeit das strukturell bedeutsame Moment. In der vorausgehenden Sequenz - Wie schätzen Sie den zweiten Kompetenzbereich ein - wurde als ein Strukturmerkmal aufgezeigt, dass der Mentor als Gesprächspartner kaum in Erscheinung tritt und nur schwer fassbar ist. Die Dynamik in Frau Steffens Ausführungen an dieser Sequenzstelle könnten auch Ausdruck einer Reaktion auf dieses fehlende InBeziehung-Treten sein. Aus adressierungsanalytischer Perspektive betrachtet erinnert die Selbstpositionierung an diejenige einer Schülerin oder Lernenden.

Frau Steffen: weil ich hätte von mir aus jetzt nie solche Texte gelesen

Mit weil wird eine Kausalität hergestellt oder eine Begründung eingeleitet. Die sprechende Person zeigt auf etwas getan zu haben, obschon ihr die intrinsische Motivation dazu fehlte. Damit begründet oder rechtfertigt sie ihr extrinsisch motiviertes Handeln und lehnt gleichzeitig Verantwortung oder Haftbarkeit für dieses ab. Mit jetzt bezieht sich die Aussage von der Interaktionslogik her auf eine spezifische Situation, jedoch nicht vorrangig auf den aktuellen Zeitpunkt. Um die Logik der Satzstruktur noch besser zu explizieren, wird im Sinne eines Gedankenexperimentes nie mit «nicht» 
kontrastiert. Dabei erhält die mit «nicht» formulierte Aussage eine leicht andere Implikation als die empirisch vorgefundene Version mit nie. Die Formulierung weil ich hätte von mir aus jetzt nicht solche Texte gelesen kann als neutrale Situationseinschätzung betrachtet werden. Demgegenüber beinhaltet weil ich hätte von mir aus jetzt nie solche Texte gelesen ein Überraschungsmoment. Der Ausdruck lässt die Option einer Inspiration oder einer positiven Erfahrung offen und ist in der Langversion mit «ich hätte es von mir aus nie gemacht, aber es war dann überraschend gut» zu umschreiben. Dieses Überraschungs- und Inspirationsmoment haftet dem empirisch vorgefundenen Sprechakt an und wird dem Mentor mitgeteilt. Die Bezeichnung solche Texte weist allerdings ein entlarvendes Moment auf. Mit solche wird etwas angedeutet und die Texte in einer unbestimmten Form markiert. Weiter wirkt solche distanzierend, was sich mit «eine solche Art von Texten ist mir fremd» paraphrasieren lässt. In dieser Fremdheit reproduziert sich Frau Steffens generell distanzierte Haltung zu theoretischen Konzepten und Überlegungen.

Der Sprechakt weist bereits in sich ein Spannungsmoment auf, das sich zwischen inspirierend-überraschend und distanzierend bewegt. Angesichts der bereits rekonstruierten Sprechakte Frau Steffens lässt sich eine inkonsistente Position gegenüber den ihr zugetragenen Aufgaben erkennen. Auf einer manifesten Ebene agiert die Studentin gesprächspolitisch korrekt und bemüht, den an sie gestellten Erwartungen zu entsprechen und die Anforderungen zu erfüllen (soziale Erwünschtheit). Sie expliziert über Themen geschrieben, Texte gelesen zu haben und das entsprechend breite Themenspektrum als wichtig zu erachten. In der Latenz scheint eine andere Implikation durch. Die Studentin vollzieht eine Distanzierungsbewegung bezogen auf das Interesse und die Identifikation mit den Themen und Inhalten, die sie bearbeiten musste.

Frau Steffen führt ihre Begründung weiter aus:

Frau Steffen: und die bringen einem sicherlich etwas weil auch den Hori/ Horizont erweitert dass man sich überhaupt Gedanken über solche Themen macht wie Autonomie //oder//

Die Formulierungen etwas bringen und den Horizont erweitern sind anschlussfähig an die Figuren der gesprächspolitischen Korrektheit und der sozialen Erwünschtheit. Die dies- 
bezügliche Auseinandersetzung bringt etwas, dieses etwas bleibt allerdings unterbestimmt und bewegt sich irgendwo zwischen viel und wenig. In der Langversion liesse sich die Aussage mit «irgendetwas wird es und muss es bringen, sich damit zu beschäftigen, selbst wenn ich dieses etwas nicht so genau erkenne» beschreiben. Das NichtErkennbare wird in der Folge mit der Floskel Horizonterweiterung gekennzeichnet. Grundsätzlich lässt sich jeder Tätigkeit und Erfahrung eine Horizonterweiterung zuschreiben, egal ob diese schlussendlich positiv oder negativ konnotiert ist. Horizonterweiterungen sind ebenfalls sozial erwünscht und als Bewertung stets passend. Allerdings wird auf einer latenten Bedeutungsebene auch klar, dass sowohl bei sicherlich etwas bringen als auch bei den Horizont erweitern für die sprechende Person der eigentliche Mehrwert kaum ersichtlich ist. Diese fehlende Ersichtlichkeit macht die entsprechend vorgenommene Kennzeichnung überhaupt notwendig.

Semantisch ist die Aussage weil auch den Hori/Horizont erweitert angesichts des fehlenden Subjekts nicht korrekt. Korrekterweise hiesse es entweder «weil sie auch den Horizont erweitern» oder aber «weil es auch den Horizont erweitert». In der ersten Version erweitern die Texte den Horizont, in der zweiten Version erweitert das Lesen der Texte diesen. Letzteres kommt einem leicht höheren Abstraktionsgrad gleich, ohne anderseits die Beteiligung und Leistung der lesenden Person auszuschliessen. Die empirisch vorgefundene Formulierung weil auch den Hori/ Horizont erweitert stellt eine Variante zwischen diesen beiden Versionen dar. Sie weist auf einen Brennpunkt hin; auf das Spannungsmoment, dass Texte womöglich den Horizont erweitern, dies aber nur in aktiver Auseinandersetzung der lesenden Person tun. Anders formuliert erweitern Texte dann den Horizont, wenn sie auch bearbeitet, erschlossen und verstanden werden. Lesen müssen lässt sich verordnen, «verstehen» jedoch nicht - was im semantisch inkorrekten Sprechakt latent durchscheint.

Als Kaschierungsfigur lässt sich der Sprechakt nicht mehr lesen, da bei einer Kaschierung kaum eine Vertiefung oder Untermauerung mit einem Beispiel vorgenommen würde. Diese Lesart findet folglich keine Fortsetzung. Viel eher reproduziert sich die Figur der sozial-erwünschten und politisch-korrekten Selbstpositionierung als folgsame und gewissenhafte Studentin, die sich mit den ihr zugetragenen Aufgaben auseinandersetzt und dies auch sichtbar macht. Frau Steffen verdeutlicht, dass sie der potenziellen Horizonterweiterung viel Gutes abgewinnt, nämlich sich Gedanken über Themen zu 
machen und intellektuelles Neuland zu betreten. "Was zu tun ist, auch noch zu mögen, als wertvoll zu betrachten und dies auch sichtbar zu machen», wäre eine Beschreibung dieser Figur.

Herr Bucher: //aber Sie// werden ja nicht besser im Unterrichten (.)

Bis zur obigen Gesprächsstelle bleiben die Aussagen von Frau Steffen vage. Just bei der vorgenommenen Konkretion durch das Ansprechen des soziologischen Konzepts der Autonomie beziehungsweise der mit oder angedeuteten Alternative wird Frau Steffen von Herrn Bucher - aber Sie werden ja nicht besser im Unterrichten (.) - unterbrochen. Durch die Unterbrechung ist ihr mit oder eingeleitetes zweites Anschauungsbeispiel nicht mehr gefragt. Die Unterbrechung wird mit aber als Einwand vorgenommen und dadurch die ursprüngliche Aussage Frau Steffens einerseits gestoppt und andererseits negiert.

Für die Satzstruktur bedeutsam ist das ja vor dem nicht, das sprechaktlogisch notwendig erscheint. Die Aussage ohne ja würde mit aber Sie werden nicht besser im Unterrichten als Setzung ungewohnt harsch klingen. Ja nicht in der Bedeutung von «dennoch nicht» oder «trotzdem nicht» glättet das Harsche im Einwand und ermöglicht ein Dementi, in Form einer Doch-Antwort - «doch ich werde schon besser im Unterrichten» -, durch die angesprochene Person.

Der Sprechakt lässt verschiedene Deutungen zu. Nach Lesart (A) wäre er eine Unterstellung wie «gebracht hat ja die Auseinandersetzung mit der Lektüre wenig. Eine Leistungssteigerung ist für mich nicht erkennbar». Die Bemerkung spielt direkt auf die Person an, die hierbei kritisiert und grundsätzlich infrage gestellt wird.

Nach einer Lesart (B) führt der Sprecher, etwas riskant formuliert, das Studium ad absurdum. "Lektürearbeit ist ja gut und recht, bringt aber grundsätzlich nichts für das Unterrichten», so die diesbezügliche Paraphrase. Im Gegensatz zu Lesart (A), nach der die angesprochene Person kritisiert wird, ist die Kritik hier an die Institution oder ans Curriculum gerichtet.

In einer dritten Auslegung (C) lässt sich im Sprechakt Ironie erkennen und dies erinnert an die Figur der paradoxen Intervention, in der das Gegenüber argumentativ herausgefordert wird. Manifest kann dabei sowohl auf die Person als auch auf die Institution abgezielt werden. Latent steht allerdings nicht das Kritisieren, sondern das Provozieren 
im Fokus. Gerade auch die kurze Pause, die Herr Bucher einschaltet, kann für sich genommen bereits als Indiz für diese dritte Lesart betrachtet werden. In einer echten Konfrontation würde eher keine Pause gemacht, sondern der Angriff direkt fortgesetzt werden.

Angesichts der aus der zweiten Sequenz - wie schätzen Sie den zweiten Kompetenzbereich ein - rekonstruierten Fallstrukturhypothese und der eingeschalteten Pause können die Lesarten (A) und (B) verworfen werden. Die verbleibende Auslegung (C) operiert erneut im Modus des «people processing». Als Anschluss wäre eine Fortsetzung der argumentativen und provokativen Herausforderung erwartbar. Diese könnte beispielsweise lauten: «Da wäre doch eine konkrete Anleitung, wie man Lernenden den Umgang mit der Schere beibringt, viel nützlicher.» Durch eine solche plakative Aussage könnte eine Diskussion über sinnvolle und weniger sinnvolle Studieninhalte lanciert werden, um die diesbezügliche Haltung der Studentin zu erfahren.

Herr Bucher: eh Sie haben es ja vorhin eh eigentlich gesagt ich müsste eigentlich noch viel genauer wissen was die Kinder wann in welchem Tempo das müssen Sie ja eigentlich technisch können

Buchers gewählter Anschluss entspricht nicht der oben elaborierten Variante und die anmoderierte Provokation mittels Abwertung wird nicht fortgeführt. Nach der kurzen Pause und einem ersten Zögern mit eh wird Frau Steffen zitiert. Auffällig ist die dreifache Wiederholung des Worts eigentlich und zwei kleine Verzögerungen mit eh, die allesamt die vorausgehende pointierte Formulierung aber Sie werden ja nicht besser im Unterrichten abmildern. Eigentlich, in der hier verwendeten Form, bedeutet «im Grunde genommen» oder «in Wirklichkeit». Auf einer latenten Ebene scheint das Eigentliche oder Wirkliche etwas anderes als das hier Vorgefundene zu sein.

Die Figur der argumentativen Herausforderung - hier ohne Provokation - findet eine Fortsetzung, indem Frau Steffen eine spezifische Position und Rolle zugeschrieben und ihr aufgezeigt wird, was sie vorhin eigentlich gesagt hat. Herr Bucher bezieht abermals keine explizite Position, womit sich das Strukturmerkmal der Vermeidung von Gegenseitigkeit reproduziert. 
Die abschliessende Formulierung - das müssen Sie ja eigentlich technisch können - wäre im Ausbildungskontext einer höheren technischen Fachschule erwartbar, wenn eine Absolventin mit der praktischen Schlussprüfung hadert und nicht weiterkommt. Der Ausbilder könnte dann bemerken: Das müssen Sie ja eigentlich technisch können. Im artikulierten Erstaunen, wie auch im darin enthaltenen Vorwurf, gewinnt eine Dynamik Gestalt, die als (Brandbeschleuniger` bezeichnet werden könnte. Die angesprochene Person wird bezüglich einer Stellungnahme, Verteidigung oder Rechtfertigung unter Zugzwang gesetzt.

Frau Steffen: (atmet hörbar aus) ja das lernen wir //ja//

Der Aussage geht ein hörbares Atmen voraus. Ja stellt manifest eine zustimmende Antwort dar und kann als «ja, ich habe diese Aussage gemacht und sie ist für mich korrekt» gelesen werden. Das zweite ja lässt sich als «wie bereits gesagt» deuten. Es lässt ebenso eine Ungeduld durchscheinen, da das bereits Thematisierte auf keine Resonanz bei Herrn Bucher gestossen ist und deshalb erneut besprochen werden muss.

Manifest verteidigt Frau Steffen die Studieninhalte. Ihr Sprechakt lässt sich als «wo liegt das Problem, wir lernen das ja im Studium» umschreiben. In der Latenz bringt das hörbare Ausatmen und die doppelte Verwendung von ja auch einen Überdruss zum Ausdruck, der sich mit «hören Sie endlich auf mit mir dieses Provokationsspiel zu spielen» paraphrasieren lässt. Frau Steffens Ausführungen werden erneut von Herrn Bucher unterbrochen und sie lässt sich stoppen, das heisst sie spricht in der Sprechüberschneidung nicht weiter.

Herr Bucher: //da//werden Sie mit Fragen zugeschüttet die auch nicht direkt in den Unterricht einfliessen

Herr Bucher bringt einen weiteren Impuls ein, obwohl die Studentin sich an dieser Stelle hätte positionieren können. Aus interaktionsdynamischer Perspektive ist das Übergehen der Gereiztheit und Ungeduld, die sich im hörbaren Ausatmen Ausdruck verschaffen, bedeutsam. Womöglich schliesst er deshalb mit seinem nächsten Impuls inhaltlich nicht an das Vorausgehende an. Die Dynamik des hier verwendeten «Brandbeschleuniger» ist 
eine andere als beispielsweise diejenige der Provokation - aber Sie werden ja nicht besser im Unterrichten -, mit der die Studentin direkt in ihrer künftigen Rolle als Lehrerin angegriffen wird.

Auch an dieser Stelle findet «people processing» im Modus der Provokation statt, wenn auch in abgewandelter Form. Für die Herausarbeitung der strukturellen Differenz wird zunächst die Formulierung zugeschüttet werden näher betrachtet. Zugeschüttet werden passt kontextungebunden zu Gruben, die mit Kies oder Beton aufgefüllt oder eben zugschüttet werden. Ebenfalls findet das Verb bei Naturkatastrophen wie Lawinenabgängen oder Murgängen Verwendung. Menschen oder Gebäude sind schutzlos dem Phänomen ausgeliefert und werden von Schnee- oder Erdmassen begraben.

Der Ausspruch mit Fragen zugeschüttet werden wäre auch bei einem Politskandal vorstellbar. Die angeklagte Politikerin wird von Journalisten mit Fragen zugeschüttet, ohne eine Chance auf adäquate Vorbereitung oder Gegenwehr zu haben.

In der Fortsetzung kommt mit die auch nicht direkt in den Unterricht einfliessen zum Ausdruck, dass die Fragen zusätzlich bearbeitet werden müssen, bevor sie in den Unterricht einfliessen. ${ }^{46}$ Hier wird im Modus von «people processing» der Anspruch auf eine Übersetzungsleistung - die Handhabbarmachung von Fragen für den Unterricht als Teil der eigenen Professionalisierung - hervorgehoben.

Die Provokation erfolgt an dieser Sequenzstelle über ein imaginiertes Opferszenario. In der Langschrift würde die Botschaft des Sprechakts lauten: «Stellen Sie sich die Situation des Zugeschüttet-Werdens bildlich vor. Was macht das mit Ihnen? Wie kommt Ihnen das vor? Positionieren Sie sich dazu.» Diese Provokationsfigur enthält mit der Perspektivübernahme eine zusätzliche Ebene. In dramatisierender Weise wird der angesprochenen Person aufgezeigt, was ihr bei der Auseinandersetzung mit Texten widerfährt. Die genutzten Übertreibungen und Zuspitzungen sind offensichtlich und nehmen fast schon eine karikierende Gestalt an.

Im Anschluss nimmt Frau Steffen folgende Positionierung vor:

\footnotetext{
${ }^{46}$ Unter Zuzug von Kontextwissen gilt es zu Herrn Buchers Bemerkung Folgendes anzufügen: Eine häufig formulierte Kritik von Studierendenseite ist, dass sie teilweise Aufgaben- oder Fragestellungen bearbeiten müssen, die aus ihrer Perspektive wenig mit ihrer künftigen Tätigkeit als Kindergarten- oder Unterstufenlehrperson zusammenhängen. Insofern ist die Vermutung zulässig, dass der Mentor hier mit dieser Kritik spielt oder anders ausgedrückt: die Studentin versucht mit ihren eigenen Waffen zu schlagen. Das für die Stelle wichtige Strukturmoment bleibt auch mit diesem kontextualisierten Zusatz das provokative Element.
} 
Frau Steffen: ich denke schon dass es in den Unterricht einfliesst weil das ist so wie (..) aso ich ich glaube mit diesen Texten haben wir uns so wie unsere eigene Meinung bilden können wie wir als Lehrperson auftreten möchten und wie wir (.) zum Beispiel mit den Eltern umgehen möchten oder wie wir mit den Kindern umgehen möchten (.) und ich denke nicht dass das jetzt auf das Fachdidaktische oder so einen Einfluss hat aber es hat auf die Haltung der Lehrperson also auf meine Haltung hat es einen Einfluss (.) je mehr ich über solche Themen weiss und je breiter dass ich ich in all diese Themen etwas darüber gelesen hab

Mit ich denke schon leitet die Studentin eine Gegenposition ein, was sich vor allem in der Verwendung von schon zeigt. Die vorgefundene Formulierung kann mit einer analogen, unter Auslassung von schon, kontrastiert werden. Ich denke würde das Anschliessende als Vermutung oder persönliche Meinung kennzeichnen. In der vorgefundenen Formulierung ich denke schon ist allerdings die Bearbeitung der vorausgehenden Provokation - mit Fragen zugeschüttet die nicht direkt in den Unterricht einfliessen - das bedeutsame Strukturmoment. Dabei gewinnt mit dem schon eine Figur der sanften Verteidigung Gestalt.

In der Fortsetzung beginnt Frau Steffen die entsprechende Begründung mit weil das ist so wie im Modus einer Setzung. Sie bricht die eingeleitete Begründung jedoch ab und reformuliert nach einer Pause die Aussage mit aso ich glaube vorsichtiger und unverfänglicher.

Die Studentin entschärft mit so wie; ich denke nicht und aso ich glaube im Verlauf des Sprechakts mehrere Formulierungen und kennzeichnet diese als subjektive Einschätzungen. Mit der gestaltgewinnenden Figur werden klare Setzungen und damit potenzielle Angriffsflächen vermieden. Frau Steffen vollzieht eine Bewegung weg von der Ebene des Unterrichts hin zur Ebene der Rolle als Lehrperson. Durch die Auseinandersetzung mit den Texten scheint sie sich über ihren Auftritt als Lehrperson klarzuwerden. Sie reagiert dabei auf die in Herrn Buchers Aussage - die auch nicht direkt in den Unterricht einfliessen - enthaltene Latenz und hebt diese in ihren Ausführungen von der latenten auf die manifeste Ebene. Dabei zeigt sie auf, dass sie die notwendigen Zwischenschritte erkennen und auch leisten kann. 
Strukturell bedeutsam scheint die Verwendung von wir und uns in ihren Ausführungen. Mutmasslich spricht sie von einem allgemeinen Wir oder auch von sich im Kreise ihrer Kommilitoninnen und Kommilitonen. Jedenfalls sucht sie Schutz im Kollektiv. Sie macht sich als Person weniger angreifbar als wenn sie sich als Individuum positionieren würde. Zusammenfassend lässt sich feststellen, dass Frau Steffen im Sprechakt auf einer Inhaltsebene die subjektive Sinnhaftigkeit der Auseinandersetzung mit solchen Texten aufzeigt. «People processing» im Modus der argumentativen Herausforderung zeigt demzufolge Wirkung. Die Studentin spricht über das, was sie beschäftigt, wie etwa die Haltung einer Lehrperson. Sie nimmt damit auch eine Selbstpositionierung vor.

Herr Bucher: Sie verteidigen jetzt das Mentorat

Bei diesem überraschenden Sprechakt ist eine feinanalytische Rekonstruktion angebracht. Kontextungebunden ist er als Bestandteil einer umfassenden Instruktion vorstellbar. Dabei indiziert das Wort jetzt, dass es bereits ein Vorher gab. Unter Auslassung des Terminus Mentorat würde der Sprechakt in dieser Lesart die Struktur einer Befehlserteilung, beispielsweise in einem militärischen Kontext, aufweisen.

Der Sprechakt wäre ebenso im Rahmen der Einnahme einer Meta-Position denkbar. Die sprechende Person zeigt der Angesprochenen auf, was diese gerade tut. Vorstellbar wäre dies in einem diskursiven Format, beispielsweise in einer Debatte zwischen Politikerinnen oder Politikern. Der Sprechakt weist als Metakommentar manifest auf etwas hin und beinhaltet ein Überraschungsmoment. Verteidigt wird möglicherweise etwas, was für die verteidigende Person wertvoll und schützenswert ist - vielleicht eine Herzensangelegenheit - oder zumindest eine Verteidigung benötigt. Dieser Akt des Verteidigens löst beim Sprecher Erstaunen aus. Gegenteilig wäre vorstellbar, dass die der Verteidigung zugrunde liegende Absicht in Zweifel gezogen wird: Sie verteidigen jetzt das Mentorat «aber das kann ja wohl nicht Ihre Absicht sein.» Das vermutbare «aber» in «aber Sie verteidigen jetzt das Mentorat» wird nur implizit durch «jetzt» angedeutet. Auf einer latenten Bedeutungsschicht gewinnt die Implikation «hab ich Sie da eben ertappt` Gestalt. Im realen Gesprächskontext wird mit dem Sprechakt Sie verteidigen jetzt das Mentorat Frau Steffens Position, nämlich diejenige einer Verteidigung, bestimmt. 
Auf einer manifesten Ebene entspricht der Sprechakt strukturell am Ehesten der zweiten oder dritten Elaboration - dem erstaunten Meta-Kommentar. Latent wird Frau Steffen durch einen Diskurs-Move erneut zu einer Stellungnahme aufgefordert. Die Provokation enthält die Erwartung sich, bezogen auf die im Raum stehende Frage - ist das Mentorat verteidigungswert -, zu positionieren. Hier scheinen folglich auch Implikationen aus der ersten elaborierten Lesart, derjenigen der Auftragserteilung, durch.

Herr Bucher: aber das könnte man ja auch abschaffen

Um die Struktur des hier formulierten Einwands herauszuarbeiten, lässt sich erneut auf das Mittel des gedankenexperimentellen Kontrasts zurückgreifen und eine Gegenüberstellung von einer kann- und einer könnte-Version vornehmen. «Aber das Mentorat kann man ja auch abschaffen» stellt eine reale Option dar. Grundsätzlich ist es möglich und denkbar das Mentorat abzuschaffen. In der realisierten Formulierung - aber das könnte man ja auch abschaffen - besteht die reale Option kaum. Die Abschaffung ist nur als Fiktion vorstellbar, was zusätzlich in der Verwendung von ja auch Gestalt gewinnt. Dieser könnte-Version haftet etwas an, das eine Implikation wie "rein theoretisch» oder «eigentlich» aufweist und die erwartete Antwort der Studentin mit einer bemerkenswerten Offensichtlichkeit aufgezeigt.

An dieser Gesprächsstelle zeigt sich, dass Herr Bucher den etablierten Modus der Prüfung und Bewährung in der Anfechtung, und dabei als Person nicht sicht- oder fassbar zu werden, nicht bruchlos umsetzen kann. Denn in der hier formulierten provokativen Steilvorlage fordert er latent eine Bestätigung für seine Art von Mentoring und für sich als Mentor ein. Als Reaktion könnte die Studentin die provokative Steilvorlage übergehen und die Kommunikation auf einer inhaltlichen Ebene fortsetzen: «lch möchte es nicht abgeschafft wissen, denn das Mentorat hilft mir nicht nur im Umgang mit den Schülerinnen und Schülern, sondern auch mit mir selbst.» Dieser Anschluss wäre rollenförmig, kooperativ und deeskalierend. Die Provokation würde ins Leere laufen.

Ebenso könnte die gebotene Steilvorlage angenommen werden - «aus meiner Sicht könnte man das schon abschaffen»-, um den Mentor ein Eigentor schiessen zu lassen. Manifest würde damit der Hypothese des Abschaffens zugestimmt, latent beinhaltet die Antwort allerdings ein Kränkungspotenzial, das Herr Bucher selber provoziert hat. 
Zu überlegen wäre, ob die hier vorgefundene Konstellation es zulässt, dass Frau Steffen in ihrem Anschluss eine Gegenstrategie fährt und Herrn Bucher als Provokateur entlarvt. Sie könnte sagen: «Okay, Sie wollen mich provozieren.» Mit diesem Anschluss würde die Rollenförmigkeit der Situation nicht aufrechterhalten.

Der Anschluss der Studentin auf diese erneute Provokation ist somit aufschlussreich bezogen auf wie sie sich im anhaltenden Provokations-Selbstbehauptungs-Spiel positioniert und dieses mitspielt. Weiter lässt sich aufzeigen, dass sich an dieser Stelle das fallspezifische Interesse Herrn Buchers reproduziert, das Setting als solches zum Thema zu machen. Dies ist in seiner Rolle als Mentor bemerkenswert. Dieser Aspekt lässt sich grundsätzlich entweder dem Habitus des Mentors oder seinen persönlichen Interessen zuschreiben. Bereits zu Gesprächsbeginn hat er das Studienangebot am Studienstandort sowie auch das Curriculum als Themen eingebracht. Habituelle Aspekte und Interessen liegen ausserhalb des eigentlichen Forschungsinteresses und werden deshalb nicht weiter berücksichtigt. Dennoch gewinnt in dieser eigenlogischen Mentoratskomposition eine weitere Dynamik, die grundsätzliche Legitimierungsproblematik betreffend, Gestalt. Wie in der vorläufigen Fallstrukturhypothese geschildert, ist eine Figur erkennbar, die Studentin zu neuen und vor allem zu den richtigen Einsichten zu führen. Dieser Figur ist zu entnehmen, auf welcher Basis das Setting für den Mentor legitimiert ist. Gleichzeitig scheint für ihn das Verlangen zu bestehen, die Legitimation des Settings mit der Studentin zu diskutieren. Diese Dynamik betreffend wird die Frage «wozu braucht es das Format «Mentoratsgespräche»» kurzfristig von der manifesten auf die latente Ebene verlagert und auf dieser aufgegriffen. Die Diskussion einer Thematik findet bei Herrn Bucher im Modus der Provokation und der Aufforderung, sich zu dieser Provokation zu verhalten, statt. Auch hier findet dieser Modus Anwendung.

Fragt man sich, weshalb Frau Steffen dazu angehalten wird die Mentoratspraxis zu besprechen, lässt sich vermuten, dass es primär um die Sichtbarwerdung der Person und ihrer Haltung geht. Dass dies konkret anhand des Beispiels der Mentoratspraxis erfolgt, scheint fallspezifisch durchaus bedeutsam. Es kann als Hinweis für eine Prekarität, die bezogen auf diese Praxis für den Mentor besteht, gelesen werden. 
Frau Steffen: das könnte man schon ((lacht)) (.)

Die Reaktion auf die Steilvorlage fällt in einer sozialen Dimension kooperativ aus. Der Ball wird aufgenommen und auf einer manifesten Ebene eloquent, schlagfertig und souverän zurückgespielt, ohne aber mit einem «Ja» explizit zuzustimmen. In der latenten Bedeutungsschicht lässt sich das nicht vorhandene «Ja» bei das könnte man schon als «red du nur, provozier du nur, ich lass mich nicht einwickeln» auslegen. Frau Steffen bleibt folglich auch ohne explizite Zustimmung mit ihrem Nicht-Einsteigen sozial anschlussfähig. Dennoch zieht ihre Entgegnung ein «Aber» im Sinne eines Einwandes oder einer Begrenzung nach sich. Das Lachen als Reaktion, wie auch die daran anschliessende kurze Pause, lassen sich als Irritation und Orientierungsunsicherheit bezogen auf die an sie gestellten Erwartungen lesen. Gleichzeitig stellt das Lachen eine Form von Distanzierung oder auch Entlarvung dar.

Die anschliessenden Ausführungen Frau Steffens werden nur punktuell feinanalytisch rekonstruiert, da sich gewisse Dynamiken reproduzieren.

Frau Steffen: aber also ich finde man hat jetzt auch vor allem durch die Gespräche dass wir unsere Meinung eben verteidigen müssen und (.) auch wenn jemand uns das Wort im Mund herumdreht dass man immer noch (.) zu seiner Meinung steht und sagen kann nein ich habe es so und so gemeint und das wird mir sicher späterlich später auch (.) auch brauchen wenn man Eltern hat die (.) irgendwie finden dass man etwas nicht gut macht muss man auch zu seiner Meinung stehen und diese vertreten können

Nach der Pause folgt erwartungsgemäss ein aber. Der vermutete Einwand wird allerdings nicht weiter ausgeführt, sondern durch ein also - in der Bedeutung einer Fortsetzung eines unterbrochenen Gedankengangs - ergänzt. Im Anschluss nimmt Frau Steffen mit der Einleitung ich finde eine subjektive Stellungnahme vor. Sie sagt an dieser Stelle, was sie bezogen auf das Format «Mentorat» zu sagen hat, ohne auf die Provokation einzusteigen. Die Studentin zieht sich dadurch elegant aus der Schlinge und lässt sich nicht fangen. 
In ihren Ausführungen scheint auch eine Kritik an der Praxis des «people processing» auf. Diese äussert sich beispielsweise in der Bemerkung auch wenn jemand uns das Wort im Mund herumdreht. Die Kritik wird mit dem Bezug jemand allgemein gehalten, das heisst sie spielt nicht direkt auf die Person. Auch spricht sie nicht von sich selbst, sondern verallgemeinernd von uns. Sie bezeichnet sich als «eine unter vielen» und nimmt eine selbstbewusste Identifikation mit der Gruppe vor.

In ihren Ausführungen scheint durch, dass sie das erfolgreiche Verteidigen der eigenen Meinung nicht durch die Lektüre von Texten, sondern in Gesprächen erworben hat. Frau Steffen verwendet den Terminus Gespräche und nicht etwa den der «Diskussionen». Eine Kontrastierung von beiden Begriffen erscheint aufschlussreich.

Strukturell gesehen finden Gespräche in Dyaden oder in Kleingruppen statt. Diskussionen sind dementgegen auch mit grösseren Personenkonstellationen und vor Publikum vorstellbar. Während sich in Diskussionen die Teilnehmenden idealerweise auf Augenhöhe begegnen, können Gespräche durchaus hierarchisch vorstrukturiert sein. Man denke hier beispielsweise an eine Mitarbeiterin, die von ihrem Vorgesetzten zu einem Gespräch unter Vier-Augen vorgeladen wird.

Frau Steffens Wortwahl lässt erkennen, wie sie das Setting erlebt: hierarchisch, verpflichtend und unausweichlich. Diese hier vorgefundene Gesprächsfigur erinnert eher an ein Verhör als an ein diskursives Setting. Auf der Interaktionsebene widerspricht die Studentin dem provokativen Einwand des Abschaffens. Sie verteidigt das Mentorat tatsächlich, wie ihr dies manifest unterstellt und latent auch zugewiesen wird.

In der Gesprächsinteraktion zeigt sich, dass «people processing» bis zu einem gewissen Grade auch tatsächlich funktioniert. Die Studentin gibt an den Wert des Richtigen zu erkennen und mithilfe der Gespräche im Rahmen des Mentorats Fähigkeiten erworben zu haben, die ihr bei der Verteidigung ihrer Meinung dienlich sind. Diese Erkenntnis teilt sie nicht nur inhaltlich mit, sondern führt sie auch performativ auf.

In ihren Ausführungen spricht die Studentin teils von ich, teils von wir. Gegen Ende ersetzt sie beide Personalpronomen durch ein distanzierteres man. Der Wechsel zwischen den Subjektbezeichnungen, im Sinne einer Situationsunsicherheit, lässt sich als Ausdruck einer Bewegung weg von der eigenen Person hin zu einer allgemeineren Gruppe der Betroffenen deuten. Analog kann die Verwendung des Plurals bei Gespräche als ein Abstand-Nehmen von der Beziehungslogik verstanden werden. Frau Steffen spricht von 
Gesprächen in einem allgemeinen und übergeordneten Kontext, nicht aber von einem individuellen oder spezifischen Gespräch.

Insgesamt gewinnt das Artifizielle und Inszenierte der Situation deutlich Gestalt. Die Form der Selbstbehauptung, der Verteidigung und der Argumentation stehen im Fokus der Intervention des Mentors. Die eigentliche Meinung der Studentin ist sekundär, selbst wenn auch diese noch einer Korrektur oder Hinführung zum Richtigen bedarf. Hinweise für das Artifizielle finden sich etwa in der Antwort von Frau Steffen. Die eigene Meinung verteidigen müssen beinhaltet als Aussage ein paradoxes Moment. Bei der Verteidigung der eigenen Meinung handelt es sich vorwiegend um eine Option, eine Berechtigung oder Gewährung, nicht aber um einen Zwang. Gewöhnlich spricht man ohne Modalverb von «seine Meinung verteidigen» oder «vertreten», allenfalls von «verteidigen können» oder «dürfen». Das Modalverb müssen mutet im Zusammenhang von Meinung verteidigen befremdlich an. Seine Verwendung deutet auf eine inszenierte Situation hin, wie sie vornehmlich in Ausbildungskontexten, beispielsweise der Schule, vorzufinden ist.

Im Unterricht üben Schülerinnen und Schüler die mündliche Erörterung ein. Typischerweise inszeniert die Lehrperson dazu ein geeignetes Setting, beispielsweise zu einem sozialpolitischen Thema. Die Schülerinnen und Schüler werden angehalten, ihre Position zu verteidigen und ihre Argumentationslinien zu schärfen. Dabei treten die eigentlichen Inhalte in den Hintergrund. Im Unterschied zum Mentoratsgespräch ist allerdings den Beteiligten im Unterrichtskontext klar, dass es sich hierbei um eine Inszenierung, eine Übungsanlage handelt.

Auch wenn im Gespräch zwischen Frau Steffen und Herrn Bucher diesbezüglich keine Transparenz besteht, scheint die Dynamik der Inszenierung, die der Einübung einer Argumentationskompetenz und der Standhaftigkeitstestung dient, sich zunehmend zu verselbständigen. Auf Seite der Studentin zeigen sich unterschiedliche und gegenläufige Bewegungen. Einerseits teilt sie mit, die «Spielanlage` als sinnvoll zu betrachten. Andererseits entlarvt sie mit ihrer einleitenden Bemerkung zum Mentorat - das könnte man schon «abschaffen» - die ihr entgegengebrachte Provokation. Eine solche Entlarvung zieht latent auch eine Umkehrung der Hierarchiestruktur nach sich. Möglicherweise scheint die Dynamik der verkehrten Rollen dennoch zu prekär, weshalb die Studentin sich umgehend davon distanziert und wieder mitspielt. Dennoch zeigt sich auf einer Beziehungsebene die Mitspielbereitschaft kleiner als auf der Interaktionsebene. Ebenso nimmt diese 
Bereitschaft im Fortschreiten des Gespräches grundsätzlich ab. Ein Indikator hierfür ist das Ersetzen von ich, als Teil der dyadischen Ich-du-Konstellation, durch man.

Mit der Verwendung der Partikel eben wird ein Bezug zur Praxis des Seine-eigeneMeinung-vertreten-Müssens hergestellt. Eben lässt sich demzufolge als Markierung und Kommentar für die Affirmation dieser Praxis lesen, die im Augenblick der Äusserung auch performativ aufgeführt wird. Mit dieser Bewegung hebt Frau Steffen erneut das Latente auf eine manifeste Ebene. Sie drückt aus, was offensichtlich ist, aber wohl nicht offensichtlich sein sollte.

Insgesamt setzt die Studentin das Mentoratsgespräch mit der Aufforderung, seine eigene Meinung verteidigen zu müssen, in einen Kausalzusammenhang. Durch Mentoratsgespräche fallen in diesem Sinne Verpflichtungen an, sich in der Kompetenz der Meinungsvertretung zu üben.

Die Gesprächspassage ist überkomplex, da Interaktions- und Beziehungsebene von den beiden Gesprächsteilnehmenden unterschiedlich und widersprüchlich bedient werden. Aus der Interaktionsperspektive sind die Äusserungen des Mentors intervenierend. Es wird eine Zeigestruktur hergestellt. Dies erinnert an ein klassisches Ausbildungssettings mit den zwei Formaten «Unterricht» und «Prüfung». Auf dieser Interaktionsebene werden die Interventionen des Mentors von der Studentin über weite Strecken kooperativ mitgetragen. Sie können deshalb weitgehend als geglückt betrachtet werden. Auf einer Beziehungsebene ist die Dynamik der Gesprächspassage eine andere. Die Studentin wird auf dieser Ebene mit einer Aufforderung konfrontiert, die zugespitzt mit «Was wir hier gerade tun, das bringt Ihnen doch etwas, oder?» paraphrasiert werden könnte. Herr Bucher fischt mit dieser Figur nach Komplimenten und Bestätigung. Selbst wenn manifest eine solche bis zu einem gewissen Grade erteilt wird, scheint latent durch, dass die Studentin diese Strategie entlarvt. Die Provokation verkommt in der Gesprächsinteraktion zum Regelfall und Frau Steffen geht, je länger das Gespräch andauert, auf der Beziehungsebene nicht mehr darauf ein.

Mit diesen Überlegungen kann die bisherige riskante Fallstrukturhypothese ausgebaut und geschärft werden. 


\subsubsection{Zusammenfassung und riskante Fallstrukturhypothese der Sequenz Sie verteidigen jetzt das Mentorat}

In der dritten Sequenz im Mentoratsgespräch zwischen der Studentin Frau Steffen und dem Mentor Herrn Bucher scheinen sich die strukturellen Spannungen fortzuschreiben und zuzuspitzen.

Der Mentor agiert einerseits weiterhin in einem an "people processing» angelehnten Modus, indem er die Studentin mit mäeutisch-sokratischen Fragen und provokativen Aussagen konfrontiert. Sie soll Stellung beziehen, um eine anschliessende Dekonstruktion zu ermöglichen. Andererseits sucht Herr Bucher Bestätigung für seine Auslegung von Mentoring und fordert diese analog im Modus der Provokation ein. Er entzieht sich kontinuierlich einer Selbstpositionierung, die er allerdings beim Gegenüber einfordert, oder aber er spielt als Teil der Inszenierung den Advocatus Diaboli.

Die Studentin bearbeitet auf einer Interaktionsebene das ihr Angediente über weite Strecken affirmativ und kooperativ. Stellenweise, wie etwa im Sprechakt ja das lernen wir ja, der durch hörbares Ausatmen eingeleitet wird, verschaffen sich Genervtheit und Überdruss über das (Provokations-Verteidigungs-Spiel〉 Ausdruck. Ihre Mitspielbereitschaft gestaltet sich in der Konsequenz inkonsistent. Bei Äusserungen wie also ich finde vor allem wichtig wie auch weil ich hätte von mir aus jetzt nie solche Texte gelesen oder und die bringen einem sicherlich etwas oder auch ich denke schon dass es in den Unterricht einfliesst ist diese Mitspielbereitschaft vorhanden. Bei Aussagen wie ja da lernen wir ja hingegen scheint auch Widerstand gegenüber dem Spiel durch. Dabei gewinnt eine Figur Gestalt, die sich mit «sozial bemüht politisch korrekt zu agieren, mit unterschiedlicher performativer Umsetzung» umschreiben lässt.

Grundsätzlich spielt Frau Steffen über weite Strecken mit, ohne sich dem Spiel gänzlich zu unterwerfen. Ihre Mitspielbereitschaft hat allerdings auch Grenzen oder rote Linien. Beispielsweise wird im Sprechakt unsere Meinung eben verteidigen müssen manifest eine Weitsichtigkeit und Abgeklärtheit - «es ist gut, dass wir dazu gezwungen werden» kundgetan. Der Sprechakt gestaltet sich der Form nach brav und kooperativ. Dennoch zeigt das Brave in der Latenz auch eine gewisse Subversion, die riskant formuliert mit «Das ist es doch, was Sie doch von mir hören wollen, oder?!» paraphrasiert werden kann. In der Fortsetzung des Sprechaktes auch wenn jemand uns das Wort im Mund herumdreht 
wird die Latenz der Bedeutungsebene offengelegt. Dabei lässt sich analytisch erkennen, wie wirkmächtig die latenten Sinnstrukturen den manifesten Interaktionsverlauf beeinflussen, schliesslich wird die Studentin ja nicht expressis verbis mit der «Spielanlage〉 vertraut gemacht, sondern hat diese zuerst als solche zu erkennen und zu entlarven.

Mit ihren Ausführungen zeigt Frau Steffen auf, was sie mit Mentoratsgespräch verbindet: sich selbst und die eigene Meinung gegen die Angriffe und Wortverdrehungen des Mentors verteidigen zu müssen. Sie fügt sich dieser Konzeption und spielt auf der Interaktionsebene souverän, wenn auch nicht ungebrochen, mit. An manchen Gesprächsstellen lässt sie sich in eine Legitimierungsnotwendigkeit verwickeln, zeigt aber gleichzeitig qua Selbstbehauptung ihre roten Linien auf. Die Markierung der roten Linien weist ein spielerisches Element auf. Dies wird beispielsweise in der Bemerkung des Wort-imMund-Umdrehens deutlich. In der vorgefundenen Art teilt sie dem Mentor mit: «Glauben Sie nicht, ich hätte das nicht durchschaut.»

\subsubsection{Fallzusammenfassung und finale Fallbestimmung: Frau Steffen \& Herr Bucher}

Auf der Basis der anhand unterschiedlicher Sequenzen rekonstruierten Strukturmerkmale und Fallstrukturhypothesen lassen sich mit Blick auf die Interaktionsdynamik im Gespräch zwischen der Studentin Frau Steffen und dem Mentor Herrn Bucher die folgenden Erkenntnisse festhalten: Das Gespräch gleicht in Gestalt und Ablauf einer Variante von «people processing», in der die Studentin durch Interventionen in Form von Provokationen, Infragestellungen und Dauerirritationen dazu aufgefordert wird, sich bezogen auf das an sie Herangetragene zu positionieren. In der Interaktionsstruktur gewinnt seitens des Mentors eine Figur Gestalt, deren Absicht darin besteht, im Rahmen eines Mentoratsgesprächs einen Lern- und Entwicklungsraum zu bieten, der eine habitualisierte Skepsis gegenüber den eigenen Handlungen und Vorstellungen bestenfalls befördert oder wenigstens anstösst. Die Interventionen des Mentors bleiben nicht erfolglos. Die Studentin setzt sich zu dem ihr Angetragenen ins Verhältnis, dies nicht zuletzt deshalb, weil ihr von der Interaktionsstruktur her kaum eine andere Wahl bleibt. 
In Differenz zu einem diskursiven Modus folgt der im vorgefundenen Gespräch Gestalt gewinnende Austausch einer mäeutisch-sokratischen Logik, die auch eine Prüfungsimplikation aufweist. Dies lässt sich auch durch die Einseitigkeit innerhalb der Gesprächslogik aufzeigen. Darin tritt der Mentor nicht mit einer «echten` Gegenposition auf, sondern setzt eine solche, wenn überhaupt, als Teil seines strategischen Arrangements für die Herstellung eines `vermeintlichen ` Diskurses ein. Die provokative Aussage aber das «Mentorat» könnte man ja auch abschaffen zeigt einerseits die konstitutiv vorhandene Machtasymmetrie auf, da der Mentor keine ernsthafte, vollumfängliche Zustimmung zum Vorschlag, das Mentorat abzuschaffen, zu erwarten hat. Ansonsten hätte er, so die plausible Annahme, die Frage nicht gestellt. Gleichzeitig wird durch die serheischtes Verneinung auch eine Unsicherheit betreffend die Legitimation des Mentoratsmandats erkennbar. Aus struktureller Sicht betrachtet, beispielsweise infolge der hierarchischen Situation zwischen der Studentin und dem Mentor, ist die Studentin bis zu einem gewissen Grade dem Mentor ausgesetzt und kann sich nur in einem beschränkten Radius positionieren. In ihrer Positionierung sucht sie ein Gleichgewicht und versucht, das 〈Provokations-Verteidigungs-Spiel` mitzuspielen, ohne sich dabei jedoch zu unterwerfen. Sie zeigt sich grundsätzlich auf eine elegante Art und Weise kooperativ und souverän und markiert dennoch ihre roten Linien. Dieser Balanceakt - mitspielen, aber nur bis zu einem gewissen Punkt - gelingt ihr mehrheitlich, wenngleich nicht vollumfänglich.

Falls die Praxis der Mentoratsgespräche aufgrund von strukturell bedingten, potenziellen Spannungen (vgl. Kapitel 2.1.3) an sich keine Fragen oder Gesprächsthemen aufwirft oder bei den Studierenden grundsätzlich keine Gesprächsanliegen vorhanden sind, lassen sich solche auch artifiziell in den kommunikativen Austausch einführen. Denn pragmatisch betrachtet besteht in einem institutionalisierten Gesprächssetting wie dem vorliegenden die Notwendigkeit, die Gesprächszeit zu füllen. Im Gespräch zwischen Frau Steffen und Herrn Bucher gewinnt eine Figur Gestalt, die darin besteht, die Bearbeitung dieses Zeitfüllens für die Entwicklung einer habitualisierten Skepsis gegenüber dem eigenen Handeln zu verwenden. Diese Figur erinnert an die Subsumtionsantinomie und an die Ungewissheitsantinomie, die Helsper für das Lehrhandeln formuliert hat (Helsper, 2002, S. 80 f.). Herr Bucher fungiert als Krisenauslöser und initiiert im Modus der Dauerirritation Destabilisierungsprozesse, die ihrerseits emergente Lernprozesse wie beispielsweise die Ausbildung einer habitualisierten Skepsis und Reflexivität ermöglichen oder anstossen. 
In der hier vorgefundenen Eigenlogik einer Prüfung und Bewährung in der Anfechtung zeigt sich eine Figur, in der Gewicht daraufgelegt wird, die Studentin auf die Gefahr der Subsumtionsantinomie im pädagogisch-professionellen Handeln vorzubereiten, da pädagogisch-professionelles Handeln nicht nur gesicherter Routinen, sondern gleichzeitig auch einer bewussten Haltung der Skepsis gegenüber diesen Routinen bedarf.

Des Weiteren verschafft sich in der vorgefundenen Intervention eine Dynamik Ausdruck, die darauf abzielt, das professionelle Selbst (Bauer, 1998) zu stärken. Die angewendeten Techniken (Dauerprovokation und -irritation) zur Stärkung des professionellen Selbst durch die Auseinandersetzung mit der eigenen Unvollkommenheit, führen auf der latenten Bedeutungsebene zu Spannungen. Diese scheinen im Gespräch zwischen Frau Steffen und Herrn Bucher als Macht- oder Hierarchieasymmetrie auf und sind auch der nicht vorhandenen Reziprozität zuzuschreiben. Der hier etablierte Gesprächsmodus weist zudem eine starke Inszenierungslogik auf. Dass das Gespräch einer entsprechenden Inszenierung bedarf, zeigt die dem Setting zugrunde liegende Strukturproblematik einer Legitimierungsbedürftigkeit auf, die auch beim Mentor latent ein gewisses Unbehagen auszulösen scheint. Bearbeitet werden dieses Unbehagen respektive mögliche grundlegende Zweifel an der Mentoringkonzeption beispielsweise durch die Provokation aber das «Mentorat» könnte man ja auch abschaffen. Wie zuvor rekonstruiert, stellt diese Aussage einerseits eine an die Studentin gerichtete Provokation als Aufforderung zur Stellungnahme dar. Andererseits scheint sich ihm eine entsprechende Frage unbewusst auch zu stellen. Dies kann als Hinweis für eine tieferliegend gesuchte Bestätigung gedeutet werden, die ebenfalls im Modus der Provokation zutage tritt. Die Provokationen und die Infragestellungen richten sich somit nicht ausschliesslich gegen die Studentin, sondern ebenso gegen das Setting an und für sich.

Abschliessend lässt sich für das Mentoratsgespräch zwischen Herrn Bucher und Frau Steffen die folgende Fallbestimmung formulieren:

In der Interaktionsdynamik des Gesprächs gewinnt eine Figur Gestalt, die eine starke Prüfungs- und Bewährungsimplikation aufweist und sich mit dem Imperativ «Zeig dich!» beschreiben lässt. Die Eigenlogik des Gesprächs erinnert demzufolge an die Anderslogik einer Prüfung und Bewährung in der Anfechtung. Es scheint, als gelte es mittels Provokation, Anfechtung und Irritation von Routinen die Etablierung einer bewussten Haltung der Skepsis zu befördern und diese zu überprüfen. Die Ungewissheit in Bezug 
darauf, ob diese produktive Öffnung erreicht wird (Helsper, 2002, S. 81), wie auch die Gefahren einer Machtantinomie respektive Symmetrieantinomie oder auch Überforderungsphänomene bleiben unauflösbare Bestandteile dieses Interaktionsmodus - auch wenn sie nicht explizit ausgespielt werden (Helsper, 2002, S. 82). Die antinomische Handlungsstruktur, die grundsätzlich in Mentoratsgesprächen aufscheint, wird fallspezifisch nicht antinomisch bearbeitet, sondern das Antinomische seitens des Mentors stillgestellt. Die Gesprächsinteraktion gestaltet sich mehrheitlich distanziert, formell, spezifisch und asymmetrisch. 


\subsection{Ingrid \& Lorenz}

Das dritte Mentoratsgespräch zwischen der Studentin Ingrid und dem Mentor Lorenz findet ebenfalls im Frühlingssemester 2017 statt. Es ist auf 12.00 Uhr angesetzt und dauert mit über einer Stunde doppelt so lange wie die drei anderen Gespräche. Die Studentin befindet sich im vierten von sechs Semestern.

\subsubsection{Eröffnungssequenz: Ich darf noch ein bisschen Kaffee trinken}

Lorenz: ich darf noch ein bisschen Kaffee trinken das stört dich nicht Ingrid: du kannst das die ganze Zeit machen das stört mich gar nicht Lorenz: ja also dann (.) begrüsse ich dich offiziell zum Mentoratsgespräch Ingrid: ja

Lorenz: ich ((lacht)) ja

Ingrid: ((lacht))

Lorenz: ja ich hab dir eins ausgedruckt

Ingrid: ah okay mhm

Lorenz: weil die meisten nehmen es gar nicht mit weil sie wissen dass ich es sowieso ausdrucke

Ingrid: $m h m$

\subsubsection{Rekonstruktion der einzelnen Sequenzen}

Lorenz: Ich darf noch ein bisschen Kaffee trinken

Für eine Interpretation der Gesprächseröffnung wird in einem ersten Zugriff Kaffee trinken mit «fernsehen» ersetzt. Der Sprechakt ich darf noch ein bisschen «fernsehen» wäre vorstellbar, wenn ein Kind seinem Vater mitteilt, dass die Mutter erlaubt hat noch etwas fernzusehen. Das Kind handelt also regelkonform, hat die Erlaubnis von einer 
höheren Instanz erhalten und bearbeitet einen potenziellen Einwand bereits im Vorfeld. Mit ein bisschen wird die angesprochene Tätigkeit abgemildert.

In der vorgefundenen Version kündigt die sprechende Person an, dass sie noch Kaffee trinken dürfe, und zwar lediglich ein bisschen. Die Abmilderung ein bisschen stuft das Kaffee trinken manifest als Nebentätigkeit ein. Gleichzeitig wird die Tätigkeit im selben Sprechakt latent zu etwas Relevantem hochstilisiert. Mit dem Sprechakt wird keine Erlaubnis eingeholt, sondern dieser ist eher als Erklärung oder Mitteilung zu verstehen. Dennoch wird das Privileg der Tätigkeit - z.B. «fernsehen» respektive im realen Gesprächskontext Kaffee trinken - nicht unkommentiert im Sinne einer Selbstverständlichkeit beansprucht. Die Information zu diesem Privileg scheint das zentrale Strukturmerkmal des Sprechaktes zu sein. Kontextfrei kann vermutet werden, dass die sprechende Person in einer subalternen Position zur angesprochenen Person steht. Diese Position wird in der Fortsetzung des Sprechaktes gedreht.

Lorenz: das stört dich nicht

Der Zusatz schliesst lückenlos an das Vorausgehende an. Die Mitteilung ist auf einer manifesten Ebene als Symmetrieangebot im Sinne von «ich trinke nicht einfach Kaffee, sondern setze dich darüber auch in Kenntnis» zu betrachten. Regelhaft wäre die Aussage das stört dich nicht mit einem fragenden «Oder» am Ende des Satzes. Selbst wenn es sich hierbei um eine rhetorische Frage handeln würde, die nur eine Verneinung seitens der angesprochenen Person zuliesse, wäre dieser Zusatz erwartbar. Hier wird allerdings weder ein «Oder» angefügt noch die Stimme zu einer Frage erhoben. Insofern entspricht die Aussage einer Setzung und stellt eine Hierarchiemarkierung dar: «lch definiere hier die Regeln und darf, im Sinne einer Selbstermächtigung, mir gewisse Privilegien, wie beispielsweise Kaffee trinken, herausnehmen.» Verstärkt wird die superiore Position des Sprechers durch die Unterstellung, dass die andere Person durch sein Kaffee trinken nicht gestört werde.

Die Setzung im Symmetrieangebot «ich tue etwas» im Zusammenspiel mit der Unterstellung «das stört dich nicht» betont allerdings die Asymmetrie geradezu. Nur aus einer superioren Position kann ein Symmetrieangebot unterbreitet werden, weshalb hierbei genau die Asymmetrie, die es womöglich zu vermeiden gelte, befestigt wird. 
Der Gesprächsauftakt beinhaltet zwei zentrale Strukturmerkmale. Zum Ersten weist die vorliegende soziale Situation eine Ambivalenz betreffend Symmetrie und Asymmetrie auf. Diese Ambivalenz drückt sich in der Kombination aus gemachter Setzung, Unterstellung und gleichzeitiger Verschleierung aus. Weiter lässt sich eine gewisse Informalisierungsbewegung erkennen, die hier über die Geste des Kaffee-Trinkens Gestalt gewinnt.

Ingrid: du kannst das die ganze Zeit machen das stört mich gar nicht

Obwohl in der Gesprächseröffnung seitens des Mentors keine Frage formuliert wird, antwortet Ingrid als hätte man ihr eine Frage gestellt. Ihr Anschluss fällt nicht devot aus, wie zum Beispiel in «selbstverständlich darfst du das», sondern lässt sich eher als Selbstbehauptungsfigur lesen. Manifest teilt sie Lorenz durch ein unterbestimmtes das mit, dass sie sich nicht irritieren oder ablenken lasse. Sie zeigt sich diesbezüglich kooperativ und tolerant. In der Latenz lässt sie Lorenz durch Formulierungen wie die ganze Zeit und gar nicht auch ins Leere laufen. Die Übermasse in die ganze Zeit und gar nicht passen eben nicht zur Nebensächlichkeit des Ein-bisschen-Kaffee-Trinkens. Dadurch distanziert sich Ingrid von der asymmetrischen Positionierung und nimmt eine Haltung der Gleichgültigkeit ein. Diese lässt sich mit «mir ist klar, dass wir beide hierarchisch unterschiedlich positioniert sind, aber das kümmert mich nicht weiter, mach du nur» paraphrasieren. Ingrids Anschluss impliziert eine gegenteilige Dynamik als die der Unterwerfung oder der Opposition. Weder lässt sie sich von der latenten Asymmetriedemonstration behelligen noch beantwortet sie diese in ihrer Re-Adressierung.

Für den spezifischen Kontext des Mentorats weist das Gespräch in der bisherigen Gesprächseröffnung auf beiden Seiten eine Informalisierungsdynamik auf. Diese stellt allerdings nicht lediglich einen Übergang vom informellen zum formellen Teil dar, wie etwa in «Ich trinke noch ganz kurz fertig, ist das okay für dich?»

Vielmehr liegt es nahe, dass die Informalisierungsbewegung für den ganzen Teil des Gespräches angedacht ist, was in der Phrase die ganze Zeit zum Ausdruck kommt. Es scheint folglich einer beidseitigen Entscheidung zu entsprechen sich als Kollegin und Kollege zu adressieren, anstelle als Studentin und Mentor. 
Lorenz: ja also dann (.) begrüsse ich dich offiziell zum Mentoratsgespräch

Ingrid: ja

Wie bereits in der Gesprächseröffnung zwischen Frau Steffen und Herrn Bucher wäre auch für diesen Sprechakt die Begrüssung an einem Elternabend ein wohlgeformter Kontext. Dafür müsste das Personalpronomen dich mit der Pluralform «Sie» ersetzt und anstelle von Mentoratsgespräch ein Platzhalter verwendet werden. Die Eltern haben sich auf den bereitgestellten Stühlen eingefunden und die Lehrperson möchte nun mit ihrem Programm beginnen. Der offiziellen Begrüssung wäre ein inoffizieller Austausch mit einzelnen anwesenden Eltern vorausgegangen.

Als Strukturbedingung für eine entsprechende Begrüssung kommt eigentlich keine dyadische Situation infrage. Denn auch ungeachtet des Personalpronomens dich ist eine sprachliche Rahmung durch den Terminus offiziell in einem Zweiersetting irritierend. Unter Berücksichtigung dieser Überlegungen ist die betont formalisierte Begrüssung für ein Mentoratsgesprächs überschüssig.

Insgesamt gewinnen im bisherigen Wortwechsel Suchbewegungen auf den Spannungsfeldern von Symmetrie und Asymmetrie wie auch Informalisierung und Formalisierung Gestalt. Initiiert werden diese Bewegungen vom Mentor, der dadurch den Schutz einer formellen, distanzierten Situation aufweicht. Der Studentin werden Nähe- und Informalisierungsangebote unterbreitet, jedoch umgehend wieder durch eine offizielle, hochformelle Begrüssung zurückgenommen. Dieses Changieren zwischen den Polen Symmetrie und Asymmetrie sowie den Polen Informalisierung und Formalisierung lässt eine Unsicherheit bei der Etablierung des Settings erkennen. Aus der Perspektive von Ingrid bleibt ungeklärt, ob das Gespräch auf einer informellen Ebene fortgesetzt oder durch die sprachliche Offizialisierung auf eine entsprechende Ebene gehoben wird. Ebenfalls ungeklärt ist, ob sich die Akteurin und der Akteur in einer primär rollenförmigen, spezifischen Situation bewegen oder ob diese Rollenförmigkeit zugunsten einer diffuseren Form aufgehoben wird oder ob das Gespräch auch im weiteren Verlauf zwischen den beiden Polen changiert.

Als Anschluss an die offizielle Begrüssungsformel von Lorenz wäre in einer dyadischen Situation ein «Danke» oder etwas ähnliches von seitens der Gesprächspartnerin 
erwartbar. Ingrid schliesst allerdings mit ja an, was keiner Annahme der Begrüssung entspricht. Sie geht auf die offizielle Begrüssung nicht ein. In ihrer Antwort ist eine Distanzierungsbewegung wie auch Ansätze von Gleichgültigkeit zu erkennen und diese lässt Lorenz erneut ins Leere laufen.

Lorenz: ich ((lacht)) ja

Ingrid: ((lacht))

Lorenz: ja ich hab dir eins ${ }^{47}$ ausgedruckt

Ingrid: ah okay $\mathrm{mhm}$

Lorenz: weil die meisten nehmen ${ }^{48}$ es gar nicht mit weil sie wissen dass ich es sowieso ausdrucke

Ingrid: $m h m$

Lorenz schliesst zunächst mit dem Personalpronomen ich an und fährt nach einem Lachen mit ja fort, was sprechaktlogisch ein adäquater Anschluss an eine Frage wäre. Das eingeschobene Lachen wird von Ingrid mit einem Lachen erwidert. Die vorgefundene Formulierung ich (lacht) ja könnte gelesen werden, als gelte es noch etwas klarzustellen. Die Fortsetzung ja ich hab dir eins ausgedruckt ist von der Strukturlogik her erneut eine Reaktion auf eine nicht existierende Frage. Diese hypothetische Frage könnte sowohl durch Ingrid gestellt worden sein - «Hast du mir eins ausgedruckt?» oder selbstvergewissernden Charakter haben - «Hab ich dir eins ausgedruckt?» Auch eine Klarstellung im Modus von «es ist tatsächlich so, dass ich dir eins mitgebracht habe» wäre denkbar. Im Zusammenspiel mit dem Lachen ist die Selbstvergewisserung respektive Klarstellung als Unsicherheitsbearbeitung zu verstehen. Die Unsicherheit in Lorenz Sprechakt könnte eine Reaktion auf Ingrids Ablehnung seines Informalisierungsangebotes - ich darf noch ein bisschen Kaffee trinken das stört dich nicht - und seiner Formalisierungsgeste ja also dann (..) begrüsse ich dich offiziell zum Mentoratsgespräch - sein.

\footnotetext{
${ }^{47}$ Zur Klärung: Eins bedeutet hier ein Exemplar der Vorbereitungsaufgabe, das der Studentin im Vorfeld des Gesprächs elektronisch zugestellt wurde und das sie dem Mentor vor dem Gespräch ausgefüllt zu retournieren hatte. Er legt ihr an dieser Stelle ein Exemplar dieses Dokumentes vor.

${ }^{48}$ Es kann vermutet werden, dass mitnehmen hier anstelle von «mitbringen» verwendet wird. Im Schweizerdeutschen werden umgangssprachlich beiden Verben synonym verwendet.
} 
Strukturell erwartbar wäre an dieser Stelle ein Dank für die Geste des Mitbringens. Analog zur Gesprächsbegrüssung bleibt das Erwartbare aus. Die Antwort Ingrids ah okay $\mathrm{mhm}$ gestaltet sich indifferent und abwartend. Gut vorstellbar ist, dass die anschliessende Erklärung, die mit weil eingeleitet wird, interaktionsdynamisch auf dieses ResonanzVakuum mit einer Vorwärtsverteidigung reagiert und eine Legitimierungsverpflichtung nahe legt.

In Lorenz Handlung - ein Exemplar ausdrucken und mitbringen - gewinnt eine paternalistische Fürsorglichkeit Gestalt. Diese Fürsorglichkeit zeigt seine Einschätzung auf, dass die Studierenden seiner Unterstützung bedürfen. Hierbei eröffnet sich ihm die Option auf Anerkennung und Dankbarkeit, wonach er, riskant formuliert, bei Ingrid ringt.

Ingrids Reaktionen fallen wiederum distanziert und gleichgültig aus. Das bereits herausgearbeitete Strukturmerkmal des Auflaufen-Lassens reproduziert sich und lässt die Formulierung einer ersten Fallstrukturhypothese für die Eröffnungssequenz zu.

\subsubsection{Zusammenfassung und riskante Fallstrukturhypothese der Eröffnungssequenz Ich darf noch ein bisschen Kaffee trinken}

Bereits in den jeweils ersten Sprechakten der beiden Gesprächspartizipierenden lässt sich eine spannungsvolle, in sich widersprüchliche Mentoratspraxis feststellen. Es zeigen sich Unsicherheiten die Symmetrie-Asymmetrie-Konstellation betreffend. Während auf der manifesten Ebene ein Symmetrieangebot unterbreitet wird - «ich informiere dich darüber, noch ein bisschen Kaffee zu trinken» -, wird in der Latenz Asymmetrie hergestellt. Dies zeigt sich einerseits darin, dass keine Frage, sondern eine Setzung formuliert wird: Ich darf noch ein bisschen Kaffee trinken anstelle von «Darf ich noch ein bisschen Kaffee trinken?» Andererseits ist die Umkehrung, die Studentin informiert den Mentor «noch ein bisschen Kaffee zu trinken, da dies den Mentor nicht störe», undenkbar. Folglich weist die vorgefundene soziale Situation strukturell keine Symmetrie auf.

Die asymmetrische Konstellation und die damit einhergehenden Implikationen können als grundsätzliche Strukturproblematik des Settings vermutet werden. Diese Problematik wird hier zunächst über Symmetrisierungsangebote und über Informalisierungsbewegungen bearbeitet, gewagter formuliert, durch solche verschleiert. In der Zuspitzung 
lässt sich vermuten, dass die strukturell vorhandene Asymmetrie eine gewisse Gefahr für die Beziehungskonstellation von Studierenden zu Mentorierenden darstellt.

Eine zweite Unsicherheit betrifft das Spannungsfeld von Informalisierung und Formalisierung. Prinzipiell lassen sich lediglich formelle Gesprächsrahmungen informalisieren. Informelle Settings bedürfen hingegen keiner Informalisierungsbemühung. Wie zuvor erwähnt transportiert die Informalisierungsbewegung des Mentors auf der manifesten Ebene einen Symmetrieanspruch respektive ein Symmetrieangebot. Dieses Angebot bricht allerdings in der Latenz zusammen, da es selbst nur im Rahmen einer bestehenden Asymmetrie oder Hierarchie unterbreitet werden kann. Gleichzeitig bietet die gegenläufige Formalisierungs- und Distanzierungsbewegung auch Schutz für die Beteiligten. In der Rollenspezifik werden sie vor möglichen Entgrenzungen ${ }^{49}$, Nähe und Diffusität abgesichert.

Die riskante Strukturhypothese der Eröffnungssequenz kann wie folgt formuliert werden: Das bezeichnende Strukturmerkmal der Gesprächseröffnung ist das Changieren zwischen den Polen Symmetrie und Asymmetrie wie auch Informalisierung und Formalisierung. Formal asymmetrische Strukturen des Settings werden durch Symmetrie- und Informalisierungsbemühungen seitens der höher gestellten Person bearbeitet. Gerade in dieser Dynamik verfestigt sich in der Latenz die asymmetrische Struktur, da die Angebote und Bemühungen nur aus einem hierarchischen Verhältnis heraus überhaupt unterbreitet werden können.

\subsubsection{Zweite Sequenz: Aber das kostet nochmals deftig}

Im weiteren Gesprächsverlauf stellt der Mentor der Studentin die Frage, die er nach eigenen Angaben immer am Anfang solcher Gespräche stellt. Diese lautet: Warum kommst du eigentlich ins Mentoratsgespräch? Der Frage folgt ein Lachen seinerseits. Die Studentin führt dazu aus, dass sie Vertrauen zu ihm habe. Sie schätze es, dass sie ihre Anliegen mit einer Bezugsperson besprechen könne und nicht mit verschiedenen

\footnotetext{
49 Der Begriff Entgrenzung wird entsprechend dem von Wernet (2003) ausgearbeitete Phänomen der pädagogischen Entgrenzung verwendet. Wernet versteht Entgrenzungsbewegungen als typische Begleiterscheinungen schulischpädagogischer Interaktion. Dabei wird die einseitige Distanzwahrung der Lehrperson verletzt (siehe ausführlich Wernet, 2003).
} 
Dozierenden interagieren müsse. Im Anschluss erteilt der Mentor der Studentin explizit die Erlaubnis, ein erstes Thema ins Gespräch einzubringen. Ingrid stellt ihm einige organisatorische und technische Fragen zur Bachelorarbeit und zu Leistungsnachweisen. Weitere Anliegen hat sie keine.

Als nächstes spricht der Mentor die Studentin auf eine Aussage an, die sie in ihrer schriftlichen Vorbereitungsaufgabe getätigt hat. Sie spricht darin ausführlich über die als sehr hoch empfundene Belastung des Studiums. Ihr bliebe folglich wenig Zeit für Familie und Privatleben. An dieser Gesprächsstelle setzt die sequenzanalytische Rekonstruktion wieder ein.

Ingrid: und das war für mich der Punkt wo ich sag ich brauch diese Gewissheit das sagen zu können und wie gesagt jetzt hatte ich drei Tage gar nichts gemacht ich brauch das Wochenende wirklich und ich bin deswegen nicht im Verzug oder in irgendwelchem Stress

Lorenz: ja

Ingrid: das geht

Lorenz: ja aber das heisst dann schon dass die Planung recht gut funktioniert Ingrid: die funktioniert ja ja

Lorenz: dass dann auch //wirklich// Freiraum so Löcher gibt für Freiraum Ingrid: //mhm//

Ingrid: ja aber das sind eben trotzdem so Erfahrungen vom letzten Mal wo ich's Studium abgebrochen habe Lorenz: ja genau Ingrid: das ist noch geblieben Lorenz: ähm darf ich beim Abbrechen bleiben? Ingrid: ja Lorenz: weil irgendwo jetzt muss ich halt suchen weil es geht jetzt nicht schön der Reihe nach aber das ist ja egal ähm irgendwo hast du geschrieben Ingrid: meinst du mit dem Leistungsnachweis Deutsch Lorenz: ja du hast ziemlich viel Zeugs aufgezäh/t was du machen musst Ingrid: ja 
Lorenz: und dann irgendwo kam das Wort abbrechen vor im Fall ah das wart jetzt ich hab's mir noch angestrichen (.) siehst du wenn man sich nicht an den Plan hält aber ich find das passt jetzt genau (..) ah genau äh schau mal bei Seite vier da was hat sich verändert dass die Bezugssystemen so ähm die die zwei letzten «anscheinend habe ich deswegen auch die Motivation das Studium weiterzumachen» anscheinend Ingrid: ja Lorenz: also offenbar schon Ingrid: $\mathrm{mhm}$ Lorenz: so wie es jetzt auch tönt oder aber Ingrid: $\mathrm{mhm}$

Lorenz: ähm auch wenn ich oft an einem Punkt des Aufgebens bin Ingrid: ja

Lorenz: da habe ich mir einen Strich reingemacht mit der Frage weisst du das sind die Antennen da ist das also ist das ein Thema für dich oder oder Ingrid: nee also ich möchte schon das Studium fertigmachen und möchte auch in dem Beruf arbeiten aber das ist einfach von ich geh jetzt auf die Vierzig zu hab extrem viel Berufserfahrung bin auch auf Arbeit in der Lehrlingsausbildung drin Lorenz: ja genau Ingrid: und für mich ist oft dann wenn es mir zu viel wird oder wenn ich merk ich habe eine schlechte Woche was auch manchmal kommt grade wenn so Leistungsnachweise anstehen welchen Sinn hat es und ich muss mich immer selber motivieren um das was ich machen will auch zu erreichen und dann kommt Lorenz: ja

Ingrid: immer so diesen hat es einen Sinn sich diesem Stress jetzt hinzuzugeben Lorenz: ja Ingrid: oder hat es keinen Sinn weil ich habe Möglichkeit ich habe eine Arbeit ich habe eigentlich ne gute Stelle aber ich möchte gerne das andere machen Lorenz: ja ja Ingrid: vom Gefühl her und da bin ich dann immer so auf dem Grat geht man ins Alte zurück wo ich weiss ich bin nicht glücklich auf Dauer oder geh ich in das Neue wo ich weiss das ist was ich machen möchte 
Lorenz: aber kostet nochmal deftig

Ingrid: krass und das ist sehr schwierig dann ja weil ich schon so lange aus der Schule raus bin aus dem lernen raus bin ähm eben den Haushalt selber machen muss alles und das ist dann immer so

Lorenz: ja

Ingrid: ein Grat wo ich sag ich muss mich selber motivieren ich muss mir selber einen Tritt in den Hintern geben sagen nein ich will das jetzt und manchmal ist das ganz gut und manchmal ist das weniger gut Lorenz: ja ja also ich kann's mir schon noch vorstellen man hat etwas Sicheres also es ging mir ähnlich übrigens also fällt mir jetzt grad auf oder und dann sagt man ich mach mal was Neues also ich bin dann mit 42 nochmals studieren gegangen

Ingrid: $m h m$

Lorenz: war supertoll aber super anstrengend und so aber war aber auch die Frage wenn ich bleibe wo ich bin dann hab ich alles schön eben Ingrid: eben

\subsubsection{Rekonstruktion der einzelnen Sequenzen}

Ingrid: und das war für mich der Punkt

Die hier formulierte Aussage lässt sich als Einleitung lesen. Die sprachliche Figur erinnert an einen gefällten Entschluss, beispielsweise eine Liebesbeziehung aufzulösen oder eine Arbeitsstelle zu kündigen. Auch Beschlüsse, wie sich einer Operation zu unterziehen oder sich eine Auszeit zu gönnen, wären für die Aussage passend. Ebenso könnte eine Angeklagte in einem Verhör zu Protokoll geben, dass «dies der Punkt war», an dem sie die Kontrolle über sich verlor. Der Sprechakt ist auch in weniger dramatische Konstellationen passend. In der Nachbetrachtung eines Kinofilmes könnte jemand verlauten lassen, dass «dies für sie der Punkt war», an dem die Story auseinanderfiel. 
Die hier angedachten Kontexte lassen sich zwei differenten Auslegungen zuordnen. Zum einen kann Punkt für einen Umbruch oder eine Wende stehen. Der Begriff stellt dann eine Kummulationsfigur in einer Krisensemantik dar, wie bei der Auflösung des Liebesverhältnisses oder dem Verhör. Zum anderen kann Punkt sich, im Sinne von springendem Punkt, auf ein herausragendes Merkmal beziehen. Man denke etwa an die Filmbesprechung.

Die sprachliche Figur referenziert eine Handlungskette, die aus drei Elementen besteht: dem Ereignis - mit das gekennzeichnet und unterbestimmt -, der Entscheidung empirisch sichtbar und mit Punkt gekennzeichnet - und der Beschreibung der Konsequenz. Letztere müsste in der Fortsetzung weiter beschrieben werden.

In den genannten Beispielen beziehen sich die getroffenen Entscheidungen in erster Linie auf die Sprecherin selbst. Im Gegensatz existieren Kontexte, in denen die Sprecherin Entscheidungen für andere Personen fällt. Dies wäre zum Beispiel der Fall, wenn die Firmeninhaberin die Schliessung eines Werkes festlegt oder die Mutter ihr Kind in ein Internat schickt.

Wird von Punkt im Sinne eines Wendepunktes gesprochen, dann markiert dieser das Ende eines Prozesses oder die Entlastung respektive Auflösung eines Belastungsmomentes. Kommt Punkt die Bedeutung von dem springenden Punkt zu, steht nicht die Wende, sondern das ausschlaggebende Kriterium im Zentrum.

Strukturell unterscheiden sich die elaborierten Situationen nicht signifikant. Sie beschreiben alle eine Stelle am Ende eines mehr oder weniger langen Prozesses. Würde im Sinne eines gedankenexperimentellen Kontrastes nicht von einem Punkt, sondern von einem "Moment» gesprochen, wäre auch eine Genese denkbar. Im Unterschied zur Auflösung würde beim Neuanfang der Fokus prospektiv und nicht retrospektiv angelegt. Im empirischen Sprechakt wird jedoch nicht «Moment» sondern Punkt verwendet, weshalb eine Schliessbewegung angenommen wird.

Die Äusserungen der Sprecherin lassen sich als nachträgliche Bilanzierung gegenüber einer Zuhörerschaft, die im Geschehen involviert oder auch nicht involviert ist, lesen. Mit das wird eine Konkretisierung vorgenommen und etwas Spezifisches markiert. Das stellt den Wendepunkt, den Entscheidungspunkt oder auch den springenden Punkt dar. Die Formulierung das war verweist eindeutig auf ein vergangenes Ereignis und der Punkt markiert einen Moment der Differenz zwischen vorher und nachher. Der Sprechakt führt 
zu etwas noch Ausstehendem hin, das eine Selbstkundgabe, eine Selbstoffenbarung, ein Geständnis, eine Erklärung oder eine Beichte sein könnte.

Der Gesprächspartner wird als Person angesprochen, die etwas Bedeutsames erfährt. Das Beziehungsverhältnis zwischen den Sprechenden lässt dies zu oder erfordert es sogar. Vorstellbar ist eine Beziehungskonstellation der mittleren Vertrautheit. Stünden die Personen in einem sehr engen, eingeweihten Verhältnis, wäre eine entsprechende Aussage kaum erwartbar. Das vorliegende Beziehungsverhältnis weist jedoch auch Elemente oder Zeitspannen auf, die der angesprochenen Person bis dato verborgen waren. Durch die Präzisierung für mich gewinnt eine Figur der Selbstbezüglichkeit Gestalt, die persönliche Bedeutsamkeit aufzeigt und nach aussen getragen wird.

Die Aussage wird mit und das war für mich der Punkt retrospektiv formuliert. Es besteht die Möglichkeit, dass bereits während der damaligen Situation der Punkt als ausschlaggebend identifiziert wurde. Denkbar ist allerdings auch, dass dieser erst in der Retrospektive als solcher erkannt wurde. Wird Punkt als Zeit- oder Wendepunkt ausgelegt, dann lässt sich der Sprechakt als Hinführung zu einer biografisch konsistenten Erzählung lesen. Mittels der Aussage präsentiert sich die Sprecherin als autonome, reflektierte und handlungsmächtige Person.

In Rahmen des tatsächlichen Kontextes bezieht Ingrid mittels ihrer Aussage Position und zeigt einen Sachzusammenhang in einer ich-bezogenen Perspektive auf. Dies stellt eine Form der Selbstöffnung dar, wie sie beispielsweise in einer therapeutischen Konstellation erwartbar wäre. Auch lässt sich die Aussage als diskursive Öffnung lesen. In diesem Fall beansprucht die Studentin die Gültigkeit des Folgenden für sich, jedoch nicht für eine Allgemeinheit.

Unter Berücksichtigung des Kontexts lassen sich in Ingrids Sprechakt beide elaborierten Deutungsmuster von Punkt wiederfinden: Der auflösende Moment einer Belastung - mit retrospektivem Zeithorizont - und der Wendepunkt als Neuanfang - mit prospektivem Zeithorizont. Beide Deutungsmuster führen zu unterschiedlichen, möglichen Fortsetzungen. Im Fall der Auflösung eines Belastungsmomentes wäre beispielsweise ein Anschluss wie «an welchem mir klar wurde, so mache ich nicht mehr mit» erwartbar. Analog wäre bei einer Deutung als Wendepunkt eine Formulierung wie «an dem ich plötzlich erkannte, was ich wirklich wollte» vorstellbar. Ebenfalls denkbar ist eine Fort- 
führung, wenn der Punkt als springender Punkt ausgelegt wird - «weshalb ich auf Strafen verteilen völlig verzichtete».

In allen drei Auslegungen würde mit einer Erklärung angeschlossen werden, was sich an diesem Punkt abspielte.

Zusammenfassend gilt festzuhalten, dass der Sprechakt die Bedeutung einer subjektiven Berichterstattung hat, in der rückblickend auf etwas geschaut wird. Die Sprecherin nimmt mit dem Sprechakt eine klare Selbstpositionierung als Person vor, die retrospektiv Bedeutungsmomente entsprechenden Situationen zuschreibt und dies auch artikuliert und adressiert.

Ingrid: wo ich sag ich brauch diese Gewissheit das sagen zu können

Auf die Hinführung und das war für mich der Punkt folgt mit dem Nebensatz wo ich sag ein Selbstkommentar, der einen neuen Gedankengang einleitet. Dabei steht $w^{50}$ für «an dem». Diese Fortsetzung läuft der Lesart des springenden Punktes zuwider, weshalb diese nicht weiterverfolgt wird.

Der bisherige Sprechakt und das war für mich der Punkt wo ich sag ich brauche diese Gewissheit das sagen zu können wäre in seiner Gänze in (subjektiv) als prekär eingestuften Situationen vorstellbar. Die Gewissheit der sprechenden Person könnte in solchen Momenten Klarheit vermitteln und beruhigend wirken. Zu deuten wäre die Aussage, als ob die Person eine gewissenhafte (Neu-)Orientierung braucht, um agieren zu können. Die sprechende Person könnte zum Beispiel an einem spezifischen Punkt die Gewissheit über ihren Beziehungsstatus brauchen. Sie müsste für sich klären, ob sie sagen könne sie hat eine Beziehung oder nicht. Die Gewissheit wird durch die sprechende Person im Modus einer Selbst-Zusprache, unter Anwesenheit der angesprochenen Person, hergestellt. Dieser Vorgang stellt folglich eine Selbstvergewisserung dar.

In einem anderen Kontext könnte eine Firmeninhaberin die Gewissheit brauchen, sagen zu können «sie habe alle Optionen geprüft, welche die Schliessung des Werks verhindert hätten». Diese Aussage würde sie als Selbstkommentar formulieren und nach aussen

${ }^{50}$ Wo-Formulierungen anstelle von «an dem» sind mündlich gesprochen in der Schweiz durchaus geläufig. 
tragen. Die darin erwähnte Gewissheit erteilt die Firmeninhaberin - im Modus einer Selbst-Legitimation oder auch Selbst-Rückversicherung - in erster Linie sich selbst.

Die soziale Bedeutung des Sprechaktes ist die Darstellung einer nach aussen getragenen Selbstvergewisserung. Die hier benötigte Gewissheit impliziert, dass ein beträchtliches Mass an Ungewissheit und Kontingenz vorhanden ist. Auch möglich wäre, dass die Gewissheit noch nicht gefestigt ist und sich im Sprechakt implizit ein Entwicklungsthema andeutet. Wenn auch sich die sprechende Person vordergründig selbstbewusst äussert, beinhaltet der Sprechakt eine latente Legitimationsfigur, die Elemente einer Anrufung zur Absolution aufweist.

Etwas sagen zu können beinhaltet grundsätzlich eine Aussenwirkung und Zielgerichtetheit, da etwas zu anderen Personen oder gegenüber einer Öffentlichkeit gesagt wird. In der Sequenz bleibt offen, wer mit dem Gesagten adressiert wird. Würde die Sprecherin die Aussage primär an sich selbst richten, hätte sie die Möglichkeit dies zu artikulieren, beispielsweise «wo ich mir sag» oder auch «diese Gewissheit, mir sagen zu können.» Angesichts der unklaren Adressierung im Zusammenspiel mit einer formulierten Selbstbezüglichkeit lässt sich die Aussage als nach aussen gekehrter innerer Dialog lesen. Manifest zeigt sich die sprechende Person souverän. Latent scheint in der Ausführungsbedürftigkeit des eigenen inneren Dialoges auch eine Unsicherheit durch.

Bemerkenswert an der Aussage ist der Tempuswechsel. Während sich das war für mich der Punkt auf die Vergangenheit bezieht, ist wo ich sag im Präsens formuliert. Letzteres weist dadurch Kommentarcharakter auf. Es wird aus aktueller Sicht kommentiert, dass dies der entscheidende Punkt war. Der Tempuswechsel kann als Hinweis gelesen werden, dass das Ereignis zwar manifest abgeschlossen, aber latent noch aktuell und nicht abschliessend verarbeitet ist.

Inhaltlich lässt sich diese Gesprächsstelle nicht gänzlich erschliessen, da einiges unbestimmt bleibt. Festzuhalten ist, dass mit dem Sprechakt eine beachtliche Dramaturgie aufgebaut wird, die auf eine gewichtige Entscheidung oder drastische Konsequenz hindeutet. Diese Überzeichnung lässt eine prekäre Selbstoffenbarung erwarten.

Der Sprechakt dient nicht, wie zuvor antizipiert, der Auflösung oder des Neuanfangs, sondern es wird lediglich die Möglichkeit ebendieser artikuliert. Demzufolge geht es um eine Erlaubnis oder um ein Zugeständnis, eine Belastung aufzulösen oder einen Neu- 
anfang einzuleiten. Die beiden verbleibenden Lesarten, die der Auflösung eines Belastungsmoments und die des Neuanfangs, müssen entsprechend angepasst werden. Der bisherige Sprechakt lässt sich als Selbstvergewisserung, Selbstoffenbarung und Legitimationsersuchung - für etwas, das trotz Explikation bereits gedanklich vollzogen wurde - lesen. Eine (implizite) Anfrage auf Unterstützung ist nicht zu erkennen. Die Sprecherin spricht souverän, selbstreflexiv und in der Gegenwartsform über etwas Vergangenes und vermeintlich Abgeschlossenes. Dieser Tempuswechsel - von das war zu wo ich sag - ist auffällig und verweist auf eine Suchbewegung. Im Ruf nach Gewissheit wird latent eine Ungewissheit artikuliert und ihr Gegenüber als Empfänger ihrer Selbstkundgabe angesprochen. Diese Adressierung enthält den Appell das Offenbarte nachzuvollziehen und gutzuheissen.

Dadurch wird das dyadische Setting zu einem Ort der Selbstvergewisserung und der Bestätigung des eigenen Selbstbildes durch jemand anderen.

Im Rahmen der hier aufgebauten Dramaturgie wäre als Fortsetzung eine brisante Äusserung vorstellbar, die erst nach Abwägung des Raumes des Sagbaren getroffen werden kann.

Ingrid: und wie gesagt jetzt hatte ich drei Tage gar nichts gemacht

Die zuvor aufgebaute Dramaturgie wird im Sprechakt nicht weiter bedient. Die Studentin verweist mit und wie gesagt auf ein bereits vorher dargelegtes Thema und erstattet darüber Bericht. Mit der Konjunktion und wird die vorausgehende Aussage ein Stück weit abgeschlossen und ein weiteres, zusätzliches Argument eingeführt.

Die implizite Norm oder Erwartung, auf die rekurriert wird, ist eine andere als drei Tage nichts zu tun. Gerade die Formulierung gar nichts zeigt auf, dass der Bezugspunkt ausserhalb der Norm liegt und die Situation von der Sprecherin als aussergewöhnlich oder zumindest als berichtenswert erachtet wird.

Aus inhaltlicher Perspektive ist das nichts bemerkenswert. Ingrid hat in diesen drei Tagen wohl einiges, wenn auch nichts bezogen auf das implizit Erwartete, getan. Dieses nichts referenziert eine Erwartung, die auf ihr ganzes Leben zugreift und nicht (mehr) zwischen der Rolle und der Privatperson unterscheidet.

Für eine ausführliche Rekonstruktion wird die Fortsetzung des Sprechaktes dazu gezogen. 
Ingrid: ich brauch das Wochenende wirklich und ich bin deswegen nicht im Verzug oder in irgendwelchem Stress

Lorenz: ja

Auch in der Fortsetzung erinnert die latente Selbstrechtfertigungsfigur, die manifest einer Berichterstattung über ihren Entwicklungsprozess nachkommt, an ein therapeutisch angelegtes Sprechen. In einer Therapiesitzung würde die Patientin über sich selbst sprechen und sich damit gegenüber ihrem Gesprächspartner öffnen. Ingrid verweist einerseits auf die Befriedigung eines Bedürfnisses - drei Tage gar nichts zu tun - und artikuliert andererseits den Vollzug der Bedürfnisbefriedigung.

Mit dem Sprechakt unterstreicht die Studentin die Richtigkeit ihrer eigenen Situationseinschätzung: Sie kann sich ein Wochenende mit nichts tun, das nota bene bei ihr drei Tage dauert, leisten und leidet anschliessend nicht unter den negativen Konsequenzen wie Stress. Auch ist deswegen nicht im Verzug.

Manifest positioniert sich Ingrid als souveräne Person, die Kenntnisse über die eigenen Bedürfnisse hat und über eine angemessene Selbsteinschätzung verfügt. Diese Kombination ermöglicht ihr die Umsetzung einer erfolgreichen Work-Life-Balance. Unter Zuzug des Mentoratskontextes bleibt erklärungsbedürftig, weshalb sie dies dem Mentor mitteilt. Wenn die Studentin bezogen auf ihr Studium weder im Verzug ist noch Stress erlebt, wäre diese Aussage nicht notwendig. Träten sich Ingrid und Lorenz ausschliesslich rollenförmig gegenüber, würde sie sich nicht auch als Privatperson rechtfertigen. Sie aber zeigt und rechtfertigt sich in der Diffusität ihrer ganzen Person. Diese diffuse Sichtbarwerdung kann als Versuch einer Absicherung oder ersuchten (Absegnung) ausgelegt werden. Dies deutet abermals auf eine latente Unsicherheit von Ingrid hin. Die unklare Rollenzuschreibung und -annahme stellt folglich ein bedeutsames, fallspezifisches Strukturmerkmal dar.

Beachtenswert ist ausserdem die Bezeichnung irgendwelcher Stress. Der möglicherweise bereits erfahrene oder auch der antizipierte Stress wird hierbei beliebig und mit einer abwertenden Konnotation besetzt. In der Äusserung gewinnt zudem ein distinktives Moment Gestalt, das sich mit «es geht also nicht um den Stress, den typischerweise alle anderen erleben, sondern meine Situation weist auch einige Besonderheiten auf» 
paraphrasieren lässt. Ingrid präsentiert sich nicht als Normalfall, sondern ihre Situation weist in ihren Augen Besonderheiten auf.

Im Verzug sein beschreibt einen zeitlichen Rückstand oder eine zeitliche Verzögerung im Rahmen einer Durchführung. Mit der Aussage orientiert sich Ingrid erneut an einer Norm und rechtfertigt gleichzeitig ihr eigenes Abweichen von dieser. Unter Zuzug von Kontextwissen scheint klar, dass sich Ingrid mit im Verzug sein inhaltlich auf die Ausbildungsinstitution und deren Anforderungen bezieht.

Die Figur der Suche nach einer Legitimation wird gefestigt. Ob sich diese Legitimation eher als Rechtfertigung nach aussen oder Selbstvergewisserung manifestiert, bleibt offen. Insgesamt zeigt sich, dass die in Ingrids Aussage zum Vorschein kommende Norm, die der permanenten Arbeit ist. Im Gegensatz dazu scheint nichts tun nicht ausschliesslich Erholung zu sein, sondern es bedarf auch einer Anstrengung, nämlich sich von Richtmassen zu emanzipieren und selbstbestimmt Auszeiten zu nehmen. Dies geht mit einer Unsicherheit einher, die nach einer Legitimation, Absicherung oder (Absegnung` wie «das ist schon okay so" trachtet.

Ingrid verhandelt in ihrer Aussage die Thematik von impliziten Normen beziehungsweise Erwartungen und Möglichkeiten der Abweichung. Diese Thematisierung ist ein Indiz, dass dem Gespräch aus studentischer Perspektive eine Kontrollfunktion - als Strukturmerkmal - anlastet, der die Studentin hier begegnet und die sie bearbeitet.

Lorenz seinerseits schliesst mit ja an, was als anerkennendes, aktives Zuhören verstanden werden kann.

Ingrid: das geht

Mit das geht wird eine Bilanzierung der vorangegangenen Aussage vorgenommen. Eine solche Kurzaussage, in der Funktion eines Kommentars, trifft man systematisch in Situationen an, in denen (Selbst-)Bedenken geäussert werden. Vorstellbar wäre eine Situation, in der eine Person mit Höhenangst eine Hängebrücke überqueren müsste. Sie startet trotz ihrer Angst einen Versuch und kommentiert nach ein paar Schritten den eigenen Erfolg mit das geht.

Bezogen auf den Mentoratskontext lässt sich die Aussage ebenfalls als bilanzierender Kommentar betrachten, der die Selbstvergewisserung fortsetzt. Mit das geht bekräftig 
Ingrid einer bestimmten Herausforderung gewachsen zu sein. Diese Bekräftigung könnte dem zuvor ausgeführten Sprechakt Nachdruck verleihen und allfällige Restzweifel - auch die von Dritten - beseitigen. Die vorgenommene Bewertung der eigenen Leistung erfolgt selbstbewusst. Eine vorhandene Skepsis, die auf einer latenten Ebene durchscheint, wird dabei nicht nur gegenüber Lorenz, sondern auch in Hinblick auf sich selbst bearbeitet.

Lorenz: ja aber das heisst dann schon dass die Planung recht gut funktioniert Ingrid: die funktioniert ja ja

Vorstellbar wäre der Anfang des Sprechaktes - ja aber das heisst dann schon dass - im Kontext eines Familientreffens. Die Tochter, die in einer anderen Stadt studiert, erzählt ihren Eltern von ihrem neuen Hobby. Der Vater fragt im Anschluss: «Ja, aber das heisst dann schon, dass du auch für die Uni arbeitest?» Die sprechende Person, im Beispiel der Vater, möchte sich versichern, dass eine Befürchtung - hier: nicht für die Uni zu arbeiten - nicht zutrifft. Das aber zeigt an, dass ein Thema noch im Raum steht. Der Vater lässt seine diesbezüglichen Erwartungen durchblicken und mittels schon erhält die Formulierung auch eine suggestive Note.

Ein therapeutisches Setting ergäbe ebenso Sinn. Der Therapeut möchte seine vorgenommene Einschätzung, die auf Schilderungen der Patientin basiert, absichern. Ein entsprechender Sprechakt könnte lauten: «Ja, aber das heisst dann schon, dass es Ihnen unterdessen gelingt mit Ihrer Familie darüber zu sprechen?»

Beide Kontexte beinhalten eine kontingente Ausgangslage inklusive Restunsicherheit und Zweifel, die mit dem Sprechakt bearbeitet werden. Die fragende Person hätte bei einem allfälligen Scheitern des Gegenübers eine gewisse Mitverantwortung, die im familiären Kontext grösser, im therapeutischen Kontext kleiner ausfällt.

Auch im vorgefundenen Kontext scheinen Zweifel, entsprechende Erwartungen und eine Form der Mitverantwortung auf Seiten des Fragenden die dominanten Merkmale des Sprechaktes zu sein. Lorenz steigt mit den Worten ja aber ein, welche formal einen Einwand respektive eine Entgegnung ankündigen. Die Teilaussage das heisst übersetzt und leitet eine Schlussfolgerung ein, die mit der Fortsetzung dann schon auch suggestiven Charakter erhält. Das manifest ausgesprochene Lob wird mit dem Prädikat recht gut relativiert und zeigt latent noch vorhandenes Entwicklungspotenzial auf. Gerade mit dem 
Aufzeigen dieses Potenzials legitimiert der Mentor die eigene Rolle, da er ein solches erstens erkennt und zweitens möglicherweise eine Entwicklung anstossen kann.

Mentoratsgespräche weisen per se strukturelle Ambivalenzen auf, wie bereits in Kapitel 2.1.3 herausgearbeitet. Empirisch lässt sich an dieser Stelle die strukturelle Spannung zwischen angeleiteter Selbstreflexion durch den Mentor und dessen Bewertungs- oder Beurteilungsfunktionen aufzeigen. Bewerten und denkprozessanregende Begleitungen, als methodische Schritte hin zur Professionalisierung, gehen konstitutiv nicht miteinander einher. Die Aussicht auf Bewertung schränkt potenziell eine echte Öffnung seitens der Studierenden ein. Wenn wie hier Bewertungselemente aufscheinen, seien sie noch so wohlwollend oder milde vorgetragen, festigen sie die strukturelle Asymmetrie der Ausbildungssituation. Sie lassen sich auch nicht durch Kooperation bearbeiten, ohne Spannungen auf beiden Seiten zu erzeugen.

Nach diesem kurzen Exkurs wird die Analyse der Gesprächssequenz fortgesetzt. Strukturell entspricht der Sprechakt - ja aber das heisst dann schon dass die Planung recht gut funktioniert - einer Absicherung gegenüber dem (Nicht-)Eintreten eines befürchteten Szenarios. Im Sprechakt ist erkennbar, wie sich die sprechende Person zu diesem Szenario verhält.

Im Gesprächskontext wird an dieser Stelle die vorgängige Aussage von Ingrid - drei Tage nichts zu tun ohne in Stress oder Verzug zu geraten - als Schlussfolgerung verortet. Bereits der Umstand, dass es den Sprechakt des Mentors braucht, kann trotz des manifest ausgesprochenen Lobes, als Indiz für eine entsprechende Unsicherheit auf seiner Seite gedeutet werden.

Lorenz changiert im Sprechakt zwischen einer Frage - «Heisst das, dass die Planung recht gut funktioniert?» und einer Bewertung - «Ich stelle fest, dass deine Planung recht gut funktioniert». Die realisierte Variante schwächt die Bewertungslogik ab. Hier verdeutlicht sich die im Exkurs angesprochene, konstitutive Ambivalenz zwischen dem Anregen von Selbstreflexion und der gleichzeitigen Bewertungs- und Beurteilungsfunktion. Es sei an dieser Stelle festgehalten, dass diese Spannung zwar empirisch erkennbar ist, jedoch lediglich dezent in Erscheinung tritt.

Insgesamt formuliert Lorenz seine Aussage in einer vermittelten Lockerheit und geringer Formalität. Der Mentor benennt den Metakontext, über den Ingrid spricht beziehungsweise über den sie beide vorher bereits gesprochen haben. Seine Anmerkung lässt sich 
als Absicherung bezogen auf die funktionierende Planung lesen. Dabei übergeht er manifest die von Ingrid eingenommene Selbstproblematisierung und spricht sie auf informalisierende Art und Weise auf der Sachebene an. Das aber in seiner Formulierung legt nahe, dass ihre Problematisierung in der Latenz dennoch aufgenommen wurde. Ingrids Selbstrechtfertigung respektive Offenbarung gegenüber Lorenz zeigt demzufolge Wirkung. Er erkennt diese an und heisst ihre Handlung als Teil ihres Entwicklungsprozesses gut.

Mit die funktioniert ja ja bestätigt Ingrid die Übersetzungsleistung und Schlussfolgerung von Lorenz. Sie entkräftet seine mehrfache Relativierung, was sich auch in der Weglassung des evaluativen gut zeigt. Die vorgefundene Formulierung lässt sich mit einer üblicheren Syntax - «ja die funktioniert» - kontrastieren. Letztere Formulierung käme einer souveränen und sachlichen Bestätigung des bereits Gesagten gleich. Hier aber ist der Sprechakt die funktioniert ja ja. Alltagssprachlich wird eine Verdoppelung von ja oftmals als Ausdruck von mangelndem Interesse gelesen, nach dem Motto «red du nur». In dieser Auslegung würde sich Ingrid der Bewertung entziehen. Jedenfalls geht sie auf Lorenz Schlussfolgerung hinsichtlich der funktionierenden Planung nicht weiter ein. Ingrid gibt auf einer manifesten Ebene zu erkennen, dass sie sich diesbezüglich als kompetent erachtet. Auf einer latenten Ebene scheint mit ja ja durch, dass die funktionierende Planung nicht das Thema darstellt, worüber sie mit ihm eigentlich sprechen möchte.

Lorenz: dass dann auch //wirklich// Freiraum so Löcher gibt für Freiraum Ingrid: //mhm//

Lorenz elaboriert was unter einer schon recht gut funktionierenden Planung zu verstehen ist: eine Planung, die Freiräume und Löcher ermöglicht. Auf eine vertiefte Rekonstruktion der Aussage wird verzichtet, da sie eher inhaltlich und nicht strukturell bedeutsam erscheint.

Im Sinne eines Zwischenresumées kann festgehalten werden, dass Ingrid sich zu Beginn der Sequenz vorrangig auf die ihr geglückte Überwindung einer krisenhaften Situation bezieht. In ihrer Selbstdarstellung zeigt sie den durchlaufenen Entwicklungsprozess auf und betont die Bedeutsamkeit von Auszeiten. 
Lorenz steigt vordergründig auf ihr Thema ein. Er nimmt ihr eingebrachter Gegenstand als Ausgangspunkt, um die von ihm identifizierte Herausforderung zum Thema zu machen. Die rhetorische Absicherung, die funktionierende Planung betreffend, kann als Hinweis für vorhandene Zweifel gelesen werden.

Weiter gilt als dominantes Merkmal der bisherigen Sequenz hervorzuheben, dass die von Ingrid und Lorenz verhandelten Themen auseinanderklaffen. Er spricht über eine funktionierende Planung, sie über die benötigte Sicherheit und die Bedeutung von Pausen. Sie zeigt die Vereinnahmung ihrer Person durch das Studium auf, wodurch ihr kaum Zeit für andere Dinge bleibt. Sie fordert etwas ein, was gar keiner Einforderung bedarf respektive wo ihr die Unterstützung - insbesondere im Kontext des Mentoratsgesprächs - grundsätzlich gewiss ist. Zugespitzt formuliert gewinnt in ihrem Vorgehen eine Figur Gestalt, die mit 〈Freiticket für meine Art des Studierens〉 betitelt werden kann.

Für das Setting «Mentoratsgespräche» strukturell bedeutsam ist, dass die Studentin keine Beratung hinsichtlich einer existentiellen Ungewissheit anfragt, sondern die für sie bedeutsame Thematik im Modus einer Absicherung oder gesuchten (Absegnung) bearbeitet. Selbst wenn die Studentin dem Mentor die Rolle als Berater oder Unterstützer systematisch aberkennt, wird er dennoch gebraucht. Er erhält dabei eine Position, die ihm ermöglicht, Empathie und Verständnis gegenüber ihren Anliegen einzubringen.

Ingrid: ja aber das sind eben trotzdem so Erfahrungen vom letzten Mal wo ich's Studium abgebrochen habe

Ingrid beginnt den Sprechakt mit ja aber - analog zu Lorenz' vorangegangener Aussage, fährt mit einer Entgegnung fort und wechselt zurück zu ihrem eigenen Thema. Ingrid und Lorenz nehmen beide den jeweils zugespielten Ball formal auf, ohne auf dessen Inhalt einzugehen und fahren mit dem für sie relevanten Thema fort. Für Ingrid ist das die Erfahrung des Studienabbruchs. Würde sie ihre Aussage ohne ja aber und trotzdem formulieren - «das sind eben so Erfahrungen vom letzten Mal wo ich's Studium abgebrochen habe» - wäre dies ein wohlgeformter Anschluss, mit dem sie sich geläutert zeigen würde. Mit der vorgefundenen Formulierung gewinnt allerdings keine (reflexive) Läuterung Gestalt, sondern diese drückt eine Unsicherheit aus. Mit so Erfahrungen und vom letzten Mal bleibt sie vage und weist darauf hin, dass es weitere Male gab oder geben 
könnte. Sie teilt Lorenz mit, dass die Anforderung das Studium mit dem persönlichen Leben zu vereinbaren für sie weiterhin, trotz funktionierender Planung, voraussetzungsreich bleibt. Mit ihren Erfahrungen legitimiert Ingrid ihre aktuellen Handlungen und Überlegungen, wie beispielsweise eine Auszeit von drei Tagen einzulegen. Im Sprechakt - ja aber das sind eben trotzdem so Erfahrungen - weist sie zudem darauf hin, dass nicht die Planung herausfordernd ist, sondern die Erfahrungen aus dem Abbruch nachwirken und potenziell krisenbehaftet sind.

Lorenz: ja genau

Ingrid: das ist noch geblieben

Lorenz: ähm darf ich beim Abbrechen bleiben?

Lorenz spricht mit ja genau eine Bestätigung aus, wobei unklar bleibt, welchem Gedanken beigepflichtet wird. In einer Auslegung werden die gemachten Erfahrungen rund um den Studienabbruch gutgeheissen, was sich mit «ich kann deine Erfahrungen nachvollziehen» paraphrasieren lässt. Lorenz positioniert sich als jemand, der Verständnis aufbringt und adressiert Ingrid entsprechend als Person, die des Verständnisses bedarf. In einer weiteren Auslegung ist denkbar, dass ja genau die Funktionen von «so meine ich es» einnimmt. Dabei bearbeitet Lorenz die semantische Anschlusskrise, indem mit ja genau eine Brücke zwischen ihrem und seinem Thema geschlagen wird.

Ingrid setzt mit das ist noch geblieben ihre Ausführung fort und verleiht ihrer Erfahrung Nachdruck. Noch deutet an, dass der Studienabbruch noch immer eine Altlast ist. Ebenfalls weist noch darauf hin, dass der Neuanfang nicht ohne Rekurs auf den Abbruch funktioniert. Für Ingrid war der Abbruch ein prägendes Ereignis, das anhaltend nachwirkt und vor dessen Hintergrund sie ihr aktuelles Erleben rahmt.

Die anschliessende Frage von Lorenz beginnt mit dem Verzögerungslaut ähm, der funktional die Fortsetzung der Äusserung anzeigt. Lorenz' Frage ist auffallend formell ähnlich wie die Frage zu Gesprächsbeginn. Er holt sich von Ingrid die Erlaubnis für eine Themenvertiefung ein. Das ist auf der Ebene der Interaktion bemerkenswert, da sie das Thema zuvor selbst eingebracht hat. Vor allem zeigt sich in der Frage, dass er die thematische Gesprächsführung nicht qua seiner Rolle bei sich festmacht. Lorenz stützt nicht nur die Fokussierung von Ingrid und übergeht die Besprechung seines Themen- 
vorschlags, sondern er holt an dieser Stelle zusätzlich ihre Erlaubnis ein, an dem von ihr eingebrachten Thema zu verweilen.

Wenn die Formulierung darf ich beim Abbrechen bleiben auch manifest eine Einwilligung einholt, wird mit ihr auf der latenten Bedeutungsebene die Expertise der sprechenden Person hervorgehoben. Gut vorstellbar wäre die Aussage beispielsweise in einer therapeutischen Konstellation, wenn der Therapeut meint den Kern eines Problems identifiziert zu haben und eine vertiefte Auseinandersetzung initiiert.

Im Rahmen des Mentoratsgesprächs lässt sich die Reaktion von Lorenz als Annäherung an ein therapeutisches Setting lesen. In diesem Setting wird interaktiv auf die noch vorhandene Krise reagiert und diese erneut aufgegriffen, da eine Vertiefung der Krisenbearbeitung lohnenswert erscheint.

Kontextgebunden überrascht seine Frage auch, da Lorenz qua seiner Rolle als Mentor keine Zustimmung der Mentee benötigt. Das formale Einholen einer Erlaubnis gleicht folglich einer rhetorischen Frage. Wie bereits bei Gesprächsbeginn wird an dieser Stelle eine Spannung zwischen augenscheinlichem Symmetrieangebot und struktureller Asymmetrie des Settings erzeugt. Die Frage lässt sich angesichts der sozialen Situation zwischen Mentor und Mentee eigentlich nur mit ja beantworten. Analog zur Eröffnungsaussage ich darf noch ein bisschen Kaffee trinken - nimmt Lorenz die Rolle des Mentors wiederholt nicht selbstverständlich und eindeutig ein. Das herausgearbeitete Strukturmerkmal der in sich brüchigen Figur einer symmetrischen und asymmetrischen Konstellation wird hier reproduziert.

Der nächste Gesprächsabschnitt befasst sich inhaltlich mit dem Aufsuchen der Stelle im Vorbereitungsdokument, die er anschliessend zitiert. Dieser Abschnitt wird nicht flächendeckend feinsequenziell, sondern in grösseren Sinnabschnitten in den Blick genommen.

Ingrid: ja

Lorenz: weil irgendwo jetzt muss ich halt suchen weil es geht jetzt nicht schön der Reihe nach aber das ist ja egal ähm irgendwo hast du geschrieben

Ingrid: meinst du mit dem Leistungsnachweis Deutsch

Lorenz: ja du hast ziemlich viel Zeugs aufgezäh/t was du machen musst Ingrid: ja 
Lorenz: und dann irgendwo kam das Wort abbrechen vor im Fall ah das wart jetzt ich hab's mir noch angestrichen (.) siehst du wenn man sich nicht an den Plan hält aber ich find das passt jetzt genau (..) ah genau äh schau mal bei Seite vier da was hat sich verändert dass die Bezugssystemen so ähm die die zwei letzten "anscheinend habe ich deswegen auch die Motivation das Studium weiterzumachen ${ }^{51}$ anscheinend

Ingrid: ja

Lorenz: also offenbar schon

Ingrid: $m h m$

Lorenz: so wie es jetzt auch tönt oder aber

Ingrid: $m h m$

Lorenz: ähm "auch wenn ich oft an einem Punkt des Aufgebens bin $»^{52}$ Ingrid: ja

Ingrid beantwortet die Anfrage von Lorenz, ob er beim Abbrechen bleiben dürfe, mit ja. Nachdem sie den Studienabbruch in ihren Ausführungen so viel Raum gegeben hat, überrascht diese Kurzantwort. Vielmehr wäre eine ausführlichere Zustimmung zu erwarten. Obwohl Ingrid manifest eine Zusage erteilt, scheint sie latent eine gegenteilige, leicht abwehrende Bewegung zu vollziehen, als ob sie das krisenhafte Thema Studienabbruch nicht seiner Führung übergeben möchte. Diese Figur der Abwehr ist anschlussfähig an die bereits herausgearbeitete Dynamik, die auf 〈Absegnung〉 und nicht auf (therapeutische) Beratung ausgerichtet ist.

Lorenz verwendet einige unpräzise und alltagssprachliche Begriffe wie irgendwie, irgendwo und ziemlich viel Zeugs. Dies weist grundsätzlich auf einen informellen Kontext hin. Der Begriff Zeugs ist umgangssprachlich, enthält eine abwertende Konnotation und steht für Belanglosigkeiten ohne Eigenwert. Oftmals wird Zeugs auch als Platzhalter gebraucht, wenn im Moment des Sprechens das präzise Wort fehlt. Die abwertende Dimension haftet allerdings auch dieser Auslegung an. Auch Wendungen wie das ist ja egal oder siehst du wenn man sich nicht an den Plan hält sind als Versuche von Informali-

\footnotetext{
51 Zitierte Textstelle aus dem Vorbereitungsdokument der Studentin

52 Zitierte Textstelle aus dem Vorbereitungsdokument der Studentin
} 
sierung und kollegialer Begegnung zu lesen. Diese Informalisierungsbemühungen stehen auf einer Interaktionsebene allerdings im Widerspruch zur formellen Frage-Darf ich beim Abbrechen bleiben? Es lässt sich folglich feststellen, dass die asymmetrische Konstellation weiterhin über Symmetrieangebote bearbeitet wird.

Die Aussage siehst du wenn man sich nicht an den Plan hält aber ich find das passt jetzt genau stellt einen sich selbsterklärenden Kommentar dar. In diesem teilt Lorenz Ingrid mit, dass er dem Gesprächsverlauf mehr Bedeutung zuschreibt als seinem ursprünglichen Gesprächsplan ${ }^{53}$. Diese Planänderung nimmt er nicht kommentarlos vor, sondern expliziert sie. Dies lässt sich als Indiz für Unentschlossenheit oder auch Unsicherheit bezogen auf den weiteren Gesprächsverlauf lesen. In typischen kollegialen, symmetrischen Gesprächen wären solche Selbstkommentare nicht erforderlich und auch nicht erwartbar. Viel eher sind sie in Situationen vorzufinden, die eine fehlende Klarheit hinsichtlich Symmetrie und Asymmetrie aufweisen. In Lorenz' Sprechakt wird das Kollegiale und Heterarchische betont. Er ist bedacht kollegial wahrgenommen und nicht zurückgewiesen zu werden.

In der Fortsetzung seiner Äusserung sucht er eine bestimmte Textstelle in der Vorbereitungsaufgabe und zitiert diese. Ingrid hat zuvor im Gespräch ausgeführt, dass die Planung ihrer Work-Life-Balance funktioniert und sie auch Arbeitspausen verantworten kann. In ihrem Vorbereitungsdokument spricht sie konträr davon oft am Punkt des Aufgebens zu sein. Auf diesen Widerspruch rekurriert Lorenz und er ersucht mit also offenbar schon und so wie es jetzt auch tönt oder aber eine Bestätigung ihres Entschlusses das Studium fortzuführen. Ingrid erteilt ihm diese zweimal mit $\mathrm{mhm}$ nur zaghaft, weshalb er, so die Vermutung, die dramatische Formulierung aus ihrer schriftlichen Vorbereitung - ähm auch wenn ich oft an einem Punkt des Aufgebens bin - vollständig zitiert. Ihm scheint es weniger um die Thematisierung des Studienabbruchs von damals, als um eine Risikoeinschätzung einen erneuten Abbruch betreffend zu gehen.

Zusammenfassend lässt sich sagen, dass das prekäre Thema Studienabbruch vom Mentor in einer bemerkenswert ungezwungenen Sprache verhandelt wird. Dies kann als Versuch der Entdramatisierung oder auch Entproblematisierung gelesen werden. Auf diese

\footnotetext{
53 Dieser Plan ist nicht gegenständlich vorhanden, jedoch erwähnt der Mentor zu Beginn und ähm ich denke wir gehen einfach so ein bisschen durch und sehen dann wo wir landen. Das Durchgehen bezieht sich, so die offensichtliche Vermutung, auf die Vorbereitungsaufgabe, die die Studentin ihm als Gesprächsvorbereitung hat zukommen lassen.
} 
bemüht ungezwungene Art und Weise der Kommunikation reagiert die Studentin mit einer Schliessfigur. Sie wird mit ihren eigenen Aussagen konfrontiert und verhält sich dazu minimalistisch und ausweichend.

Nachfolgend wird mit der kleinschrittigen Rekonstruktion fortgefahren.

Lorenz: da habe ich mir einen Strich reingemacht mit der Frage weisst du das sind die Antennen da ist das also ist das ein Thema für dich oder oder

Im Sprechakt wird eine Belanglosigkeit expliziert. Lorenz zeigt auf, was er getan hat, nämlich einen Strich reingemacht mit einer Frage. Durch die Erläuterung der gesetzten Markierung gibt Lorenz Ingrid Einblicke in seine Arbeitsweise und positioniert sich als Experte. Er erwähnt eine Frage, ohne diese explizit zu stellen. Anschliessend fügt er einen Einschub an, in dem er eine Erklärung - die Antennen - für das Aufgreifen des Themas ausführt. Die Formulierung weisst $d u$ das sind die Antennen scheint bedeutsam. Ein angelehnter Sprechakt wäre vorstellbar, wenn beispielweise der Grossvater, mit zitternden Händen, zu seinem Enkelkind sagt: «Weisst du, das sind die Nebenwirkungen der Medikamente.» Die Aussage erklärt eine Handlung und ermöglicht gleichzeitig, sich von dieser zu distanzieren. Die Handlung erfolgt aus der Perspektive des Sprechers als Automatismus und lässt sich nicht unterdrücken. Nach dieser Lesart wird mit weisst $d u$ das sind die Antennen durch eine Erklärung Verständnis für eine Handlung ersucht. Ein anderer Kontext wäre: «Weisst du, das ist meine fürsorglichen Art.» Hier geht es nicht allein um Verständnis für eine Handlung, sondern mehr noch um Anerkennung oder gar Bewunderung einer Eigenschaft.

Die daran anschliessende Frage weist durch ihre rhetorische Struktur - die beiden oder ein vergewisserndes Moment auf - «Meine Antennen zeigen das schon richtig an, oder?» Das bereits diskutierte Anerkennungsersuchen wird reproduziert.

Mit der Metapher der Antennen baut Lorenz manifest eine gewisse Distanz zur eigenen Handlung auf. Gleichzeitig positioniert er sich als Experte, der mit Antennen als Werkzeug arbeitet. Im übertragenen Sinne streckt er seine Antennen aus und empfängt Signale, die auf problembehaftete Textstellen hindeuten. Auf einer latenten Ebene enthält der Sprechakt noch eine weitere Implikation: Die erhoffte Anerkennung für seine Arbeitsweise und seine Fähigkeiten gewinnt erneut Kontur. Er signalisiert, dass er bei der 
Bearbeitung der aufgedeckten Probleme Hilfestellung leisten kann und wird. Die Aussage von Lorenz gerät bei da ist das also ist das in eine syntaktische Schieflage und lässt sich als Suchbewegung nach einer passenden Formulierung lesen.

Im Vorbereitungsdokument schreibt Ingrid: «Anscheinend habe ich deswegen auch die Motivation das Studium weiterzumachen, auch wenn ich oft an einem Punkt des Aufgebens bin.» Lorenz wiederholt beim Zitieren der Textpassage das Wort anscheinend anscheinend habe ich deswegen auch die Motivation das Studium weiterzumachen anscheinend -, obwohl es im Originaltext nur einmal vorkommt. Hier scheint eine Irritation seinerseits durch, die sich in der Wiederholung von anscheinend Ausdruck verschafft. Anscheinend bedeutet "soweit man das, nach Kenntnissen der Tatsachen, sagen kann» und wird für Vermutungen, die sich auf Sachverhalte beziehen, verwendet. Der Begriff findet im Regelfall keine Anwendung, wenn über eigenes Empfinden oder persönliche Entscheidungen gesprochen wird, wie Ingrid dies in ihrem Text tut.

Die konkrete Verwendung im Text kann als Distanzierung von der eigenen Entscheidung gedeutet werden. Denkbar ist, dass diese Distanzierungsbewegung bei Lorenz eine Irritation auslöst und er Ingrid darauf anspricht. Er positioniert sich gegenüber Ingrid als jemand, der nicht nur jenes aufnimmt und deutet, was ihm schriftlich oder mündlich mitgeteilt wird, sondern auch auf Details zurückgreift, die seine Antennen zwischen den Zeilen wahrnehmen. Diese Selbstpositionierung als Experte verweist auf einer latenten Bedeutungsebene allerdings auf eine Unsicherheit mit der eigenen Rolle. Er unternimmt dahingehend Bemühungen seine Rolle zu legitimieren.

Abschliessend gilt es zu konstatieren, dass im vorgefundenen Sprechakt der Fokus auf der Selbstdarstellung des Sprechers und seiner Arbeitsweise liegt.

Ingrid: nee also ich möchte schon das Studium fertigmachen und möchte auch in dem Beruf arbeiten aber das ist einfach von ich geh jetzt auf die Vierzig zu hab extrem viel Berufserfahrung bin auch auf Arbeit in der Lehrlingsausbildung drin Lorenz: ja genau

Auch in Ingrids Anschluss reproduziert sich der strukturelle Aspekt der Selbstdarstellung. Die Erwähnung des eigenen Alters lässt sich als Akt der Selbstzuordnung zu den späten Bildungsaufsteigerinnen verstehen. Diese Zuordnung ist für sie bedeutsam. Nebst dem 
Alter erwähnt sie auch ihre Berufserfahrung, die sie als aussergewöhnlich darstellt, sowie ihre Tätigkeit in einem schulnahen Berufsfeld. Mit ihren Ausführungen unterstreicht sie die Besonderheit ihrer Person argumentativ. Dies löst bei ihrem Gegenüber eine Form von Resonanz aus, die in ja genau Ausdruck findet. Anhand des bisherigen Gesprächsverlauf gewinnt eine Figur Gestalt, in der auf ein entsprechendes Verständnis und Einfühlungsvermögen beim Gegenüber spekuliert wird.

Der Anschluss ja genau, als bestätigende Aussage, wäre im Allgemeinen erwartbar, wenn zuvor eine Meinung formuliert oder Argumentationslinie komplett dargelegt wurde. An der vorgefundenen Stelle führt Ingrid jedoch ihre Situation aus, weshalb der Anschluss ja genau irritiert und einer Erklärung bedarf. Dies umso mehr, als dass Lorenz bereits an eine vorherige Aussage von Ingrid mit ja genau anschloss, ohne inhaltlich anzuknüpfen. Ja genau nimmt demzufolge eher die Funktion einer gutheissenden und beipflichtenden Geste ein, mit der ein grundsätzliches Verständnis gegenüber Ingrid und ihrer Situation kundgetan wird.

Die Gesprächspassage stellt eine Selbstoffenbarung oder Selbstkundgabe der Studentin dar, in der sie gegenüber dem Mentor ihre Erfahrungen, Gedanken und Gefühle aufzeigt. Eine Selbstoffenbarung weist je nach Kontext unterschiedliche Dynamiken auf. Denkbar ist beispielsweise, dass die sich offenbarende Person beim Gegenüber Unterstützung sucht. In dieser Konstellation verfügt die adressierte Person über einen Wissens- oder Erfahrungsvorsprung, den sich die Gesprächspartnerin zu Nutze macht. Diese Konstellation trifft beispielsweise für eine therapeutische Situation zu.

Eine Selbstoffenbarung löst möglicherweise beim Gegenüber Verständnis aus. Dies wäre zum Beispiel der Fall, wenn in einem hierarchischen Setting die Praktikantin ihren Vorgesetzten über die eigenen prekären Familienverhältnisse informiert. Die Selbstoffenbarung fungiert hier als Klarstellung, um mögliche Fehleinschätzungen zu berichtigen, ihnen vorzubeugen oder das Urteil des Gegenübers abzumildern. In eher heterarchisch geprägten Situationen lassen sich durch Selbstoffenbarung auch Nähe und verbindende Elemente herstellen, wenn beispielsweise eine Person in ein Beziehungsgeheimnis eingeweiht wird.

Diese Ausführungen zeigen, dass sich in einer Selbstoffenbarung grundsätzlich unterschiedliche Motivlagen Ausdruck verschaffen können. Wenn die Selbstoffenbarung beispielweise in der Funktion eines Ventils eingesetzt wird, könnte sich für die offen- 
barende Person eine persönliche Entlastung oder Erleichterung einstellen. Dabei würde die Wirkung in erster Linie die Person selbst betreffen. Die Selbstoffenbarung könnte aber auch strategische Ziele verfolgen, um vornehmlich beim Gegenüber eine der oben angedachten Wirkungen zu erzeugen.

Die Beziehungskonstellation zwischen Ingrid und Lorenz ermöglicht es der Studentin ihren Mentor in selbstoffenbarender Form anzusprechen. Dabei wird Lorenz als Person adressiert, die Verständnis für ihre Situation zeigt. Ob diese Adressierung auch strategisch motiviert ist, lässt sich nicht abschliessend klären. Ebenso ist denkbar, dass die Selbstoffenbarung für sie selbst entlastend ist. Zugespitzt lässt sich die hier vorgefundene Interaktionsfigur - aus Ingrids Perspektive - als Entlastung qua 〈Absegnung〉 beschreiben. Diese Figur steht allerdings in einem Spannungsverhältnis zu Lorenz' eigenem Umgang mit der Offenbarung. Die Offenbarung scheint bei ihm eine Dynamik auszulösen, sich in die Rolle des Therapeuten, der von Ingrid gebraucht wird, zu begeben. Als Therapeut ist sein Expertenstatus nicht befestigt. Deshalb befindet er sich in anhaltender Unsicherheit und ringt nach Anerkennung und Legitimierung seiner Rolle. Im Weiteren versteht Lorenz Ingrids Offenbarung als Näheangebot, das er entsprechend reziprok erwidert.

Lorenz zeigt sich als zuständig für die signalisierten Probleme und bietet Unterstützung an, indem er diese aufgreift. Seine Reaktion ist non-direktiv, verständnisvoll und wohlwollend.

Ingrid führt die Facetten ihrer Krise, die für sie eine Herausforderung darstellen, in der Folge weiter aus:

Ingrid: und für mich ist oft dann wenn es mir zu viel wird oder wenn ich merk ich habe eine schlechte Woche was auch manchmal kommt grade wenn so Leistungsnachweise ${ }^{54}$ anstehen welchen Sinn hat es und ich muss mich immer selber motivieren um das was ich machen will auch zu erreichen und dann kommt Lorenz: ja Ingrid: immer so diesen hat es einen Sinn sich diesem Stress jetzt hinzuzugeben Lorenz: ja

\footnotetext{
54 Unter Leistungsnachweis wird hier eine schriftliche Studienleistung verstanden, die die Studierenden in einem Studienseminar oder einem Unterrichtsmodul verfassen müssen, um die entsprechenden ECTS-Punkte zu erwerben.
} 
Ingrid: oder hat es keinen Sinn weil ich habe Möglichkeit ich habe eine Arbeit ich habe eigentlich ne gute Stelle aber ich möchte gerne das Andere machen Lorenz: ja ja

Ingrid: vom Gefühl her und da bin ich dann immer so auf dem Grat geht man ins Alte zurück wo ich weiss ich bin nicht glücklich auf Dauer oder geh ich in das Neue wo ich weiss das ist was ich machen möchte Lorenz: aber kostet nochmal deftig Ingrid: krass und das ist sehr schwierig dann ja weil ich schon so lange aus der Schule raus bin aus dem lernen raus bin ähm eben den Haushalt selber machen muss alles und das ist dann immer so

Lorenz: ja

Ingrid: ein Grat wo ich sag ich muss mich selber motivieren ich muss mir selber einen Tritt in den Hintern geben sagen nein ich will das jetzt und manchmal ist das ganz gut und manchmal ist das weniger gut Lorenz: ja ja also ich kann's mir schon noch vorstellen man hat etwas Sicheres also es ging mir ähnlich übrigens also fällt mir jetzt grad auf oder und dann sagt man ich mach mal was Neues also ich bin dann mit 42 nochmals studieren gegangen

Ingrid: $m h m$

Lorenz: war supertoll aber super anstrengend und so aber war aber auch die Frage wenn ich bleibe wo ich bin dann hab ich alles schön eben Ingrid: eben

Bereits in der gerafften Betrachtung des weiteren Gesprächsprotokolls lässt sich erkennen, dass Ingrid ihre Selbstoffenbarung fortsetzt. Ihr fällt es schwer sich bei erhöhtem Stress und Druck für das Studium zu motivieren. Sie lässt Lorenz wissen, dass sie die Sinnhaftigkeit der Ausbildung grundsätzlich infrage stellt, da sie sich bereits erfolgreich beruflich etabliert hat. Sie würde einerseits zwar gerne das Andere machen - wie sie an dieser Stelle das Unterrichten oder die Tätigkeit als Lehrerin unterbestimmt bezeichnet -, schreckt aber andererseits vor der inhärenten Unsicherheit zurück. Ingrid befindet sich auf einer Gratwanderung zwischen der Sicherheit des Altbekannten und der Verheissung des Neuen. Ihre diesbezügliche Zerrissenheit und die Fragen, mit denen sie sich konfron- 
tiert sieht, schildert sie ausführlich und mit einer gewissen Dramaturgie. Die Formulierung sich dem Stress hingeben versinnbildlicht ein Gefühl des Ausgeliefert-Seins und des SichUnterwerfens unter institutionelle Rahmenbedingungen. Ingrid adressiert Lorenz mit der Schilderung ihrer Zerrissenheit und zeigt sich dadurch als Person, die nach Verständnis und 〈Absegnung i ihres Handelns sucht.

Lorenz reagiert auf die Schilderung mit aktivem Zuhören und kurzen Kommentaren. Mit der kumpelhaften und informellen Formulierung aber das kostet nochmals deftig zeigt er Verständnis auf. Gleichzeitig unterbreitet er ein Näheangebot und ist um Vergemeinschaftung bemüht.

Aus einem anderen Blickwinkel erlangt auch ein selbstbezogenes Motiv des GemochtWerdens Gestalt. Das Aufscheinen dieses Motivs verweist auf die grundsätzliche Prekarität des Settings. Der Status von Lorenz ist nicht strukturell durch das Setting gewährleistet, sondern erfordert eine interaktive Aufrechterhaltung. Dies zeigt sich unter anderem in der bereits besprochenen Aussage - das kostet nochmals deftig -, mit der er Ingrids Dramaturgie übernimmt und entsprechend untermauert.

Ingrids Fortführung bleibt mit krass im umgangssprachlichen Modus. Die Kombination aus aber kostet nochmal deftig mit dem Anschluss krass wäre beispielsweise in einer Stammtischrunde mit Bezug auf einen Dritten vorstellbar. Eine Person erzählt in lockerer Sprache, dass bei ihrem Nachbarn im Haus nachträglich ein Pool eingebaut wird. Ob sich das Investment wirklich lohnt, scheint für den Sprecher ungewiss. Mit krass bestätigt die angesprochene Person diese Einschätzung. Aus beider Sicht ist das Unterfangen aufwendig und extravagant. Gleichzeitig scheint eine gewisse Bewunderung für das Unterfangen durch.

Wendet man diese strukturellen Merkmale auf den vorliegenden Kontext, sind die Elemente der Bewunderung und der Ungewissheit, ob sich die Investition tatsächlich auszahlt, übertragbar. Der Anschluss krass von Ingrid legt nahe, dass sie genau jenes vom Mentor bekommt, was sie sucht: Bestärkung, Bewunderung und Verständnis für ihre Situation. Diese Bestätigung wird noch verstärkt, wenn Lorenz mit ja ja ich kann's mir schon noch vorstellen und seinen weiteren Ausführungen ihre Schilderung affirmiert. Sein Einfühlungsvermögen und die Fähigkeit der Perspektivübernahme führt er auf eigene biografische Erfahrungen zurück. 
Die Anerkennung, Bewunderung und Anteilnahme gebühren auf einer manifesten Ebene Ingrid und ihrer anspruchsvollen Situation. In der Latenz fordert Lorenz diese Attribute allerdings auch für sich selbst ein, da er sich Anfang Vierzig in einer vergleichbar anspruchsvollen Situation befand und diese - so die Deutung des Sprechakts - erfolgreich meisterte.

Bei flächiger Sichtung des erhobenen Materials gewinnt verdichtet Gestalt, dass der Mentor ein für die Studentin bedeutsames Thema identifiziert hat und sichtlich bemüht ist, sie bei der Bearbeitung des Themas zu unterstützen. Diese Unterstützung erfolgt mehrheitlich durch Anerkennung und Anteilnahme, die in der Interaktion über ein Ansprechen und Aufgreifen stattfindet. Die Strukturlogik der Interaktion lässt sich als Selbstoffenbarung beschreiben, die beim Empfänger auf Resonanz stösst. Diese Empathie und das Verständnis werden der Senderin kollegial entgegengebracht.

\subsubsection{Zusammenfassung und riskante Fallstrukturhypothese der Sequenz Aber kostet nochmal deftig}

Die in sich brüchige Gesprächsfigur, die zwischen einer diffusen und einer rollenförmigen, spezifischen Sozialbeziehung sowie zwischen informell und formal changiert, hat als deskriptiver Befund weiterhin Bestand.

Es lässt sich festhalten, dass die Anliegen, die für die Studentin subjektiv relevant sind, im Gespräch Gehör finden. Zu Beginn der Sequenz nehmen die Studentin und der Mentor auf unterschiedliche Referenzrahmen Bezug. Während sie vordergründig von der Aufrechterhaltung einer Work-Life-Balance spricht, thematisiert er mehrheitlich das Wie des Planens. Der bereits durchlebte Studienabbruch stellt ein Stigma für die Studentin dar, das zunächst im Raum steht. Dieses Stigma und dessen mögliche Konsequenzen für ihr aktuelles Studium werden im Verlauf des Gesprächs aufgegriffen.

In einer latenten Bedeutungsschicht zeigt sich eine Figur, die nicht ausschliesslich das Studium oder die Vermeidung eines erneuten Studienabbruchs betrifft. Vielmehr ringen beide Partien um Legitimation, Anerkennung und (Absegnung) ihrer Person als Ganzes. Mit anderen Worten werden die gesetzten Themen nicht innerhalb der Rollen - Studentin und Mentor - bearbeitet, sondern die Lebensgeschichten der Gesprächspartner mitein- 
bezogen. Diese Ausdehnung kann mit Wernet (2003) als «entgrenzend» beschrieben werden.

Lorenz' Empathie für ihre Situation und seine lockere, non-direktive Sprache lassen sich als Näheangebot deuten. Ebenso sind sie indikativ für eine Suche nach Anerkennung seiner Person und für eine Legitimation seiner Art des Mentoring. Ingrid steigt insofern auf dieses Näheangebot ein, als dass sie sich inm weiter offenbart. Diese Selbstoffenbarung ist als Rechtfertigungs- und Absicherungsbemühung zu verstehen. Hinweise auf Beratungs- oder Unterstützungsbedarf sind nicht erkennbar.

Ingrid gibt sich einerseits entschlossen ihr Studium fortzuführen, schützt sich andererseits aber entschieden gegen eine komplette Vereinnahmung ihrer selbst durch das Studium. Diese Haltung bedarf sowohl einer Selbstlegitimation als auch einer (Absegnung) durch ihren Mentor, die sie sucht und bekommt. Die Suchbewegung nach Legitimation findet im Modus der Dramatisierung statt.

Bei einer professionellen Beratung oder Begleitung, wie dies beispielsweise in Mentoringoder Coachingsettings angedacht ist, wäre das Einhalten einer adäquaten emotionalen Distanz (Neukom et al., 2011) zwischen den Beteiligten die Norm. An mehreren Stellen gibt es Hinweise für ein Unterschreiten dieser Distanz durch den Mentor: wenn Lorenz betont informelle Sprache verwendet, wenn er Ingrid kollegial und diffus adressiert, wenn er sich despektierlich über Studieninhalte äussert oder wenn er sich Sinne von «ich kenne das, ich kenne deine Probleme aus eigener Erfahrung» mit der Studentin solidarisiert. Fallspezifisch gewinnt eine Figur Gestalt, in der der Mentor durch das Zollen von Anerkennung eine spiegelbildliche Anerkennung einfordert. Die hier aufscheinende Anerkennungsdynamik lässt Rückschlüsse auf eine grundlegende Anerkennungs- und Legitimierungsproblematik zu.

In Bezug auf das ihr unterbreitete Näheangebot verhält sich Ingrid opportunistisch. Sie lässt Nähe zu und fordert sie ein, wenn diese in Anerkennung und Verständnis resultiert. An mehreren Punkten lässt sie Lorenz aber auch ins Leere laufen, wenn sie durch Schliessoder Distanzierungsbewegungen nicht auf seine Angebote einsteigt. Allerdings treibt sie an anderen Stellen selbst Entgrenzungen voran, indem sie Persönliches in einer dramatisierenden Weise offenbart. 
Mit Blick auf die fallspezifische Interaktion innerhalb des Formats «Mentoratsgespräche» lassen sich folgende Schlussfolgerungen ziehen: Der bisherige Gesprächsverlauf zeigt eine Variante des Mentoratsgesprächs, in der beidseitig ein beachtlicher Aufwand betrieben wird, um die eigene Position zu legitimieren. Die Suche nach gegenseitiger Anerkennung und Legitimierung bezieht sich nicht allein auf die spezifischen Rollen - die der Mentor an gewissen Stellen in Anlehnung an eine Therapie interpretiert - , sondern auf die involvierten Personen an sich. Es finden Entgrenzungen statt durch die beide die gesuchte Anerkennung erhalten: der Mentor in seinem selbstbezogenen Gemocht-Werden und die Studentin in ihrem Verlangen nach (Absegnung). Die hier vorgefundene Gesprächsdynamik läuft in der Konsequenz Gefahr spezifisch vorstrukturierte Rollen mittels Entgrenzung diffus werden zu lassen.

Gekoppelt an dieses Changieren zwischen einer diffusen und einer spezifischen Sozialbeziehung ist die weiter oben geschilderte Spannung zwischen Symmetrie und Asymmetrie. Die asymmetrische Disposition des Formats steht in einer Spannung zu den Symmetrieangeboten des Mentors. Beide Pole gleichzeitig zu bedienen führt zu einer Situationsunsicherheit, die hier mit Verschleierungsdynamiken der asymmetrischen Konstellation bearbeitet wird.

\subsubsection{Dritte Sequenz: Das wäre jetzt prinzipiell ein Angebot}

Bevor der Gesprächsabschluss als weitere Sequenz rekonstruiert wird, sei hier der restliche Gesprächsverlauf in aller Kürze zusammengefasst. Die grundlegende Struktur des Gesprächs bleibt auch im weiteren Verlauf erhalten. Den einzelnen Traktanden aus dem Vorbereitungsdokument folgend, werden diverse Themen aus dem Studium angesprochen. An mehreren Stellen zeigt Ingrid auf, wie viel Zeit und Eigenmotivation sie das Studium kostet und wie sie gelernt hat sich von den Anforderungen und dem hohen Workload partiell abzugrenzen. Unterdessen wird sie - so ihre diesbezüglichen Ausführungen - auch nicht mehr von einem schlechten Gewissen verfolgt, falls sie den institutionellen Ansprüchen nicht genüge. Persönliche Grenzerfahrungen wie eine Gürtelrose und ein Fast-Burnout ${ }^{55}$ haben ihr die Notwendigkeit einer Abgrenzung aufgezeigt.

\footnotetext{
${ }^{55}$ Die Bezeichnung Fast-Burnout ist ein Zitat aus Ingrids Ausführungen.
} 
Lorenz begegnet ihr während des ganzen Gesprächs mit Verständnis und Empathie. Immer wieder erzählt er auch von eigenen ähnlichen Erfahrungen und seinem persönlichen Umgang mit Stress. Ebenfalls spricht er von den in seinen Augen überhöhten Anforderungen manch anderer Dozierender bei schriftlichen Arbeiten.

Die folgende Sequenz ist der inhaltliche Abschluss im Gespräch zwischen Ingrid und Lorenz. Daran anschliessend findet nur noch die formale Verabschiedung statt.

Lorenz: ah ich habe noch ganz kurz etwas ich hab's ein bisschen jetzt vergessen (.) während des Gesprächs

Ingrid: $m h m$

Lorenz: ist vielleicht auch kein Zufall ähm was ich noch Studierenden anbiete die wollen wenn ich so auch mit Stress oder so höre Ingrid: $\mathrm{mhm}$ Lorenz: dann kann wenn ich wenn jemand will auch ein Stressverarbeitungstest mit jemandem machen

Ingrid: $\mathrm{mhm}$

Lorenz: und dann kann ich eine Rückmeldung/also ist ein psychologischer Test Ingrid: $\mathrm{mhm}$ Lorenz: nicht irgendwie so dann kann ich auch eine Rückmeldung geben wie man so reagiert in wie wie also soll ich sagen in schwierigen oder stressigen Situationen also welche Bewältigungsmechanismen Ingrid: $\mathrm{mhm}$ Lorenz: man offenbar ähm anwendet und wie hilfreich die sind Ingrid: ja Lorenz: das wäre jetzt prinzipiell ein Angebot hab ich mir überlegt könnt ich dir machen Ingrid: $m h m$ Lorenz: also wenn du sowas machen möchtest Ingrid: $\mathrm{mhm}$ Lorenz: dürftest du das gerne machen dich würde es ungefähr eine halbe Stunde ausfüllen mal kosten Ingrid: $\mathrm{mhm}$ 
Lorenz: die Fragen ich würd's dann auswerten und dann gäb's ein Gespräch so irgendwie eine Stunde

Ingrid: ja

Lorenz: also prinzipiell wenn dich das interessiert wobei so wie du erzählt hast bist du da richtig also (.) machst du das top oder aber wenn dich das interessiert Ingrid: na aber hundertprozentig geben ich kann auch mal fünfzig geben ((lacht)) Lorenz: ja also dann kannst du dir mal überlegen

Ingrid: $m h m$

Lorenz: ob du so was machen möchtest dann kannst du's mir einfach sagen Ingrid: $m h m$

Lorenz: also dann können's wir einfach mal irgendwann

Ingrid: $m h m$

Lorenz: wenn sich's ergibt machen

Ingrid: ja

Lorenz: das überlegst du dir

Ingrid: ja

Lorenz: und sagt du mir also wenn du das möchtest

Ingrid: $m h m$

Lorenz: ich würde mitmachen das wäre sicher noch interessant aber nicht dass du jetzt das Gefühl hast der muss da irgendwie eine Notfallmassnahme Ingrid: $m h m$

Lorenz: weil das ist jetzt drum kommt's mir wirklich jetzt am Schluss noch in den Sinn

Ingrid: $m h m$

Lorenz: das überlegst du dir (.)

Ingrid: mach ich (.) sag dir Bescheid

Lorenz: ja

Ingrid: super 


\subsubsection{Rekonstruktion der einzelnen Sequenzen}

Lorenz: ah ich habe noch ganz kurz etwas

Auch ohne Kontextwissen lässt sich vermuten, dass dem Sprechakt eine kommunikative Situation vorausgeht, die sich dem Ende neigt und durch den Sprechakt neu eröffnet wird. Mit ah nimmt Lorenz Bezug auf etwas Flüchtiges, wie einen Gedanken oder eine Assoziation. Etwas ist dem Sprecher noch in den Sinn gekommen. Dieses etwas im zeitlichen Umfang durch noch ganz kurz begrenzt.

Gut vorstellbar wäre der Sprechakt in einem Gespräch zwischen einem Vorgesetzten und einer Mitarbeiterin, wenn das eigentliche Thema bereits besprochen ist. Beispielsweise könnte der Vorgesetzte noch mitteilen, dass eine ausstehende Anschaffung genehmigt wurde: Ah ich habe noch ganz kurz etwas. «Der Antrag für die Beschaffung von Stehpulten wurde bewilligt. Diese Bestellung kann umgehend ausgeführt werden.»

Manifest suggeriert ah Spontaneität, als wäre das dem Sprecher eben erst wieder eingefallen. Auf der latenten Ebene wird mit dem Sprechakt ein thematischer Wechsel eingeleitet und etwas, was in einem gewissen Spannungsverhältnis zum Vorhergesagten steht, angekündigt. Es gibt offensichtlich noch ein Thema, das nicht einfach ohne entsprechende Einleitung besprochen werden kann.

Kontrastiert man die realisierte Variante - ah ich hab noch ganz kurz etwas - mit einer Formulierung im Konjunktiv - «ah ich hätte noch ganz kurz etwas» -, wird deutlich, dass nur letztere einer (expliziten) Zustimmung bedarf. In dem Fall könnte die adressierte Person auch ablehnend reagieren: «Wäre dies auch zu einem anderen Zeitpunkt möglich? Mir kommt das gerade sehr ungelegen.» Die Formulierung im Konjunktiv wäre eher aus einer subalternen Position heraus erwartbar. Bei der vorgefundenen Version mit haben ist die Zustimmung bereits impliziert.

Im Kontext des Mentoratsgesprächs stellt dies eine Bemühung um Symmetrie dar. Allerdings liegt kein Anliegen vor, das noch gutzuheissen ist, sondern dieses wird lediglich angekündigt. Auf diese Weise entsteht eine spannungsvolle und in sich widersprüchliche Dynamik mit Strukturierungsansprüchen und Symmetriebemühungen. 
Die Figur weist zum einen Parallelen zur Eröffnungssequenz auf. Dort hat Lorenz mit der Aussage ich darf noch ein bisschen Kaffee trinken das stört dich nicht einerseits eine Symmetriebewegung vorgenommen und andererseits aus der Asymmetrie des Settings heraus der Studentin unterstellt, dass sie sich daran nicht störe. Zum anderen konnten analoge Bemühungen auch in der zweiten Sequenz - beispielsweise bei ähm darf ich beim Abbrechen bleiben? - festgemacht werden. Dieses Spannungsverhältnis zwischen Symmetriebemühungen und struktureller Asymmetrie scheint sich als dominantes Strukturmerkmal zu reproduzieren.

Anhand der bisherigen Überlegungen lässt sich Folgendes festhalten. Mit dem Sprechakt wird das anschliessende Thema als ausserplanmässige Kleinigkeit eingeführt und eine zeitliche Selbstbeschränkung vorgenommen. Allerdings ist diese Einführung in sich selbst widersprüchlich, denn würde es sich bei dem Thema tatsächlich um eine Kleinigkeit handeln, wäre eine explizite Ankündigung überflüssig.

Lorenz: ich hab's ein bisschen jetzt vergessen (.) während des Gesprächs Ingrid: $m h m$

Das etwas entspricht offenbar keiner spontanen Eingebung des Mentors, denn diese könnte nicht ein bisschen jetzt vergessen werden. Auffällig und für die Gesprächsdynamik bedeutsam ist die gewählte Formulierung ein bisschen jetzt vergessen, die in einem deutlichen Kontrast zu gängigeren Formulierungen wie etwa "fast vergessen» oder «beinahe vergessen» steht. Vorstellbar wäre ein solcher Sprechakt allenfalls in einem schulischen Szenario, wenn ein Schüler gegenüber der Lehrerin eingesteht, dass er seine Hausaufgaben ein bisschen jetzt vergessen habe. Der Schüler hofft auf «mildernde Umstände), da er die Hausaufgaben ja nur ein bisschen und nicht ganz vergessen hat. Mit ein bisschen vergessen wird folglich der Vorgang kleingeredet.

Im Rahmen des Mentoratsgesprächs stellt sich die Frage, weshalb der Mentor ein Eingeständnis - etwas ein bisschen vergessen zu haben - abgibt und weiter noch, weshalb er nicht eine üblichere Formulierung - «Da fällt mir noch etwas ein. Ich hab während des Gesprächs gar nicht mehr daran gedacht.» - wählt. Lorenz ringt mit seinem Eingeständnis um Verständnis. Die ihm zur Verfügung stehende Zeit läuft gerade ab und er intendiert den verbleibenden Zeitrahmen noch ganz kurz zu dehnen. Mit ganz kurz und ein bisschen 
enthält die Aussage gleich zwei diminuierende Figuren, die vordergründig das Folgende in seiner Relevanz einordnen. Jenes, was folgt, ist nicht das wichtigste Traktandum des Gespräches, darf aber dennoch nicht ausgelassen werden und ist demzufolge auch keine Marginalie.

Zusammenfassend lässt sich sagen, dass der bisherige Sprechakt eine Ausdehnung des Gespräches ankündigt. Die Ausdehnung ist manifest sowohl zeitlich begrenzt als auch von sekundärer Wichtigkeit. Auf einer latenten Ebene schreibt sich in diese Bagatellisierungsbemühung auch etwas Brisantes ein. Die Ankündigung der zeitlichen Ausdehnung des Gesprächs ist keine strikte Setzung, sondern sie benötigt eine soziale Abfederung. An dieser Stelle reproduzieren sich Bemühungen der Asymmetrievermeidung.

Ingrid stimmt der Ausweitung des Gesprächs minimal mit $m h m$ zu. Dadurch stellt sie eine gewisse Gesprächssymmetrie her, die Lorenz ihr zuvor angeboten hat.

Lorenz: ist vielleicht auch kein Zufall

Unabhängig vom empirischen Kontext könnte ein solcher Sprechakt als Kommentar mit altklugem Unterton fallen, beispielsweise in Form von «ist vielleicht auch kein Zufall, dass das gerade jetzt passiert ist».

Die Aussage wirkt aufgeladen und kann mit «alles hat einen tieferen Sinn» paraphrasiert werden. Der Sprecher erkennt diesen tieferen Sinn und weiss ihn zu deuten. Er schreibt sich selbst eine entsprechende Expertise - «ich hab's durchschaut» - zu.

Im Zusammenspiel mit dem vorausgehenden Sprechakt - ich hab's ein bisschen jetzt vergessen - wird deutlich, dass das Vergessen unter den vorgefundenen Umständen nicht sonderlich überraschend ist. Wenn etwas vielleicht auch kein Zufall ist, ist es im Gegenzug schicksalhaft, erwartbar oder geplant. Der Fast-Fehler des Ein-bisschen-jetzt-Vergessens wird teilweise begründet. Die Verwendung von vielleicht und auch beschränken die Ausweitung der eigenen These und schliessen andere Erklärungen, wie etwa Zufall, nicht aus.

Bis zu dieser Stelle ist Lorenz noch nicht zum propositionalen Gehalt dessen, was er noch ganz kurz einbringen möchte, vorgedrungen. 
Lorenz: ähm was ich noch Studierenden anbiete die wollen

Zu Beginn der Sequenz ist die Rede von etwas haben. In der Fortsetzung wird nun etwas angeboten. Ein solches Angebot könnte etwa in einer Marktsituation unterbreitet werden. Dort wäre allerdings die Formulierung «was ich noch für Kundinnen und Kunden anbiete, die das wünschen» oder "allen Kundinnen und Kunden anbiete, die das wünschen» naheliegend. Das Angebot gestaltet sich in dieser Form offiziell und für alle Interessierten offen.

In der realisierten Variante ist das Angebot exklusiv für gewisse Studierende zu verstehen. Ihm liegt ein individuelles Auswahlmotiv zugrunde. Von der Interaktionslogik her ist davon auszugehen, dass Ingrid eine dieser Auserwählten ist, wenn auch das nicht explizit formuliert wird. Die Exklusivität des Angebotes wird durch die Ergänzung die wollen zusätzlich erhöht. In der Marktmetapher würde das mit der Aussage «nur für ganz gute Kundinnen und Kunden» korrespondieren. Zugespitzt liesse sich auch sagen: «Wer nicht will, der hat schon.» Unabhängig davon, ob Ingrid das Angebot annimmt oder nicht, es ist klar wie Lorenz als Anbieter dazu steht.

Das Angebot gilt nicht ausschliesslich für Ingrid, sondern potenziell für all jene, die wollen. Dieses explizite Wollen scheint wichtig, Lorenz zeigt sich bemüht, das Angebot an eine Nachfrage zu koppeln. Dennoch würde womöglich eine direkte Unterbreitung des Angebotes auf Basis der sich vollziehenden Zweierbeziehung - «ich biete auch dir X an, falls dich das interessiert» -einen höheren Druck zur Annahme aufweisen als die vorgefundene Version. In der realisierten Variante - Studierenden [..] die wollen - ist die konkrete Aufforderung weniger offensichtlich. Diese Bewegung wird allerdings durch die Exklusivität des Angebots auch wieder zurückgenommen. Das Zusammenspiel aus exklusivem Angebot und vermeintlicher Unverbindlichkeit ist spannungsvoll. Auf der einen Seite steht die Erwartung, dass Ingrid als Auserwählte auf das Angebot eingeht, auf der anderen Seite die sie entlastende Unverbindlichkeit. Nun wird auch klar, was Lorenz ein bisschen vergessen hat, nämlich Ingrid dieses Exklusivangebot zu unterbreiten.

Die im Angebot enthaltene Aufforderung wird manifest durch eine betonte Unverbindlichkeit und eine informalisierende Art der Angebotsunterbreitung bearbeitet. Auf einer latenten Ebene überführt gerade dieser Zusatz die wollen die Aufforderung in eine suggestivere Form. 
Lorenz: wenn ich so auch mit Stress oder so höre

Ingrid: $m h m$

Lorenz Sprechakt weist semantisch mehrere Brüche auf. Die Wendung so auch mit Stress oder so höre drückt eine Fremdheit und Distanzierung, vielleicht sogar Ablehnung, aus. Als Kontrast wäre die Formulierung - «wenn ich Stress höre» - beispielsweise aus dem Munde eines Stressexpertin vorstellbar. Hingegen lässt die realisierte Version nicht auf eine Expertise bezogen auf die Stressthematik schliessen, sondern rückt den zuvor eingeleiteten Prozess der Angebotsunterbreitung ins Zentrum.

«Stress» als Terminus wird in der vorgefundenen Formulierung umfassend und unspezifisch verstanden. Dies verschafft sich gleich mehrfach, beispielsweise in der doppelten Verwendung von so wie auch in der Präposition mit, Ausdruck. Das auf einer manifesten Ebene hervorgebrachte Exklusivangebot verweist auf einer latenten Ebene auf eine gegenteilige Bewegung. Das Angebot kommt niederschwellig zum Zug, wenn der Mentor etwas mit Stress oder so identifiziert. Dabei scheint durch, dass die Artikulation des Angebots auch ein eigenes Anliegen von Lorenz bedient: das Angebot unterbreiten zu können.

Bereits zuvor variieren die Sprechakte in ihrer sprachlichen Ausdrucksweise: Von geläufig und sozial anschlussfähig - ich hab noch ganz kurz etwas - und - vielleicht auch kein Zufall - bis zu holprig - ich hab's ein bisschen jetzt vergessen - und - was ich noch Studierenden anbiete die wollen wenn ich so auch mit Stress oder so höre. Diese Varianz ist als Indiz dafür zu verstehen, dass die Unterbreitung des Angebotes keiner Routine folgt, sondern für den Mentor eine Herausforderung darstellt.

Der Anschluss von Ingrid fällt mit $\mathrm{mhm}$ erneut minimalistisch aus und lässt sich analog zu ihrer vorherigen Bestätigung deuten. 
Lorenz: dann kann wenn ich wenn jemand will auch ein Stressverarbeitungstest ${ }^{56}$ mit jemandem machen

Ingrid: $m h m$

Der Sprechakt wäre grundsätzlich ohne das erste wenn erwartbar und würde lauten «dann kann ich, wenn jemand will ...». In der realisierten Version schleust sich vor das ich ein wenn ein. Mit dieser eingeschobenen Bedingung hängt das Angebot des Stressverarbeitungstests vom persönlichen Wohlwollen des Mentors ab. Obwohl es sich manifest noch immer um ein Angebot handelt, lassen sich zugespitzt formuliert Elemente von Willkür erkennen, die im Einschub wenn ich latent durchscheinen. Dazu gesellt sich ein Moment des Selbstbezugs, auch wenn er sich anschliessend korrigiert.

Markant ist die zweifache Verwendung von jemand respektive jemandem, wodurch die direkte Ansprache Ingrids und damit die direkte Unterbreitung des Angebotes erneut vermieden wird. Wäre die Freiwilligkeit, die Annahme des Angebots betreffend, bereits als Voraussetzung gegeben, müsste diese von Lorenz nicht wiederholt betont werden. Gerade durch die Hervorhebung - wenn jemand will - wird ein direktives Moment in suggestiver Weise eingeführt und gleichzeitig durch die Art und Weise der Formulierung verschleiert.

Die bisherigen Befunde lassen die Formulierung einer ersten riskanten Fallstrukturhypothese dieser Sequenz zu. Die Gesprächsinteraktion zeigt ein Spannungsverhältnis auf, da der Studentin manifest ein freiwilliges Angebot unterbreitet wird, das aber gerade durch die mehrmalige Betonung der Freiwilligkeit auf einer latenten Bedeutungsebene eine suggestive und die Studentin «bearbeitende` Implikation erhält.

Es scheint, als gehe die Unterbreitung des Angebots mit einer gewissen Prekarität einher. Dies zeigt sich beispielsweise in der indirekten Ansprache mittels jemand oder auch in den teils umständlichen Formulierungen. Gerade der Metakommentar - ich hab's ein bisschen jetzt vergessen ist vielleicht auch kein Zufall - legt nahe, dass die Unterbreitung des

\footnotetext{
56 Der Stressverarbeitungsfragebogen (SVF) ermöglicht die Erfassung von Bewältigungs- bzw. Verarbeitungsmassnahmen in belastenden Situationen. Der Test stellt kein singuläres Testinstrument dar, sondern ein Inventar von Methoden, die sich auf verschiedene Aspekte der Stressverarbeitung beziehen und aus denen je nach Untersuchungsziel respektive -fragestellung einzelne Verfahren ausgewählt werden können. Der SVF wurde von Erdmann und Janke erstellt und erschien 2008 in der vierten, überarbeiteten und erweiterten Auflage.
} 
Angebots eine Belastung für den Mentor und eine mögliche Ablehnung des Angebots ein Risiko für ihn darstellen.

In der Fortsetzung wird das Angebot weiter ausgeführt und näher erläutert. Die Strukturlogik reproduziert sich weitestgehend, weshalb sich die Rekonstruktion auf eine knappe Skizzierung der zentralen Bedeutungsmerkmale beschränkt.

Lorenz: und dann kann ich eine Rückmeldung/also ist ein psychologischer Test Ingrid: $\mathrm{mhm}$

Lorenz: nicht irgendwie so dann kann ich auch eine Rückmeldung geben wie man so reagiert in wie wie also soll ich sagen in schwierigen oder stressigen

Situationen also welche Bewältigungsmechanismen

Ingrid: $\mathrm{mhm}$

Lorenz: man offenbar ähm anwendet und wie hilfreich die sind

Ingrid: ja

Lorenz: das wäre jetzt prinzipiell ein Angebot hab ich mir überlegt könnt ich dir machen

Ingrid: $\mathrm{mhm}$

Lorenz: also wenn du sowas machen möchtest

Ingrid: $\mathrm{mhm}$

Lorenz: dürftest du das gerne machen dich würde es ungefähr eine halbe Stunde ausfüllen mal kosten

Ingrid: $\mathrm{mhm}$

Lorenz: kosten die Fragen ich würd's dann auswerten und dann gäb's ein Gespräch so irgendwie eine Stunde Ingrid: ja

Lorenz' Ausführungen weisen semantisch an mehreren Stellen Brüche auf. Er bricht angefangene Sätze ab und setzt neu an. Dabei reproduziert sich das Strukturmerkmal der herausfordernden Angebotsunterbreitung. Ebenfalls reproduziert sich die gezeigte Unverbindlichkeit des Angebots. Dies gewinnt in den häufigen Verwendungen von Konjunktiven und kann-Formulierungen Gestalt. Insgesamt suggerieren die gewählten Formulierungen - mit den Wagheitsmarkierern so, irgendwie, offenbar und prinzipiell - 
Lockerheit und Informalität. In der Latenz wird dabei auch die Unsicherheit bearbeitet, die aus der Prekarität des unterbreiteten Angebots resultiert. Dies zeigt sich beispielsweise in der Formulierung wie wie soll ich sagen.

Der vorgeschlagene Stressverarbeitungstest wird explizit als ein psychologischer verortet. Er weist nicht irgendwie so ein beliebiges Format auf. Diese Formulierung ist anschlussfähig an die Figur des Marktes: Einer auserwählten Kundin wird ein exklusives Angebot unterbreitet. Der Test wird hier durch das Prädikat psychologisch aufgewertet. Dadurch findet auch eine (Selbst-)Aufwertung des Testadministrators in seiner Expertise statt. Die konkrete Unterbreitung des Angebots - das wäre jetzt prinzipiell ein Angebot hab ich mir überlegt könnt ich dir machen - wird weiterhin nicht vollzogen, sondern lediglich angedacht. Auf einer manifesten Bedeutungsebene hebt diese Aussage die Attraktivität des Angebots an und betont die darin enthaltene Grosszügigkeit des Mentors. Auf einer latenten Ebene reduziert sich der Grad der Exposition des Sprechers, da das Angebot nur bei signalisiertem Interesse auch tatsächlich unterbreitet wird. Das Risiko einer Zurückweisung und einer damit einhergehenden Kränkung wird dadurch begrenzt. Der implizite Imperativ des Sprechaktes lautet «Zeig dein Interesse! Frag mich, ob ich dir das Angebot unterbreite!»

Lorenz positioniert sich nicht rollenspezifisch als Mentor, sondern diffus als ganze Person. Sein Interesse das Angebot unterbreiten zu können ist ein persönliches, das in einer bemerkenswerten Deutlichkeit und auch mit einer Penetranz verfolgt wird. Gleichzeitig haftet der Formulierung also prinzipiell eine Willkür an. Lorenz kann nach eigenem Dafürhalten entscheiden wem und wann er das Angebot unterbreitet. Dies wertet das Angebot einerseits abermals auf, anderseits verstärkt sich mit dieser machtvollen Komponente die Ambivalenz seiner Position in Bezug auf die Spannungsfelder von Symmetrie und Asymmetrie und Heterarchie und Hierarchie.

Die Sprechakte Ingrids fallen mit $\mathrm{mhm}$ und ja minimal, monoton und auffallend neutral aus. Mit Blick auf die Art und Weise wie sie von Lorenz als Auserwählte angesprochen wird, kann ihre Reaktion als verhalten bezeichnet werden. Im Lichte der Marktmetapher 〈beisst) sie beim verlockenden Angebot nicht an. Mit ihren Kurzantworten erteilt sie der sozialen Situation eine minimale Zustimmung, bezogen auf das eigentliche Angebot ist allerdings keine solche erkennbar. 
Lorenz: also prinzipiell wenn dich das interessiert wobei so wie du erzählt hast bist du da richtig also (.) machst du das top oder aber wenn dich das interessiert

Die Formulierung - also prinzipiell wenn dich das interessiert - leitet mit einer gewissen Jovialität etwas ein, das gewichtig ist. Erneut wird dabei die Artikulation des Angebotes an das Interesse der Gegenseite gekoppelt und dabei dennoch deutlich aufgezeigt, dass das Angebot zu interessieren hat.

In der vorgefundenen Situation würde eine Absage Ingrids Lorenz kränken. Die Wenndann-Satzstruktur stellt einen Zusammenhang zwischen Bedingung und Konsequenz her - wenn dich das interessiert, «dann machen wir diesen Test». Lorenz vollendet die Wenndann-Satzstruktur nicht, sondern ergänzt den begonnenen Satz mit einem Einwand. Dieser entspricht einem Kompliment und macht das Angebot im Grunde genommen obsolet. Das Kompliment basiert auf Ingrids bisherigen Erzählungen - wobei so wie du erzählt hast. Ob sich diese mit den Einschätzungen von Lorenz decken, bleibt ungeklärt. Zum wiederholten Male scheint die Prekarität der Angebotsunterbreitung in Hinblick auf Ingrids Reaktion durch. Würde Ingrid das Angebot ablehnen, liesse sich dies für Lorenz damit erklären, dass Ingrid die Notwendigkeit des Testes nicht erkannt hat. Sie hätte die Verantwortung für ihr Versäumnis allein zu tragen. Lorenz könnte ihre Absage mit so wie du erzählt hast bist du da richtig (.) machst du das top vor sich selbst legitimieren, selbst wenn er die Situation anders einschätzt. Insofern bearbeitet er mit dieser Formulierung bereits präventiv seine Angst, die Situation nicht richtig erkannt zu haben. Lorenz kann nicht falsch liegen: nimmt Ingrid das Angebot an, hat er ihr Problem erkannt; lehnt sie das Angebot ab, lässt sich dies über ihre Selbsteinschätzung das top zu machen erklären. Der erneute Einwand - aber wenn dich das interessiert - weist beinahe schon den Charakter einer Beschwörung auf.

Insgesamt ist im Sprechakt eine Dynamik zu erkennen, die in der Überbetonung der Unverbindlichkeit auf eine gegenteilige Bewegung hinweist. Diese Gegenbewegung lässt sich mit «es hat dich zu interessieren, dein Interesse wäre hier angebracht» umschreiben. Gleichzeitig beugt Lorenz mit der Aussage wobei so wie du erzählt hast bist du da richtig also (.) machst du das top einer möglichen Absage, die für ihn auch ein Scheitern und eine Zurückweisung bedeuten würde, vor. 
Ingrid: na aber hundertprozentig geben ich kann auch mal fünfzig geben ((lachen))

Ingrids Anschluss auf Lorenz' Aussage überrascht und bedarf einer genauen Betrachtung. Kontextfrei wäre der Sprechakt na aber hundertprozentig als Antwort auf die Frage «Bist du dir sicher?» vorstellbar. Die Antwort könnte beispielsweise «na aber hundertprozentig, absolut sicher» lauten und die erlebte Sicherheit beschreiben. Dem vorgefundenen Sprechakt geht allerdings keine Frage voraus, vielmehr scheint na aber einen Einwand einzuleiten. Der Sprechakt lautet na aber hundertprozentig geben, obwohl die sprachlich gebräuchliche Formulierung «hundert Prozent geben» wäre. Um die strukturelle Differenz zwischen hundertprozentig und «hundert Prozent» geben herauszuarbeiten, wird geben durch Verben ersetzt, die in der Kombination mit hundertprozentig üblich sind: «versprechen», «verlassen», "garantieren» oder auch «funktionieren». All diese Kombinationen beantworten die Frage nach dem Wie oder nach der Qualität. Hundert Prozent bezieht sich hingegen auf ein Ausmass oder eine Quantität. Ingrid spricht demzufolge nicht über das Wie des Gebens, sondern über das Wieviel.

Ingrid führt den Gedanken des hundertprozentig geben mit ich kann auch mal fünfzig geben gefolgt von einem Lachen weiter aus. Der Zusatz der Prozente fällt im zweiten Teilsatz weg.

Kontextfrei wäre der Sprechakt ich kann auch mal fünfzig geben in einer Situation passend, wenn die sprechende Person jeweils weniger als fünfzig eingebracht hat, ihren Anteil aber problemlos auf fünfzig erhöhen kann. Die Erhöhung scheint keine besondere Rolle zu spielen und weist auf eine gewisse Beliebigkeit hin. Dem Sprechakt kommt die Bedeutung zu, dass die sprechende Person nach eigenem Gutdünken mehr oder weniger einbringen und auf dieser Klaviatur problemlos spielen kann, was sich auch im Lachen bestätigt.

Im Zusammenspiel mit na aber hundertprozentig geben ist der Sprechakt wie folgt zu lesen: Na aber stellt eine Einschränkung dar, die hier für eine Minderung des vollen Einsatzes, das heisst für hundertprozentig geben steht. Für Ingrid scheint im Vergleich dazu auch mal fünfzig geben eine Option zu sein, beispielsweise in Analogie zu «auch mal eine Fünf geradestehen lassen». Inhaltlich teilt Ingrid Lorenz mit, dass sie ihren Einsatz für das Studium problemlos um die Hälfte reduzieren könne. Dies würde den Stressverarbeitungstests und damit Lorenz' Angebot erübrigen. Interaktionsdynamisch nimmt sie 
dabei eine selbstbestimmte Positionierung vor. Diese lässt sich mit «ich weiss schon, was für mich gut ist. Ich kann, muss aber nicht immer hundertprozentig geben» paraphrasieren. In ihrer Äusserung verschafft sich erneut ein Spannungsverhältnis zwischen der manifesten und latenten Selbstdarstellung Ausdruck. Manifest zeigt sie ihren Einsatz auf, der so gross ist, dass sie ihn auch halbieren könnte. Latent gewinnt abermals die geforderte (Absegnung) respektive ein Freiticket für den Einsatz, den sie zu leisten bereit ist, Gestalt. Auf die Option der Angebotsunterbreitung nimmt Ingrid in keiner Weise Bezug. Aus Sicht der Beziehungsdynamik lässt dies bereits eine indirekte Absage vermuten.

Im Fortgang des Gesprächs wird seitens Lorenz nicht auf ihre Äusserung eingegangen, sondern es findet eine Fortsetzung der Angebotsunterbreitung statt, die keine grundsätzlich neuen Erkenntnisse der Strukturdynamik aufzeigt. Deshalb werden die nächsten Wortwechsel erneut flächig rekonstruiert:

Lorenz: ja also dann kannst du dir mal überlegen

Ingrid: $\mathrm{mhm}$

Lorenz: ob du so was machen möchtest dann kannst du's mir einfach sagen Ingrid: $\mathrm{mhm}$

Lorenz: also dann können's wir einfach mal irgendwann

Ingrid: $\mathrm{mhm}$

Lorenz: wenn sich's ergibt machen

Ingrid: ja

Lorenz: das überlegst du dir

Ingrid: ja

Lorenz: und sagst du mir also wenn du das möchtest

Ingrid: $\mathrm{mhm}$

Lorenz: ich würde mitmachen das wäre sicher noch interessant aber nicht dass du jetzt das Gefühl hast der muss da irgendwie eine Notfallmassnahme Ingrid: $\mathrm{mhm}$

Lorenz: weil das ist jetzt drum kommt's mir wirklich jetzt am Schluss noch in den Sinn

Ingrid: $m h m$ 
Lorenz: das überlegst du dir (.)

Ingrid: mach ich (.) sag dir Bescheid

Lorenz: ja

Ingrid: super

Zum ersten Mal wird die Studentin mit dann kannst du dir mal überlegen direkt angesprochen. Es folgen zwei weitere kann-Formulierungen, bevor bei der darauffolgenden Ansprache das Optionale, das heisst die kann-Formulierung wegfällt und mit das überlegst du dir und und sagst du mir also wenn du das möchtest eine Aufforderung formuliert wird. Diese leichte Verschärfung wird umgehend mit einer Gegenbewegung unterlegt. Die Formulierung ich würd mitmachen das wäre sicher noch interessant erstaunt. Als eine Lesart wird hier der Angesprochen in den Mund gelegt, was der Sprecher von ihr als Antwort erhofft. Nach einer anderen Auslegung wäre dies ein Versuch das non-direktive Auftreten zu untermalen.

Ingrids Reaktionen fallen an dieser Gesprächsstelle mit ja und $m h m$ knapp und unverbindlich aus. Die Vermutung liegt nahe, dass Lorenz seine Aufforderungen deshalb wiederholt. Nach einer kurzen Pause kommt Ingrid der erneuten Aufforderung - das überlegst du dir - nach und bestätigt, dies zu tun und anschliessend ihre Überlegungen zu kommunizieren. Dass Ingrid auf das aufdringliche und penetrante Angebot antworten muss, wird aufgrund des bisherigen Gesprächsverlaufes und in der sozialen Situation eines dyadischen Mentoratsgesprächs als gegeben erachtet und gilt auch der Höflichkeit geschuldet. Ingrid hält sich bezogen auf eine konkrete Zu- oder Absage mit mach ich (.) sag dir Bescheid weiterhin bedeckt. Es bleibt unklar, ob sich das super von Ingrid auf Lorenz' Vorschlag bezieht oder aber der Tatsache geschuldet ist, dass er sich mit ihrem Versprechen zufriedengibt. In der Latenz lässt sich Ingrids Antwort als erteilte Absage lesen.

Im Anschluss leitet Lorenz das Gesprächsende und die Verabschiedung ein. Deshalb endet die Rekonstruktion an dieser Stelle und es folgt eine Zusammenfassung und Formulierung der weiter ausdifferenzierten Fallstrukturhypothese. 


\subsubsection{Zusammenfassung und riskante Fallstrukturhypothese der Sequenz Das wäre jetzt prinzipiell ein Angebot}

In expliziter Form unterbreitet der Mentor der Studentin das Exklusivangebot eines psychologischen Stressverarbeitungstests. Die Hinführung zum vorgelegten Angebot changiert zwischen einer Lappalie, die beinahe in Vergessenheit geriet, und einer betonten Exklusivität. Die Bedeutung des Angebotes lässt sich auch in der Adressierung Ingrids als potenziell Auserwählte erkennen, falls sie das zur Angebotsunterbreitung benötigte Interesse signalisiert. Die Struktur dieser Unterbreitung, die im Markt von Angebot und Nachfrage konstitutiv eine Unverbindlichkeit aufweist, gerät in der vorgefundenen Überschüssigkeit in Schieflage. Dabei vermischen sich non-direktive, symmetrische Anteile mit direktiven, asymmetrischen Elementen. Die Bemühungen des Mentors, eine mögliche Asymmetrie zu vermeiden oder mindestens zu relativieren, lassen sich als Hinweise für die Verschleierung der therapeutischen und pädagogischen Anteile lesen, die der Situation durch die dringliche Empfehlung des Angebots immanent anhaften. Auch die Metakommentare, in denen der Mentor sich und seine Handlungen expliziert, zeigen die angestrebte Asymmetrievermeidung, wie auch ein Motiv des Gemocht-Werdens auf. Für dieses Gemocht-Werden, so die plausible Annahme, wird eine Bewegung in Richtung symmetrischer Beziehungsstruktur angestrebt. Zugespitzt formuliert bewegt sich das unterbreitete Angebot zwischen unverbindlich und übergriffig, obwohl das Letztere klar gegenintentional erscheint, da ja gerade eine unbedingte Vermeidung dieser Übergriffigkeit Gestalt gewinnt. Dennoch scheint auf latenter Ebene durch, wie gross sein Anliegen ist, die Studentin für diesen Stressverarbeitungstest zu gewinnen. Was in einer manifesten Betrachtungsweise als Angebot zu ihrem Nutzen dargestellt wird, verkommt in der Latenz zu einem Angebot, das die Motivlage des Mentors bedient. Dies zeigt sich in seinen Bemühungen, gemocht und gebraucht zu werden oder - pointiert und ex negativo formuliert - seiner (Not), nicht gemocht oder gebraucht zu werden. Diese Legitimierungsbedürftigkeit führt zu einer Prekarität, in der eine Verschleierung des asymmetrischen Handelns bei gleichzeitigem Buhlen um Anerkennung Gestalt gewinnt. Riskant formuliert weist das Setting «Mentoratsgespräche» Spannungen und Schwierigkeiten mit asymmetrischen, hierarchischen und direktiven Strukturen auf. Fallspezifisch scheinen diese 
Schwierigkeiten in Selbstvergewisserungs- und Selbstcharismatisierungsdynamiken und einer Bedürftigkeit des Mentors auf.

Die grundsätzliche Problematik auf der Legitimations- und Anerkennungsebene führt dazu, dass solche Dynamiken innerhalb des Settings interaktiv hergestellt, bearbeitet und gefestigt werden. Dies zeigt sich konkret im Buhlen um Anerkennung in der Diffusität der ganzen Person und in der Verschleierung von Asymmetrie.

Mit Ausnahme der überraschenden Äusserung na aber hundertprozentig geben ich kann auch mal fünfzig geben ((lachen)) sind die Redebeiträge der Studentin ausschliesslich Kurzantworten, meist lediglich in Form von $\mathrm{mhm}$ oder ja. Dies lässt sich als Schliessbewegung bezogen auf das Angebot deuten, das heisst die Studentin zeigt diesbezüglich kein Interesse auf.

\subsubsection{Fallzusammenfassung und finale Fallbestimmung: Ingrid \& Lorenz}

Die Strukturmerkmale, die sich bereits in der Eröffnungssequenz andeuten und sich in den beiden weiteren analysierten Sequenzen reproduzieren, führen zu folgenden Befunden: Die Interaktionsdynamik des Gespräches zwischen der Studentin Ingrid und dem Mentor Lorenz lässt eine legitimierungsbedürftige Positionierung des Mentors erkennen. Diese Legitimierungsbedürftigkeit zeigt sich in unterschiedlichen Dynamiken, die allerdings auch ineinander spielen. So erinnert einerseits die im Gespräch etablierte Eigenlogik an die Anderslogik eines therapeutischen Settings und es scheint, als würde sich die Legitimation auch aus der Nähe zu diesem Setting ableiten. Andererseits sind markante Symmetriesierungstendenzen erkennbar und deuten darauf hin, dass das etablierte Setting eine Schwierigkeit bei einer asymmetrischen Bearbeitung aufweist, und dies, obwohl der sozialen Situation eines Mentoratsgesprächs eine grundsätzliche Asymmetrie innewohnt. Indizien für Symmetrisierungsbemühungen zeigen sich in Nähe- und Vergemeinschaftungsangeboten und gewinnen auch in Dynamiken der Asymmetrievermeidung und -verschleierung Gestalt. In der Latenz allerdings befestigen sie die asymmetrische Beziehungskonstellation zwischen der Studentin und dem Mentor, da nur in der Asymmetrie entsprechende Vermeidungs- respektive Symmetriebewegungen erforderlich sind. Fallspezifisch scheinen diese Bemühungen um eine symmetrische 
Beziehungsstruktur für den Mentor auch ein Bedürfnis nach Anerkennung, sowohl spezifisch für seine Art von Mentoring und seinen Status als Experte als auch diffus, das heisst für ihn als Person, zu bedienen. Darin verschaffen sich ein Motiv des GemochtWerdens und eine Bedürftigkeit des Mentors Ausdruck. Hinweise für diese Bedürftigkeit zeigen sich beispielsweise in Grenzüberschreitungen hinsichtlich einer professionellen Distanz- und Rollenwahrnehmung.

Des Weiteren lassen sich bei der interaktiven Bearbeitung der Pole Diffusität und Spezifität Changierfiguren erkennen. Für die Diskussion der Diffusität-SpezifitätAntinomie kann das von Oevermann (2008, S. 71) für die Therapie formulierte Gebot der Abstinenz - «Sei genügend spezifisch. Halte die Grenzen der rollenförmigen Beziehung ein»-beigezogen werden. Denn wie in der Rekonstruktion herausgearbeitet, wird dieses Gebot vom Mentor an mehreren Gesprächsstellen überschritten. Dies lässt sich als Hinweis dafür lesen, dass das Modell der Abstinenzregel fallspezifisch nicht nahtlos auf die vorgefundene Situation im Mentoratsgespräch übertragen werden kann. Dies mag daran liegen, dass es sich nicht um ein therapeutisches Setting handelt, sondern lediglich eine Anlehnung an ein solches erfolgt. Wenn der Impuls für eine Therapie nicht von der Klientin ausgeht, sondern der Mentor sich als Therapeut im Sinne von «habe (Not) und brauche mich» anbietet oder, pointiert formuliert, aufdrängt, scheint die Einhaltung des Gebots der Abstinenz prekär und wird fallspezifisch nicht eingehalten. Hierbei zeigt sich, dass die Befestigung, die Sicherung oder die Legitimation des Settings nicht wie bei der Therapie gewährleistet ist, sondern interaktiv hergestellt werden muss. Diese Herstellungsfigur scheint auf Nähe, Symmetrie, eine diffuse Beziehungsstruktur und ein Gemocht-Werden durch das Gegenüber angewiesen zu sein.

Im Mentoratsgespräch zwischen Ingrid und Lorenz beruht diese Dynamik des Angewiesen-Seins allerdings auf einer Gegenseitigkeit, wenngleich sich dies bei der Studentin in differenter Form zeigt. In der Interaktion gewinnt eine Figur Gestalt, in der die Studentin das Setting «Mentoratsgespräche» und den Mentor Lorenz als Vertreter des Settings dazu nutzt, sich hinsichtlich ihrer Art des Studierens, ihres Zeitmanagements und ihrer etablierten Work-Life-Balance abzusichern und eine entsprechende Gutheissung einzuholen. Die seitens der Studentin aufscheinende Rechtfertigungsimplikation lässt sich als Hinweis auf Normen und Erwartungen lesen, mit denen sie sich konfrontiert sieht. Gerade diese Figur der gegenseitigen Angewiesenheit weist eine Differenz zu einem therapeu- 
tischen Setting auf, da der Therapeut in einem solchen Setting nicht auf die Klientin angewiesen ist, sondern die gemeinsam zu bearbeitende «Not〉 der Klientin das Setting in seiner Funktion legitimiert.

Der Ausbildungsanspruch, der sich in einer an Therapie angenäherten Gesprächslogik manifestiert, lässt sich mit Selbstoptimierung innerhalb eines grundsätzlich krisenhaften Ausbildungsprozess beschreiben. Diese Figur baut auf der Idee auf, dass der Mentor das Krisenhafte innerhalb dieses Ausbildungsprozesses erkennt und mit seiner therapeutischen Unterstützung auffängt oder gar «heilt». Dadurch lässt sich auch seine eigene Position legitimieren.

Aufgrund der konstitutiv asymmetrischen Beziehungskonstellation und damit einhergehend einer gewissen Abhängigkeit ist die Studentin diesen diffusen Ausdehnungen und Entgrenzungstendenzen bis zu einem gewissen Grade ausgesetzt. Gleichzeitig weisen die Spannungsfelder von Diffusität und Spezifität wie auch Symmetrie und Asymmetrie auf die Studentin bezogene Entsprechungen auf. So gewinnen bei ihr (Absegnungsbemühungen` Gestalt. Dieses (Freiticket) verlangt sie nicht lediglich für die spezifische Rolle der Studentin, sondern diffus für ihre ganze Person. Eine 〈Absegnung` wird grundsätzlich aus einer asymmetrischen, abhängigen Konstellation heraus erteilt. Die Studentin fordert diese analog zum Mentor über Nähe-, Symmetrie- und Diffusitätsdynamiken ein. Da er aufgrund vorhandener Anerkennungsbemühungen auf einer latenten Ebene ebenfalls in einem Abhängigkeitsverhältnis zur Studentin steht, findet die Studentin bezüglich dieser Forderung auch beim Mentor Resonanz. Deshalb lässt sich von beidseitigen Entgrenzungen sprechen.

In der Zusammenführung und Kurzform lässt sich für das Mentoratsgespräch zwischen der Studentin Ingrid und dem Mentor Lorenz die folgende Fallbestimmung formulieren: Das strukturelle Problem der Legitimierungsbedürftigkeit wird im Gespräch über die Etablierung einer Eigenlogik bearbeitet, die sich an die Anderslogik der Therapie anlehnt. Das hierfür notwendige Interesse respektive das Bedürfnis seitens der Studentin, sich im Gespräch von Lorenz quasitherapeutisch begleiten zu lassen, ist allerdings nicht erkennbar. Diese fehlende Resonanz seitens der Studentin führt zu Anerkennungs- und Symmetrisierungsbemühungen aufseiten des Mentors. Diese lassen sich, pointiert formuliert, mit «habe «Not» und brauche mich» fassen. 
Die in der Gesprächsinteraktion aufscheinenden Grenzüberschreitungen hinsichtlich einer professionellen Distanz- und Rollenwahrnehmung zeigen auf, dass der Mentor auf eine gewisse Nähe, Symmetrie und diffuse Beziehungsstruktur und ein Gemocht-Werden der Studentin angewiesen ist. Diese Dynamik des Aufeinander-angewiesen-Seins allerdings beruht auf Gegenseitigkeit. Auch die Studentin ist auf Anerkennung und 〈Absegnung) in ihrer Rolle als Studentin und in der Diffusität der ganzen Person angewiesen. 


\subsection{Salomé \& Edith}

Das vierte Gespräch zwischen der Studentin Salomé und der Mentorin Edith findet ebenfalls im Frühlingssemester 2017 statt. Es ist auf 11.00 Uhr angesetzt und dauert eine gute halbe Stunde. In der Nähe der Türe steht ein Flipchart, auf dem die Mentorin folgende Punkte notiert hat:

- Rückblick Partnerschuljahr

- Kompetenzfelder

- weitere Anliegen

In einer Ecke des Raumes gibt es einen Tisch, auf dem die Mentorin sieben laminierte Karten im A4-Format hingelegt hat, vier Karten in der oberen Reihe, drei Karten in der unteren Reihe

obere Reihe, von links nach rechts:

Karte 1: Diagnose \& Beurteilung

Karte 2: Planung \& Durchführung von Unterricht

Karte 3: Sicherung der Qualität und professionelle Weiterentwicklung

Karte 4: Kommunikation \& Zusammenarbeit

untere Reihe, von links nach rechts:

Karte 5: Institutionelles Handeln, Schule \& Gesellschaft

Karte 6: Lernen \& Entwicklung

Karte 7: Teilhabe am professionsspezifischen Fachdiskurs

Die Studentin und die Mentorin sitzen an zwei zusammengeschobenen Tischen in der Raummitte. Die Studentin und die Mentorin tauschen im Vorfeld des Gesprächs einige informelle Sätze aus. An dieser Stelle erteilt mir die Mentorin mit einem Zeichen die Erlaubnis, das Aufnahmegerät einzuschalten. 


\subsubsection{Eröffnungssequenz: Und wie läuft's im Partnerschulprojekt}

Edith: und wie läuft's im Partnerschulprojekt?

Salomé: sehr gut

Edith: schön

Salomé: ich geniesse es also es ist echt toll wir lernen viel wir dürfen viel ausprobieren wir haben alleine Lektionen gemacht auch zusammen und auch zu dritt Edith: $\mathrm{mhm}$ ((bejahend))

Salomé: also es ist eine coole Klasse es ist ganz ganz toll

Edith: schön

Salomé: ja ((lacht)) also ich merke auch so langsam wirklich dass ich ein bisschen sicherer werde ehm vor der Klasse zu stehen weil ich wirklich merke ehm so langsam jetzt geht es aufs Ende zu und ich merke wirklich es ist so langsam (..) doch schon besser als am Anfang weil am Anfang ist man doch immer so ein bisschen unsicher nervös und so

Edith: ((unverständlich))

Salomé: und mittlerweile ehm ich mache meine Präp und dann (.) hab ich's im Kopf und mache meinen Unterricht und (.) kann auch spontan ausweichen und so (.) also ehm ja

Edith: gut wir schauen heut mal zurück das ist ja gar nicht mehr so lange es sind jetzt die letzten Schulferien im Partnerschulprojekt

Salomé: unglaublich ((lacht))

\subsubsection{Rekonstruktion der einzelnen Sequenzen}

Edith: und wie läuft's im Partnerschulprojekt?

Der Sprechakt und wie läuft's im [.] impliziert eine gewisse Vertrautheit, da nach einem spezifischen Gegenstand gefragt wird. Mit dem und als Ausgangspunkt der Konversation im Modus eines lockeren Gesprächseinstieges wird das Setting tendenziell zu einem 
informellen Setting gemacht. Eine mögliche Situation aus einem privaten, familiären Kontext für diese Frage wäre beispielsweise, wenn die Grossmutter von ihrer Enkelin wissen möchte «Und wie läuft's in der Schule?» Die Kontaktaufnahme der Grossmutter weist darauf hin, dass die beiden in keinem innigen Verhältnis zueinander stehen. Vorstellbar ist, dass trotz einer grundsätzlichen, verwandtschaftlichen Nähe auch eine gewisse Distanz zwischen den beiden besteht und dieses Spannungsfeld zwischen Nähe und Distanz mit dem Sprechakt bearbeitet wird.

Ein weiterer vorstellbarer Kontext wäre ein beruflicher, beispielsweise ein Gespräch zwischen einer Vorgesetzten und einer Mitarbeiterin. Die Vorgesetzte fragt beispielsweise, wie es mit dem Hausbau laufe und zeigt dadurch auch Interesse auf, das über den beruflichen Kontext hinaus geht. Die Frage ist in hierarchischen, wie auch in egalitären Settings vorstellbar. Sie hat als «Türöffner` die Funktion einer informalisierenden Kontaktaufnahme, beinhaltet eine Erzählaufforderung und dient der Herstellung von Vertrautheit. Die angesprochene Person hat eine breite Auswahlmöglichkeit darauf zu reagieren, auch weil die direkte, persönliche Ansprache - «Und wie läuft es bei dir?» - ausbleibt.

Der alltagssprachliche, informelle Gesprächseinstieg bietet die Grundlage für ein Gespräch, welches ein Interesse an der subjektiven Einschätzung der angesprochenen Person oder an ihrer Befindlichkeit vermittelt. Gleichzeitig scheint auch eine Figur durch, in der der Gesprächseinstieg als Mittel zum Zweck der Kontaktaufnahme eingesetzt wird. Im real vorgefundenen Kontext dieses Mentoratsgesprächs signalisiert der Sprechakt einen Übergang vom Gespräch ohne Aufnahme zum Gespräch mit Aufnahme. Dieser Wechsel kommt ohne sprachliche Formalisierung aus. Das Gespräch bleibt mit der Einstiegsfrage weiterhin informell. Würde die Frage lauten «und wie läuft's mit dem Partnerschulprojekt» wäre klar, dass die Frage auf eine Berichterstattung bezogen auf das Projekt zielt. Bei der realisierten Version - und wie läuft's im Partnerschulprojekt - bleibt hingegen offen, welche Art von Antwort erwartet wird. Die Mentorin interessiert sich entweder für die studentische Meinung zum Projekt, für die Arbeit im Projekt oder für Salomés Befinden innerhalb des Projektes.

Zusammenfassend lassen sich folgende Strukturmerkmale für die Eröffnungsfrage festhalten:

Das Gespräch wird der Form nach alltagssprachlich und informalisierend eröffnet. Die Einstiegsfrage zielt auf eine pauschale Evaluation ab. Beispielsweise könnte mit einer 
Kurzantwort wie "gut», "ganz gut» oder ähnlichen Antworten angeschlossen werden. Dies käme in dieser knappen Form einer Formalisierungsbewegung respektive ReUniversalisierungsbewegung gleich. Der Anschluss wäre formal korrekt und würde dennoch eine schliessende Bewegung darstellen. Salomé könnte ebenso gegenteiligen im Modus einer Öffnung auf die Gesprächsaufforderung reagieren. Die Antwort könnte wie folgt beginnen: «Anfangs war ich eher skeptisch, weil ich sehr viel lieber nochmals ein Praktikum im Kindergarten gemacht hätte, aber nur bis ich die Klasse kennenlernte.» Im Spektrum möglicher Beziehungen von keiner Vertrautheit bei fremden Personen und hoher Vertrautheit in familiären, freundschaftlichen oder intimen Beziehungen, begegnet man hier einer mittleren Vertrautheit in einer in gewisser Weise bereits etablierten Beziehung. Aus dieser mittleren Vertrautheit heraus erwächst eine Grundspannung, die im Sprechakt ihren Ausdruck findet. Programmatisch speist sich das Setting «Mentoratsgespräche» primär aus einem formalen, organisatorischen und rollenförmigen Interesse zwischen Studierenden und Mentorierenden. Gleichzeitig ist eine Gesprächseröffnung erkennbar, die eine Informalisierungsbewegung beinhaltet.

Salomé: sehr gut

Die Antwort sehr gut fällt betont positiv aus. Da die Selbsteinschätzung nicht begründet wird, erhält diese ein schliessendes und distanzierendes Moment. In der Kurzantwort «super» wäre die schliessende Komponente bereits weniger ausgeprägt, da sie eine Rückfrage wie «Na erzähl mal, was ist denn so super?» vermuten liesse. Würde hingegen eine Anmoderation wie beispielsweise «eigentlich sehr gut» vorgenommen, stünde das «eigentlich» für etwas noch zu Explizierendes. Dadurch würde der Sprechakt den Charakter der Schliessung, wie er bei sehr gut vorliegt, verlieren. Wie vorgenommen, scheint das schliessende Element bedeutsam.

Edith: schön

Der Anschluss ist der Form nach schlicht und bringt Freude zum Ausdruck. Die studentische Antwort scheint den Erwartungen der Mentorin zu entsprechen. Schön ratifiziert Salomés Antwort in positiver, aber auch unspezifischer Weise. In einer Lesart 
kann dies als Tolerierung der Schliessbewegung verstanden werden und bedeutet: «Du willst mir nicht mehr oder Genaueres erzählen, ich akzeptiere das.»

Auch möglich ist, dass Edith mit schön zuwartet und Salomé unaufdringlich die Option zuspielt, ihre positive Evaluation weiter auszuführen und den Gesprächsball doch noch aufzunehmen. Denn aus interaktionslogischer Perspektive betrachtet liegt der Ball an dieser Stelle bei Salomé. Sie entscheidet, wie sie die informalisierende Kontaktaufnahme bearbeiten möchte. Die vorgefundene Ratifizierung mit schön ändert nichts an dieser Ausgangslage. Der erste Gesprächsimpuls ist somit bearbeitet und der erste Ball hin und her gespielt.

Salomé: ich geniesse es

Ohne Berücksichtigung des Kontextes sind Begebenheiten, die sich als Fortsetzung anbieten «ein gutes Glas Wein zu trinken» oder «mit meinen Enkelkindern Zeit zu verbringen». Um geniessen zu können, muss man sich seiner sicher sein, denn Genuss impliziert eine gewisse Gelassenheit und Souveränität. Die Aussage ich geniesse es ist zum einen eine bewusste Handlung, die sich über eine Selbstzuschreibung definiert, und zum andern eine begrenzte Erfahrung, die etwas Nicht-Routiniertes und Besonderes für die geniessende Person markiert.

Gewendet auf den Kontext «Mentoratsgespräche» wäre eine allfällige Auslegung der Aussage des Geniessens die starke Identifikation mit dem Partnerschulprojekt und den im Projekt stattfindenden studentischen Tätigkeiten. Eine weitere Auslegung wäre, es zu geniessen, im Praxisfeld zu sein im Sinne einer Differenzfigur zu anderen studentischen Tätigkeiten.

Abschliessend lässt sich festhalten, dass das signifikante Merkmal im Sprechakt von Salomé die Übersteigerung ist, die sich im Geniessen Ausdruck verschafft.

Salomé: also es ist echt toll wir lernen viel wir dürfen viel ausprobieren

Mit also leitet Salomé eine Begründung ihrer Genussempfindung ein und präzisiert das mit es zuvor allgemein Gehaltene. Sie führt aus, welche Elemente des Partnerschul- 
projektes sie mit Genuss verbindet. Dies ist bemerkenswert, da viel lernen nicht à priori mit der Zuschreibung von Genuss assoziiert wird.

Eine inhaltliche Auslegung wird an dieser Stelle nicht weiterverfolgt. Vielmehr scheint für die Gesprächsstelle bedeutsam, was in den übersteigerten Formulierungen wie sehr gut, ich geniesse es und es ist echt toll Gestalt gewinnt. Das Näheangebot der Gesprächseröffnung - und wie läuft's - wird ‘sanft) abgewendet. Der Sprechakt beinhaltet eine ReSpezifizierungstendenz und eine Distanzierungsbewegung. Würde das Näheangebot angenommen, wäre eine konkrete Erklärung anstelle der ^floskelhaften` Formulierung wir lernen viel wir dürfen viel ausprobieren zu erwarten.

Das Verb dürfen impliziert eine Vorinstanz, die diese Erlaubnis erteilt. Die Formulierung viel ausprobieren dürfen kann kontextgebunden bezüglich Praktika als stereotype Redewendung gelesen werden. Diese zeigt auf, dass das viel ausprobieren dürfen für Studierende als attraktiv gilt.

Als Strukturmoment kommt in Salomés Sprechakt das Unproblematische und uneingeschränkt Positive der Situation zum Ausdruck. Das zuvor unterbreitete Näheangebot wird mit einer Schliessbewegung - «bei mir ist alles gut, du brauchst nicht weiter nachzufragen» - bearbeitet.

Salomé: wir haben alleine Lektionen gemacht auch zusammen und auch zu dritt

Edith: $m h m$ ((bejahend))

Salomé: also es ist eine coole Klasse es ist ganz ganz toll

Die Ausführungen Salomés beziehen sich inhaltlich auf die Thematik des Unterrichtens, über die sie im Modus der Retrospektive erzählt. Da sich, bezogen auf die Gesprächsinteraktion zwischen ihr und der Mentorin, wenig daraus erschliessen lässt, wird der erste Teil nicht feinsequenziell rekonstruiert. Bemerkenswert im zweiten Teil von Salomés Aussage ist die nochmalige Steigerung in ihrer Formulierung. Kontextunabhängig wäre die Aussage also es ist eine coole Klasse es ist ganz ganz toll aus dem Munde einer Schülerin zu vermuten, wenn nach einem Wohnortwechsel im Anschluss an den ersten Schultag begeistert von der neuen Klasse erzählt wird. Auch eine erwachsene Person, die einen Sprachkurs absolviert, könnte dies zu einer Freundin sagen. Ebenfalls denkbar wäre die Umkehrsituation, dass eine Lehrperson, die nach den Sommerferien eine neue Klasse 
übernimmt, eine solche Formulierung wählt. Allen Geschichten gemein ist eine Situation, die Begeisterung auslöst und als herausragend dargestellt wird. Im Kontext der beruflichen Bewährung innerhalb eines Praktikums teilt die Studentin mit, dass sich für sie die Arbeit in der Klasse friktionsfrei und problemlos gestaltet und bei ihr Enthusiasmus auslöst. Inhaltlich bleibt die Aussage vage, eine Begründung, weshalb die Klasse so cool ist, wird nicht geliefert. Dies ist allerdings interaktiv auch nicht zu erwarten, da die Studentin mit der informalisierenden Frage - und wie läuft's im Partnerschulprojekt? auch pauschal und unverbindlich angesprochen wird.

Das Strukturmerkmal des Sprechaktes ist die Fortsetzung des Zeigens von Begeisterung und die Positionierung als eigenständig und kooperativ. Mit dem bejahenden $\mathrm{mhm}$ zwischen Salomés Ausführungen bekräftigt Edith ihre aktive Zuhörerschaft und lässt Salomé Raum für weitere Ausführungen.

Edith: schön

Edith quittiert Salomés Ausführungen erneut mit schön. Möglicherweise hat sich der Begriff schön auf der Ebene einer Sprachgewohnheit bei Edith etabliert. Wie hier angewendet dient schön der Bezugnahme und Bestätigung der aufmerksamen Zuhörerschaft. Allerdings wäre dies auch mit "gut» oder "okay» gegeben, weshalb dem schön womöglich eine weitere Bedeutung zukommt. Schön schafft, mehr als «okay» oder «gut», auch etwas Gemeinsames und Anteilnehmendes und weist eine Bedeutung auf, die sich mit «ich finde es schön, dass es dir so gut gefällt» oder «ich freue mich mit dir» paraphrasieren lässt.

Auf einer latenten Bedeutungsebene gewinnt mit der Antwort schön auch etwas Gestalt, dass sich alltagssprachlich formuliert mit ‘anknüpfen ohne anzuknüpfen` umschreiben lässt. Gesprächspragmatisch weist schön ein schliessendes Element auf oder bietet zumindest keinen Anlass für etwas Öffnendes.

Die nächsten Ausführungen von Salomé und der unverständliche Anschluss von Edith werden als Ganzes rekonstruiert. 
Salomé: ja ((lacht)) also ich merke auch so langsam wirklich dass ich ein bisschen sicherer werde ehm vor der Klasse zu stehen weil ich wirklich merke ehm so langsam jetzt geht es aufs Ende zu und ich merke wirklich es ist so langsam (..) doch schon besser als am Anfang weil am Anfang ist man doch immer so ein bisschen unsicher nervös und so.

Edith: ((unverständlich))

Salomé: und mittlerweile ehm ich mache meine Präp und dann (.) hab ich's im Kopf und mache meinen Unterricht und (.) kann auch spontan ausweichen und so (.) also ehm ja

Ohne auf die einzelnen Aussagen im Detail einzugehen, scheint die Aussage von Salomé dahingehend auffällig, als dass gleich dreimal das Verb merken, in der Bedeutung von «wahrnehmen» oder «reflexiv feststellen», verwendet wird. Diese Formulierung zeigt sich in zwei beinahe identischen Satzkonstruktionen - ich merke auch so langsam wirklich wie auch in - wirklich merke ehm so langsam.

Im Sprechakt wird von der Selbstwahrnehmung eines Fortschritts berichtet. Dieser wird mit so ein bisschen sicherer werde zwar bescheiden dargestellt, aber dennoch mit Nachdruck ausgesprochen. Auf einer manifesten Bedeutungsebene werden dadurch allfällige Zweifel ausgeräumt. Gerade diese Hervorhebung verweist auf einer latenten Ebene darauf, dass womöglich noch Zweifel vorhanden sind. Es kann angenommen werden, dass für Salomé die Thematik der Sicherheitsgewinnung weiterhin relevant bleibt, selbst wenn - oder gerade weil - sie gegenüber Edith ihre Fortschritte darlegt. Allerdings zeigt sich in der vorgenommenen Fortschrittsbekundung, dass sie die Thematik nicht mit Edith zu besprechen gedenkt, sondern lediglich ihren Fortschritt dokumentiert. Dennoch bestünde für Edith die Möglichkeit, auf die von Salomé geschilderte Fortschrittsbekundung einzugehen. Ediths Kurzantwort, die sich akustisch nicht erschliessen lässt, scheint Salomés Ausführungen nicht zu unterbrechen.

Zusammenfassend lässt sich sagen, dass Salomé im Sprechakt eine Verdichtung ihres Gesamteindruckes und Grundgefühls vornimmt und ihre Fortschrittsbekundung aufzeigt. Auf einer gesprächspragmatischen Betrachtungsebene ist erneut eine schliessende Komponente erkennbar. Salomé zeigt auf, dass diesbezüglich kein Gesprächsbedarf besteht. 
Edith: gut wir schauen heut mal zurück das ist ja gar nicht mehr so lange es sind jetzt die letzten Schulferien im Partnerschulprojekt

Salomé: unglaublich ((lacht))

Auf die Erfolgsmeldung bezogen auf ihre Unterrichtspräparation und die Fähigkeit, spontan von dieser abzuweichen, reagiert Edith mit einem abschliessenden gut als Ratifizierungsmarkierung. Daran anschliessend leitet sie das erste Gesprächsthema, ein gemeinsames Zurückschauen, ein. Edith konstituiert an dieser Stelle das Mentoratsgespräch als Retrospektive. Dies ist unter dem Gesichtspunkt, dass die vorherige Evaluation auch rückblickend war, bemerkenswert. Es muss sich demzufolge bei der angekündigten Rückschau um eine andere Form einer rückblickenden Perspektive handeln. Edith adressiert Salomé mit einer Wir-Formulierung und zeigt auf, dass sie sich gemeinsam über diese Rückschau beugen.

Die bisher erarbeiteten Strukturmerkmale lassen die Formulierung einer ersten Fallstrukturhypothese zu:

\subsubsection{Zusammenfassung und riskante Fallstrukturhypothese der Eröffnungssequenz Und wie läuft's im Partnerschulprojekt?}

Die Analyse der Eröffnungssequenz des Mentoratsgesprächs zeigt einen informalisierenden Gesprächseinstieg seitens der Mentorin. Im Fokus dieser Kontaktaufnahme steht die Herstellung von Reziprozität. Dies lässt sich vergleichen mit der gängigen Begrüssungsfrage "wie geht's», die der Kontaktaufnahme, nicht aber der Analyse des Gemütszustandes der angesprochenen Person dient. Eine ähnliche Dynamik findet sich auch in der vorgefundenen Gesprächseröffnung, auch hier steht die soziale Funktion einer Kontaktaufnahme im Zentrum. Allerdings - und dies stellt ein Gegensatz zum diffusen Gesprächsstimulus «wie geht's» dar - wird spezifisch nach dem Partnerschulprojekt gefragt. Im Sprechakt lässt sich folglich eine Verschränkung von diffusen und spezifischen Anteilen erkennen.

Die Studentin ihrerseits steigt auf die informalisierende Kontaktaufnahme ein, indem sie der Mentorin in übersteigerter Sprache - ich geniesse es, es ist echt toll, es ist ganz ganz 
toll - über ihre Befindlichkeit im Partnerschulprojekt berichtet. Gesprächspragmatisch betrachtet indizieren die Übersteigerungen etwas, was auf eine Fragilität in der sozialen Situation hinweist. Mögliche Diffusitätsanforderungen, die mit dem Näheangebot der Mentorin einhergehen, werden seitens der Studentin bearbeitet. Ihre Schilderungen beinhalten eine Schliessbewegung bezogen auf die soziale Situation, als ob es einer potenziellen Diffusität auszuweichen respektive diese zu (umschiffen` und re-spezifizieren gelte.

Die Studentin Salomé zeigt in der Sequenz ihren durchlaufenen Entwicklungsprozess auf. Ihre Ausführungen bieten wenig Anlass für Rückfragen. Dabei zeigt sie sich einerseits bescheiden - ein bisschen sicherer werde -, andererseits auch als in ihrem künftigen Berufsfeld angekommen und einsozialisiert - mache meinen Unterricht und (.) kann auch spontan ausweichen und so. Die Struktur ihrer Selbstdarstellung lässt sich mit «ich zeig mich jetzt hier nicht wirklich» paraphrasieren.

Ediths Bezugnahmen sind erfreut und wohlwollend. Ihre Kurzantworten weisen die soziale Bedeutung auf, an Salomés Ausführungen sohne eigentliche Anknüpfung anzuknüpfen ». Mit anderen Worten wird formal, nicht aber inhaltlich darauf eingegangen. Selbst wenn Edith zu Beginn ein Näheangebot unterbreitet, folgen ihre weiteren Sprechakte ebenfalls einer Schliessbewegung.

Jenseits der manifest informalisierend gestalteten Kontaktaufnahme konturiert sich auf der latenten Ebene eine Vermeidung eines diffus Miteinander-in-Kontakt-Tretens. Diese Vermeidungsfigur lässt sich jedoch nicht als ausschliesslich distanziert und rollenförmig beschreiben, sondern betrifft oder vereinnahmt dennoch die ganze Person.

Im Zusammenspiel dieser beiden gegenläufigen Bewegungen zeigt sich eine gewisse Fragilität, das sich Ausweichen scheint nicht ganz einfach und (geschmeidig) vonstattenzugehen, sondern bedarf beidseitig einer Bearbeitung.

Die Anfangsinteraktion lässt sich auf folgende Figur einer riskanten Fallstrukturhypothese verdichten:

Auf einer manifesten Bedeutungsebene begegnen sich die Studentin und Mentorin mit gewissen Diffusitätspotenzialen, die sich bei der Mentorin zu Beginn in Nähe- und Informalisierungsangeboten zeigen und bei der Studentin Übersteigerungen in der Sprache hervorrufen. Auf einer latenten Ebene allerdings vermeiden oder (umschiffen) beide eine mögliche Nähe oder eine diffuse Kontaktaufnahme, indem sie wenig 
Anknüpfungspunkte für eben diese liefern. Dadurch verschafft sich ein Sich-gegenseitigin-Ruhe-Lassen Ausdruck. Die Bearbeitung des Spannungsfeldes von Diffusität und Spezifität wie auch von Nähe und Distanz, so die Fallstrukturhypothese der Gesprächseröffnung, führt zu beidseitigen Umschiffungsbewegungen, die riskant formuliert einer Tanzfigur um ein unsichtbares Feuer gleichen. In dieser Metapher steht das unsichtbare Feuer für potenzielle Nähe und Diffusität, die vermieden werden müssen und gleichzeitig auch nicht vermieden oder umgangen werden können.

\subsubsection{Zweite Sequenz: Also eigentlich sind jetzt alle Ziele abgearbeitet}

In den folgenden Minuten führt Salomé ihre Schilderungen betreffend des Partnerschulprojektes fort. Sie zeigt auf, was sie im Rahmen des Projektes erlebt und wie sie für ihre künftige Tätigkeit profitiert. Salomés Sprechanteil ist in dieser Passage höher als derjenige von Edith. Edith bestätigt ihre aktive Zuhörerschaft ab und an mit $\mathrm{mhm}$ oder schön. In einer Sprechpause fordert Edith Salomé auf, mit ihr zum Tisch in der Ecke zu gehen. Auf diesem Tisch liegen Karten mit den aufnotierten Kompetenzfeldern ${ }^{57}$ auf. Edith beginnt mit der Aufrufung des zweiten Kompetenzfeldes - «Planung \& Durchführung von Unterricht» - das in der Gesprächspassage verhandelt wird. Hier setzt die detaillierte Rekonstruktion ein.

Salomé: ehm das hatte ich dann allerdings bereits gegen Ende des Praktikums besser im Griff auch durch Tipps von von X [Name der PLP; S.H.] und wollte dies jetzt einfach nochmals verfeinern und jetzt würde ich einfach sagen das ist erledigt

Edith: also das hast du jetzt (.) gut schön

Salomé: also eigentlich sind jetzt alle Ziele abgearbeitet also für das P4 werde ich Neue machen

\footnotetext{
57 In der Rahmenkonzeption der Berufspraktischen Studien am Institut KU der PH FHNW wird die Arbeit mit den Kompetenzfeldern wie folgt beschrieben: «Ziel des Mentorats ist es, die eigenen Kompetenzen in der regelmässigen Auseinandersetzung mit den acht Kompetenzfeldern im Sinne professioneller Standards abzugleichen und Belege zu diesen Kompetenzfeldern zu finden und zuzuordnen" (siehe: https://web.fhnw.ch/ph/praxis/kindergartenunterstufe/leitfaeden/vertiefungsphase/leitfaden-vertiefung-19_20; eingesehen am 29. Juni 2020).
} 
Edith: ganz neue Ziele das hört sich gut an (.) dann kann ich ja grad davon ein bisschen weggehen und geh einfach nochmals durch "Diagnose \& Beurteilung» Salomé: ehm durch den Leistungsnachweis Fachdidaktik Deutsch ist das bearbeitet worden ehm ich musste eine Diagnose machen und Arbeit schreiben

\subsubsection{Rekonstruktion der einzelnen Sequenzen}

Salomé: ehm das hatte ich dann allerdings bereits gegen Ende des Praktikums besser im Griff

Aus dem Gesprächstranskript geht hervor, dass Salomé mit das ihr gesetztes Ziel anspricht. Dieses lautet «den Überblick über die ganze Klasse zu behalten».

Sucht man nach naheliegenden Situationen, in denen der obige Sprechakt vorstellbar wäre, muss - um Kontextfreiheit zu gewährleisten - Praktikum durch einen Platzhalter ersetzt werden. Erwartbar wäre diese Aussage beispielsweise von einer Automobilistin, die in England den Linksverkehr gegen Ende ihres Aufenthaltes besser im Griff hat. Zum Zeitpunkt des Gespräches hat sie diesen zwar nicht vollständig, jedoch besser im Griff. Das Strukturmerkmal der Aussage ist das Aufzeigen der angestrebten Beherrschbarkeit, wenn auch die Situation als nicht vollständig beherrschbar erfahren wird. Irritierend ist die Verwendung von bereits in Kombination mit dem Ausdruck gegen Ende. Sprechaktlogisch weist die Verwendung von bereits darauf hin, dass ein Umstand früh, früher als erwartet, eintritt. Bereits würde von der Logik her in Verbindung mit «zu Beginn» oder «inmitten» einer Periode, nicht aber erst gegen deren Ende stehen. Im vorgefundenen Kontext könnte dies beispielsweise lauten: «Das hatte ich allerdings bereits nach der ersten Praktikumswoche besser im Griff.» Dieser logische Bruch lässt folglich durchscheinen, dass eine Differenz zwischen der abgelegten Rechenschaft und den tatsächlichen Gegebenheiten besteht, die sich im sprechaktlogischen Widerspruch von bereits und gegen Ende des Praktikums Ausdruck verschafft. In Salomés Rechenschaftslegung zeigt sich erneut eine Schliess- und Distanzierungsbewegung, die wenig Anlass für 
Nachfragen bietet. Gleichzeitig ist erkennbar, dass diese Rechenschaftslegung nicht durchgängig (geschmeidig) und bruchlos vorgenommen werden kann.

Salomé legt im Sprechakt Rechenschaft bezogen auf Fortschritte, die ihre Individualnorm betreffen, ab. Die hier vorgenommene Form der Adressierung zeigt auf, dass Salomé in Edith eine Person sieht, für die ihre Selbstbeschreibung als Beweislage eine Gültigkeit aufweist.

Salomé: auch durch Tipps von von X [Name der PLP; S.H.]

In Salomés Ergänzung lohnt sich eine genaue Analyse von Tipps. Diese beschreiben kleinere, universalistische Kurzratschläge, Anleitungen oder Hinweise. Sie weisen eine Allgemeingültigkeit auf, sehen von einer Fallspezifik ab und bleiben unverbindlich. Tipps setzen nicht zwingend eine kognitive Durchdringung voraus und sind einfach in der Handhabung. Sie haben zwar den Charakter eines Rates, vermeiden jedoch gleichzeitig das in einem Rat oder Ratschlag virulent erkennbare Problem der Asymmetrie, der Hierarchie und der Verantwortung (Paris, 2005, S. 364).

Im konkreten Kontext scheint die Studentin die Unaufdringlichkeit und Unterkomplexität dieser Hinweise ihrer Praxislehrperson zu begrüssen. Wie hier aufgezeigt, hat sie ihr gesetztes Ziel gegen Ende des Praktikums nicht ausschliesslich, aber auch wegen diesen Tipps erreicht. Sie empfand die von ihrer Praxislehrperson erteilten Tipps für angebracht und hilfreich. Im hier vorgefundenen Zusatz teilt Salomé Edith auf einer latenten Ebene mit, wem gegenüber sie Nähe zulässt und Zugang zu ihrer eigenen Person gewährt. Gleichzeitig zeigt sie auf, dass es sich bei den Dingen, die es im Griff zu haben gilt, um solche handelt, die mit Tipps bereits handhabbar gemacht werden. Darin findet erneut eine doppelte Schliess- und Distanzierungsbewegung gegenüber Edith statt, die sich mit «es gibt nur kleinere Probleme und die löse ich gerne mit meiner Praxislehrperson» umschreiben lässt. Die potenziell vorhandenen Diffusitätsansprüche werden interaktiv im Vermelden einer Erfolgsberichtserstattung bearbeitet. Die Hypothese der Schliess- und Distanzierungsbewegungen reproduziert sich. 
Salomé: und wollte dies [das Ziel: den Überblick über die ganze Klasse behalten; S.H:] jetzt einfach nochmals verfeinern und jetzt würde ich einfach sagen das ist erledigt

Aus kontextungebundener Perspektive betrachtet will man den Detaillierungsgrad erhöhen, wenn man etwas nochmals verfeinern will. Dies wäre beispielsweise bei einem Zeitplan oder bei einem Prozedere wie der Verzierung des Hochzeitskleides mit Pailletten und Perlen möglich. Verfeinern lässt sich hier als graduelles Phänomen lesen.

Kontextgebunden hat sich Salomé das Ziel gefasst, «den Überblick über die ganze Klasse zu behalten» nochmals zu verfeinern. Im Sprechakt zeigt Salomé ihre subjektive Einschätzung auf, eine Stufe erreicht zu haben, die sie zufriedenstellt. Mit der Verwendung des Selbstkommentars - jetzt würde ich sagen - bleibt sie mit der Formulierung im Konjunktiv dennoch vorsichtig.

An dieser Stelle bietet sich ein Kurzexkurs zu den Zielen an, die Studierende an der PH FHNW jeweils für die kommende Praxisphase in einer Perspektivplanung festhalten müssen. In dieser Planung beantworten die Studierenden unter Professionalisierungsvorhaben des/der Studierenden in einem leeren Feld folgende Frage: «An welchen Aspekten und Kompetenzen möchte ich in diesem Praktikum arbeiten?» Die Studierenden definieren demzufolge im Praktikum Professionalisierungsbereiche, die sie selbst oder ihre Praxislehrpersonen oder Mentorierenden - oftmals im Sinne von Schwächen und Defiziten - identifiziert haben. Diesem Vorgang begegnet man an dieser Stelle. Die Studentin zeigt auf, welche Nachjustierung sie für ihr Ziel vorgenommen hat. Im Modus einer Rechenschaftslegung kennzeichnet sie selbstbestimmt und selbstbewusst ihr gesetztes Ziel als erledigt. Erneut bieten ihre Ausführungen mit der vorgefundenen Schliessbewegung keinen Anlass für weitere Rückfragen.

Edith: also das hast du jetzt (.) gut schön

Edith startet ihre Aussage mit also, einem Partikel, der eine minimale zeitliche Verzögerung nach sich zieht. Sie bezieht sich auf die Ausführungen von Salomé und wiederholt und bestätigt diese. Die kurze Pause nach also das hast du jetzt lässt sich gedankenexperimentell mit verschiedenen Verben wie «erledigt», «erreicht», 
"geschafft» oder "entschieden» füllen. Im real vorgefundenen Sprechakt wird allerdings keine Ergänzung vorgenommen, sondern anhand von gut schön mitgeteilt, dass Edith die vorgenommene Schliessung zur Kenntnis nimmt und ohne darauf Bezug zu nehmen als «abgehakt» akzeptiert.

Salomé: also eigentlich sind jetzt alle Ziele abgearbeitet also für das $P 4^{58}$ werde ich Neue machen

Analog zu Edith startet Salomé ihre Aussage mit also, hier aber in der Bedeutung einer Bilanzierungseröffnung. Betrachtet man den ersten Teil ihrer Aussage, fällt auf, dass sie nicht das für den Terminus «Ziele» geläufige Verb «erreicht», sondern abgearbeitet verwendet. Die Formulierung Ziele abarbeiten anstelle von «Ziele erreichen» als Fokussierungsmetapher bedarf einer genaueren Betrachtung. Fragt man sich kontextfrei, wann man von abarbeiten spricht, wäre dies beispielsweise bei einem Stapel Unterlagen oder Korrekturarbeiten, ebenso bei Traktanden, Punkten oder Aufgaben möglich. Zur Abarbeitung sind nebst Fleiss auch Ausdauer und Beharrlichkeit notwendig und eine gewisse Monotonie ist der Tätigkeit nicht abzusprechen. Abgearbeitet wird, was man aufgegeben bekommt.

Der zweite Teil der Aussage ist von der Satzstruktur her analog aufgebaut. Während das erste also eine rückblickende Aussage einleitet, folgt hier mit also eine Aussage, die den nächsten Schritt betrifft. Dieses Vorgehen bestärkt die Abarbeitungslogik, in der Punkt für Punkt, Aufgabe für Aufgabe erledigt werden. Im zweiten Teil verwendet Salomé erneut ein für den Terminus «Ziele» ungebräuchliches Verb. Sie spricht von «neue Ziele machen», während Ziele üblicherweise "gesetzt», «festgelegt» oder «definiert» werden. Salomé wird für ihr letztes Praktikum neue Ziele «herstellen`. Die empirisch vorgefundene Formulierung lässt die Deutung zu, dass keine Ziele aus dem Prozess heraus generiert werden können, sondern solche eigens angefertigt oder eben gemacht werden müssen. Über die Formulierungen Zielen abarbeiten und Ziele machen gewinnt eine Inszenierung Kontur, die notwendig wird, um der Erfüllungslogik, respektive der Rechenschaftslegung

\footnotetext{
58 P4 ist die Abkürzung für das vierte und letzte Praktikum im Zwischensemester des letzten Studienjahrs des Bachelorstudienganges zur Kindergarten- und Unterstufenlehrperson an der PH FHNW.
} 
Folge zu leisten. Mit abarbeiten anstelle von «erreichen» und machen anstelle von «definieren» wird die in der Interaktion auftauchende Dynamik des «Abarbeitens» von Themen im Modus einer Rechenschaftslegung kurzzeitig von der latenten auf die manifeste Ebene gehoben.

Fragt man nach Anschlussmöglichkeiten, die dem Sprechakt folgen könnten, wäre beispielsweise eine Nachfrage «Hast du dir diesbezüglich schon konkrete Gedanken gemacht?» oder ein allgemeiner Kommentar wie «da bin ich aber mal gespannt» vorstellbar. Von der Strukturlogik her würde Salomé in beiden Varianten durch Ediths gezeigtem Interesse aufgefordert, ihre neuen Ziele auszuführen.

Edith: ganz neue Ziele das hört sich gut an (.) dann kann ich ja grad davon ein bisschen weggehen und geh einfach nochmals durch «Diagnose \& Beurteilung»

Der Sprechakt ganz neue Ziele das hört sich gut an könnte in einer Situation fallen, wenn die Sprecherin Wohlwollen gegenüber den Vorsätzen oder Zielen der anderen Person äussert, ohne sich inhaltlich auf diese zu beziehen oder mehr darüber zu erfahren. In der Formulierung das hört sich gut an ist erneut eine Distanzierungsbewegung zu erkennen. Die Sprecherin vermeidet eine Stellungnahme und bleibt in ihrer Äusserung universalistisch, zugespitzt formuliert auch (floskelhaft). Gleichzeitig gewinnt im Sprechakt auch eine Erleichterung Gestalt. Es scheint, als wäre die Sprecherin erleichtert und entlastet, davon weggehen zu können.

Kontextgebunden zeigt der Sprechakt auf, dass der Agendapunkt reibungslos und erfolgreich abgearbeitet wurde und die Mentorin diesen hinter sich lassen kann.

Das Strukturmerkmal der Eröffnungssequenz reproduziert sich an dieser Stelle. Denn es lässt sich eine gewisse Prekarität bezogen auf den Spannungsbogen von Diffusität und Spezifität, wie auch von Nähe und Distanz erkennen. Einerseits gilt es, ein Zuviel an Nähe und Diffusität zu vermeiden. Andererseits scheint dieses Vermeiden, oder wie bereits zuvor elaboriert, 〈Umschiffen〉 auch prekär zu sein, da in einer dyadischen Gesprächssituation auch gewisse Ansprüchlichkeiten auf Nähe und Interesse an der ganzen Person bestehen. Folglich zeigt sich eine ausschliesslich distanzierte und spezifische Bearbeitung der sozialen Situation ebenfalls als anspruchsvoll und wird gleichermassen vermieden oder 〈umschifft). 
Weiter ist in der von Edith angesprochenen Kausalität - ganz neue Ziele [..] dann kann ich ja grad davon ein bisschen weggehen - eine Bestätigung der Abarbeitungslogik erkennbar. Dies lässt sich als «Traktandum X abgehakt, nun folgt das nächste Traktandum» lesen.

Beachtenswert ist die gewählte Formulierung ein bisschen weggehen. Ganz verlassen kann sie das Thema nicht oder noch nicht. Diese Formulierung weist eine Paradoxie auf, denn entweder man bleibt und verweilt oder man geht weg, ein bisschen weggehen ist wörtlich genommen nicht möglich. In der brüchigen Formulierung zeigt sich, dass Edith die Gesprächssituation durch die Bewegung «weitergehen und abarbeiten» bearbeitet. Dabei verzichtet sie auf Rekursivität. Auch sucht sie nach einer Begründung, die das Weitergehen legitimiert. Diese findet sie in der hergestellten Kausalität zwischen neue Ziele und ja grad davon weggehen zu können.

Im letzten Teil ihrer Aussage teilt Edith mit, dass sie einfach nochmals durch «Diagnose \& Beurteilung" gehe und verwendet erneut die Metapher des Gehens. Die Formulierung nochmals zeigt auf, dass das Thema bereits zu einem früheren Zeitpunkt angesprochen wurde. Im Modus einer Sorgfalt wird das Traktandum erneut aufgegriffen und erhält sowohl durch die abgekürzte Formulierung ja grad, als auch durch die Partikel einfach eine Leichtigkeit und Unverbindlichkeit. Eine naheliegende, kontextfreie Situation, in der der Sprechakt ausgesprochen werden könnte, wäre eine Person, die einfach nochmals, unverbindlich und ohne konkrete Erwartung, durch die Möbelausstellung schlendert. Konfrontiert mit dem realen Kontext zeigt Edith in der Aussage auf, einfach nochmals durch die Punkte "Diagnose \& Beurteilung» zu gehen, nicht als vertiefte Auseinandersetzung, sondern der Logik eines Rapports folgend. Somit wird eine Vollständigkeit und Verbindlichkeit bezüglich der Bearbeitung gewährleistet, da - metaphorisch gesprochen - alle Punkte durchgegangen oder gestreift werden.

Als Strukturmerkmal lässt sich folgender Interaktionsmodus festmachen: Die Mentorin ruft ein Thema, konkret ein Kompetenzfeld, auf und fordert die Studentin auf, sich diesbezüglich zu äussern. Nach erfolgter Auskunft wird das nächste Kompetenzfeld in analogem Modus bearbeitet. Es zeigt sich eine klare Übernahme der Gesprächsführung und Prozessverantwortung seitens der Mentorin. Dieser Prozess wird in der vorgefundenen Form pflichtgetreu und einer Vollständigkeitslogik folgend gestaltet. 
Salomé: ehm durch den Leistungsnachweis Fachdidaktik Deutsch ist das

bearbeitet worden ehm ich musste eine Diagnose machen und Arbeit schreiben

Der hier vorgefundene Sprechakt stellt eine erneute Bestätigung des bereits elaborierten Strukturmerkmals der Rechenschaftslegung dar. Die eingeforderte Vollständigkeitslogik wird auch von Salomé mitgetragen, indem sie in auffallend distanzierender Passivformulierung - ist das bearbeitet worden - aufzeigt, wie sie zu «Diagnose \& Beurteilung» gearbeitet hat. Hier reproduziert sich die zuvor aufgezeigte Interaktionslogik. Deshalb wird an dieser Stelle und unter Einbezug der Befunde aus der Eröffnungssequenz eine riskante Fallstrukturhypothese für den bisherigen Gesprächsverlauf im Mentoratsgespräch zwischen Edith und Salomé formuliert.

\subsubsection{Zusammenfassung und riskante Fallstrukturhypothese der Sequenz Also eigentlich sind jetzt alle Ziele abgearbeitet}

Die rekonstruierte Interaktion weist die Struktur einer Rechenschaftslegung auf, die der Logik der Bearbeitung institutioneller Verbindlichkeiten folgt. Die Mentorin fordert die Studentin durch das Aufrufen von Kompetenzfeldern - hier konkret «Planung \& Durchführung von Unterricht» wie auch «Diagnose \& Beurteilung» - zur Rechenschaftslegung auf. Die Studentin kommt der Aufforderung nach, bezieht Position und schreibt sich in den aufgerufenen Bereichen hinreichende Kompetenzen zu. Ihre Erfolgsmeldungen weisen eine schliessende Komponente auf und machen eine interaktive Nachfrage seitens der Mentorin unwahrscheinlich.

Die sichtbarwerdende ambivalente Dynamik, die in der Eröffnungssequenz mit einem «Tanz um ein unsichtbares Feuer` beschrieben wurde, ist weiterhin erkennbar. Einerseits lässt sich eine beidseitig distanzierende oder zumindest Diffusität und Nähe vermeidende oder (umschiffende` Dynamik feststellen. Es scheint, als würden diffuse Ausdehnungen und potenzielle Nähe eine Gefahrenzone bilden, die es zu umgehen gilt. Andererseits wird eine ausschliesslich spezifische und rollenförmige Begegnung zwischen der Studentin und der Mentorin ebenfalls vermieden, da sich auf eine eigentümliche Art und Weise auch diffuse Ansprüchlichkeiten im dyadischen Setting «Mentoratsgespräche» bemerkbar machen. In dieser Changierbewegung begegnen sich die Studentin und die Mentorin 
beidseitig im Modus des Sich-gegenseitig-in-Ruhe-Lassens und Sich-keine-ProblemeMachens. Es scheint als gäbe es etwas abzuarbeiten und möglichst schadlos hinter sich zu bringen. Dieser Abarbeitungsmodus wird von der Mentorin initiiert und von der Studentin mitgetragen. Die Rechenschaftslegung folgt einer Vorwärtsbewegung, die sich mit «immer weiter und ausschliesslich nach vorne» beschreiben lässt und ohne Rekursivität oder Anknüpfungspunkte auskommt.

Das zentrale Strukturmoment scheint das vollständige Abarbeiten der ihnen zugetragenen Ansprüche (Arbeit an den acht Kompetenzfeldern und den formulierten Zielen), denen sie im Modus einer Vertragserfüllung nachkommen. Weiter kann festgehalten werden, dass diese Vorgehensweise von beiden Akteurinnen gleichermassen aufgezeigt wird und die Studentin wie auch die Mentorin eine gemeinsame Form des Durchgehens gefunden haben. So greift die Mentorin als Prozessverantwortliche ein Kompetenzfeld oder ein Thema auf, die Studentin ihrerseits reagiert darauf und bezieht Stellung. Diese Stellungnahme wird von der Mentorin inhaltlich unkommentiert zur Kenntnis genommen und zum nächsten Kompetenzfeld fortgeschritten. Bei der Gesprächsstrukturierung oder, pointiert formuliert, beim 〈Füllen〉 der Gesprächszeit nimmt die Mentorin eine klare, rollenförmige Gesprächsführung und Prozesssteuerung vor und bleibt gleichzeitig inhaltlich ohne Bezugnahmen.

\subsubsection{Dritte Sequenz: Das hab ich auch der Praxislehrperson zu verdanken}

Im weiteren Gesprächsverlauf werden die verbleibenden Karten mit den Aufschriften "Institutionelles Handeln», "Schule \& Gesellschaft», "Lernen \& Entwicklung», "Sicherung der Qualität und professionelle Weiterentwicklung» und "Teilhabe am professionsspezifischen Fachdiskurs» analog zu zuvor von der Mentorin aufgerufen und die Studentin zeigt auf, wie sie diese Themen bearbeitet. Die nächste feinsequenzanalytische Rekonstruktion setzt bei der Aufrufung der nächsten Karte ein. 
Edith: gut Kommunikation \& Zusammenarbeit haben wir vorhin schon angeschaut mit dem Tandem mit deiner Tandempartnerin und jetzt mit Praxislehrpersonen gibt's noch irgendwas was wir jetzt nicht (.) genannt haben was dir noch auf der Seele liegt vielleicht (...)

Salomé: nein (.) nein

Edith: alles gut?

Salomé: $\mathrm{mhm}$ ((bejahend))

Edith: dann seh ich dass deine Perspektivplanung wirklich umfassend (.) abgehakt sein kann das sag ich immer ein bisschen ungern weil ich denke immer das ((unverständlich)) du hast gut dran gearbeitet intensiv dran gearbeitet

\subsubsection{Rekonstruktion der einzelnen Sequenzen}

Edith: gut Kommunikation \& Zusammenarbeit haben wir vorhin schon angeschaut mit dem Tandem mit deiner Tandempartnerin und jetzt mit Praxislehrpersonen

Wie bereits bei der zuvor aufgerufenen Karte wird auch hier mit einem schliessenden gut der vorausgehende Sprechakt abgeschlossen. Gut nimmt an dieser Stelle die Bedeutung einer Zäsur ein. In Analogie zu «so weit so gut» ist dies im Sinne eines Konklusionsmarkers zu verstehen und kündigt einen neuen Sinnabschnitt an. Dem daran anschliessenden Sprechakt kommt die Bedeutung einer Information oder Kenntnisnahme zu, aus Sicht der Sprecherin wurde das entsprechende Thema bereits in mehreren Konstellationen verhandelt und gilt als «erledigt». Der aufgerufene Punkt «Kommunikation \& Zusammenarbeit» wird formal eröffnet und umgehend wieder geschlossen. Hierbei zeigt sich eine Befugnis der Sprecherin auf, dies in Eigenregie vorzunehmen. Es ist eine Vorwärtsdynamik ersichtlich, wodurch sich das bereits elaborierte Strukturmerkmal des Abarbeitungsmodus - immer weiter und ausschliesslich nach vorne - reproduziert. 
Edith: gibt's noch irgendwas was wir jetzt nicht (.) genannt haben was dir noch auf der Seele liegt vielleicht (...)

Im Anschluss führt Edith einen unvermittelten Übergang aus. Sie leitet vom Modus der Rechenschaftslegung und Abarbeitung zum Modus der Seelsorge und Fürsorglichkeit über und erteilt Salomé die Möglichkeit, noch etwas Entsprechendes anzufügen. Dabei spricht sie von wir im Sinne einer Gesinnungsgemeinschaft und stellt somit, bezogen auf den durchlaufenen Abarbeitungsprozess, ein Gemeinschaftsgefühl her.

An dieser Stelle gilt es zu klären, welche soziale Bedeutung dem unerwarteten Übergang von der universalistischen Abarbeitung und Rechenschaftslegung zur partikularen Nachfrage nach seelischen Belastungsmomenten zukommt. Auf den ersten Blick scheint die Frage ein Näheangebot darzustellen, die fragende Person zeigt sich interessiert, zu erfahren was die angefragte Person belastet. Allerdings wäre bei zwei sich nahestehenden Personen eine solche Frage nicht vorstellbar. Obschon manifest eine gewisse Nähe und ein Interesse an der ganzen Person suggeriert wird, scheint auf einer latenten Bedeutungsebene gegenteilig die Distanz durch, welche die ‘floskelhafte` Fragestellung beinhaltet. Anders formuliert zeigt die formale Frage eine Distanz auf, die verringert oder zumindest bearbeitet wird, ohne dadurch Nähe und Diffusität zuzulassen. Weiter bezieht sich die Frage lediglich auf was wir jetzt nicht (.) genannt haben. Der Modus der Abarbeitung mit dem Ziel der Vollständigkeit haftet dem Sprechakt weiterhin an.

Mit dem Sprechakt wird aufgezeigt, dass das Kompetenzfeld «Kommunikation \& Zusammenarbeit» formal als erledigt gilt. Das nächste Traktandum steht zur Bearbeitung an, falls Salomé Edith grünes Licht erteilt und das aktuelle abgeschlossen werden kann. Die folgende Gesprächspassagen wird an gewissen Passagen feinsequenziell, vorwiegend jedoch summarisch interpretiert, bevor anschliessend die bereits erstellte, riskante Fallstrukturhypothese weiter ausformuliert und ergänzt wird.

Salomé: nein (.) nein

Edith: alles gut?

Salomé: $\mathrm{mhm}$ ((bejahend)) 
Edith: dann seh ich dass deine Perspektivplanung wirklich umfassend (.) abgehakt sein kann das sag ich immer ein bisschen ungern weil ich denke immer das ((unverständlich)) du hast gut dran gearbeitet intensiv dran gearbeitet

Die doppelte Verneinung Salomés wird durch eine Pause unterbrochen, was sich als Hinweis für ein minimales Zögern oder sich nochmals Vergegenwärtigen lesen lässt. Möglicherweise fragt Edith deshalb nochmals nach und fährt erst nach einer erneuten Bestätigung fort.

Kontextfrei weist der Satzanfang dann seh ich dass eine strukturierende Funktion auf. Im Modus eines Metakommentars wird eine Schlussfolgerung eingeleitet. Die Sprecherin positioniert sich als Entscheidungsträgerin, die eine solche Zäsur bezogen auf den Aushandlungsgegenstand vornimmt. Gleichzeitig ist auch eine Bewegung im Sprechakt erkennbar, die sich erneut als Erleichterung deuten lässt. Etwas als abgehakt sehen zu können und nicht weiter verhandeln zu müssen scheint eine entlastende Implikation aufzuweisen. Perspektivplanung ist ein Terminus technicus der PH FHNW, so wird ein Formular betitelt, in dem die Ziele für die nächste Praktikumsphase festgelegt werden. Der Begriff Perspektivplanung als Code für dieses Formular, hat sich in der Zusammenarbeit von Studierenden und Hochschulpersonal etabliert. Die Ambivalenz, die sich in die Wortschöpfung einschreibt - Perspektiven können nicht geplant werden - , wird nicht weiterverfolgt. Diese weist auf eine strukturelle Spannung und Fragilität im Ausbildungssetting hin, betrifft jedoch nicht die eigentliche Interaktionsdynamik des Gesprächs.

In sich brüchig ist auch die Formulierung umfassend abgehakt sein kann. Abgehakt als Begriff folgt der Logik «trifft zu - abgehakt, der Haken ist gesetzt» oder «trifft nicht zu, der Haken ist (noch) nicht gesetzt und fehlt». Eine Steigerung von abgehakt zu umfassend abgehakt ist irritierend. Umfassend enthält ein evaluatives Moment und folgt der Abarbeitungslogik. Das Ziel wurde umfassend erreicht und kann abgehakt werden. Die vorgefundene Formulierung scheint sich in der Ambivalenz zwischen einem lenkenden und einem entwicklungsoffenen Umgang zu verfangen. Dem lenkenden Aspekt kommt die Bedeutung von «lass uns das Thema abhaken und weitergehen» zu. Diese klare Lenkung bedarf eines Konsenses, der hier im übertragenen Sinne von «Siehst du das auch so?» eingeholt wird und ein entwicklungsoffenes Element enthält. 
Es ist davon auszugehen, dass sich ein bisschen ungern sagen auf abhaken bezieht, eine andere Option erschliesst sich aus dem Kontext nicht. Edith bearbeitet mit ihrem Begleitkommentar den latenten Widerspruch, der in «Perspektivplanung abhaken» steckt. Durch die Anmerkung das sag ich immer ein bisschen ungern scheint durch, dass abhaken als Vorgang im Zusammenhang mit Perspektivplanung etwas bei ihr auslöst, das sie als unangenehm erlebt. Dennoch verwendet sie das Verb abhaken und hebt kurzfristig die eigens etablierte Gesprächsgestaltung (Kompetenzfelder abhaken) von der latenten auf die manifeste Ebene.

In der Fortsetzung der Aussage lobt Edith Salomé für ihre Arbeitsweise. Das Lob erhält durch die Betonung der Adjektive gut und intensiv, vor allem durch die repetitive Satzstruktur dran gearbeitet, eine spezifische und formelle Dynamik. Der Sprechakt wirkt distanzierend und beinahe andächtig. Die Position und Macht der lobenden Person und entsprechend die Position der für ihre Arbeitsweise belobten Person wird unmissverständlich eingenommen respektive zugewiesen. Während die Aussage das sag ich immer ein bisschen ungern etwas über die sprechende Person in ihrer Ganzheit sagt, wird dies umgehend wieder durch die klare Einnahme der Rolle als Lob-Aussprechende spezifiziert und eine mögliche Diffusität «entschärft). Da sich das Changieren auf dem Spannungsbogen von Diffusität und Spezifität wiederholt, wird die bereits etablierte riskante Fallstrukturhypothese geschärft:

\subsubsection{Zusammenfassung und riskante Fallstrukturhypothese der Sequenz Das hab ich auch der Praxislehrperson zu verdanken}

Verdichtet ist auch in dieser Sequenz eine Dynamik zu erkennen, in der sich die Grundfigur der Abarbeitung, Rechenschaftslegung und Vorwärtsbewegung reproduziert. Analog reproduzieren sich die klare Agendasetzung durch die Mentorin, wie auch die Logik der Bearbeitung von Erwartungen an die Studentin und daran anschliessend die Ratifizierung der Berichterstattung. Auffällig ist dabei die routinierte und einvernehmliche Art der Beoder Abarbeitung. Die einzelnen Kompetenzfelder aufzurufen und abzuarbeiten, selbst ohne inhaltliche Durchdringung, scheint für die Mentorin wie auch für die Studentin eine Passung aufzuweisen. 
Typologisch zeigt sich hier ein Modus, mit der Spannung von Diffusität und Spezifität, oder wie das Oevermann für das therapeutische Arbeitsbündnis formuliert, mit der «widersprüchlichen Einheit» (Oevermann, 1996b, S. 119) von Handeln als ganze Person und von Rollenhandeln, umzugehen, der beide Pole gleichzeitig bedient und auch umgehend wieder entkräftet. So gewinnen Ansprüchlichkeiten auf Diffusitätselemente Gestalt, als gelte es ein Zuviel an Spezifität und Rollenförmigkeit im Gespräch zu vermeiden. Zeitgleich werden diese Diffusitätsbemühungen, wie beispielsweise Ansprüchlichkeiten auf sichtbarwerdende Zugewandtheit und Fürsorge, wieder mit der antinomischen Gegenbewegung in Richtung Spezifität bearbeitet. Anders formuliert lassen die latent im Raum stehenden Ansprüchlichkeiten eine ausschliesslich spezifische Bearbeitung nicht zu. Beide Pole scheinen je auch eine Riskanz zu beinhalten, weshalb sie potenziell vermieden oder eben «umschifft» werden.

Diese Gleichzeitigkeit, die sich nicht in eine Richtung auflösen lässt, manifestiert sich empirisch auch auf dem Spannungsbogen von Nähe und Distanz. Auch hier zeigen sich Parallelitäten zur Näheantinomie, die Helsper (z.B. 2004, S. 77) beschrieben hat. Näheund Diffusitätselemente lassen sich beispielsweise in der Aussage gibt's noch irgendwas was wir jetzt nicht (.) genannt haben was dir noch auf der Seele liegt vielleicht (...) finden, als gelte es Nähe zur und Interesse an der ganzen Person zu signalisieren. Dieses Näheangebot wird jedoch umgehend und gleichzeitig durch eine Distanzierungs- und Spezifizierungsbewegung wieder ausgehebelt, da es lediglich und spezifisch nur um das, was «sie» jetzt nicht (.) genannt haben, geht.

Insgesamt lässt sich festhalten, dass sich das Gesprächsverhältnis zwischen der Studentin und der Mentorin im Ton verbindlich, freundlich, wohlwollend und in der Sache unmissverständlich gestaltet. Die Konstellation Studentin und Mentorin zeigt sich als eingespielt, vor allem bezogen auf das Sich-in-Ruhe-Lassen und ebenfalls bezogen auf die distanzierenden Bezugnahmen oder Schliessbewegungen. Diese werden beidseitig vorgenommen, was sich beispielsweise bei der Frage, ob es noch etwas gäbe, dass Salomé auf der Seele liege und daran anschliessend bei Salomés Antwort nein (.) nein zeigt.

Die sich reproduzierenden Strukturmerkmale lassen folgende Fallstrukturhypothese zu: Gewisse Ansprüchlichkeiten auf Nähe und Diffusität stehen im Raum, die eine ausschliesslich spezifische und distanzierte Interaktion bei der Abarbeitung der Agendapunkte respektive der Kompetenzfelder nicht zulassen. Diese Diffusitätsansprüchlich- 
keiten rufen Dynamiken des Vermeidens oder (Umschiffens) hervor, die beidseitig eingespielt bearbeitet werden.

\subsubsection{Vierte Sequenz: Was kannst du selber dafür tun}

Die Karten mit den aufnotierten Kompetenzfeldern sind nun allesamt vollständig abgearbeitet. Im weiteren Gesprächsverlauf wird über den Praktikumsausfall von Salomé infolge einer hartnäckigen Erkältung gesprochen. Für Salomé war der Ausfall misslich, allerdings sieht sie darin auch etwas Positives. So erhielt ihre Kommilitonin die Möglichkeit, alleine zu unterrichten.

An dieser Stelle setzt die sequenzanalytische Rekonstruktion einer längeren Sequenz ein.

Diese wird in Teilen flächig und in anderen Teilen feinsequenziell rekonstruiert:

Edith: jede Situation hat ihre Schattenseiten und ihre Sonnenseiten (..) ehm Perspektive nach vorne wenn du jetzt mal anschaust deinen gesamten Professionsweg seit dem $P 1^{59}$ seit dem ersten Semester bis heute und jetzt mal überlegst und jetzt hast du das dritte Studienjahr und da ist das P4 noch vor dir was würdest du jetzt sagen könnte jetzt noch passieren was du für's Portfolio ergänzt oder vielleicht noch ne Vertiefung wo du sagst ah da würd ich gern nochmal hingucken aus diesen und diesen Gründen also wenn wir jetzt ein Wunschkonzert aufmachen könnten was was würdest du dir wünschen? Salome: also was ich was ich noch sehen will quasi oder machen will Edith: $m h m$ ((bejahend)) im P4 genau

Salome: also sicher noch mehr Elternarbeit weil Elternarbeit kommt einfach so viel zu kurz in der ganzen Ausbildung wir haben jetzt ein Seminar in EW Pädagogik altersgemischte Schulsettings dort geht es ein bisschen um Elternarbeit aber auch nicht der ganze Kurs das waren glaub zweimal von achtmal also ehm ich finde einfach das ist etwas das in der ganzen Ausbildung so fest fehlt

\footnotetext{
${ }^{59}$ P1 steht für das erste Praktikum. Zwischen dem ersten und zweiten Semester absolvieren die Studierenden ein erstes 3-wöchiges Praktikum in einem Kindergarten oder einer Schulklasse der ersten drei Primarschuljahre.
} 
Edith: wa::s kannst du selbst dafür tun um //das//

Salome: //Praktikum// nachfragen ob ich zuschauen darf

Edith: also mir würde noch eine andere Option einfallen

Salome: in einem Reflex

Edith: es gibt ehm die Notwendigkeit eine Bachelorarbeit zu schreiben

Salome: klar ja ehm auf jeden Fall

Edith: und in einer Bachelorarbeit könnte man ja auch so vorgehen und sagen das ist jetzt ein Bereich wo du ein Defizit oder ein Loch oder wo sich ne Lücke auftut und dann könntest du dich ja auch interessengeleitet das ist ja offensichtlich ein Interesse von dir da hinsetzen und sagen gut du erarbeitest jetzt mal das Thema Elternarbeit (.) ehm (.) über die Bachelorarbeit noch zusätzlich Salomé: ja ich bin jetzt eigentlich dort schon eher auf dem gestalterischen Weg ((lacht)) ehm aber (.) vielleicht kann man das ja auch einbauen

Edith: vielleicht kann man's kombinieren?

Salomé: auf jeden Fall mhm ((bejahend)) es ist ja nicht so dass dass es dass ich finde ich hätte gar keine Ahnung aber es ist einfach so (.) ich meine wenn ich überleg wenn ich fertig bin bin ich 23 und ich stehe vor diesen Vätern und ich sie grüezi ich bin die neue Lehrperson von Ihrem Kind ((lacht))

Edith: und der Vater ja der ist unter Umständen vierzig ((lacht)) wunderbar ((lacht))

Salomé: und das ist irgendwie so ein bisschen (.) wir haben einfach (.) ich fände es ehm noch cool wenn es irgendwie ein Seminar gäbe oder keine Ahnung ein Semester von mir aus wo wo einfach (.) wie führe ich einen Elternabend durch wie leite ich ein Elterngespräch wie spreche ich mit den Eltern ohne dass ich das Kind schlecht mache oder auf die Wolke hinaus hebe oder so und und ich finde das das ist so etwas da werden wir einfach darauf losgelassen und das ist einfach auch ein wichtiger Teil unserer Arbeit Edith: $m h m$ ((bejahend)) ja da musst du jetzt aufpassen du darfst jetzt nicht deine Erfahrung verallgemeinern weil andere Studierende haben (.) vielleicht zwei drei Elterngespräche ein mit und ein ohne Kinder oder so was erlebt oder waren schon an Elternabenden mit dabei ehm offensichtlich ist dir das noch nicht begegnet 
Salomé: ja oder nicht genügend so dass ich es später selber machen könnte (.) also wenn ich mir jetzt überlege ich müsste jetzt (.) also wenn ich jetzt vor einer Klasse stehen würde und ich müsste unterrichten wäre das für mich kein Problem (..) aber wenn ich jetzt ein Elterngespräch führen müsste alleine dann wäre das für mich ein grosses Problem (.) jetzt (.) also so Edith: das ist also jetzt eine Perspektive für's nächste Studienjahr oder die nächsten zwei Semester was gibt es noch für (.) Anliegen die du jetzt mit der Perspektive nach vorne verbindest?

\subsubsection{Rekonstruktion der einzelnen Sequenzen}

Edith: jede Situation hat ihre Schattenseiten und ihre Sonnenseiten (..)

Auf den ersten Blick und kontextungebunden erinnert die Aussage an einen Kalenderspruch, der eine universale Gültigkeit beansprucht und als Metapher auch etwas (Plattitüdenhaftes) aufweist. Bei genauerer Betrachtung allerdings fällt auf, dass bei einem Kalenderspruch die Sonnenseiten an erster Stelle erwähnt würden. Die Aussage hiesse eher «jede Situation hat ihre Sonnen- und Schattenseiten». Die vorgefundene primäre Erwähnung des Negativen ist kontra-intuitiv. Denkbar wäre, dass es sich beim Sprechakt um einen empathischen, tröstlichen Kommentar zu einer erläuterten Gegebenheit handelt. In diesem Falle wäre allerdings ein sprechaktlogischer Zusatz mit einer persönlichen Bezugnahme wie beispielsweise ein «na ja» oder «weisst du» im Sinne eines ¿Geschmeidigmachers` erwartbar. In der realisierten Variante scheint demzufolge nicht das Empathische und auch nicht die metaphorische Aussage, sondern die Ankündigung eines Themenwechsels in der Bedeutung von «lass uns weitermachen und vorwärtsschreiten» im Vordergrund zu stehen. Die Dynamik der Aussage lässt die Vermutung zu, dass die Sprecherin im Begriffe ist, etwas abzuschliessen und nicht weiter darauf einzugehen und dabei eine sozialverträgliche Formulierungsform verwendet. Mit Blick auf die soziale Situation der Interaktion wird das Spezifische oder Konkrete negiert oder weggespielt, was einer Distanzierungsbewegung und Schliessfunktion gleichkommt. 
Unter Zuzug des Kontextes lässt sich der Sprechakt als Reaktion auf Salomés Ausführung bezogen auf das alleinige Unterrichten ihrer Kommilitonin lesen. Diese Ausführungen schliesst Edith mit ihrem Sprechakt ab.

Edith: ehm Perspektive nach vorne

Ehm scheint den Übergangsmarkierer zwischen der vorherigen Aussage und der hier folgenden darzustellen. Eine Perspektive benennt einen Standpunkt, von dem aus etwas gesehen wird. Im alltagssprachlichen Gebrauch richtet sich eine Perspektive häufig in die Zukunft. In der Doppelung Perspektive nach vorne schleicht sich ein, dass das zuvor Verhandelte unmissverständlich hinter sich gelassen wird.

Unter Zuzug der vorherigen Aussage der Schattenseiten und Sonnenseiten wird die Lenkungsbewegung und der pragmatische Abschluss des zuvor verhandelten Themas noch deutlicher erkennbar.

Edith: wenn du jetzt mal anschaust deinen gesamten Professionsweg seit dem P1 seit dem ersten Semester bis heute

Der Sprechakt lässt sich als Einstimmung auf etwas, hier konkret auf einen Rückblick, lesen. Die Einstimmung wird kleinschrittig vorgetragen und dabei mehrere Anhaltspunkte aufgerufen. Die Sprecherin teilt ihre Aussage in vier Fragmente auf - wenn du jetzt mal anschaust - deinen gesamten Professionsweg - seit dem P1 - seit dem ersten Semester bis heute. Die vorgenommene Sequenzierung ist ausschliesslich in die Vergangenheit gerichtet, was in einem logischen Widerspruch zur Ankündigung Perspektive nach vorne steht. Dieses Faktum bestärkt erneut die Vermutung, dass der gesuchte Abschluss das dominante Strukturmerkmal des vorausgehenden Sprechaktes darstellt.

Im Sprechakt wird ein fürsorgliches an die Hand nehmen mithilfe einer Gedankenreise anmoderiert. Diese wird in der nächsten Sequenz weiter ausgeführt und anschliessend als Einheit rekonstruiert. 
Edith: und jetzt mal überlegst und jetzt hast du das dritte Studienjahr und da ist das P4 noch vor dir was würdest du jetzt sagen könnte jetzt noch passieren was du für's Portfolio ergänzt oder vielleicht noch ne Vertiefung wo du sagst ah da würd ich gern nochmal hingucken aus diesen und diesen Gründen also wenn wir jetzt ein Wunschkonzert aufmachen könnten was was würdest du dir wünschen?

Edith leitet mit dem angewendeten didaktischen Tool einen Denkprozess ein. Mit der Formulierung aus diesen und diesen Gründen wird klar, dass die Vertiefung manifest einer Begründung bedarf. Auf einer latenten Ebene erhält diese durch die vorgefundene 〈Platzhalterformulierung〉 auch etwas Willkürliches. Spricht jemand von diesen und diesen Gründen, werden die Gründe nicht expliziert, sondern nur als Aufzählung erwähnt. Im Gesprächskontext scheint hierbei durch, dass Edith zwar eine Begründung einfordert, diese aber für sie kaum eine Bedeutung aufweist.

Als gedankenexperimenteller Kontrast könnte Edith Salomé fragen, wie sie sich zu ihrem letzten Studienjahr stelle, welche Themen bei ihr noch anstehen und sie vertiefen möchte. Sie allerdings zieht die Situationsdramaturgie interaktiv hoch, spricht von einem Wunschkonzert, das sie beide aufmachen können. Die Metapher ein Wunschkonzert aufmachen ist bemerkenswert, da die mit Wunschkonzert geläufige Redewendung gewöhnlich ex Negativum formuliert wird und meist «das Leben ist kein Wunschkonzert» oder ähnlich lautet. Auch sonst ist die Formulierung Wunschkonzert aufmachen ungewohnt, viel eher lassen sich «Wundertüten aufmachen» oder «Wunschkonzerte veranstalten». Mit der vorgefundenen Formulierung erhält Frage nach den noch anstehenden Themen ein theatralisches, inszeniertes Moment und kann sich von der gegenteiligen Bewegung: «das Leben - oder hier die Ausbildung - ist kein Wunschkonzert» nicht ganz lösen. Dies lässt sich als Hinweis auf eine weitere Spannung lesen: einerseits wird explizit nach Wünschen gefragt, andererseits scheint es nicht primär um deren Einlösung oder Entsprechung zu gehen. Eine ähnliche Spannung ist auch in der Formulierung aus diesen und diesen Gründen zu erkennen: einerseits wird eine Begründung verlangt, andererseits scheinen diese Gründe wenig Bedeutung aufzuweisen. Die Frage nach dem Wunschkonzert enthält dadurch auch eine ritualisierte Implikation. Der Frage kommt mehr Bedeutung zu als der Antwort, die auf die Frage folgt. 
Bis dato hat Edith das Mentoratsgespräch weitgehend durch Aufrufung von Agendapunkten gestaltet, zu denen sich Salomé zu äussern hat. An dieser Sequenzstelle ändert sich der Interaktionsmodus. Edith verlässt die Abarbeitungskonstitution und moderiert eine Gedankenreise an. Das Tool der Gedankenreise, die mit dem Wunschkonzert ausgeführt wird, bewegt sich erneut auf dem Spannungsbogen von Nähe und Distanz. Durch das wir als Vergemeinschaftungsangebot wird eine Bewegung in Richtung Nähe aufgezeigt, die gleichzeitig auch nicht zugelassen oder sogar vermieden wird. Diese Vermeidung lässt sich daran erkennen, dass die Frage in einer diffusen und nahen Konstellation nicht vorstellbar wäre, würde man doch eine gute Freundin nichts Entsprechendes fragen. Dennoch zeigt sich eine Dynamik, die sich als Annäherung und aufgezeigtem Interesse an der ganzen Person beschreiben lässt.

Edith spricht, wie Salomé denken könnte, formuliert ihr also gewissermassen die Sätze vor. Dies allerdings erscheint für die Inszenierung einer Gedankenreise nicht ungewöhnlich.

Abschliessend ist festzuhalten, dass an dieser Stelle die Rechenschaftslegung durch Elemente zur Initiierung von Denkprozessen ergänzt wird. Mit der vorgefundenen Dynamik erhält die in den vorausgehenden Sequenzstellen relativ stabil im Abarbeitungsmodus aufgebaute Gesprächsrahmung eine grundlegende Modifikation. Die Modifikation betrifft allerdings nicht die Bearbeitung des Spannungsfeldes von Nähe und Distanz respektive von Diffusität und Spezifität, da diese Pole weiterhin wechselseitig bedient und zeitgleich auch vermieden werden.

Salome: also was ich was ich noch sehen will quasi oder machen will

Salomés Anschluss ist eine Rückfrage im Sinne einer Rückversicherung. Diese Rückfrage zeigt, dass die von Edith vorgenommene Modifikation des Gesprächsmodus für Salomé eine Irritation darstellt. Salomé ersetzt das in Ediths Fragestimulus verwendete hingucken durch sehen. Sie steigt weder auf die Gedankenreise noch auf das Wunschkonzert ein, sondern formuliert ihre Rückfrage allgemein gehalten. Salomés Sprechakt weist eine ReUniversalisierungsbewegung als Reaktion auf das irritierende Moment der Modifikation in Ediths Gesprächsmodus auf. Es ist, als würde das Näheangebot von Edith mit einem Impuls in Richtung Distanz beantwortet. 
Edith: $m h m$ ((bejahend)) im P4 genau

Salome: also sicher noch mehr Elternarbeit weil Elternarbeit kommt einfach so viel zu kurz in der ganzen Ausbildung wir haben jetzt ein Seminar in EW 60

Pädagogik altersgemischte Schulsettings dort geht es ein bisschen um

Elternarbeit aber auch nicht der ganze Kurs das waren glaub zweimal von achtmal also ehm ich finde einfach das ist etwas das in der ganzen Ausbildung so fest ${ }^{61}$ fehlt

Edith beharrt nicht auf ihrer Inszenierung der Gedankenreise, sondern ratifiziert implizit Salomés Re-Universalisierung. Salomés formuliert in ihrer Fortsetzung, was sie sich noch wünschen würde. Sie startet ihre Aussage mit also sicher noch mehr und zeigt die Offensichtlichkeit ihres Wunsches auf. Auch leitet sie mit dieser Formulierung eine Begründung ein und unterstreicht ihr Anliegen mit betont ausgesprochenen so und ganz, die ihre Begründung zusätzlich untermauern. Salomé beschreibt ein institutionelles Anliegen und kein persönliches Problem. Sie schildert ihre Erwartungen an die Pädagogische Hochschule, umfassend ‘fit` für ihren künftigen Beruf gemacht zu werden und identifiziert diesbezüglich ein Versäumnis, das sie im Modus einer (An-)Klage gegenüber der Institution formuliert. Das Ausbildungsdefizit schreibt sie dem Curriculum der Hochschule zu und vermeidet dabei dezidiert, Edith als Vertreterin der Hochschule in einer Mitverantwortung für dieses Versäumnisses zu adressieren.

Edith könnte öffnend auf Salomés Ausführungen reagieren und diese Einschätzung bestätigen oder auch zurückweisen, beispielsweise mit «lch höre das immer wieder von Studierendenseite, weiss aber von Dozierenden, dass sie sich in ihren Modulen sehr wohl der Thematik widmen». Ihr Anschluss würde das Anliegen aufgreifen und Salomé auffordern, die Einschätzungsdiskrepanz zu bearbeiten. Analog ist eine schliessende Bewegung vorstellbar, in der Edith Salomé keinen Anknüpfungspunkt liefert. Sie könnte sagen: «Elternarbeit, ein Dauerthema, höre ich oft von Studierenden, gibt es noch andere?» Dies wäre eine klare Signalisierung, dass Edith die Problematik erkennt und

\footnotetext{
${ }^{60}$ EW ist die von den Studierenden häufig verwendete Kurzform von Erziehungswissenschaften.

${ }^{61}$ Im Schweizerdeutschen wird im mündlichen Sprachgebrauch häufig fest anstelle von «sehr» verwendet.
} 
kennt, dennoch mit einer Wegbewegung aufzeigt, dass sie Salomé keinen Raum für die Weiterbearbeitung des Anliegens bietet.

Edith: wa::s kannst du selbst dafür tun um //das//

Im Anschluss bezieht sich Edith auf das von Salomé identifizierte Ausbildungsdefizit und spielt ihr den Ball retour. Sie schreibt die Verantwortung für die Bearbeitung des Defizits Salomé zu und modifiziert es damit zu ihrer individuellen Aufgabe. Ihre Frage folgt dem Imperativ «Hilf dir selbst und sag mir, wie du das zu tun gedenkst!» Sie adressiert Salomé mit der Aufforderung zum selbständigen Denken und zur Übernahme von Eigenverantwortung und führt dabei eine Selbstpositionierung als Nicht-Zuständige aus. Dies kann als Vorwärtsverteidigung betrachtet werden, um Versäumnisse - eigene wie auch institutionelle - nicht diskutieren zu müssen. Denn, indem die Studentin auf ihre Eigenverantwortung verwiesen wird, kann sich die Mentorin gleichzeitig gegenüber den Ansprüchen der Studentin entlasten. Diese Figur erinnert an die «instrumentelle Autonomie» oder auch an die «entlastende Autonomie» zwischen Lehrenden und Lernenden, wie sie von Helsper (2002, S. 90) beschrieben wurde. Die Figur zeigt eine "paradoxe Form einer Simulation von Autonomie» wie auch eine «Entlastung durch Autonomiezuschreibung» auf, wenn Lehrende zwischen Förderung von Autonomie und Überforderung durch Autonomie ausbalancieren müssen (Helsper, 2002, S. 90). Eine gewisse Parallele zu eben diesen Autonomien scheint zulässig, da die Art und Weise, wie Salomé hier adressiert wird, an ein klassisches Lehrerin-Schülerin-Setting erinnert. Wäre die Frage, als kontrastierendes Gedankenexperiment, ohne dafür formuliert und das Fragepronomen wa::s nicht in die Länge gezogen, könnte sie als «echte` Frage verstanden werden. Wie hier vorgefunden, weist die Frage nebst einer pädagogischen und mäeutisch-sokratischen Implikation diesen entlastenden Autonomie-Bias auf, eine gesuchte und in diesem Sinne «richtiges Antwort hervorzulocken.

Zusammenfassend lässt sich sagen, dass Edith nach der Pflichtabarbeitung der Kompetenzbereiche Salomé ermöglicht noch eigene Themen zu positionieren. Salomé nutzt diese Option und weist auf ein Ausbildungsdefizit im Bereich Elternarbeit hin. Edith reagiert auf Salomés Anliegen mit einer Rückfrage und spielt ihr dabei den Ball umgehend retour. Sie weist eine mögliche Zuständigkeit seitens der Hochschule dezidiert zurück. Die 
Verantwortungszuschreibung an die Studentin wird im Sprechakt zum zentralen Strukturelement.

Salome: //Praktikum// nachfragen ob ich zuschauen darf

Salomé geht davon aus, die gesuchte Antwort zu kennen und fällt Edith ins Wort. Ihre Antwort Praktikum formuliert sie ohne Artikel oder Präposition. Dies lässt sich als Eifer lesen, umgehend zu antworten und sich als Wissende zu zeigen. Die daran anschliessenden Ausführungen entsprechen der von Salomé im bisherigen Gesprächsverlauf dominant gezeigten Priorisierung der Praxis. Für die Gesprächsdynamik ist diese Priorisierung sekundär, da sie nicht die Gesprächsinteraktion betrifft. Deshalb wird nicht detailliert darauf eingegangen.

Edith: also mir würde noch eine andere Option einfallen

Um die Interaktionsdynamik des Sprechaktes herauszuarbeiten, wird der vorgefundene Sprechakt mit einer Version ohne würde-Formulierung kontrastiert. «Also mir fällt noch eine andere Option ein» wäre in einer Situation vorstellbar, wenn gemeinsam nach Optionen gesucht wird, einige bereits genannt wurden und der Sprecherin gerade noch eine weitere einfällt. Diese zusätzliche Option scheint eine weitere unter ihresgleichen zu sein. Anders lässt sich der Sprechakt lesen, wenn er - wie vorgefunden - mit würde formuliert wird. Hier scheint er die Bedeutung einzunehmen, die sich mit «also wenn ich an deiner Stelle wäre, würde mir noch eine andere Option einfallen» paraphrasieren lässt. Dabei wird die vorausgehende Antwort von Salomé weder als richtig noch als falsch taxiert. Salomé erfährt, dass die Antwort eine mögliche, nicht aber die gesuchte darstellt. Die Formulierung im Präsens suggeriert auf einer manifesten Ebene, dass Edith diese Option gerade während des Sprechens einfällt oder eben einfallen würde, wäre sie an Salomés Stelle. Latent scheint jedoch durch, dass es sich nicht um ein gemeinsames Suchen handelt, sondern Salomé eine zweite Chance für die richtige Antwort erhält. Mit dieser Implikation erinnert die Gesprächssequenz erneut an die mäeutisch-sokratische Fragetechnik. In der Andeutung, dass es noch eine andere Option gäbe, wird die Fragetechnik sehr offensichtlich eingesetzt. Ediths Sprechakt enthält noch eine weitere Note 
und könnte in der Langversion mit «es gibt da noch eine andere Option, auf die du anscheinend nicht kommst» umschrieben werden. Hierbei ist auch eine leichte Implikation einer (Retourkutsche` auf die von Salomé ausgeübte Kritik betreffend des Ausbildungsdefizites zu erkennen.

Salome: in einem Reflex ${ }^{62}$

Edith: es gibt ehm die Notwendigkeit eine Bachelorarbeit zu schreiben

Salomé ergreift die zweite, ihr zugespielte, Chance und versucht sich ohne Zögern mit einer weiteren Antwortoption. Mit Ediths definitorischen Anschluss, den sie mit es gibt einleitet, findet das mäeutisch-sokratische Fragen keine Fortsetzung, sondern das 〈versteckte Osterei ` ${ }^{63}$ wird aufgedeckt. Der zuvor aufgezogene Spannungsbogen lässt sich klären: die gesuchte Antwort respektive was ihr noch einfallen würde, ist die Notwendigkeit eine Bachelorarbeit zu schreiben.

In Ediths Aussage gewinnt eine weitere Implikation Gestalt, als Salomé lediglich auf die Notwendigkeit der Bachelorarbeit hinzuweisen. Vielmehr lässt sich erneut ein Datum der Verantwortungszuschreibung an Salomé finden. Der bisher bemerkenswert harmonische und einvernehmliche gegenseitige Umgang gerät ins Wanken. Denn es zeigt sich erstmalig eine inhaltliche Differenz zwischen Salomé und Edith. Für Salomé wäre das von ihr auf Nachfrage hin identifizierte Defizit primär durch Vermittlung der Hochschule oder ansonsten durch Beobachtung der Praxislehrperson zu bearbeiten. Edith hingegen schlägt eine eigenverantwortliche, theoriebasierte und wissenschaftliche Bearbeitung vor.

Aus der Gesprächsbewegung heraus markant ist, dass Edith Salomé an dieser Stelle nicht direkt anspricht, sondern mit es gibt eine indirekte Formulierung wählt. Um die Dynamik dieser indirekten Ansprache herauszuarbeiten, wird erneut auf das Mittel des gedankenexperimentellen Kontrastes zurückgegriffen. Edith könnte Salomé wie folgt ansprechen: «Du schreibst ja im letzten Studienjahr eine Bachelorarbeit, wäre es für dich allenfalls eine Option.....». Sie hingegen spricht universell von einer vorhandenen Notwendigkeit und

\footnotetext{
62 Reflex ist eine gängige studentische Abkürzung für Reflexionsseminar, ein Begleitformat der Pädagogischen Hochschule für die einzelnen Praxisphasen, das sich zwischen der Wissenschaftspraxis der Hochschule und des Berufsfeldes positioniert.

63 Die Metapher des «versteckten Ostereis` wird im ersten Mentoratsgespräch zwischen Tina und Michael ausgeführt.
} 
hält dadurch das dyadische Element minimal und nimmt sich selbst als Person vollumfänglich heraus. Dies lässt sich als weiterer Hinweis für einen schliessenden und distanzierenden Bearbeitungsmodus lesen. Zudem markiert Edith damit auch eine asymmetrische Dimension zwischen ihr und Salomé und verweist sie in den Rang der noch nicht fertig ausgebildeten Studentin, der noch das Schreiben einer Bachelorarbeit bevorsteht. Hier scheint sich eine Reproduktion des Elements eines «subtilen Retourkutschenmomentes` zu zeigen. Das von Salomé identifizierte Ausbildungsdefizit wird von Edith mit dem Defizit Salomés, noch keine Bachelorarbeit geschrieben zu haben, beantwortet. Abschliessend gilt festzuhalten, dass Edith Salomé auf mögliche Vertiefungsbedürfnisse explizit mit der Aufforderung ein Wunschkonzert aufzumachen angesprochen hat und deshalb die Hinführung zu einer Bearbeitung in Eigenverantwortung irritierend ist.

\section{Salome: Klar ja ehm auf jeden Fall}

Edith: Und in einer Bachelorarbeit könnte man ja auch so vorgehen und sagen das ist jetzt ein Bereich wo du ein Defizit oder ein Loch oder wo sich ne Lücke auftut und dann könntest du dich ja auch interessengeleitet das ist ja offensichtlich ein Interesse von dir da hinsetzen und sagen gut du erarbeitest jetzt mal das Thema Elternarbeit (.) ehm (.) über die Bachelorarbeit noch zusätzlich

Salomé stimmt Ediths Aussage vollumfänglich zu. Ihr Anschluss lässt sich als kooperative Bearbeitung der Differenz zwischen Edith und ihr lesen. Daran anschliessend verbalisiert Edith den Prozess, den Salomé durchlaufen könnte: die eigenen Defizite und Lücken identifizieren und daraus Bearbeitungsschritte ableiten. Bemerkenswert ist die dreifache Beschreibung desselben Phänomens: Defizit als eher technischer Begriff, der mit dem alltagssprachlichen, aber auch dramatischen Begriff Loch ergänzt wird, gefolgt von einer sich öffnenden Lücke, die den Vorgang im metaphorischen Sinn beschreibt. Anfangs spricht Edith universell von man, wechselt während der Aussage auf $d u$ und spielt dadurch direkt auf die Person. Da Edith im Sinne eines Selbstzitates vorspricht, was Salomé sagen könnte, wäre von der Syntax her keine $d u$-, sondern eine Ich-Formulierung zu erwarten. Mit der Verwendung von $d u$ anstelle des logischen «lchs» lässt sich ein weiteres empirisches Datum finden, in dem die spezifische Struktur von Ediths Distanzierung und Verantwortungszuschreibung an Salomé Gestalt gewinnt. 
Fragt man sich, in welcher Situation eine analoge Aussage, unter Auslassung von Elternarbeit und Bachelorarbeit, getätigt werden könnte, denkt man an ein schulisches Setting, wenn die Lehrperson die Schülerin bei einem Projekt coacht. Die Schülerin interessiert sich beispielsweise für Fische. Die Lehrperson könnte entsprechend sagen - und dann könntest du dich ja auch interessengeleitet das ist ja offensichtlich ein Interesse von dir da hinsetzen und sagen gut du erarbeitest jetzt mal das Thema "vom Aussterben bedrohte Fische» noch zusätzlich. Die Lehrperson lenkt asymmetrisch und fürsorglich zu einer individualisierten Aufgabenstellung hin. Allerdings passt die Formulierung eher zu einer Aufgabe, die zeitlich und aufwandmässig überschaubar ist, was sich in Worten wie hinsetzen, jetzt mal und noch zusätzlich Ausdruck verschafft, nicht aber für eine fundierte Auseinandersetzung. Das steht im Kontrast zu der vorherig aufgezogenen Notwendigkeit und zu Defizit und Loch, die auf eine grössere Tragweite anspielen. Unter Zuzug des Kontextes steht das auch im Kontrast zu einer Bachelorarbeit, die eine umfassende wissenschaftliche Arbeit am Ende des Bachelorstudiums darstellt.

Bemerkenswert ist die Aussage das ist ja offensichtlich ein Interesse von dir. Wäre das wirklich offensichtlich, müsste es kaum als offensichtlich deklariert werden. In der Formulierung scheint durch, dass es bei der Thematik nicht um ein Interessengebiet der Sprecherin handelt. Weiter wird mit dem Sprechakt bearbeitet, dass Salomé Elternarbeit nicht primär im Rahmen ihrer Interessen, sondern im Rahmen der Ausbildungsdefizite erwähnt. Edith scheint darauf zu reagieren und transformiert das Defizit, Loch oder die Lücke zu einem Interesse von Salomé. Dieser Transformation begegnet sie mit dem Kommentar das ist ja offensichtlich ein Interesse von dir und unterstellt ihr dabei ein solches.

Zusammenfassend für die bisherige Gesprächsinteraktion an dieser Sequenzstelle gilt festzuhalten, dass die Studentin auf den ihr zugespielten Ball, was sie sie noch wünschen würde, das Thema Elternarbeit identifiziert, das sie von institutioneller Seite als ungenügend thematisiert erachtet. Die Frage nach einem Wunsch verkommt bei der Mentorin zuerst zu einem Defizit und anschliessend zu einem Interesse. Das Anliegen wird mit einer wegspielenden Bewegung der Eigenverantwortung der Studentin zugeschrieben, indem ihr die Option der Bearbeitung im Rahmen der Bachelorarbeit aufgezeigt wird. Diese Verantwortungszuschreibung lässt sich als Ausdruck einer Erwartung an die Studierende lesen, die sich mit dem Imperativ «Interessiere dich selber!» paraphra- 
sieren lässt. Das von Salomé identifizierte Ausbildungsdefizit wird in ein persönliches Defizit von Salomé transformiert. Mit dem Nachschub das ist ja offensichtlich ein Interesse von dir erhält die Thematik Elternarbeit eine leicht abwertende und klar wegweisende Implikation. In der Zuspitzung lässt sich dies mit «wenn dich das Thema Elternarbeit so interessiert, dann schau selber, wie du das in deine Ausbildung einbauen kannst» umschreiben.

Das Sich-gegenseitig-in-Ruhe-Lassen bricht auf und es zeigt sich, dass das stetige Ausbalancieren der bereits identifizierten Spannungsbögen von diffus und spezifisch sowie nah und distanziert beidseitig eine hohe Anstrengung erfordert. Diese wird nicht ungebrochen durchgehalten und führt zu neuen Dynamiken der Abgrenzung und Verantwortungszuschreibung.

Salomé: ja ich bin jetzt eigentlich dort schon eher auf dem gestalterischen Weg ((lacht)) ehm aber (.) vielleicht kann man das ja auch einbauen Edith: vielleicht kann man's kombinieren?

Mit ja in der Bedeutung von «ja das könnte man» formuliert Salomé gleich zu Beginn eine Bestätigung und bezieht interaktionsdynamisch in der Fortsetzung dennoch Position gegen Ediths Vorschlag. Mit einer Reihe von Füllwörtern - jetzt, dort, schon - und Abtönungspartikel - eigentlich, eher - und mit der Wegmetapher umgeht sie es, dem Vorschlag eine direkte Absage zu erteilen. Auf dem Weg sein bedeutet bildhaft gesprochen, dass eine Entscheidung vorerst getroffen, jedoch nicht in Stein gemeisselt ist, da Wege Abbieger enthalten und Richtungsänderungen nicht ausschliessen. Die soziale Spannung, die durch Salomés Nicht-Einsteigen auf den Vorschlag entsteht, wird mit einem Lachen von Salomé bearbeitet. Salomé begegnet der Idee von Edith mit Diplomatie und stellt die Kultur der Einigkeit nach der inhaltlichen Differenz sozial wieder her. Mit dem Sprechakt vielleicht kann man das ja auch einbauen zeigt sich Salomé im Ton verbindlich, in der Sache jedoch unverbindlich.

Edith wiederholt die Aussage weitgehend, wenn auch sie eine Frage formuliert und einbauen zu kombinieren transformiert. Dennoch zeigt sie auf, dass sie an ihrem Vorschlag der eigenverantwortlichen Bearbeitung grundsätzlich festhält. 
Salomé: auf jeden Fall mhm ((bejahend)) es ist ja nicht so dass dass es dass ich finde ich hätte gar keine Ahnung aber es ist einfach so (.) ich meine wenn ich überleg wenn ich fertig bin bin ich 23 und ich stehe vor diesen Vätern und ich sie grüezi ich bin die neue Lehrperson von Ihrem Kind ((lacht))

Edith: und der Vater ja der ist unter Umständen vierzig ((lacht)) wunderbar ((lacht))

Obwohl Salomé die Frage umgehend bejaht, geht sie nicht weiter auf die Möglichkeit einer Kombination ein. Vielmehr nimmt sie auf der manifesten Ebene eine Selbsteinschätzung betreffend ihrer Kompetenz bezogen auf Elternarbeit vor. In der Latenz erhält ihr Anschluss eine andere Implikation. Er zeigt auf, dass es ihr um etwas anderes als um das Schreiben einer Bachelorarbeit geht. Bedeutsam in diesem Zusammenhang ist, dass Salomé mit wenn wir jetzt ein Wunschkonzert aufmachen könnten was was würdest du dir wünschen auch nach etwas anderem gefragt wurde.

Im Sprechakt legt Salomé ihre Bedenken dar. Diese betreffen nicht das fehlende Wissen, sondern die fehlende Lebenserfahrung, die Altersdifferenz und Genderthematik. Ohne inhaltliche Vertiefung wird an dieser Stelle deutlich, dass sich auch Salomé abgrenzt und nicht mehr nur (geschmeidig) anschliesst. Die Brisanz dieser neuen Dynamik der Abgrenzung und Selbstpositionierung wird interaktiv mit einer theatralischen Einlage ich stehe vor diesen Vätern und ich sie grüezi ich bin die neue Lehrperson von Ihrem Kind ((lacht)) - bearbeitet und sozial entschärft. Erneut doppelt Edith die getätigte Aussage nach, analog zu Salomé auf eine humoristische Art und Weise. Sie stellt sich die Situation bildhaft vor, was bei ihr Heiterkeit auslöst und zu einem mit Lachen unterlegten wunderbar führt. Die Prekarität, die das Aufbrechen des (geschmeidigen` Anschliessens und Sich-in-Ruhe-Lassens mit sich bringen, wird beidseitig in einem ähnlichen Modus (hier: humoristisch und theatralisch) bearbeitet. Als kontrastierendes Gedankenexperiment wäre auch folgende Bezugnahme der Mentorin vorstellbar: «Wenn man sich in die Rolle des 40-jährigen Vaters versetzt, was sind wohl seine Gefühle, wenn er vor einer jüngeren Lehrerin steht?» In dieser oder ähnlicher Bezugnahme liessen sich mögliche Emotionen der Studentin freilegen. Eine solche bleibt jedoch aus. Vielmehr agiert die Mentorin vergemeinschaftend und mit höherem Diffusitätspotenzial, indem sie sich mit Salomés Einschätzung solidarisiert. Die vorgefundene Aussage wäre beispiels- 
weise von einer guten Freundin erwartbar. Der Spannungsbogen von Diffusität und Spezifizität erfährt erneut eine Changierbewegung, hier in die diffuse Richtung.

Salomé: und das ist irgendwie so ein bisschen (.) wir haben einfach (.) ich fände es ehm noch cool wenn es irgendwie ein Seminar gäbe oder keine Ahnung ein Semester von mir aus wo wo einfach (.) wie führe ich einen Elternabend durch wie leite ich ein Elterngespräch wie spreche ich mit den Eltern ohne dass ich das Kind schlecht mache oder auf die Wolke hinaus hebe oder so und und ich finde das das ist so etwas da werden wir einfach darauf losgelassen und das ist einfach auch ein wichtiger Teil unserer Arbeit

Der erste Teil von Salomés Anschluss wird abgebrochen und bleibt inhaltlich unklar. Die Fortsetzung - wir haben einfach - lässt eine Kritik wie «kaum je über Elternarbeit gesprochen» oder eine entsprechende Formulierung vermuten. Allerdings wird keine Kritik ausgesprochen, sondern die Aussage nach dem Abbruch unverbindlich reformuliert. Die Gesprächsdynamik erinnert erneut an ein Gespräch zwischen zwei vertrauten Personen, was sich unter anderem in Formulierungen wie irgendwie, keine Ahnung wie auch in ich fände es ehm noch cool festmachen lässt. Gleich viermal verwendet Salomé das Wort einfach. Auf einer manifesten Ebene stellt einfach eine Leichtigkeit und Bedenkenlosigkeit dar. Latent weist der Sprechakt auch eine kritische Tönung auf. Die Formulierung da werden wir einfach darauf losgelassen und das ist einfach auch ein wichtiger Teil unserer Arbeit weist auf eine Bedenkenlosigkeit seitens der Institution hin. Denn die Metapher einfach darauf losgelassen erinnert an ein Rudel freigesetzter, wilder Tiere und zeigt den mit der Freisetzung einhergehenden Kontrollverlust wie auch das Risiko zu scheitern auf.

Salomés weitere Ausführungen werden nicht weiter analysiert, da sie nicht primär die Interaktionsdynamik des Gespräches betreffen.

Abschliessend ist festzuhalten, dass Salomé erneut Position bezieht. Da sie jedoch bei Edith mit der Zuschreibung der Verantwortung an die Hochschule zuvor (abgeblitzt) ist, wählt sie eine Form, die zwar Kritik ausübt, jedoch die Beziehung zu Edith nicht oder zumindest kaum gefährdet. Dies zeigt sich auch dadurch, dass Salomé ihr Anliegen unverfänglich und humorvoll artikuliert und nicht auf die Person spielt. 
Edith: $m h m$ ((bejahend)) ja da musst du jetzt aufpassen du darfst jetzt nicht deine Erfahrung verallgemeinern weil andere Studierende haben (.) vielleicht zwei drei Elterngespräche ein mit und ein ohne Kinder oder so was erlebt oder waren schon an Elternabenden mit dabei ehm offensichtlich ist dir das noch nicht begegnet Salomé: ja oder nicht genügend so dass ich es später selber machen könnte (.) also wenn ich mir jetzt überlege ich müsste jetzt (.) also wenn ich jetzt vor einer Klasse stehen würde und ich müsste unterrichten wäre das für mich kein Problem (..) aber wenn ich jetzt ein Elterngespräch führen müsste alleine dann wäre das für mich ein grosses Problem (.) jetzt (.) also so

Edith weist Salomé auf einen Gefahrenpunkt hin, dem sie gerade erliegt, nämlich persönliche, eigene Erfahrungen zu verallgemeinern. Im Modus einer Zurechtweisung teilt sie Salomé mit, was sie tun muss und nicht tun darf. Die Bemerkung offensichtlich ist dir das noch nicht begegnet enthält auf der latenten Ebene erneut eine Kritik, als ob ihr das hätte begegnen müssen.

An dieser Stelle gewinnt wiederholt eine Dynamik Gestalt, die das Anliegen von Salomé zu ihrem persönlichen Problem transformiert. Es scheint, als wäre Salomés Anliegen an ein Kränkungsmotiv bei Edith gekoppelt. Wenn Salomés Anliegen zu einem persönlichen Problem gemacht wird, lässt sich für Edith die Kritik abwenden. Was in Ediths Aussagen zuvor bereits durchscheint, wird nun unmissverständlich formuliert: Das von Salomé identifizierte Ausbildungsdefizit ist ein persönliches, kein allgemeines und bedarf einer eigenverantwortlichen Bearbeitung. Damit gilt die Aussage da werden wir einfach darauf losgelassen als entkräftet, die Kritik wird abgewendet, die Kränkung revanchiert und die Verantwortung an Salomé zurückgespielt.

Salomé bleibt in ihrem Anschluss souverän und bestätigt auf einer sachlichen Ebene Ediths Einschätzung, dass ihr dies nicht respektive nicht genügend begegnet sei, sie sich jedenfalls aus heutiger Sicht nicht zutraue, dies später selber machen zu können. Analog zu Ediths vorheriger Inszenierung startet nimmt eine Gedankenreise in ihre berufliche Zukunft vor. Im Sinne eines Eingeständnisses zeigt sie auf, dass die Vorstellung, ein Elterngespräch alleine zu führen, für sie ein grosses Problem darstellt. Performativ gerät ihre Aussage ins Stocken, was sich den Sprechpausen und der Suche nach einer Formulierung 
bei (.) jetzt (.) also so zeigt. Dies lässt sich auf die Anforderung zurückführen, die Kränkung von Edith zu bearbeiten und gleichzeitig auch gesichtswahrend das eigene Anliegen zu positionieren.

Abschliessend und zusammenfassend ist festzuhalten, dass sich auf einer latenten Bedeutungsebene ein Kränkungsmotiv Ausdruck verschafft, das bei Edith eine Bewegung in Richtung Gegenangriff auslöst. Salomé muss mit ihrem Anschluss auf das von Edith wahrgenommene Kränkungsmotiv reagieren, ohne dieses zu bestätigen.

Edith: das ist also jetzt eine Perspektive für's nächste Studienjahr oder die nächsten zwei Semester was gibt es noch für (.) Anliegen die du jetzt mit der Perspektive nach vorne verbindest?

Edith geht mit ihrem Anschluss erneut nicht auf das Anliegen ein, sondern zeigt Salomé auf, dass sie ihr Anliegen im nächsten Studienjahr zu bearbeiten hat. Aus der Betonung eine Perspektive lässt sich schliessen, dass es noch andere geben könnte. Mit der Identifizierung dieser Perspektive ist für Edith das Thema abgehakt. Sie hat das Anliegen an Salomé zurückgespielt und ihr aufgezeigt, dass sie für deren Bearbeitung eigenverantwortlich ist.

Edith fährt im Gespräch fort, indem sie nach weiteren Anliegen in Verbindung mit Perspektive nach vorne fragt. An der Stelle lässt sich die Fallstrukturhypothese für diese letzte Sequenz formulieren:

\subsubsection{Zusammenfassung und riskante Fallstrukturhypothese der Sequenz Was kannst du selber dafür tun}

Mithilfe einer Gedankenreise wird ein Denkprozess anmoderiert und die Studentin aufgefordert, Wünsche oder Anliegen für die ihr verbleibende Studienzeit zu identifizieren. Bis dato wird das Mentoratsgespräch relativ stabil im Abarbeitungsmodus durch Aufrufung von Agendapunkten gerahmt, zu dem sich die Studentin Salomé zu äussern hat. Hier aber findet ein Moduswechsel statt: Die Frage nach dem Wunschkonzert bedient eine andere Ebene als die vorausgehenden Fragen. Das dabei verwendete Tool der Gedankenreise bewegt sich erneut auf den Polen von Nähe und Distanz sowie Diffusität 
und Spezifität, da Interesse an der ganzen Person aufgezeigt wird. Dies drückt sich beispielsweise in der Wir-Formulierung - wenn wir ein Wunschkonzert aufmachen könnten - aus. Gleichzeitig erinnert die Frage an ein Ritual, bei dem allerdings das Formulieren der Frage und nicht die Antwort der angefragten Person im Fokus steht. Dies lässt sich auch daran erkennen, dass ein entsprechender Sprechakt in einer (echt) diffusen und nahen Beziehungskonstellation unvorstellbar wäre. Das Angebot des Wunschkonzertes changiert folglich zwischen Sein-Sollen und Nicht-sein-Dürfen. Diese Dynamik zeigt sich bereits in vorausgehenden Sequenzstellen. Eine vergleichend spannungsvolle Dynamik lässt sich auch erkennen, wenn - wie ausgeführt - einerseits explizit nach Wünschen gefragt wird, es andererseits jedoch nicht um die Einlösung dieser Wünsche geht.

Der aufscheinende Moduswechsel führt bei der Studentin zu einer Irritation. Sie fragt deshalb zuerst nach, bevor sie anschliessend ihre Wünsche oder Anliegen, die sie als institutionelle Ausbildungsdefizite identifiziert, aufzeigt und auch der eingeforderten Begründungspflicht nachkommt.

Das dominante Strukturmerkmal des bisherigen Interaktionsverlaufs - das Sich-gegenseitig-in-Ruhe-Lassen - bricht durch die Frage nach dem Wunschkonzert auf. Ebenfalls zeigt sich, dass die Pendelbewegung - einerseits eine Distanz aufrechtzuerhalten und andererseits Nähe und Diffusität dennoch nicht vollumfänglich vermeiden zu können nicht mehr in Schwingung ist. Es scheint, als stosse das von Salomé identifizierte Ausbildungsdefizit bei Edith auf ein Kränkungsmotiv und treffe sie diffus als ganze Person. Die Bearbeitung dieser Kränkung führt dazu, dass das Ausbildungsdefizit in ein persönliches Defizit von Salomé transformiert wird und auf diese Weise eine eigenverantwortliche Aufarbeitung erfordert. Der in der Verantwortungszuschreibung subtil angebrachten Kritik begegnet Salomé souverän, indem sie ihre Situations- und Selbsteinschätzung rollenspezifisch als Studentin verteidigt.

Die Änderung in der Interaktionsdynamik scheint beidseitig Dynamiken der Gesichtswahrung hervorzurufen. Diese Dynamiken begleiten den aufgeführten Tanz, der sich sowohl um Nähe als Gefahr, als auch um Distanz als Riskanz dreht. Es scheint, als gelte es Nähe und Diffusität tendenziell zu vermeiden. Gleichzeitig birgt eine Distanz auch das Risiko, als Gleichgültigkeit oder Negation des Besonderen verstanden zu werden und bringt wieder Bewegungen und Schritte in Richtung Nähe und Diffusität hervor. 


\subsubsection{Fallzusammenfassung und finale Fallbestimmung: Salomé \& Edith}

In der Zusammenführung der Fallstrukturhypothesen der rekonstruierten Sequenzen ergibt sich der folgende Befund für die Interaktionsdynamik des Mentoratsgesprächs zwischen der Studentin Salomé und der Mentorin Edith: Der typologisch prägnante Modus des Gespräches beruht auf Rechenschaftsablegung und Vertragserfüllung, in dessen Rahmen die Kompetenzfelder der PH FHNW vollständig, routiniert und einvernehmlich abgearbeitet werden. Der mechanische Modus - Kompetenzfeld aufrufen durch Mentorin und Rechenschaftsablegung der Bearbeitung des Kompetenzfeldes durch Studentin - gestaltet sich primär rollenförmig und vermeidet eine Kontaktaufnahme auf einer diffusen Beziehungsebene. Viel eher lässt sich in der Interaktion ein Element erkennen, das eine Bewegung aufzeigt, die mit «immer weiter nach vorn» beschrieben werden kann. Diese Vorwärtsbewegung weist eine gewisse Geschmeidigkeit auf, die sich darin manifestiert, dass sich die beiden Akteurinnen gegenseitig keine Probleme bereiten, sondern sich in Ruhe lassen, ganz so, als ob es gelte, das Gespräch weitgehend schadlos hinter sich zu bringen.

In der vorgefundenen Gesprächsinteraktion scheint sich allerdings auch eine gewisse Erwartungshaltung an eine dyadische Gesprächskonstellation Ausdruck zu verschaffen, die eine ausschliesslich rollenförmige und spezifische Bearbeitung nicht zulässt. Dieser Erwartung wird auf einer manifesten Bedeutungsebene beispielsweise durch Nähe- und Informalisierungsangebote begegnet und so auch eine gewisse Diffusität innerhalb der sozialen Situation zugelassen. Zugleich scheint es jedoch, als würden gerade diese diffusen Ausdehnungen und die damit einhergehende Nähe auf der Latenzebene eine Gefahrenzone bilden, die es zu vermeiden respektive zu (umschiffen〉 gilt. Diese Gleichzeitigkeit des Zulassens und Vermeidens von Diffusität und Spezifität wie auch Nähe und Distanz lässt die Gesprächskonstellation zu einer anspruchsvollen und fragilen Angelegenheit werden und führt zu Changierbewegungen respektive zu einem ‘Tanz um ein unsichtbares Feuer), als gelte es Diffusität zu vermeiden und synchron auch zu bedienen. 
Der auf Stabilität ausgerichtete Abarbeitungsmodus bricht an der Stelle auf, an der die Rechenschaftslegung durch eine offene Frage nach einem persönlichen Anliegen ergänzt wird. Die bis zu diesem Gesprächszeitpunkt stabil gehaltene Instabilität von Sein-Sollen und Nicht-sein-Dürfen gerät hinsichtlich der Pole Diffusität und Spezifität sowie Nähe und Distanz ins Wanken. Auf einer manifesten Ebene beantwortet die Studentin lediglich die von der Mentorin gestellte Frage nach einem Wunsch, auf einer latenten Bedeutungsschicht scheint die Formulierung eines Wunsches (nach mehr Ausbildungsinhalten bezogen auf Elternarbeit) jedoch einer impliziten Verletzung der Spielregel des Sichgegenseitig-in-Ruhe-Lassens und Sich-keine-Probleme-Machens zu entsprechen. Darin gewinnt ein Kränkungsmotiv, das sich auf eine emotionale Zurückweisung zurückführen lässt, Gestalt. Diese Getroffenheit der Mentorin führt in der Interaktionsdynamik dazu, dass das studentische Bedürfnis in ein persönliches Defizit der Studentin modifiziert wird, das einer eigenverantwortlichen Bearbeitung bedarf und in der Latenz auch ein Element der Kritik an die Studentin aufweist. Mit der Zuschreibung des studentischen Anliegens zur eigenverantwortlichen Bearbeitung ist die Abarbeitungsstruktur wiederhergestellt und auch dieses Traktandum erledigt.

Als abschliessende Fallbestimmung lässt sich festhalten, dass im Gespräch zwischen der Studentin Salomé und der Mentorin Edith eine Interaktionsdynamik sichtbar wird, die ihren Fokus mehrheitlich auf eine verwaltungsförmige und pragmatische Abarbeitung des Gesprächs richtet und die Legitimation des Settings vor allem durch die formale Erfüllung und weniger durch die Ingeltungsetzung der sozialen Rollen gewährleistet. Die Aufrechterhaltung dieses Abarbeitungsmodus scheint jedoch nicht bruchlos möglich zu sein, da nebst der Bearbeitung der Kompetenzfelder trotz der weitgehend problemlos verlaufenden und vertrauten dyadischen Gesprächskonstellation auch potenzielle Ansprüche oder Erwartungen an Nähe und Diffusität im Raum stehen. Diese Gleichzeitigkeit äussert sich in einer gewissen Fragilität und in Changierbewegungen zwischen den Polen Diffusität und Spezifität wie auch Nähe und Distanz. 


\section{$5 \quad$ Fallkontrastierung}

In der folgenden Kontrastierung sollen der Forschungsgegenstand, das Strukturproblem von Mentoratsgesprächen und die daraus resultierenden fallspezifischen Interaktionsdynamiken der Bearbeitung dieses Strukturproblems einer weiteren und vergleichenden Klärung und Systematisierung unterzogen werden. Das Ziel dieser Systematisierung besteht darin, eine idealtypische Zuspitzung von Varianten der Einrichtung und Bearbeitung des Settings vorzunehmen und diese jeweils in ihrer Eigenlogik zu beschreiben. Diese vier Eigenlogiken bringen ihrerseits allgemeine und fallübergreifende Strukturen hervor, obwohl sich diese im konkreten Einzelfall unterschiedlich manifestieren. Die daraus resultierenden typischen Modi der Bearbeitung bilden die Grundlage für die Theoretisierung des Gegenstands "Strukturproblem des Formats «Mentoratsgespräche»» und der entsprechenden Interaktionsdynamiken, das heisst für die Theoretisierung der fallspezifischen, eigenlogischen Ausdrucksgestalten dieses Strukturproblems. Diese beruhen auf empirisch begründeten Aussagen und bauen auf den Ergebnissen der Rekonstruktion der einzelnen Falldarstellungen in Kapitel 4 auf.

Bereits die theoretische Auseinandersetzung mit der konzeptionellen Rahmung des Mentorats hat auf potenzielle Schwierigkeiten bei der funktionalen Etablierung des Formats hingewiesen. Wie sich diese Schwierigkeiten allerdings im Konkreten zeigen, war aufgrund der theoretischen Überlegungen nicht oder nur vage antizipierbar. Die Differenz zwischen der antizipierten Problemlage und der konkreten Umsetzung bildet den Ausgangspunkt respektive die Ausgangsfrage der empirischen Untersuchung und wird in der nachfolgenden Kontrastierung diskutiert.

Die Rekonstruktionsergebnisse beginnen aus darstellungslogischen Überlegungen heraus mit der Beschreibung der geteilten und relativ abstrakten strukturellen Problemlage des Settings. Diese Umdrehung, das heisst die Vorwegnahme der Verallgemeinerung der Ergebnisse, erlaubt es, den Umgang mit dem Strukturproblem anhand von vier Modi der Bearbeitung wie vertiefende Plausibilisierungen darzustellen und zu diskutieren.

Als Vorwegnahme der Kernthese lässt sich aus der Untersuchung der faktischen Strukturiertheit des Settings «Mentoratsgespräche» eine Problemlage feststellen, die sich auf die Legitimierung des Settings bezieht. Das Setting ist seit geraumer Zeit formal 
institutionalisiert wie auch legitimiert und als Ausbildungsformat in einem personalisierten Studium nicht wegzudenken. Allerdings indizieren die empirischen Befunde, dass das Format eine Eigenlogik aufweist, die von den beteiligten Akteurinnen oder Akteuren, das heisst von den Studierenden und den Mentorierenden, in irgendeiner Weise 〈bespielt» respektive bearbeitet werden muss. Die Ergebnisse der Fallrekonstruktionen zeigen, dass eine nur rein formale und institutionalisierte Legitimierung nicht ausreicht und das Setting die Schwierigkeit der funktionalen Etablierung erkennen lässt. Zudem scheint das Setting auf etwas aufzubauen oder etwas vorauszusetzen, was nicht ohne Weiteres vorliegt. Dieses Etwas, zum Beispiel ein Gesprächsbedarf, ein konkreter Anlass oder auch ein mit der Mentorin oder dem Mentor zu besprechendes Problem oder Anliegen, das die Legitimation des Gespräches bilden könnte, kann nicht zwingend als gegeben erachtet werden.

Im Folgenden gilt es, die fallspezifischen Ausdrucksgestalten im Umgang mit der Legitimierungsproblematik aufzuzeigen und in ihrer Eigenlogik zu beschreiben. Für die Darstellung der jeweiligen Eigenlogiken bieten sich an verschiedenen Stellen Anderslogiken aus differenten sozialen Situationen an, die zu den Eigenlogiken ins Verhältnis gesetzt werden. Als Anderslogiken kennzeichne ich in dieser Arbeit Interaktionslogiken, die Strukturmerkmale aufweisen, die in spezifischen sozialen Kontexten als normal oder üblich gerahmt und insofern vertraut erscheinen. Sie werden in dieser Arbeit verwendet, um Strukturähnlichkeiten der untersuchten Mentoratsgespräche zu lebensweltlich vertrauten Interaktionskonstellationen sichtbar zu machen.

Die Diskussion der Rekonstruktionsergebnisse ist wie folgt aufgebaut: In einem ersten Schritt wird die strukturelle Problemlage von Mentoratsgesprächen diskutiert. Im Anschluss daran gilt es, aufzuzeigen, wie sich diese Problemlage in den Gesprächsinteraktionen konkret äussert respektive welche Dynamiken dabei aufscheinen. Für die Darstellung dieser Interaktionsdynamiken werden zwei analytische Perspektiven separat betrachtet: Als Erstes die Aspekte und Modi der Bezugnahme auf das Setting, die sich auf die Frage beziehen, wie die Akteurinnen und Akteure das Format gestalten und was sie interaktiv hervorbringen. Die zweite Perspektive betrifft die Aspekte und Modi der Bezugnahme aufeinander und fokussiert die Frage, wie sich die Akteurinnen und Akteure interaktiv aufeinander beziehen und zueinander ins Verhältnis setzen. 
Diese beiden Analysefoki oder -perspektiven stehen in einer Wechselbeziehung zueinander, da die betrachteten Phänomene in den Gesprächen in sozialer Hinsicht aufeinander einwirken. Denn die Art und Weise, in der sich die Mentorin oder der Mentor beispielsweise auf das Format bezieht, findet Resonanz darin, wie die Studentin adressiert und wie auf sie Bezug genommen wird. Folglich handelt es sich um ein in der Praxis nicht auftrennbares (Interaktions-)Geschehen, das lediglich darstellungstechnisch einer analytischen Separierung unterzogen wird. Anders formuliert werden in Analogie zu einem Scheinwerfer unterschiedliche Aspekte und Modi der Bezugnahmen in diesem Zusammenspiel ins Licht gerückt. Im Zuge der Rekonstruktion wurde deutlich, dass sich diese Analysefoki oder -perspektiven einzeln, aber auch im Zusammenspiel als geeignet erweisen, um die strukturelle Verfasstheit respektive das Strukturproblem von Mentoratsgesprächen in einer möglichst differenzierten Deutung aufzuzeigen. Abgeschlossen wird die Fallkontrastierung mit einer knappen Zusammenfassung und Verallgemeinerung der empirisch gewonnenen Erkenntnisse.

\subsection{Strukturelle Problemlage von Mentoratsgesprächen}

Die in den Mentoratsgesprächen erkennbare Problemlage, das heisst die Legitimierungsbedürftigkeit des Settings, findet Ausdruck in den Gesprächsinteraktionen und lässt sich auf dieser Ebene auch empirisch zugänglich machen. Angelegt ist das Problem aber bereits auf der strukturellen Ebene des Gesprächsformats, nämlich in deren Rahmung mit den entsprechenden institutionellen, formellen und organisatorischen Vorgaben. Deshalb gilt es, im ersten Schritt der Analyse aufzuzeigen, welche Eigenlogiken diese Rahmung hervorruft. Es geht darum, aufzuzeigen, wie das Gesprächssetting durch die Akteurinnen und Akteure gestaltet wird, und dabei insbesondere darum, welche Beziehungsformen interaktiv hergestellt werden und wie in diesen Gesprächen handelnd an die institutionellen Vorgaben und Bedingungen angeknüpft wird. Dabei interessiert die Frage, wie die beteiligten Akteurinnen und Akteure, namentlich die Mentorierenden, die empirisch erkennbare Legitimierungsbedürftigkeit bearbeiten. Als theoretischer Bezugsrahmen der dyadischen Interaktion bieten sich für diese Diskussion erneut Oevermanns therapeu- 
tisches Arbeitsbündnismodell und die entsprechenden theoretischen Überlegungen an. Diese haben im Sinne von sensibilisierenden Konzepten die Fallrekonstruktionen begleitet und werden nachfolgend als Vergleichsfolie für die kontrastierenden Betrachtungen herangezogen.

Gemäss der Mentoratskonzeption der PH FHNW (siehe Kapitel 2.1.2) finden die Mentoratsgespräche regelmässig einmal pro Semester statt. Diese Mentoratsgespräche reagieren nicht auf ein spezifisches studentisches Anliegen oder einen konkreten Anlass, sondern werden - formal und institutionalisiert eingerichtet - als Bestandteil der Ausbildung durchgeführt. ${ }^{64}$ Dennoch lässt sich in diesen Gesprächen auch ein Anspruch auf quasi-supervisorische Anteile feststellen; das heisst, konzeptionell ist in den Gesprächen auch die Besprechung konkreter studentischer Anliegen, individueller Themen und Professionalisierungsfragen angelegt.

Wie bereits in den theoretischen Vorüberlegungen in den Kapiteln 2.3.3.3 und 2.4 aufgezeigt wurde, fusst die Legitimation des Arbeitsbündnisses in einem therapeutischen Setting auf der individuellen «Not` der Klientin oder des Klienten. Diese «Not` gilt es im Rahmen einer Therapie mittels stellvertretender Krisenbewältigung zu mindern, damit die Klientinnen und Klienten auf diese Weise ihre autonome Lebenspraxis zurückgewinnen können. Während also in der Therapie die individuelle (Not) eine Conditio sine qua non und somit eine notwendige Bedingung für das Arbeitsbündnis zwischen der Therapeutin oder dem Therapeuten und der Klientin oder dem Klienten darstellt, gestaltet sich das Arbeitsbündnis im Mentorat, wie ebenfalls bereits ausführlich dargelegt, markant anders. So kann weder Gesprächsbedarf noch eine entsprechende «Not` seitens der Studierenden als gesichert vorausgesetzt werden. Deshalb lässt sich das Gespräch nicht durch Rekurs darauf legitimieren. Wenn kein Gesprächsbedarf vorhanden ist, weist das Format «Mentoratsgespräche» respektive das Arbeitsbündnis im Mentorat folglich eine Legitimierungsschwierigkeit auf. Pointiert formuliert lässt sich das Gesprächsformat in diesen Fällen mit (legitimierungsbedürftig, aber nicht legitimiert) charakterisieren. Die Dynamik dieser Bedürftigkeit stellt das wesentliche strukturelle Problem des Arbeitsbündnisses im Mentorat dar. Im Grunde genommen schreibt der Gesprächsanlass jeder

\footnotetext{
${ }^{64}$ Wie in Kapitel 3.3.1 ausgeführt wurde, befinden sich im Sample Gespräche, die sich nicht primär mit der Besprechung der studentischen Portfolioarbeit befassen, sondern diese Arbeit höchstens marginal thematisieren.
} 
und jedem einzelnen Studierenden einen Unterstützungsbedarf zu und konstituiert auf diese Weise eine allgemeine und individuelle Unterstützungsbedürftigkeit, die das Vorhandensein oder zumindest das zeitweise Auftreten von Nöten oder Notlagen impliziert.

Ansatzweise zeigt sich in einem Gespräch im Zusammenhang mit der Thematik «Elternarbeit» Gesprächsbedarf seitens der Studentin. In den anderen rekonstruierten Gesprächsprotokollen ist dieser empirisch kaum ersichtlich und wird deshalb artifiziell und interaktiv an die Gesprächssituationen «herangetragen`. Diese kaum vorhandene Bedürftigkeit stellt für das Setting letztendlich eine unlösbare Herausforderung respektive Anforderung dar. Denn mit Blick auf die Interaktion zeigt sich, dass sich die Studierenden und Mentorierenden nicht jenseits des Strukturproblems dieser Legitimierungsbedürftigkeit bewegen können, sondern dieses Problem beidseitig bearbeiten respektive sich dazu verhalten müssen. Abstrakt formuliert lässt sich festhalten, dass sich die im Mentoratsgespräch stattfindende Interaktion dafür legitimieren muss, dass sie stattfindet. Obwohl sich diese Dynamik als Interaktion beschreiben lässt und folglich beide Beteiligten betrifft, gestaltet sie sich für die Beteiligten dennoch different. So scheint die Mentorin oder der Mentor einen Modus oder eine Form der Bearbeitung dieser Legitimierung zu initiieren, während sich die Studentin auf individuelle Weise zum initiierten Modus verhält. Für die Studentin gestaltet sich die Legitimierungsproblematik auch insofern anders, als die Verpflichtung zur Teilnahme und die damit einhergehende Gutschrift eines ECTS-Punkts für sie eine Form von Legitimation darstellen. Deshalb muss sich die Studentin lediglich zur interaktiven Legitimierungsdynamik des Stattfindens, nicht aber jener des Herstellens verhalten.

Wenngleich die Dynamiken in den vier rekonstruierten Mentoratsgesprächen auf unterschiedliche Weise Gestalt gewinnen, zeigt sich in allen Gesprächen als gemeinsamer Fluchtpunkt die Schaffung von situativen Anforderungen, die die Legitimierung des Gesprächsanlasses bearbeiten. Die jeweilige Eigenlogik einer fallspezifischen Bearbeitung erinnert oder lehnt sich mehr oder weniger deutlich an eine bereits bestehende Anderslogik an, beispielsweise an ein therapeutisches oder auch schulisches Setting.

Diese Anlehnung an ein differentes, in anderen Kontexten etabliertes Setting findet allerdings nicht bruchlos statt, sondern weist Inkonsistenzen auf. Diese Inkonsistenzen lassen sich damit erklären, dass es sich um ein Mentoratsgespräch mit seiner Eigenlogik 
und nicht um ein Therapie- oder Unterrichtsgespräch, das ebendieser Anderslogik folgt, handelt. ${ }^{65}$

In Ermangelung eines gesichert vorliegenden Gesprächsanlasses, eines Gesprächsbedarfs und Dringlichkeit oder Notwendigkeit wird ebensolches geschaffen. Diese geschaffenen situativen Anforderungen stellen interaktiv Bedeutsamkeit und Legitimation her oder bearbeiten zumindest die gegenteilige Dynamik, das heisst, sie beziehen sich auf die Vermeidung einer möglichen Bedeutungslosigkeit der Gespräche. Obschon der Gesprächsanlass durch die Studienordnung und somit durch eine formale Verpflichtung geregelt ist, zeigt sich interaktiv, dass noch etwas anderes, etwas Zusätzliches, zur formalen Verpflichtung «nachgeliefert` werden muss. Diese strukturelle Problemlage das nicht grundsätzliche Vorliegen eines Gesprächsbedarfs - stellt das zentrale Unterscheidungsmerkmal zu einem therapeutischen Setting dar. Dies führt zu Dynamiken, die sich so interpretieren lassen, dass die oder der oder Studierende gleichsam einer $\langle\text { Not }\rangle^{66}$ bedarf. Hinsichtlich des Bedarfs oder der Notwendigkeit einer 〈Not〉 verhält sich das Setting «Mentoratsgespräche» analog zu einem Therapiegespräch, denn auch in einem solchen wird das Setting als Setting - wie bereits mehrfach ausgeführt - durch die (Not) der Klientin oder des Klienten legitimiert. Im Unterschied zum Mentoratssetting liegt die 〈Not` in der Therapie jedoch bereits vor, weshalb die Therapie überhaupt erst in Anspruch genommen wird. Das Nichtvorliegen im Mentorat verlangt demgegenüber nach einer Bearbeitung während der Gesprächsinteraktion und führt beispielsweise zu Umschiffungsdynamiken, Entgrenzung ${ }^{67}$ oder auch Informalisierungsrespektive gegenteiligen Formalisierungsbewegungen, die im nachfolgenden Kapitel diskutiert und miteinander kontrastiert werden.

\footnotetext{
${ }^{65}$ An dieser Stelle ist anzumerken, dass auch in Therapie- oder Unterrichtsgesprächen prekäre Situationen, Brüche und Inkonsistenzen entstehen können und auch häufig anzutreffen sind. Es geht hier daher nicht primär um diese grundsätzliche Prekarität, sondern um die Prekarität, die sich aus der Anlehnung an eine Anderslogik ergeben.

66 «Not» ist in diesem Zusammenhang im erweiterten Sinn des Wortes, das heisst auch im Sinne eines Anliegens oder einer Problemlage, zu verstehen.

${ }^{67}$ Unter «Entgrenzung» wird im vorliegenden Zusammenhang eine Form von persönlich-diffuser Nähe verstanden, die sich jenseits der für das Mentoratssetting erwartbaren Grenze oder (roten Linie) befindet und diese Grenze somit überschreitet. Diese Verwendung von «Entgrenzung» steht unter anderem in Differenz zu Kowalskis (2020) Entgrenzungsbegriff, der sich damit auf eine dezidiert übergriffige, aufgezwungene, egoistische Nähe bezieht, die sich durch das Bedürfnis nach emotionaler Zuwendung, Anerkennung und Aufmerksamkeit derjenigen Person, welche die Entgrenzung vornimmt, erklären lässt (Kowalski, 2020, S. 477).
} 
Im Sinne eines Zwischenfazits lässt sich somit festhalten, dass Mentoratsgespräche unter beiden Bedingungen, das heisst mit und ohne Gesprächsbedarf seitens der Studierenden, stattfinden. Im letzteren Fall erfordert die Leerstelle des fehlenden Bedarfs eine interaktive Bearbeitung oder 〈Füllung〉. Daraus lässt sich als erster Kernbefund auf einer höheren Abstraktionsebene Folgendes ableiten: Die Legitimierung des Mentoratsgesprächs speist sich nicht bereits aus seiner formalen Rahmung, sondern muss während des Gesprächsverlaufs performativ hergestellt und gefestigt werden. Anders formuliert verlangt die Leerstelle in der Etablierung und der Ermächtigung nach einer Legitimierung. Wie diese Dynamik der Legitimierung empirisch Gestalt gewinnt, gilt es weiter zu klären.

\subsection{Modi der Bearbeitung}

Die Umgangsweisen oder mit anderen Worten die Modi der Bearbeitung werden nachfolgend ausformuliert und beschrieben. ${ }^{68}$ Gemeinhin ist nicht davon auszugehen, dass sich in einem Mentoratsgespräch mehrere respektive grundlegend differente Modi der Bearbeitung zeigen, da sich aus strukturtheoretischer Perspektive Strukturen grundsätzlich reproduzieren (Oevermann, 1983, S. 270). Auf einer theoretischen Ebene lässt sich dies jedoch nicht bereits von vornherein ausschliessen.

Die Diskussion der Modi der Bearbeitung orientiert sich an den folgenden heuristischen Fragen:

- Wie und als was wird das Setting «Mentoratsgespräche» hervorgebracht?

- Welche (impliziten) handlungsleitenden Modi der Bearbeitung des Strukturproblems zeigen sich latent im Handeln der Akteurinnen und Akteure?

- Wie lassen sich die Interaktionsdynamiken mit ihren Phänomenen und Facetten typologisch prägnant darstellen? Auf welchen Dimensionen spielen sich diese Dynamiken $a b$ ?

\footnotetext{
68 Der Anspruch dieser Arbeit ist die Beschreibung der erkennbaren Interaktionsdynamiken dieser Modi der Bearbeitung. Dies gilt als hinreichendes Kriterium für die Darstellung. Ob die einzelnen Modi die konzeptionellen Anforderungen des Formats "Mentoratsgespräche» erfüllen und, wenn ja, in welchem Masse oder in welcher Rangfolge, steht ausserhalb des Forschungsinteresses und wird nicht thematisiert.
} 
Wie bereits erläutert dürfte es sich zu Analysezwecken als aufschlussreich erweisen, wenn die Aspekte und Modi der Bezugnahme auf das Setting und die Aspekte und Modi der Bezugnahme der beteiligten Akteurinnen und Akteure aufeinander separat diskutiert werden.

\subsubsection{Aspekte und Modi der Bezugnahme auf das Setting}

Die allgemeine, fallübergreifende Legitimierungsbedürftigkeit steht in einer wechselseitigen Relation zur Gesprächsinteraktion. Sie findet Resonanz darin, wie sich die Individuen, hauptsächlich die Mentorin oder der Mentor, das Format oder Setting aneignen und sich auf dieses beziehen. Bei der Diskussion dieses Punktes gilt es aufzuzeigen, wie die jeweils einzelnen Anwesenden den Modus ausgestalten.

Die empirisch anzutreffenden Modi der Bezugnahmen auf das Setting erinnern in gewissen Aspekten an gängige und etablierte Anderslogiken. Diese Anderslogiken können auf einer deskriptiven Ebene als Orientierung herangezogen und zur Charakterisierung der empirisch vorgefundenen Eigenlogiken genutzt werden. Die nachstehenden Kurzbeschreibungen zeigen für die vier rekonstruierten Gesprächsprotokolle auf, wie sich die beteiligten Akteurinnen und Akteure auf das Setting «Mentoratsgespräche» im Allgemeinen und auf die in Abschnitt 5.1 dargelegte Legitimierungsbedürftigkeit im Besonderen beziehen und an welche Anderslogik der interaktiv hervorgebrachte Gesprächsmodus erinnert. Auf eine Fallzusammenfassung wie auch auf eine empirische Hinterlegung der Ergebnisse mit Daten und Textbausteinen wird an dieser Stelle verzichtet, da die Kurzbeschreibungen die Funktion einer Verdichtung mit Verallgemeinerungspotenzial haben.

Tabelle 1 zeigt, an welche vier Anderslogiken die in den Analysen herausgearbeiteten interaktiven Modi in einer idealtypischen Zuspitzung erinnern. Die aufscheinenden Formen der Eigenlogik und die darin erinnerten Anderslogiken lassen sich jedoch nicht auf wenige Begriffe reduzieren. 
Tabelle 1: Idealtypische Zuspitzung der Interaktionsmodi

\begin{tabular}{|l|l|}
\hline Gespräch & $\begin{array}{l}\text { Modus der Bezugnahme auf das Setting erinnert an die } \\
\text { Anderslogik von }\end{array}$ \\
\hline Tina \& Michael & Unterricht im Modus von Zeigen, Lehre, Wissensvermittlung \\
\hline Frau Steffen \& Herr Bucher & Prüfung \& Bewährung in der Anfechtung \\
\hline Ingrid \& Lorenz & Therapie \\
\hline Salomé \& Edith & $\begin{array}{l}\text { Abarbeitung einer Agenda und verwaltungsförmige Rechenschafts- } \\
\text { ablegung }\end{array}$ \\
\hline
\end{tabular}

\section{Modus der Bezugnahme: Anderslogik «Unterricht»}

Im Gespräch zwischen der Studentin Tina und dem Mentor Michael wird interaktiv ein Setting hervorgebracht, das in seiner Eigenlogik an die Anderslogik von schulischem Unterricht, konkret an Zeigen, an Lehre oder an Wissensvermittlung, erinnert. Der strukturellen Problemlage der Legitimierungsbedürftigkeit wird in diesem Gespräch insofern begegnet, als ein Modus hervorgebracht wird, die Parallelen zu Pranges Figur des Zeigens (Prange, 2012, S. 65) aufweist und die Situation zu einer pädagogischen (macht). Mit dieser Figur des Zeigens, die die Grundformen pädagogischen Handelns wie Unterrichten, Informieren, Beraten, Arrangieren und Animieren beinhaltet (Giesecke, 2015), nimmt der Mentor die Lehrerrolle ein und etabliert zwischen der Studentin und sich selbst eine Lehrer-Schülerin-Beziehung mit entsprechenden Implikationen wie beispielsweise einer wohlwollenden und fürsorglich-anleitenden Zuneigung. Es lässt sich somit ein Anspruch seitens des Mentors erkennen, der Studentin etwas zu vermitteln oder zu zeigen respektive ihr einen Weg aufzuzeigen. Bezeichnend ist hierbei der Gesprächsauftakt des Mentors. Mit der Formulierung ich zeige dir wo wir hingehen hebt er diese latente Zeigedynamik und die Wegmetapher zu Gesprächsbeginn kurzfristig auf die manifeste Ebene. Diese empirisch vorgefundene Zeigedynamik ist eine mehrfache: Der Studentin wird etwas gezeigt, wodurch sich der Mentor mit seiner Expertise zeigt und wobei sich zugleich zeigt, dass diese Expertise der studentischen Anerkennung bedarf. Denn in Differenz zur Logik des schulischen Unterrichts kommt im Mentoratssetting eine Notwendigkeit oder ein Anliegen zum Ausdruck, sich in der Position des Mentors mit einer 
entsprechenden Erfahrungs- und Expertisedifferenz in Geltung zu setzen. Im schulischen Unterricht scheint diese Ingeltungsetzung durch das Format «Unterricht» oder den Modus des Unterrichtens bereits weitgehend gesichert zu sein. Gerade weil das Mentoratsgespräch kein Unterrichtsgespräch ist, gestaltet sich die Ingeltungsetzung hierbei fragiler und bedarf einer performativen Bearbeitung respektive Bestätigung.

\section{Modus der Bezugnahme: Anderslogik «Prüfung \& Bewährung»}

Der Mentor Herr Bucher und daran anschliessend die Studentin Frau Steffen nehmen in einer Eigenlogik Bezug auf das Setting «Mentoratsgespräche», die gewisse Ähnlichkeiten zu einer weiteren Anderslogik aus dem Schul- oder Ausbildungskontext aufweist, nämlich zu einer Prüfungssituation. Im vom Mentor etablierten Modus geht es jedoch nicht primär um Bewertung von Wissen oder Leistung und auch nicht um Selektion. Vielmehr werden der Studentin mittels provokativer Fragen die eigenen Unzulänglichkeiten ihrer (Lehrerinnen-)Persönlichkeit aufgezeigt, zu denen sie sich zu verhalten hat. Ein Ausbildungsformat wie das hier vorliegende Setting «Mentoratsgespräche» folgt programmatisch einem Ausbildungsanspruch. Fragt man sich, welcher Anspruch in der vorliegenden Gesprächsdynamik empirisch Gestalt gewinnt, lässt sich dieser mit Bewährung in der Anfechtung und entsprechend mit dem Imperativ «Zeig dich!» beschreiben. Diese Bewährungsfigur lässt sich dem Konzept von Personenoptimierung, in der Fallrekonstruktion auch als «people processing» bezeichnet, zuschreiben. Die Figur «Lehrperson werden bedarf einer Prüfung und Bewährung in der Anfechtung» - , die zu Veränderungen und Optimierung der Persönlichkeit der (angehenden) Lehrerinnen und Lehrer genutzt werden soll, schwingt beim hier gezeigten somit latent Gesprächsmodus mit. Im Kontrast zu den anderen drei Gesprächsmodi "Unterricht», "Therapie» und «Abarbeitung» zeigt sich diese Arbeit an der Personenoptimierung oder diese BeArbeitung der studentischen Persönlichkeit hier jedoch modusspezifisch ausgeprägter.

Allerdings steht diese Idee oder dieser Anspruch von «people-processing» respektive von Personenoptimierung in einem Missverhältnis zum empirisch sichtbar werdenden Modus, der, wie bereits aufzeigt, an die Anderslogik von Prüfung oder Bewährung erinnert. Eine Prüfungs- oder Bewährungssituation ist ein gängiges Element in einem Ausbildungskontext. Prüfung und Bewährung verfolgen jedoch nur insofern einen Ausbildungs- 
anspruch, als sie den Ausbildungsprozess überprüfen. Einem Personenoptimierungs- oder Vermittlungsanspruch kommen sie hingegen nicht nach, denn in einer Prüfungs- oder Bewährungssituation wird dezidiert nicht gefördert, optimiert, vermittelt oder verbessert, sondern der Prüfungskandidatin oder dem Prüfungskandidaten werden lediglich die eigenen Zulänglichkeiten respektive Unzulänglichkeiten bezogen auf die Prüfungsinhalte dargelegt. Im direkten Vergleich mit dem Gespräch zwischen der Studentin Tina und dem Mentor Michael wird im Gespräch zwischen der Studentin Frau Steffen und dem Mentor Herrn Bucher nichts gezeigt; vielmehr muss sich die Studentin im «ProvokationsVerteidigungs-Spielı selbst zeigen und bewähren.

\section{Modus der Bezugnahme: Anderslogik «Therapie»}

Die interaktiv hervorgebrachte Eigenlogik im Gespräch zwischen der Studentin Ingrid und dem Mentor Lorenz lehnt sich an die Anderslogik eines therapeutischen Settings an, und zwar vor allem was dessen Etablierung oder Hervorbringung durch den Mentor betrifft. Die Gesprächsdynamik fokussiert auf Probleme und Problemlösungen, wobei der Studentin auch ein Testangebot unterbreitet wird. Es gewinnt eine Figur Gestalt, die sich wie folgt beschreiben lässt: «Ich zeige dir dein Problem und zeige dir zugleich, wie ich dich bei der Bearbeitung oder Lösungsfindung unterstützen kann.» Die strukturelle Problemlage der Legitimierungsbedürftigkeit wird somit bearbeitet, indem der Mentor der Studentin ein aus seiner Sicht vorhandenes Problem aufzeigt und ihr eine Be- oder Verarbeitung des Problems nahelegt. Denn - so lässt sich die dahinterliegende Dynamik ausformulieren - würde die Studentin ihr Problem oder ihre 〈Not` erst einmal erkennen und anerkennen, liesse sich in der Folge ein Arbeitsbündnis zwischen dem Mentor und ihr einrichten. Dieses Arbeitsbündnis würde analog zu einem therapeutischen Arbeitsbündnis auf dem Mandat einer stellvertretenden Krisenbewältigung und auf dem Wunsch der Klientin, ihre bestehende (Not) zu mildern und dadurch ihre Autonomie wieder zurückzugewinnen, basieren. So liesse sich die grundsätzliche Problemlage der Legitimierungsbedürftigkeit bearbeiten, denn ein therapeutisches Setting unterliegt dem Problem der Legitimierungsbedürftigkeit nicht, da die 〈Not` der Klientin oder des Klienten und die Freiwilligkeit im Wunsch, diese 〈Not` zu lindern, die entsprechende Legitimation bereits «liefern». 
Fallspezifisch wird die Unterbreitung des Testangebots des Mentors mit der Unterbreitung eines Näheangebots ergänzt. Hier scheinen sich ein hohes eigenes Anerkennungsbedürfnis und ein Bedürfnis des Gemocht-Werdens Ausdruck zu verschaffen. Das hier etablierte Setting kann sich nicht von der - pointiert formulierten - Figur «habe 〈Not) und brauche mich» oder, in anderen Worten, «bitte lass mich dir dein Problem zeigen lass mich dir helfen", die auf Nähe, diffuse Beziehungsstruktur und ein GemochtWerden angewiesen ist, lösen.

Dieses Angewiesen-Sein beruht allerdings auf Gegenseitigkeit, da sich dies auch bei der Studentin zeigt, wenngleich in differenter Form. Daraus ergibt sich eine weitere Dynamik, die darin besteht, dass die Studentin das Setting «Mentoratsgespräche» und den Mentor als Vertreter des Settings dazu (verwendet), sich hinsichtlich ihrer Art des Studierens, ihres Zeitmanagements wie auch ihrer Work-Life-Balance abzusichern und eine entsprechende Gutheissung respektive ‘Absegnung〉 einzuholen. Die aufseiten der Studentin aufscheinende Rechtfertigungsimplikation lässt sich als Hinweis auf Normen, Erwartungen und Kontrollen lesen, mit denen sie sich konfrontiert zu sehen scheint.

Gerade diese Figur der gegenseitigen Angewiesenheit weist einen Bruch zur Anderslogik der Therapie auf, da die Therapeutin oder der Therapeut in einem therapeutischen Setting nicht auf die Klientin oder den Klienten angewiesen ist, sondern die gemeinsam zu bearbeitende «Not` das Setting in seiner Funktion legitimiert.

Der Ausbildungsanspruch, der sich in einer an Therapie angenäherten Gesprächslogik manifestiert, lässt sich als Selbstoptimierung innerhalb eines grundsätzlich krisenhaften Ausbildungsprozess beschreiben. Der Mentor scheint das Krisenhafte zu erkennen und er könnte mit seiner Unterstützung einen Beitrag zur «Heilung〉 leisten.

\section{Modus der Bezugnahme: Anderslogik "Abarbeitung»}

Die Studentin Salomé und die Mentorin Edith bringen interaktiv einen Gesprächsmodus hervor, der der Abarbeitung entlang einer Agenda entspricht, die sich an vorgegebenen Kompetenzfeldern orientiert. Die hier vorgefundene Eigenlogik lässt sich mit verwaltungsförmiger Rechenschaftsablegung und Vertragserfüllung hinsichtlich institutioneller Verbindlichkeiten beschreiben und kommt einem Grundsatz von Vollständigkeit und Sorgfaltspflicht nach. Die in der Gesprächsinteraktion Gestalt gewinnende Bewegung 
weist eine mechanische Komponente auf, die sich mit «immer weiter nach vorn» fassen lässt.

Der Ausbildungsanspruch oder das Ausbildungsanliegen scheint in diesem Gespräch eine andere Position einzunehmen als in den drei zuvor analysierten Gesprächen. Während sich der Anspruch im Gespräch von Tina und Michael eher auf Wissensvermittlung bezieht, der Fokus bei Frau Steffen und Herrn Bucher auf Bewährung und Personenoptimierung gerichtet wird und im Gespräch zwischen Ingrid und Lorenz die Persönlichkeitsbildung (oder (Heilung)) im Zentrum steht, stellt sich dies bei Salomé und Edith anders gelagert dar. Denn während sich alle drei zuvor beschriebenen Modi der Bearbeitung mit dem Prozessieren der Person der Studentin befassen, zielt der Modus im Gespräch zwischen Salomé und Edith vielmehr auf die Aufrechterhaltung des Settings. Durch die formale Erfüllung oder Bearbeitung seiner selbst legitimiert sich das Setting «Mentoratsgespräche» im vorliegenden Gesprächsmodus gleichsam selbst. Die beiden Akteurinnen verfolgen mit der Abarbeitung eine pragmatische Version von Mentoratsgespräch und lassen sich hierbei gegenseitig «in Ruhe`.

Im Gespräch zwischen Salomé und Edith lässt sich allerdings auch ein Bruch im Bezugnahmemodus feststellen, und zwar an der Stelle, an der die Mentorin die Studentin fragt, ob es noch andere Dinge gebe, die ihr auf der Seele lägen und sie besprechen wolle. Das studentische Anliegen, das Thema «Elternarbeit» im Curriculum des Studiums stärker zu berücksichtigen, lässt sich nicht bruchlos im zuvor etablierten und bis zu diesem Zeitpunkt übereinstimmend oder abgestimmt verfolgten Abarbeitungsmodus behandeln. Das Anliegen wird der Studentin in der Folge zur individuellen Be- oder Abarbeitung zugeschrieben, wodurch die Abarbeitungsstruktur des Gesprächs wiederhergestellt wird. Insgesamt lässt sich festhalten, dass in den ersten drei Gesprächen ein Gesprächsanliegen hergestellt wird, das fallspezifisch unterschiedlich ausfällt. Im letzten Gespräch hingegen wird ein solches Anliegen am Gesprächsende zwar aufgeworfen, der Studentin jedoch zur eigenverantwortlichen Bearbeitung übergeben. Nicht das Thema an sich, sondern die Verantwortungszuschreibung wird dadurch zum bearbeiteten Gegenstand des Gespräches. 


\section{Modi der Bezugnahme auf das Setting: Zwischenfazit}

Im Sinne eines Zwischenfazits lässt sich an dieser Stelle festhalten, dass die strukturelle Problemlage der Legitimierungsbedürftigkeit mit unterschiedlichen Modi bearbeitet wird, die Ähnlichkeiten zu anderen, etablierten Settings wie Unterricht, Prüfung und Bewährung oder Therapie aufweisen oder der Anderslogik einer vertragserfüllenden, rechenschaftsablegenden Abarbeitung folgen. Das Format «Mentoratsgespräche» ist Bestandteil einer Ausbildung; aus programmatischer Perspektive betrachtet kann einem solchen Format ein Anspruch auf Personenoptimierung zugeschrieben werden. Diesem Anspruch des Prozedierens einer personalen Entwicklung wird empirisch in je spezifischer Weise begegnet. So wird dieser durch Dynamiken angestossen, die an Vermittlungs-, Prüfungs- oder auch Selbstoptimierungssituationen erinnern. In einem Modus ist dieser Anspruch allerdings empirisch kaum erkennbar, da hier eher das Format und nicht die Person prozediert wird. Zugleich gilt es noch einmal explizit darauf hinzuweisen, dass die herausgearbeiteten Modi der Bezugnahme als idealtypische Zuspitzungen zu verstehen sind. In der konkreten Gesprächsinteraktion manifestieren sie sich weniger prägnant und lassen sich weniger distinkt von anderen Logiken oder Interaktionsmodi abgrenzen als dies in der vorhergehenden analytisch ausgerichteten Darstellung der Fall war.

Die nachfolgende Diskussion erfolgt entlang der Typologien, die aufscheinen, wenn Eigenlogiken sich in Teilen und Aspekten Anderslogiken wie «Unterricht», "Prüfung \& Bewährung», "Therapie» und «Abarbeitung» bedienen und nicht mehr wie bisher auf der Grundlage einer Betrachtung der Einzelfälle.

\subsubsection{Aspekte und Modi der Bezugnahme aufeinander}

Bei den Aspekten und Modi der Bezugnahme aufeinander gilt es zu klären, in welcher Form das soziale Miteinander oder die soziale Interaktion Gestalt gewinnt. Diesbezüglich interessiert besonders die Frage, wie sich die am Gespräch Beteiligten aufeinander beziehen und wie sie das Gegenüber jeweils adressieren, re-adressieren und sich der vorgenommenen Adressierung entsprechend selbst positionieren. 
In allen erhobenen Gesprächsdaten wird das Gespräch von der Mentorin oder dem Mentor initiiert. Dies ist aus interaktionslogischer Perspektive auch zu erwarten, weil sie oder er die hierarchisch höher gestellte Position einnimmt. Die Mentorin oder der Mentor verantwortet das Gespräch institutionell wie auch organisatorisch und wird von der Hochschule auch für diese Aufgabe mandatiert. Mit der Gesprächseröffnung wird für die Studentin ein Möglichkeitsraum eröffnet, zu dem sie sich ihrerseits mit einer ReAdressierung verhalten muss. Daran schliesst die Mentorin oder der Mentor erneut an und auf diese Weise setzt sich die Interaktion fort. Dadurch gestaltet sich der Gesprächsmodus wechselseitig und als gemeinsame Konstruktion, obwohl die Strukturierungsmacht näher bei der Mentorin oder dem Mentor zu verorten ist.

In Anlehnung an Oevermanns Begriff der «widersprüchlichen Einheit» zwischen spezifischen und diffusen Beziehungskomponenten, den er für die Bestimmung des Arbeitsbündnisses zwischen der Therapeutin oder dem Therapeuten und der Klientin oder dem Klienten verwendet (Oevermann, 1996b, S. 115), lässt sich in den analysierten Mentoratsgesprächen in der gegenseitigen Bezugnahme eine widersprüchliche Einheit von Sein-Sollen und Nicht-sein-Dürfen erkennen. Interaktiv gewinnen Anforderungen Gestalt, die Dynamiken im Sinne von sowohl-als-auch und weder-noch, das heisst das eine tun, ohne das andere zu lassen, beinhalten. Das Spannungsvolle und entsprechend auch Komplexe dabei ist die gleichzeitige Aufrechterhaltung zweier Logiken, die sich grundsätzlich ausschliessen oder widersprechen. Diese Widersprüchlichkeit lässt sich nicht auflösen, sondern lediglich in der Gesprächsinteraktion bearbeiten. Empirisch kommt diese Gleichzeitigkeit beispielsweise in Vermeidungsbewegungen oder aber auch in changierenden Bewegungen zum Ausdruck, die in den Gesprächen in fallspezifisch unterschiedlichen Figuren und Ausprägungen aufscheinen.

Für die Diskussion dieser Vermeidungs- und Changierfiguren bieten sich Gegensatzpaare aus Helspers antinomischen Handlungsstrukturen (z.B. 1996, 2002) an, die er exemplarisch für das Lehrhandeln formuliert hat (siehe Kapitel 2.3.3.2). Da auch in Mentoratsgesprächen antinomische Handlungen aufscheinen, werden Helspers Antinomien im Sinne einer Vergleichsfolie für die Diskussion herangezogen.

Auch weitere Begriffspaare, die sich auf ein Paradoxon beziehen oder in sich eine Widersprüchlichkeit aufweisen, lassen sich für die Diskussion hinzuziehen. So lässt das Setting «Mentoratsgespräche» beispielsweise sowohl Informalisierung als auch 
Formalisierung zu und erfordert beides bis zu einem gewissen Grad, versucht zugleich jedoch, das eine oder andere zu vermeiden. Die Komplexität rührt in diesem Beispiel somit aus der Notwendigkeit, zugleich informalisierend wie auch formalisierend zu handeln. Beide antinomischen Pole sind aus strukturtheoretischer Perspektive grundlegend erforderlich und auch gleichwertig (Helsper, 1996, 2004; Oevermann 1996b).

Die Dimensionen dieser Antinomien, Paradoxien oder Widersprüchlichkeiten werden nachfolgend aus darstellungstechnischen Überlegungen in Verbindung mit der analytischen Kategorie «Aspekte und Modi der Bezugnahme aufeinander» diskutiert, da sich diese als Bewegungen und Dynamiken primär in diesem Zusammenhang Ausdruck verschaffen. Zugleich ergeben sich aber auch Implikationen hinsichtlich der analytischen Kategorie «Aspekte und Modi der Bezugnahme auf das Setting» respektive mit Blick auf die die bereits im vorangehenden Kapitel dargelegte Art und Weise, in der die beteiligten Akteurinnen und Akteure das Setting hervorbringen. Aus der Empirie abgeleitet bieten sich die folgenden Dimensionen an, um die Spannungsfelder und antinomischen Strukturen dieser Gespräche zu diskutieren:

- Informalisierung versus Formalisierung;

- Diffusität versus Spezifität;

- Symmetrie versus Asymmetrie.

Andere Spannungsfelder und Antinomien, die sich bei einer theoretischen Betrachtung des Formats als relevant erweisen würden (siehe Kapitel 2.1.3), bieten sich in Anbetracht der empirischen Daten nicht explizit als Kategorien zur Diskussion der strukturellen Problemlage an. Als Beispiele für weitere, aus theoretischer Perspektive vermutbare Spannungsfelder können unter anderem der Bewertungsaspekt oder auch die fixe und nicht frei wählbare Zuteilung zu Mentoratspersonen genannt werden. In den Gesprächsinteraktionen lassen sich jedoch wie bereits festgehalten keine Hinweise darauf finden, dass diese theoretisch antizipierbaren Problematiken in den Gesprächen tatsächlich manifest werden, weshalb sie hier nicht diskutiert werden.

Die Dimensionen «Informalisierung-Formalisierung», «Diffusität-Spezifität» und «Symmetrie-Asymmetrie» unterliegen keiner hierarchischen Ordnung. Das heisst, die 
gewählte Reihenfolge dient einzig der systematischen Strukturierung der Ausführungen, soll jedoch keine Wertung oder Gewichtung implizieren. Des Weiteren sind die Dimensionen lediglich als analytisch distinkt aufzufassen, da sie meist miteinander verwoben sind und deutliche Überschneidungen aufweisen. So geht eine formale Adressierung der Studentin beispielsweise eher mit spezifischen und nicht mit diffusen Beziehungsanteilen einher. Dennoch lässt sich mit einer analytischen Trennung eine Fokussierung vornehmen, die mit Blick auf den Erkenntnisgewinn einen Mehrwert aufweist. So bezieht sich die Dimension «Symmetrie-Asymmetrie» auf ein hierarchisch strukturiertes Phänomen, während die Dimension «Diffusität-Spezifität» die Rolleneinnahme in den Blick nimmt und die Dimension «Informalisierung-Formalisierung» den Fokus eher auf die Einrichtung des Formats lenkt.

Ähnlich wie bei den Begriffspaaren «hell» und «dunkel» existieren auch bei diesen Dimensionen Zwischentöne. Allerdings geht es in der Kontrastierungsdiskussion nicht primär um das Aufzeigen, wo die vier Modi auf den jeweiligen Dimensionen zu verorten sind. Der Fokus richtet sich vielmehr darauf, modusspezifisch darzustellen, wie das Dynamische, das Spannungsvolle, das Sowohl-als-auch beispielsweise bei «SymmetrieAsymmetrie» in einem Gesprächsmodus zum Ausdruck kommt. Hier gilt es zu zeigen, wie die Gleichzeitigkeit im Spannungsfeld zwischen den Polen interaktiv bearbeitet wird.

Wie sich die Dimensionen konkret in den Daten manifestieren, wird in Kapitel 5.2.2.4 anhand einer tabellarischen Darstellung illustriert. Diese Übersicht beruht auf den nachfolgenden Einzelbetrachtungen der drei als Konstrukte zu verstehenden Dimensionen. Das Ziel besteht darin, die Eigenlogiken der vier Modi mit Blick auf die jeweiligen Spannungsfelder aufzuschlüsseln, damit in der Tabelle schliesslich eine Reduktion oder Zuspitzung jedes einzelnen Modus in Bezug auf die drei Dimensionen vorgenommen werden kann. Dabei geht es dezidiert nicht darum, das Zusammenspiel der aus der Eigenund Anderslogiken erwachsenen Strukturen theoriearchitektonisch stillzustellen und einseitig zu schliessen, sondern das Dynamische innerhalb der drei Dimensionen beizubehalten. 


\subsubsection{Dimension «Informalisierung-Formalisierung»}

Zwischen den Polen der Informalität und der Formalität erstreckt sich ein Kontinuum, innerhalb dessen sich Informalisierung und Formalisierung als Bewegung und Dynamik manifestieren. Die Formalisierung bezieht sich primär auf die Art und Weise, wie das Gegenüber angesprochen und adressiert wird, oder darauf, wie die Interaktion mit dem Gegenüber aufgebaut ist. Die formalisierende Adressierung orientiert sich an vorgegebenen Verfahrensweisen: Tätigkeiten laufen reguliert, vorschriftsmässig, bürokratisch und, alltagssprachlich formuliert, geordnet ab. Diese Konzeption ist auf den Organisationstheoretiker Weber und sein Bürokratiemodell zurückzuführen (Weber, 1925). Wesentlich in Webers idealtypischem Modell sind die Regelgebundenheit und die Unpersönlichkeit der Amtsführung sowie die Aktenmässigkeit der verwaltungstechnischẹ Abläufe (Weber, 1925). Der gegenüberliegende Pol der Informalisierung lässt sich ex negativo als Nichtformalisierung bestimmen.

Mit der in im Begriff des Kontinuums implizierten Gleichzeitigkeit von variierenden Graden an Informalisierung und Formalisierung scheinen auch ein Motiv der Nähewahrnehmung wie auch ein Motiv der Distanzwahrnehmung einherzugehen. Da die Dimension «Informalisierung-Formalisierung» - wie bereits ausgeführt - eine starke Überschneidung mit der Dimension «Diffusität-Spezifität» aufweist, wird der Fokus bei der Einzelbetrachtung nachfolgend vor allem auf das Regulativ, die Verfahrensabläufe und die interaktiv etablierte Ordnung gerichtet.

Modusübergreifend zeigt sich die Schwierigkeit in Bezug darauf, das dyadische Gespräch informell oder informalisierend zu gestalten, was in unterschiedlichen Ausprägungen von Formalisierungsbewegungen Gestalt gewinnt. Diese entlasten das Gespräch zugleich von Nähe und Diffusität oder kontrollieren solche Dynamiken zumindest. Das Formalisierende scheint mehr Sicherheit zu geben oder eine potenzielle Unsicherheit bereits im Vorfeld einzugrenzen. Bei drei von vier aufgezeigten Gesprächsmodi zeigt sich allerdings, dass formalisierenden und formalisierten Gesprächsanteilen umgehend mit Informalisierungsbewegungen begegnet wird, wodurch interaktive Changier- und Vermeidungsdynamiken sichtbar werden.

Nachfolgend wird konkret aufgezeigt, wie sich diese Dynamiken in den Modi Ausdruck verschaffen. Zu diesem Zweck wird auch die Gesprächseröffnung und die darin enthaltene 
erste Adressierung der Studentin und die darin erkennbare Informalisierungs- respektive Formalisierungsbewegung miteinbezogen.

\section{Anderslogik «Unterricht»}

Im Gespräch, das sich einer an Unterricht angenäherten Anderslogik bedient, werden bereits in der Gesprächseröffnung ich zeige dir wo wir hingehen Formalisierung und Strukturierung erkennbar. Diese Gesprächseröffnung kontrolliert respektive begrenzt eine potenzielle Ungewissheit oder Unkontrollierbarkeit der dyadischen Gesprächssituation. Solche auch im weiteren Gesprächsverlauf aufscheinenden Formalisierungsbewegungen verschaffen sich unter anderem in Verallgemeinerung, beispielsweise in Man- und Passivformulierungen, Ausdruck und verweisen auf gültige Normen. Allerdings scheint es, als würden solche Formalisierungsbewegungen umgehend durch gegenteilige Informalisierungsbewegungen bearbeitet. In Letzteren gewinnt eine fürsorglichanleitende, Empathie bekundende und vergemeinschaftende Adressierung Gestalt, die an die Anderslogik von Unterricht erinnert.

\section{Anderslogik «Prüfung \& Bewährung»}

Mit der Gesprächseröffnung ich begrüsse Sie recht herzlich wird ein hochoffizielles Setting installiert, das für eine dyadische Gesprächssituation auffällig formell und distanziert anmutet. Mit diesem Gesprächseinstieg gewinnen etwas Überschüssiges und zeitgleich auch ein Motiv der Formalisierung wie auch der Distanzwahrnehmung Gestalt. Die Gesprächssituation scheint dadurch von informalisierenden und diffusen Anteilen, die eine dyadischen Situation konstitutiv beinhaltet, entlastet zu sein. Auch im weiteren Gesprächsverlauf wird mit der Installation einer Prüfungs- und Bewährungsanlage die antinomische Handlungsstruktur von Informalisierung und Formalisierung weitgehend formalisierend und distanzierend bearbeitet, wodurch potenzielle Informalisierungstendenzen stillgestellt werden.

\section{Anderslogik «Therapie»}


Der Mentor steigt mit der Gesprächseröffnung ich darf noch ein bisschen Kaffee trinken das stört dich nicht informalisierend in das Gespräch ein. In der Gesprächsfortsetzung sind jedoch Changierdynamiken zwischen Informalisierung und Formalisierung erkennbar. Dies zeigt sich, indem eine informalisierende, Nähe herstellende Geste umgehend mit einer formalisierenden, Distanz herstellenden Geste bearbeitet respektive ausgeglichen wird. In dieser Gesprächsinteraktion gewinnt eine Dynamik Gestalt, in der ein Testangebot unterbreitet wird, das Bestandteil eines therapeutischen Settings sein respektive zu einem therapeutischen Setting führen kann. Gleichzeitig wird jedoch die Offensichtlichkeit dieser Angebotsunterbreitung und damit einhergehend die Einnahme der Rolle des Therapeuten vermieden. Diese Gleichzeitigkeit des Ein-Angebot-sein-Sollens und Kein-Angebot-sein-Dürfens löst eine Bewegung in Richtung Informalisierung aus. Die vorgefundenen Informalisierungsdynamiken können als Versuche gelesen werden, ein Setting aufzubauen, das Nähe und Vertrauen schafft, eine mögliche Distanz verringert und so die Unterbreitung des Angebots eher zulässt oder ermöglicht. Mentor und nicht Therapeut zu sein und dennoch ein Angebot zu unterbreiten scheint somit ein Bemühen um Informalisierung zu erfordern, ist gleichzeitig aber auch auf gegenteilige, formalisierende Bewegungen angewiesen, weil es sich nicht um ein therapeutisches Setting handelt und es deshalb gilt, die informalisierenden Dynamiken auch wieder zu bearbeiten und entkräften.

\section{Anderslogik «Abarbeitung»}

Auch die Gesprächseröffnung und wie läuft's im Partnerschulprojekt lässt sich als informalisierendes Kontaktaufnahmeangebot und kollegiale Adressierung lesen. Die Gleichzeitigkeit von Informalisierung und Formalisierung zeigt sich im Abarbeitungsmodus wie folgt: Die Formalisierungsdynamiken lassen sich vor allem in der geordneten und vollständigen Abarbeitung von Agendapunkten erkennen, während die Informalisierungsdynamiken mehrheitlich die Art und Weise der beidseitigen Adressierung betreffen. Diese erfolgt auf einer manifesten Bedeutungsebene zwar informell und kollegial, (umschifft) auf einer latenten Bedeutungsebene innerhalb der Kollegialität jedoch Nähe und vermeidet eine «echtes Kontaktaufnahme. 


\subsubsection{Dimension «Diffusität-Spezifität»}

Moderne Organisationen oder Individuen wählen - idealtypisch betrachtet - bewusst oder unbewusst zwischen dichotomen Entscheidungs- oder Orientierungsalternativen, die Parsons in Anlehnung an die und Erweiterung der Merkmale des weberschen Bürokratiemodells mit «pattern variables» bezeichnete (Parsons et al., 1954). Eine solche Alternative ist diejenige der funktionalen Diffusität im Unterschied zur funktionalen Spezifität, die nachfolgend näher betrachtet wird.

Das von Oevermann (z.B. 2002b, S. 40) beschriebene Phänomen der diffusen und spezifischen Sozialbeziehungen entstammt ursprünglich Parsons Theorie (ausführlich zu «diffus» und «spezifisch» siehe Kapitel 2.3.3.2). Die beiden antagonistischen Orientierungsalternativen sind entweder die der Form nach rollenförmige Bezugnahme, das heisst der Versuch einer Versachlichung der Beziehung, oder aber die Orientierung an der ganzen Person. Wie umfassend sich das Interesse an der anderen Person innerhalb einer sozialen Interaktion gestaltet, ist aus struktureller Perspektive betrachtet eine Frage der Angemessenheit. Auch hier bewegen sich die beteiligten Akteurinnen und Akteure auf einem Kontinuum zwischen dem Pol der Diffusität, der sich in einer Adressierung der gesamten anderen Person ausdrückt, und dem Pol der Spezifität, bei dem die andere Person in einem definierten Bereich und in begrenztem Umfang und somit in ihrer Rolle adressiert wird (Parsons, z.B. 1951, S. 65 f.). Den Mittelweg, der innerhalb des Kontinuums möglich wird, charakterisiert Patry gemäss Aristoteles' mesotischem Prinzip mit «nicht zu viel und nicht zu wenig» (Patry, 1997, S. 75, zitiert nach Nerowski, 2012, S.142). Oevermann wiederum betont, dass eine mangelnde Professionalisierung im Sinne einer «distanzlosen Verkindlichung» oder des «technologischen, wissensmässigen und verwaltungsrechtlichen Expertentums» vorliege (Oevermann, 1996b, S. 155), wenn der Spagat, den die «widersprüchliche Einheit von diffusen und spezifischen Sozialbeziehungen» (Oevermann, 1996b, S. 148) verlange, nicht vollführt werden könne (Oevermann, 1996b, S. 155). Darin zeigt sich die Fragilität dieses Balanceaktes zwischen einem Zuviel und einem Zuwenig an Diffusität oder Spezifität respektive der 
Herausforderung eines angemessenen und professionellen Umgangs mit diesen beiden Polen.

Um aufzeigen zu können, wie sich diese widersprüchliche Einheit von Diffusität und Spezifität im Arbeitsbündnis zwischen Studierenden und Mentorierenden zeigt, wurde in Kapitel 2.4 das Arbeitsbündnis der Therapie, in dem diese widersprüchliche Einheit institutionalisiert abgesichert ist, mit dem Arbeitsbündnis im Mentorat ins Verhältnis gesetzt und verglichen. Auf einer theoretischen und konzeptionellen Betrachtungsebene scheint das Ausbildungsformat "Mentoratsgespräche» grundsätzlich eher spezifisch disponiert zu sein. Gleichzeitig präfiguriert ein Vieraugengespräch auf einer programmatischen Ebene auch Diffusitätspotenziale. Diese widersprüchliche und ungeklärte Disposition, das heisst das Potenzial einer Ausdehnung der Diffusität in einem tendenziell eher spezifisch angelegten Setting, wird in den vier Interaktionsmodi sehr unterschiedlich bearbeitet und kontrolliert und nachfolgend modusspezifisch dargelegt.

\section{Anderslogik «Unterricht»}

Die formalisierte Gesprächseröffnung ich zeige dir wo wir hingehen lässt wenig Raum für Nähe und Spontaneität. Vielmehr werden die in einem dyadischen Setting potenziell angelegten diffusen Anteile mit dem vorliegenden Gesprächseinstieg eingegrenzt und handhabbar gemacht. Die Absicherung einer diffusen Begegnung scheint nicht bereits institutionell gewährleistet zu sein, sondern erfordert eine performative Bearbeitung oder Instandsetzung eines Gleichgewichts zwischen Diffusität und Spezifität. Einerseits werden mithilfe dieser Changierfigur diffuse Gesprächsanteile verfahrensförmig zurückgefahren und andererseits scheinen auch Dynamiken einer pädagogisch-fürsorglichen Nähe gegenüber der Studentin auf. Diese Nähe weist auch spezifische, rollenförmige Elemente auf, das heisst, der an die Anderslogik von Unterricht angenäherte Interaktionsmodus bedient die beiden antinomischen Pole Nähe und Distanz wie auch Diffusität und Spezifität gleichzeitig. In Differenz zur Anderslogik des schulischen Unterrichts sind die Studierenden in der vorliegenden sozialen Situation von Mentoratsgesprächen als erwachsene Personen jedoch in der Lage, zwischen diffusen und rollenförmigen Sozialbeziehungen zu unterscheiden. Den diesbezüglichen Schutz der hierarchisch höher gestellten Person brauchen sie deshalb weniger als im schulischen Kontext. Die hier 
sichtbar werdende Changierbewegung lässt sich folglich nicht mit der widersprüchlichen Anforderung, sowohl «emotional-diffuse, partikulare [...] und spezifisch-rollenförmige Haltungen» einzunehmen (Helsper, 2002, S. 75) erklären; vielmehr lässt sich darin eine Schwierigkeit der sozialen Situation von Mentoratsgesprächen erkennen. Mit anderen Worten beziehen sich die hier aufscheinenden widerstreitenden Anforderungen auf die Schwierigkeit, eine pädagogische Situation unter Erwachsenen zu gestalten, sich zeigendfürsorglich und gleichzeitig kollegial auf Augenhöhe zu begegnen. Die Changierbewegung scheint somit eine etwas andere Motivlage zu bedienen als diejenige des schulischen Kontextes.

\section{Anderslogik «Prüfung \& Bewährung»}

Die grundsätzlich antinomische Anlage der Handlungsstruktur, das heisst das gleichzeitige Sowohl-als-auch und Weder-noch, wird in Modus «Prüfung \& Bewährung» vom Mentor gerade nicht antinomisch bearbeitet, sondern das Antinomische wird in diesem Modus weitgehend stillgelegt oder vermieden. Dies zeigt sich auch in den beiden anderen Dimensionen «Informalisierung-Formalisierung» und «Symmetrie-Asymmetrie». Die Stillegung oder Vermeidung tritt jedoch in der Dimension «Diffusität-Spezifität» besonders ausgeprägt in Erscheinung und entlastet die Gesprächssituation von dyadischen Anteilen und der Involviertheit des Mentors als ganzer Person.

Die «Spielanlage` des Modus «Prüfung \& Bewährung» in der Anfechtung ist einseitig auf die Studentin ausgerichtet; eine Gegen- oder Wechselseitigkeit ist nicht erkennbar. Dabei hat sich die Studentin grundsätzlich in der Diffusität ihrer ganzen Person zu bewähren und zu verteidigen. Sie spielt das Bewährungsspiel über weite Strecken mit, ohne sich diesem jedoch ganz zu unterwerfen. Im Fortgang des Gesprächs zeigen sich zunehmend Dynamiken, die es ihr ermöglichen, sich in der Diffusität der ganzen Person weniger angreifbar zu machen und die ihr zugetragene Rolle im Spiel einzunehmen, ohne sich diffus zu involvieren. Dies gelingt ihr in unterschiedlichem Ausmass. Zugleich zeigen sich allerdings auch bei ihr Distanzierungsfiguren bezogen auf Interesse und Identifikation, die eine diffuse Beziehungsstruktur vermeiden.

Wie bereits aufgezeigt, wird die antinomische Handlungsstruktur im Bezugnahmemodus, der an die Anderslogik «Prüfung \& Unterricht» erinnert, beinahe stillgestellt. Der Mentor 
verhält sich über weite Strecken rollenförmig und spezifisch und vermeidet diffuse Beziehungsanteile mehrheitlich. Dennoch weist auch dieser Modus Brüche oder Changierdynamiken auf. Dies zeigt sich beispielsweise dann, wenn der Mentor mit der Provokation aber das [Mentorat S.H.] könnte man ja auch abschaffen diffus eine Bestätigung seiner selbst und spezifisch für seine Art von Mentoring einfordert.

\section{Anderslogik «Therapie»}

Wie bereits ausgeführt, legitimiert sich in einem therapeutischen Arbeitsbündnis die diffuse Begegnung durch die (Not〉 der Klientin oder des Klienten und ihr oder sein Bedürfnis, diese «Not` mithilfe der Therapeutin oder des Therapeuten im Sinne einer stellvertretenden Krisenbewältigung zu lindern. Im Gegensatz dazu können die Voraussetzungen für eine entsprechende diffuse Begegnung im vorliegenden Modus nicht als institutionell gesichert erachtet werden, da es sich hierbei nicht um ein Therapie-, sondern um ein Mentoratsgespräch handelt. Dies führt zu Diffusitätsbemühungen respektive zu einer permanenten Bearbeitung dieser Problematik der nicht gesicherten, diffusen Begegnungsmöglichkeit. Hierbei lässt sich aufseiten des Mentors eine Figur des Buhlens um Anerkennung und Gemocht-Werden, einerseits diffus als ganze Person mit eigenen, persönlichen Erfahrungen, andererseits auch in der spezifischen Rolle des Therapeuten mit seiner diesbezüglichen Expertise, feststellen. Insgesamt zeigt sich, dass die emotionale Bedürftigkeit des Mentors die vermeintlich professionelle Beziehung diffundieren und emotionalisieren lässt. Die Studentin verhält sich zur Problematik der diffusen Ausdehnung opportunistisch. Sie lässt diese zu respektive fordert sie sogar ein, wenn ihr diese eine Bestätigung bezogen auf ihre Art des Studierens bietet. Gleichzeitig lässt sie den Mentor mittels Schliess- und Distanzierungsbewegungen auch ins Leere laufen, indem sie auf seine Vergemeinschaftungsangebote nicht eingeht.

\section{Anderslogik «Abarbeitung»}

Im Abarbeitungsmodus lässt sich primär eine beidseitig distanzierende oder zumindest Diffusität wie auch Nähe vermeidende oder cumschiffendes Dynamik erkennen. Gleichzeitig gewinnt ein Bedarf nach und ein Anspruch auf sichtbar gemachte Zugewandtheit oder auch Fürsorge Gestalt. Infolgedessen stehen auch diffuse Potenziale im Raum und 
lassen eine ausschliesslich spezifische Bearbeitung nicht zu. Diese Gleichzeitigkeit führt zu Widersprüchlichkeiten und Spannungen, da sowohl Diffusitätsbemühungen als auch Notwendigkeiten einer spezifischen Bearbeitung aufscheinen. Im Konkreten kann das bedeuten, dass auf eine sichtbar werdende Bemühung einer Begegnung in der Diffusität der ganzen Person umgehend eine rollenförmige, schliessende und distanzierende Gegenbewegung folgt. Diese Bewegung und Gegenbewegung um die «Gefahrenzone` von Nähe und Diffusität lassen sich metaphorisch mit einem «Tanz um ein unsichtbares Feuer` beschreiben.

Die Gleichzeitigkeit eines Vermeidens und synchronen Bedienens von Diffusität einerseits und eines Vermeidens und synchronen Bedienens von Spezifität andererseits führt zu Changierbewegungen an beiden Polen. Diese Gleichzeitigkeit von Sein-Sollen und Nichtsein-Dürfen manifestiert sich empirisch auch auf dem Spannungsbogen von Symmetrie und Asymmetrie» (siehe Kapitel 5.2.2.3).

In der dritten Sequenz gewinnt im bisher etablierten und problemlos durchgeführten Abarbeitungsmodus eine neue Dynamik Gestalt: Das von der Studentin identifizierte Ausbildungsdefizit (Elternarbeit) stösst bei der Mentorin auf ein Kränkungsmotiv. Diese Kränkung zeigt sich in der betonten Nicht-Zuständigkeit der Mentorin. Eine Kränkung trifft die Diffusität der ganzen Person. An dieser Stelle zeigt sich, dass es der Mentorin nicht gelingt, eine rollenförmige und sachliche Gegenbewegung aufzuführen. Die geschmeidige Tanzfigur scheint kurzfristig gefährdet zu sein. Mit der Zuschreibung der eigenverantwortlichen Bearbeitung dieses Ausbildungsdefizit an die Studentin findet die Mentorin jedoch umgehend in ihre Rolle zurück, da eine solche Zuschreibung nur aus einer rollenförmigen und hierarchisch höher gestellten Position heraus vorgenommen werden kann. In der Folge kann die diffus-spezifische Tanzfigur als wiederhergestellt und auch diese Thematik als abgearbeitet betrachtet werden.

\subsubsection{Dimension «Symmetrie-Asymmetrie»}

Bei der Beschreibung der Dimension «Symmetrie-Asymmetrie» kann an Helspers Symmetrieantinomie (z.B. 2016, S. 55) angeschlossen werden. Die Dimension bezieht sich auf die antinomische Struktur der überlegenen Position der oder des Professionellen 
einerseits und des Anspruchs auf eine symmetrische Begegnung andererseits. Diese konstitutive Asymmetrie gilt sowohl für die Anderslogik einer Beziehung zwischen einer Therapeutin oder einem Therapeuten und einer Klientin oder einem Klienten als auch für die Anderslogik der Beziehung zwischen einer Lehrperson und ihren Schülerinnen und Schülern, die beide bereits zuvor für die Beschreibung der Modi der Bezugnahme auf das Setting hinzugezogen wurden. Folgt man Helsper, braucht es gerade im LehrpersonenLernenden-Setting für Lern- und Entwicklungsprozesse Begegnungen auf Augenhöhe, da lediglich solche Begegnungen entsprechende Prozesse erst ermöglichen (Helsper, 2016, S. 112). In einem solchen Setting muss beispielsweise die grundlegende Asymmetrie, das heisst das Gefälle von Wissen, Macht, Erfahrung und Kompetenz zwischen Lehrperson und Lernenden, immer wieder «kontrafaktisch symmetrisiert» werden, damit Lernen und Entwicklung stattfinden können (Helsper et al., 2007, S. 509). Eine vergleichbare Dynamik zeigt sich auch für Symmetriebemühungen in einer therapeutischen Konstellation.

Bei der Diskussion der Dimension «Symmetrie-Asymmetrie» in Mentoratsgesprächen kann an die Beziehung zwischen Lehrperson und Lernenden angeschlossen werden, da es sich in beiden Settings um eine strukturell asymmetrische Situation handelt, die eine Schwierigkeit mit der Asymmetrie aufweist oder in der diese Asymmetrie zumindest einer Bearbeitung bedarf. Wenn man Helspers Konzept der Symmetrisierungsbemühungen auf das Mentoratssetting überträgt, bedeutet dies, dass die asymmetrische Disposition auch hier einer Bearbeitung bedarf respektive Symmetriebemühungen erfolgen müssen, um entsprechende Professionalisierungsprozesse zu initiieren. Denn wie bereits im Zusammenhang mit der Dimension «Diffusität-Spezifität» aufgezeigt (siehe Kapitel 5.2.2.2), stellt das Mentorat - trotz asymmetrischer Struktur - eine erwachsenenpädagogische Situation dar. Unter Erwachsenen gilt es möglicherweise auch, Kollegialitätsanforderungen zu bearbeiten, was sich ebenfalls in Symmetriebemühungen manifestiert. Zumindest in den vorliegenden Daten zeigt sich empirisch eine Problemlage, die darin besteht, das Setting ausschliesslich asymmetrisch zu gestalten. Dies führt insgesamt zu Dynamiken der Verschleierung dieser grundsätzlich asymmetrischen Konstellation, die allerdings auch wieder brüchig sind, weil faktisch eine Asymmetrie vorliegt.

Als zentraler Befund zur Dimension «Symmetrie-Asymmetrie» lässt sich festhalten, dass die faktisch vorliegende asymmetrische Beziehungsstruktur zwischen Studierenden und 
Mentorierenden eine gewisse Prekarität aufweist. Dies führt beispielsweise zu Dynamiken zur Vermeidung der offensichtlich vorhandenen Asymmetrie und damit einhergehend zu Symmetrisierungsbemühungen. Auch Changierbewegungen innerhalb des Spannungsfeldes sind in unterschiedlichen Ausprägungen erkennbar. In einem Modus, und zwar demjenigen der an eine Prüfungs- und Bewährungslogik erinnert, ist die Asymmetrie bereits strukturell angelegt und kann über weite Strecken bruchlos installiert und aufrechterhalten werden. Dieser Gesprächsmodus erscheint allerdings ungewohnt machtvoll und ihm haftet auch ein leicht unfaires Element an. In den Rekonstruktionen der anderen drei Modi der Bezugnahme ist demgegenüber eine Bearbeitung dieser Asymmetrieantinomie in Richtung Symmetrisierung zu erkennen, wenngleich diese Dynamik modusspezifisch unterschiedlich ausfällt. Wie diese Asymmetrie in den einzelnen Modi konkret bearbeitet respektive verschleiert wird, welche Bemühungen um die Herstellung von Symmetrie erfolgen und wie die Expertise- und Erfahrungsdifferenz unterschiedlich in Geltung gesetzt wird, wird nachfolgend dargelegt.

\section{Anderslogik «Unterricht»}

Im Bezugnahmemodus, der an die Anderslogik von Unterricht erinnert, sind die Changierund Suchbewegungen hinsichtlich der Dichotomie «Symmetrie-Asymmetrie» besonders ausgeprägt erkennbar. Die grundsätzlich asymmetrische und hierarchische Situation im Mentoratsgespräch wird in diesem Modus befestigt und mit Symmetriebemühungen zugleich auch verschleiert. Bei der Herstellung des Arbeitsbündnisses gewinnt eine deutliche Sowohl-als-auch und Weder-noch Dynamik Gestalt. Dies bedeutet, dass in der Beziehungsstruktur zwischen dem Mentor und der Studentin sowohl symmetrische als auch asymmetrische und weder symmetrische noch asymmetrische Anteile sichtbar werden. Dabei zeigt sich, dass die Expertise- und Erfahrungsdifferenz, die der Legitimierung des Settings dienlich ist, nicht bereits durch die Struktur des Settings geklärt ist, wie dies beispielsweise bei Unterricht der Fall wäre. Vielmehr bedarf diese Differenz einer interaktiven Herstellung und Bekräftigung, was hier in Anlehnung an die Anderslogik des Unterrichts erfolgt. So werden die Expertise und auch die Erfahrung des Mentors durch Zeigen, Lehre und Wissensvermittlung aufgezeigt, wodurch an der Anerkennbarkeit des Status des Mentors gearbeitet wird. Dieser Status scheint grundsätzlich unsicher und prekär zu sein, jedenfalls prekärer als derjenige der Studentin, und wird in einem relativ 
aufwendigen Prozedere legitimiert und aufrechterhalten. Die Asymmetriebekräftigung seitens des Mentors findet hauptsächlich in der Latenz statt, während sich eine Asymmetrieverschleierung eher auf einer manifesten Ebene erkennen lässt.

\section{Anderslogik «Prüfung \& Bewährung»}

Die Asymmetrie und damit einhergehend der für eine asymmetrische Situation vermutbare Expertisevorsprung wird vom Mentor über die Installation einer asymmetrischen Anlage bearbeitet, die an eine Prüfungs- und Bewährungssituation erinnert. In diesem Modus werden Symmetrieanforderungen oder auch entsprechende Ansprüchlichkeiten von vornherein ausgeschaltet respektive umgangen und der grundsätzlich vorliegenden antinomischen Handlungsstruktur wird einseitig asymmetrisch begegnet. Die Expertise des Mentors tritt in der Prüfungs- und Bewährungssituation nicht inhaltlich zu Tage, sondern sein Expertenstatus ist bereits durch die soziale Situation der Prüfung gewährleistet. Innerhalb dieser «Spielanlage` wird die Studentin mit wie schätzen Sie $X Y$ ein mehrfach als Expertin und aus einer vermeintlichen Symmetrie heraus angesprochen. Gerade durch diese Adressierung im Modus einer Anfrage an ihre Expertise erhält die Interaktion eine klar asymmetrische Prüfungs- und Bewährungsimplikation, die allerdings verschleiert wird.

\section{Anderslogik «Therapie»}

Im Bezugnahmemodus, der an die Anderslogik der Therapie erinnert, gewinnt eine Gleichzeitigkeit Gestalt, die sowohl die überlegene Position des Professionellen als auch den Anspruch einer Begegnung auf Augenhöhe bedient. Gerade diese aufscheinenden Symmetrisierungsbemühungen festigen allerdings die asymmetrische Konstellation, da sie nur in der Asymmetrie, die es gerade zu kaschieren gibt, möglich und nötig sind. Interaktiv wird seitens des Mentors ein beachtlicher Aufwand betrieben, um die eigene Position zu legitimieren und die gesuchte respektive erhoffte Anerkennung - einerseits für seine Art von Mentoring und andererseits für ihn als Person - zu erlangen. Dazu werden sowohl die fachliche Expertise als auch die persönliche Erfahrung in Geltung gesetzt.

\section{Anderslogik «Abarbeitung»}


Analog zu den in Kapitel 5.2.2.2 festgestellten Ansprüchlichkeiten hinsichtlich diffuser Elemente lassen sich im Bezugnahmemodus, der an eine "Abarbeitung» erinnert, auch Ansprüchlichkeiten auf symmetrisierende Elemente feststellen. Diese Bemühungen um Symmetrie, die sich möglicherweise auch aus Kollegialitätsanforderungen speisen, werden zeitgleich mit der Aufrechterhaltung von Asymmetrienotwendigkeiten bearbeitet, da asymmetrische Strukturen den Vertragserfüllungs- und Abarbeitungsmodus festigen.

Im vorliegenden Abarbeitungsmodus zeigt sich ein Bedürfnis der Mentorin nach Anerkennung als Rechenschaftsablegerin und Vertragserfüllerin, das weitgehend ohne persönliche Involviertheit auskommt. Gleichzeitig und ambivalent dazu sind auch Reziprozitätsherstellungsbemühungen seitens der Mentorin erkennbar. Dies zeigt sich in Dynamiken, mit deren Hilfe Asymmetrie möglichst umgangen und Symmetrie aufgezeigt wird. Das Legitimierungsproblem des Arbeitsbündnisses zwischen Mentorin und Studentin wird somit durch 'Umschiffung) der vorhandenen Asymmetrie bearbeitet. Im Vergleich zu den anderen drei Modi scheinen hierbei sowohl der Asymmetrieanspruch als auch die Symmetriebemühungen weniger dominant aufzutreten. Dies könnte darauf zurückzuführen, dass nicht primär die Person oder Rolle der Mentorin in Geltung gesetzt werden muss, sondern sich der Gesprächsfokus vorranging auf die Sache, auf die Abarbeitung und Vertragserfüllung richtet.

Allerdings zeigt sich diese Dynamik im Gesprächsverlauf auch brüchig, und zwar an der Stelle, wo der Abarbeitungsmodus nicht mehr aufrechterhalten werden kann und die persönliche Involvierung der Mentorin in das Gespräch zunimmt. Die Mentorin fasst den studentischen Wunsch nach mehr Elternarbieit im Curriculum als Kritik auf. Durch die damit einhergehende gerät die abzuarbeitende Sache in den Hintergrund. Gleichzeitig rücken die beiden beteiligten Personen in den Fokus, wobei die Mentorin jedoch wiederum versucht, die grundsätzlich asymmetrischen Situation zu festigen. An der Stelle dieses Moduswechsels erfolgt die Bearbeitung der Antinomie die Symmetrie und Asymmetrie betreffend analog zu den anderen drei Modi. 


\subsubsection{Tabellarische Darstellung der Aspekte und Modi der Bezugnahme}

Tabelle 2 ist als Reduktion der ausgeführten Bezugnahmemodi zu verstehen. Die Darstellung ermöglicht einen Vergleich der Dimensionen innerhalb eines Modus wie auch einen Vergleich einer Dimension zwischen den verschiedenen Modi. Die Sigel «M» steht dabei für «Mentorin» oder «Mentor» und «S» für «Studentin». Dabei sind diese empirisch herausgearbeiteten Spannungslinien als dynamisches, aber auch in sich brüchiges und fragiles Konstrukt zu verstehen. So lassen sich die analytisch herausgearbeiteten, aus dem Zusammenspiel von Eigen- und Anderslogiken erwachsenen dynamischen Strukturen nicht auf einen Pol hin schliessen, sondern die antinomische Dynamik wird beibehalten.

Tabelle 2: Aspekte und Modi der Bezugnahme

\begin{tabular}{|c|c|c|c|}
\hline $\begin{array}{l}\text { Modus } \\
\text { erinnert an } \\
\text { Anderslogik }\end{array}$ & $\begin{array}{l}\text { Informalisierung- } \\
\text { Formalisierung }\end{array}$ & $\begin{array}{l}\text { Diffusität- } \\
\text { Spezifität }\end{array}$ & $\begin{array}{l}\text { Symmetrie- } \\
\text { Asymmetrie }\end{array}$ \\
\hline Unterricht & $\begin{array}{l}\text { - Formalisierungs- } \\
\text { bewegung } \\
\text { kontrolliert dyadische } \\
\text { Gesprächssituation } \\
\text { - Informalisierungs- } \\
\text { bewegung ermöglicht } \\
\text { fürsorglich-anleitende } \\
\text { und vergemeinschaf- } \\
\text { tende Adressierung }\end{array}$ & $\begin{array}{l}\text { - Spezifitätsbewegung } \\
\text { hegt potenzielle } \\
\text { Diffusität ein und } \\
\text { fährt diese ver- } \\
\text { fahrensförmig zurück } \\
\text { - } \quad \text { (Pädagogisches Nähe } \\
\text { bedient beide Pole } \\
\text { der Antinomie von } \\
\text { Nähe und Distanz } \\
\text { gleichzeitig } \\
\text { Changierfigur durch } \\
\text { Instandsetzung und } \\
\text { Aufrechterhaltung } \\
\text { eines Gleichgewichts } \\
\text { zwischen Diffusität } \\
\text { und Spezifität }\end{array}$ & $\begin{array}{l}\text { - Asymmetriebewe- } \\
\text { gung befestigt } \\
\text { Hierarchie } \\
\text { - Symmetriebewegung } \\
\text { verschleiert Hierar- } \\
\text { chie } \\
\text { - Herstellung und } \\
\text { Bekräftigung von } \\
\text { Expertise und } \\
\text { Asymmetrie finden in } \\
\text { der Latenz statt, } \\
\text { manifeste Asym- } \\
\text { metrieverschleierung } \\
\rightarrow \text { Sowohl-als-auch } \\
\text { und Weder-noch } \\
\text { Dynamik }\end{array}$ \\
\hline $\begin{array}{l}\text { Prüfung \& } \\
\text { Bewährung }\end{array}$ & $\begin{array}{l}\text { ¿Über -Formalisie- } \\
\text { rungsbewegung } \\
\text { vermeidet dyadische } \\
\text { Interaktion und stellt } \\
\text { antinomische } \\
\text { Handlungsstruktur } \\
\text { weitgehend still } \\
\text { - Universalistische } \\
\text { Adressierung }\end{array}$ & 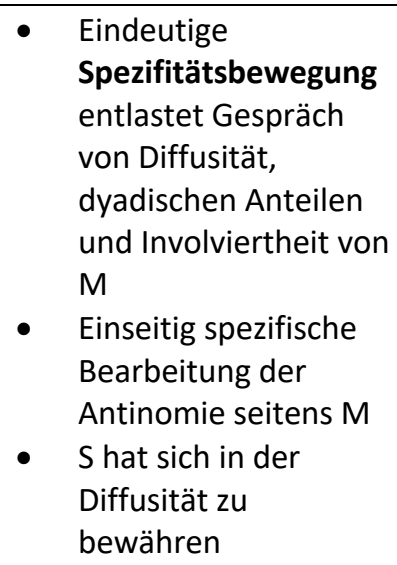 & $\begin{array}{ll}\text { - } & \text { Asymmetrische } \\
\text { Prüfungs- und } \\
\text { Bewährungsanlage } \\
\text { Einseitig spezifische } \\
\text { Bearbeitung der } \\
\text { Antinomie seitens M; } \\
\text { keine Symmetrie- } \\
\text { bemühungen }\end{array}$ \\
\hline
\end{tabular}




\begin{tabular}{|c|c|c|c|}
\hline Therapie & $\begin{array}{l}\text { Informalisierungs- } \\
\text { bewegung verringert } \\
\text { Distanz und schafft } \\
\text { Nähe und Vertrauen } \\
\text { - Formalisierungs- } \\
\text { bewegung bearbeitet } \\
\text { den Umstand, dass es } \\
\text { sich nicht um } \\
\text { therapeutisches } \\
\text { Gespräch handelt }\end{array}$ & $\begin{array}{l}\text { Diffusitätsbewegung } \\
\text { und -bemühung } \\
\text { schafft Voraussetzung } \\
\text { für ein therapeu- } \\
\text { tisches Arbeitsbündnis } \\
\text { Diffusitätsbewegung } \\
\text { zielt auf Anerkennung } \\
\text { der Person } \\
\text { - Spezifitätsbewegung } \\
\text { befestigt Setting } \\
\text { «Mentorat» } \\
\text { Spezifitätsbewegung } \\
\text { zielt auf Anerkennung } \\
\text { in der Rolle } \\
\text { S verhält sich zur } \\
\text { Changierbewegung } \\
\text { opportunistisch }\end{array}$ & 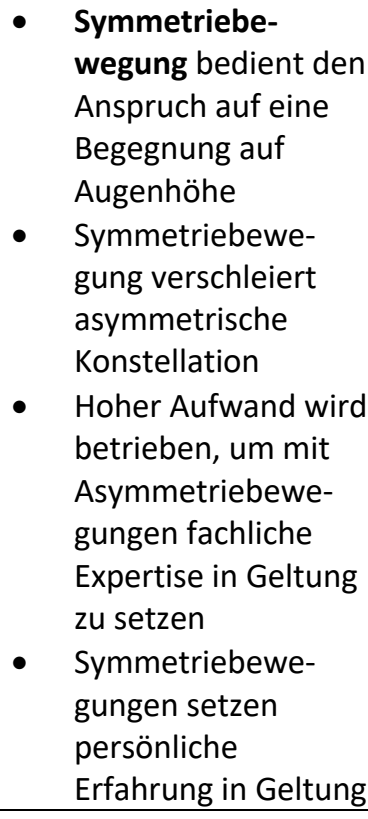 \\
\hline $\begin{array}{l}\text { Abarbei- } \\
\text { tung }\end{array}$ & $\begin{array}{l}\text { - Informalisierungs- } \\
\text { bewegung stellt } \\
\text { Kollegialität her und } \\
\text { vermeidet innerhalb } \\
\text { dieser Kollegialität } \\
\text { dennoch rechtes Nähe } \\
\text { und Kontaktaufnahme } \\
\text { - } \quad \text { Formalisierungbewe- } \\
\text { gung setzt eine } \\
\text { geordnete und } \\
\text { vollständige } \\
\text { Abarbeitung der } \\
\text { Agendapunkte durch }\end{array}$ & $\begin{array}{l}\text { - Spezifitätsbewegung } \\
\text { sumschifft } \\
\text { Gefahrenzone von } \\
\text { Nähe und Diffusität } \\
\text { - Zugleich zeigt sich ein } \\
\text { Anspruch auf } \\
\text { Zugewandtheit und } \\
\text { Fürsorge. Dies führt zu } \\
\text { Spannungen, da } \\
\text { sowohl Diffusitäts- } \\
\text { bemühungen als auch } \\
\text { Spezifierungsnot- } \\
\text { wendigkeiten im Raum } \\
\text { stehen } \\
\text { Gleichzeitigkeit von } \\
\text { Sein-Sollen und Nicht- } \\
\text { sein-Dürfen führt zu } \\
\text { beidseitig zu } \\
\text { Changierbewegungen }\end{array}$ & 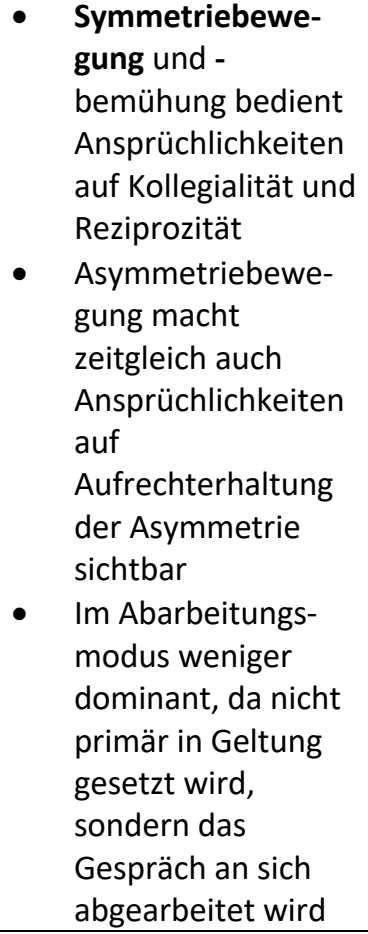 \\
\hline
\end{tabular}




\subsection{Zusammenfassung und Verallgemeinerung der empirischen Ergebnisse}

Der rekonstruktive sequenzanalytische Zugang zur Interaktionsdynamik von Mentoratsgesprächen unter Anwendung der Methode der objektiven Hermeneutik bot die Möglichkeit, die Eigenlogik der vier ausgewählten Gespräche herauszuarbeiten und zu beschreiben. Auf diese Weise konnten in der Analyse unterschiedliche Modi der Bezugnahme auf das Setting, aber auch der Bezugnahme der am Gespräch beteiligten Akteurinnen und Akteure aufeinander aufgezeigt und zueinander ins Verhältnis gesetzt werden. Auf der Basis der Forschungsbefunde wird nachfolgend eine resümierende Verallgemeinerung der Erkenntnisse vorgenommen.

Als Charakteristikum des Settings «Mentoratsgespräche» sind fallübergreifend Strukturdynamiken erkennbar, die an Anderslogiken anderer sozialer Situationen erinnern. Diese Annäherungsbewegungen an bestehende und etablierte Formate wie "Unterricht», «Prüfung \& Bewährung», "Therapie» und «Abarbeitung» sind als Ausdruck eines Strukturproblems dieses Formats zu verstehen. So scheint es dem Format nicht zu gelingen, sich innerhalb der normativen Vorstellung der programmatischen Idee von Mentoring in der (Aus-)Bildung von Lehrerinnen und Lehrern zu etablieren und zu legitimieren. Diese fallübergreifende Legitimierungsproblematik kann als Hinweis darauf betrachtet werden, dass das Setting die beteiligten Akteurinnen und Akteuren konzeptionell letztendlich ein Stück weit vor unauflösbare Anforderungen stellt, die sich wie folgt beschreiben lassen: Um im Rahmen eines dyadischen Gespräches gemeinsam ein ‘echtes` Anliegen bearbeiten zu können, muss ein solches Anliegen auch tatsächlich bestehen. Ein Gesprächsanliegen oder -bedarf ist zwar möglicherweise, nicht aber zwingend vorhanden und kann deshalb nicht als vorausgesetzt betrachtet werden. Denn wenn die studentischen Anliegen sehr praxisnahe Handlungsoptionen oder didaktische Fragestellungen des Unterrichtens betreffen, besteht für Studierende bereits die naheliegende Möglichkeit, diese Fragen mit ihren Praxislehrpersonen zu besprechen. In Fällen wiederum, in denen die studentischen Anliegen den Charakter eines persönlichen Problems aufweisen, gäbe es ebenfalls bereits ein passendes und auch legitimiertes Format, nämlich dasjenige der Therapie oder auch dasjenige einer institutionell an- 
gebotenen studentischen Beratung. Insofern besteht eine Schwierigkeit bezogen auf die Frage, welche Anliegen als Ausgangspunkt von Mentoratsgesprächen dienen und eine (Daseins-)Berechtigung die Gespräche darstellen.

Vor diesem Hintergrund ermöglicht die interaktive Ausrichtung auf eine bereits etablierte Anderslogik Aufschlüsse darüber, welche Struktur die studentischen Anliegen - aus der Sicht der Mentorierenden - aufzuweisen haben. Der an Unterricht angenäherte Modus der Bezugnahme auf das Mentoratssetting richtet sich an didaktischen oder pädagogischen Fragestellungen und Anliegen der Studierenden aus, während der an einer Therapie orientierte Modus Raum für persönliche Probleme der Studierenden anbietet. In den an Prüfung und Bewährung respektive Abarbeitung angenäherten Modi werden primär die Anliegen der Mentorierenden, nicht aber diejenigen der Studierenden fokussiert.

Wenngleich diese Anderslogiken in den empirischen Daten zumeist relativ stark aufscheinen, gilt es dennoch festzuhalten, dass sich innerhalb der Modi auch gewisse Changierbewegungen feststellen lassen. Die Anlehnungen an Anderslogiken scheinen folglich auch von Prekarität und Fragilität geprägt sein und können nicht bruchlos vorgenommen werden. An gewissen Passagen der Gesprächsinteraktionen entsteht der Eindruck, es gelte diese Anderslogiken, an die sich der Gesprächsmodus auf einer latenten Ebene annähert, auf einer manifesten Ebene geradezu zu vermeiden.

Diese Changier- und Vermeidungsbewegungen beziehen sich auf verschiedene antinomische Dimensionen wie «Informalisierung-Formalisierung», «DiffusitätSpezifität» und "Symmetrie-Asymmetrie», wobei diese Dimensionen jedoch wie in Kapitel 5.2.2 bereits festgehalten gewisse Überschneidungen und Abgrenzungsprobleme aufweisen. Modusspezifisch sind sie in unterschiedlichen Ausprägungen erkennbar; in einem der vier identifizierten Modi werden sie sogar weitgehend stillgelegt.

Oevermann betrachtet «die für die primäre konkrete Lebenspraxis stellvertretend expertenhaft vorzunehmende Krisenbewältigung» als grundsätzlichen Strukturkern des professionellen Handelns (Oevermann, 2002b, S. 24). Empirisch zeigt sich in den Mentoratsgesprächen ein strukturell angelegtes Problem, das darin besteht, das Arbeitsbündnis zwischen Mentorierenden und Studierenden zu etablieren. Die stellvertretende Krisenbewältigung erweist sich für die Herstellung eines authentischen Arbeitsbündnisses zwischen Mentorierenden und Studierenden als anspruchsvoll und prekär, da gewisse Ausgangsbedingungen, wie beispielsweise die Freiwilligkeit bei der Errichtung des 
Arbeitsbündnisses, auf einer institutionellen Ebene nicht gewährleistet sind. Dieses Strukturproblem der Legitimierungsbedürftigkeit des Settings «Mentoratsgespräche» lässt sich als gemeinsamer Fluchtpunkt der vier untersuchten Gespräche auffassen, wobei der Umgang damit allerdings sehr unterschiedlich ausfällt. 


\section{Reflexion, Ausblick und Desiderate für Folgestudien}

Im die Untersuchung abschliessenden Kapitel gilt es, in Kürze die Grenzen und Limitationen des Forschungsvorhabens aufzuzeigen, Anschlussfragen für Folgestudien zu formulieren und auf mögliche Konsequenzen für die Mentoratskonzeption hinzuweisen.

Das Ziel der vorliegenden Forschungsarbeit bestand darin, einen empirischen Beitrag zur Analyse der Strukturlogik und den Interaktionsdynamiken von Mentoratsgesprächen zu leisten. Auf einer Datenbasis von 25 Mentoratsgesprächen wurden vier in mehreren Aspekten maximal kontrastierende Kernfälle ausgewählt und einige Gesprächspassagen aus diesen Gesprächen rekonstruiert. Dies ermöglichte eine detaillierte Rekonstruktion des Strukturproblems und den zentralen Interaktionsdynamiken, woraus sich vier unterschiedliche Modi des Umgangs oder der Bearbeitung dieses Strukturproblems ableiten liessen. Angesichts der zunehmenden Sättigung scheint die Annahme plausibel zu sein, dass sich das identifizierte Strukturproblem bei einer Erhöhung der Grundgesamtheit reproduziert hätte. Das Spektrum der Bearbeitungsmodi hätte sich jedoch vermutlich noch weiter ausdifferenzieren lassen. Die Beschränkung auf vier Kernfälle muss folglich mit forschungsökonomischen Überlegungen begründet werden, denn eine Erweiterung der Analysen um weitere Fälle hätte den Rahmen des Vorhabens gesprengt und bleibt deshalb weiteren Studien vorbehalten.

Des Weiteren gilt es festzuhalten, dass das Sample ausschliesslich «konzeptionskonforme` Mentoratsgespräche mit regulärem Verlauf umfasst. Unberücksichtigt blieben infolgedessen einerseits offensichtlich problem- oder krisenbehaftete Konstellationen zwischen Mentorierenden und Studierenden, obwohl die Interaktionsdynamiken in solchen Mentoratsgesprächen für die Beschreibung der strukturellen Verfasstheit durchaus aufschlussreich wären. Diesbezüglich lässt sich jedoch vermuten, dass sich Dyaden, die sich mit solchen kritischen Gesprächskonstellationen konfrontiert sehen, nicht auf den Aufruf für eine freiwillige Teilnahme an einem Forschungsprojekt gemeldet hätten. Um auch krisenbehaftete Gesprächskonstellationen für Forschende zugänglich zu machen, müsste daher ein anderes Forschungsdesign konzipiert werden. Ebenfalls unberücksichtigt blieben andererseits Mentoratsgespräche, die sich jenseits der konzeptionellen 
Vorgaben bewegten und einen markant differenten Weg bezogen auf das «Füllen der Gesprächszeit` verfolgten.

Artefakte wie Gesprächsvorbereitungsunterlagen, Gesprächsleitfäden oder weitere Dokumente wie das in den Gesprächen mehrfach erwähnte Dokument der Kompetenzfelder der PH FHNW waren nicht Bestandteil dieser Untersuchung. Die Rekonstruktion solcher Artefakte könnte jedoch durchaus aufschlussreiche Erkenntnisse liefern, insbesondere auch bezogen auf die Strukturlogik der Gespräche, und stellt deshalb ein weiteres Forschungsdesiderat dar.

Da sich die dem Forschungsvorhaben zugrunde liegende Datenbasis ausschliesslich auf Gesprächsinteraktionen aus dem IKU der PH FHNW bezieht, stellt sich unweigerlich die Frage, ob sich die empirischen Befunde auf andere Pädagogische Hochschulen (aus studienarchitektonischen Überlegungen heraus primär auf Ausbildungsinstitute in der Schweiz) übertragen liessen. Es wäre daher erforderlich, zu klären, welche Befunde als institutsspezifisch zu betrachten sind und welche Interaktionsdynamiken sich für solche Settings verallgemeinern lassen. Um belastbare Aussagen zu möglichen Verallgemeinerungen formulieren zu können, wären entsprechende Untersuchungen an weiteren Instituten und Pädagogischen Hochschulen mit jeweils differenten Mentoringprogrammatiken und -konzeptionen durchzuführen. Solche weiterführenden Untersuchungen in weiteren Instituts- und Hochschulkulturen würden allerdings voraussetzen, dass Artefakte wie die an den Institutionen jeweils erstellten Konzeptpapiere zu Mentoring oder einem entsprechenden Begleitformat ebenfalls in die Analysen einbezogen würden. Durch diese Komplexitätssteigerung liessen sich auch kontrastierende Befunde zu den Wechselwirkungen von Konzeption und empirischer Realität erarbeiten.

Gemäss der Kernthese der Untersuchung weist das Setting «Mentoratsgespräche» ein Strukturproblem hinsichtlich seiner Legitimierung auf. Gerade weil es sich dabei um ein strukturelles Problem handelt, ist dieses nicht lös-, sondern lediglich reflexiv bearbeitbar. Wenn als Konsequenz dieser Forschungsbefunde Gestaltungsmöglichkeiten für die Bearbeitung dieser Legitimierungsproblematik oder die daraus resultierenden Spannungen aufgezeigt würden, dann würde dies die Lösbarkeit einer Problemlage suggerieren, obwohl es keine Lösung gibt. Möglicherweise gelänge es, mit anderen Gestaltungsansätzen (wie beispielsweise der Einführung der Freiwilligkeit des Mentorats) 
gewisse Spannungsmomente zu variieren oder abzubauen. Welche neuen Schwierigkeiten oder Spannungen sich dadurch ergeben würden, wäre jedoch empirisch zu klären. Aus diesem Grund verzichte ich auf eine entsprechende Diskussion. Auch würde diese Diskussion eine Fokusverschiebung in der Forschungsanlage bedeuten, da die Untersuchung ausschliesslich problemerschliessend ausgerichtet ist.

Gleichwohl steht jenseits der skizzierten Möglichkeiten für Folgeuntersuchungen am Ende eines Vorhabens wie des vorliegenden die Frage nach den konkreten, auch hochschulbezogenen Implikationen im Raum, das heisst im vorliegenden Fall danach, welche Konsequenzen sich aus den Befunden für die Konzeption und Gestaltung des Mentorats ergeben. Muss man, ausgehend von der Analyse der Spannungsfelder und der Legitimationsproblematik, letztendlich Herrn Buchers Provokation Recht geben, und das Mentorat abschaffen?

Die Schlussfolgerung, dass ein Setting oder Format nur dann beibehalten werden soll, wenn darin keine ambivalenten, spannungsvollen oder widersprüchlichen Implikationen aufscheinen, scheint der Idee einer widerspruchslosen und spannungsfreien Welt zu folgen, die ich als Illusion und gesellschaftlich nicht tragfähig betrachte. ${ }^{69}$ Gerade für die Arbeit im Mentorat scheint nicht (Spannungsfreiheit) als Wert an sich anzustreben, sondern vielmehr der reflexive Umgang mit auftretenden Spannungen lohnenswert zu sein.

Man muss oder soll das Mentorat nicht abschaffen, nur weil darin auch Spannungen und Ambivalenzen aufscheinen......

Vielmehr lässt sich als Ausblick und Richtungsweiser festhalten, dass ein differenziertes Problembewusstsein für die Strukturlogik und die Interaktionsdynamiken dieser sozialen Praxis neue Folien und Perspektiven schafft, die es ermöglichen, sich das Setting mit all seinen Spannungen und Ambivalenzen zu erschliessen. Aus diesem Grund dürfte ein Zuwachs an Wissen und Befunden über die für die Gespräche charakteristischen Interaktionsdynamiken nicht ausschliesslich aus einer Forschungsperspektive interessant sein. Des Weiteren können diese Erkenntnisse zu einem reflexiven und idealerweise

69 Die Diskussion der eher philosophisch angelegten Frage, weshalb Spannungen, Ambivalenzen und Widersprüchlichkeiten die Sinnhaftigkeit und die Legitimation eines Settings tangieren oder ein solches sogar grundsätzlich infrage stellen, scheint mir durchaus lohnenswert. Allerdings liegt auch dies ausserhalb des Rahmens des vorliegenden Vorhabens und wird deshalb nicht weiterverfolgt. 
reflexionssteigernden Umgang mit den aufscheinenden Dynamiken beitragen und- im günstigen Fall - in einem weiteren Schritt Bearbeitungsspielräume für alle an dieser sozialen Praxis beteiligten Akteurinnen und Akteure, das heisst für Studierende, vor allem aber auch für Mentorierende, aufzeigen.

Für mich steht unter der Prämisse, dass die Lehrerinnen- und Lehrerbildung auch aus der Perspektive der Persönlichkeitsbildung gedacht wird, ausser Frage, dass Studierende eine persönliche Ansprechperson brauchen, und zwar ungeachtet der Erkenntnis, dass diese Beziehungskonstellation respektive dieses Arbeitsbündnis gewisse, nicht unbedeutende strukturelle Probleme aufweist. Vor diesem Hintergrund möchte ich abschliessend die These formulieren, dass das Setting «Mentoratsgespräche» für Studierende aus strukturtheoretischen Überlegungen ein Lernfeld für den Umgang mit antinomischen Spannungen darstellt. Aus dieser Perspektive betrachtet scheint das Setting durchaus funktional, wenngleich nicht notwendigerweise reflexiv zu sein. Die Frage, wie sich diese Funktionalität in der Praxis manifestiert und auf welche Weise sie empirisch erfasst werden kann, bleibt als Forschungsdesiderat allerdings weiterführenden Studien vorbehalten. 


\section{Literaturverzeichnis}

Arnold, K.-H. (2014). Schulpraktika in der Lehrerbildung: Theoretische Grundlagen, Konzeptionen, Prozesse und Effekte. Waxmann.

Bauer, K.-O. (1998). Pädagogisches Handlungsrepertoire und professionelles Selbst von Lehrerinnen und Lehrern. Zeitschrift für Pädagogik, 44, 343-361.

Baumert, J., \& Kunter, M. (2006). Stichwort: Professionelle Kompetenz von Lehrkräften. Zeitschrift für Erziehungswissenschaft, 9(4), 469-520.

Blömeke, S. (2002). Universität und Lehrerausbildung. Julius Klinkhardt.

Blömeke, S. (2014). Vorsicht bei Evaluationen und internationalen Vergleichen. Unterschiedliche Referenzrahmen bedrohen die Validität von Befragungen zur Lehrerausbildung. Zeitschrift für Pädagogik, 60, 109-131.

Boettcher, W., \& Bemerich-Vos, A. (1987). "Kollegiale Beratung» in Schule, Schulaufsicht und Referendarausbildung. Peter Lang.

Boettcher, W., \& Bremerich-Vos, A. (1985). Pädagogische Beratung: Zur Unterrichtsnachbesprechung in der 2. Phase der Lehrerausbildung. In W. Kallmeyer (Hrsg.), Kommunikationstypologie: Handlungsmuster. Textsorten. Situationstypen (S. 246-279). Schwann.

Bortz, J., \& Döring, N. (2015). Forschungsmethoden und Evaluation für Human- und Sozialwissenschaftler (4., überarb. Aufl.). Springer.

Bräu, K. (2008). Die Betreuung selbständigen Lernens: Vom Umgang mit Antinomien und Dilemmata. In F. Schütze \& G. Breidenstein (Hrsg.), Paradoxien in der Reform der Schule (S. 179-199). VS.

Breidenstein, G., Hirschauer, S., Kalthoff, H., \& Nieswand, B. (2020). Ethnografie: Die Praxis der Feldforschung: $B d$. 3979. Sozialwissenschaften, Kulturwissenschaften (3., überarb. Aufl.). UVK.

Britzmann, D. P. (2009). The Very Thought of Education: Psychoanalysis and the Impossible Professions. Suny Press.

Büchmann, G., Robert-Tornow, W., \& Hofmann, W. (2007). Geflügelte Worte: Der klassische Zitatenschatz (unveränderte Taschenbuchausgabe der 43., neu bearb. Ausgabe). Ullstein.

Bühler, P. (2012). Negative Pädagogik. Ferdinand Schöningh.

Bundesministerium Bildung, Wissenschaft, Forschung. (n. d.). PädagogInnenbildung NEU. Abgerufen am 24. Dezember 2020 von https://www.bmbwf.gv.at/Themen/ schule/fpp/ausb/pbneu.html

Combe, A., \& Helsper, W. (1994). Was geschieht im Klassenzimmer? Perspektiven einer hermeneutischen Schul- und Unterrichtsforschung: Zur Konzeptualisierung der Pädagogik als Handlungstheorie. Deutscher Studien-Verlag. 
Crasborn, F., \& Hennissen, P. (2010). The skilled mentor: Mentor teachers' use and acquisition of supervisory skills. Technische Universiteit Eindhoven.

Dammerer, J., Wiesner, C., \& Windl, E. (Hrsg.). (2020). Mentoring im pädagogischen Kontext. Professionalisierung und Qualifizierung von Lehrpersonen (Bd. 10). Studienverlag.

Diekmann, A. (2017). Empirische Sozialforschung: Grundlagen, Methoden, Anwendungen (11. Aufl., Bd. 55678). Rowohlt.

Dietrich, F. (2014). Professionalisierungskrisen im Referendariat: Rekonstruktionen zu Krisen und Krisenbearbeitungen in der zweiten Phase der Lehrerausbildung. Springer.

Dzengel, J. (2016). Schule spielen: Zur Bearbeitung der Theorie-Praxis-Problematik im Studienseminar. Springer.

Dzengel, J. K., Kunze, K., \& Wernet, A. (2012). Vom Verschwinden der Sache im pädagogischen Jargon: Überlegungen zu einem Strukturproblem in der Ausbildungskultur im Studienseminar. Pädagogische Korrespondenz, 45, 20-44.

Dzengel, J., Kunze, K., \& Wernet, A. (2011). Zwischen Theorie und Praxis (AKuRat) [Forschungsbericht].

Fietze, B. (2011). Profilbildung und Statuskonkurrenz. Zur Bedeutung der Distinktionsstrategien im Professionalisierungsprozess von Supervision und Coaching. In E.M. Graf, Y. Aksu, I. Pick, \& S. Rettinger (Hrsg.), Beratung, Coaching, Supervision. Springer VS.

Fraefel, U. (2018). Die Wende zum kollaborativen Mentoring in Schulpraktika. Überblick zu empirischen Befunden, theoretischen Verortungen und Strategien der Transformation. In G. Bellenberg, G. Im Bram, \& C. Reintjes (Hrsg.), Mentoring und Coaching als Beitrag zur Professionalisierung angehender Lehrpersonen (Bd. 3, S. 41-67). Waxmann.

Futter, K. (2016). Lernwirksame Unterrichtsbesprechungen im Praktikum: Nutzung von Lerngelegenheiten durch Lehramtsstudierende und Unterstützungsverhalten der Praxislehrpersonen. (Dissertation) Universität Zürich.

Garz, D. (2015). Theorie der Lebenspraxis: Einführung in das Werk Ulrich Oevermanns. VS.

Giesecke, H. (2015). Pädagogik als Beruf. Grundformen pädagogischen Handelns (12. Aufl.). Juventa.

Glaser, B. G., \& Strauss, A. L. (2010). Grounded Theory: Strategien qualitativer Forschung (3., unv. Aufl.). Huber.

Groeben, N., \& Erb, E. (2005). Menschenbilder. In J. Straub (Hrsg.), Psychologie: Eine Einführung: Grundlagen, Methoden, Perspektiven (5. Aufl., Bd. 36204). Deutscher Taschenbuch-Verlag.

Gruschka, A. (2003). Von der Kritik zur Konstruktion ist oft nur ein Schritt: Der der Negation. Pädagogische Korrespondenz, 30, 71-79. 
Habermas, J. (1998). Vorstudien und Ergänzungen zur Theorie des kommunikativen Handelns (3. Aufl.). Suhrkamp.

Häcker, T. (2017). Grundlagen und Implikationen der Forderung nach Förderung von Reflexivität in der Lehrerinnen- und Lehrerbildung. In Berndt, C., Häcker, T., \& Leonhard, T. (Hrsg.), Reflexive Lehrerbildung revisited. Traditionen - Zugänge Perspektiven (S. 21-45). Julius Klinkhardt.

Haghanipour, B. (2013). Mentoring als gendergerechte Personalentwicklung. Wirksamkeit und Grenzen eines Programms in den Ingenieurwissenschaften. Springer.

Hascher, T. (2012). Lernfeld Praktikum. Evidenzbasierte Entwicklungen in der Lehrer/innenbildung. Zeitschrift für Bildungsforschung, 2(1), 109-129.

Helsper, W. (1996). Antinomien des Lehrerhandelns in modernisierten pädagogischen Kulturen. Paradoxe Verwendungsweisen von Autonomie und Selbstverantwortlichkeit. In A. Combe \& W. Helsper (Hrsg.), Pädagogische Professionalität. Untersuchungen zum Typus pädagogischen Handelns (S. 521570). Suhrkamp.

Helsper, W. (2000). Antinomien des Lehrerhandelns und die Bedeutung der Fallrekonstruktion - Überlegungen zu einer Professionalisierung im Rahmen universitärer Lehrerbildung. In E. Cloer, D. Klika, \& H. Kunert (Hrsg.), Welche Lehrer braucht das Land? Notwendige und mögliche Reformen der Lehrerbildung (S. 142-177). Juventa.

Helsper, W. (2002). Lehrerprofessionalität als antinomische Handlungsstruktur. In M. Kraul, W. Marotzki, \& C. Schweppe (Hrsg.), Biographie und Profession (S. 64-102). Julius Klinkhardt.

Helsper, W. (2004). Antinomien, Widersprüche, Paradoxien. Lehrerarbeit - Ein unmögliches Geschäft? In B. Koch-Priewe, F.-U. Kolbe, \& J. Wildt (Hrsg.), Grundlagenforschung und mikrodidaktische Reformansätze zur Lehrerbildung (S. 49-98). Julius Klinkhardt.

Helsper, W. (2010). Pädagogisches Handeln in den Antinomien der Moderne. In W. Helsper \& H.-H. Krüger (Hrsg.), Einführung in die Grundbegriffe und Grundfragen der Erziehungswissenschaft (S. 15-34). Springer.

Helsper, W. (2016). Antinomien und Paradoxien im professionellen Handeln. In M. Dick, W. Marotzki, \& H. Mieg (Hrsg.), Handbuch Professionsforschung (S. 50-62). Julius Klinkhardt.

Helsper, W., Grasshoff, G., Höblich, D., Jung, D., Stelmaszyk, B., \& Ullrich, H. (2007). Autorität und Schule. VS.

Helsper, W., \& Tippelt, R. (2011). Ende der Profession und Professionalisierung ohne Ende? Zwischenbilanz einer unabgeschlossenen Diskussion. In W. Helsper \& R. Tippelt (Hrsg.), Pädagogische Professionalität. Beiheft der Zeitschrift für Pädagogik, 57, 268-288.

Hericks, U. (2006). Professionalisierung als Entwicklungsaufgabe: Rekonstruktionen zur Berufseingangsphase von Lehrerinnen und Lehrern. VS. 
Herzog, S., Leonhard, T., \& Peyer, R. (2017). Im Modus individueller Unterstützung. Zur Begleitung von Professionalisierungsprozessen im Mentoring. In U. Fraefel \& A. Seel (Hrsg.), Konzeptionelle Perspektiven Schulpraktischer Studien. Partnerschaftsmodelle - Praktikumskonzepte - Begleitformate (Bd. 2, S. 163175). Waxmann.

Hobson, A., Ashby, P., Malderez, A., \& Tomlinson, P. (2009). Mentoring beginning teachers: What we know and what we don't. Teaching and Teacher Education, 25(1), 207-216.

Hughes, E. C. (1963). Professions. Daedalus, 92(4), 655-668.

Hummrich, M. (2016). Was ist der Fall?: Kasuistik und das Verstehen pädagogischen Handelns. VS.

Jackson, D. D., Bavelas, J. B., \& Watzlawick, P. (1996). Menschliche Kommunikation (9., unv. Aufl.). Huber.

Kallmeyer, W. (2000). Beraten und Betreuen. Zeitschrift für qualitative Bildungs-, Beratungs- und Sozialforschung, 2, 25.

Kant, I. (1803/1982). Über Pädagogik. In Ders. Werkausgabe. Bd. XII. Hrsg. v. W. Weischedel. Suhrkamp.

Kelle, U., \& Kluge, S. (2010). Vom Einzelfall zum Typus. VS.

Keller-Schneider, M. (2010). Entwicklungsaufgaben im Berufseinstieg von Lehrpersonen. Beanspruchung durch berufliche Herausforderungen im Zusammenhang mit Kontext- und Persönlichkeitsmerkmalen. Waxmann.

Keller-Schneider, M. (2012). «Nun bin ich im Beruf angekommen - aber es war anstrengend!» Prädiktoren der Kompetenz und der Beanspruchung von Lehrpersonen Ende des ersten Berufsjahres. In T. Hascher \& G. H. Neuweg (Hrsg.), Forschung zur (Wirksamkeit der) Lehrer/innen/bildung: Bd. 8 (S. 221-238).

Keller-Schneider, M., \& Hericks, U. (2011). Forschung zum Berufseinstieg. Übergang von der Ausbildung in den Beruf. In E. Terhart, H. Bennewitz, \& M. Rothland (Hrsg.), Handbuch der Forschung zum Lehrerberuf (S. 296-313). Waxmann.

Kolbe, F.-U., \& Combe, A. (2008). Lehrerbildung. In W. Helsper \& J. Böhme (Hrsg.), Handbuch der Schulforschung (2. Aufl., S. $\left.{ }^{\circ} 877-901\right)$. VS.

Košinár, J. (2018). Das Mentorat zwischen Indvidualisierung und Standardisierung - Eine empirie- und theoriebasierte Konzeption. In C. Reintjes, G. Bellenberg, \& G. Im Brahm (Hrsg.), Mentoring und Coaching als Beitrag zur Professionalisierung angehender Lehrpersonen (Bd. 3, S. 67-84). Waxmann.

Kowalski, M. (2020). Nähe, Distanz und Anerkennung in Pädagogischen Beziehungen: Rekonstruktionen Zum Lehrerhabitus und Möglichkeiten der Professionalisierung. VS.

Kreis, A. (2012). Produktive Unterrichtsbesprechungen: Lernen im Dialog zwischen Mentoren und angehenden Lehrpersonen (Bd. 17). Haupt. 
Kreis, A., \& Staub, F. (2011). Fachspezifisches Unterrichtscoaching im Praktikum. Eine quasi-experimentelle Interventionsstudie. Zeitschrift für Erziehungswissenschaft, 14(1), 61-84.

Kunze, K. (2011). Professionalisierung als biographisches Projekt. Professionelle Deutungsmuster und biographische Ressourcen von Waldorflehrerinnen und lehrern. Springer VS.

Kunze, K. (2014). Wenn der Fall zum Vorfall wird. Beiträge zur Lehrerinnen- und Lehrerbildung, 32(1), 13.

Kunze, K., Bartmann, S., \& Silkenbeumer, M. (2019). Teamgespräche als Adressierungsgeschehen. Methodische Überlegungen zur Rekonstruktion pädagogischer Zuständigkeitsformationen. In P. Cloos, M. Fabel-Lamla, K. Kunze, \& B. Lochner (Hrsg.), Pädagogische Teamgespräche methodische und theoretische Perspektiven eines neuen Forschungsfeldes. Beltz Juventa.

Kurtz, T. (2011). Der Professionsansatz von Niklas Luhmann. Soziale Systeme, 17(2), 3152.

Lazarides, L., R., \& Mohr, S. (2015). Lehramtsstudierende im Orientierungspraktikum. Wie wird die universitäre Praktikumsbetreuung wahrgenommen? Beiträge zur Lehrerinnen- und Lehrerbildung, 33(3), 380-392.

Leonhard, T. (2019). Leitfaden Vertiefungspraktikum an Partnerschulen 2019/2020. Berufspraktische Studien am IKU, PH FHNW.

Liegmann, A., Artmann, M., Berendonck, M., \& Herzmann, P. (2018).

Professionalisierung in Praxisphasen der Lehrerbildung (Bd. 4). Julius Klinkhardt.

Lippmann, E. (2006). Coaching. Angewandte Psychologie für die Praxis. Springer.

Luhmann, N. (1983). Medizin und Gesellschaftstheorie. Medizin, Mensch, Gesellschaft: $M M G, 8,168-175$.

Mead, G. H. (1968). Geist, Identität und Gesellschaft aus der Sicht des Sozialbehaviorismus. Suhrkamp.

Megginson, D., \& Clutterbuck, D. (2008). Die Beratungsbeziehung aufbauen und erhalten. In D. Megginson \& D. Clutterbuck (Hrsg.), Coaching und Mentoring. Individuelle Beratung für individuelle Berufskarrieren (S. 19-41). Spektrum.

Moroni, S., Gut, R., Niggli, A., \& Bertschy, B. (2014). Verbindung von Theorie und Praxis bei der Begleitung von Praxisphasen in der Lehrerbildung. Lehrerbildung auf dem Prüfstand, 7, 24-45.

Müller, B. (2011). Professionelle Beziehungen in Zwangskontexten. Online-Texte der Evangelischen Akademie Bad Boll. Abgerufen am 16. Januar 2019 von https://www.ev-akademie-boll.de/fileadmin/res/otg/doku/520111-Mueller.pdf

Nerowski, C. (2012). Handlungen der Lehrkräfte zwischen Diffusität und Spezifität. In C. Nerowski, T. Hascher, M. Lunkenbein, \& D. Sauer (Hrsg.), Professionalität im Umgang mit Spannungsfeldern der Pädagogik (S. 141-154). Julius Klinkhardt. 
Neukom, M., Schnell, K., \& Boothe, B. (2011). Die Arbeitsbeziehung im Coaching - ein Stiefkind der Forschung. Organisationsberatung, Supervision, Coaching, 18(3), 317.

Neuweg, G. H. (2013). Lehrerinnen- und Lehrerbildung durch Wissenschaft: Zur Vielschichtigkeit einer zeitgenössischen Einigungsformel. Beiträge zur Lehrerbildung, 31(3), 301-309.

Nienhaus, D., Pannatier, G., \& Töngi, C. (2005). Akademische Seilschaften. Mentoring für Frauen im Spannungsfeld von individueller Förderung und Strukturveränderung. eFeF.

Niggli, A. (2001). Ein Mentoring-Programm mit Coaching-Anteilen für die Ausbildung von Lehrpersonen. Beiträge zur Lehrerinnen- und Lehrerbildung, 19, 244-250.

Niggli, A. (2005). Unterrichtsbesprechungen im Mentoring. Sauerländer.

Niggli, A., Gerteis, M., \& Gut, R. (2008). Wirken - erkennen - sich selbst sein: Validierung unterschiedlicher Interessen von Studierenden und Praxislehrpersonen in Unterrichtsbesprechungen. Beiträge zur Lehrerbildung, 26(2), 140-153.

Oevermann, U. (1983). Hermeneutische Sinnrekonstruktion. In D. Garz \& K. Kraimer (Hrsg.), Brauchen wir andere Forschungsmethoden? (S. 113-155). Skriptor.

Oevermann, U. (1993). Die objektive Hermeneutik als unverzichtbare methodologische Grundlage für die Analyse von Subjektivität. Zugleich eine Kritik der Tiefenhermeneutik. In T. Jung \& S. Müller-Doohm (Hrsg.), Wirklichkeit im Deutungsprozess: Verstehen und Methoden in den Kultur- und Sozialwissenschaften (S. 106-189). Suhrkamp.

Oevermann, U. (1996a). Krise und Muse. Struktureigenschaften ästhetischer Erfahrung aus soziologischer Sicht. Vortrag am 19.06.1996 in der Städelschule.

Oevermann, U. (1996b). Theoretische Skizze einer revidierten Theorie professionalisierten Handelns. In A. Combe \& W. Helsper (Hrsg.), Pädagogische Professionalität. Untersuchungen zum Typus pädagogischen Handelns (S. 70183). Suhrkamp.

Oevermann, U. (2000). Die Methode der Fallrekonstruktion in der Grundlagenforschung sowie der klinischen und pädagogischen Praxis. In K. Kraimer (Hrsg.), Die Fallrekonstruktion. Sinnverstehen in der sozialwissenschaftlichen Forschung (1. Aufl., S. 58-156). Suhrkamp.

Oevermann, U. (2002a). Klinische Soziologie auf der Basis der Methodologie der objektiven Hermeneutik - Manifest der objektiv hermeneutischen Sozialforschung. Abgerufen am 16. März 2020 von https://www.ihsk.de/publikationen/Ulrich_ Oevermann-Manifest_der_objektiv_hermeneutischen_Sozialforschung.pdf

Oevermann, U. (2002b). Professionalisierungsbedürftigkeit und Professionalisiertheit pädagogischen Handelns. In M. Kraul (Hrsg.), Biographie und Profession (S. 1963). Julius Klinkhardt.

Oevermann, U. (2003). Brauchen wir heute noch eine gesetzliche Schulpflicht und welches wären die Vorzüge ihrer Abschaffung? Pädagogische Korrespondenz, 30, 54-70. 
Oevermann, U. (2006). Zur Behinderung pädagogischer Arbeitsbündnisse durch die gesetzliche Schulpflicht. In T. Rihm (Hrsg.), Schulentwicklung. Vom Subjektstandpunkt ausgehen (S. 69-92). VS.

Oevermann, U. (2010). Strukturprobleme supervisorischer Praxis: Eine objektiv hermeneutische Sequenzanalyse zur Überprüfung der Professionalisierungstheorie (3. Aufl., Bd. 2). Humanities Online.

Oevermann, U. (2013a). Die Problematik der Strukturlogik des Arbeitsbündnisses und der Dynamik von Übertragung und Gegenübertragung in einer professionalisierten Praxis von Sozialarbeit. In R. Becker-Lenz (Hrsg.), Professionalität in der Sozialen Arbeit. VS.

Oevermann, U. (2013b). Objektive Hermeneutik als Methodologie der Erfahrungswissenschaften von der sinnstrukturierten Welt. In P. C. Langer (Hrsg.), Reflexive Wissensproduktion (S. 69-98). Springer.

Oevermann, U., Allert, T., Konau, E., \& Krambeck, J. (1979). Die Methodologie einer "objektiven Hermeneutik» und ihre allgemeine forschungslogische Bedeutung in den Sozialwissenschaften. In H.-G. Soeffner (Hrsg.), Interpretative Verfahren in den Sozial- und Textwissenschaften (S. 352-434). Metzler.

Offermanns, M. (2004). Braucht Coaching einen Coach? Eine evaluative Pilotstudie. ibidem.

Oser, F., \& Oelkers, J. (2001). Die Wirksamkeit der Lehrerbildungssysteme in der Schweiz. Rüegger.

Paris, R. (2005). Raten und Beratschlagen. Sozialer Sinn: Zeitschrift für hermeneutische Sozialforschung, 6(2), 353-388.

Parsons, T. (1951). The Social System. Free Press.

Parsons, T. (1964). Die akademischen Berufe und die Sozialstruktur. In D. Rüschemeyer (Hrsg.), Beiträge zur soziologischen Theorie (S. 160-179). Luchterhand.

Parsons, T., Shils, E., Edward C. Tolman, \& Gordon W. Allport. (1954). Toward a general theory of action (3. Aufl.). Harvard University Press.

Peters, S., Genge, F., \& Willenius, Y. (2006). Flankierende Personalentwicklung durch Mentoring. Rainer Hampp.

Petersen, R., Budde, M., Brocke, P. S., Doebert, G., Rudack, H., \& Wolf, H. (2017). Praxishandbuch Mentoring in der Wissenschaft. Springer.

Pille, T. (2013). Das Referendariat. Eine ethnographische Studie zu den Praktiken der Lehrerbildung. Transkript.

Prange, K. (1986). Bauformen des Unterrichts: Eine Didaktik für Lehrer (2. Aufl.). Julius Klinkhardt.

Prange, K. (2012). Die Zeigestruktur der Erziehung: Grundriss der operativen Pädagogik (2. Aufl.). Schöningh.

Radtke, F.-O. (2004). Der Eigensinn pädagogischer Professionalität jenseits von Innovationshoffnungen und Effizienzerwartungen. Übergangen Einsichten aus der Wissensverwendungsforschung für die Organisation der Universitären 
Lehrerbildung. In B. Koch-Priewe, F.-U. Kolbe, \& J. Wildt (Hrsg.), Grundlagenforschung und mikrodidaktische Reformansätze zur Lehrerbildung (S. 99-149). Julius Klinkhardt.

Rauen, C. (2014). Coaching: Bd. 2 (3. Aufl.). Hogrefe.

Reh, S. (2008). «Reflexivität der Organisation» und Bekenntnis. Perspektiven der Lehrerkooperation. In W. Helsper, S. Busse, M. Hummrich, \& R.-T. Kramer (Hrsg.), Pädagogische Professionalität in Organisationen. Neue Verhältnisbestimmungen am Beispiel der Schule (S. 163-183). VS.

Reh, S., \& Ricken, N. (2012). Das Konzept der Adressierung. Zur Methodologie einer qualitativ-empirischen Erforschung von Subjektivation. In I. Miethe (Hrsg.), Qualitative Bildungsforschung und Bildungstheorie (S. 36-56). Barbara Budrich.

Reintjes, C., \& Bellenberg, G. (2017). Reflexive Professionalisierung im verkürzten Vorbereitungsdienst in NRW. Zur Qualität und Quantität von mentorierten Lerngelegenheiten und ihrer Nutzung. In Berndt, C., Häcker, T., \& Leonhard, T. (Hrsg.), Reflexive Lehrerbildung revisited. Traditionen - Zugänge - Perspektiven (S. 116-132). Julius Klinkhardt.

Reintjes, C., Bellenberg G., \& Im Brahm, G. (2018). Mentoring und Coaching als Beitrag zur Professionalisierung angehender Lehrpersonen. Bd. 3. Waxmann.

Ribolits, E. (2004). Vom Lehrer zum Lerncoach? In E. Ribolits \& J. Zuber (Hrsg.), Pädagogisierung. Die Kunst Menschen mittels Lernen immer dümmer zu machen (S. 101-113). Studienverlag.

Ricken, N. (2015). Pädagogische Professionalität - Revisited. Eine anerkennungstheoretische Skizze. In J. Böhme, M. Hummrich, \& R.-T. Kramer (Hrsg.), Schulkultur. Theoriebildung im Diskurs (S. 137-159). VS.

Rotering-Steinberg, S. (2009). Unterschiede und Gemeinsamkeiten von Coaching und Mentoring. In H. Stöger, A. Ziegler, \& D. Schimke (Hrsg.), Mentoring: Theoretische Hintergründe, empirische Befunde und praktische Anwendungen (S. 31-51). Pabst Science Publishers.

Ryter, A. (2018). Coaching als neuer Trend in der Lehrerinnen- und Lehrerbildung Eine erste Bestandesaufnahme. In C. Reintjes, G. Bellenberg, \& G. Im Brahm (Hrsg.), Mentoring und Coaching als Beitrag zur Professionalisierung angehender Lehrpersonen (S. 23-40). Waxmann.

Schäfer, C. (2013). Platon-Lexikon: Begriffswörterbuch zu Platon und der platonischen Tradition (2., durchgesehene und bibliografisch aktualisierte Auflage). WBG.

Schnebel, S. (2009). Beratungsprozesse zwischen Praktikanten und Mentoren - eine Studie zu den Unterrichtsbesprechungen. In M. Dieck, G. Dörr, D. Kuckarz, O. Küster, K. Müller, B. Reinhofer, T. Rosenberger, S. Schnebel, \& T. Bohl (Hrsg.), Kompetenzentwicklung von Lehramtsstudierenden während des Praktikums (S. 67-93). Schneider.

Schnebel, S. (2010). Organisationsinterne Beratung in Ausbildungssituationen - Prozesse zwischen angehenden Lehrkräften und ihren MentorInnen. In M. Göhlich, S. Weber, W. Seitter, \& T. Feld (Hrsg.), Organisation und Beratung (S. 91-102). VS. 
Schön, D. (1987). Educating the Reflective Practitioner. Towards a New Design for Teaching and Learning in the Professions. Jossey-Bass.

Schreyögg, A. (2004). Supervision. VS.

Schüpbach, J. (2007). Über das Unterrichten reden. Die Unterrichtsnachbesprechung in den Lehrerpraktika-Eine "Nahtstelle von Theorie und Praxis»? Haupt.

Schütze, F. (1996). Organisationszwänge und hoheitsstaatliche Rahmenbedingungen im Sozialwesen: Ihre Auswirkungen auf die Paradoxien des professionellen Handelns. In A. Combe, \& W. Helsper (Hrsg.), Pädagogische Professionalität. Untersuchungen zum Typus pädagogischen Handelns (S. 183-275). Suhrkamp.

Stangl, W. (o. J.). Stichwort: Mäeutik-Maieutik. In Online Lexikon für Psychologie und Pädagogik. Abgerufen am 27. August 2019 von https://lexikon.stangl.eu/7898/ maeutik-maieutik

Stender, P. (2016). Wirkungsvolle Lehrerinterventionsformen bei komplexen Modellierungsaufgaben. Springer.

Stichweh, R. (1996). Professionen in einer funktional differenzierten Gesellschaft. In A. Combe \& W. Helsper (Hrsg.), Pädagogische Professionalität. Untersuchungen zum Typus pädagogischen Handelns (S. 49-69). Suhrkamp.

Stichweh, R. (2013). Wissenschaft, Universität, Professionen. Soziologische Analysen (2. Aufl.). Transcipt.

Strauss, A. L., \& Corbin, J. M. (1996). Grounded theory: Grundlagen qualitativer Sozialforschung. PsychologieVerlagsUnion.

Strübing, J., Hirschauer, S., Ayass, R., Krähnke, U., \& Scheffer, T. (2018). Gütekriterien qualitativer Sozialforschung. Ein Diskussionsanstoß. Zeitschrift für Soziologie, 47(2), 83-100.

Struck, P., \& Würtl, I. (2015). Lehrer der Zukunft: Vom Pauker zum Coach (2. Aufl.). Wissenschaftliche Buchgesellschaft.

Terhart, E. (1992). Lehrerberuf und Professionalität. In B. Dewe, W. Ferchhoff, \& F.-O. Radtke (Hrsg.), Erziehen als Profession. Zur Logik professionellen Handelns in pädagogischen Feldern (S. 103-131). Leske und Budrich.

Terhart, E. (2000). Lehrerbildung und Professionalität. Strukturen, Probleme und aktuelle Reformtendenzen. In J. Bastian, W. Helsper, S. Reh, \& C. Schelle (Hrsg.), Professionalisierung im Lehrerberuf. on der Kritik der Lehrerrolle zur pädagogischen Professionalität (S. 73-85). Leske und Budrich.

Terhart, E. (2001). Lehrerberuf und Lehrerbildung. Forschungsbefunde, Problemanalysen, Reformkonzepte. Beltz.

Terhart, E. (2011). Lehrerberuf und Professionalität: Gewandeltes Begriffsverständnis Neue Herausforderungen. Beiheft der Zeitschrift für Pädagogik, 57, 202-224.

von Felten, R. (2005). Lernen im reflexiven Praktikum: Eine vergleichende Untersuchung. Waxmann. 
von Felten, R., \& Herzog, W. (2001). Von der Erfahrung zum Experiment. Angehende Lehrerinnen und Lehrer im reflexiven Praktikum. Beiträge zur Lehrerbildung, 19(1), 29-42.

von Schlippe, A., \& Schweitzer, J. (2011). Lehrbuch der systemischen Therapie und Beratung II. Vandenhoeck \& Ruprecht.

Wagner, H.-J. (1998). Eine Theorie pädagogischer Professionalität. DtStudien-Verlag.

Wahl, D. (2013). Lernumgebungen erfolgreich gestalten. Vom trägen Wissen zum kompetenten Handeln (1. Aufl.). Julius Klinkhardt.

Weber, M. (1925). Wirtschaft und Gesellschaft (2. Aufl., Bd. 3). Mohr.

Wenzl, T. (2014). Elementarstrukturen unterrichtlicher Interaktion. Springer.

Wepfer, M. (2017). Erfolgsfaktoren für Berufseinstieg und berufliche Orientierung. Bildung Schweiz, 9, 11.

Wernet, A. (2003). Pädagogische Permissivität: Schulische Sozialisation und pädagogisches Handeln jenseits der Professionalisierungsfrage. Leske und Budrich.

Wernet, A. (2009). Einführung in die Interpretationstechnik der objektiven Hermeneutik (3. Aufl., Bd. 11). VS.

Wernet, A. (2014). Überall und nirgends. Ein Vorschlag zur professionalisierungstheoretischen Verortung des Lehrerberufs. In S. Leser (Hrsg.), Zuneigung. Pädagogik und Widerspruch (S. 77-96). Barbara Budrich.

Wernet, A. (2018a). Entgrenzung. In M. Proske \& K. Rabenstein (Hrsg.), Kompendium Qualitative Unterrichtsforschung: Unterricht beobachten - Beschreiben Rekonstruieren (S. 240-258). Julius Klinkhardt.

Wernet, A. (2018b). Über das spezifische Erkenntnisinteresse einer auf die Rekonstruktion latenter Sinnstrukturen zielenden Bildungsforschung. In M. Heinrich (Hrsg.), Rekonstruktive Bildungsforschung (S. 125-139). Springer Fachmedien.

Wernet, A. (2019). Wie kommt man zu einer Fallstrukturhypothese? In D. Funcke \& T. Loer (Hrsg.), Vom Fall zur Theorie (S. 57-84). Springer.

Windl, E. (2020). Mentoring in den Pädagogisch-praktischen Studien - Von der Meisterlehre zum kollaborativen Mentoring. In J. Dammerer, C. Wiesner, \& E. Windl (Hrsg.), Mentoring im pädagogischen Kontext. Professionalisierung und Qualifizierung von Lehrpersonen (S. 27-37). Studienverlag.

Ziegler, A., Stöger, H., \& Schimke, D. (2009). Mentoring: Theoretische Hintergründe, empirische Befunde und praktische Anwendungen. Pabst Science Publishers. 


\section{Tabellenverzeichnis}

Tabelle 1: Idealtypische Zuspitzung der Interaktionsmodi

Tabelle 2: Aspekte und Modi der Bezugnahme 


\section{$\S 12$ Versicherung}

1. Die Gelegenheit zum vorliegenden Promotionsvorhaben ist mir nicht kommerziell vermittelt worden. Insbesondere habe ich keine Organisation eingeschaltet, die gegen Entgelt Betreuerinnen und Betreuer für die Anfertigung von Dissertationen sucht oder die mir obliegenden Pflichten hinsichtlich der Prüfungsleistungen für mich ganz oder teilweise erledigt.

2. Ich versichere, dass ich die eingereichte Dissertation

\section{Studierende im Mentorat Strukturlogiken und Interaktionsdynamiken eines}

Hochschulformats zur Unterstützung individueller Professionalisierung in der Lehrerinnen- und Lehrerbildung

selbständig und ohne unerlaubte Hilfsmittel verfasst habe; fremde Hilfe habe ich dazu weder entgeltlich noch unentgeltlich entgegengenommen und werde das auch künftig so halten. Anderer als der von mir angegebenen Hilfsmittel und Schriften habe ich mich nicht bedient. Alle wörtlich oder sinngemäss den Schriften anderer Autoren entnommenen Stellen habe ich kenntlich gemacht.

3. Die eingereichte Dissertation habe ich nicht bereits in einem anderen Prüfungsverfahren vorgelegt.

4. Des Weiteren ist mir bekannt, dass Unwahrheiten hinsichtlich der vorstehenden Erklärung die Zulassung zur Promotion ausschliessen beziehungsweise später zum Verfahrensabbruch oder zur Rücknahme des erlangten Titels berechtigen.

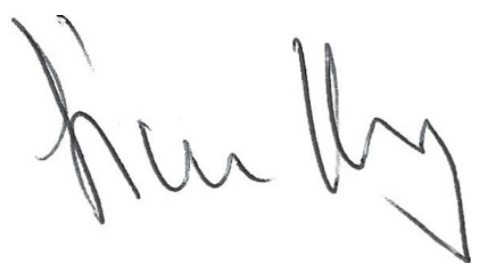




\section{Curriculum Vitae von Simone Herzog}

Geburtsdatum und -ort

Adresse

Staatsangehörigkeit

Aus- und Weiterbildung

$2017-2021$

$2016-2017$

$2013-2015$

$2004-2007$

$2003-2004$

$1996-1998$

$1993-1996$

$1991-1993$

$1986-1991$

$1982-1986$

$1977-1982$

\section{Berufstätigkeit}

seit 2015
16. März 1970, Zürich

Walther-Hauser-Str. 4b

8820 Wädenswil

Schweiz

Schweiz

Doktorandin an der Universität Göttingen bei Prof. Dr. Katharina Kunze

CAS in Mentoring und Coaching der Universität, Zürich

Studium an der Universität Basel

«Master of Arts in Educational Sciences»

Schwerpunkt:

Bildungstheorie und Bildungsforschung

Studium an der Fachhochschule Nordwestschweiz zur Stufenlehrperson Sekundarstufe I für die Fächer Deutsch, Französisch und Englisch «Bachelor of Arts in Educational Sciences» Ergänzungsstudium zur Englisch-Fachlehrperson für die Sekundarstufe I an der Fachhochschule Nordwestschweiz

Ausbildung zur Primarlehrerin an der Höheren Pädagogischen Lehranstalt in Zofingen

Ausbildung zur Juniororganisatorin bei der UBS Einsatz im Produktmanagement Firmenkundengeschäft als Assistentin des Sektionsleiters

Allroundpraktikum bei der Schweizerischen Bankgesellschaft (UBS) in Zürich, Hauptsitz Kantonsschule in Baden (Matura Typus E) Bezirksschule in Mutschellen Primarschule in Oberwil-Lieli

Pädagogische Hochschule Zürich - Studiengang Sekundarstufe I

Mentorin

Dozentin für Berufliche Orientierung, Unterrichtsqualität und

Kommunikation

Betreuung von Masterarbeiten und 
Vertiefungsarbeiten

Betreuung von Vertiefungsarbeiten

Verantwortliche für die Portfolioarbeit und das

Lernvikariat

Pädagogische Hochschule Nordwestschweiz Institut Kindergarten und Unterstufe

Wissenschaftliche Mitarbeiterin

Mentorin, Reflexionsseminarleitende

Betreuung einer Partnerschule

Dozentin für Forschungsmodule

$2010-2015$

Bezirksschullehrerin in Seengen für die

$2008-2015$

Fächer Französisch, Englisch und Deutsch

Praxislehrerin Sekundarstufe I

$2005-2010$

Bezirksschullehrerin in Spreitenbach für die

$2002-2005$ Fächer Französisch, Englisch und Deutsch

Primarlehrerin in Baden-Rütihof

Weiterbildung zur Praxislehrerin

$1998-2002$

Primarlehrerin in Oberwil-Lieli

$1991-1996$

UBS Zürich, Firmenkundengeschäft

\section{Sprachkenntnisse}

Deutsch

Französisch

Muttersprache

Französisch

Diplôme d'Études en langue française (DALF), 2005

Cambridge Certificate in Proficiency, 2012 
Erstgutachterin:

Weitere Betreuer:

Weitere Mitglieder der Prüfungskommission:

Tag der mündlichen Prüfung:
Prof. Dr. Katharine Kunze, Göttingen

Prof. Dr. Tobias Leonhard, Solothurn

Prof. Dr. Kerstin Rabenstein, Göttingen 